\title{
Selection of Dominant
}

Radionuclides for Phase I of

the Hanford Environmental

Dose Reconstruction Project

B. A. Napier

July1991
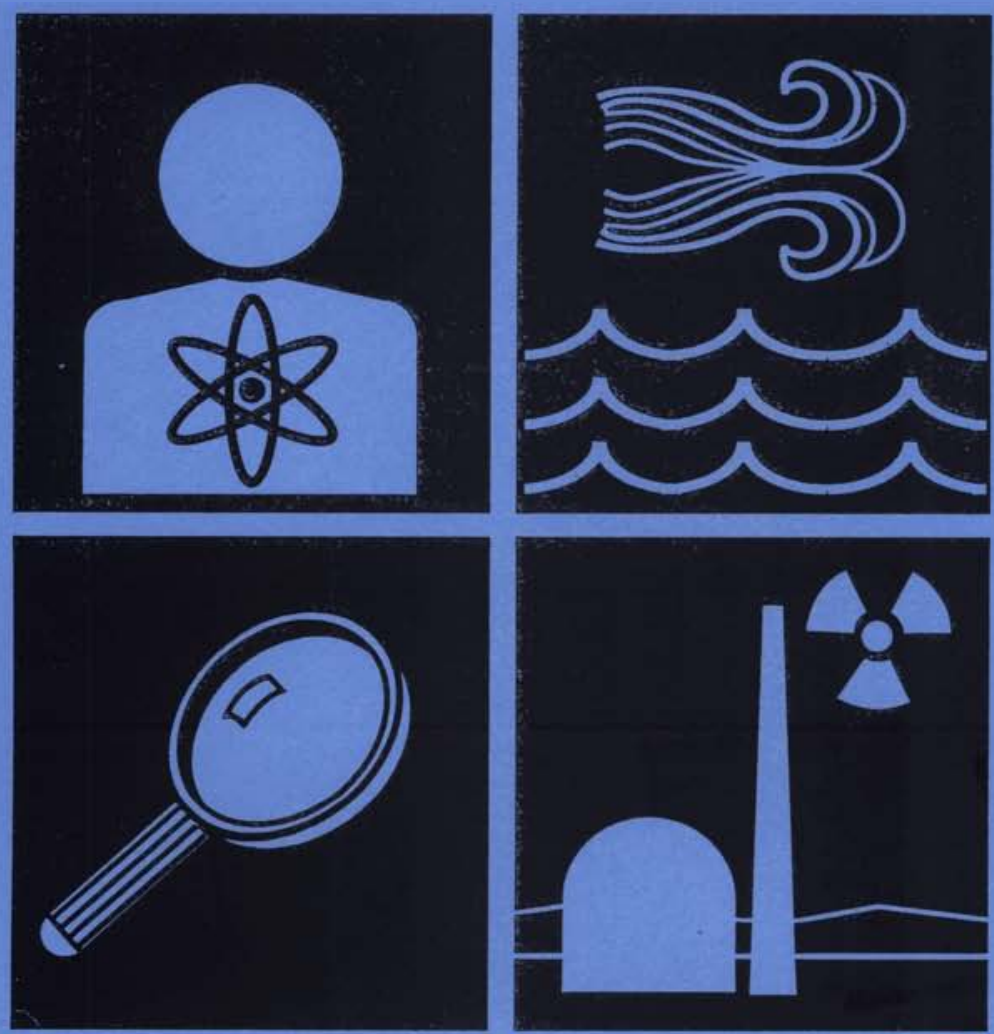

Prepared for the Technical Steering Panel 


\title{
DISCLAIMER
}

This report was prepared under the direction of the HANFORD ENVIRON MENTAL DOSE RECONSTRUCTION PROJECTTechnical Steering Panel by Battelle Memorial Institute's Pacific Northwest Laboratories operating the Pacific Northwest Laboratory for the U.S. Department of Ener:y (DOE). While funding for the work wa provided by DOE, the work is not under W E direction or control. The views and opinions of the authors expressed in this document do not necessarily reflect those of the United States Covernment or any agency thereof. Reference herein to any specific commencial product, process or service by trade name, trademark, manufacturer or otherwise does not necessarily constitute or imply its endorsement, recommendation or favoring by the US. Covernment or any agency thereof, nor by Battelle Memorial Institute.

\author{
Printed in the United States of America \\ Avalable to DOE and DOE contractors from the \\ Ofince of Scientific and Technical Information, P.O. Dox 62, Oak Ridge, TN 37831 ; \\ prices available from (615) 576-8401. FTS 626-8401. \\ Available to the public from the National Technical Information Service, \\ US. Departinent of Commerce, 5285 Port Royal Rd, Springfield, VA 22161.
}


PNL-7231 HEDR

UC-707

SELECTION OF DOMINANT RADIONUCLIDES

FOR PHASE I OF THE HANFORD ENVIRONMENTAL

DOSE RECONSTRUCTION PROJECT

HANFORD ENVIRONMENTAL DOSE

RECONSTRUCTION PROJECT

B. A. Napier

July 1991

Prepared for

the Technical Steering Panel

Pacific Northwest Laboratory

Richland, Washington 99352 



\section{SELECTION OF DOMINANT RADIONUCLIDES FOR PHASE I OF THE HANFORD ENVIRONMENTAL DOSE RECONSTRUCTION PROJECT}

July 1991

This document has been reviewed and approved by the Technical SteeringPanel.

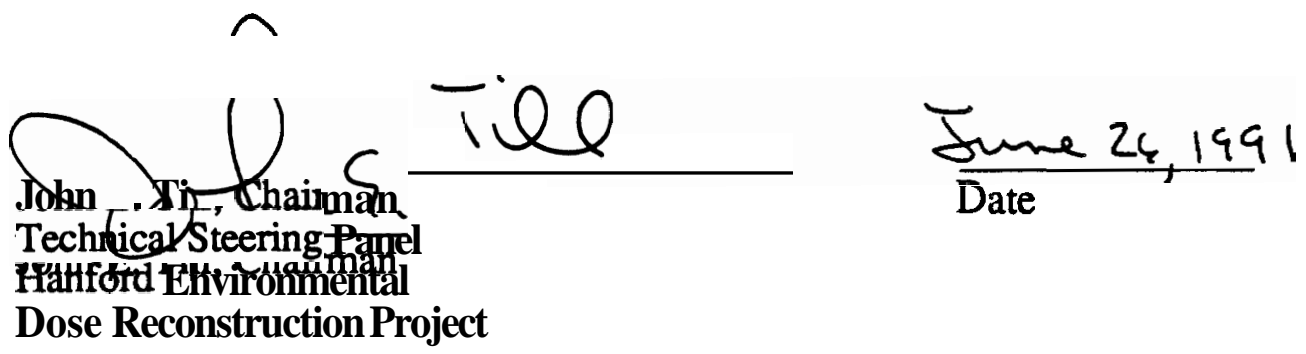





\section{PREFACE}

In recent years, concern has been mounting about possible health effects to the public from over 40 years of operations at the Hanford Site. This concern prompted the Washington State Nuclear Waste Board and the Indian Health Service to request the Centers for Disease Control to convene a Hanford Health Effects Review Panel to review and evaluate epidemiological and environmental data relevant to Hanford operations. In 1986, based on this review, the Panel recommended that two studies be initiated: one to reconstruct radiation doses potentially received by the public residing in the vicinity of Hanford and a separate study of thyroid morbidity in the same population. The Hanford Environmental Dose Reconstruction (HEDR) Project is the outgrowth of that recommendation. The project is directed by an independent pane7 of scientists and experts cal7ed the Technical Steering Pane7 (TSP). Scientists at the Pacific Northwest Laboratory conduct the work.

The HEDR Project is divided into the following technical tasks. These tasks address each of the primary steps in the path from radioactive releases to dose estimates:

Source Terms

- Environmental Transport

Environmental Monitoring Data

- Demography, Food Consumption and Agricu7ture

- Environmental Pathways and Dose Estimates.

The Source Terms Task will develop estimates of radioactive emissions from Hanford facilities since 1944. These estimates will be based on historical measurements and production information.

The Environmental Transport Task will reconstruct the probable movement of radioactive materials from the areas of release to populations. Movement via the atmosphere, surface water (Columbia River), and groundwater will be studied.

The Environmental Monitoring Data task will assemble, evaluate, and report historical monitoring data. 
The Demography, Food Consumption and Agriculture Task wi 11 develop the data needed to determine which population groups could have been affected by the releases. Population and demographic information wi 11 be developed for the general population within the study area. This information will also be developed for several special population groups including the Native American tribes in the study area, military personnel stationed at Hanford, Hanford construction workers, and migrant farm workers. The food and water consumption patterns and practices and sources of food and water must also be estimated.

Historical dairy farming practices and milk distribution systems will be studied because milk is a significant pathway for iodine-131 to enter the human body. Cows could have eaten vegetation contaminated with this radionuclide.

The Environmental Pathways and Dose Estimates Task will use the information produced by the other tasks to estimate the radiation doses people could have received from Hanford operations.

This report is the result of a collaboration of the Source Terms and Environmental Pathways and Dose Estimates Tasks. Additional information was provided by the Demography, Food Consumption and Agriculture Task. Selection of dominant radionuclides cannot be made without consideration of the resulting doses, and these in turn are functions of the types of human exposures assumed. As more detailed information becomes available, the estimates of relative importance of these radionuclides will be reviewed. 


\section{FOREWORD}

Appendix $F$ is a record of TSP comments and Battelle's responses; the TSP has reviewed and approved these responses to their comments. Comment numbers appear in this report in the 7eft margin next to the paragraph in which the corresponding comment was addressed. Changed text is shown in italics. In addition to changes to address TSP comments, source text has been changed to correct errors and for further clarification. 

SUMMARY

22. 23

The objective of the Hanford Environmental Dose Reconstruction (HEDR)

24,25 Project is to estimate the radiation dose that individuals could have received as a result of emissions from nuclear operations at Hanford since their inception in 1944. The purpose of this report is to identify the most important radionuclides released to the environment from Hanford in terms of contribution to potential off-site radiation dose. This information will be used to determine which radionuclides should be included in subsequent studies to determine doses from air and surface water that were received by the population around Hanford. For the time periods of 1944-1947 for atmospheric releases and 1964-1966 for releases to surface water (termed HEDR's Phase I time periods), certain radionuclides tend to dominate the dose estimates. Dose calculations were made with preliminary information for the Phase I time periods, permitting rankings of radionuclides. The measure of dose used for rankings is effective dose equivalent, a measure that incorporates doses to various organs in the body into a single unit.

It was found that for the air releases, ${ }^{131}$ I accounted for more than 90\% of all doses received via all pathways of exposure. Addition of the longerlived radioisotopes of ruthenium $\left({ }^{106} \mathrm{Ru}\right.$ and $\left.{ }^{103} \mathrm{Ru}\right)$ accounted for approximately 99\% of the dose. For releases to surface water, the results are less definitive, but inclusion of only 5 to 10 radionuclides accounted for the great majority of the dose to the more highly exposed individuals. It was determined that for Phase I, inclusion of at least ${ }^{32} \mathrm{p},{ }^{239} \mathrm{~Np},{ }^{65} \mathrm{Zn},{ }^{76} \mathrm{As}$, and ${ }^{64} \mathrm{Cu}$ is essential, with the inclusion of ${ }^{56} \mathrm{Mn},{ }^{24} \mathrm{Na},{ }^{46} \mathrm{Sc}$, and ${ }^{51} \mathrm{Cr}$ highly desirable. 



\section{ACKNOMEDGMENIS}

The author would like to acknowledge the efforts of C. M. Heeb for providing the series of ORIGEN2 (Croff 1980a) source runs, L. G. Morgan for estimates of release fractions for various radionuclides in the early years, J. E. Till for suggesting the use of the National Council on Radiation Protection (NCRP) screening factors as a second approach, and K. Rhoads for implementing the spreadsheet application of the ORIGEN2 results with the NCRP screening factors. 

Ac actinium

Ag silver

Al aluminum

Am americium

Ar argon

As arsenic

At astatine

$\mathrm{Au}$ gold

B boron

$\mathrm{Ba}$ barium

Be beryliium

$\mathrm{Bi}$ bismuth

Bk berkelium

$\mathrm{Br}$ bromine

C carbon

Ca calcium

Cd cadmium

Ce cerium

Cf californiurn

Cl chlorine

$\mathrm{Cm}$ curium

Co cobalt

$\mathrm{Cr}$ chrornium

Cs cesium

Cu copper

Dy dysprosium

Er erbium

Es einsteinium

Eu europium

$F$ fluorine

Fe iron

Fm ferrnium

$\mathrm{Fr}$ francium

Ga gallium

Gd gadolinium
Ge germani um

$\mathrm{H}$ hydrogen

He helium

$\mathrm{Hf}$ hafnium

$\mathrm{Hg}$ mercury

Ho holmium

I iodine

In indium

Ir iridium

$K$ potassium

$\mathrm{Kr}$ krypton

La lanthanurn

Li lithium

Lr lawrencium

Lu lutetium

Md mendelevium

$\mathrm{Mg}$ magnesi um

$\mathrm{Mn}$ manganese

Mo rnolybdenurn

$\mathrm{N}$ nitrogen

$\mathrm{Na}$ sodium

$\mathrm{Nb}$ niobium

Nd neodymi un

$\mathrm{Ne}$ neon

$\mathrm{Ni}$ nickel

No nobelium

$\mathrm{Np}$ neptunium

0 oxygen

os osmium

P phosphorus

$\mathrm{Pa}$ protactinium

$\mathrm{Pb}$ lead

Pd palladium

Pm proniethium

Po polonium
Pr praseodymium

Pt platinum

$\mathrm{Pu}$ plutonium

$\mathrm{Ra}$ radium

$\mathrm{Rb}$ rubidium

$\mathrm{Re}$ rhenium

Rh rhodium

$\mathrm{Rn}$ radon

$\mathrm{Ru}$ ruthenium

$S$ sulfur

$\mathrm{Sb}$ antimony

Sc scandium

$\mathrm{Se}$ selenium

SF spontaneous

fission

Si silicon

$\mathrm{Sm}$ samarium

Sn tin

Sr strontium

Ta tantalum

Tb terbium

Tc technetium

$\mathrm{Te}$ tellurium

Th thorium

Ti titanium

TI thallium

Tm thulium

U uranium

$\checkmark$ vanadium

$W$ tungsten

$\mathrm{Xe}$ xenon

$Y$ yttrium

$Y b \quad y t t e r b i u m$

Zn zinc

Zr zirconium 

CONTENTS

PREFACE ................................. $i$...

FOREWORD . . . . . . . . . . . . . . . . . . . . . . . . . . . . v v

SUMMARY ............................ ... . . . . . . . . . . .

ACKNOWLEDGMENTS ...................... . . $\mathbf{i x}$

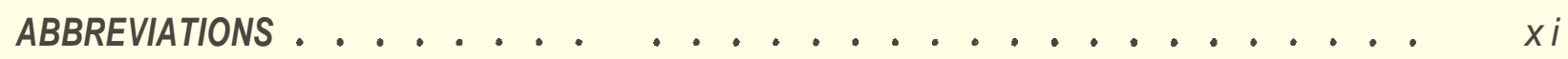

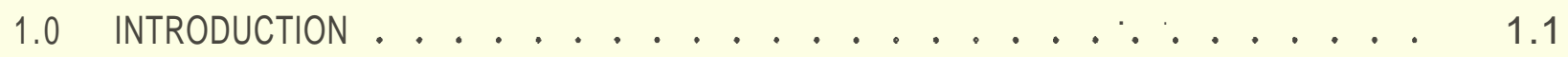

1.1 NEED FOR RADIONUCLIDE SELECTION . . . . . . . . . 1.2

1.2 MODELS USED FOR CALCULATIONS . . . . . . . . . 1.3

2.0 PRELIMINARY SOURCE TERM NORMALIZATION . . . . . . . . 2.1

2.1 ATMOSPHERIC RELEASES . . . . . . . . . . . 2.1

2.2 SURFACE WATER AND GROUNDWATER RELEASES . . . . . 2.3

3.0 ENVIRONMENTAL EXPOSURE PATHWAYS . . . . . . . . . . 3.1

3.1 ATMOSPHERIC RELEASE SIMULATIONS . . . . . . . . . 3.2

3.2 SURface WATER RELEASE SIMULATIONS . . . . . . . . . 3.4

3.3 EXPOSURE PATHWAY MODELS . . . . . . . . . . . . . . 3.4

3.4 THE NCRP SCREENING FACTORS . . . . . . . . . . 3.5

4.0 RADIONUCLIDE RANKINGS ....................... 4.1

4.1 ATMOSPHERIC RELEASES . . . . . . . . . . . 4.1

4.2 SURFACE WATER RELEASES . . . . . . . . . . 4.8

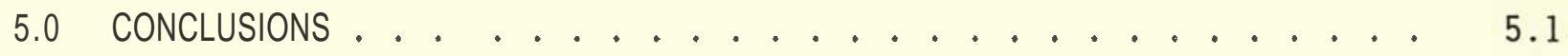

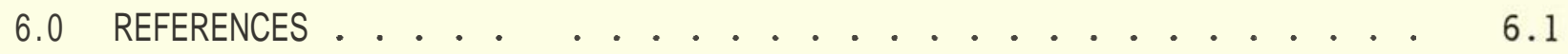

APPENDIX A - ORIGEN2 RESULTS FOR 500 MWd/t FUEL . . . . . . A A.1

APPENDIX B - GENII RESULTS FOR ATMOSPHERIC RELEASES ....... B. . .

APPENDIX C - GENII RESULTS FOR SURFACE WATER RELEASES ....... C. C.1

APPENDIX D - NCRP SCREENING FACTOR RESULTS . . . . . . . . D. D 


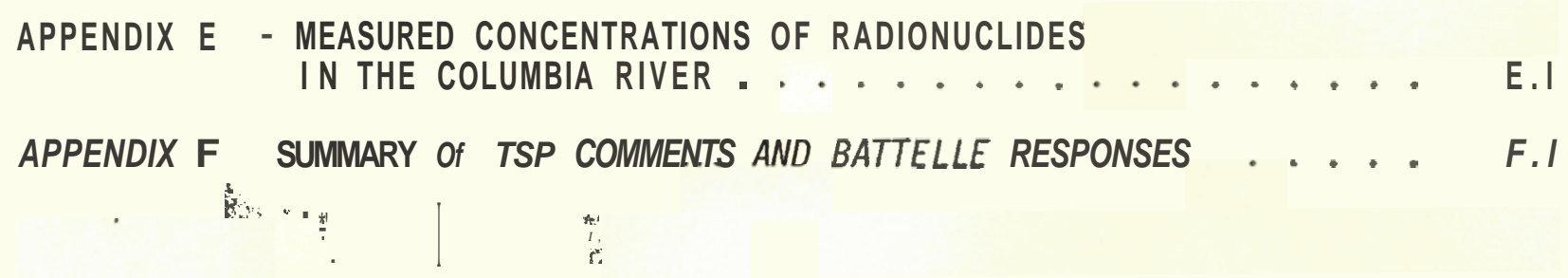




\section{FIGURES}

3.1 Various Generic Exposure Pathways . . . . . . . . . . . 3.2

4.1 Dose as a Function of Level Burnup ............... . 4.5

4.2 Dose as a Function of Cooling Time . . . . . . . . 4.6 


\section{TABLES}

2.1 Average Monthly Cooling Times for Fuel Before Dissolution . . . 2.2

3.1 Dietary and Exposure Parameters for the Hanford Maximally Exposed Individual ....................

4.1 Dominant Radionuclides in Atmospheric Releases as a Function of Fuel Burnup (30-Day Cooled Fuel) Ranked by Percent of Contribution to Effective Dose Equivalent . . . . . . . .

4.2 Dominant Radionuclides in Atmospheric Releases as a Function of Fuel Cooling Time ( $500-$ MWd/t Fuel) Ranked by Percent of Contribution to Effective Dose Equivalent ...........

4.3 Dominant Radionuclides in Atmospheric Releases Calculated

By Three Different Techniques for 500-MWd/t Fuel Cooled 30 Days, Ranked by Percent of Contribution to Effective Dose Equivalent

4.4 Dominant Radionuclides in Atmospheric Releases for Three Exposure Scenarios for 500-MWd/t Fuel Cooled 30 Days, Ranked by Percent of Contribution to Effective Dose Equivalent . . . . . . . .

4.5 Dominant Radionuclides from Atmospheric Releases for Two Dose Integration Techniques for 500-MWd/t Fuel Cooled 30 Days, Ranked by Percent of Contribution to Effective Dose Equivalent . .

4.6 Dominant Radionuclides for Surface Water Releases for a Maximall y Exposed Individual, Calculated from Concentrations Measured in 1956, 1964, and 1968 and Ranked by Percent of Contribution to Effective Dose Equivalent . . . . . . . .

4.7 Dominant Radionuclides for Surface Water Releases for Three Scenarios of Exposure, Calculated from Concentrations Measured in 1968 and Ranked by Percent of Contribution to Effective Dose

Equivalent . . . . . . . . . . . . . . . . .

4.8 Dominant Radionuclides Associated with a Hypothetical Release of 1 1b. of 500-MWd/t Fuel to Surface Water Calculated for Drinking and All-Pathways Scenarios and Ranked by Percent of Contribution to Effective Dose Equivalent 


\subsection{INTRODUCTION}

1.9 The objective of the Hanford Environmental Dose Reconstruction (HEDR) Project is to estimate the radiation dose that individuals could have received as a result of emissions from nuclear operations at Hanford since their inception in 1944. (a) A vital step in the estimation of radiation doses is the determination of the "source term," that is, the quantities of radionuclides that were released to the environment from the various Hanford operations. Hanford operations have at various times involved hundreds of different radionuclides, some in relatively large quantities. Those radionuclides present in the largest quantities, although significant from an operational handling point of view, may not necessarily have been those of greatest concern for offsite radiation dose. This report documents the selection of the dominant radionuclides (those that may have resulted in the largest portion of the received doses) in the source term for Phase I of the HEDR Project, that is, for atmospheric releases from 1944 through 1947 and for surface water releases from 1964 through 1966.

In response to a directive from HEDR's TSP, the reconstruction of doses to offsite populations began with an initial "development and testing" phase. The objective of Phase $I$ is to demonstrate through calculation that adequate models and support data exist or can be developed to allow estimation of realistic doses to individuals from releases of radionuclides to the environment that occurred as long as 45 years ago. Much of the data being used in Phase I is preliminary or approximate, and therefore the doses calculated must also be considered to be preliminary approximations. Within the constraints of Phase I, the number of radionuclides investigated in detail is small. As more information is developed, more radionuclides will be addressed and the dose estimates will become more reliable. In addition, as the project addresses times later than the 1944-47 period for atmospheric releases, when effluent control technologies were improved, the release fractions for the various species will change. This difference will result in large changes in the relative significance of the various radionuclides. Additional time periods

(a) The project is being managed and conducted by Pacific Northwest Laboratory (PNL) under the direction of an independent Technical Steering Panel (TSP) . 
will also be addressed for the surface water pathways, which may result in the need to revise the selection of radionuclides of interest for that pathway. Therefore, studies such as this will need to be performed throughout the HEDR Project to address changing information requirements.

\subsection{NEED FOR RADIONUCLIDE SELECTION}

Nuclear processes at Hanford have resulted in the production of hundreds of different radionuclides. Most of these radionuclides have had no significant impact on individuals because the released quantities have been small., the radiological half-lives have been short, or the human exposure to them has been minimal. However, it is important to determine which radionuclides have had the potential to result in radiation doses to offsite human populations.

Because of the large number of facilities and processes that have been used at Hanford over the past 45 years, it is difficult to adequately simulate the releases of all radionuclides. Historical environmental monitoring has investigated either gross radioactivity or prominent tracer isotopes. It was not possible to measure many of the nuclides at the concentrations present in the effluent or the environment. Therefore, in order to productively limit the amount of data to be reconstructed, it is beneficial to focus on the radionuclides of most importance in the dose calculations. (a)

The dominant radionuclide selection process could eventually encompass the dominant radionuclides from each Hanford Site operating area and from the specific facilities located in each area. The dominant radionuclides will differ for emissions to the air, surface water, and groundwater. For Phase I, attention has been focused on the facilities where the major releases occurred: for the atmospheric releases, on the nuclear fuel chemical separation facilities; and for the surface water releases, on the production reactors. By using monitoring data on Columbia River concentrations downstream of the Hanford Site, any potential groundwater releases that might have reached

(a) This limitation applies not only to the results of the source term task of the HEDR project, but also to the data investigated in detail by the monitoring task, the modeling of transport of minor constituents by the environmental transport task, and the data and data-handling requirements of the Environmental Pathways and Dose Estimates Task. 
the river are automatically included. Thus, groundwater pathways have not been addressed explicitly, but are included in surface water calculations.

\subsection{MODELS USED FOR CALCULATIONS}

The work described in this report was conducted in accordance with the requirements of ANSI/ASME NQA-1 1986 Edition (ASME 1986), Oualitv Assurance Program Reauirements for Nuclear Facilities, as interpreted by the PNL Quality Assurance (QA) program.

All procedures used to support this report were written and controlled in accordance with PNL QA program requirements. Records that support the data in this report were created and stored in accordance with applicable HEDR Project record control requirements. These included requirements on the generation of computer output, verification of computer codes, and review of all intermediate output. The ORIGEN2 code (Croff 1980a, 1980b), a generally accepted reactor fuel simulation model, was verified before use. The GENII code (Napier et a7. 1988) was developed at PNL, also under the procedures of ASME/ANSI NQA-1. It is maintained by the Hanford Dose Overview Program, and only authorized versions are distributed for use.

For the convenience of those who may wish to perform additional analyses of the basic data used to draw the conclusions presented in this report, all intermediate calculations are presented in the appendices to this document.

Drafts of this document underwent internal independent technical review. Review comments were satisfactorily resolved, and there were no controversial resolutions to the comments. 



\subsection{PREIMINARY SOURCE TERM NORMALVATION}

Although estimation of the total source term has not yet been completed, it is possible to estimate dominant radionucl ides. The early releases from the Hanford operations were largely continuous with each radionuclide retaining about the same relative fractional contribution to the total released activity. That is, the relative contribution of each radionuclide to the total dose was nearly constant. Therefore, it is not necessary to know the total quantity released in order to rank the radionuclides by importance. A unit source term can be assumed to be released and the radiation doses calculated from that source term can be ranked to determine the overall importance of each radionuclide. This method of normalizing source term contributions from radionucl ides is used in the following sections for atmospheric and surface water releases.

\subsection{ATMOSHHERC RELEASES}

The results of the study to date indicate the largest releases of radionuclides to the atmosphere during the Phase I study period occurred during the chemical separation of irradiated fuel in the T-Plant and B-Plant facilities (Ballinger and Hal 1 1991). The quantities of each radionuclide avai lable for release at any time are functions of the amount of irradiated fuel being dissolved, the irradiation history of the fuel, and the time elapsed since the fuel was removed from the reactor. The amount actually released further depends on the separation process used and on the efficiency of the facility effl uent controls.

The Hanford production reactors were originally designed to operate at a power level of about $275 \mathrm{MW}$ and they rapidly reached and maintained that lev'el during the Phase I period (Hanthorn 1957). The reactor power level prior to fuel discharge determines the levels of short-lived radionuclides in the fuel. The total fuel irradiation (fuel power level $x$ time) determines the quantities of long-lived radionuclides produced. The period of time following discharge from the reactor provides the opportunity for the shorter-lived radionucl ides to decay to lower concentrations in the fuel. Optimal production of plutonium product (high in the desired ${ }^{239} \mathrm{Pu}$ but low in contaminant 
${ }^{240} \mathrm{Pu}$ ) is obtained at a fuel irradiation level ("burnup") of between 300 and 500 megawatt-days per ton (MWd/t), although much of the fuel in the first several years was irradiated to somewhat lower levels than this (DeNeal 1965). As illustrated in Table 2.1, the time elapsed between fuel removal from the reactor and start of dissolution (cooling times) for the Phase I time frame ranged from about 30 to about 90 days, with the potential for a few batches at shorter or longer times. The potential for variations in the fuel composition at dissolution was investigated through a series of burnup calculations using the ORIGEN2 computer code (Croff 1980a, 1980b).

TABLE 2.1. Average Monthly Cooling Time (Days) for Fuel Before Dissolution

\begin{tabular}{|c|c|c|c|c|}
\hline Month & $\underline{1944}$ & $\underline{1945}$ & $\underline{1946}$ & $\underline{1947}$ \\
\hline January & $\ldots(a)$ & 39 & 62 & 62 \\
\hline February & -- & 53 & 67 & 69 \\
\hline March & -- & 52 & 70 & 69 \\
\hline April & -- & 42 & 62 & 68 \\
\hline May & -- & 36 & 62 & 68 \\
\hline June & -. & 35 & 73 & 77 \\
\hline July & -- & 40 & 68 & 78 \\
\hline August & -- & 39 & 63 & 82 \\
\hline September & -- & 38 & 59 & 84 \\
\hline October' & -- & 44 & 65 & 90 \\
\hline November & -- & 52 & 65 & 95 \\
\hline December & 49 & 47 & 62 & 95 \\
\hline
\end{tabular}

(a) Dashes (--) indicate that no fuel was
dissolved in that month. 
The ORIGEN2 code was run with Hanford reactor parameters for a single ton of fuel for burnups ranging from 100 to $1000 \mathrm{MWd} / \mathrm{T}$, and with cooling times ranging from 10 to 90 days. A sample portion of the resulting output is displayed in Appendix A. These calculated inventories per ton were used in the atmospheric release calculations described in section 4.0.

The effluent monitoring technology in existence in the mid-1940s was not capable of differentiating various radionuclides. Only estimates can be made of the fractions of the various chemicals available in the dissolving fuel mixture that escaped from the facilities. These are approximately $100 \%$ for nonreactive noble gases, $50 \%$ to $85 \%$ for isotopes of iodine, about $1 \%$ for isotopes of ruthenium, and about $0.001 \%$ for all other nonvolatile species. A representative value of $70 \%$ was used in the following calculations for iodine in this report.

\subsection{SURFACE WATER AND GROUNDWATER RELEASES}

For Phase I calculations, surface water (Col umbia River) concentrations were evaluated, based on measured values. Contributions from groundwater migration to the river are implicit in these measured values for surface water. Therefore, no additional calculations were performed for groundwater releases.

The original eight plutonium production reactors were cooled with water from the Columbia River. The water was withdrawn from the river, treated to remove dirt and to provide optima7 water chemistry for reactor operations, passed through the reactors to coot the fuel, and returned to the river following holdup in basins or tanks for a period of up to a few hours. Various compounds in the river water, including naturally occurring dissolved uranium, passed through the reactors, absorbed neutrons, and became radioactive. Other materials formed very thin films on the inner walls of the cooling tubes or outer walls of the reactor fuel elements, and remained in the reactor long enough to also become radioactive. The discharge of water and dislodged films resulted in release of up to several thousand curies of short-lived materials per day. The radioactive constituents were functions of the treatment given the entering water, the flow rate, reactor construction materials, and operating parameters. Thus, calculation of the actual mix of discharged 
radionuclides is very difficult. However, a comprehensive effluent monitoring system developed over time, and during the later years of reactor operation, detailed records of measurements were maintained of the effluent water and of water downstream in the river. For this report, actual measurements of river concentrations are used for determining dominant radionuclides. '

Routine river water monitoring in the mid-1960s addressed about 10 radionuclides in regular detail (AEC 1965b). Numerous special studies of water flow rates, dilution, sediment transport, etc. (AEC 1965a) usually used 5 to 10 easily measured radionuclides. Concentrations of about 70 additional radionuclides were measured occasionally. Three detailed radiochemical analyses of reactor effluent water and Columbia River water were performed in 1958, 1964, and 1968. Records of these analyses were furnished in a private communication from J. P. Corley to J. K. Soldat on March 20, 1969, for use in a draft report on river-water concentrations that was never published. The original copy of that report was recovered from J. K. Soldat's files and used for this study. The concentrations derived from the Soldat draft are given in Appendix $\varepsilon$.

Operations with eight production reactors can be considered a nearly continuous process that results in a relatively constant rate of radionuclide input to the Columbia River. One or more reactors could be off-line at any one time for maintenance or refueling, but this would result in changes in river concentrations of only a few tens of percent. Only for one period during a labor strike, in mid-1966, were all reactors off-line at one time. Occasional events, such as reactor purges or fuel element failures, would add incremental increases of some radionuclides (Koop 1956; Jerman et a7. 1965). These increases were monitored through the river continuous monitoring systems, and these radionuclides are included in the source terms used in the calculations in Appendix B. A calculation based on an acute release of $11 \mathrm{~b}$. of uranium and its associated fission products, as predicted using the ORIGEN2 code, is also provided for perspective. 


\subsection{ENMRONMENTAL EXPOSURE PATHWAYS}

Potential routes through whi'ch people may be exposed to radionuclides or radiation are called exposure pathways. Examples of general pathways are external exposure, inhalation, and ingestion. The pathways are the ways people could be exposed in a given circumstance. Thus, an appropriate collection of exposure pathways can be considered to be the definition of an exposure scenario. Because a conceptual ization of the types of exposure must be made before the pathways can be fully defined, scenarios are usually defined before the parameters are selected for the pathways.

Various generic exposure pathways are illustrated in Figure 3.1. This figure illustrates how known or assumed values may be propagated through defined steps to estimate radiation doses. In most calculations, the known quantity is generally the release amount, called source terms in Figure 3.1. These terms may take the form of units of activity per unit of time for radioactivity released to the air, surface water, or groundwater. For each pathway, the source terms are converted via appropriate models to concentrations in air or water. Finally, human exposure parameters are factored in, and the dose is estimated.

The intent of this document is to indicate the radionuclides important to dose across a range of conditions. For the air pathway, this report investigates a range of potential fuel burnups, a range of potential fuel cooling times, and a range of potential exposure pathway conditions and individual exposures. This information is supplemented with independent estimates of the radionuclide impact embodied in the National Council on Radiation Protection (NCRP) screening factors. For the river pathway, it investigates a range of potential exposure pathway conditions and individual exposure mechanisms, and considers potential variability in the 'source term by applying monitoring data for a number of years (thus implicitly covering a range of reactor power levels and fuel conditions). The results can be thought of as a matrix of possible answers. The selection of dominant radionuclides is made based on those that frequent7y occur in the resulting 1ists. 

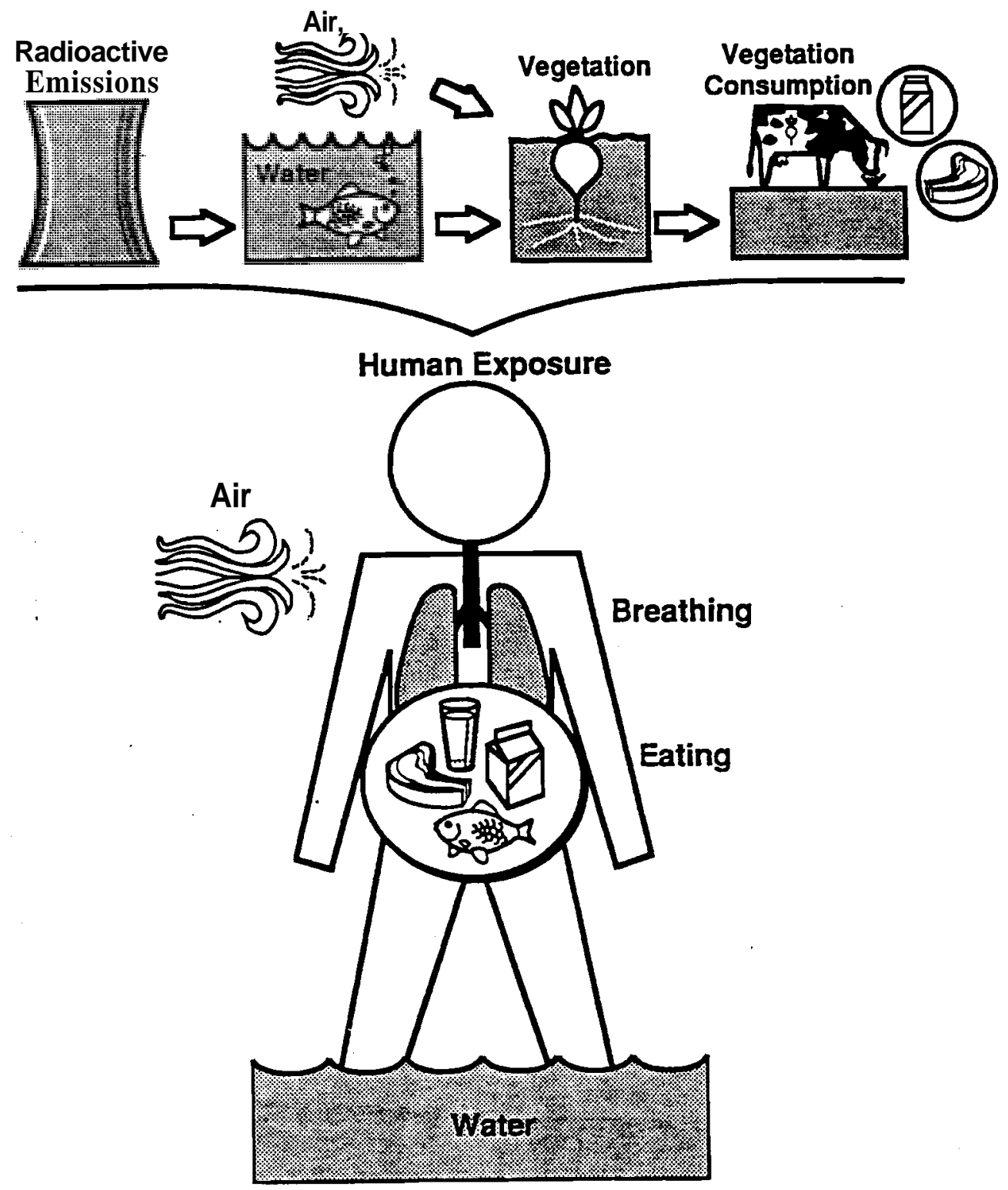

FIGURE 3.1. Various Generic Exposure Pathways

\subsection{ATMOSPHERIC RELEASE SIMULATIONS}

For releases to the atmosphere, the pathways considered in this report are submersion in contaminated air, inhalation, exposure to radionuclides deposited on the ground, ingestion of contaminated food crops, and consumption 
of contaminated animal products, such as milk and eggs. Only doses to individuals were calculated; the standard Hanford parameters for a "maximally exposed individual" were used. Phis set of parameters is based on the assumption that the individual maintains a constant residence at a single location; grows a large fraction of his or her personal food consumptian of vegetables, fruits, and grains; and raises dairy and beef cattle and poultry at that location. The dietary and exposure parameters, which are taken from McCormack et al. (1984), are listed in Table 3.1.

The parameters for the maximally exposed individual do not describe a reasonable lifestyle for many people who may not produce their own food products. Therefore, a number of additional variations of the pathways were also investigated. The first was to eliminate milk from the diet, to simulate the

TABLE 3.1. Dietary and Exposure Parameters for the Hanford Maximall y Exposed Individual (adapted from McCormack et al. 1984)

Consumption Rates, $\mathrm{kg} / \mathrm{vr}$

\begin{tabular}{|c|c|}
\hline $\begin{array}{l}\text { Leafy vegetables } \\
\text { Other vegetables } \\
\text { Fruits } \\
\text { Cereal grains } \\
\text { Meat } \\
\text { Poultry } \\
\text { Cow milk } \\
\text { Eggs } \\
\text { Fish } \\
\text { Drinking water }\end{array}$ & $\begin{array}{r}30 \\
220 \\
330 \\
80 \\
80 \\
18 \\
270 \\
30 \\
40 \\
730\end{array}$ \\
\hline \multicolumn{2}{|c|}{ Residencv Parameter, $\mathrm{h} / \mathrm{yr}$} \\
\hline $\begin{array}{l}\text { Inhalation exposure } \\
\text { A ir submersion } \\
\text { Exposure to ground }\end{array}$ & $\begin{array}{l}8760 \\
8760 \\
4380\end{array}$ \\
\hline \multicolumn{2}{|c|}{ Recreational Parameters, $\mathrm{h} / \mathrm{yr}$} \\
\hline $\begin{array}{l}\text { Swimming } \\
\text { Boating } \\
\text { Shoreline activities }\end{array}$ & $\begin{array}{l}100 \\
100 \\
500\end{array}$ \\
\hline
\end{tabular}


possibility that some people either did not drink fresh milk or obtained it from locations distant from Hanford. The second variation was to eliminate a11 local foods, leaving only submersion, inhalation, and ground exposure to simulate the possibility of someone consuming a completely imported diet. Results from these simulations are provided in Section 4.0.

\subsection{SURFACE WATER RELEASE SIMULATIONS}

For releases to surface water, many of the same pathways as used for atmospheric releases are considered and others are added. The pathways of drinking contaminated water, recreation in or near contaminated water (swimming, boating, or shoreline activities), and consumption of fish are included, as well as pathways from irrigation with contaminated river water. Irrigation-related pathways include consumption of contaminated produce and animal products (contaminated both by drinking the water and eating contaminated fodder), exposure to soils contaminated by the water, and inhalation of resuspended dusts from such soils.

For surface water release, as for the atmospheric releases, basic parameters for exposure were taken from McCormack et al. (1984) for the Hanford maximally exposed individual. Additional calculations to identify radionuclides important for lesser-exposed types of people were also made. The first variation involved consideration of drinking water only; the second involved only external exposures from shoreline and river recreational activities (although the swimming portion included ingestion of a small amount of contaminated water while swimming). The third variation was to change the ingestion parameters to represent more closely the consumption patterns of someone using Columbia River fish as a mainstay of their diet. The consumption rates for this scenario were taken from Hunn and Bruneau (1989).

\subsection{EXPOSURE PATHNAY MODES}

Because the planned stochastic simulation model for the HEDR Project is still under development, the majority of the calculations were performed using the GENII computer software system (Napier et al. 1988). The GENII system is composed of seven linked computer programs and their associated data libraries. The computer codes are of three types: user interfaces (i.e., 
interactive, menu-driven programs to assist the user with scenario generation and data input), internal and external dose factor generators, and the environmental dosimetry programs. Ali steps of the code development have been documented and tested.

\subsection{NCRP SCREENING FACTORS}

A check of the GENII results for atmospheric releases was performed using radiation dose screening factors developed by the NCRP. The U.S. Environmental Protection Agency (EPA) requested that the NCRP help develop a simple system for small users of radionuclides to show compliance with EPA and U.S. Nuclear Regulatory Commission (NRC) requirements. The NCRP provided a system of factors which may be used to conservatively estimate potential doses to individuals as a result of atmospheric releases (NCRP 1989). These factors are based on a scenario of exposure similar to the one described above for the Hanford maximally exposed individual. In their simplest form, they directly relate releases to doses, incorporating many assumptions about atmospheric transport, consumption rates, and other exposure parameters. They provide a simple tool for performing the ranking of the radionuclides identified in Section 2.1. The product of the quantity released and the NCRP screening factor gives a quantity that may be used directly for ranking.

Although three levels of screening are presented in the NCRP system, that used in this report is the simplest, Level I, which is highly conservative (that is, the screening overestimates the potential radiation doses). The estimate of effective dose equivalent (EDE) for Level I screening assumes an atmospheric concentration based upon the radionuclide concentration at the point of emission. Exposure from all pathways is expressed by a single parameter, the Level I screening factor. The radionuclide concentration is multiplied by this factor to obtain an EDE.

Two approaches were taken to using the NCRP screening factors. The simplest was to multiply the NCRP factors by the radionuclide inventory, which was calculated using ORIGEN2. A second approach was to calculate the product of the ORIGEN2 inventory estimate, the release fraction for that radionuclide, and the NCRP factor. The first technique can highlight any radionuclides that might be omitted if the release fractions are incorrect, and the second 
technique relates directly to the GENII results. It should be emphasized that the calculation performed for this ranking involves only the inventory. and the Level I screening factor: no additional terms to account for environmental concentration or dilution were used. Thus, the resulting values are not strictly doses, but are indicators of what the rank of the doses would be if dispersion and deposition were included. 


\subsection{RADIONUCLIDE RANKINGS}

This section provides comparisons of the relative rankings of radionuclide importance calculated in various ways. In each case, the rankings are based on the EDE, a concept which uses risk-based weighting factors applied to the doses calculated for each organ to provide a "weighted" whole-body equivalent. For the atmospheric releases, the thyroid is the critical organ in all tables provided; for surface water releases the gastrointestinal tract is usually the critical organ. If rankings had been made on the basis of organ doses, they would be slightly different, but the most important radionuclides would be the same. The relative importance of various radionuclides ranked on organ doses would be actually more pronounced than that calculated with the EDE.

\subsection{ATMOSPHERIC RELEASES}

Rankings of radionuclides found to be dominant in atmospheric releases are shown in Tables 4.1 through 4.5. These tables Iist the radionuclides that resulted in the largest portion of the EDE received for each of the listed scenarios. These rankings were derived from the calculations with the GENII codes provided in Appendix B.

Table 4.1 shows relative rankings by order of importance for the radionuclides found in atmospheric releases in the mid-1940s, assuming the standard scenario for the maximally exposed individual, the ORIGEN2 inventory, and the release fractions described in Section 3.1. Table 4.1 orders the radionuclides for three fuel burnups $(100,500$, and $1000 \mathrm{MWd} / \mathrm{t}$ ) at an average cooling time of 30 days. This ranking is derived from the doses calculated with the GENII code. It is noteworthy that the top five radionuclides are the same

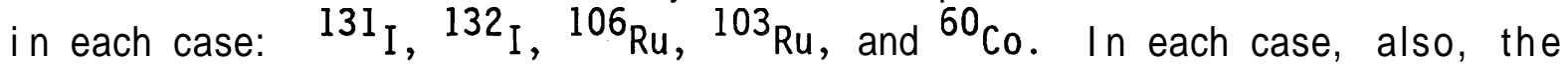
fifth-ranked radionuclide provides less than $1 / 100,000$ times the dose of the first-ranked radionuclide.

The same information is graphically shown in Figure 4.1. This figure i7lustrates the stabilization of short-lived ${ }^{131} I$ content in the fuel at 
TABLE 4.1. Dominant Radionuclides in Atmospheric Releases as a Function of Fuel Burnup (30-Day Cooled Fuel), Ranked by Percent of Contribution to Effective Dose Equivalent

\begin{tabular}{|c|c|c|c|c|c|}
\hline \multicolumn{2}{|c|}{$100 \mathrm{MWd} / \mathrm{t}, \% \mathrm{DDE}$} & \multicolumn{2}{|c|}{$500 \mathrm{MWd} / \mathrm{t}, \% \mathrm{EDE}$} & \multicolumn{2}{|c|}{$1000 \mathrm{MWd} / \mathrm{t}, \% \mathrm{DE}$} \\
\hline $131_{I}$ & 99 & ${ }^{131_{I}}$ & 98 & $131_{I}$ & 96 \\
\hline${ }^{106} \mathrm{Ru}$ & $<1$ & ${ }^{106} \mathrm{Ru}$ & 1 & ${ }^{106} \mathrm{Ru}$ & 3 \\
\hline${ }^{103} \mathrm{Ru}$ & $<1$ & ${ }^{103} \mathrm{Ru}$ & $<1$ & ${ }^{103} \mathrm{Ru}$ & 1 \\
\hline${ }^{132} \mathrm{I}$ & $<1$ & ${ }^{60} \mathrm{Co}$ & $<1$ & ${ }^{60} \mathrm{CO}_{\mathrm{O}}$ & $<1$ \\
\hline${ }^{60} \mathrm{Co}$ & $<1$ & ${ }^{132} I$ & $<1$ & 132 I & $<1$ \\
\hline${ }^{133} \mathrm{Xe}$ & $<1$ & ${ }^{133} \mathrm{Xe}$ & $<1$ & ${ }^{144} \mathrm{Ce}$ & $<1$ \\
\hline${ }^{144} \mathrm{Ce}$ & $<1$ & ${ }^{144} \mathrm{Ce}$ & $<1$ & ${ }^{239} \mathrm{Pu}$ & 1 \\
\hline${ }^{239} \mathrm{Pu}$ & $<1$ & ${ }^{239} \mathrm{Pu}$ & $<1$ & ${ }^{133} x \mathrm{e}$ & $<1$ \\
\hline $129 \mathrm{~m}_{\mathrm{Te}}$ & $<1$ & $91_{Y}$ & $<1$ & $129 \mathrm{~m}_{\mathrm{Te}}$ & 1 \\
\hline $9 l_{Y}$ & $<1$ & $129 m_{\mathrm{Te}}$ & $<1$ & $91_{Y}$ & 1 \\
\hline
\end{tabular}

21 TABLE 4.2. Dominant Radionuclides in Atmospheric Releases as a Function of Fuel Cooling Time (500-MWd/t Fuel), Ranked by Percent of Contribution to Effective Dose Equivalent

10 Davs, \% EDE

${ }^{131} \mathrm{I}$

${ }^{106} \mathrm{Ru} \quad<1$

${ }^{103} \mathrm{Ru} \quad<1$

132 $1<1$

${ }^{133} \mathrm{Xe}<1$

${ }^{60} \mathrm{CO}_{0}<1$

${ }^{129 \mathrm{~m}_{\mathrm{Te}}} \quad<1$

${ }^{144} \mathrm{Ce} \quad<1$

${ }^{239} \mathrm{Pu} \quad<1$

$91_{Y}$
30 Days, $\%$ EE

${ }^{131}$ I

${ }^{106} \mathrm{Ru}$

$103_{\mathrm{Ru}}$

${ }^{60} \mathrm{Co}$

132 I

${ }^{133} \mathrm{xe}$

${ }^{144} \mathrm{Ce}$

${ }^{239} \mathrm{Pu}$

$91_{Y}$

${ }^{129 m_{T e}}$
98

1

$<1$

$<1$

$<1$

$<1$

$<1$

$<1$

$<1$

$<1$
90 Davs, \% DEE

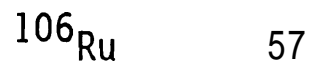

$131_{\text {I }} \quad 28$

${ }^{103} \mathrm{Ru} \quad 13$

${ }^{60} \mathrm{Co}$

${ }^{144} \mathrm{Ce} \quad<1$

${ }^{239} \mathrm{Pu} \quad<1$

$95 \mathrm{Zr} \quad<1$

$91_{Y} \quad<1$

$129 m_{\mathrm{Te}} \quad<1$

${ }^{89} \mathrm{Sr} \quad<1$ 
$21 \quad$ TABLE 4.3. Dominant Radionuclides in Atmospheric Releases Calculated by Three Different Techniques for 500-MWd/t Fuel Cooled 30 Days, Ranked by Percent of Contribution to Effective Dose Equivalent

Environmental Calculation, \% 日一

$\begin{array}{lcl}{ }^{131_{\mathrm{I}}} & 98 & { }^{131_{\mathrm{I}}} \\ { }^{106} \mathrm{Ru} & 1 & { }^{103_{\mathrm{Ru}}} \\ { }^{103_{\mathrm{Ru}}} & <1 & { }^{106}{ }_{\mathrm{Ru}} \\ { }^{60} \mathrm{Co} & <1 & { }^{60}{ }_{\mathrm{Co}} \\ 132_{\mathrm{I}} & <1 & { }^{95} \mathrm{Zr} \\ 133_{\mathrm{Xe}} & <1 & 132 \mathrm{I} \\ { }^{144} \mathrm{Ce} & <1 & 144 \mathrm{Ce} \\ 239_{\mathrm{Pu}} & <1 & { }^{137} \mathrm{Cs} \\ 91_{\mathrm{Y}} & <1 & 90_{\mathrm{Sr}} \\ 129 \mathrm{~m}_{\mathrm{Te}} & <1 & 91_{\mathrm{Y}}\end{array}$

NCRP Screening,

NCRP Screening

.

21 TABLE 4.4. Dominant Radionuclides in Atmospheric Releases for Three Exposure Scenarios for 500-MWd/t Fuel Cooled 30 Days, Ranked by Percent of Contribution to Effective Dose Equivalent

\begin{tabular}{cc}
\multicolumn{3}{c}{$\begin{array}{c}\text { Standard } \\
\%\end{array}$} \\
\hline${ }^{131_{\mathrm{I}}}$ & 98 \\
${ }^{106} \mathrm{Ru}$ & 1 \\
${ }^{103} \mathrm{Ru}$ & $<1$ \\
${ }^{60} \mathrm{Co}$ & $<1$ \\
${ }^{132} \mathrm{I}$ & $<1$ \\
${ }^{133} \mathrm{Xe}$ & $<1$ \\
${ }^{144} \mathrm{Ce}$ & $<1$ \\
${ }^{239} \mathrm{Pu}$ & $\mathrm{t} \mathrm{I}$ \\
${ }^{91} \mathrm{Y}$ & $<1$ \\
$129 \mathrm{~m}_{\mathrm{Te}}$ & $<1$
\end{tabular}

Standard Without Milk Consumption Pathway. \% EDE

$\begin{array}{lr}{ }^{131} \mathrm{I} & 92 \\ { }^{106} \mathrm{Ru} & 3 \\ 103_{\mathrm{Ru}} & 2 \\ { }^{132} \mathrm{I} & <1 \\ { }^{60} \mathrm{Co} & <1 \\ 133_{\mathrm{Xe}} & <1 \\ 144 \mathrm{Ce} & <1 \\ { }^{239} \mathrm{Pu} & <1 \\ 129 \mathrm{~m}_{\mathrm{Te}} & <1 \\ 91_{\mathrm{Y}} & <1\end{array}$

\begin{tabular}{cc}
$\begin{array}{c}\text { Minimal } \\
\%\end{array}$ & Exposure \\
\hline${ }^{131_{\mathrm{I}}}$ & 47 \\
${ }^{106} \mathrm{Ru}$ & 38 \\
${ }^{103_{\mathrm{Ru}}}$ & 14 \\
${ }^{60} \mathrm{Co}$ & $<1$ \\
${ }^{132} \mathrm{I}$ & $<1$ \\
${ }^{133} \mathrm{Xe}$ & $<1$ \\
${ }^{239} \mathrm{Pu}$ & $<1$ \\
${ }^{144} \mathrm{Ce}$ & $<1$ \\
${ }^{91} \mathrm{Y}$ & $<1$ \\
${ }^{95} \mathrm{Zr}$ & $<1$
\end{tabular}


TABLE 4.5. Dominant Radionuclides from Atmospheric Releases for Two Dose Integration Techniques for 500-MWd/t Fuel Cooled 30 Days, Ranked by Percent of Contribution to Effective Dose Equivalent

\begin{tabular}{|c|c|c|c|}
\hline \multicolumn{2}{|c|}{$\begin{array}{c}\text { 1-Yr Exposure } \\
\text { Lifetime Integration, } \\
\% \text { EDE } \\
\end{array}$} & \multicolumn{2}{|c|}{$\begin{array}{c}\text { Life time Exposure } \\
\text { Lifetime Integration, } \\
\% \text { EDE } \\
\end{array}$} \\
\hline $131_{1}$ & 98 & $131_{\mathrm{I}}$ & 97 \\
\hline $106 \mathrm{Ru}$ & 1 & ${ }^{106} \mathrm{Ru}$ & 1 \\
\hline${ }^{103} \mathrm{Ru}$ & $<1$ & ${ }^{103} \mathrm{Ru}$ & 1 \\
\hline${ }^{60} \mathrm{Co}_{0}$ & $<1$ & ${ }^{60} \mathrm{Co}$ & $<1$ \\
\hline${ }^{132}$ I & $<1$ & 132 I & $<1$ \\
\hline${ }^{133} \mathrm{Xe}$ & $<1$ & ${ }^{133} \mathrm{Xe}$ & $<1$ \\
\hline${ }^{144} \mathrm{Ce}$ & $<1$ & ${ }^{144} \mathrm{Ce}$ & $<1$ \\
\hline${ }^{239} \mathrm{Pu}$ & $<1$ & & $<1$ \\
\hline $91_{Y}$ & $<1$ & ${ }^{239} \mathrm{Pu}$ & $<1$ \\
\hline${ }^{129} \mathrm{~m}_{\mathrm{Te}}$ & $<1$ & ${ }^{129 \mathrm{~m}_{\mathrm{Te}}}$ & $<1$ \\
\hline
\end{tabular}

higher burnups for a constant power level, while the longer-lived ruthenium isotopes $\left({ }^{103} \mathrm{Ru}\right.$ and ${ }^{106} \mathrm{Ru}$ ) are shown still exponential ly reaching equilibrium between production and decay.

Table 4.2 contains relative rankings of the order of importance of the radionuclides, assuming the same exposure scenario and a constant fuel burnup, but varying the fuel cooling times. Based on GENII results, the rankings show some change when longer cooling times are reached, but 8 of the top 10 radionuclides at only 10 days cooling are the same as at 3 months cooling, and the top four radionuclides, which are the same in each case, always provide more than $99.9 \%$ of the total dose.

This information is graphically shown in Figure 4.2. This figure illustrates the reduction in dose as a result of the exponential decay of each radionuclide with its own half-7ife. The shorter-1ived ${ }^{131}$ I decreases in the fuel at a faster rate than the longer-lived ruthenium isotopes, with the result being a change in relative importance of the releases after a long cooling time. 


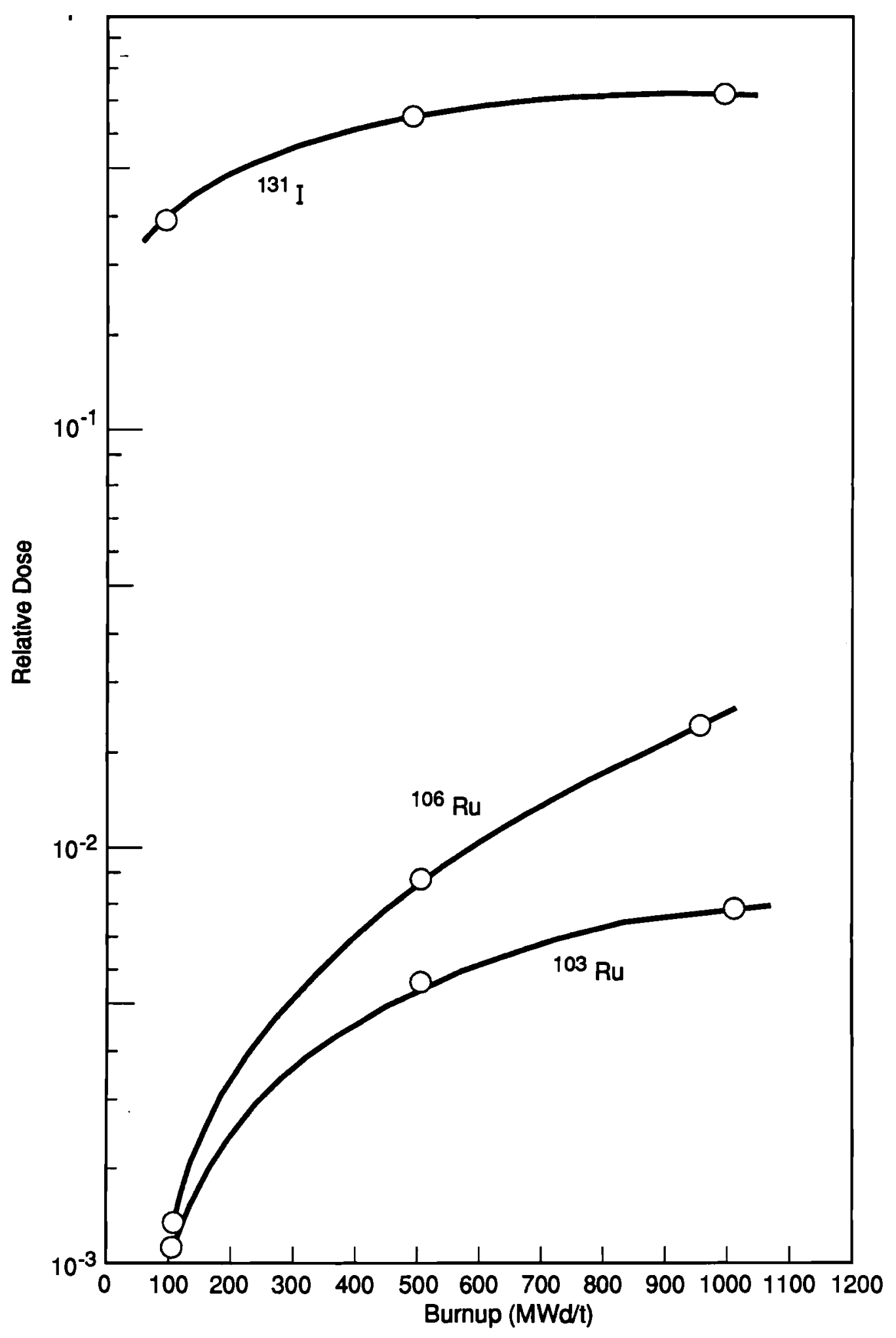

S9105030.2

FIGURE 4.1. Dose as a Function of Level Burnup 


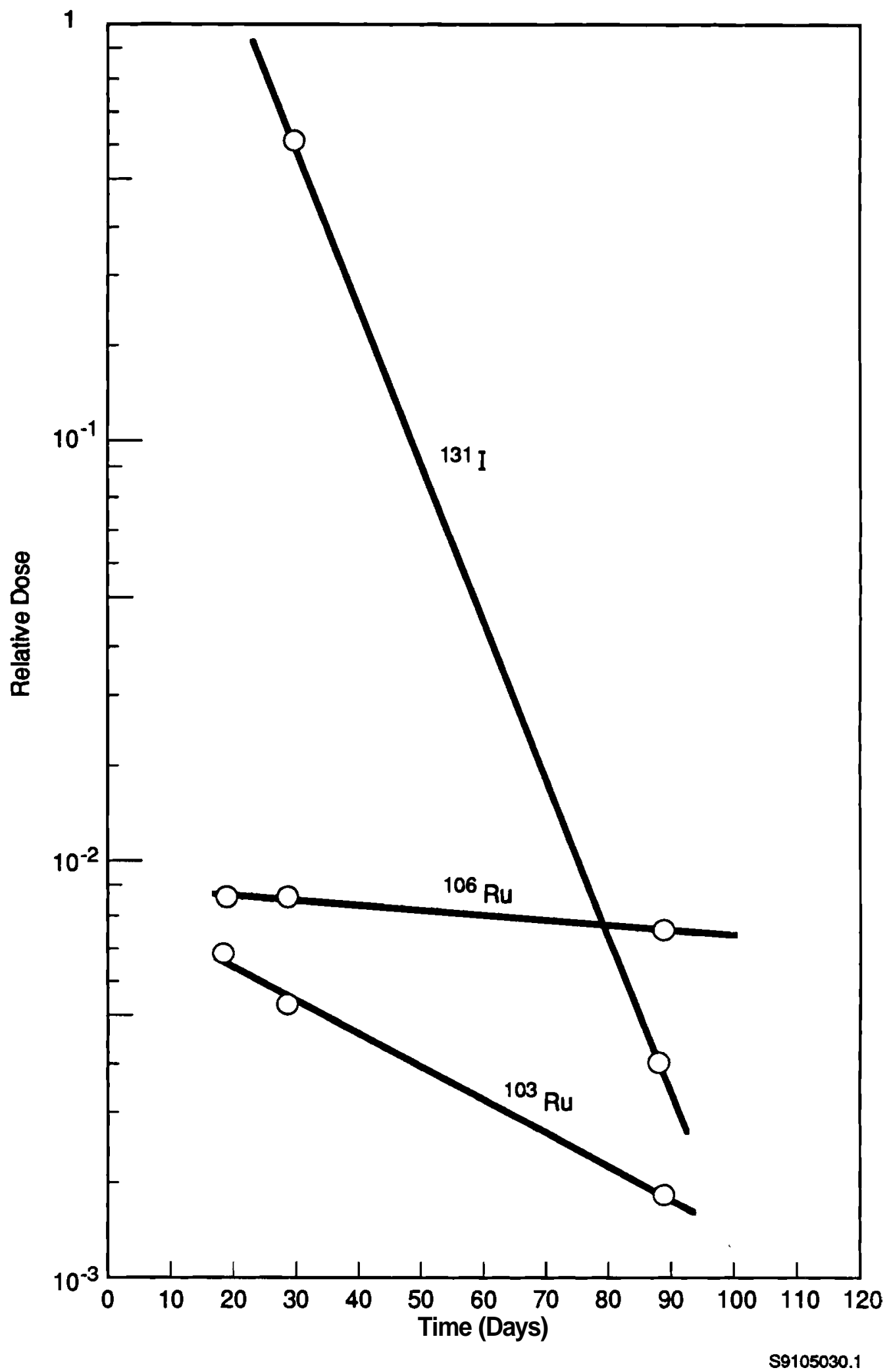

FIGURE 4.2. Dose as a Function of Cooling Time

$$
4.6
$$


In Table 4.3, rankings calculated with GENII are compared to those calculated with the NCRP screening factors. When the release fractions are included in the NCRP calculation, 7 of the top 10 radionuclides are the same. Not shown in Table 4.3 (but evident in the complete results in Appendix B) is that the NCRP results for all of the other radionuclides in the GENII-derived list are within the next six calculated in the NCRP scheme. The absolute magnitude varies only over a factor of three from the lowest percent contribution listed in Table 4.3, while the range of those shown in Table 4.3 is over five orders of magnitude. More than $99.9 \%$ of the dose is accounted for with only the top four radionuclides in these lists. The column of NCRP results calculated without release fractions shows only radionuclides that were also identified in the other columns, indicating that even gross errors in the estimation of the release fractions would have no impact on selection of the top few radionuclides for investigation.

The robustness of the above results is illustrated by Table 4.4, which shows the impacts of changing the assumptions of the exposure scenario. Ranking in this table are derived from GENII calculations for fuel irradiated to $500 \mathrm{MWd} / \mathrm{t}$ and cooled 30 days, with the release fractions described in Section 3.1. Dropping the dominant milk-consumption pathway from the scenario, the rankings are essentially unchanged from the basic scenario, which includes a milk-consumption pathway. Finally, considering only the essentially unavoidable exposures of submersion, inhalation, and groundshine, 9 of the 10 original radionuclides are the same. These radionuclides account for a range of four orders of magnitude in relative importance (i.e., the top nine radionucl ides account for $99.99 \%$ of the dose).

A final check on the results is a comparison of a simple committed EDE (1 year of exposure and 50 years of dose integration) with a cumulative "environmental dose commitment" ( 1 year of release, an additional 49 years of exposure to environmental residuals, and 50 years of dose integration). This result, presented in Table 4.5, shows that ${ }^{90} \mathrm{Sr}$, considered to be long-1ived in the environment, appears on the list but only at a level five orders of magnitude lower than the controlling nuclide, ${ }^{131} \mathrm{I}$ 


\subsection{SURFACE WATR RELEASES}

Table 4.6 ranks radionuclides in order of importance for surface water releases in the 1960s, assuming the standard Hanford scenario for the maximally exposed individual. The table contains results for three measurement periods: 1956, 1964, and 1968. These rankings are based on doses calculated with the GENII code, using the monitoring data described in Section 3.2. There is somewhat more variability in the radionuclide rankings from year to year than for the atmospheric release rankings, but the same five or six radionuclides occur repeatedly, always making up greater than $95 \%$ of the total dose. Although not shown in Tables 4.6 through 4.8, the fish consumption pathway provides about $85 \%$ of the dose to the dominant organ (the lower large intestine) and more than half of the dose to all organs. The contribution of fish consumption to total dose is evident in the data in the appendixes of this report.

The surface water exposure scenario yielded the rankings of radionuclides shown in Table 4.7, which is divided into scenarios of external exposure, untreated drinking water, and an enhanced diet of fish. For consistency, these scenarios are based on the 1968 water measurements. Although there is some variability in the radionuclide listings, the top six, which contribute over $90 \%$ of the doses, are the same radionuclides shown in the more restrictive scenarios involving fish.

Finally, Table 4.8 provides radionuclide rankings for the a17-pathway and drinking-only exposures for an acute release to the river of $11 \mathrm{~b}$. of uranium, irradiated to $500 \mathrm{MWd} / \mathrm{t}$ with minimal holdup time. One pound of uranium is roughly the amount released in the largest fuel failures (Hall 1963). (a) Although the radionuclides indicated in Table 4.8 are significantly different than those in the other surface water tables, the absolute magnitude of the dose is much lower than the results from the chronic releases. The radionuclides listed in Table 4.8 were also included in the chronic release source terms used for the calculations leading to the lists of

(a) The average loss for each of the 97 reported fuel failures in 1964 was 25 grams, or about one ounce (Jerman et al. 1965). 
21 TABLE 4.6. Dominant Radionuclides for Surface Water Releases

for a Maximally Exposed Individual, Calculated from Concentrations Measured in 1956, 1964, and 1968 and Ranked by Percent of Contribution to Effective Dose Equivalent

\begin{tabular}{|c|c|c|c|c|c|}
\hline \multicolumn{2}{|c|}{ 1956, \% $\mathrm{DDE}$} & \multicolumn{2}{|c|}{ 1964. \% $\mathrm{DEE}$} & \multicolumn{2}{|c|}{ 1968, \% EDE } \\
\hline${ }^{65} \mathrm{Zn}$ & 34 & $32 p$ & 37 & $32 p$ & 53 \\
\hline $32 p$ & 19 & ${ }^{65} \mathrm{Zn}$ & 22 & ${ }^{65} \mathrm{Zn}$ & 18 \\
\hline${ }^{76}$ As & 16 & ${ }^{64} \mathrm{Cu}$ & 12 & ${ }^{46} \mathrm{Sc}$ & 5 \\
\hline${ }^{64} \mathrm{Cu}$ & 15 & ${ }^{239} \mathrm{~Np}$ & 8 & ${ }^{76}$ As & 4 \\
\hline${ }^{239} \mathrm{~Np}$ & 13 & ${ }^{76}$ As & 6 & ${ }^{239} \mathrm{~Np}$ & 3 \\
\hline${ }^{72} \mathrm{Ga}$ & 3 & ${ }^{24} \mathrm{Na}$ & 3 & ${ }^{59} \mathrm{Fe}$ & 3 \\
\hline${ }^{24} \mathrm{Na}$ & 2 & ${ }^{72} \mathrm{Ga}$ & 2 & ${ }^{144} \mathrm{Ce}$ & 2 \\
\hline $69 m_{\mathrm{Zn}}$ & 1 & ${ }^{153} \mathrm{Sm}$ & 1 & ${ }^{24} \mathrm{Na}$ & 2 \\
\hline${ }^{136} \mathrm{Cs}$ & t I & ${ }^{56}{ }_{\mathrm{Mn}}$ & $<1$ & ${ }^{131} \mathrm{I}$ & 1 \\
\hline${ }^{232} \mathrm{Th}$ & $<1$ & ${ }^{232} \mathrm{Th}$ & $<1$ & ${ }^{72} \mathrm{Ga}$ & 1 \\
\hline
\end{tabular}

21 TABLE 4.7. Dominant Radionuclides for Surface Water Releases for Three Scenarios of Exposure, Calculated from Concentrations Measured in 1968 and Ranked by Percent of Contribution to Effective Dose Equivalent

\begin{tabular}{lr}
\multicolumn{2}{c}{$\begin{array}{c}\text { External } \\
\text { Exposure } \\
\% \text { Only } \\
\text { \% }\end{array}$} \\
\hline${ }^{24} \mathrm{Na}$ & 53 \\
${ }^{46} \mathrm{Sc}$ & 28 \\
${ }^{56} \mathrm{Mn}$ & 5 \\
${ }^{65} \mathrm{Zn}$ & 3 \\
${ }^{51} \mathrm{Cr}$ & 1 \\
${ }^{64} \mathrm{Cu}$ & 1 \\
${ }^{95} \mathrm{Zr}$ & 1
\end{tabular}

\begin{tabular}{lr}
$\begin{array}{c}\text { Untreated Drinking } \\
\text { Water Only, } \\
\% \text { EDE }\end{array}$ \\
\hline${ }^{46} \mathrm{SC}$ & 19 \\
${ }^{32} \mathrm{p}$ & 14 \\
${ }^{239} \mathrm{~Np}$ & 12 \\
${ }^{76} \mathrm{As}$ & 11 \\
${ }^{24} \mathrm{Na}$ & 9 \\
${ }^{65} \mathrm{Zn}$ & 6 \\
${ }^{144} \mathrm{Ce}$ & 4
\end{tabular}

Enhanced Fish Consumption, $\%$ DE

\begin{tabular}{lr}
\hline${ }^{32} \mathrm{p}$ & 72 \\
${ }^{239} \mathrm{~Np}$ & 9 \\
${ }^{65} \mathrm{Zn}$ & 8 \\
${ }^{76} \mathrm{As}$ & 5 \\
${ }^{64} \mathrm{Cu}$ & 5 \\
${ }^{24} \mathrm{Na}$ & 1 \\
${ }^{56} \mathrm{Mn}$ & $<1$
\end{tabular}


TABLE 4.8. Dominant Radionuclides Associated with a Hypothetical Release of $1 \mathrm{7b}$. of $500-\mathrm{MWd} / \mathrm{t}$ Fuel to Surface Water Calculated for Drinking and A17-Pathways Scenarios and Ranked by Percent of Contribution to Effective Dose Equivalent

\begin{tabular}{|c|c|c|c|}
\hline \multicolumn{2}{|c|}{$\begin{array}{c}\text { Drinking Water } \\
\text { Scenario, } \\
\% \text { EDE } \\
\end{array}$} & \multicolumn{2}{|c|}{$\begin{array}{c}\text { Al1-Pathways } \\
\text { Scenario, } \\
\% \text { EDE }\end{array}$} \\
\hline${ }^{129 m_{\mathrm{Te}}}$ & 50 & ${ }^{129 m_{\mathrm{Te}}}$ & \\
\hline${ }^{131}$ I & 15 & ${ }^{132} \mathrm{Te}$ & 7 \\
\hline${ }^{132} \mathrm{Te}$ & 6 & ${ }^{137} \mathrm{Cs}$ & \\
\hline $140_{\mathrm{Ba}}$ & 5 & $140_{\mathrm{La}}$ & \\
\hline${ }^{140} \mathrm{La}$ & 5 & ${ }^{131_{I}}$ & 3 \\
\hline${ }^{133}$ I & 3 & ${ }^{140} \mathrm{Ba}$ & \\
\hline $91_{Y}$ & 2 & ${ }^{239} \mathrm{~Np}$ & \\
\hline${ }^{97} \mathrm{Zr}$ & 2 & ${ }^{132} \mathrm{I}$ & \\
\hline${ }^{143} \mathrm{Ce}$ & 2 & ${ }^{143} \mathrm{Ce}$ & \\
\hline${ }^{99} \mathrm{Mo}_{0}$ & 2 & ${ }^{144} \mathrm{Ce}$ & \\
\hline
\end{tabular}

Tables 4.6 and 4.7. The dominant radionuclides from the acute releases were largely isotopes of iodine and their short-lived precursors, which also appear (relatively far down) in the routine monitoring numbers. The radionucl ide producing the highest individual organ dose was ${ }^{131} \mathrm{I}$. For this reason, concern by individuals responsible for overseeing reactor operations in the 1950s and 1960s focused on iodine during fuel element failure episodes (Hall 1963). Because the radioiodines and their precursors dominate the acute release doses and the overall magnitude of the acute dose is relatively low, it is apparent that over the long term, the routine releases control the total dose.

Thus, we may infer that radionuclides released during purging of the reactors do not significantly alter the dominant radionuclide distributions. The total dose is controlled by the routine releases, the constituents of which are analyzed in Tables 4.6 and 4.7. The same general activation products observed during routine releases are only enhanced during the purges (Koop 1956). The overall public doses were estimated in 1956 to be increased by less than $5 \%$ as a result of purges (Koop 1956). 
Dominant radionuclides for the surface water are selected for final recommendation based on both the frequency in which they appear in Tables 4.6 and 4.7 and the relative magnitude of the doses presented in the appendixes. For this reason, the top five radionuclides are fairly obvious, and the second group represents a judgment selection of those that must be addressed at some point in the dose reconstruction but that generally should each contribute only a few percent to the total dose to most individuals. 



\subsection{CONCUSIONS}

For the Phase I time periods of 1944-1947 for atmospheric releases and of 1964-1966 for surface water releases, a small group of radionucl ides dominate the dose estimates. For the air releases, ${ }^{131}$ I by itself generally accounts for much more than $90 \%$ of all dose received via all pathways of exposure. Addition of the longer-1ived radioisotopes of rutheni um ( ${ }^{106} \mathrm{Ru}$ and ${ }^{103} \mathrm{Ru}$ ) accounts for $99 \%$ of the dose. For the surface water releases, the results are less definitive, but inclusion of only 5 to 10 radionuclides accounts for the great majority of the dose to the most highly exposed individuals. For Phase I, inclusion of at least ${ }^{32} \mathrm{P},{ }^{239} \mathrm{~Np},{ }^{65} \mathrm{Zn},{ }^{76} \mathrm{As}$, and ${ }^{64} \mathrm{Cu}$ in the dose calculations is essential, with ${ }^{56} \mathrm{Mn},{ }^{24} \mathrm{Na},{ }^{46} \mathrm{Sc}$, and ${ }^{51} \mathrm{Cr}$ highly desirable additions. 



\subsection{REFERENCES}

American Society of Mechanical Engineers (ASME). 1986. Quality Assurance Rrogram, Requirements for Nuclear Faci,ities. ANSI/ASME NQA-1, American
National Standards mstitute, New York, New York.

Atomic Energy Commission (AEC). 1965a. Hanford Radioloqical Sciences Besearch and Development Annual Report for 1964. BNWL-36, Battelle, Pacific Northwest Laboratories, Richland, Washington.

Atomic Energy Commission (AEC). 1965b. Evaluation of Radiological Conditions in the Vicinitv of Hanford for 1964. BNWL-90, Battelle, Pacific Northwest Laboratories, Richland, Washington.

Ballinger, M. Y., and R. B. Hall. 1991. A Historv of Major Hanford Operations Involving Radioactive Material. PNL-6964, Pacific Northwest Laboratory, Richland, Washington.

Croff, A. G. 1980a. ORIGEN2 - A Revised and Updated Version of the Oak Ridqe Isotope Generation and Depletion Code. ORNL-5621, Oak Ridge National Laboratory, Oak Ridge, Tennessee.

Croff, A. G. 1980b. A User's Manual for the ORIGEN2 Computer Code. ORNL/TM-7175, Oak Ridge National Laboratory, Oak Ridge, Tennessee.

DeNea1, D. L. $1965 . \quad$ Historical Events - Reactors and Fuel Fabrication. RL-REA-2247, Hanford Site, Richland, Washington.

Hall, R. B. 1963. Environmental Effects of a Fuel Element Failure. HW-79073, Hanford Atomic Products Operation, Richland, Washington.

Hanthorn, H. E. 1957. Hanford Historv, Technologv, Expansion and Present Efforts. HW-51188, Hanford Laboratories Operation, Richland, Washington.

Hunn, E. S., and C. L. Bruneau. 1989. Estimations of Traditional Native American Diets in the Columbia Plateau. PNL-SA-17296, Pacific Northwest Laboratory, Richland, Washington.

Jerman, P. C., W. N. Koop, and F. E. Owen. 1965. Release of Radioactivitv to the Columbia River from Irradiated Fuel Element Ruptures. RL-REA-2160, Hanford Atomic Products Operation, Richland, Washington.

Koop, W. N. 1956. Influence of Purges During Reactor Operation on the Concentration of Radioisotopes in the Columbia River. HW-45125, Hanford Atomic Products Operations, Richland, Washington.

McCormack, W. D., J. V. Ramsdell, and B. A. Napier. 1984 . Hanford Dose Overview Program: Standardized Methods and Data for Hanford Environmental Dose Calculations. PNL-3777, Rev. 1, Pacific Northwest Laboratory, Richland, Washington. 
Napier, B. A., R. A. Peloquin, D. L. Strenge, and J. V. Ramsdell. 1988. GENII - The Hanford Environmental Radiation Dosimetry Software System. PNL-6584, Vols. 1-3, Pacific Northwest Laboratory, Richland, Washington.

National Council on Radiation Protection and Measurements (NCRP) . 1989. Screening Techniaues for Determining Compliance with Environmental Standards. NCRP Commentary No. 3, NCRP, Washington, D.C. 
APPENDIX A

ORIGEN2 OUTPUT FOR $500 \mathrm{MWd} / \mathrm{t}$ FUEL 


\section{$-$}


ORIGEN INVENTORIES OF RADIONUCLIDES, INCLUDES ACTIVATION PRODUCTS, FISSION PRODUCTS, ACTINIDES + DAUGHTERS

- OIIN-MKIVO 600 MNO/TOM

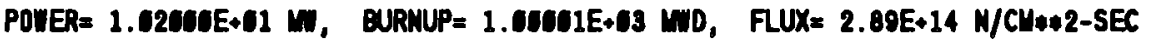

7 MUCLIDE TABLE: RADIOACTIVITY, CURIES

$$
\text { SHORT TON (2010 LB) }
$$

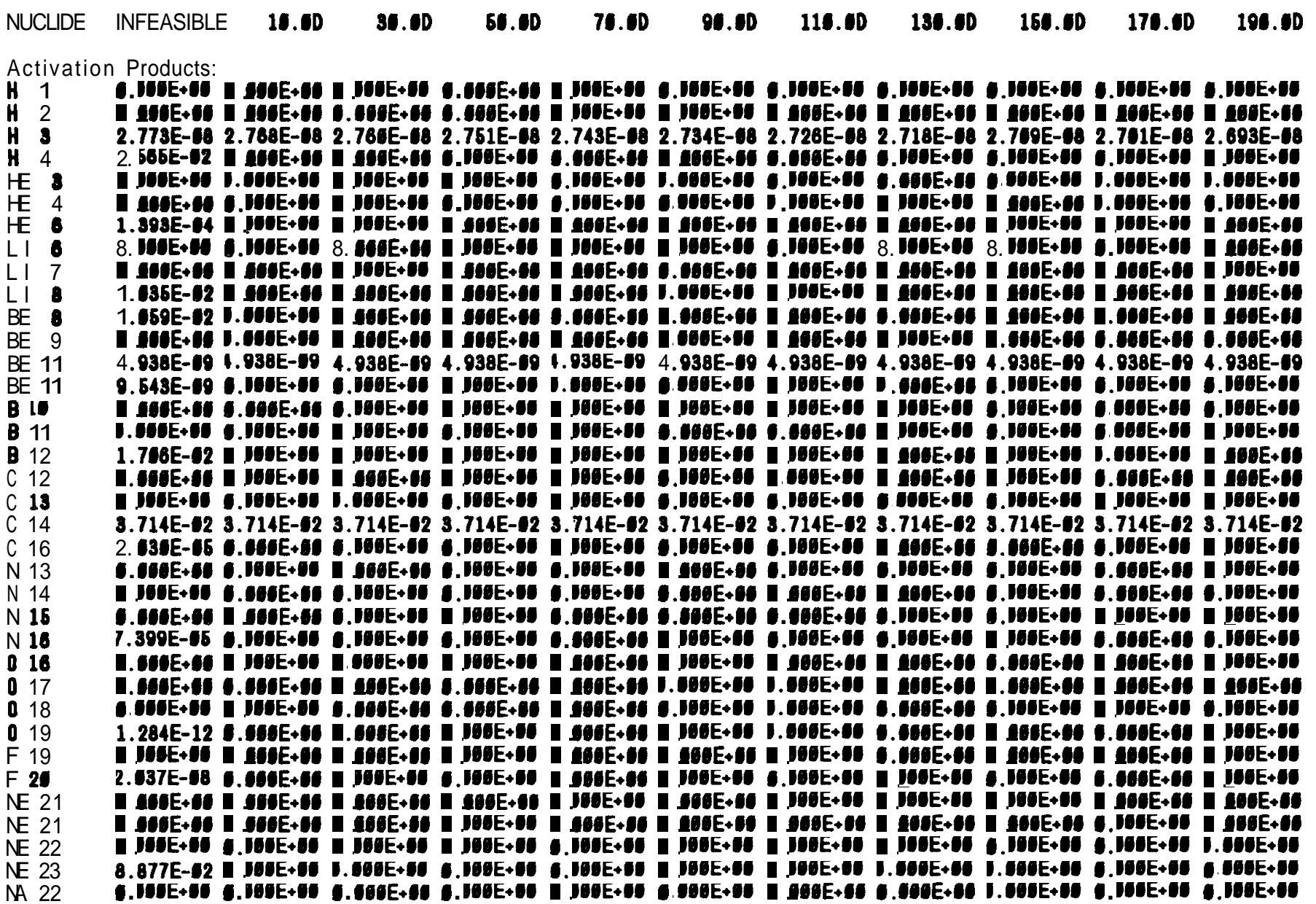


NA 23

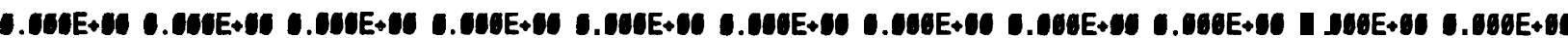

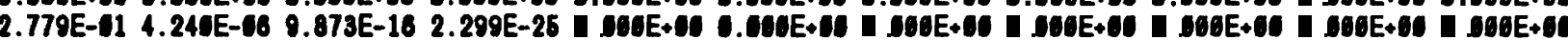

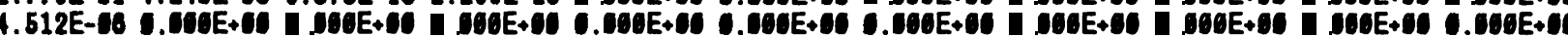

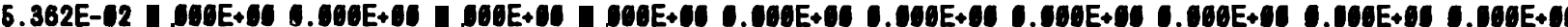

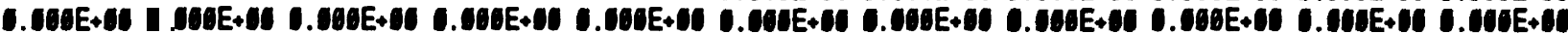
-

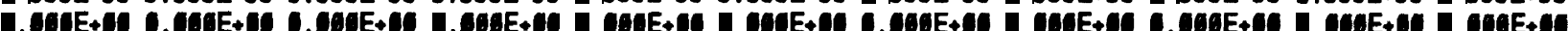
3.677E-11 I.

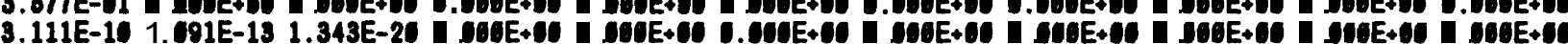

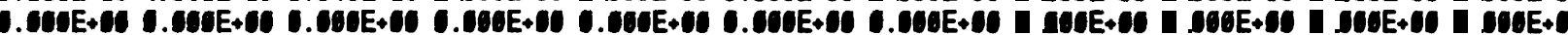

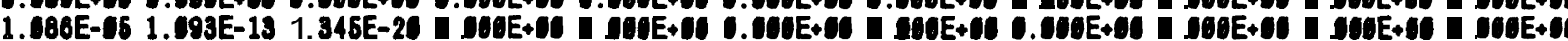

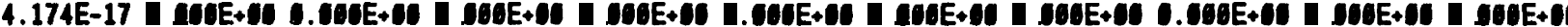

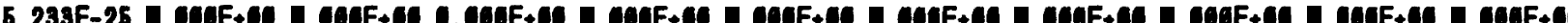
. 4 GOE +

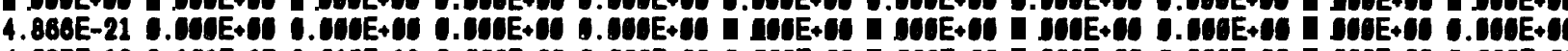

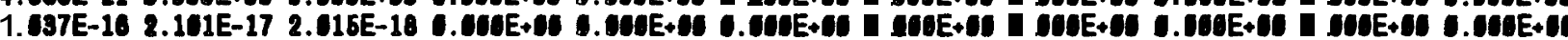

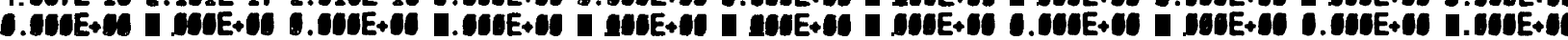

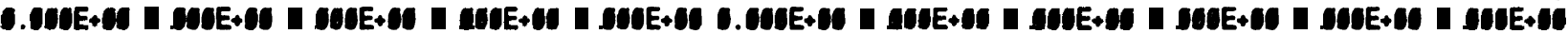

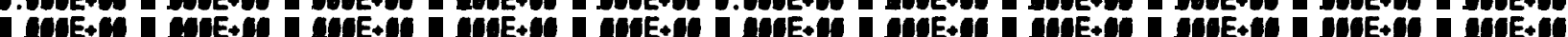
G

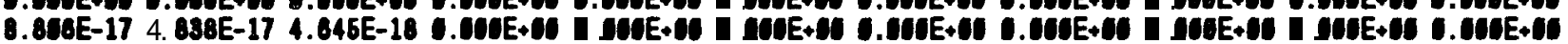

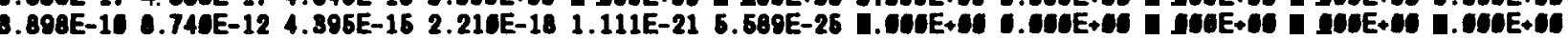

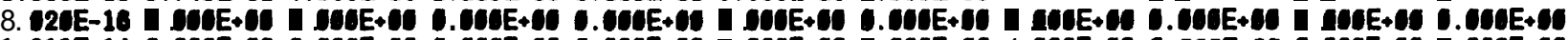

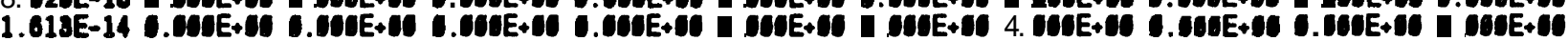

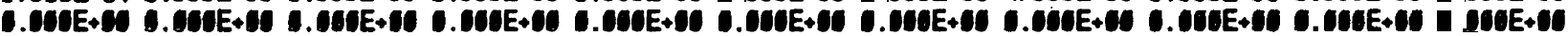

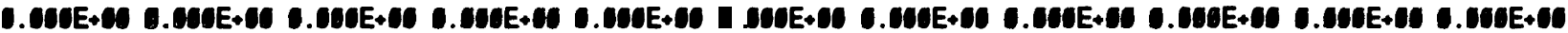
. $M D E+C$ - ME G.

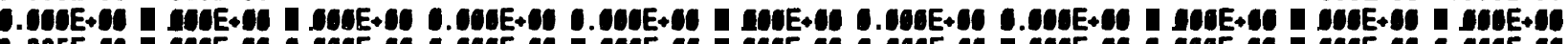

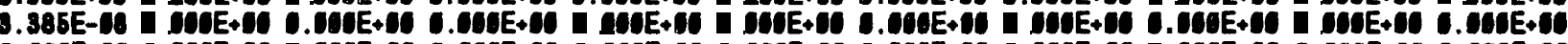

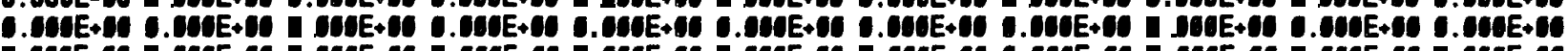

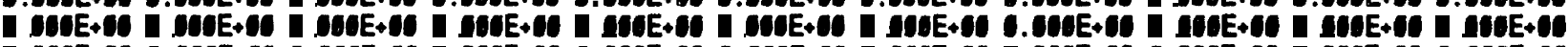

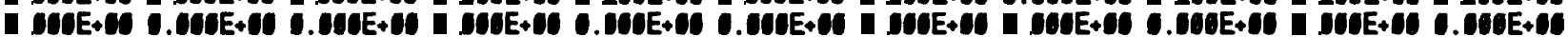

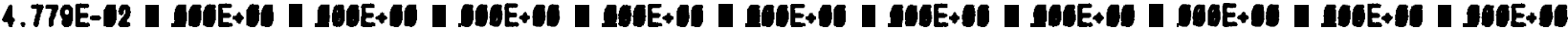

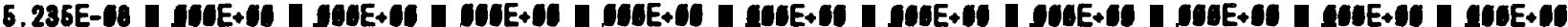
1.892 - $_{10}$ -

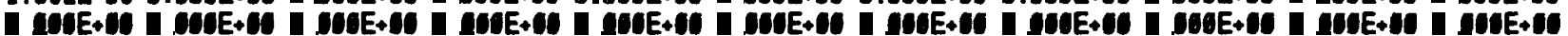

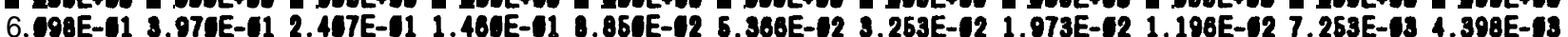

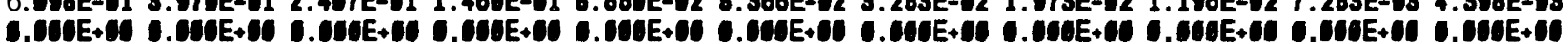

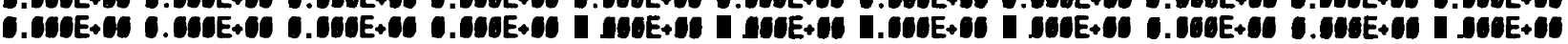

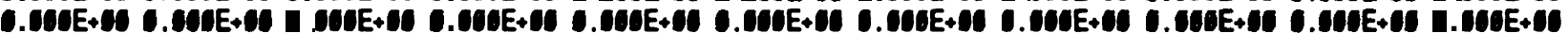

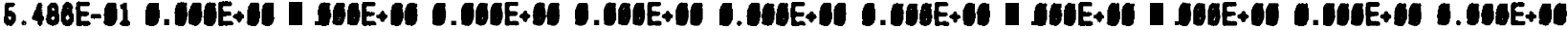

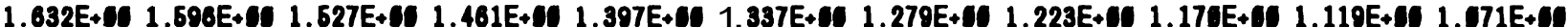

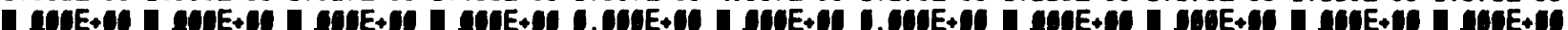

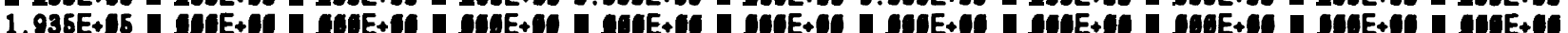

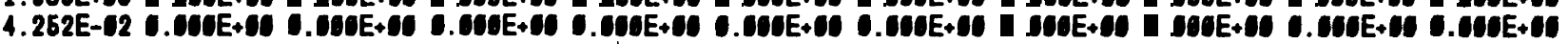

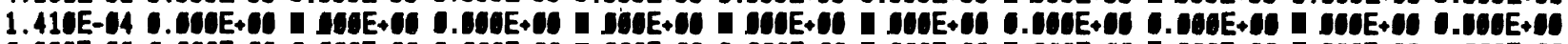

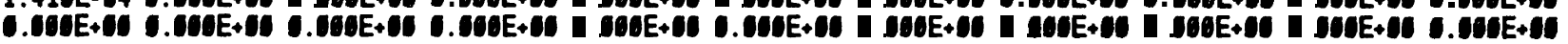


FE 55

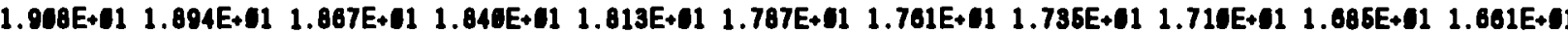

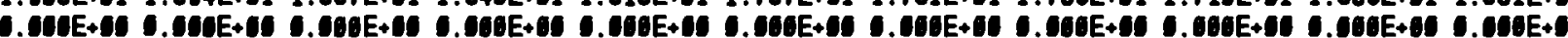

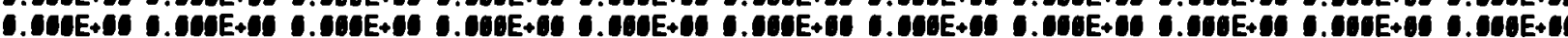

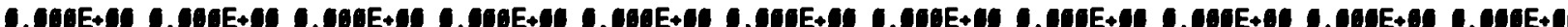

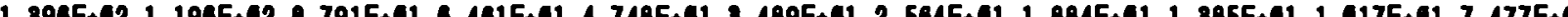
(1)

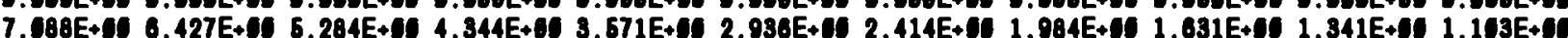

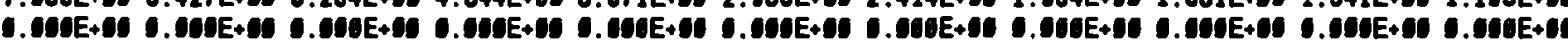

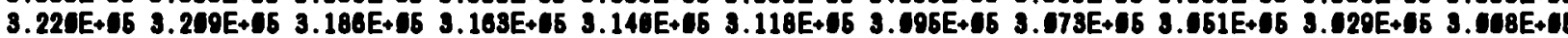

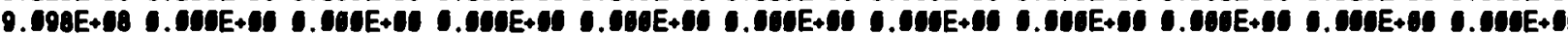

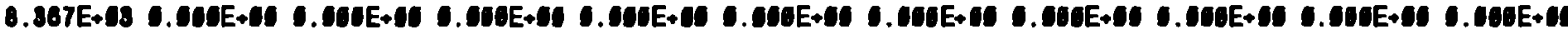

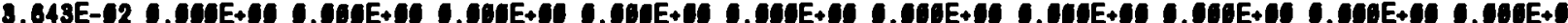
S.

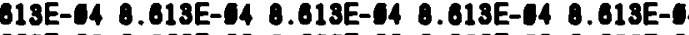

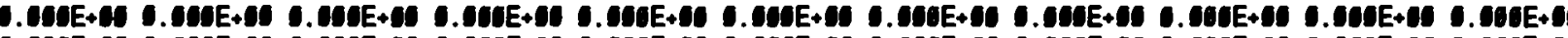

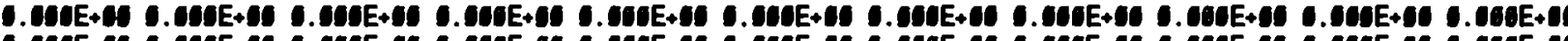

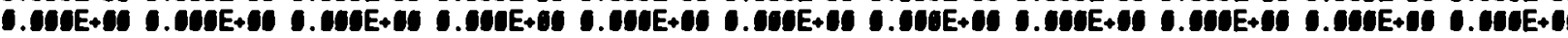

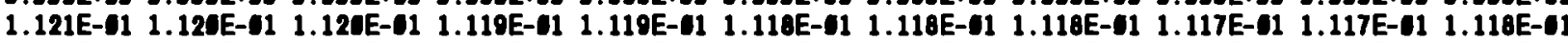

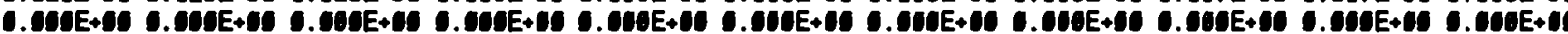

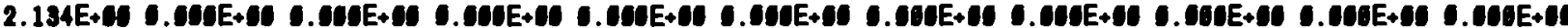

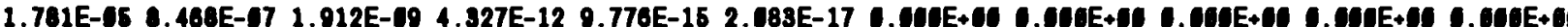
-

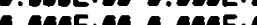

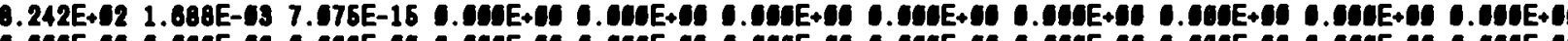

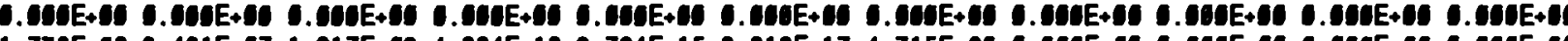

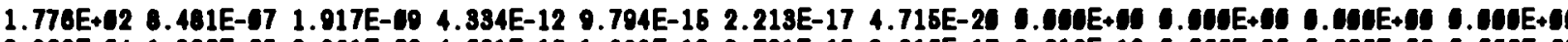

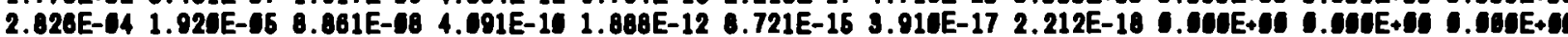

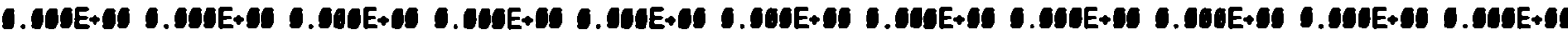

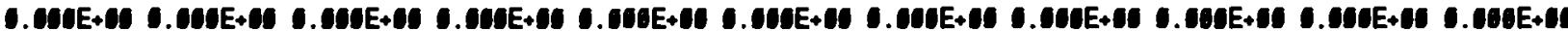

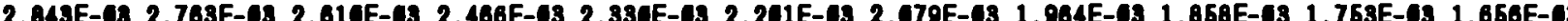
D. G

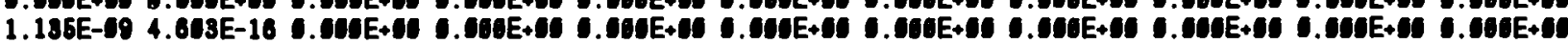

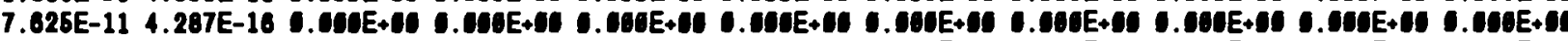

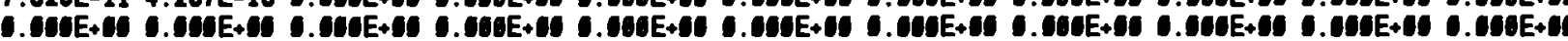

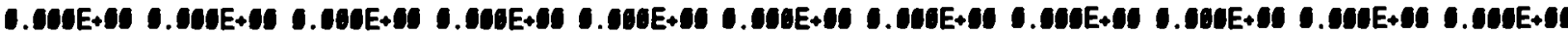

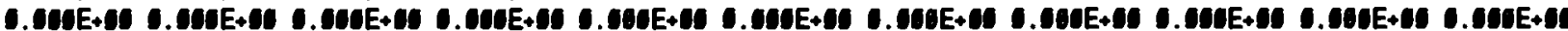

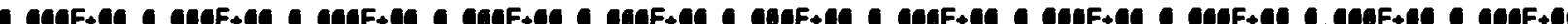
.

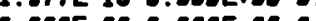

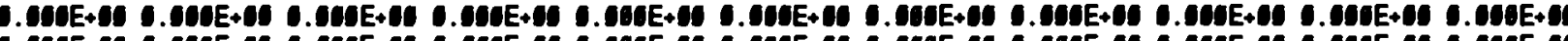

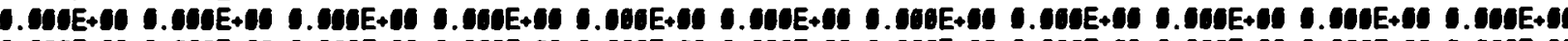

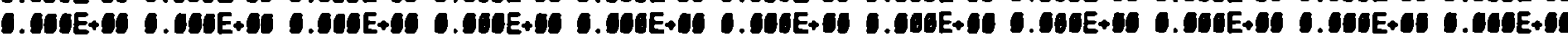

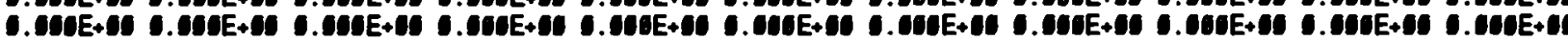

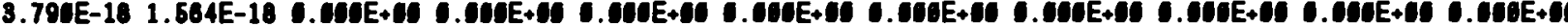

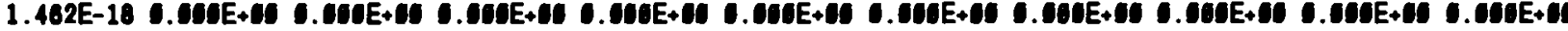
$3.689 E-10$ G 3.

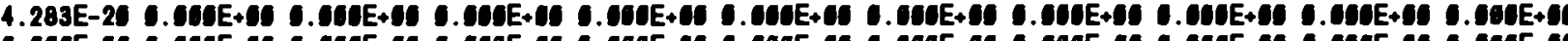

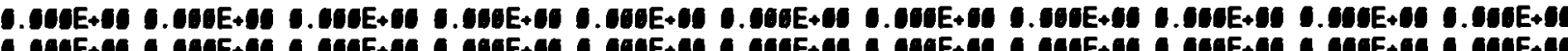

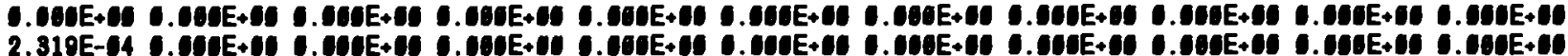


RH104M

9.90E+19 $2.081 E-103.157 E-12$ 2 580E-16 $2.122 E-201.74 \mathrm{E}-24$.

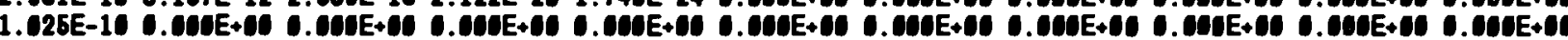

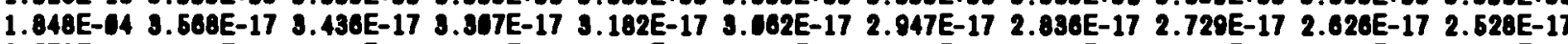

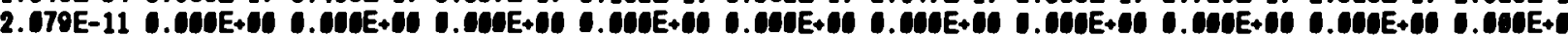

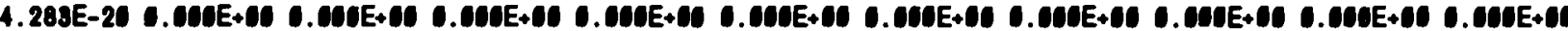

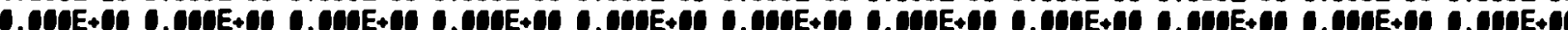

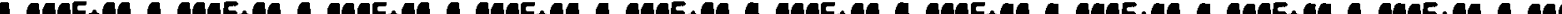

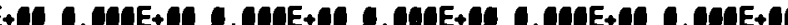

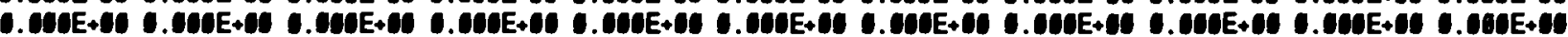

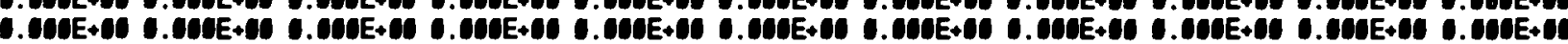

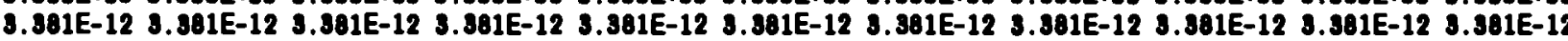

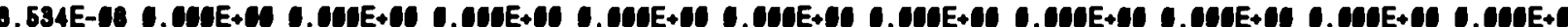

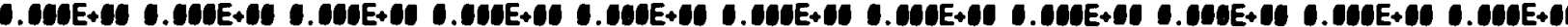

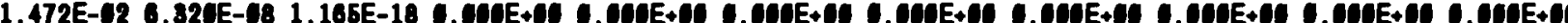
CISE+CS

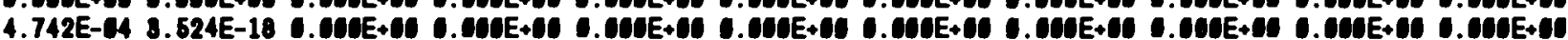

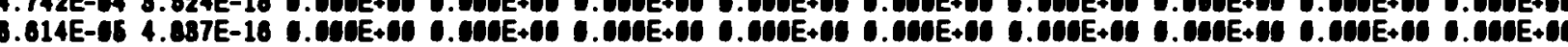

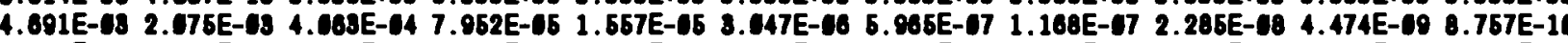

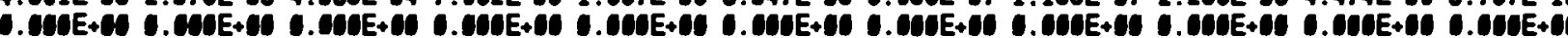
$6.288 E+11$ 8.070E-14 3.078E-14 3.078E-04 3.077E-14 3.076E-14 8.076E-104 8.074E-04 8.073E-04 3.072E-04 3.071E-04

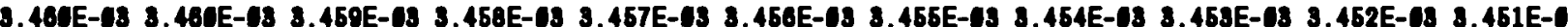

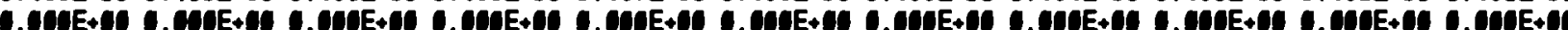

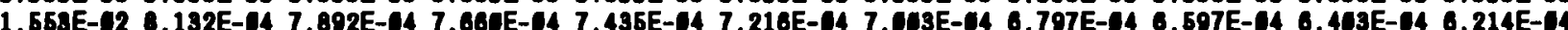

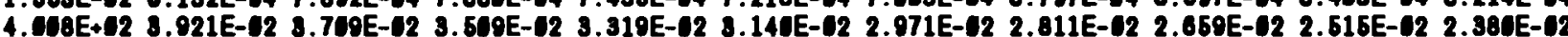

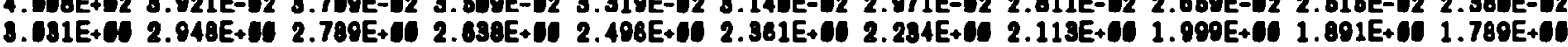

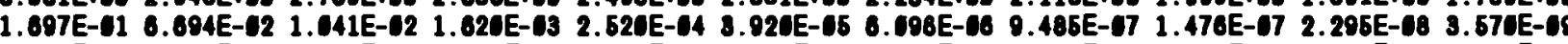

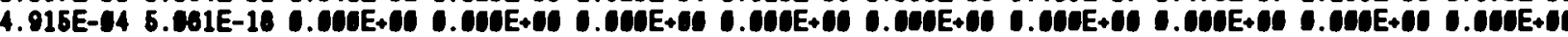

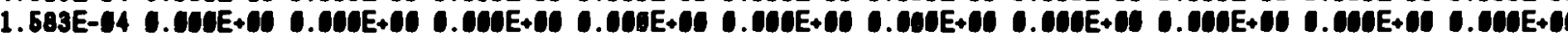

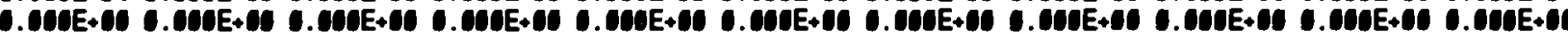

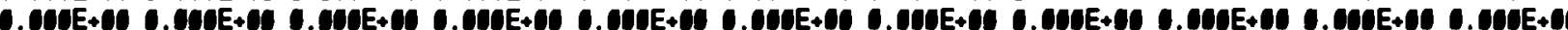
(1) (6.214E-64

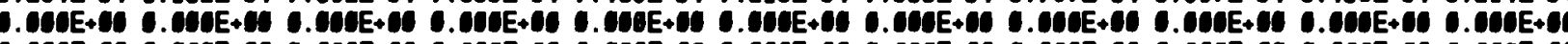

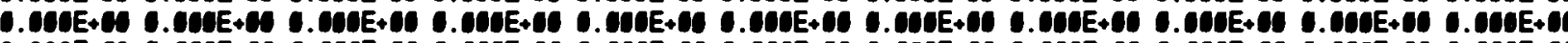

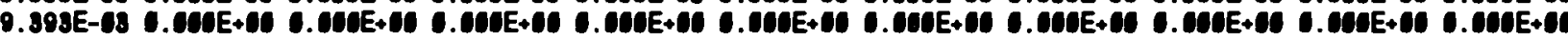

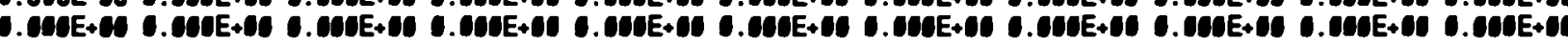

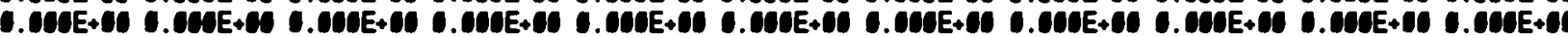

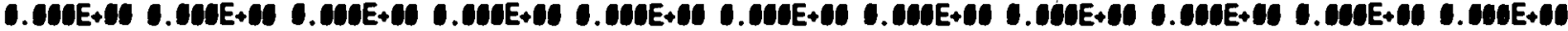

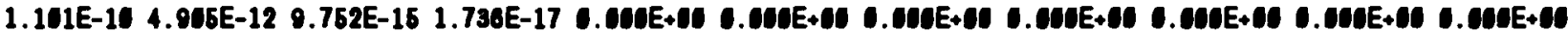
1. 648E-12 1.410E-12 1.034E-12 7.574E-18 5. 551E-18 4.068E-18 2.981E-18 2.184E-18 1.651E-13 1.173E-13 8.598E-14

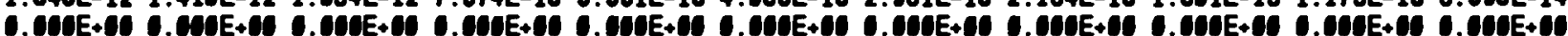

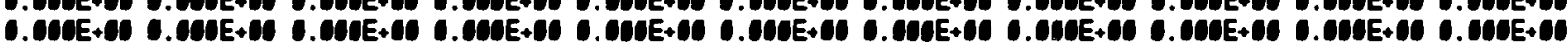

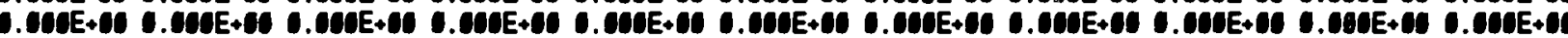

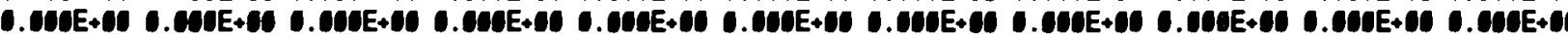

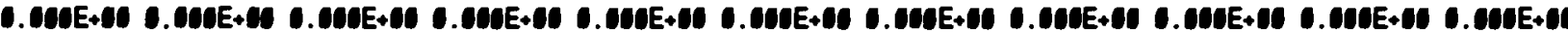
D. COAE .

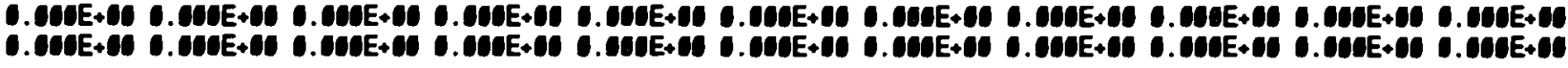




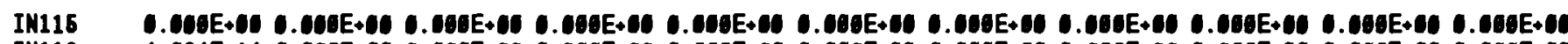

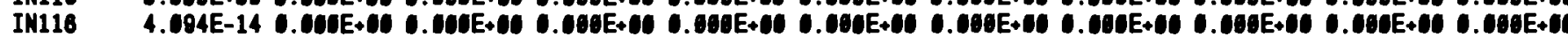
IM118U 3.228E-14 Actinides + Daughters:

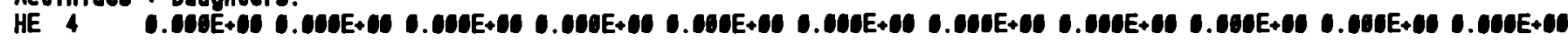

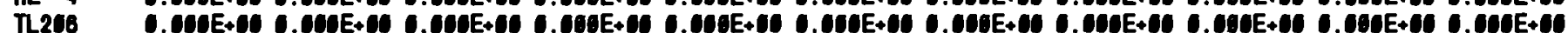

TL201 1.0018 .

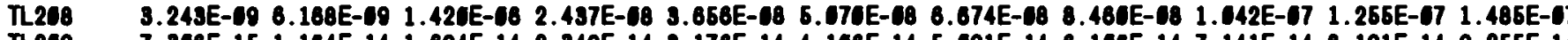

TL200 7.308E-16 1.164 E-14 1.694E-14 2.349E-14 3.176E-14 4.166E-14 5.601E-14 6.106E-14 7.141E-14 8.191E-14 9.256E-14

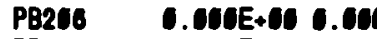

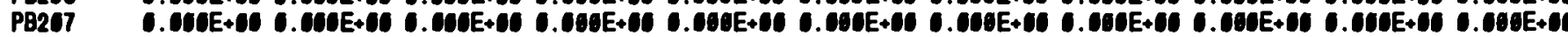

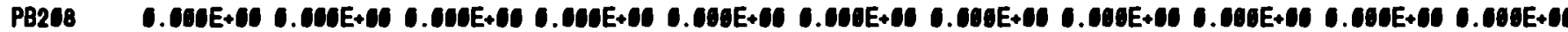

PB200 3.383E-13 $5.387 E-13$ 7.842E-13 $1.088 E-12$ 1.470E-12 $1.981 E-12 \quad 2.357 E-12$ 2.827E-12 $3.398 E-12 \quad 3.792 E-12$ 4.285E-12

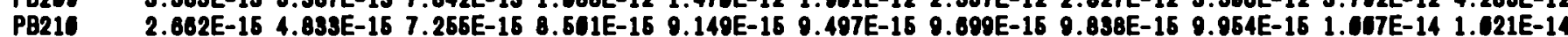

PB211 2.862E-16 $1.033 E-16$ 7.256E-16 8.861E-16 9.149E-16 9.497E-16 9.690E-16 9.838E-16 9.964E-16 $1.017 \mathrm{C}-14$ 1.021E-14

PB211 1.438E-11 2.026E-11 $6.011 \mathrm{E}-11$ 1.273E-16 2.160E-16 3.296E-16 4.716E-16 6.411E-16 8.384E-16 1.064E-69 1.318E-68

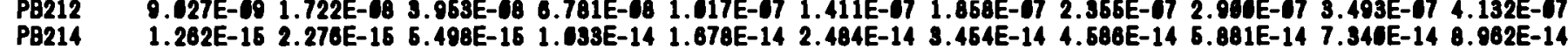

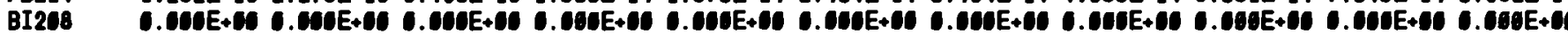

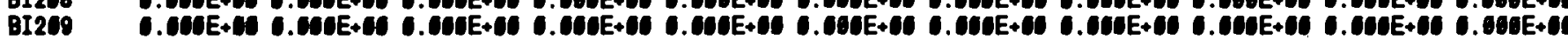

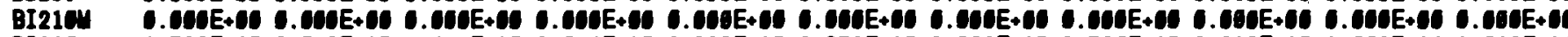

BI210 1.736E-16 3.628E-16 6.461E-16 8.084E-16 8.932E-15 9.378E-16 9.628E-16 9.790E-16 9.912E-16 $1.013 \mathrm{EE}-141.016 \mathrm{E}-14$

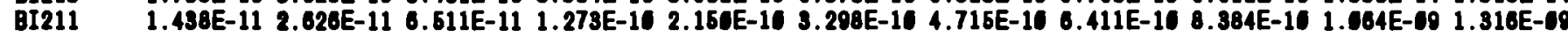

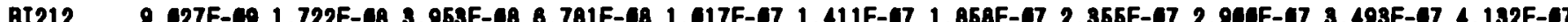

BI214 1.202E-16 2.270E-16 $5.408 E-16$ 1.033E-14 1.678E-12 $2.001 E-12$ 2.367E-12 $2.827 E-12$ 3.308E-12 $8.702 E-124.285 E-12$

P0216 7.353E-17 1.978E-16 6.692E-16 1.358E-15 1.999E-15 2.684E-15 3.335E-15 3.943E-15 4.508E-15 5.027E-15 5.510E-16

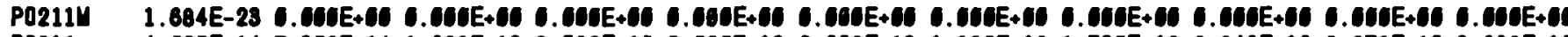

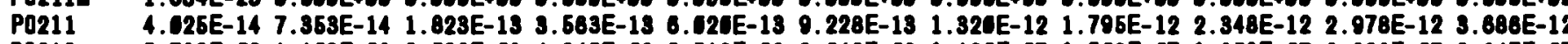

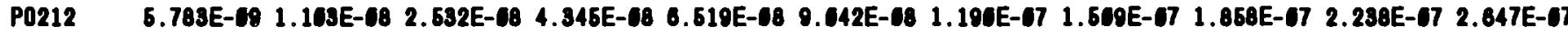

P0213 3.369E-13 6.271E-13 7.672E-13 1.064 E-12 $1.439 E-12$ 1.86EE-12 2.308E-12 2.786E-12 3.234E-12 3.710E-12 4.192E-12

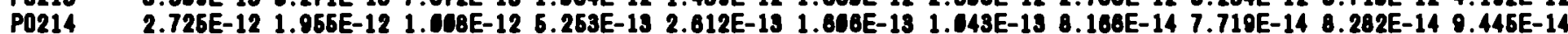

P0215 1.438E-11 2.026E-11 6.511E-11 $1.273 E-10$ 2.160E-10 3.298E-10 4.716E-10 6.411E-16 8.384E-10 $1.084 E-001.316 E-69$

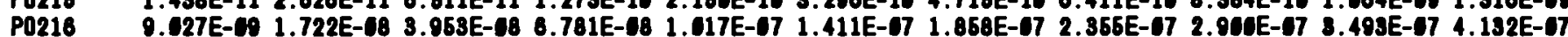

P0218 1.262E-16 $2.276 E-16$ 6.600E-16 $1.033 E-14$ 1.678E-14 2.486E-14 $3.464 E-14$ 4.687E-14 $6.882 E-14$ 7.341E-14 8.984E-14

AT217 3.382E-13 5.387E-13 7.842E-13 $1.088 E-12$ 1.47EE-12 $1.901 E-12 \quad 2.357 E-12$ 2.827E-12 $3.348 E-12 \quad 3.792 E-12$ 4.286E-12

RN218 2.724E-12 $1.953 E-12$ 1.003E-12 $5.150 E-13$ 2.845E-13 $1.358 E-13$ 6.974E-14 $3.581 E-14$ 1.839E-14 $9.443 E-15$ 4.849E-15

RN219 1.438E-11 2.028E-11 6.611E-11 1.273E-10 2.160E-10 3.296E-10 4.716E-16 6.411E-16 8.384E-10 1.064E-69 1.316E-09

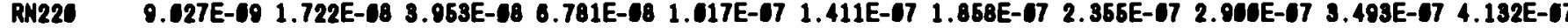

RN222 1.262E-15 2.276E-15 5.409E-15 1.033E-14 1.678E-14 2.486E-14 3.454E-14 4.587E-14 5.882E-14 7.341E-14 8.964E-14

FR221 8.382E-13 5.387E-13 7.842E-13 $1.088 E-12$ 1.470E-12 $1.901 E-12$ 2.357E-12 2.827E-12 $3.308 E-12 \quad 3.792 E-12$ 4.285E-12

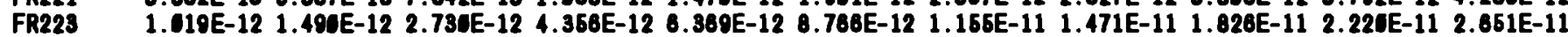

RA222 2.724E-12 $1.963 E-12$ 1.003E-12 $5.160 E-13$ 2.846E-13 $1.358 E-13$ 8.074E-14 $3.681 E-14$ 1.839E-14 $9.443 E-16$ 4.849E-16

RA223 1.438E-11 2.028E-11 6.611E-11 $1.273 \mathrm{E}-10$ 2.160E-10 3.208E-10 4.716E-16 6.411E-10 8.384E-10 1.084E-60 $1.316 \mathrm{E}-08$

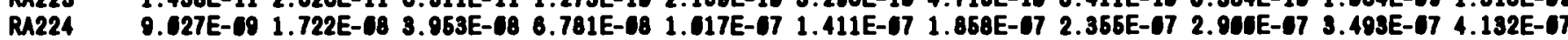

RA225 7.495E-13 7.468E-13 9.696E-13 1.339E-12 $1.787 \mathrm{E}-12$ 2.223E-12 2.691E-12 3.169E-12 3.854E-12 4.145E-12 4.642E-12

RA226 1.716E-15 2.946E-15 6.608E-15 1.189E-14 1.878E-14 2.730E-14 3.744E-14 4.022E-14 6.262E-14 7.768E-14 9.434E-14

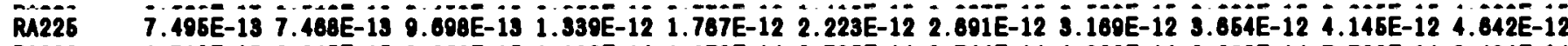

RA226 1.718E-16 2.946E-16 6.608E-16 1.189E-14 1.878E-14 2.730E-14 3.744E-14 4.922E-14 8.262E-14 7.788E-14 9.434E-14

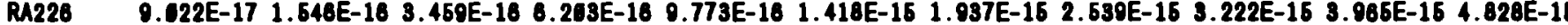

AC225 3.382E-13 5.387E-13 7.841E-13 1.087E-12 1.47 EE-12 $1.951 E-12 \quad 2.357 E-12$ 2.827E-12 $3.348 E-12 \quad 3.792 E-124.284 E-12$

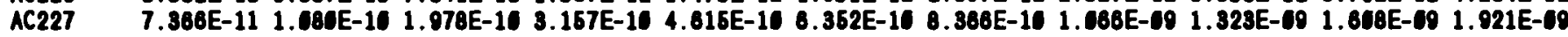

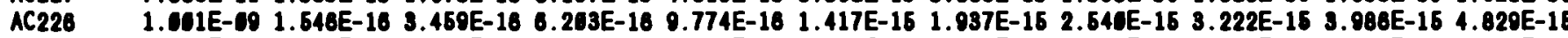

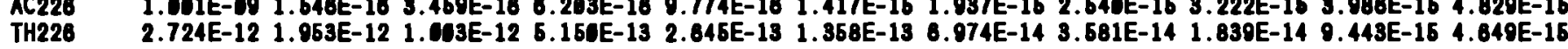




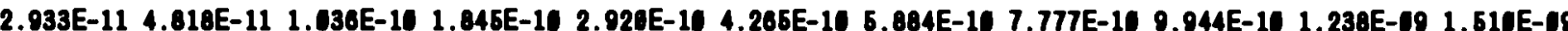

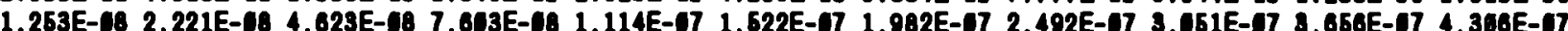

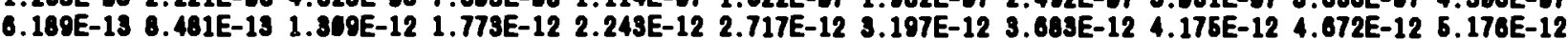

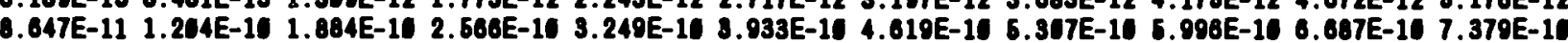

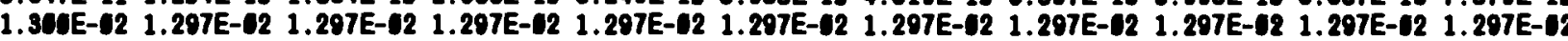

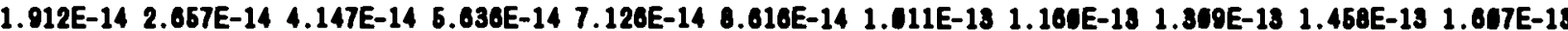

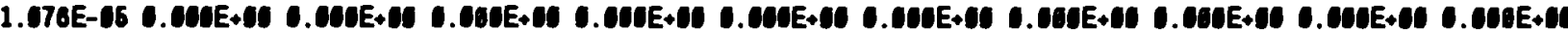

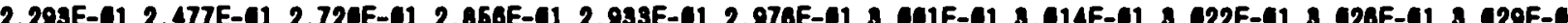
.

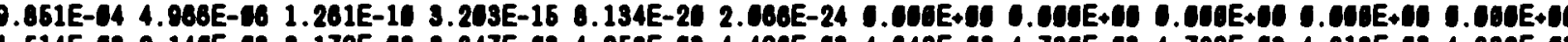

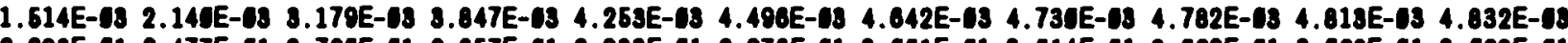
2.293E-01 2.477E-01 2.720E-01 2.867E-01 2.933E-01 2.070E-01 3.001E-01 3.014E-01 s.022E-01 8.026E-01 3.029E-01

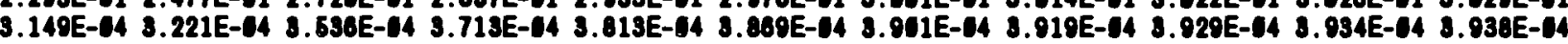

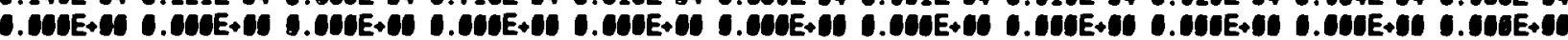

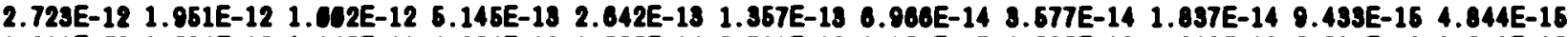
$1.611 E-60$ S 1.011E J.

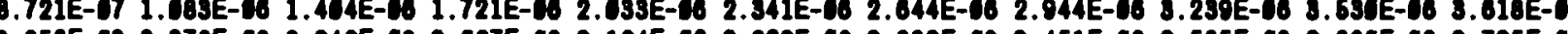

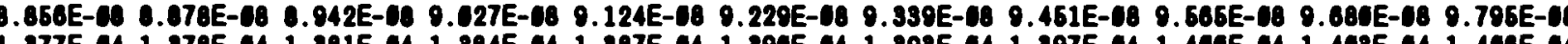

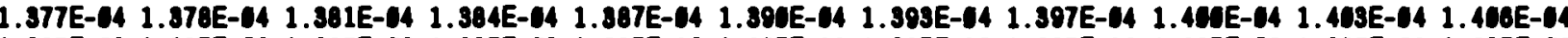

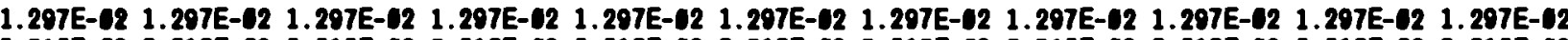

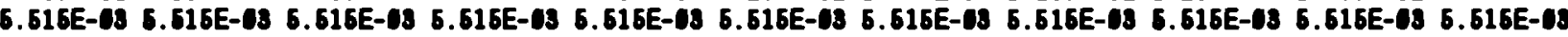

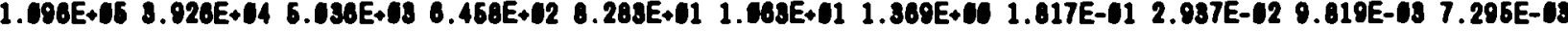

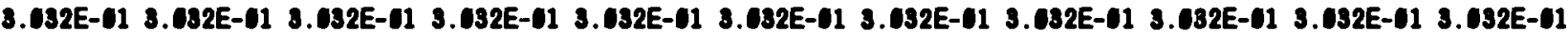

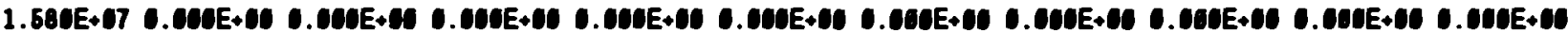
$1.680 E+17$. 8.468E+11 2.698E-14 6.905E-13 6.810E-13 6.818E-13 5.818E-13 6.818E-13 6.818E-13 6.818E-13 $5.818 E-13$ 5.818E-13

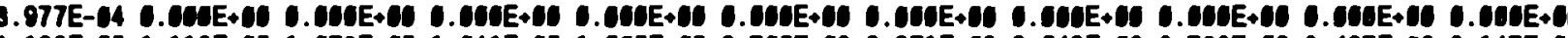

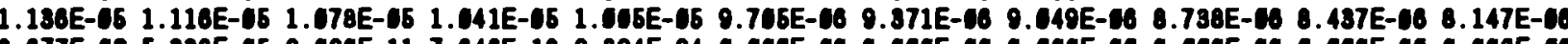

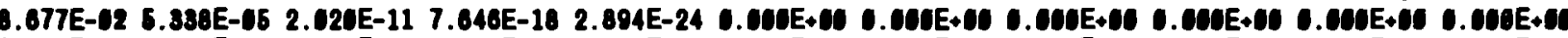

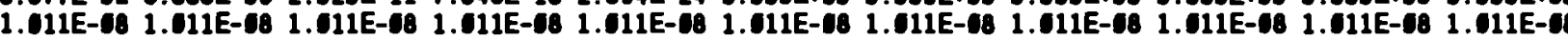

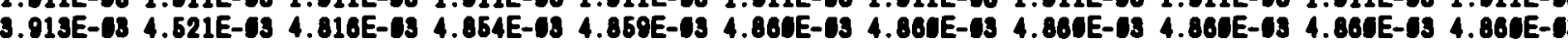
3.305E+08 1.251E+12 1.850E-01 2.679E-04 1.102E-16 7.360E-07 7.362E-07 7.361E-07 7.349E-07 7.347E-07 7.346E-07

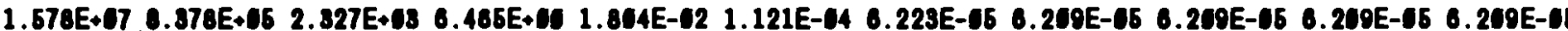

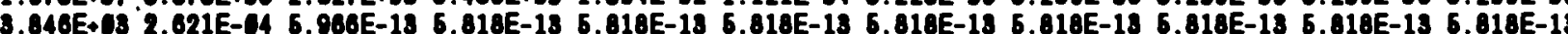

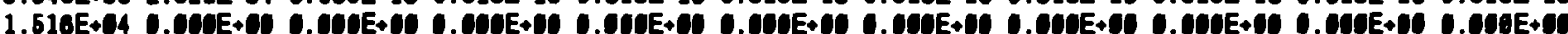

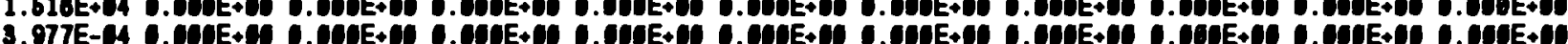

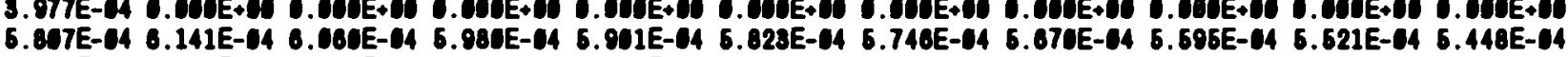
4.123E-08 3.642E-18 2.013E-18 1.028E-08 1.423E-08 1.060E-08 7.746E-14 5.716E-04 4.217E-04 3.111E-04 2.290E-04

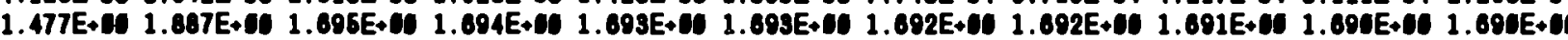

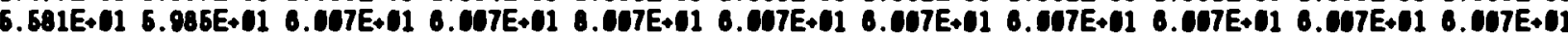

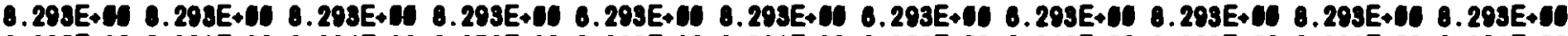
2.805E+12 2.091E+12 2.884E+12 2.876E+12 2.889E+12 2.861E+12 2.853E+12 2.846E+12 2.638E+12 2.031E+02 2.024E+12

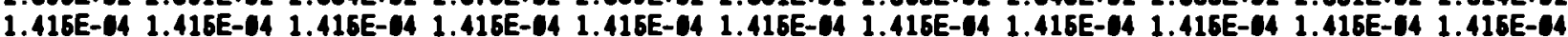

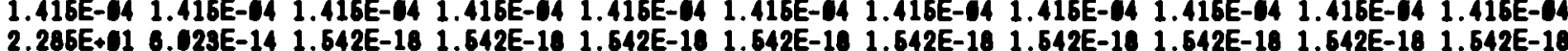

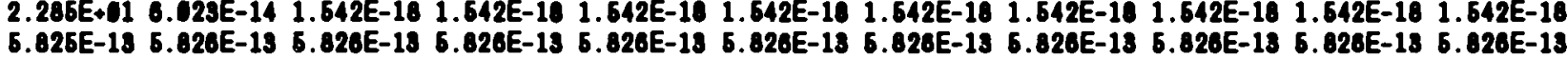

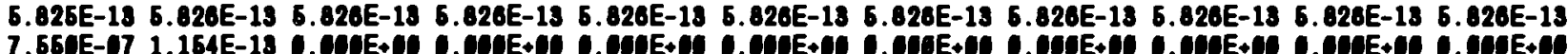

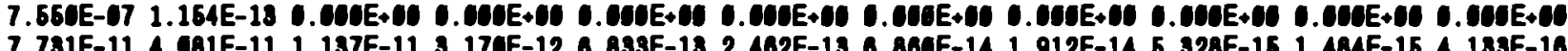
7.731E-11 4.081E-11 1.137E-11 3.170E-12 6.833E-13 2.462E-13 6.860E-14 1.912E-14 $5.328 E-16$ 1.484E-15 4.133E-16

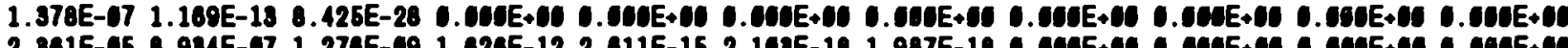

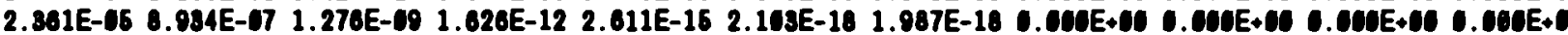
1.583E-02 2.853E-02 5.388E-02 7.016E-02 $1.044 E-01$ 1.295E-01 $1.546 E-01$ 1.706E-01 2.046E-01 2.294E-01 2.542E-01

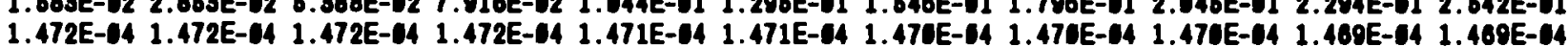

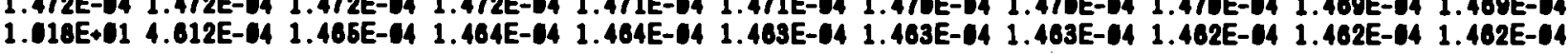




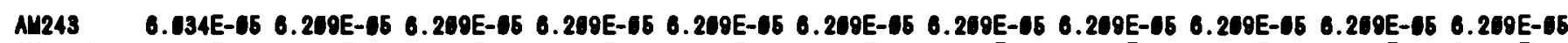

AN244I

AN244

AM245

AM246

Cu241

CH242

Cu243

Cu244

CU245

CH246

CU247

C)248

Cu249

CM25:

Cu251

BK249

BK250

BK251

CF249

CF250

CF251

CF252

I

CF253

CF254

CF255

ES2544

ES254

ES255
SF25

FF25:

H 8

LI 6

LI 7

BE 9

BE 10

C 14

NI 68

CU 86

2N 86

CU 67

IN 67

2N 68

2N 89

2N 894

24 68

2N 69

2N 69

an 69

2N 70

a 70

OE 70

2N 71

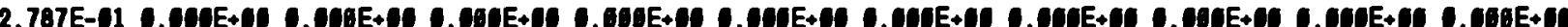

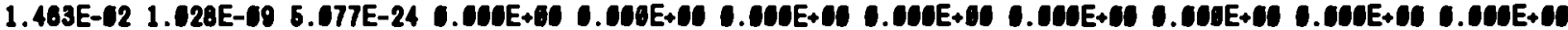
7. $550 \mathrm{E}-07$ 1.434E-13 $5.846 \mathrm{E}-22$ 5.692E-22 $5.350 \mathrm{E}-22$ 5.118E-22 $4.897 \mathrm{E}-22$ 4.085E-22 $4.482 \mathrm{E}-22$ 4.287E-22 $4.102 \mathrm{E}-22$ 7.734E-11 4.088E-11 1.139E-11 3.176E-12 8.847E-13 2.408E-13 0.871E-14 1.916E-14 5.337E-16 $1.487 E-16$ 4.142E-16

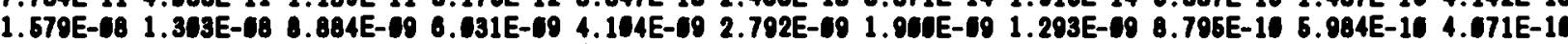

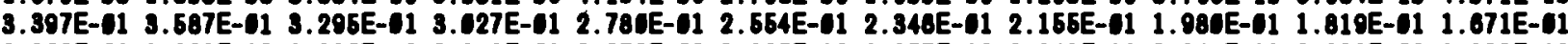

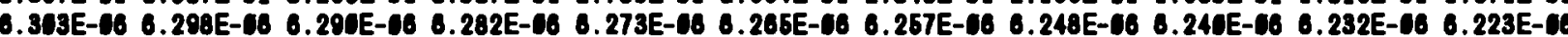

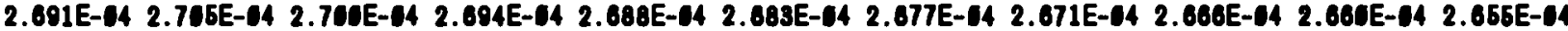

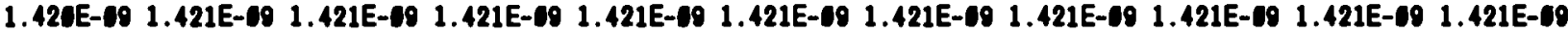

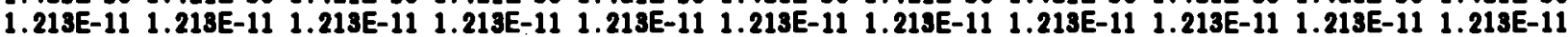

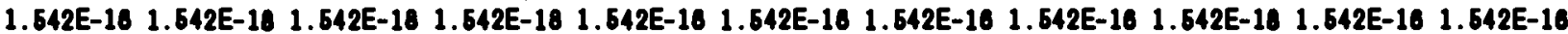

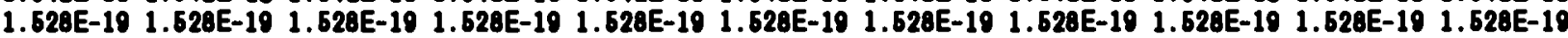

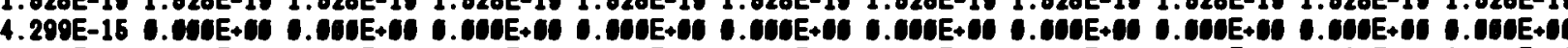

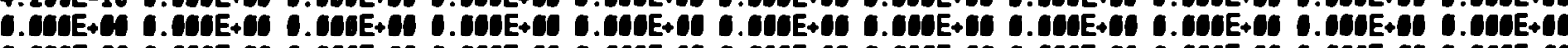

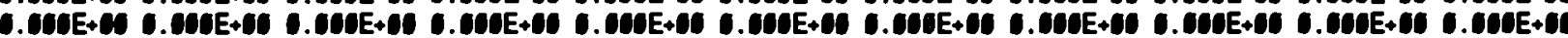
$4.242 E-17$ 4.260E-17 4.026E-17 3.852E-17 3.085E-17 3.620E-17 3.373E-17 3.227E-17 3.087E-17 2.953E-17 2.825E-17

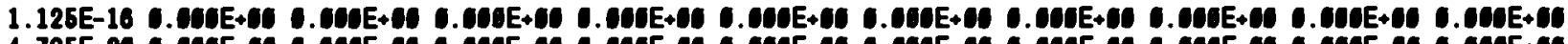

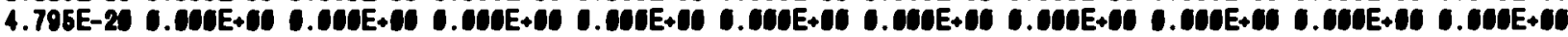

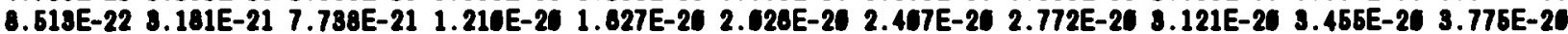
. (.040 .

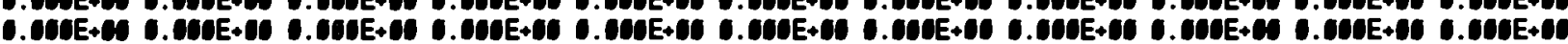

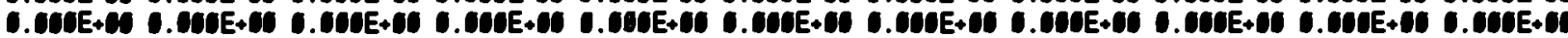

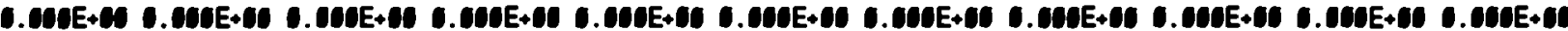

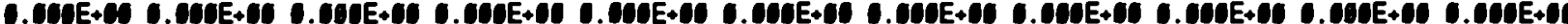

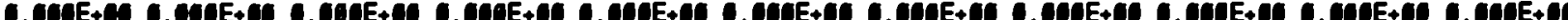
-

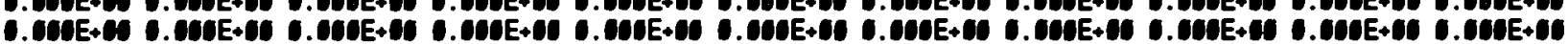
Products:

$6.548 E+408.635 E+108.609 E+108.483 E+108.468 E+108.431 E+09$

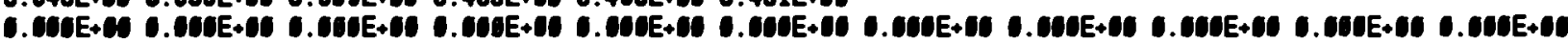

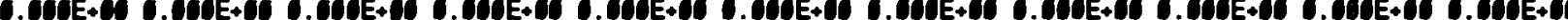

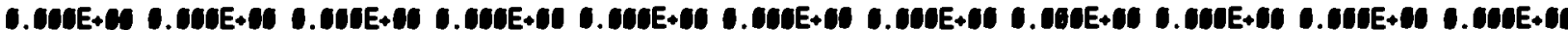

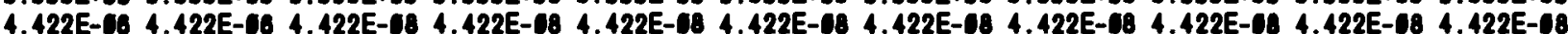

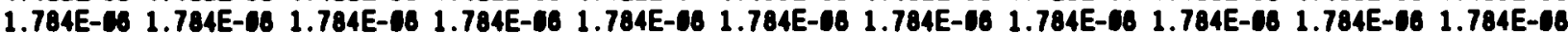
1.00E+CS

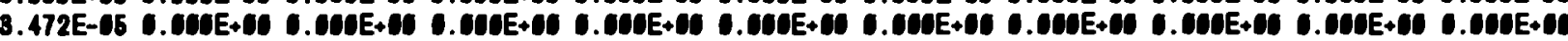

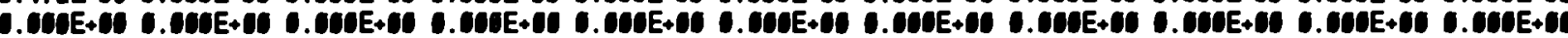

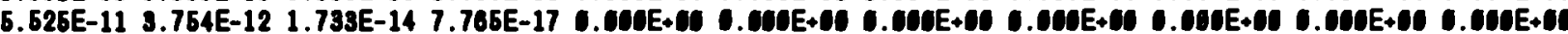

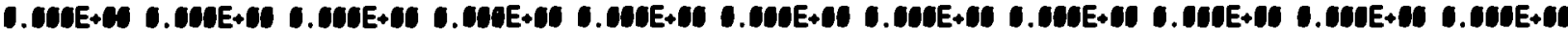

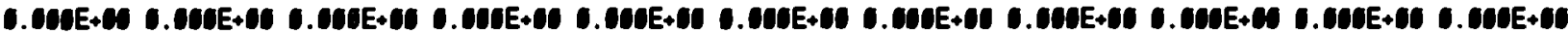

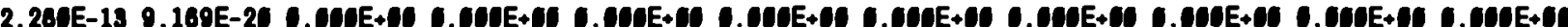

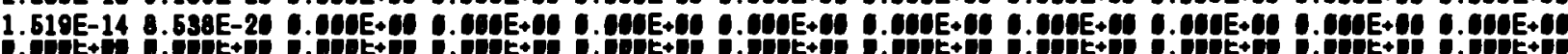

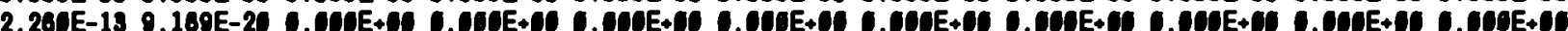

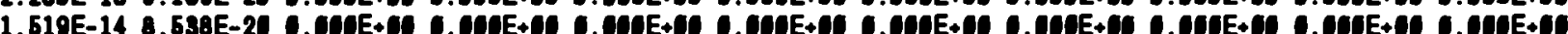

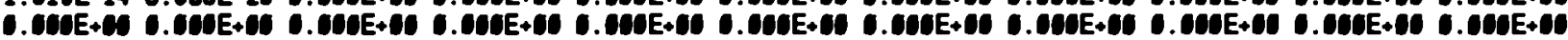

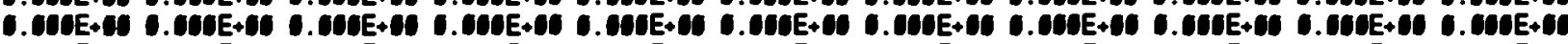

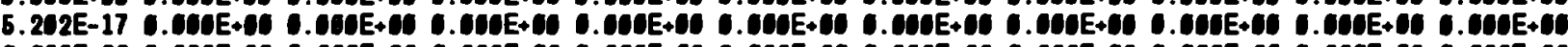

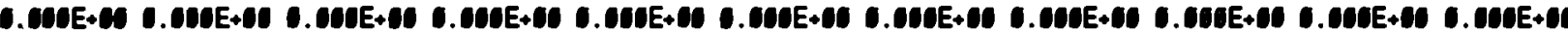

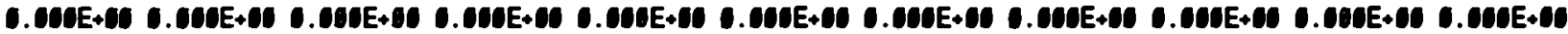




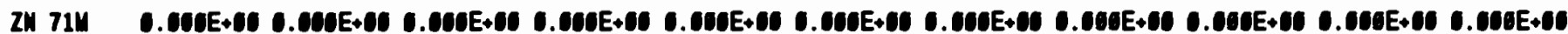
Q 71 G

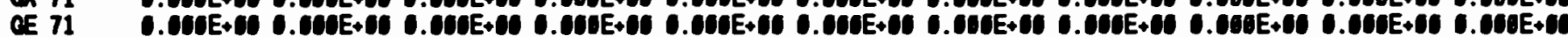

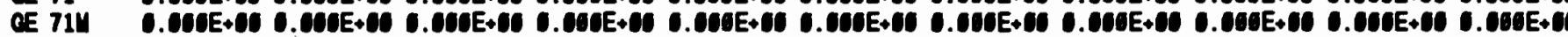

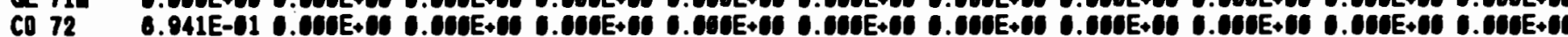

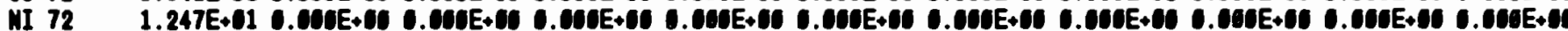

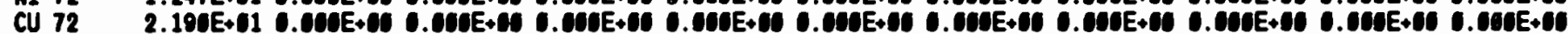

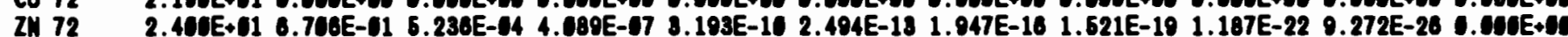

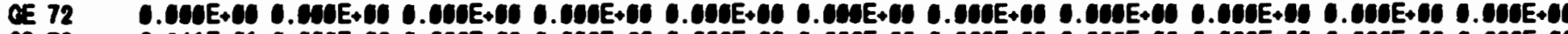

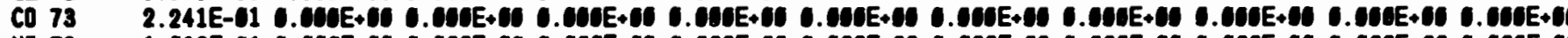

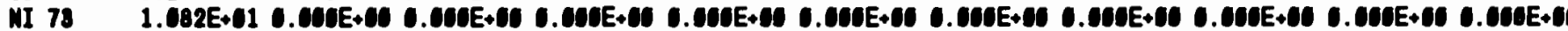

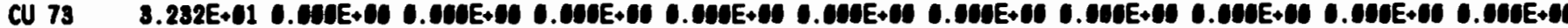

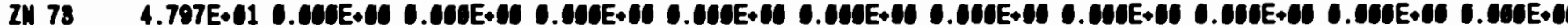

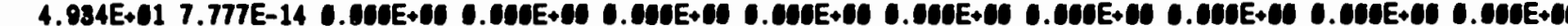

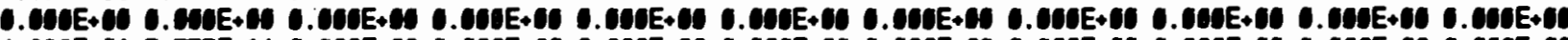

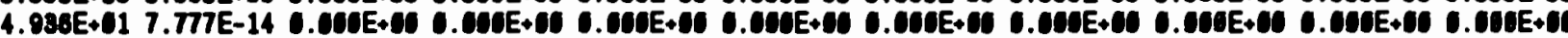

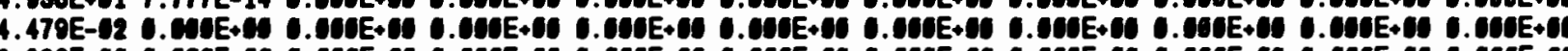

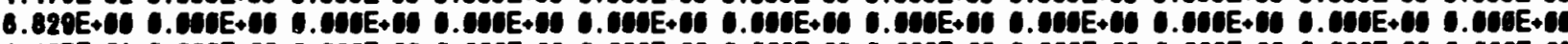

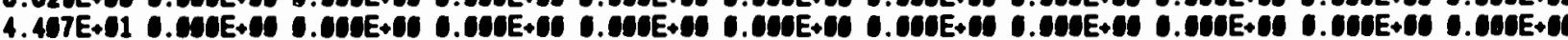

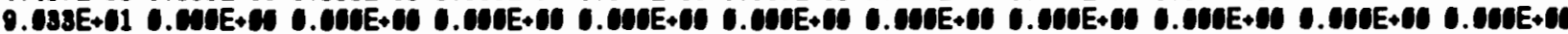

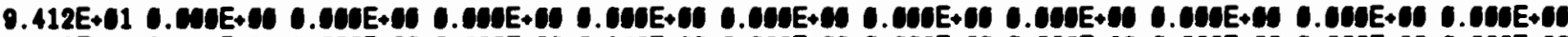

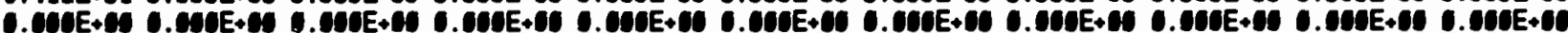

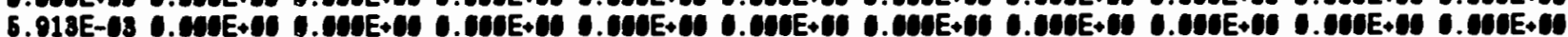
2.765E+C . . $2.766+1$. . $4.463 E+91$
$1.705 E+12$

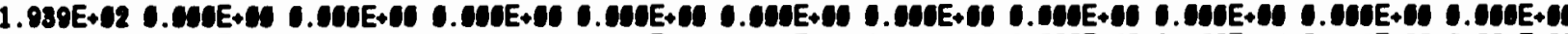

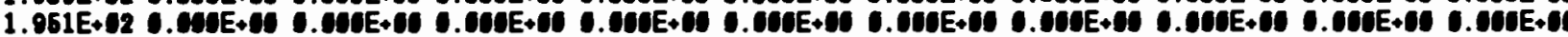

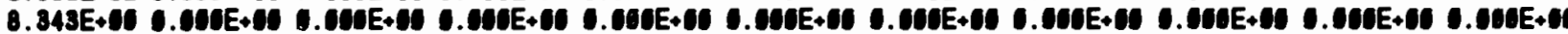

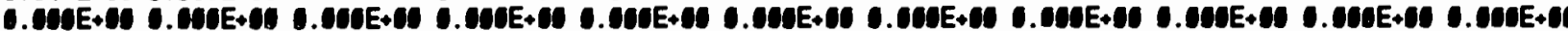

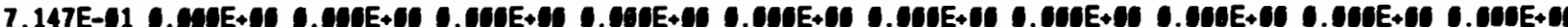

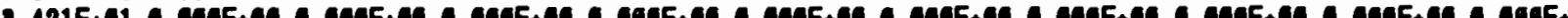
3.062E+02 0.

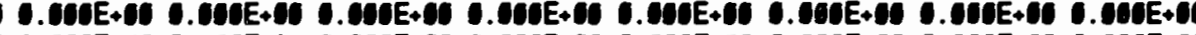

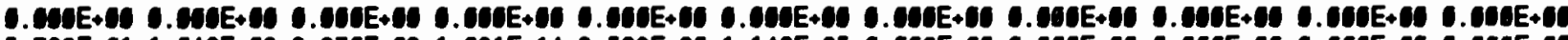

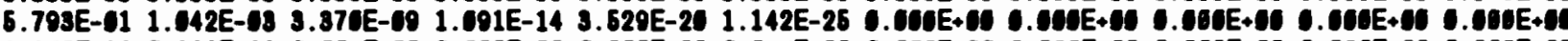

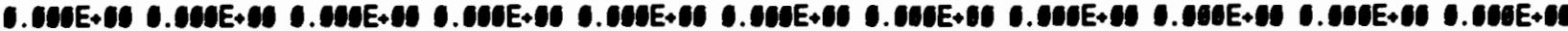

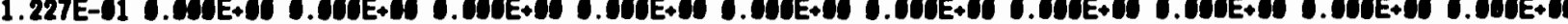

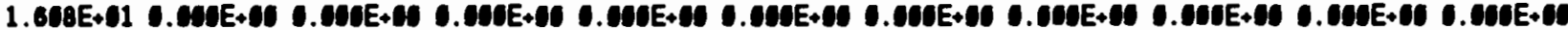

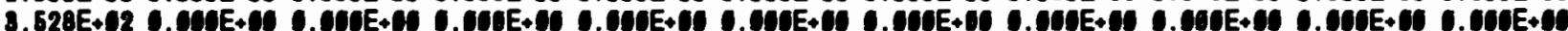

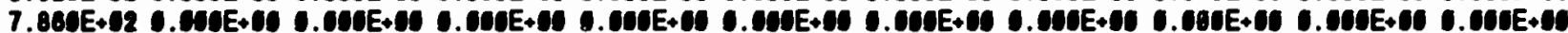

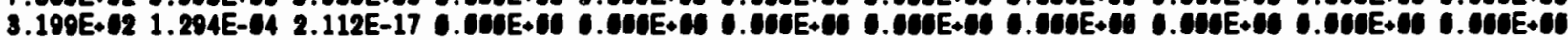

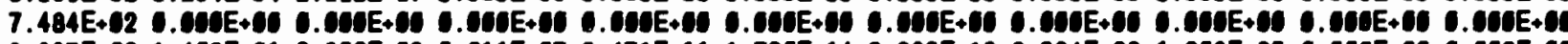

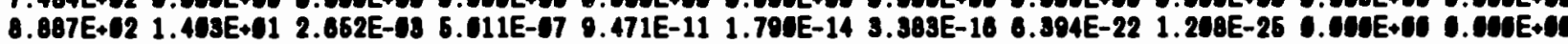

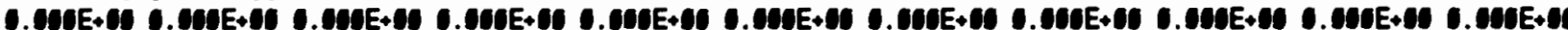

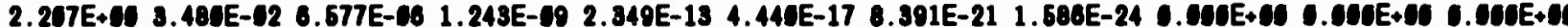

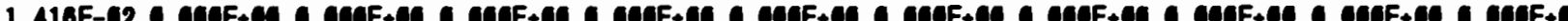

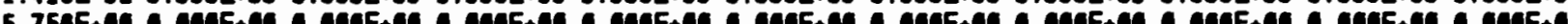
6.760E+

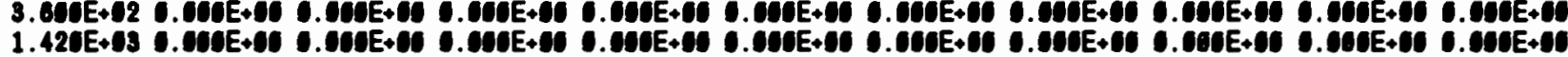




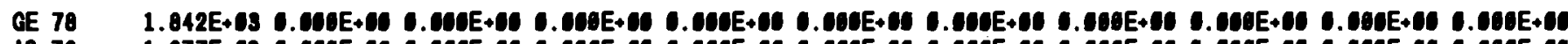

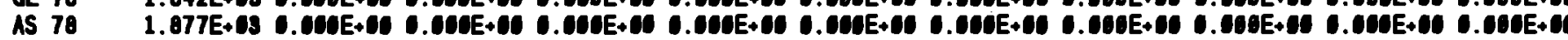

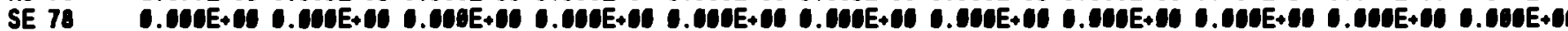

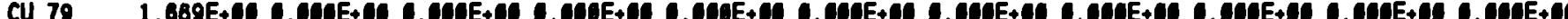

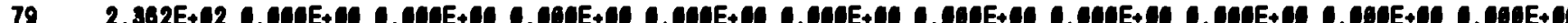

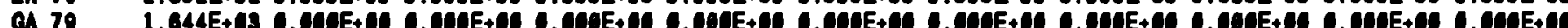

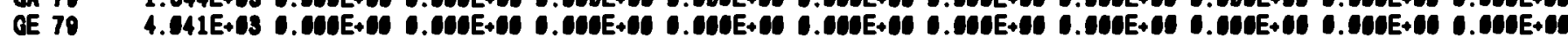

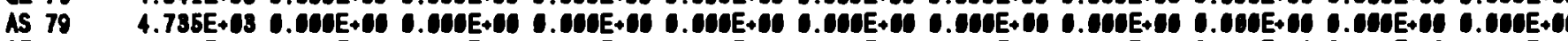

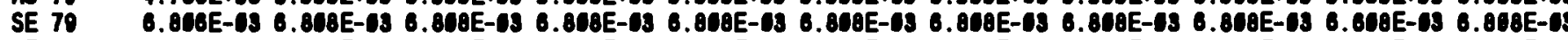

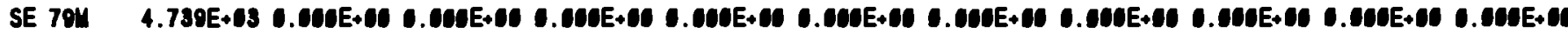

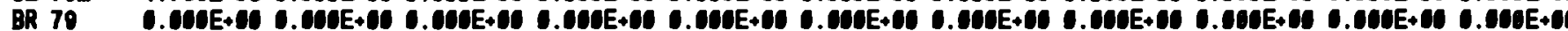

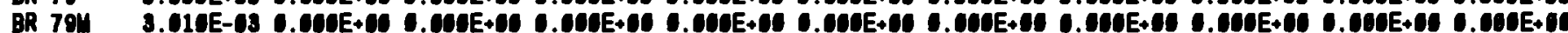
KR $79 \quad .732 E-195.728 E-11$ 4.147E-15 3.003E-19 $2.174 E-23$. O.DOUE 1.77EE-1 . .

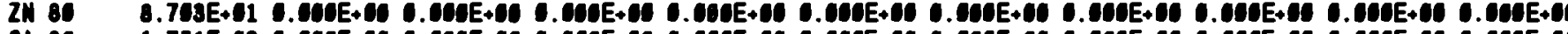

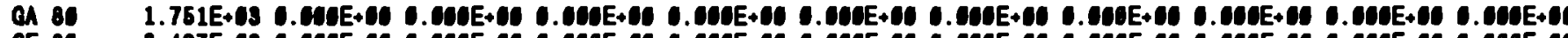

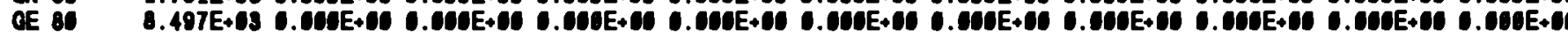

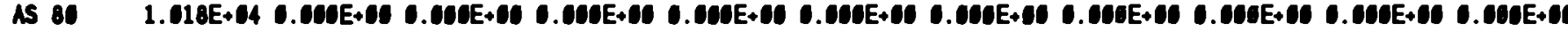

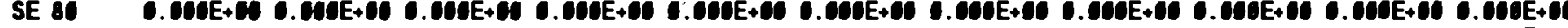

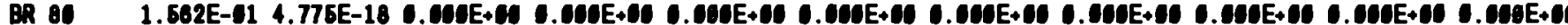
BQ $8 \mathrm{MM}$ O

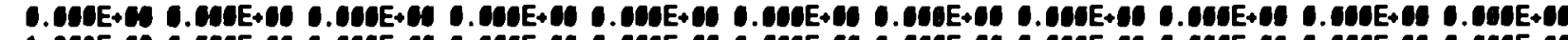
1.398E-12 :.9SE+10

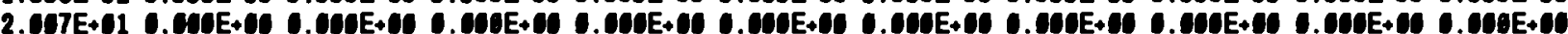

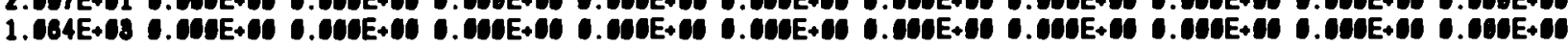

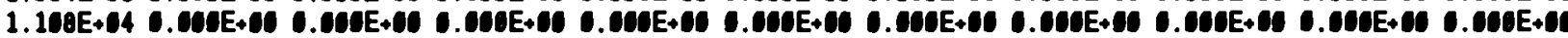

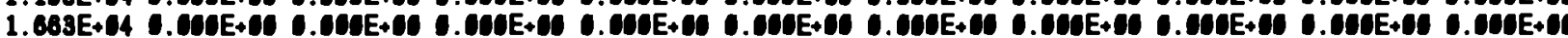

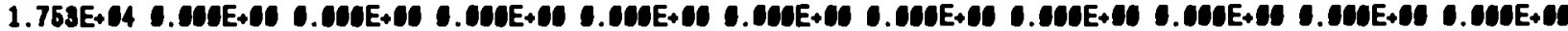

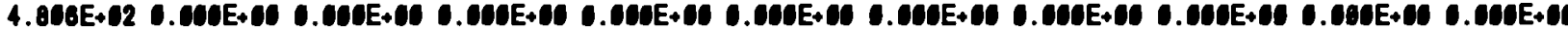

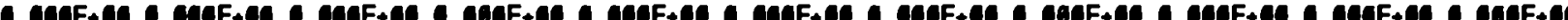
$1.731 E-09$ 1.731E-09 1.731E-09 1.731E-09 $1.731 E-09$ 1.731E-09 $1.731 E-09$ 1.731E-09 $1.731 E-091.731 E-091.731 E-09$

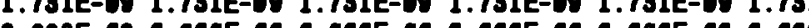

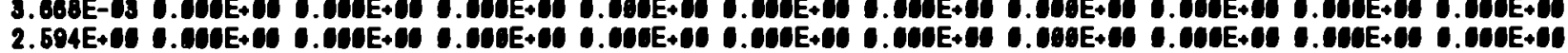

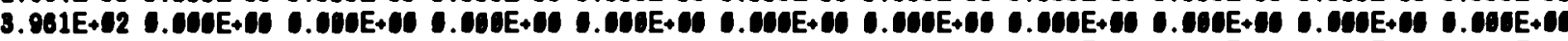

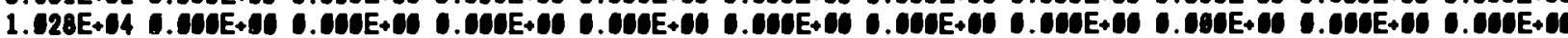

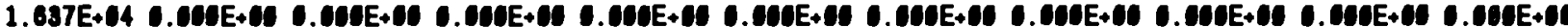

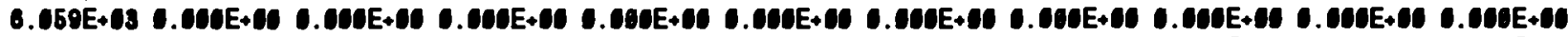

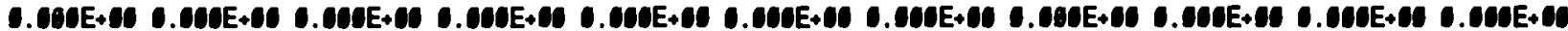

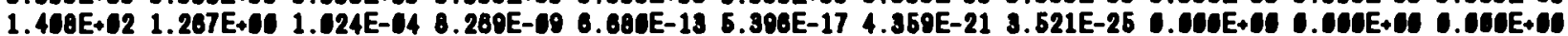

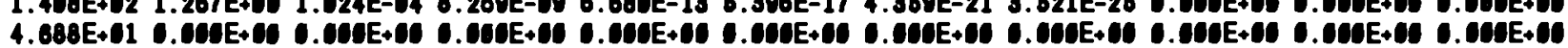

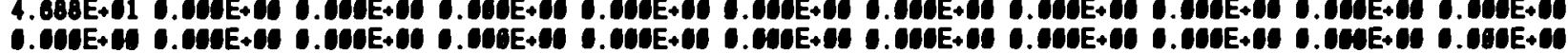

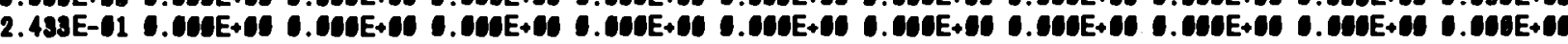

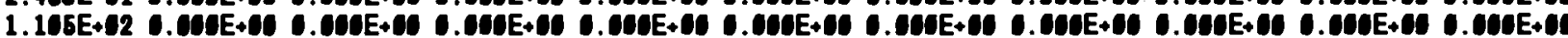

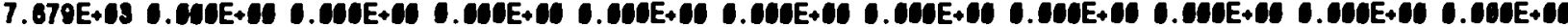

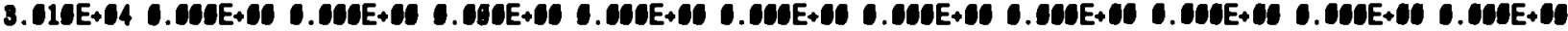

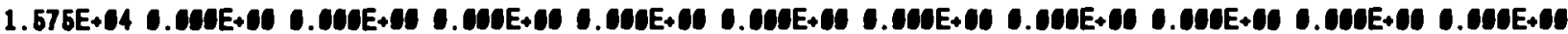

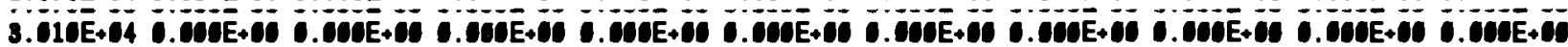

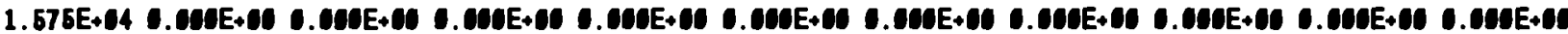

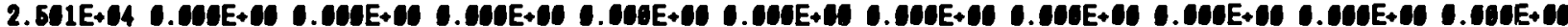

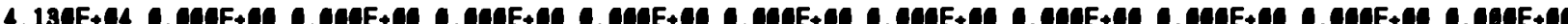
-. C.

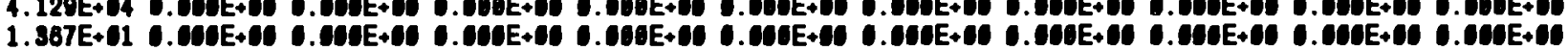




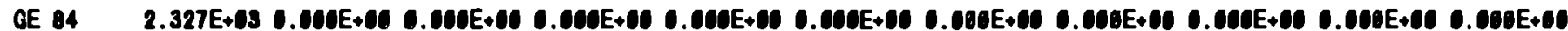
IS 84 2.

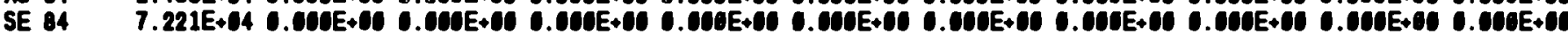

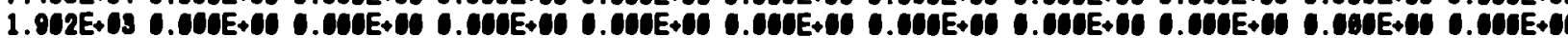

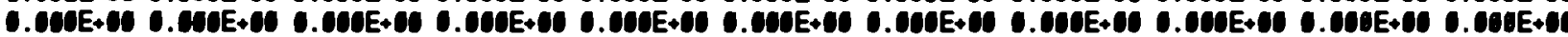

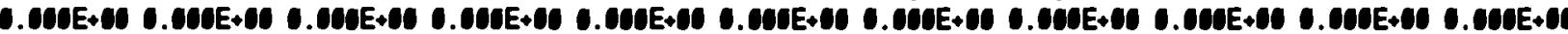
B. 190 E.02 (1515E.04 O.

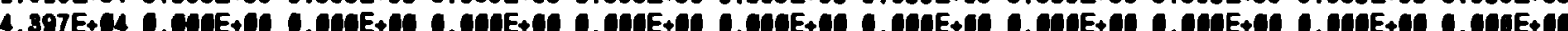

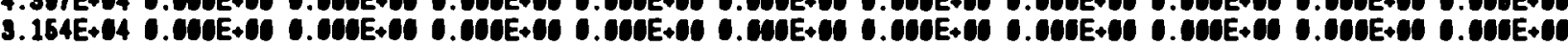
$8.927 E+14$ 0.00E+01 $1.748 E+12 \quad 1.754 E+12 \quad 1.748 E+12 \quad 1.742 E+12 \quad 1.736 E+12 \quad 1.730 E+12 \quad 1.724 E+62 \quad 1.718 E+021.711 E+12 \quad 1.705 E+121.099 E+102$

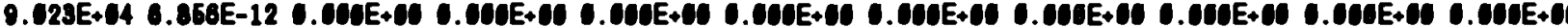

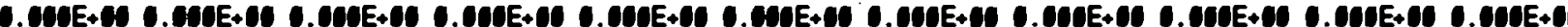
$1.101 E+20$.

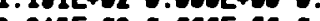

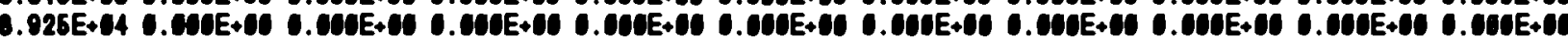
$6.679 E+14$.CDE+ $0.021 E+C 4$ J.WOE+CS

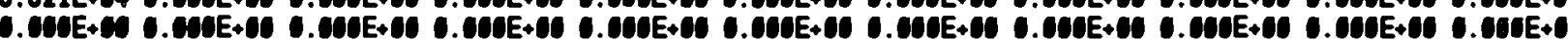

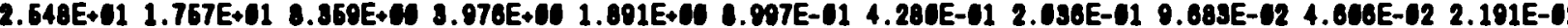

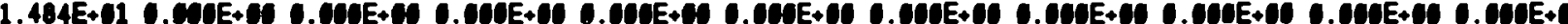
$1.712 E+119$.

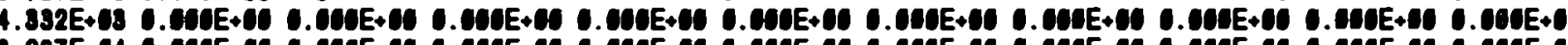

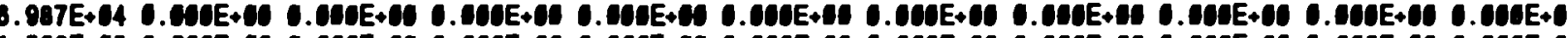

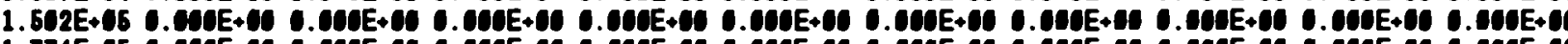

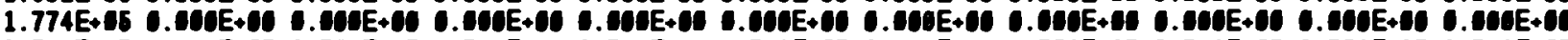

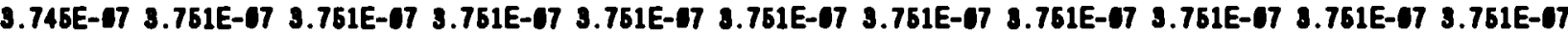

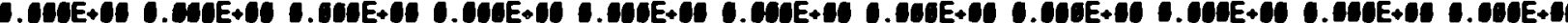

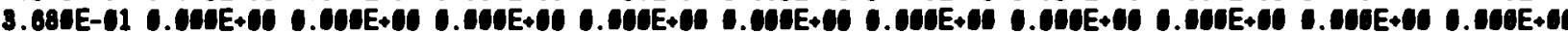

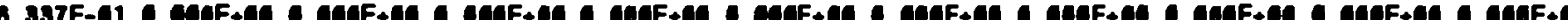

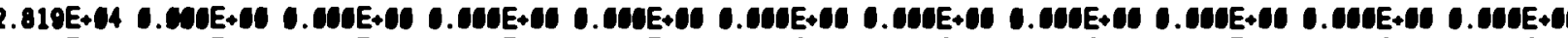

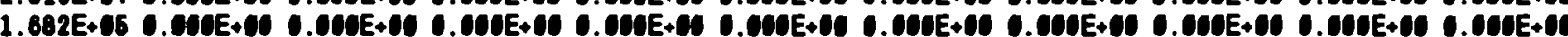

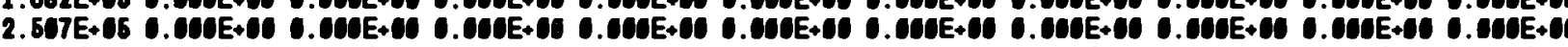

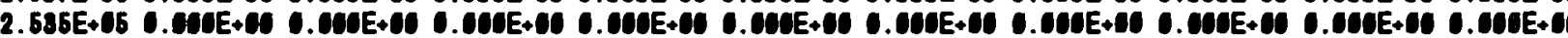

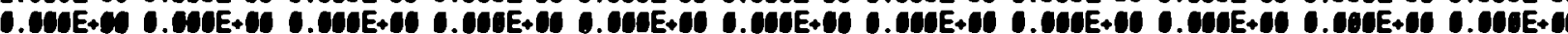

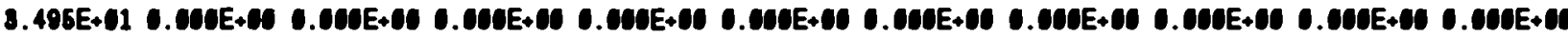

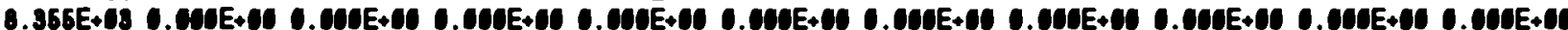
$1.244 E+15$.

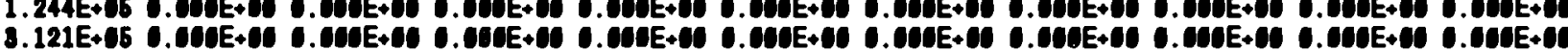

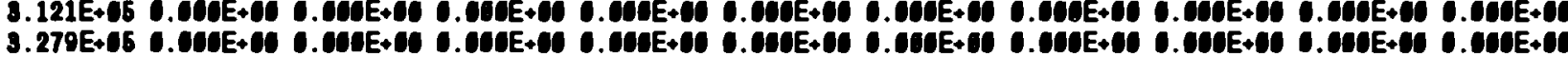

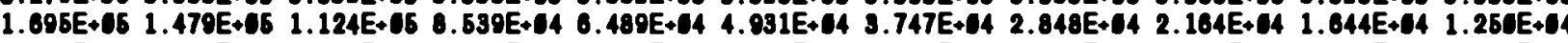

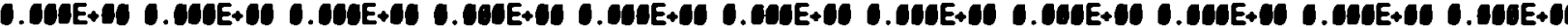

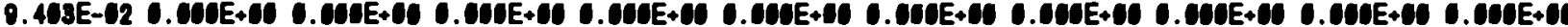

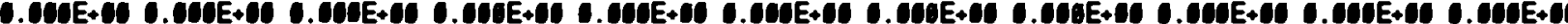

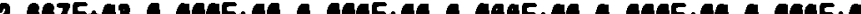
2.007E

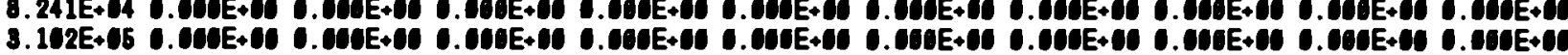


RB 95

RB OOU

SR 96

$Y 9$

Y ovy

ZR 90

ZR OUY

SE 91

BR 91

KR 91

RB 91

SR 91

$Y 01$

Y 911

2R 91

NB 91

SE 92

BR 92

KR 92

RP 92

Y 92

ZR 92

NB 92

SE 93

BR 93

KR 98

RB 98

SR 98

$Y 98$

ZR 98

NB 98

NB 994

BR 94

KR 94

RB 94

SR 94

$Y 94$

Y 94

ZR 94

NB 94

NB 941

BR 95
KR 95

RB 95

SR 95

Y 95

3R 95

$Y 95$

ZR 95

NB 95

NB 95M

MO 95
BR 96

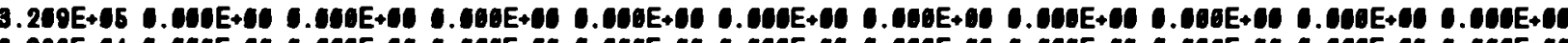

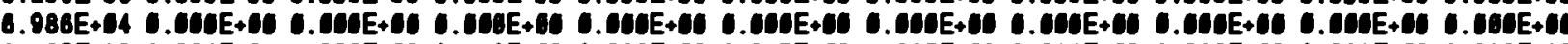
$1.326 E+031.324 E+03 \quad 1.322 E+031.321 E+031.319 E+031.317 E+03 \quad 1.316 E+031.314 E+031.312 E+031.310 E+031.300 E+03$

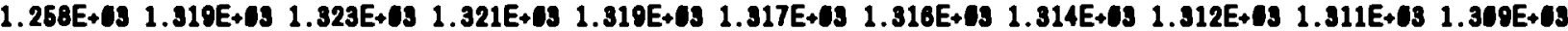

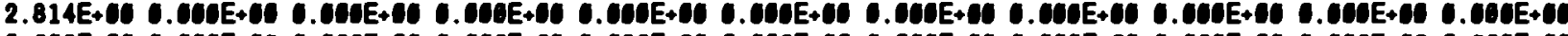

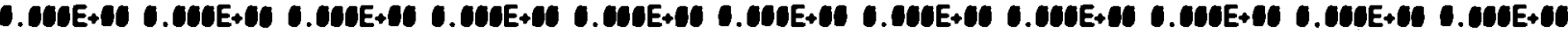

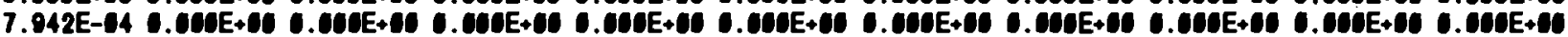

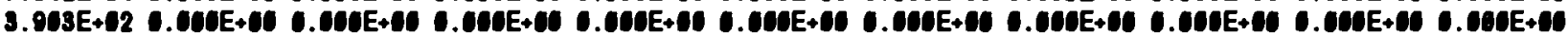

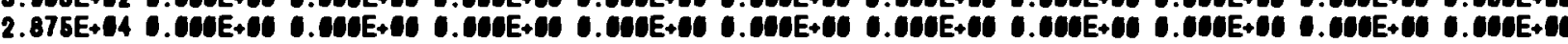

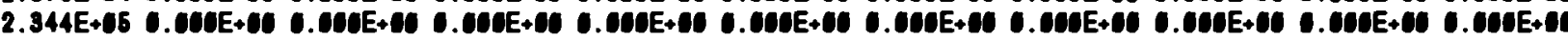

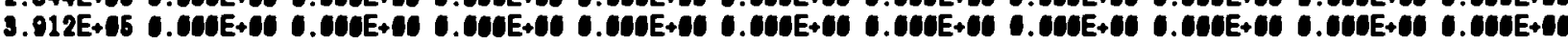

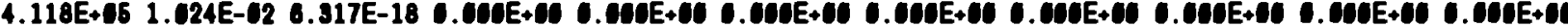

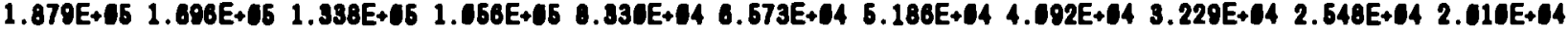

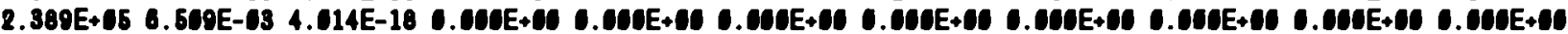
0.000 6.

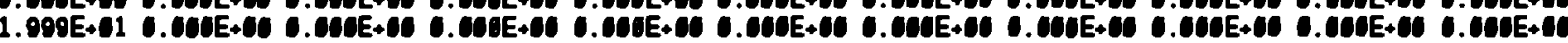

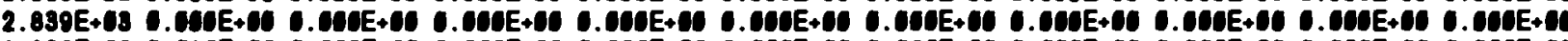

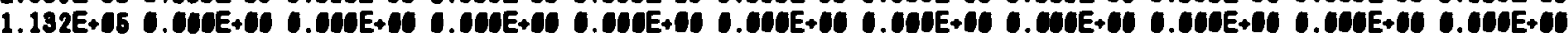

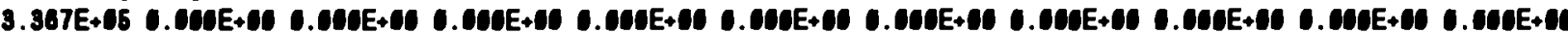

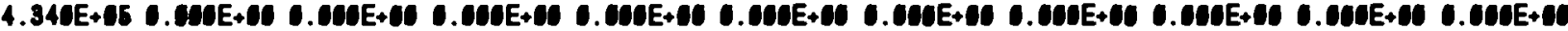

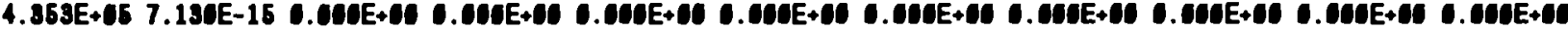
1.

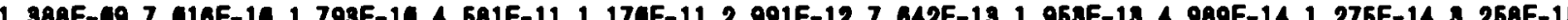
(1)

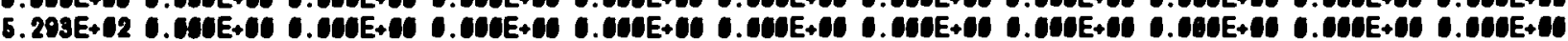

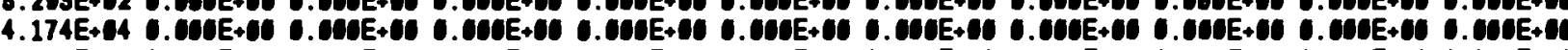

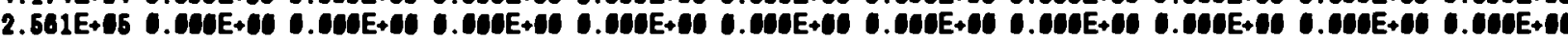

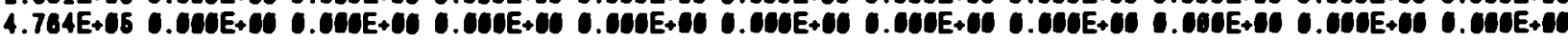

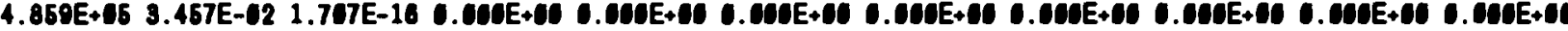

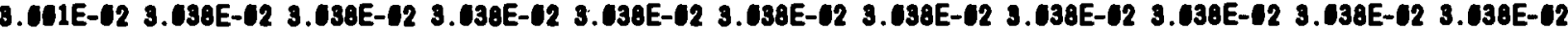

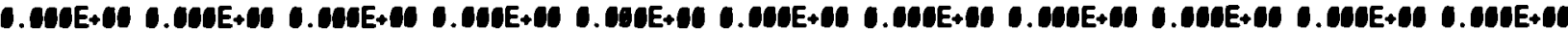

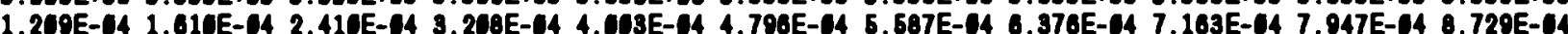

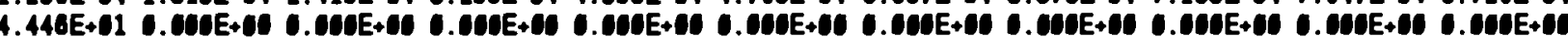

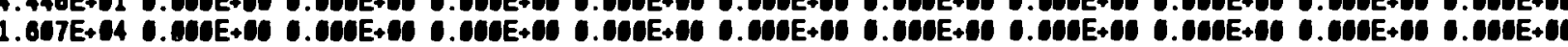

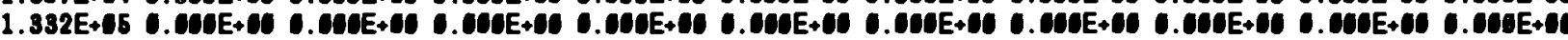

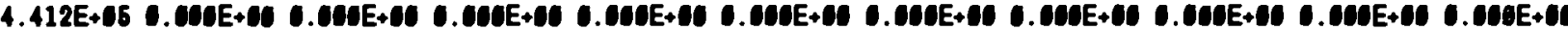

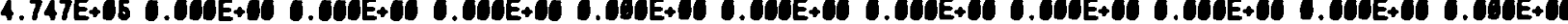

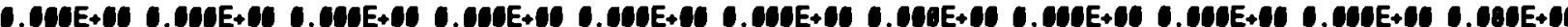

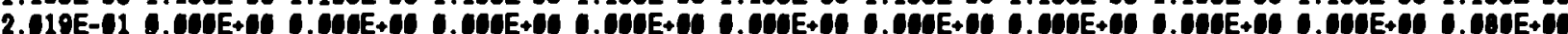

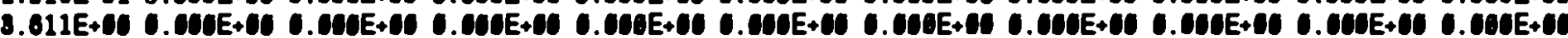
2.036E+18

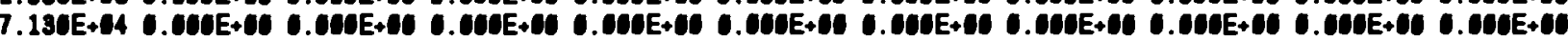

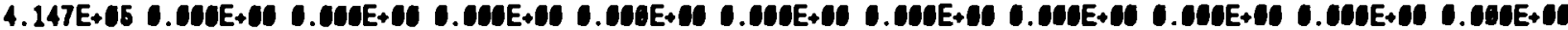

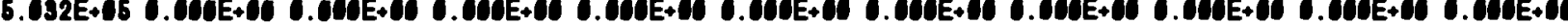

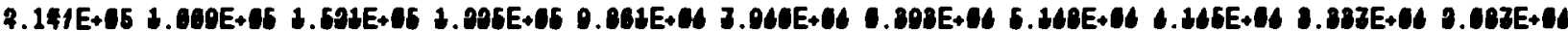

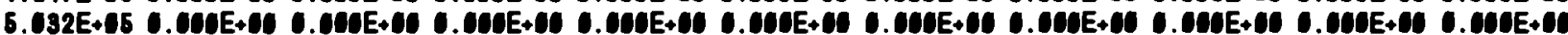

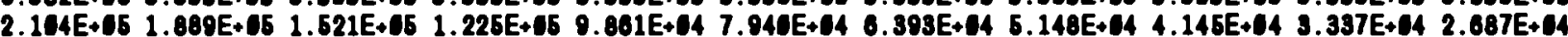

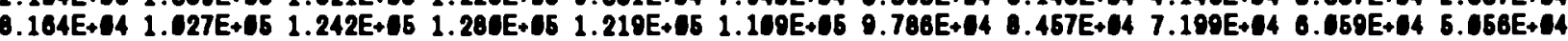

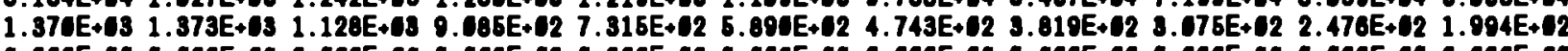

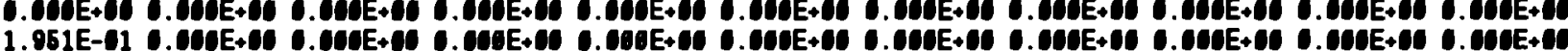




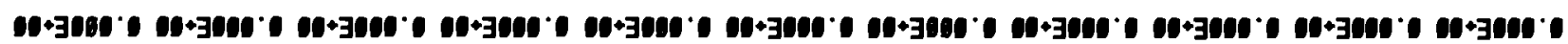

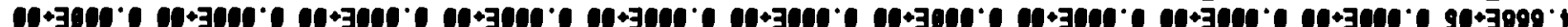

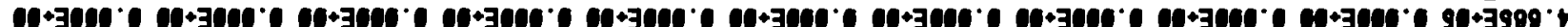

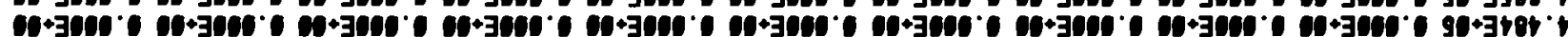

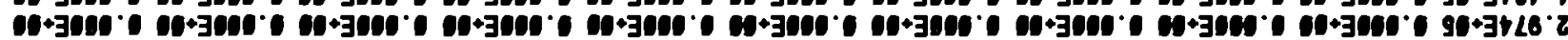

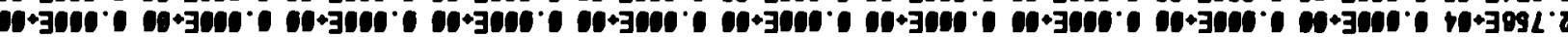
21+3010.0 10+3000.

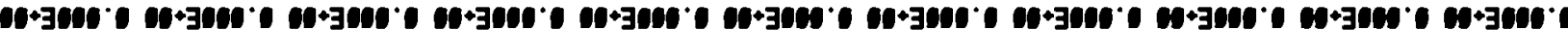

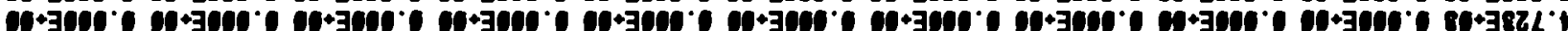

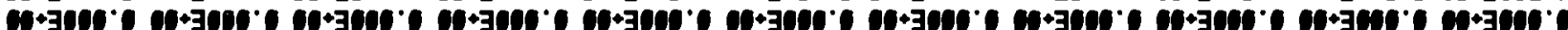

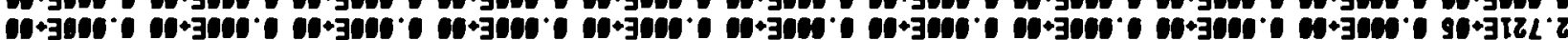

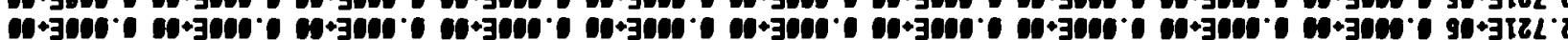

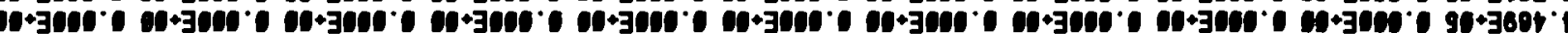
(1)+300.

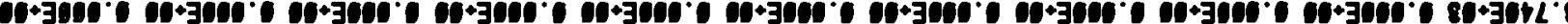

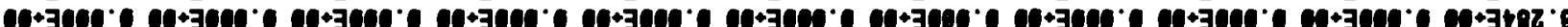

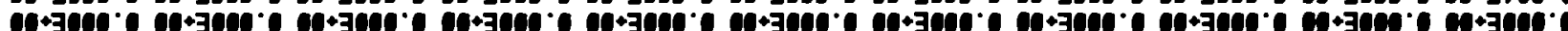

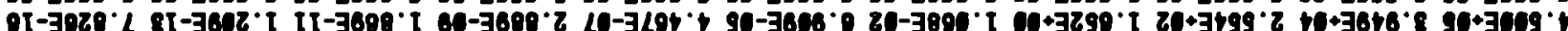

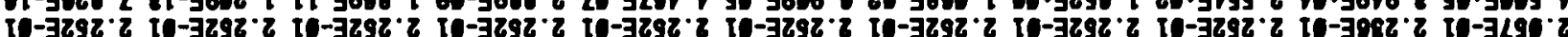

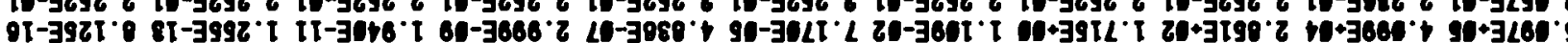

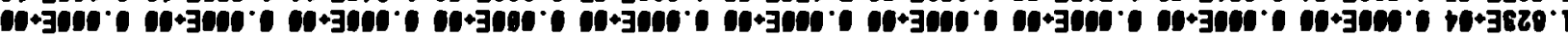

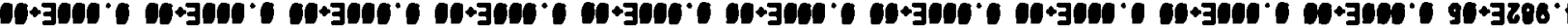

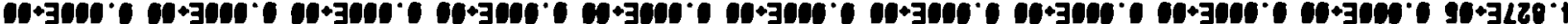

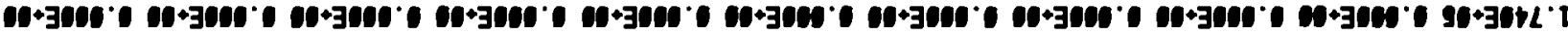

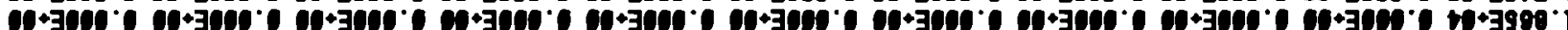

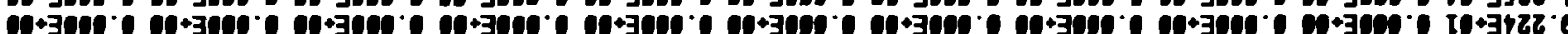

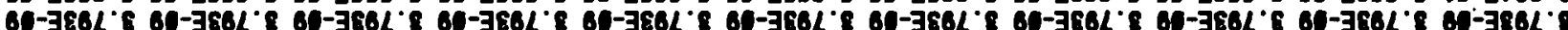
-

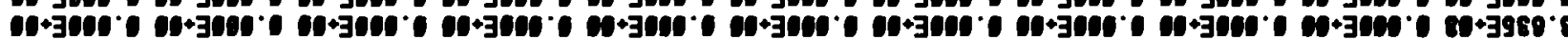

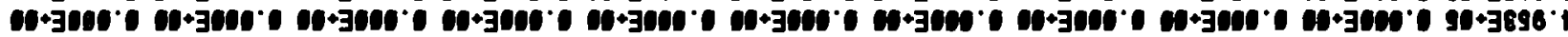

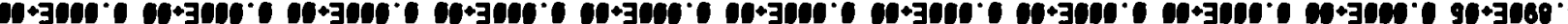

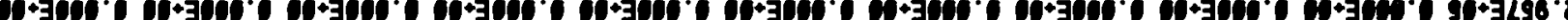

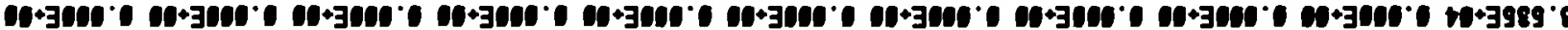

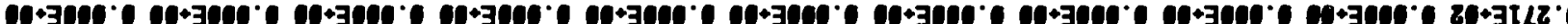
$00+300$ -

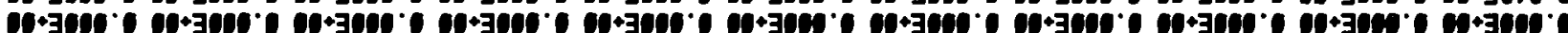

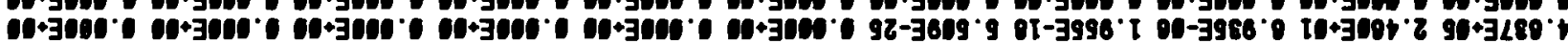

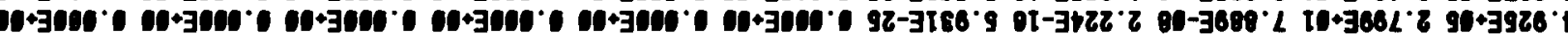
10+3001. 11+310. (1)

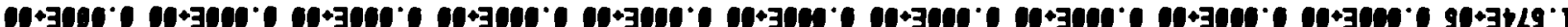

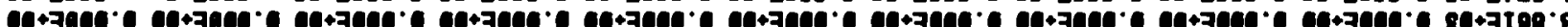

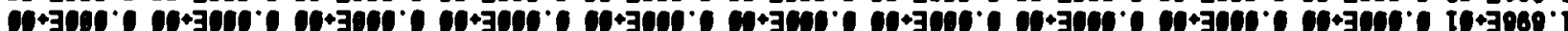

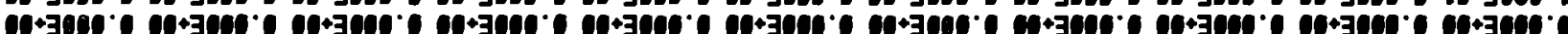

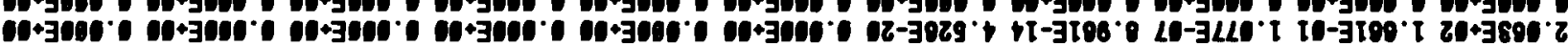

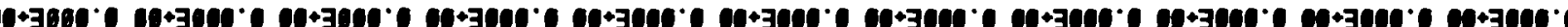

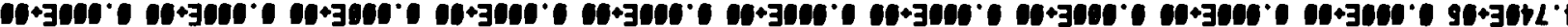

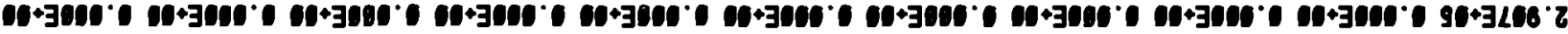

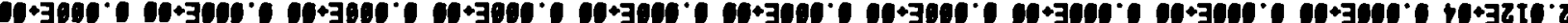

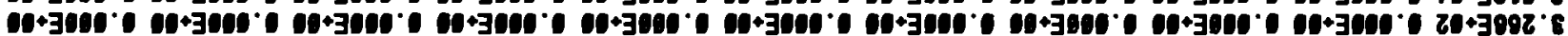




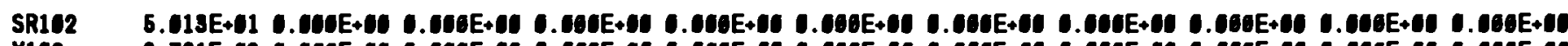

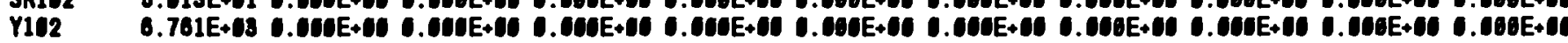

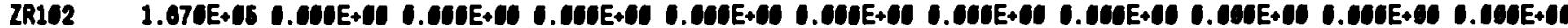

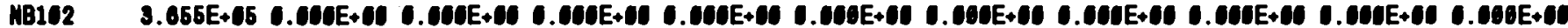

TC1024

RU102

RH102

PD102

SR10

Y10s

ZR109

NB108

TC108

TC108
RU108

RH103

Pringy

Y194

Yic4

ZR10

NB10

TC104

TC104
RU104

RH104

RH104Y

Y105

ZR105

NB105
Mo105

vo105
TC105

RU105

RH105

RH1054

PD105

Y106

ZR106

NB1Cs
Mo14s

TC168

Ru19.

RH10:

RH1064

RA10s

RH1084

PDI06

Ne108

Y107

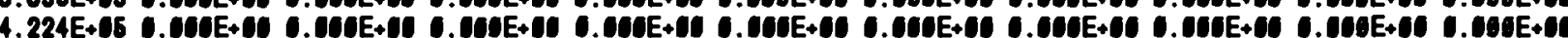
$4.228 E+15$.

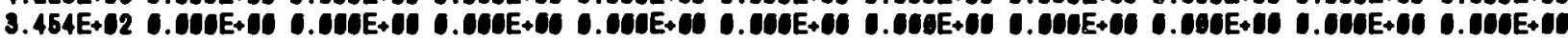

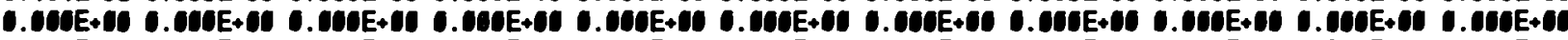
3.200E-04 3.239E-04 3.197E-04 3.156E-04 3.114E-14 3.074E-04 3.034E-04 2.094E-04 2.056E-04 2.917E-04 2.879E-04

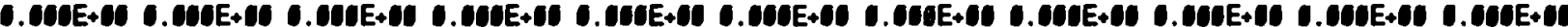

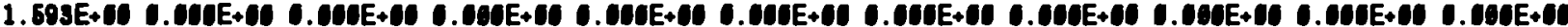

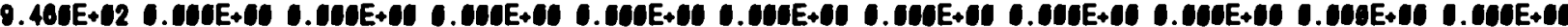

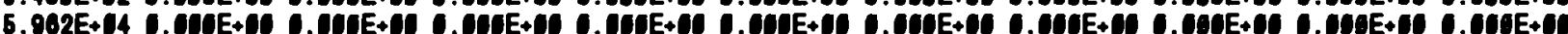
$2.302 E+05$.

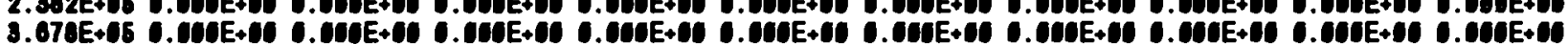

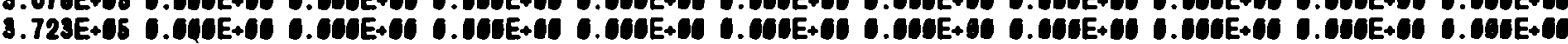

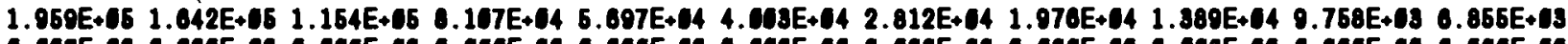

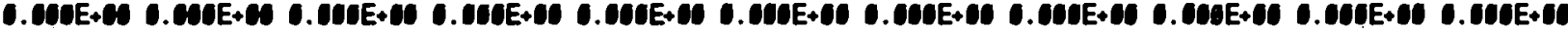

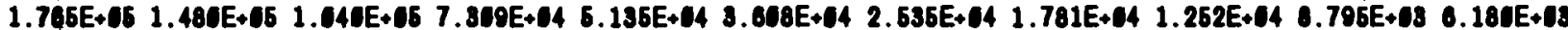

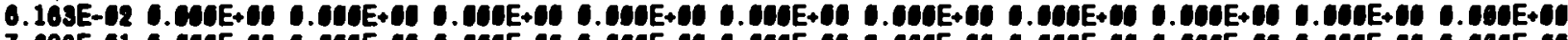

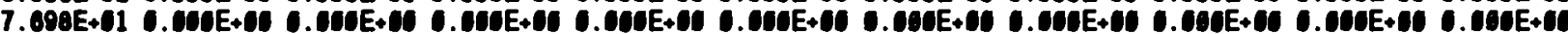

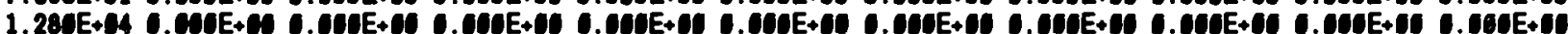
$1.20 E+05$. .

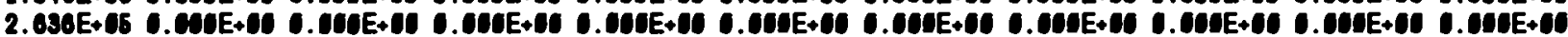

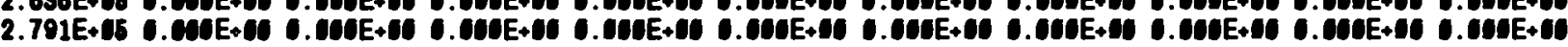

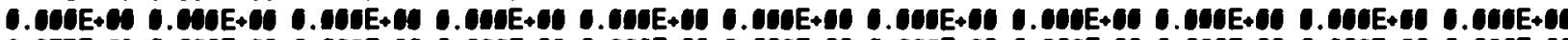

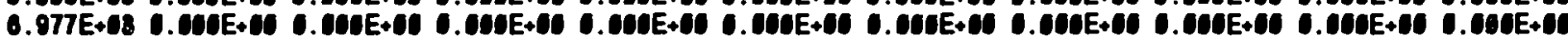

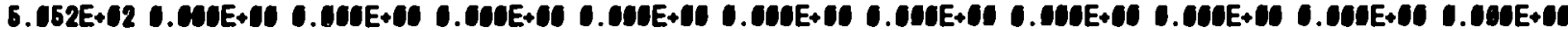

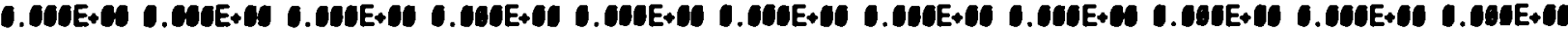

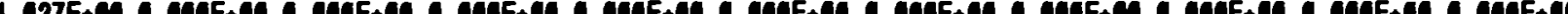
4.702E. O.

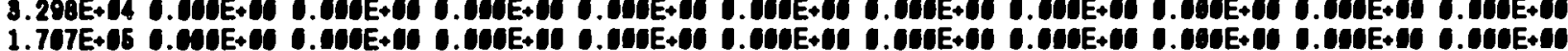

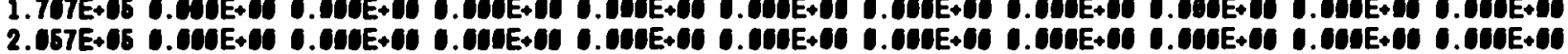

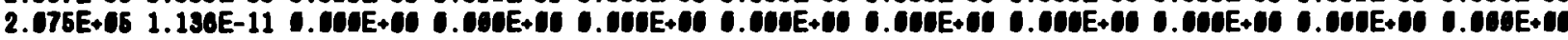

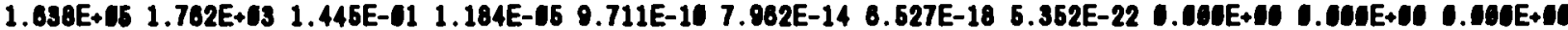

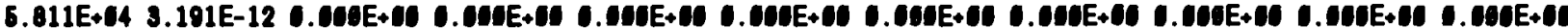

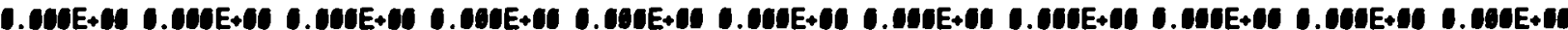

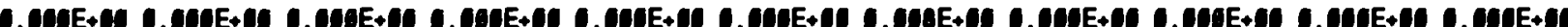

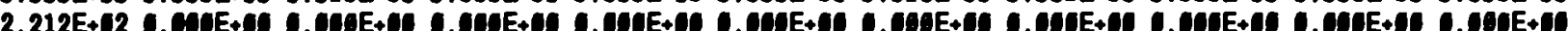

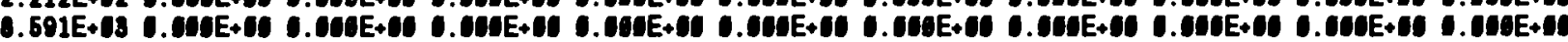

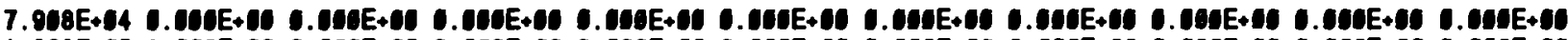

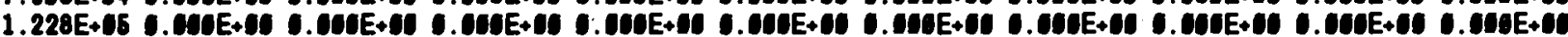

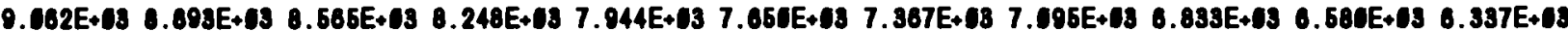

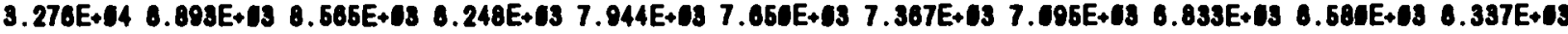

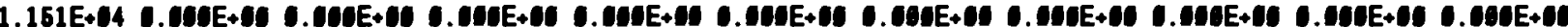

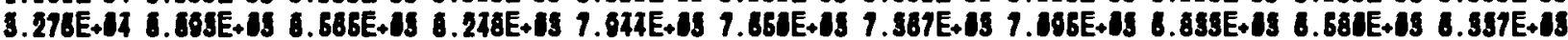

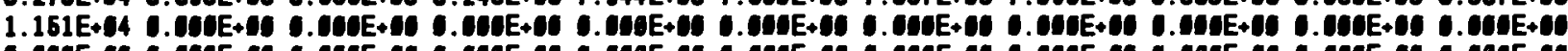

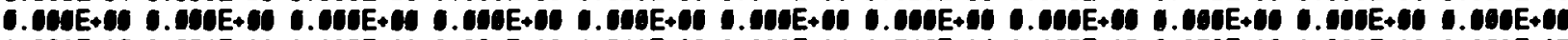

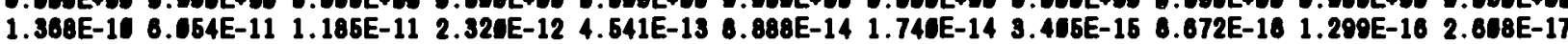

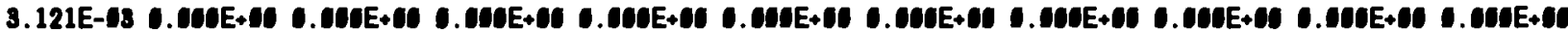

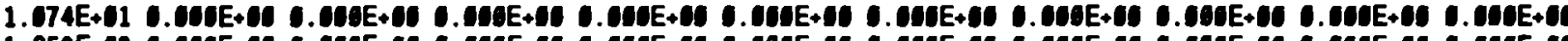

MB107

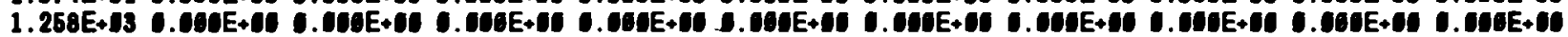


M0107

TC107

RH107

PD107

PD1074

A0107

TC108

puice

RH1C8

RH1084

PD108

Ao1say

CD106

ZR1e9

MB1C9

M109

TC169

RH109

RH1004

PD160

PD1cer

Aa109

Aaleor

CD1Co

N8110

mo110

TC110

RN110

RH110

RH11E

PDIIO

Ne110

nelien

CD110

Ma111

TC111

PUIII

RH111

PDIII

PDIIII

A0111

Ao111M

CDIII

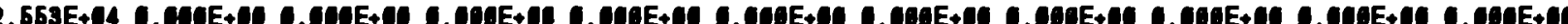
.

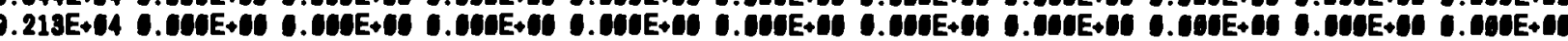

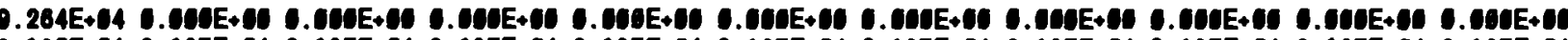

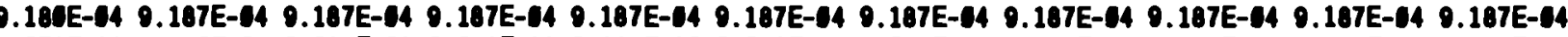

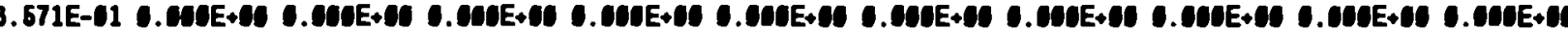

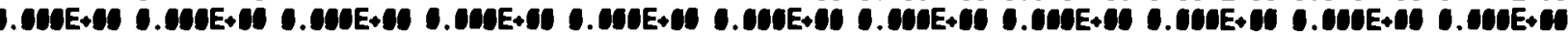

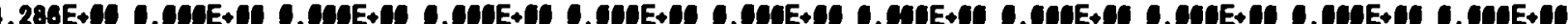

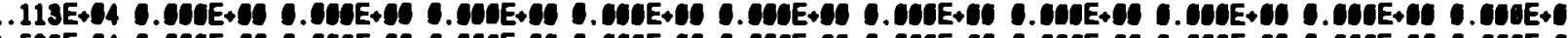

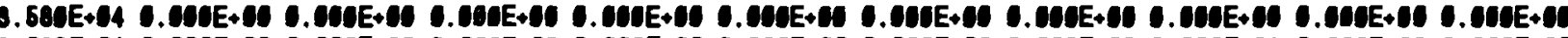

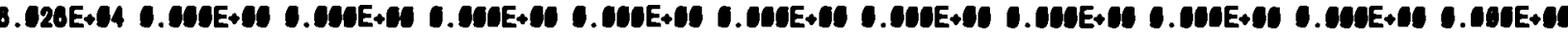

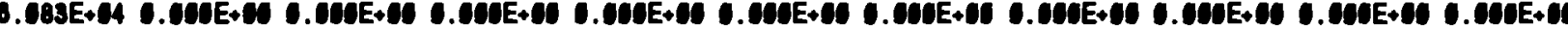

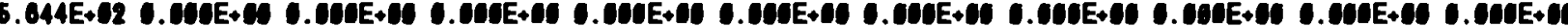

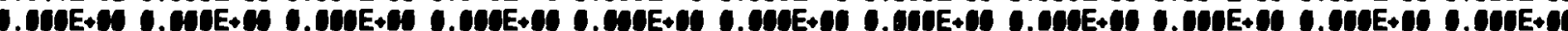
$1.601 E-18$ 1.600E-10

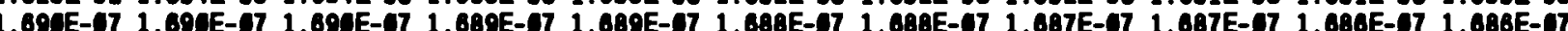

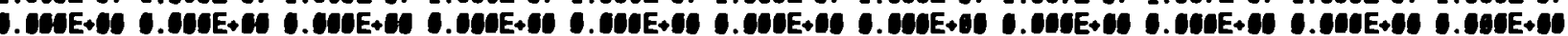

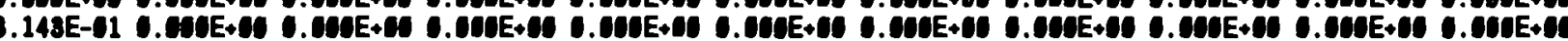

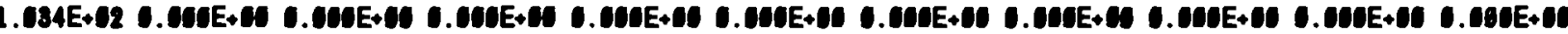

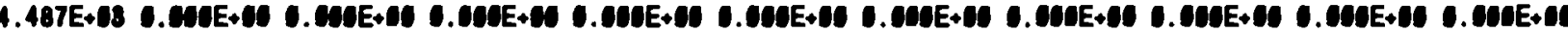

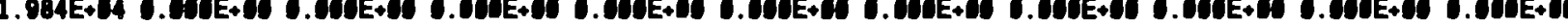

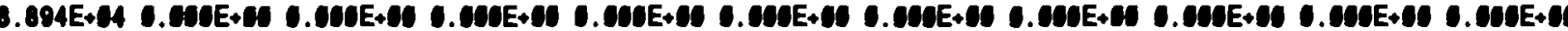
4.011 E.g4 $\mathrm{C}$. 4.

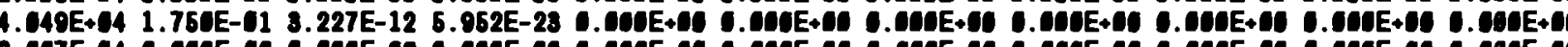

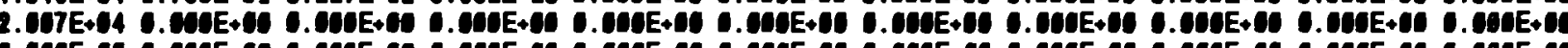

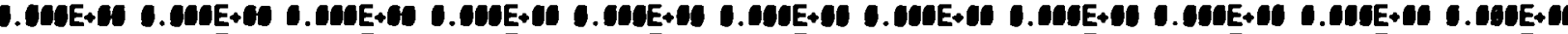

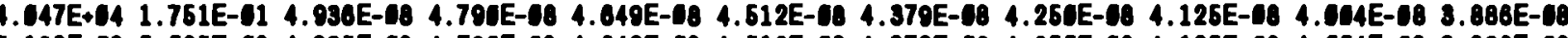

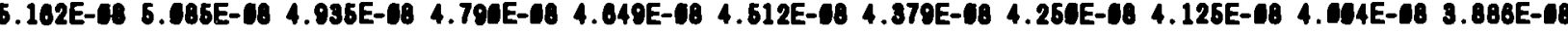

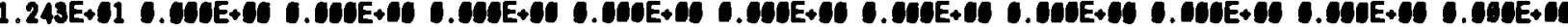

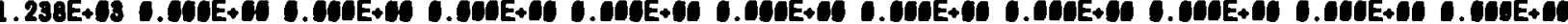
1. $.000+90$ O.001E+CO 2 CO2E+C4 COAE $C$ S

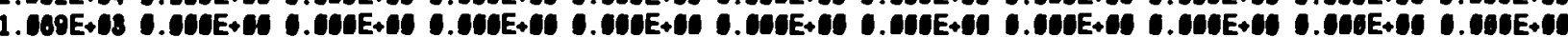

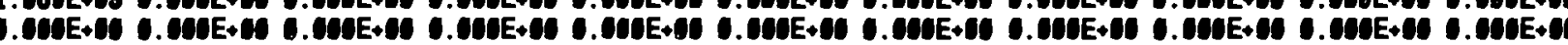

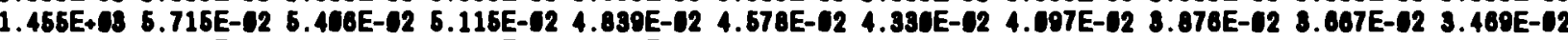

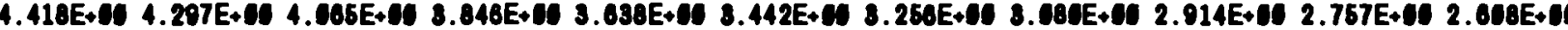

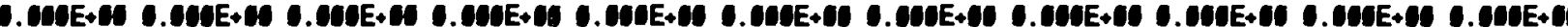

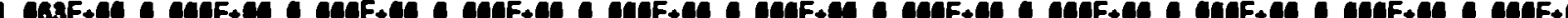

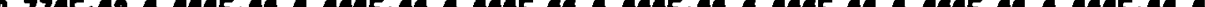

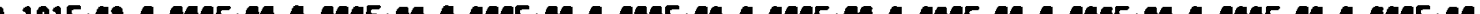

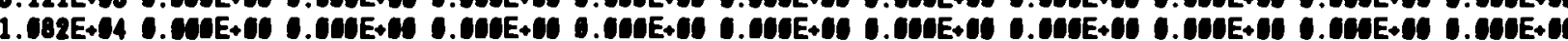

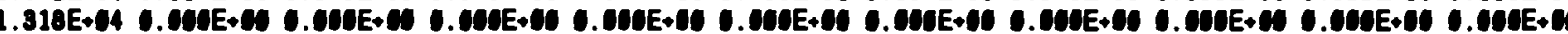

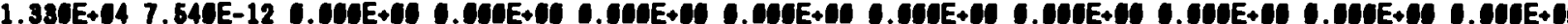

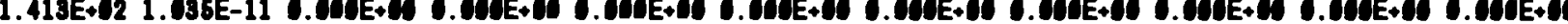

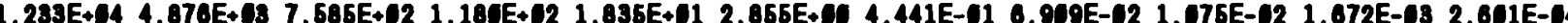

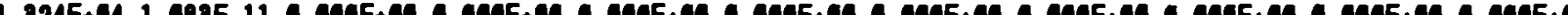

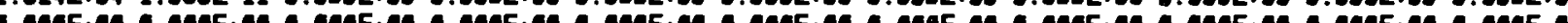

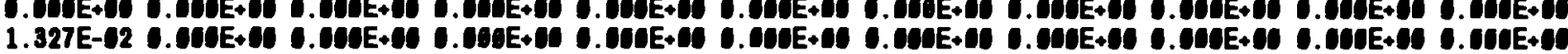




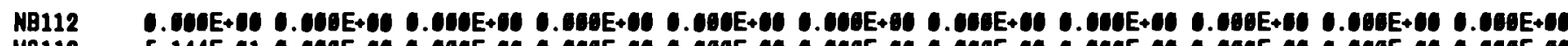

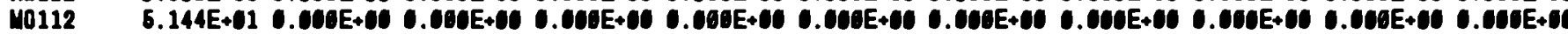

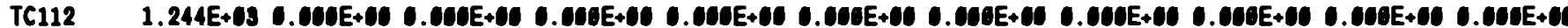

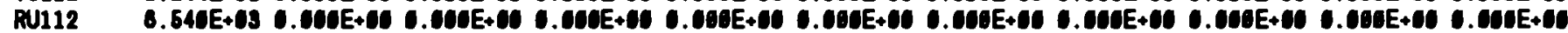

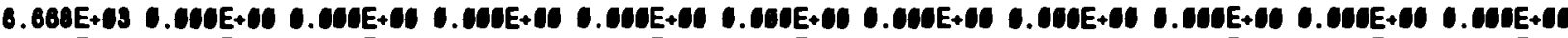

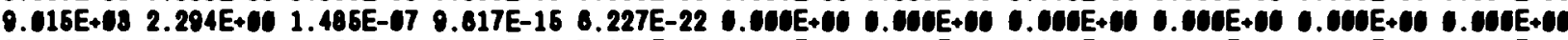

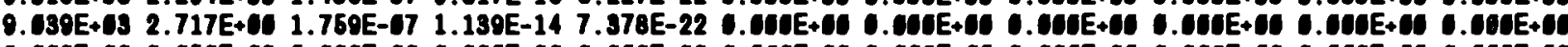

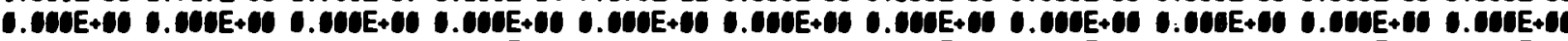

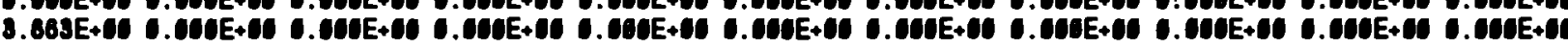

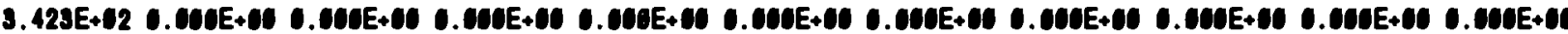

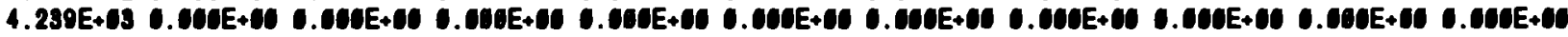

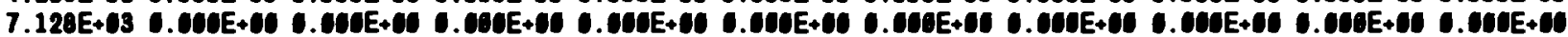

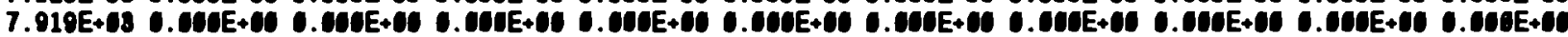

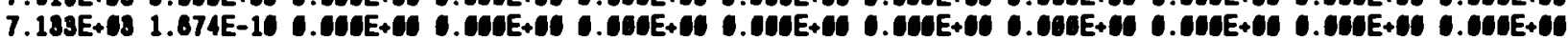

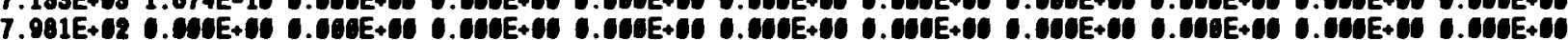

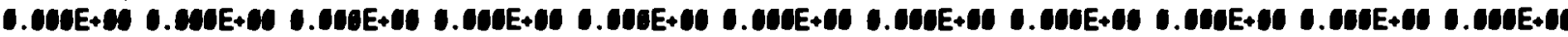

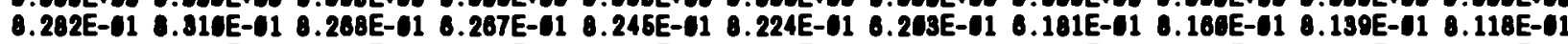

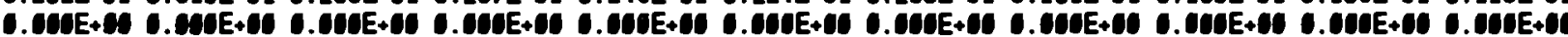

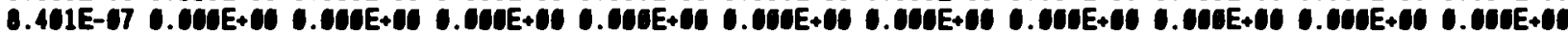

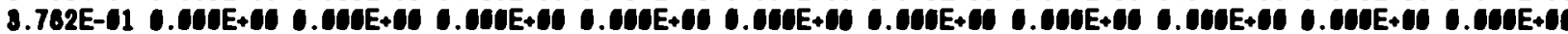

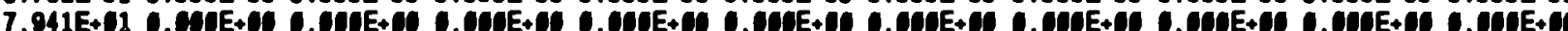
T.OUE 2.216E+18 C.00E+ $4.914 E+18$ 6.

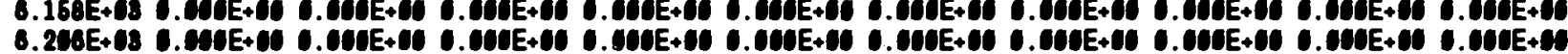

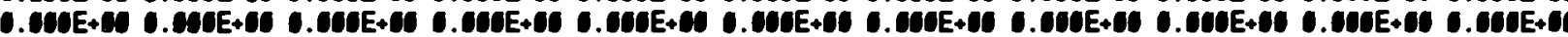

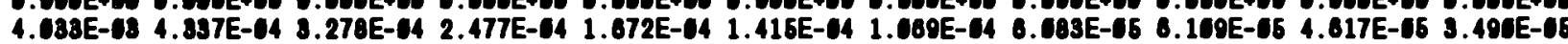

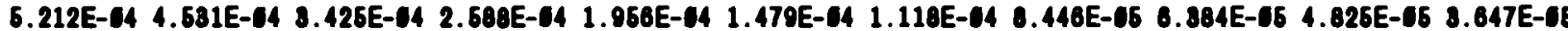

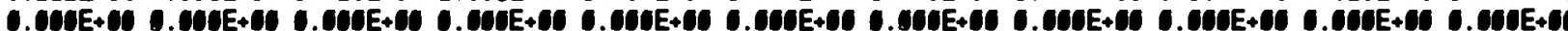
$2.522 E-C 2$ C CSE 2.622E- 12 . $1.891 E+11$.

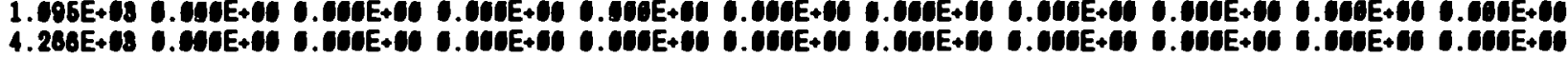

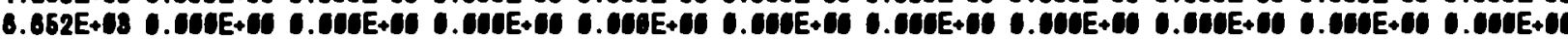

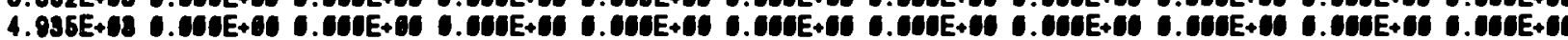

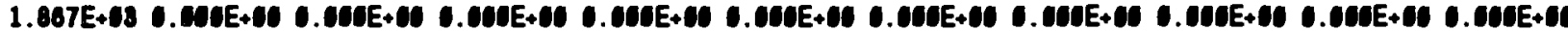
6.173E+18 2.765E+12 5.479E-61 1.087E-18 2.158E-68 4.280E-69 8.490E-12 1.084E-14 3.013E-17 2.022E-10

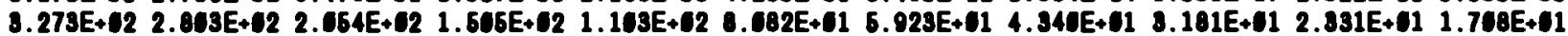

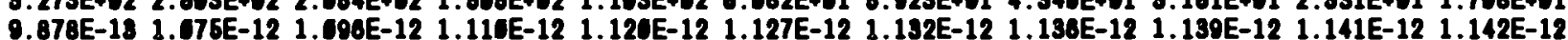
$0.217 E+18$ 8.

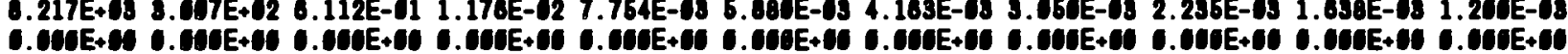

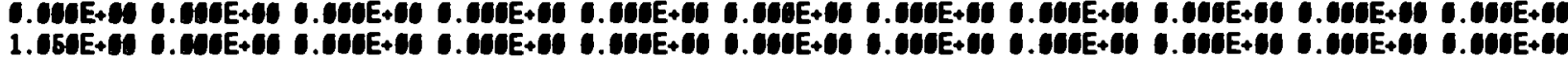

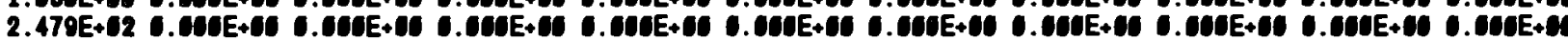

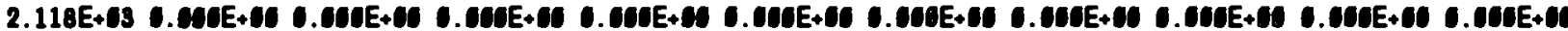

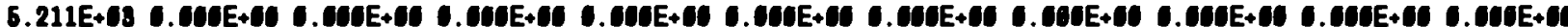

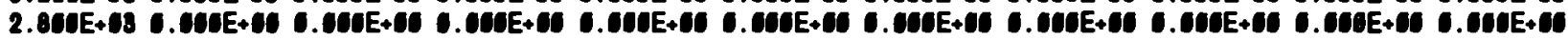

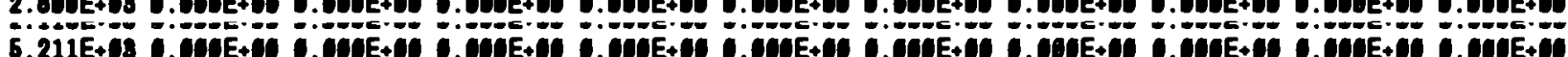

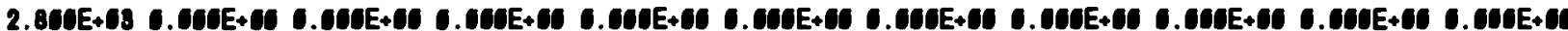

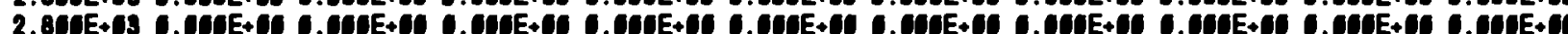
2.

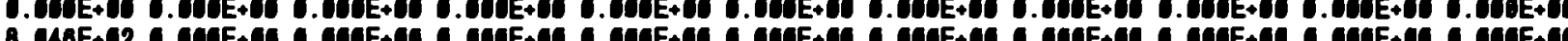

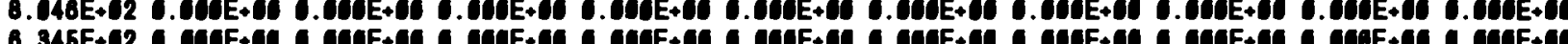

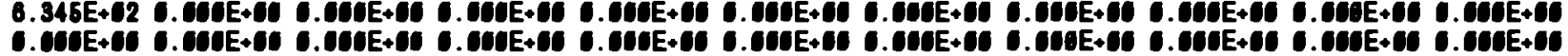


TC117

RU117

RH117

PD117

A0117

CD117

CD117

IM117

IN117M

SN117

SN117"

TC118

Pul18

RH118

Pol18

A0118

nal1en

CD118

IM110

IN118M

SN118

Ru119

?

on

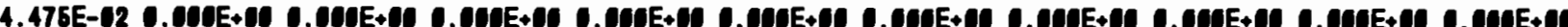

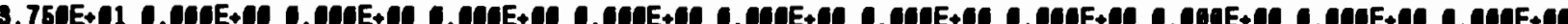

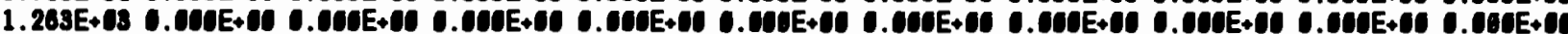

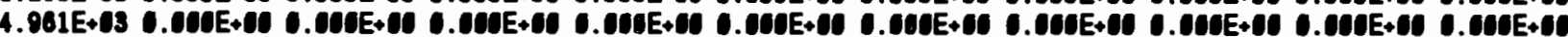

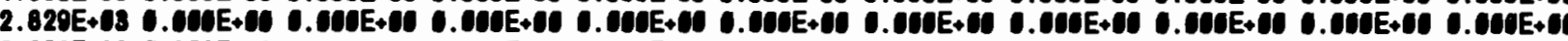

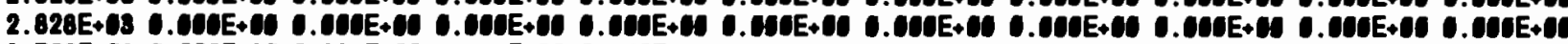

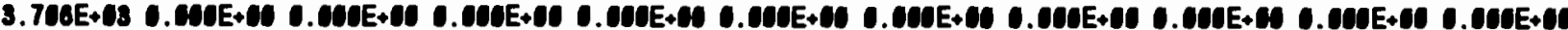

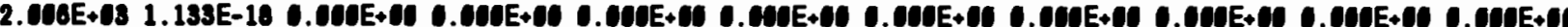

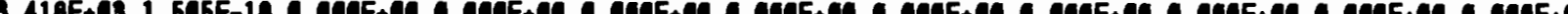
4. . 1.222E-01 7.462E-12 2.709E-02 1.029E-02 8.824E-03 1.421E-03 5.282E-04 1.083E-04 7.204E-06 2.711E-05 1.007E-06

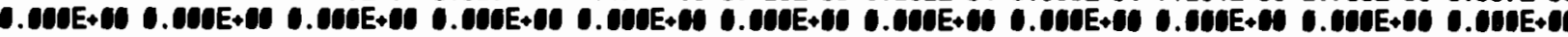

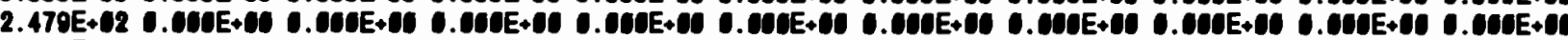

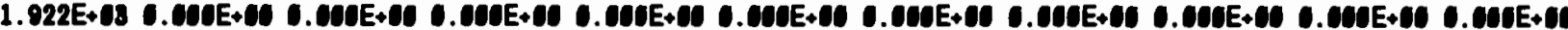

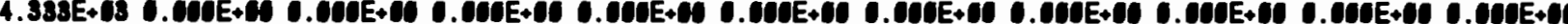

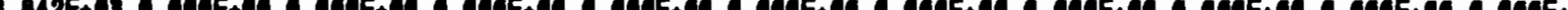
3.042E. 2.003E+18

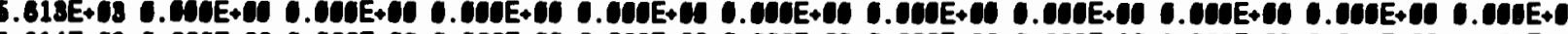

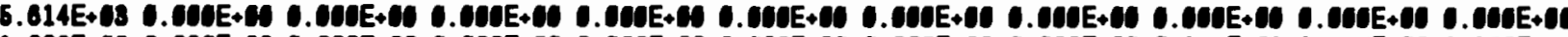

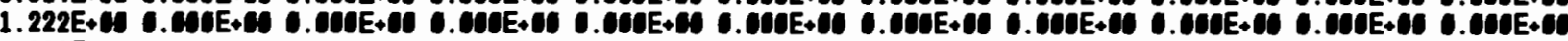

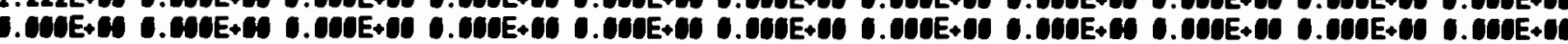

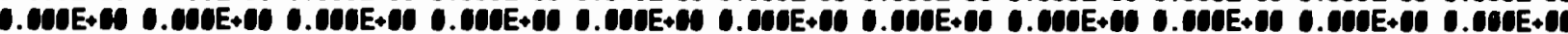

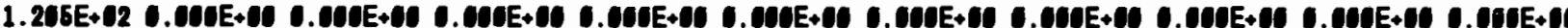
$2.609 E+G 8$ S

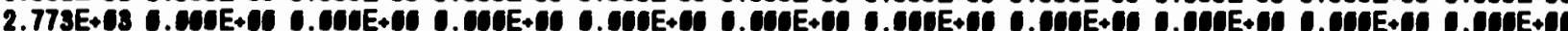

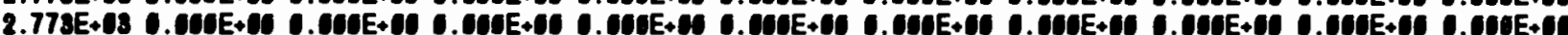

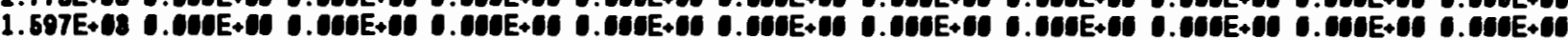

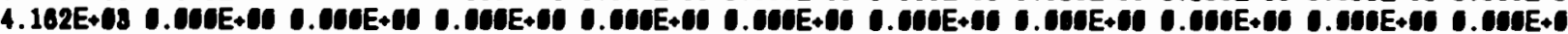

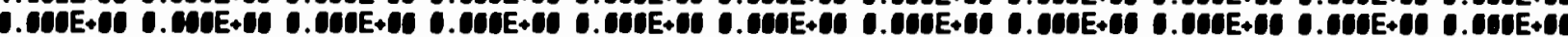

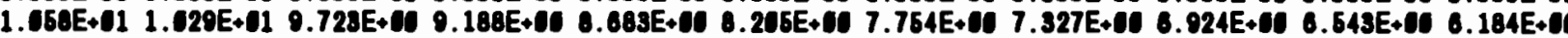

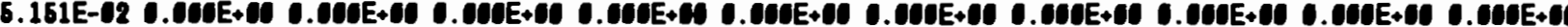

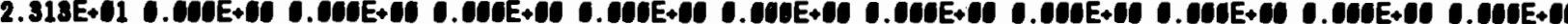
1.2015 (a) D.

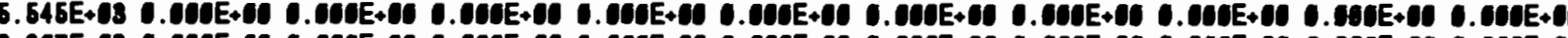

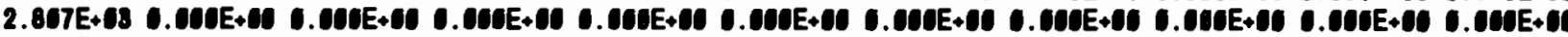

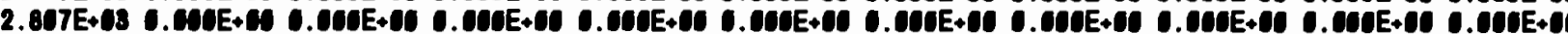

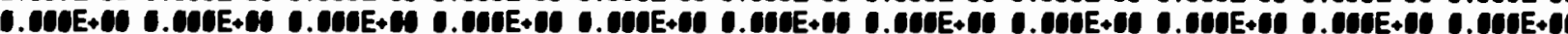

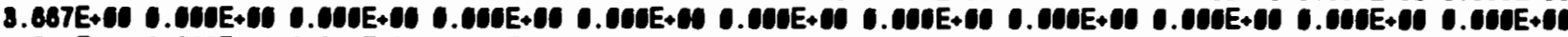

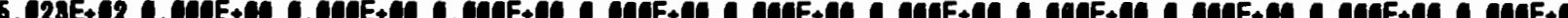

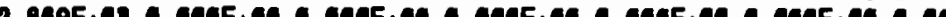

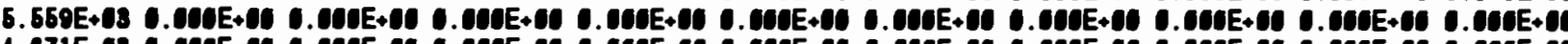

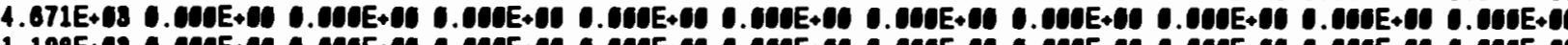

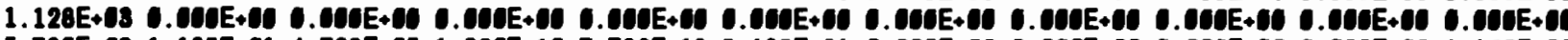

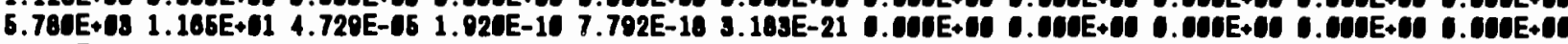
2.086E-08 2.085E-08 2.083E-08 2.082E-03 2.086E-08 2.079E-08 2.077E-03 2.076E-08 2.074E-08 2.072E-03 2.071E-08

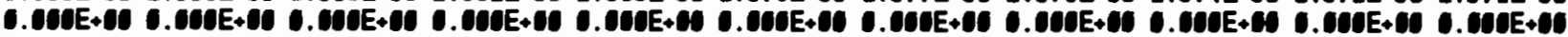

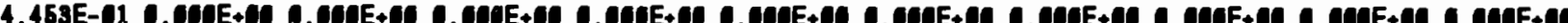

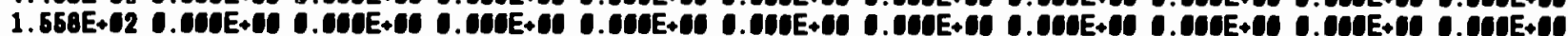




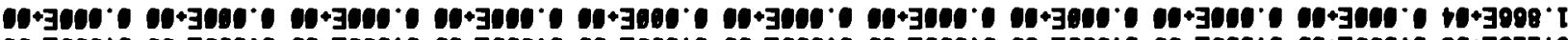

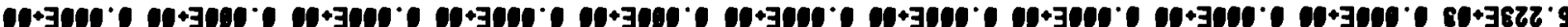

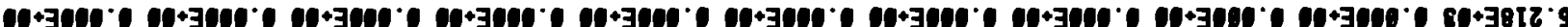

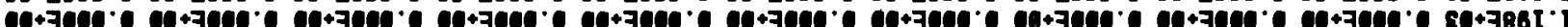

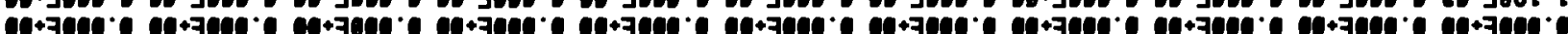

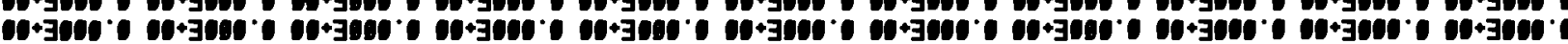

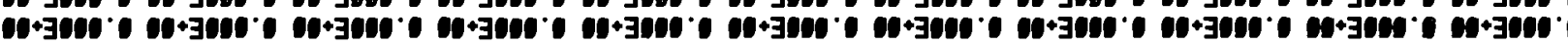

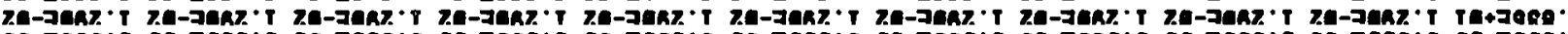
•

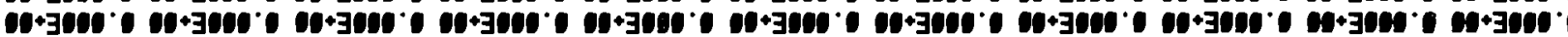

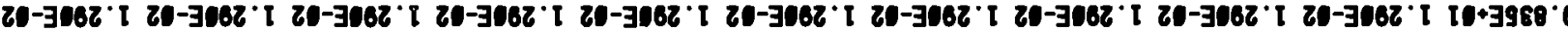

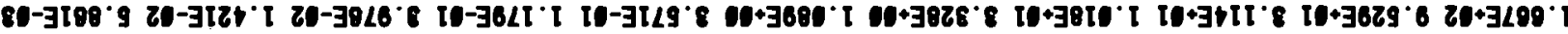

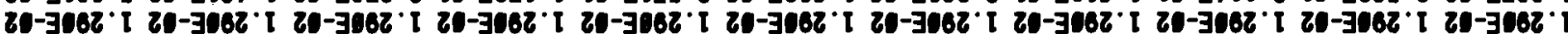
$60+3090$ -

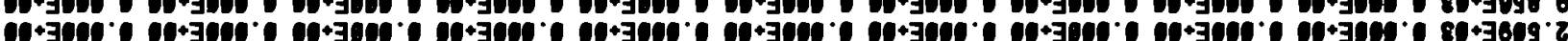

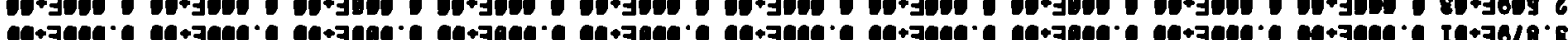
(6)

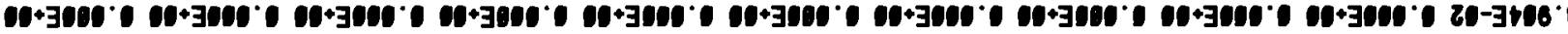

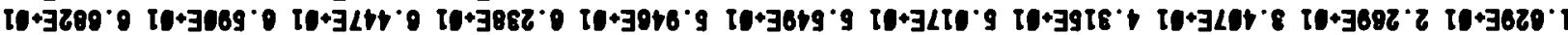

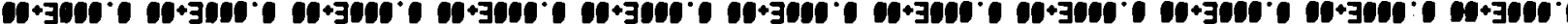

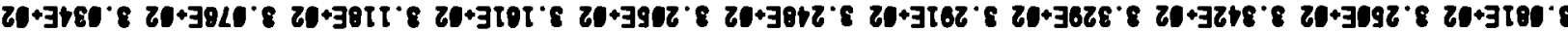

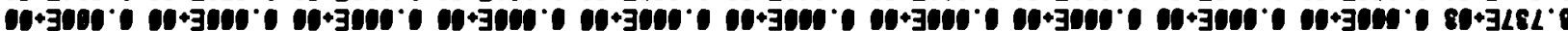

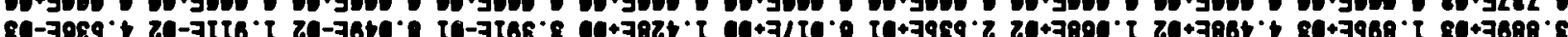

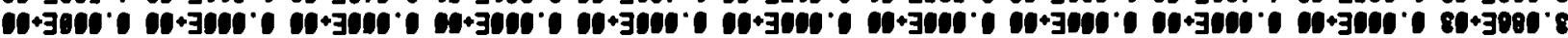

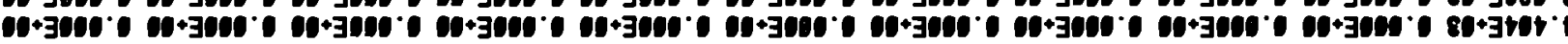

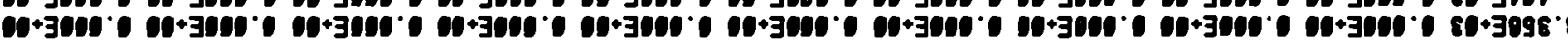

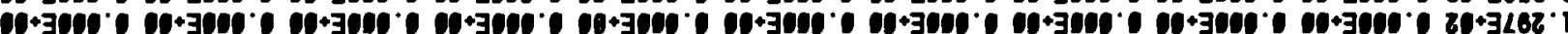

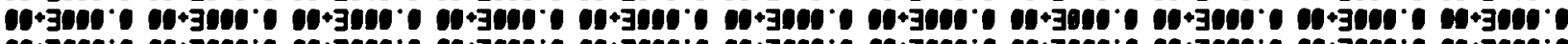

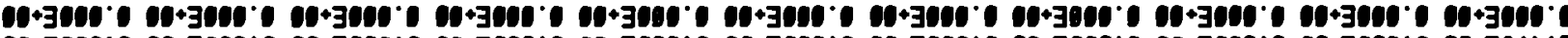

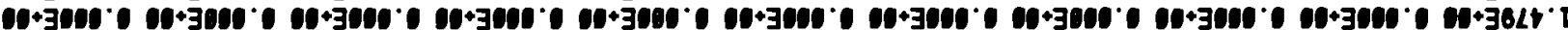

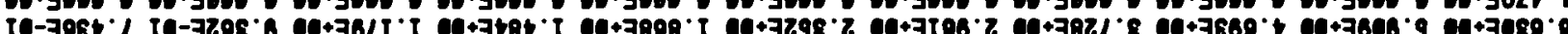

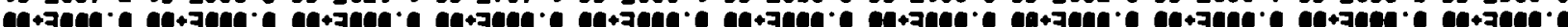

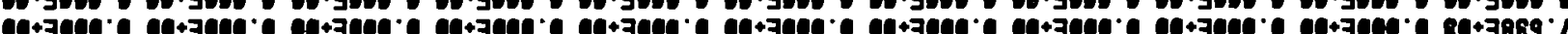

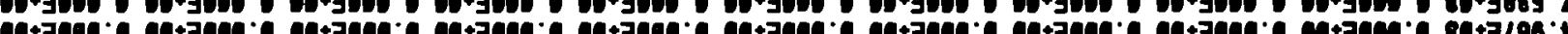

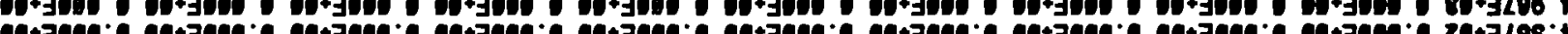

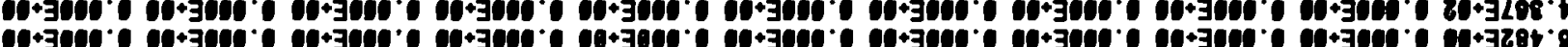

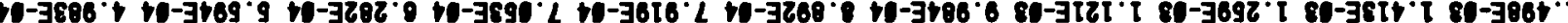

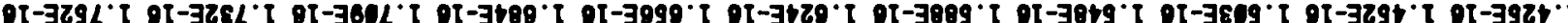

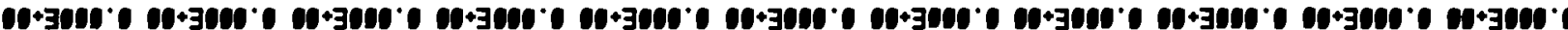
$10+3000$ - $00 \cdot 3$ (1) 10+3000 -

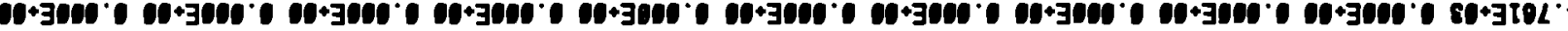

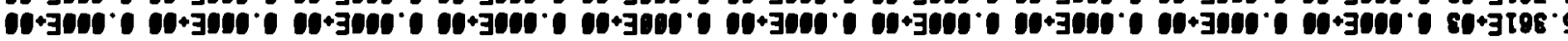

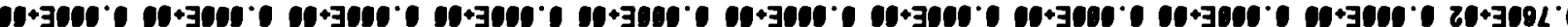

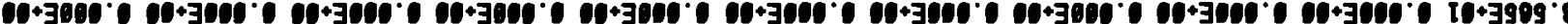

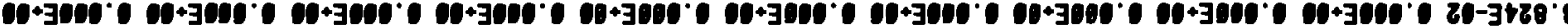

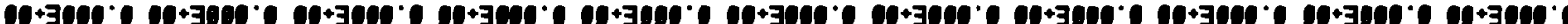

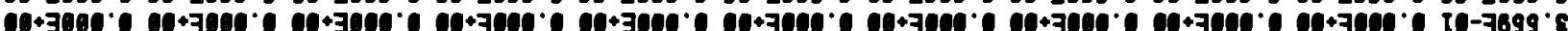

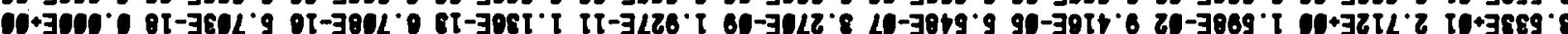

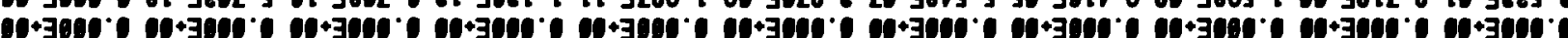

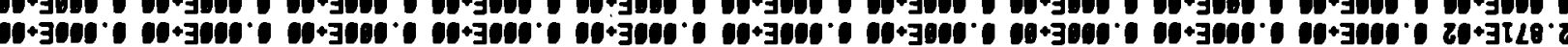

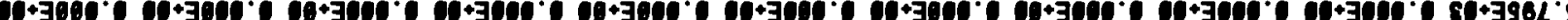

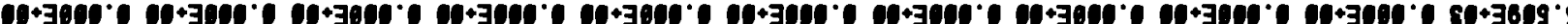

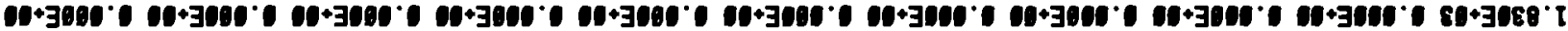




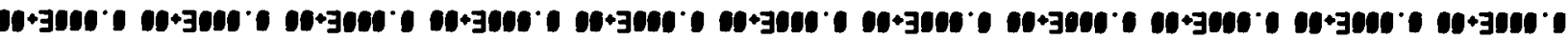

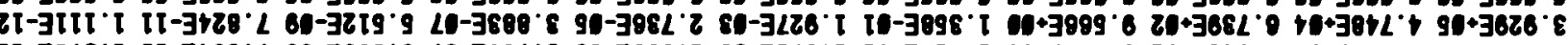

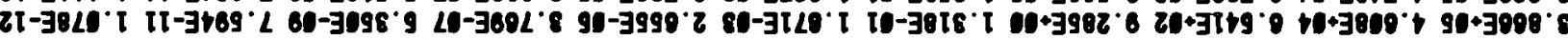

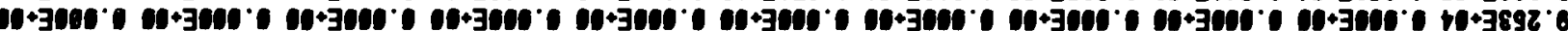

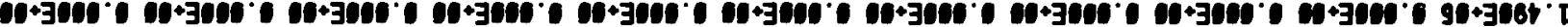

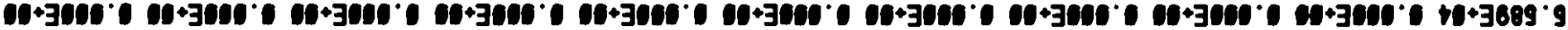

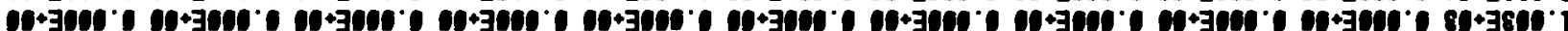

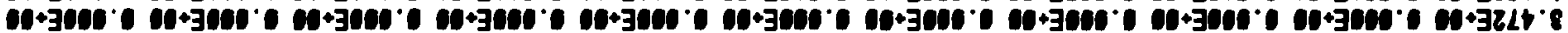

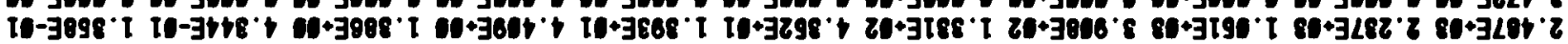

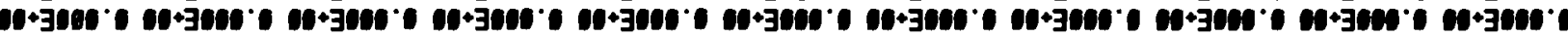

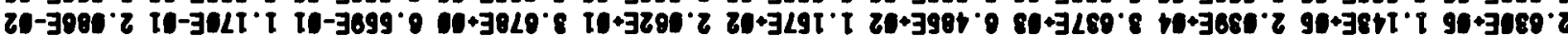

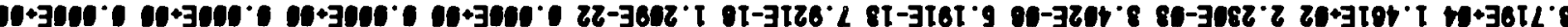

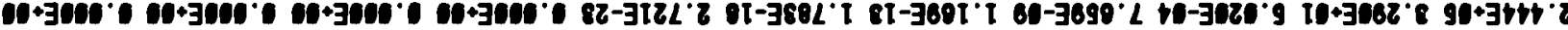

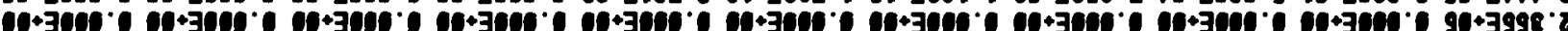

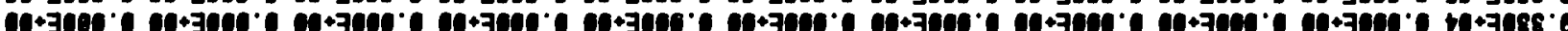

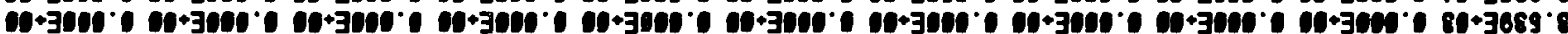

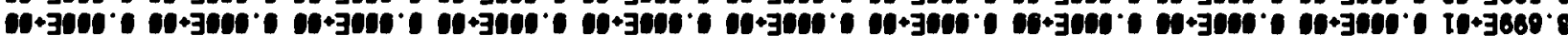

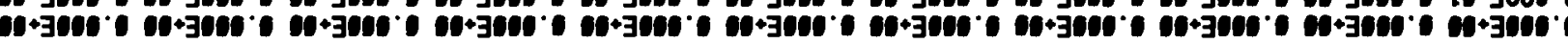

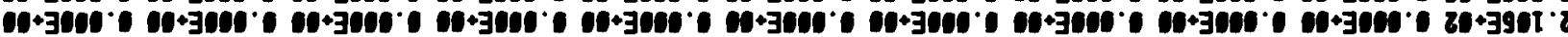

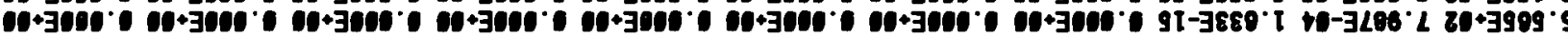

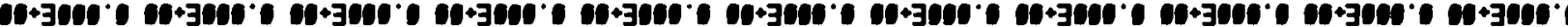

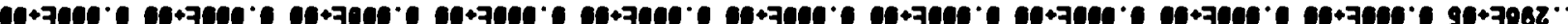
ל ' -

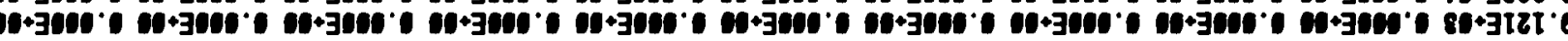

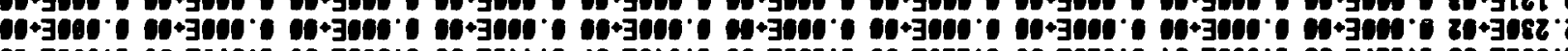

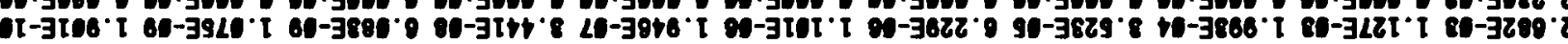

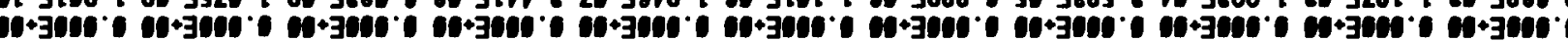

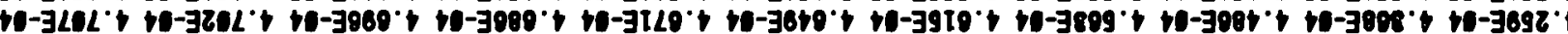

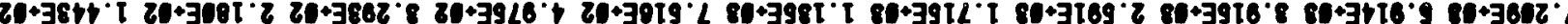

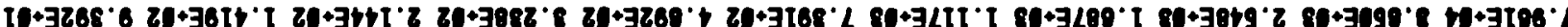

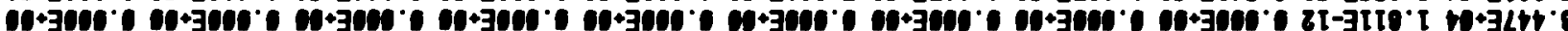

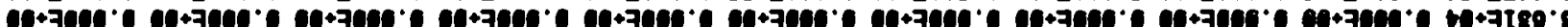

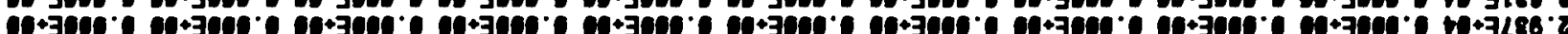

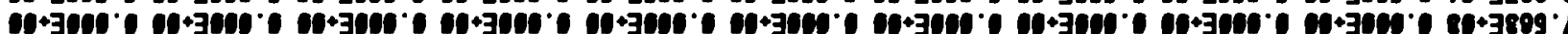

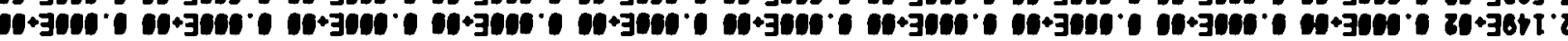

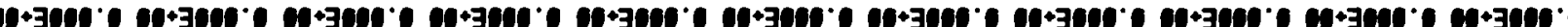

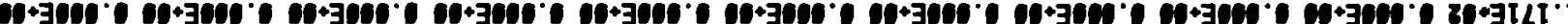

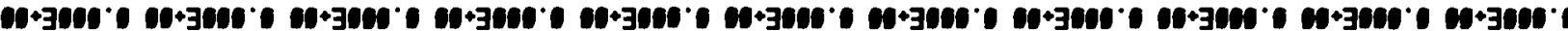

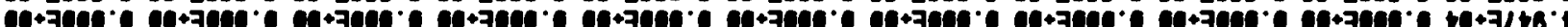

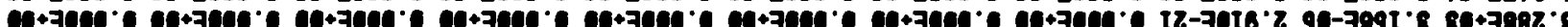

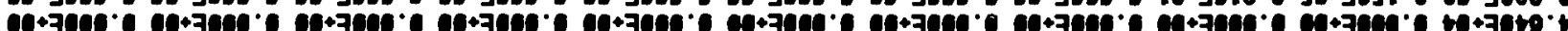

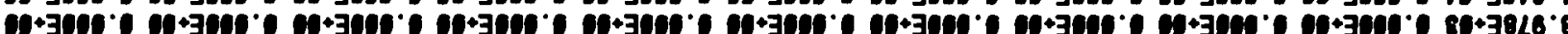
10+300e - a

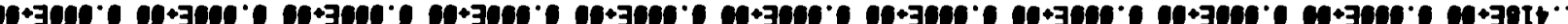

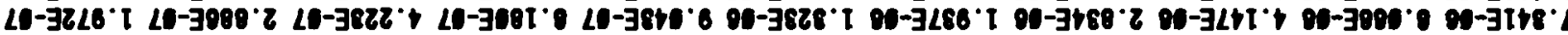

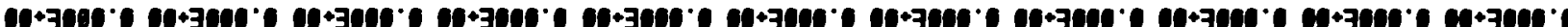

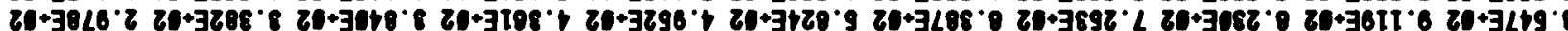

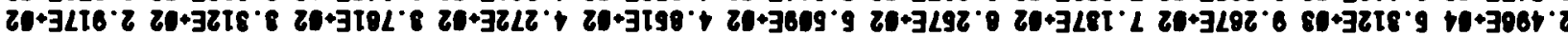
[I-

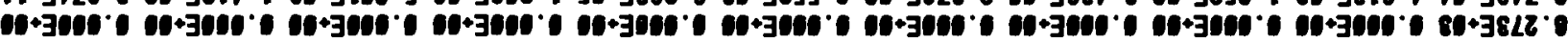

ZEIBS

ZEINS

ZETMI

28105

nist3X

I8I

ตICTI

I8191

I818S

IETuS

TETMI

IEI0J

extax

nastI

อย

nerigs

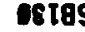

อeINS

๓eINI

neztaj

EIIJX

621

NoETII

6819

orI

MozINS

EIINS

62INI

จ213X

82 II

จะI]

ขอ151

ozI8S

ozINS

ดzINI

ดztoว

ดzToY

Let

221

L2TI

L2t8 
CS132 2.812E+00 9.041E- 11 1.133E-01 1.331E-02 1.665E-03 1.839E-04 2.161E-05 2.540E-06 2.085E-07 3.608E-06 4.122E-00

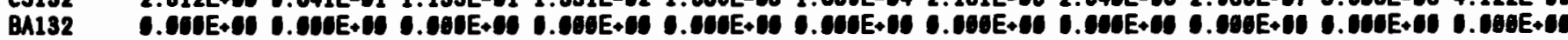

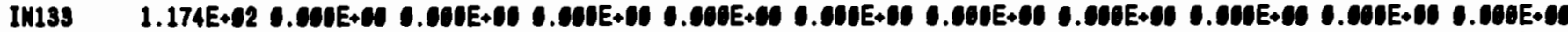

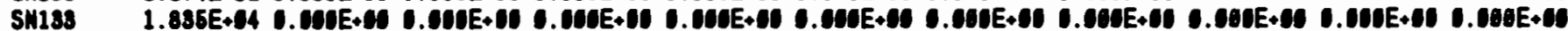

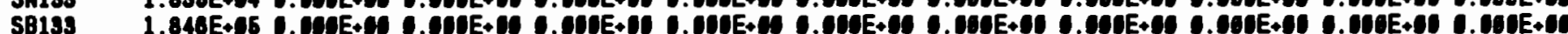
TE133

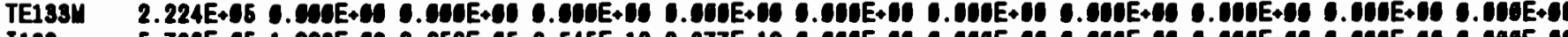

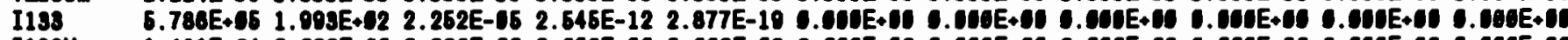

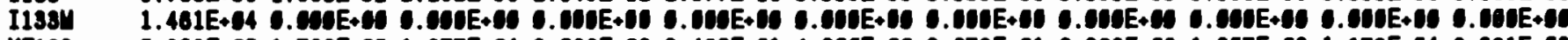

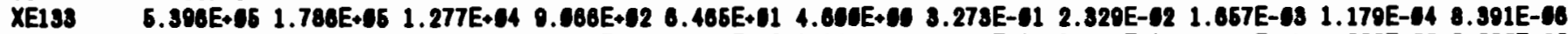

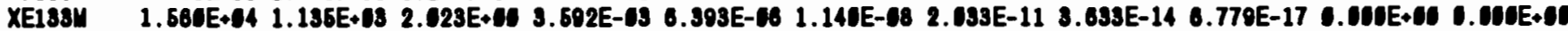

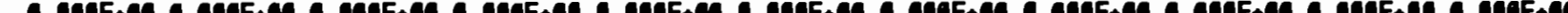

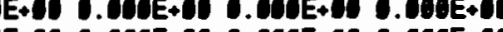
IN134 O.

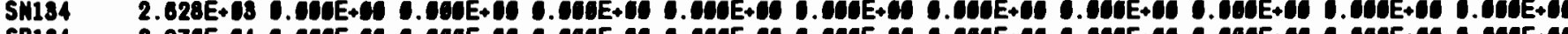

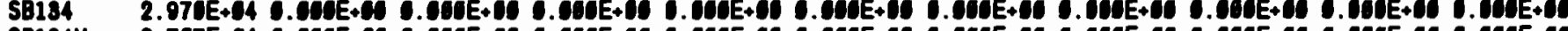

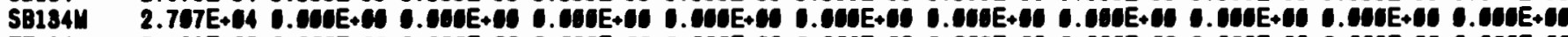

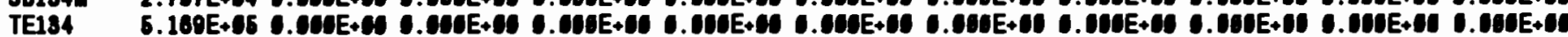

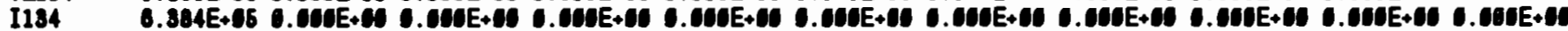

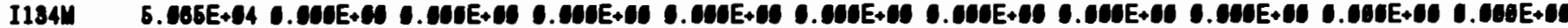
XE134 XE134I S.

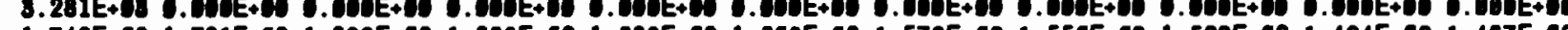

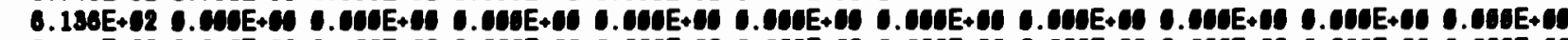

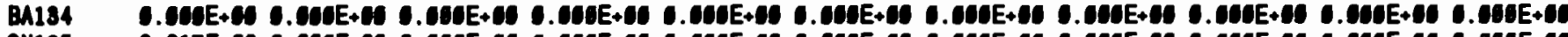

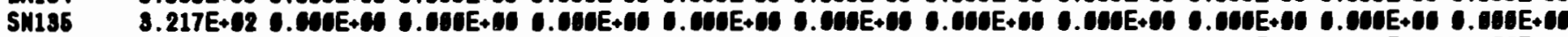

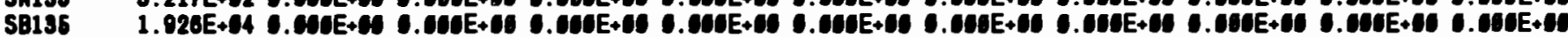

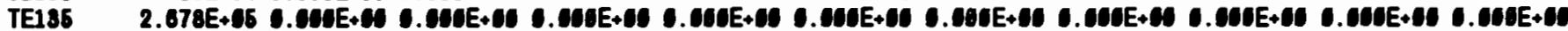

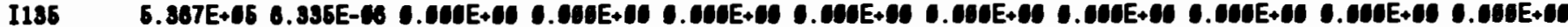

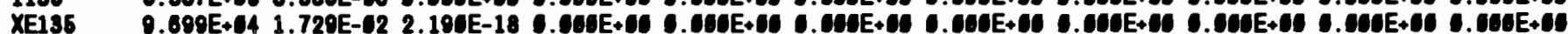

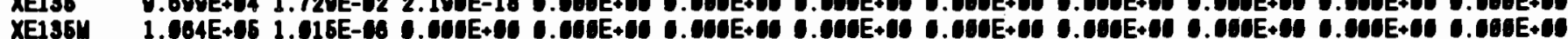
CS135

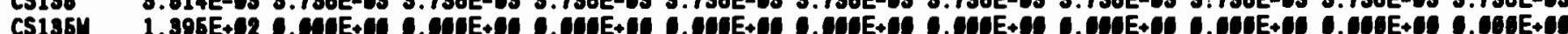
$\mathrm{B}$
$\mathrm{B} 135$

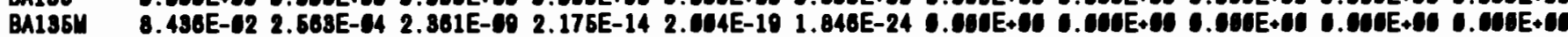

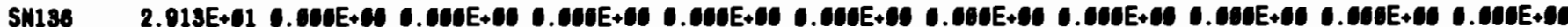

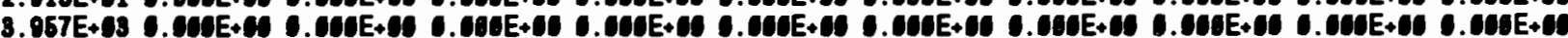

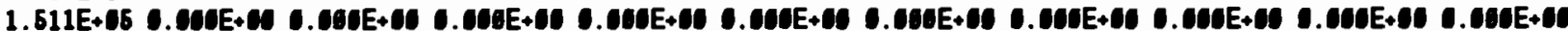

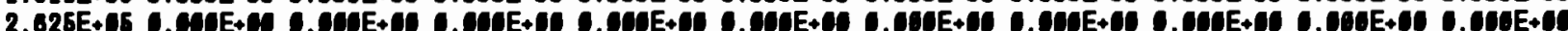

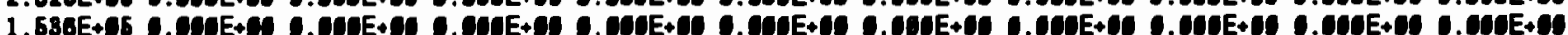

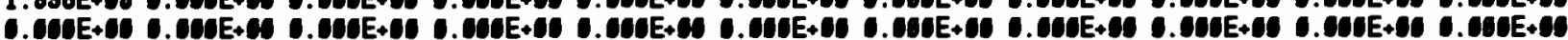

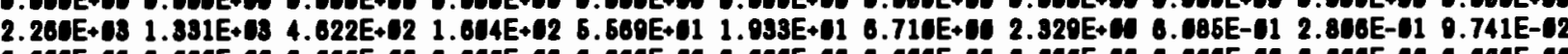

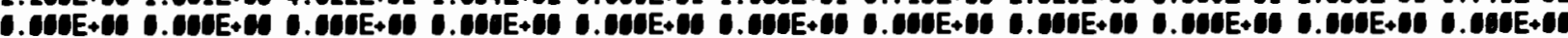

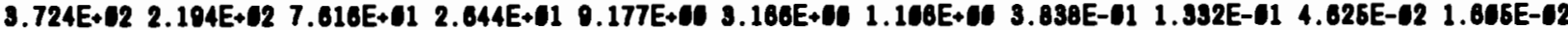

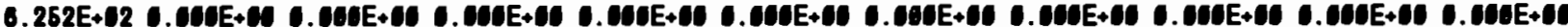

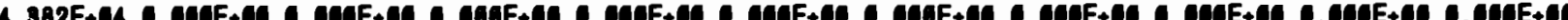

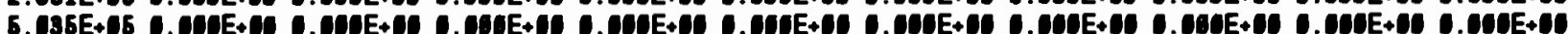

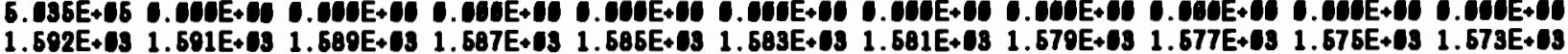

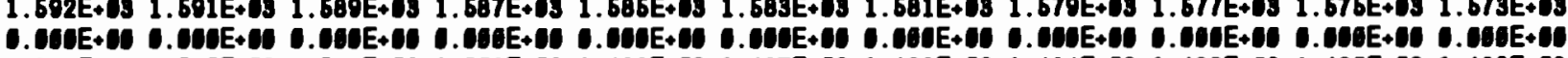

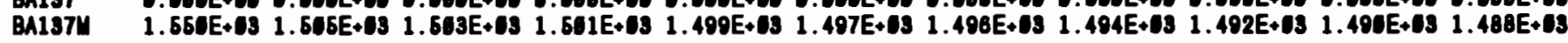




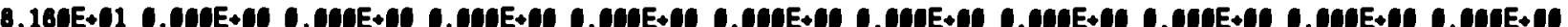

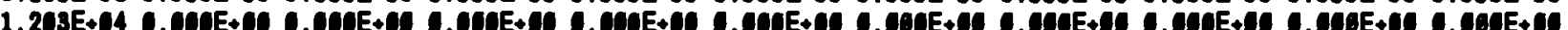

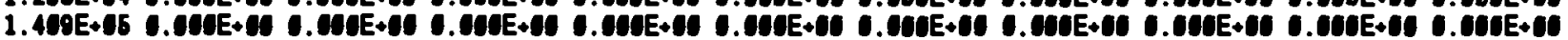

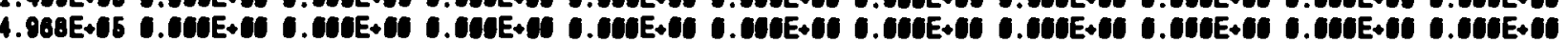

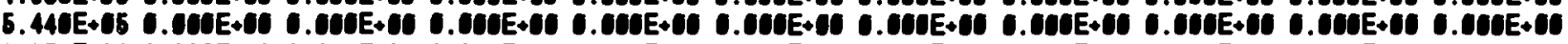

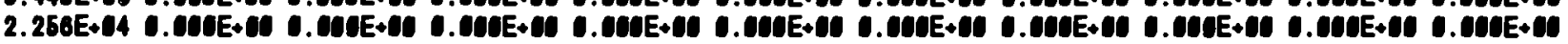

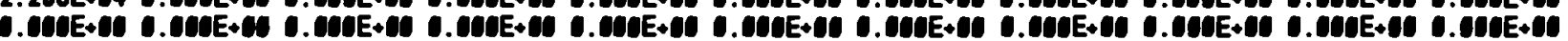
2.588E-12 2.588E-12 2.668E-12 2.568E-12 2.568E-12 2.568E-12 2.568E-12 2.588E-12 2.568E-12 2.568E-12 2.568E-12

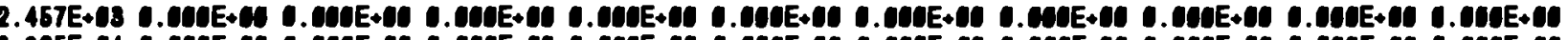

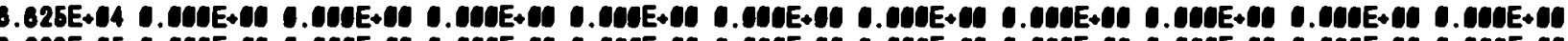

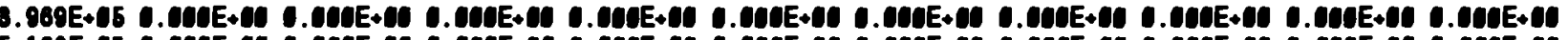

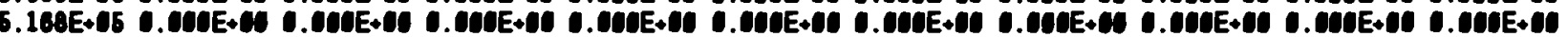

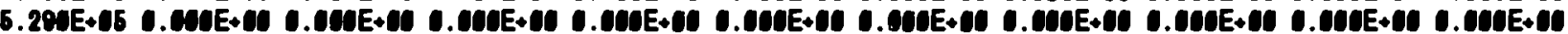

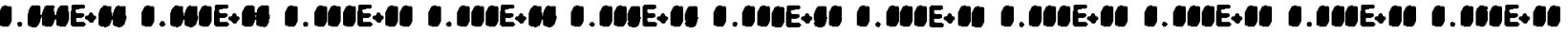

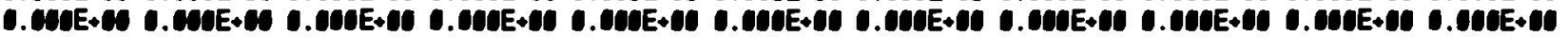
O.

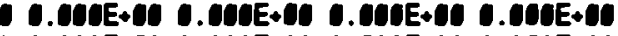

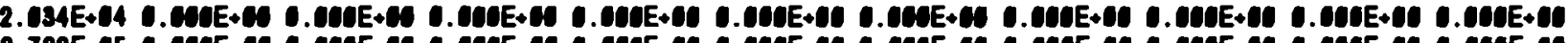

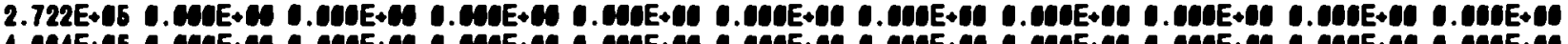

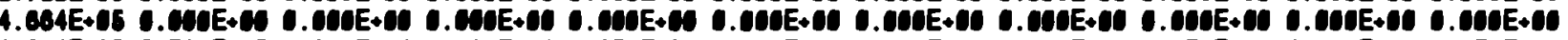

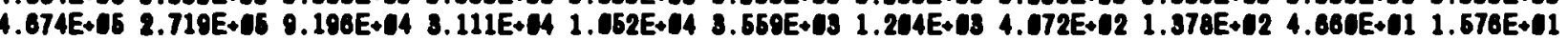

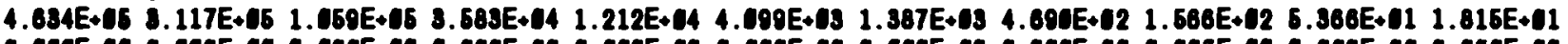

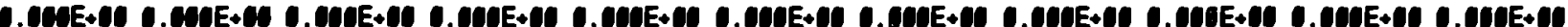
1.280E-A1 O

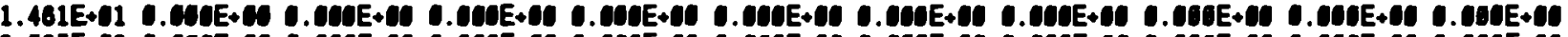

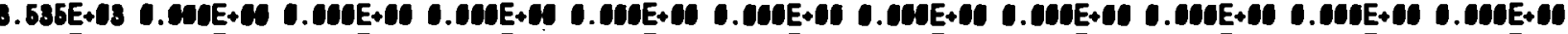

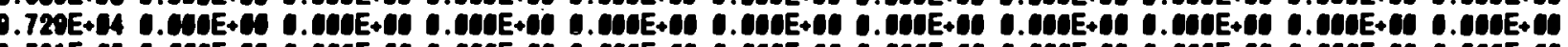

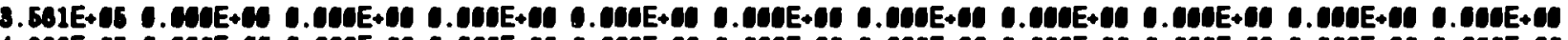

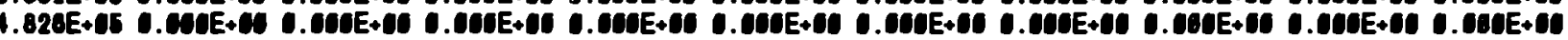

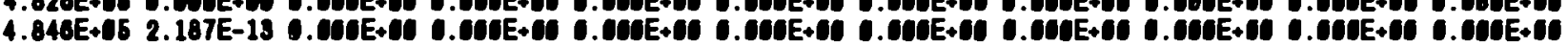

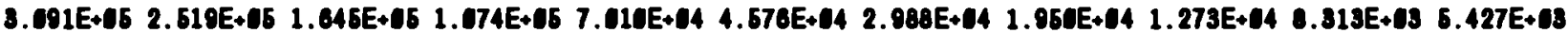

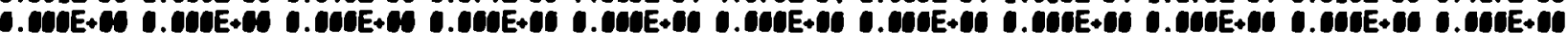

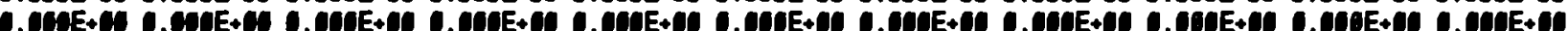
- .

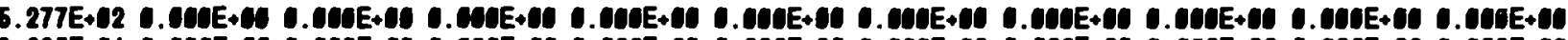

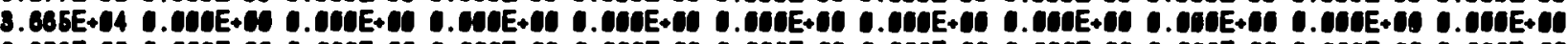

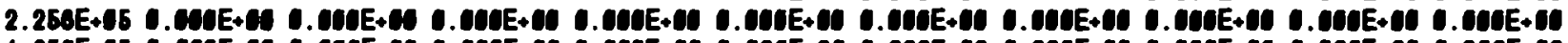

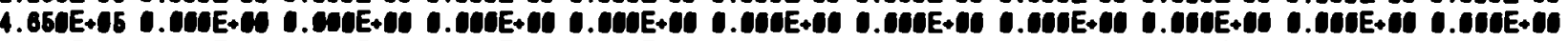

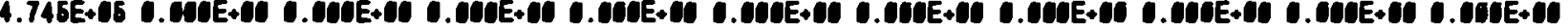
4.196E-07 4.206E-07 4.206E-07 4.206E-07 4.206E-07 4.206E-07 4.206E-07 4.206E-07 4.206E-07 4.206E-07 4.205E-07

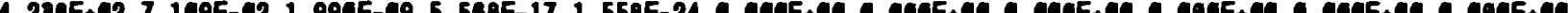

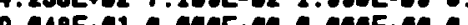

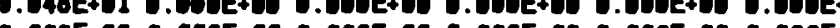

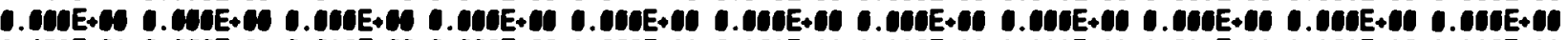

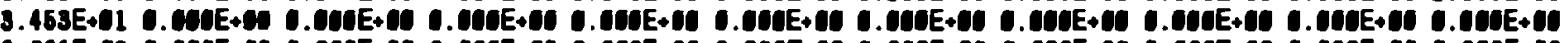

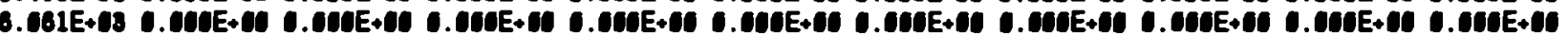

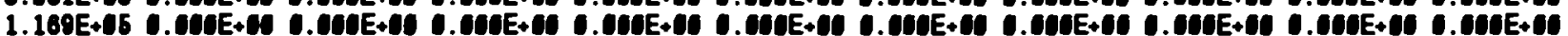

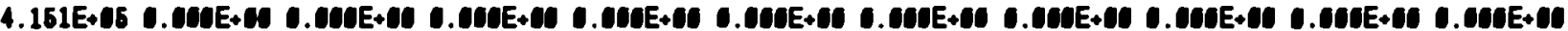

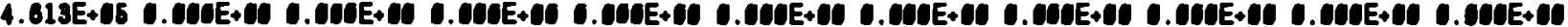

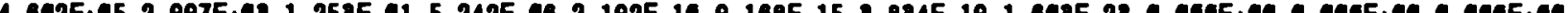

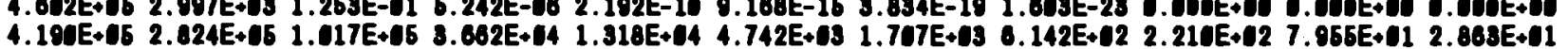




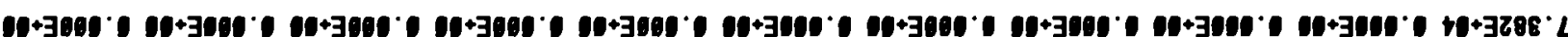

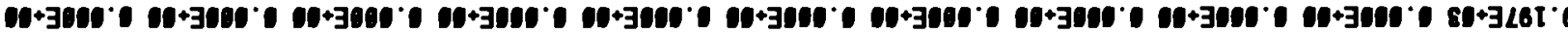

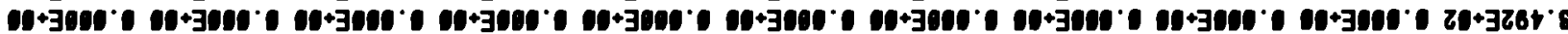

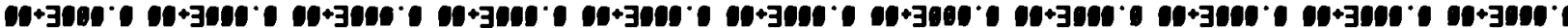

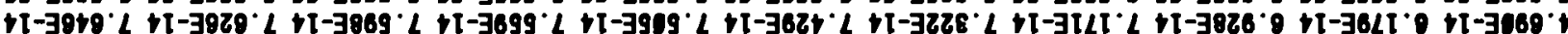

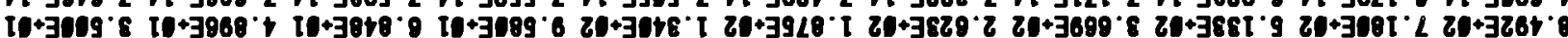

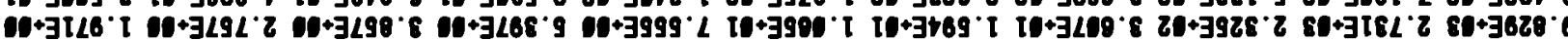

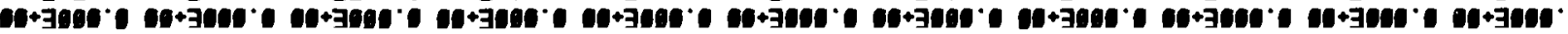

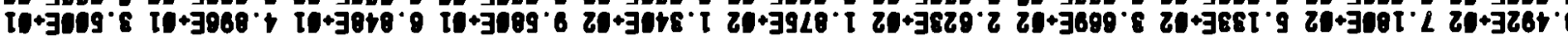

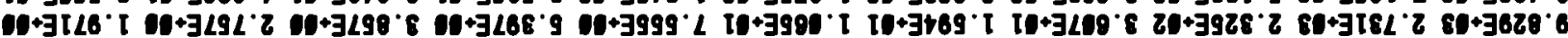

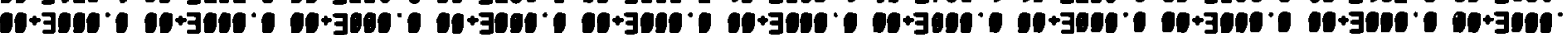
19+1 19+3919. (1)

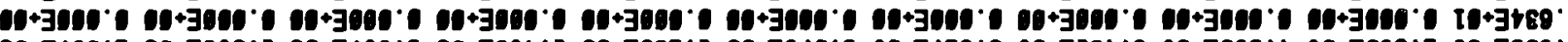

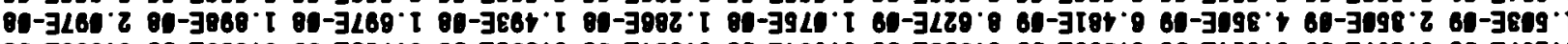

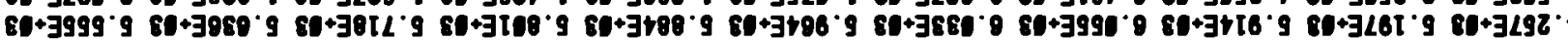

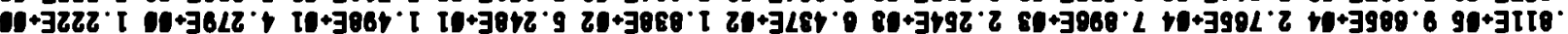

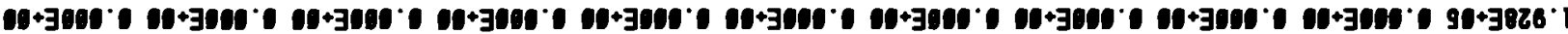

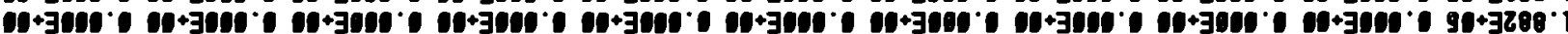

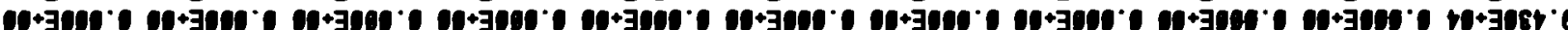

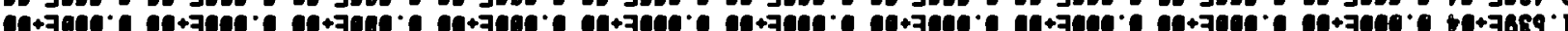

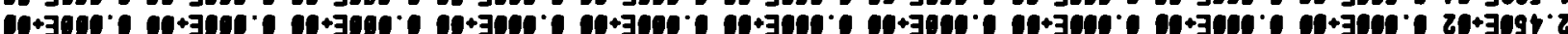

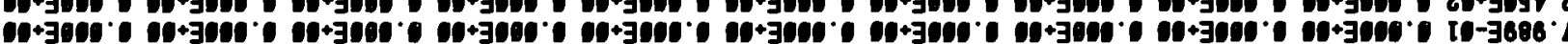

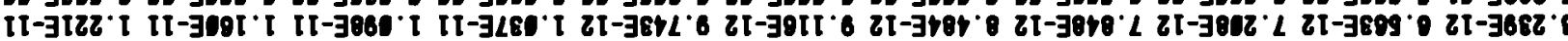

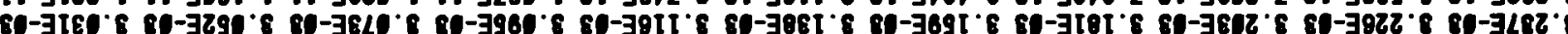
פ'

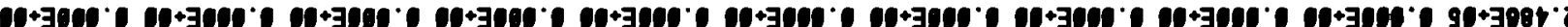
$+3000 \cdot 90^{\circ}+38<1^{\circ} \cdot 2$

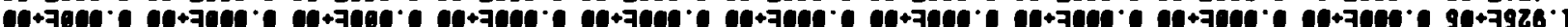

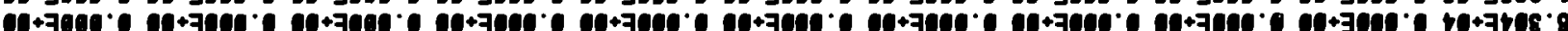

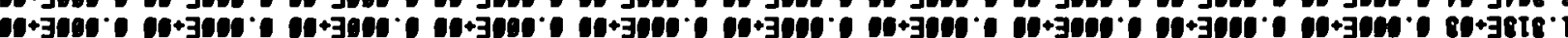

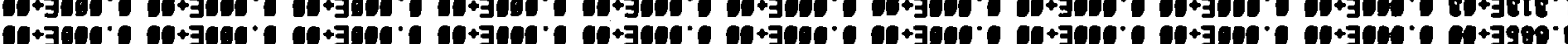

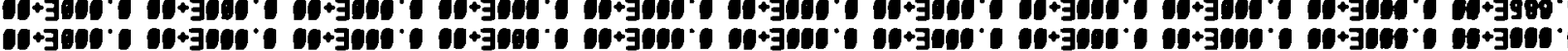

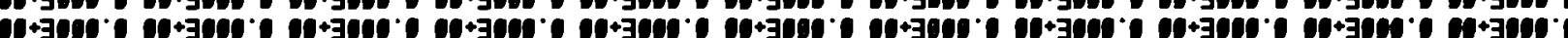
e

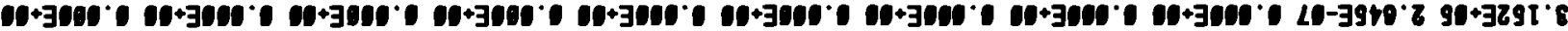
(8)

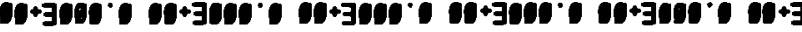

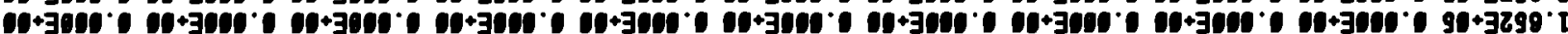

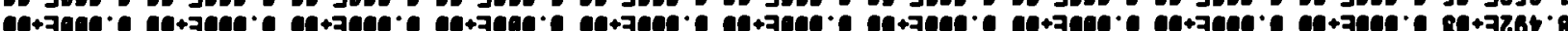

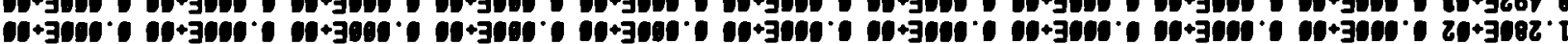

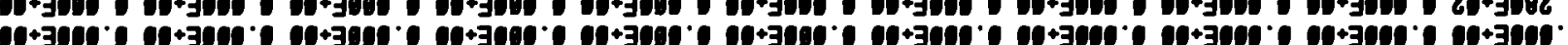

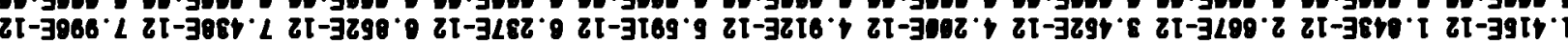

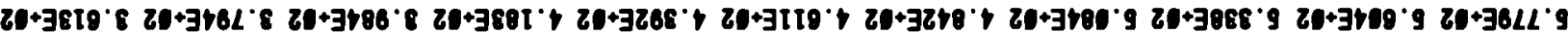

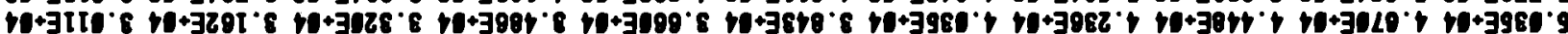

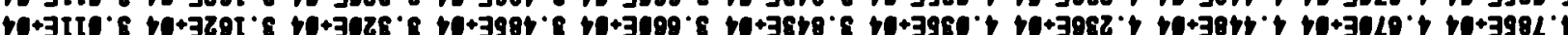

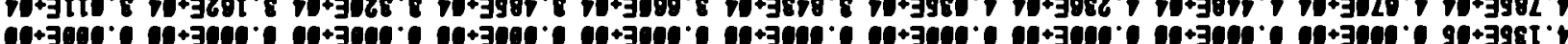

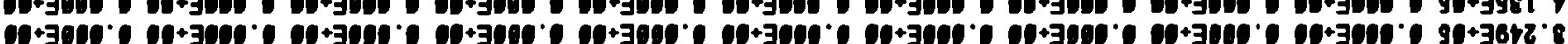

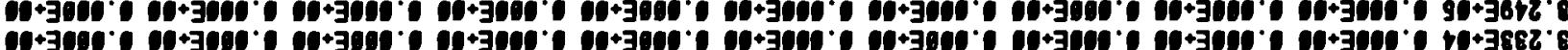

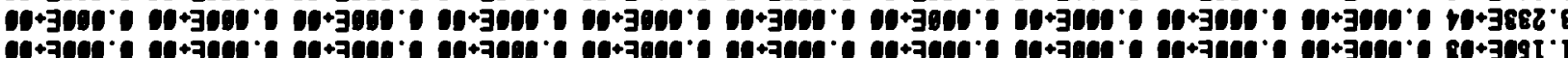

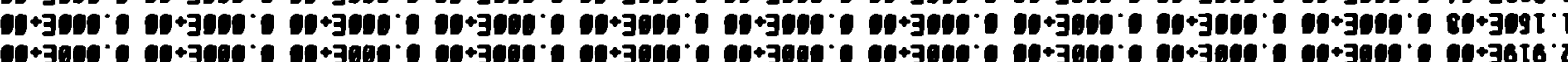

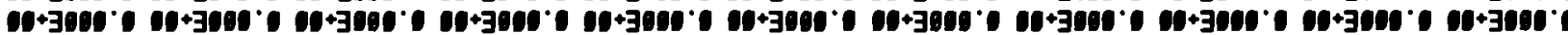


-

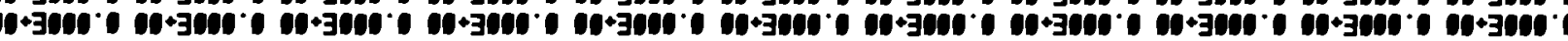

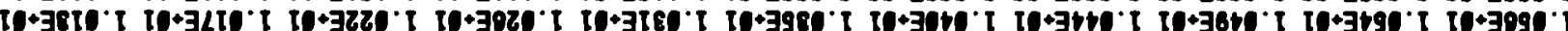

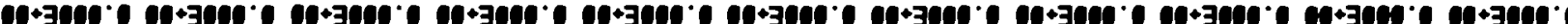

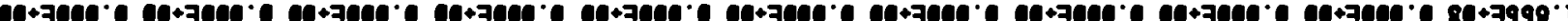

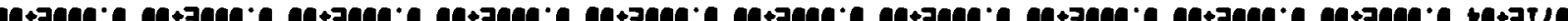
-

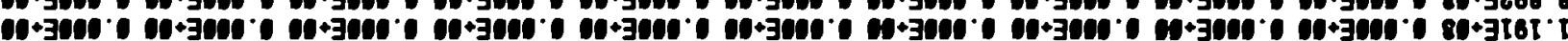

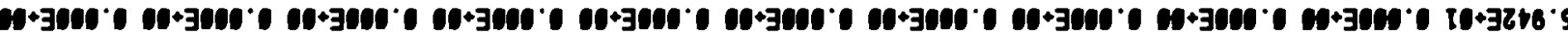

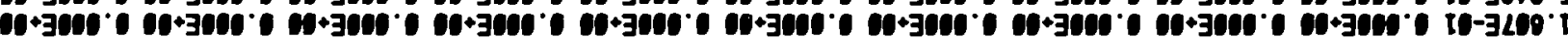

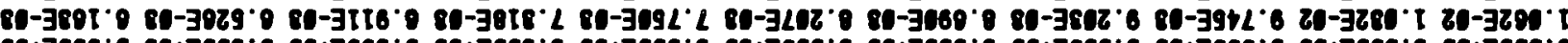

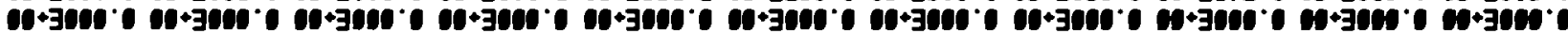

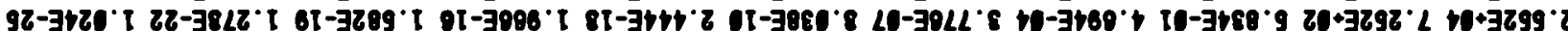

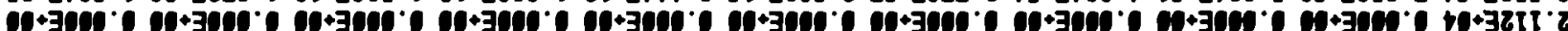

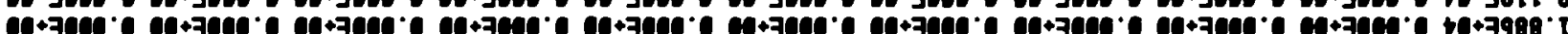

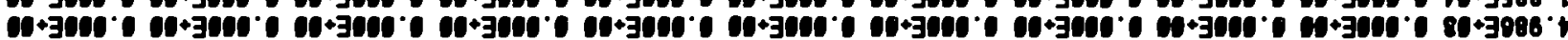

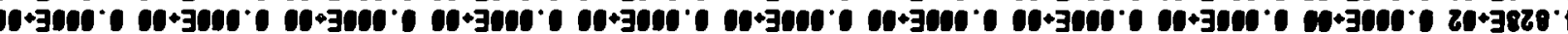

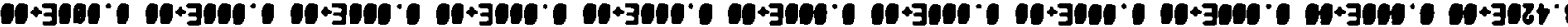

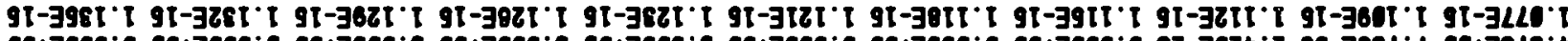

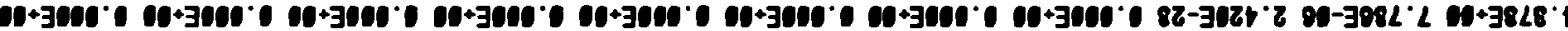

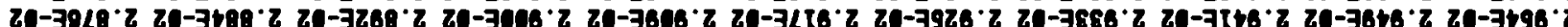

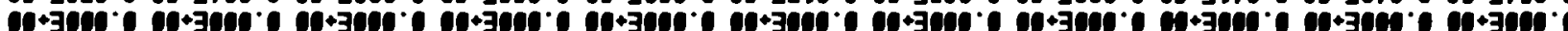

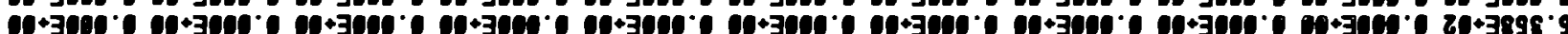

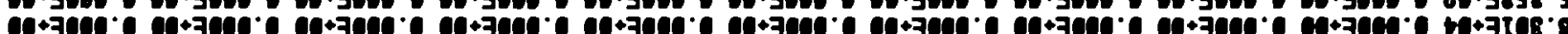

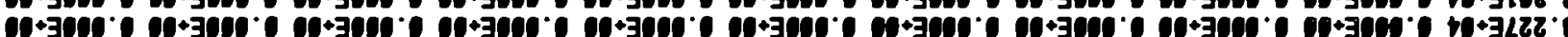

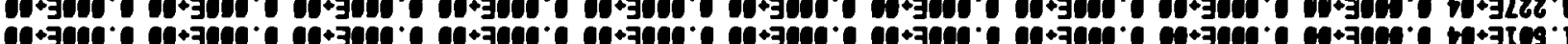

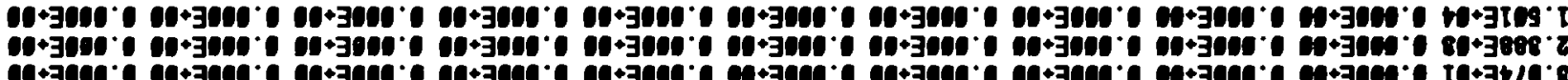

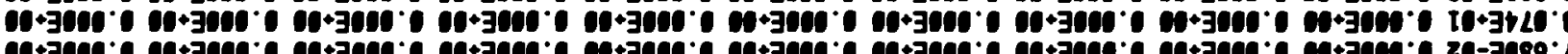

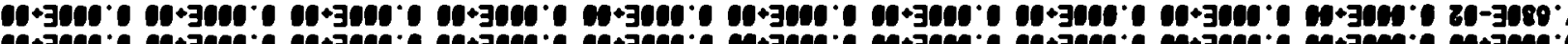

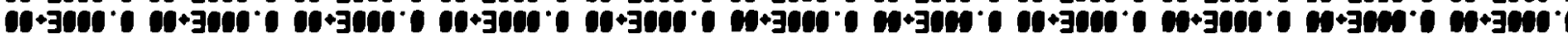

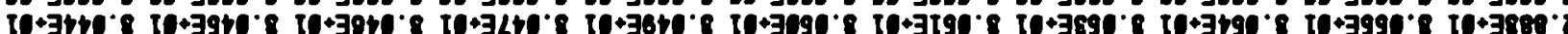

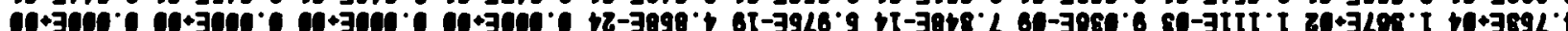

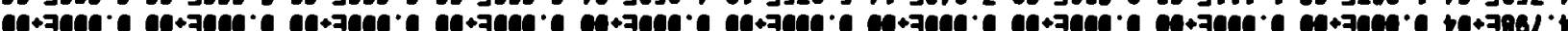

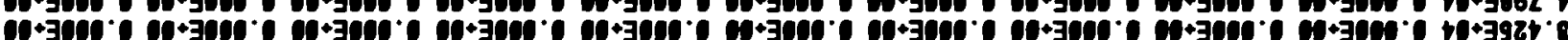

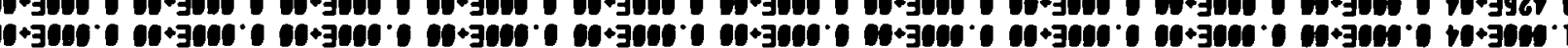

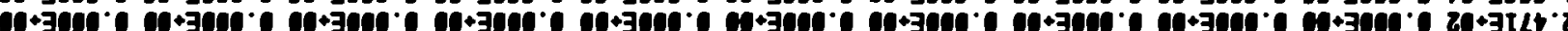

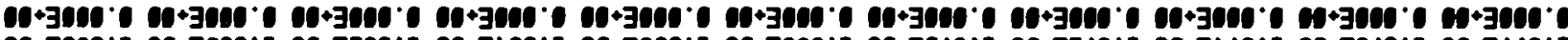

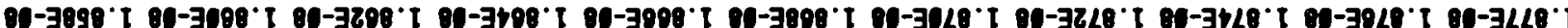

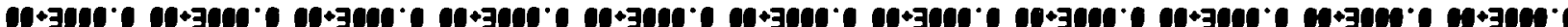

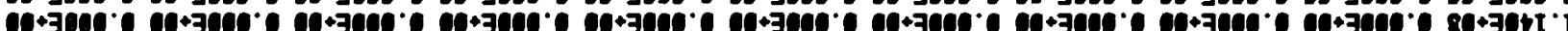

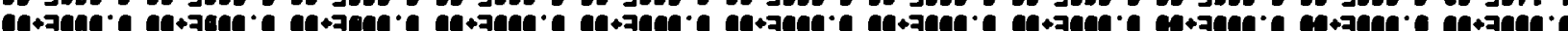

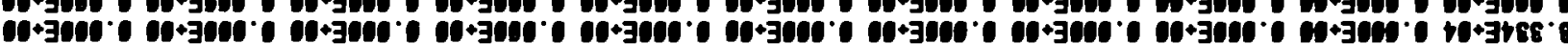

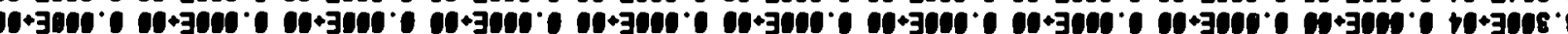

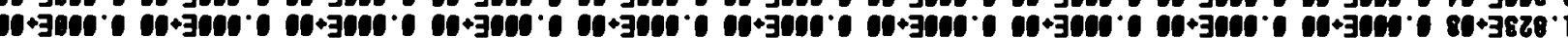

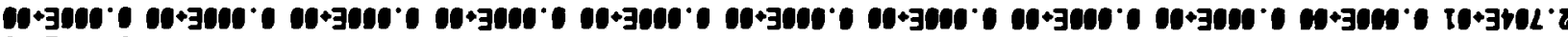

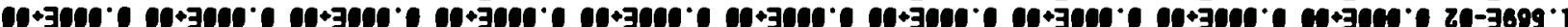

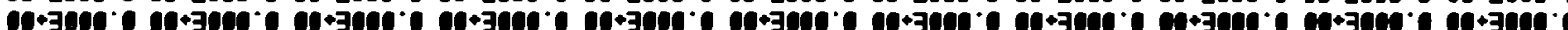

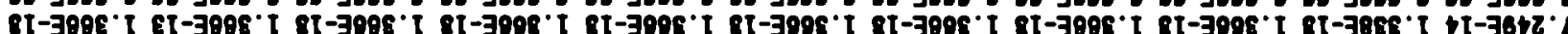

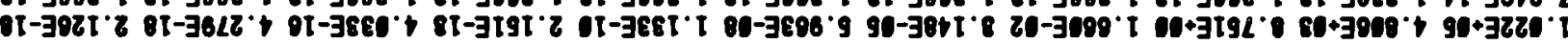

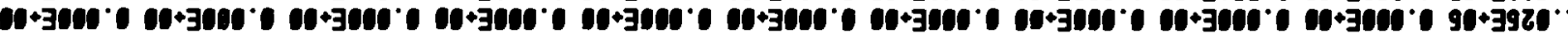

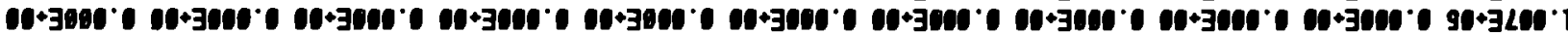

t9rol

t9rad

เ9โว?

tgiv

$89 t 00$

89 tกี

89 ths

8gthd

89זעd

89เอ

89tr

29100

megtก

89 זก



negthd

zgro

2978d

E9TV

z9tv日

Iงt/E

Igths

Igthd

Igtol
Igryd

I9เง

I9IV

t9IV

องtก

อ9tns

องTn

ogron

องเว

องIr

ogtve

องt5ว

BrIT

ortind

ortan

briyd 


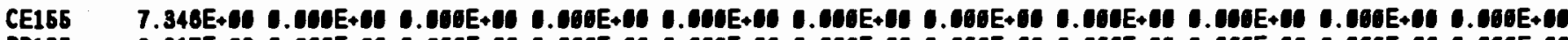

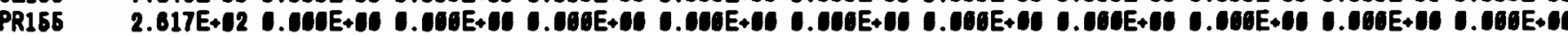

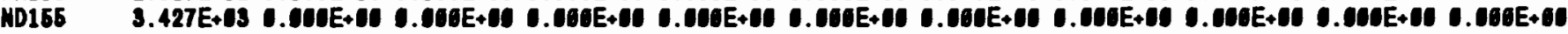

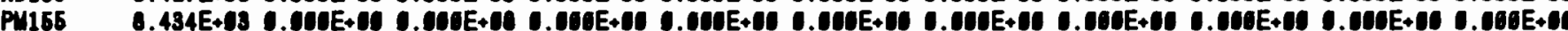

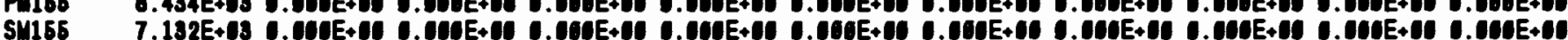

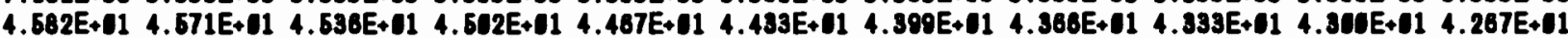

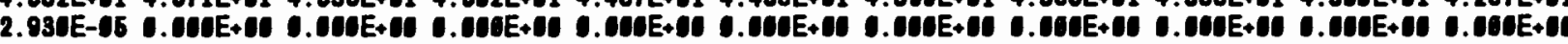

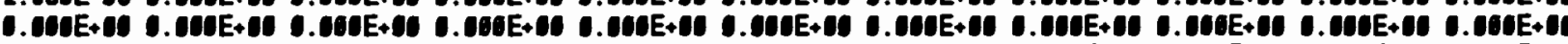

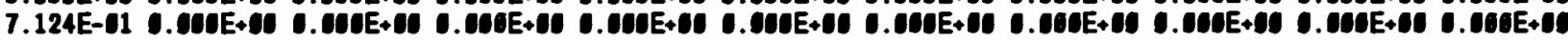

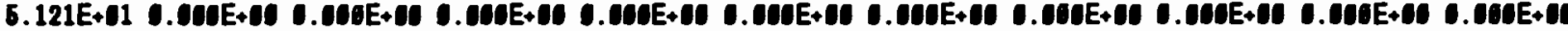

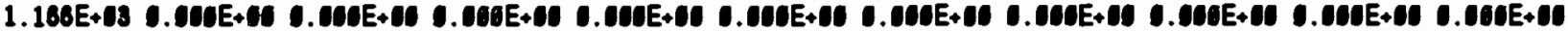

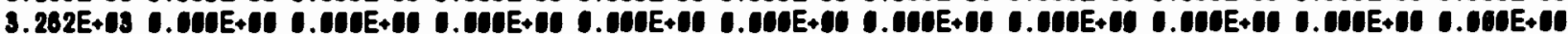
3.202E+13 . 1.287E+

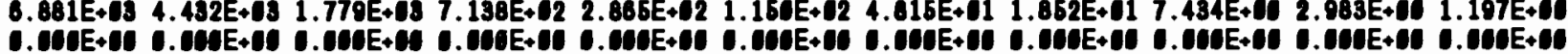

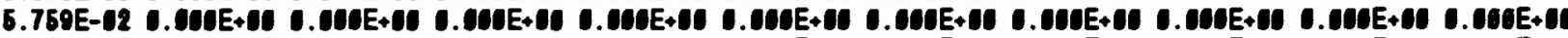

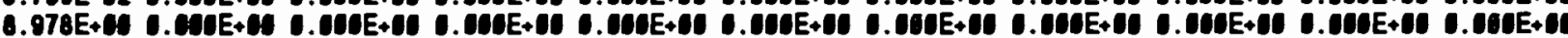

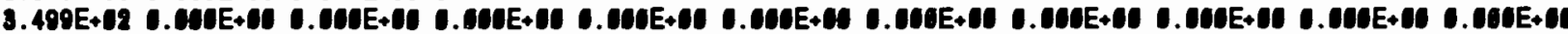

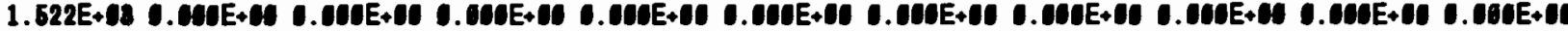

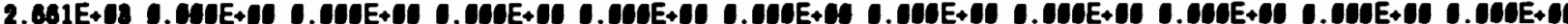
2.

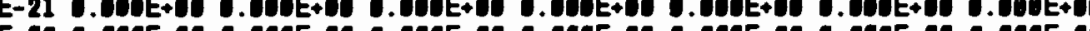
G.

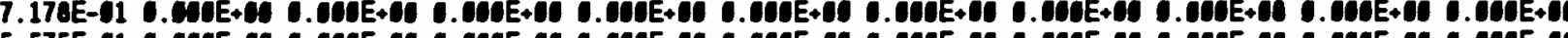

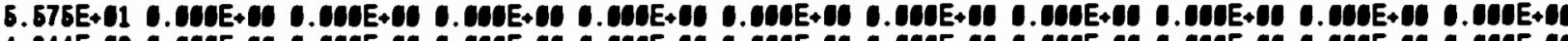

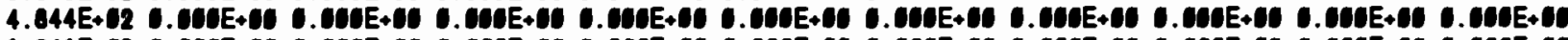

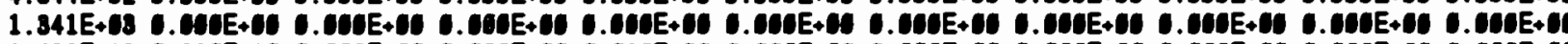

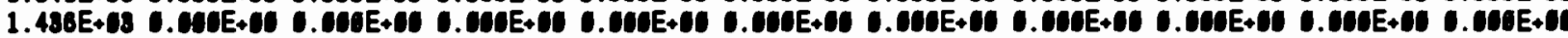

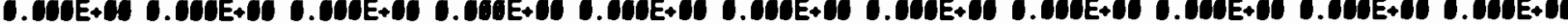

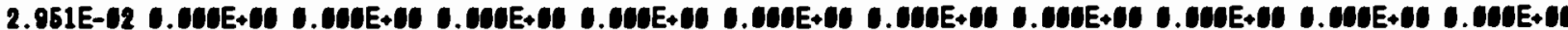

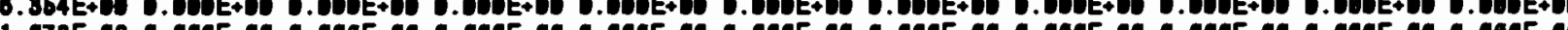
1.073E.

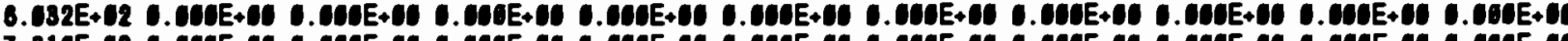

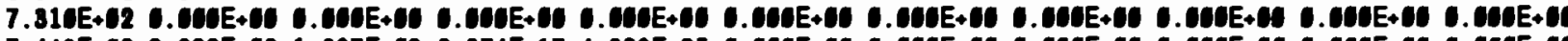

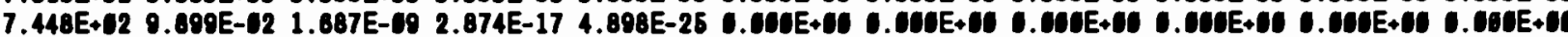

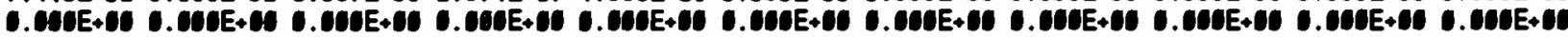

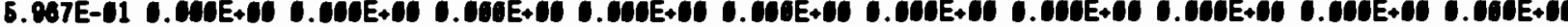

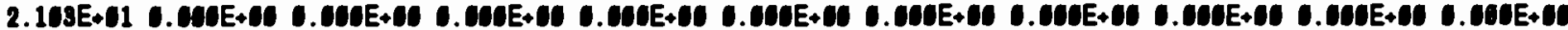

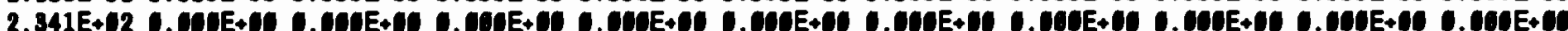
3.475E+ 12 C.

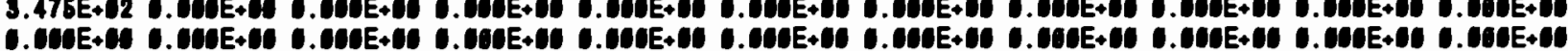

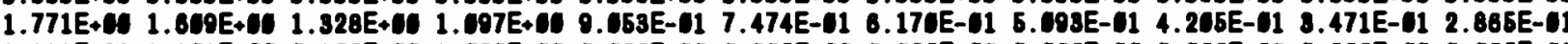

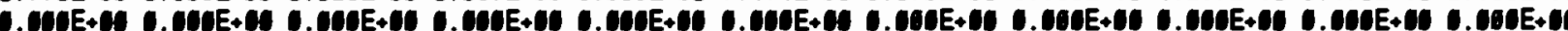

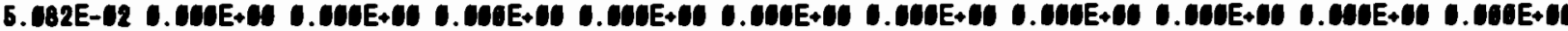

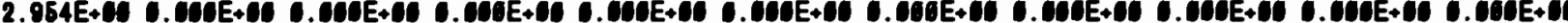

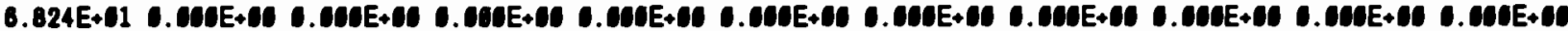

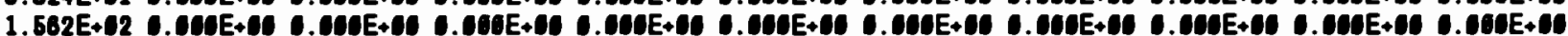

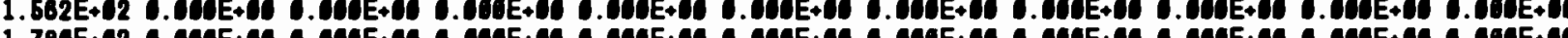

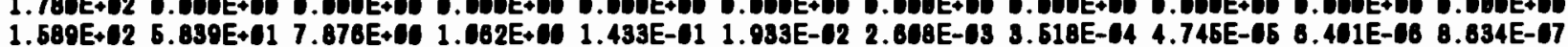
$1.680 E+02$
$0.000 \mathrm{E}+0039 \mathrm{E}+01$

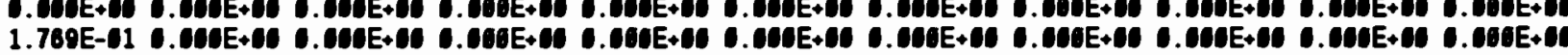

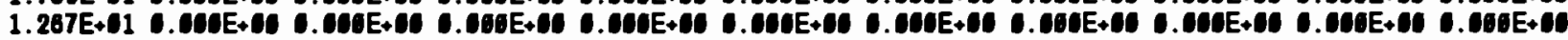




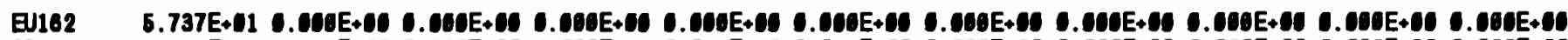

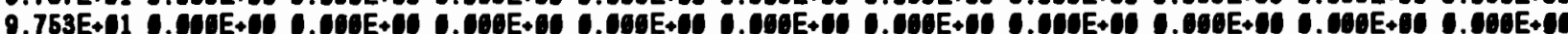

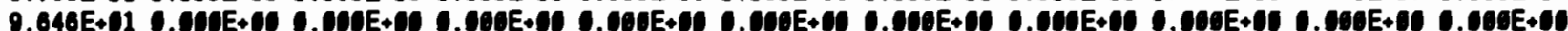
$2.80 \mathrm{E}+\mathrm{G}$.

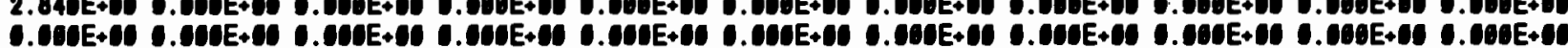

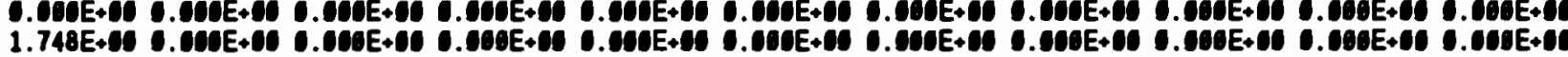

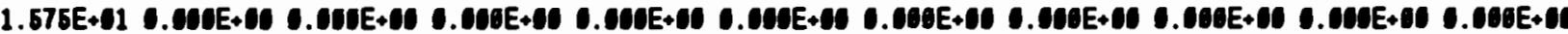

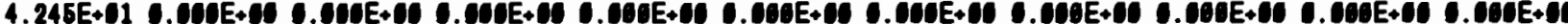

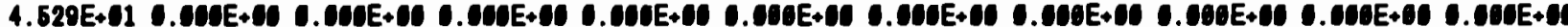
C. 1.

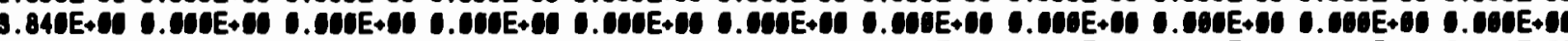

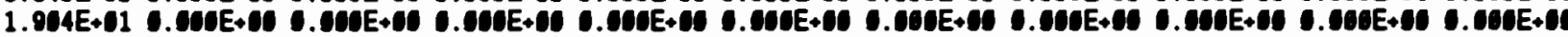

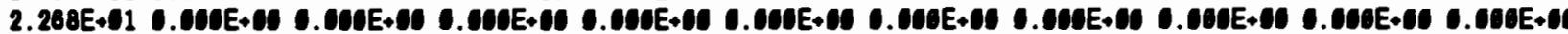

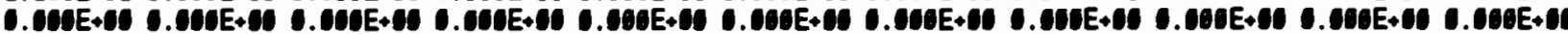

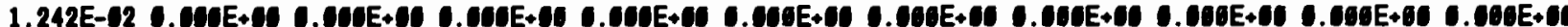

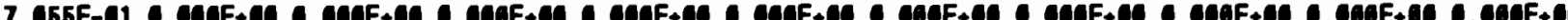
$7.949 E, C D$ O.

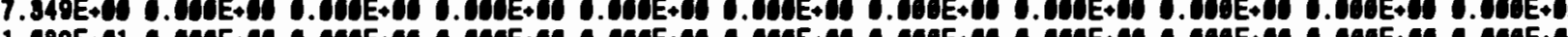
$1.788 E+11$.

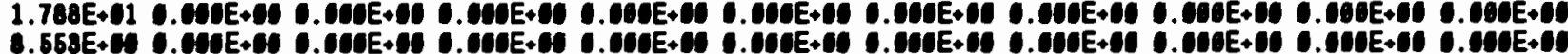

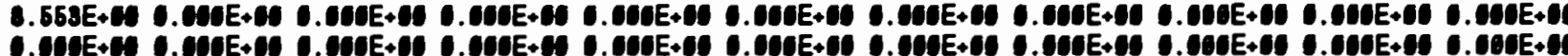

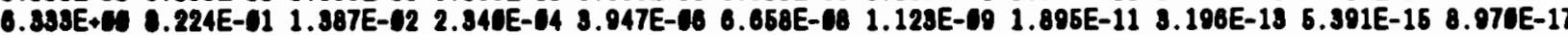
6.653E+19 1.220E+11 2.088E-12 3.488E-14 5.883E-18 0.923E-88 1.674E-69 2.823E-11 4.763E-13 8.034E-15 $1.355 E-16$

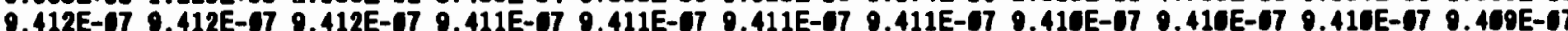

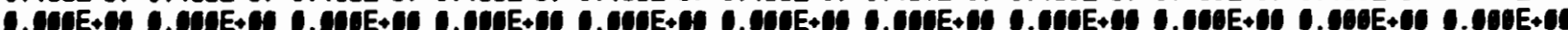

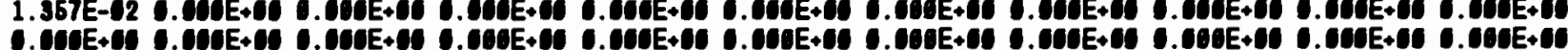

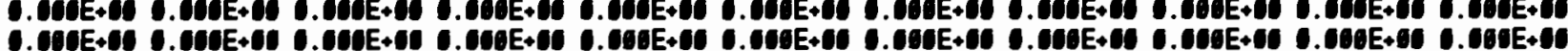

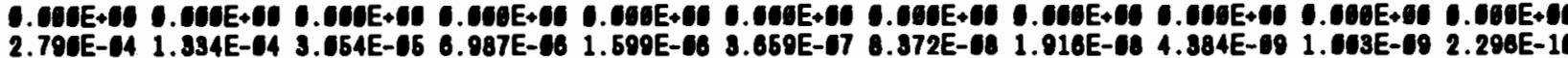

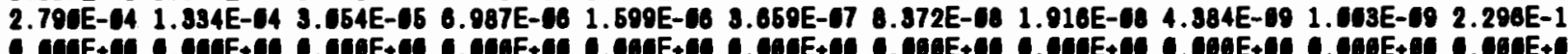

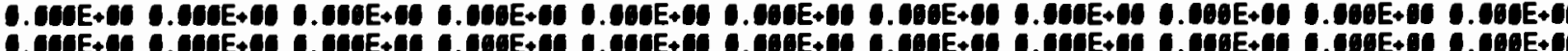

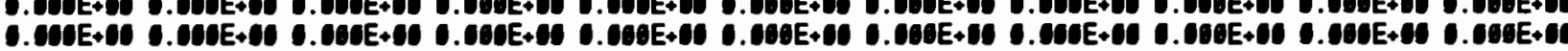

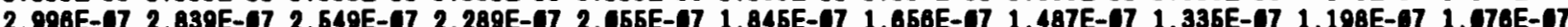
3 . $18 E-07$ COCE

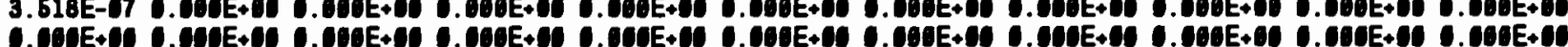

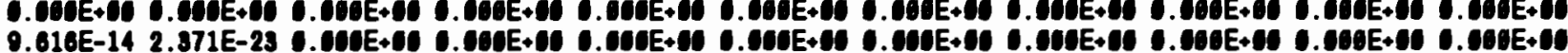

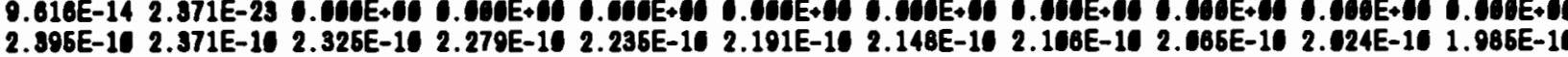
$.009 E+60$ COSE+00

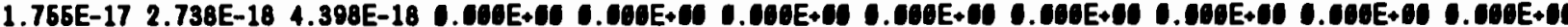

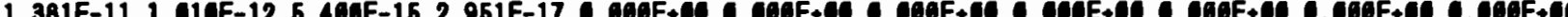

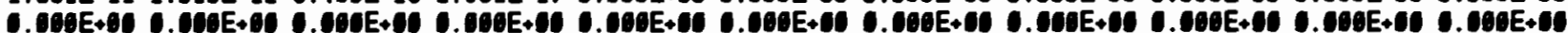

TM172 
APPENDIX B

GENII RESULTS FOR ATMOSPHERIC RELEASES 

GENII RESULTS FOR ATMOSPHERIC RELEASES

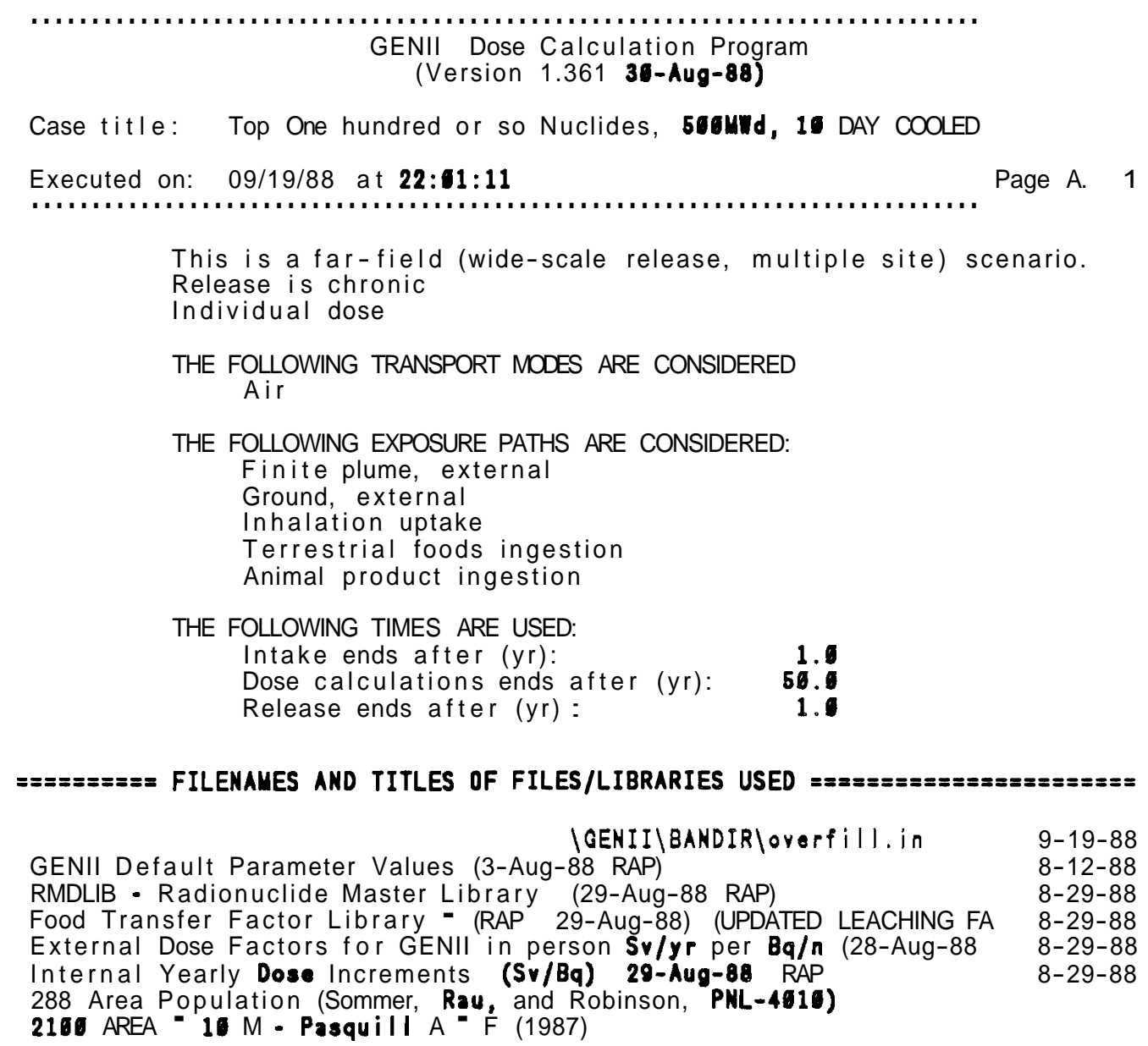

THE FOLLOWING TIMES ARE USED: Intake ends after (yr): Dose calculations ends after (yr): $\mathbf{5 0 . 0}$ Release ends after (yr): 


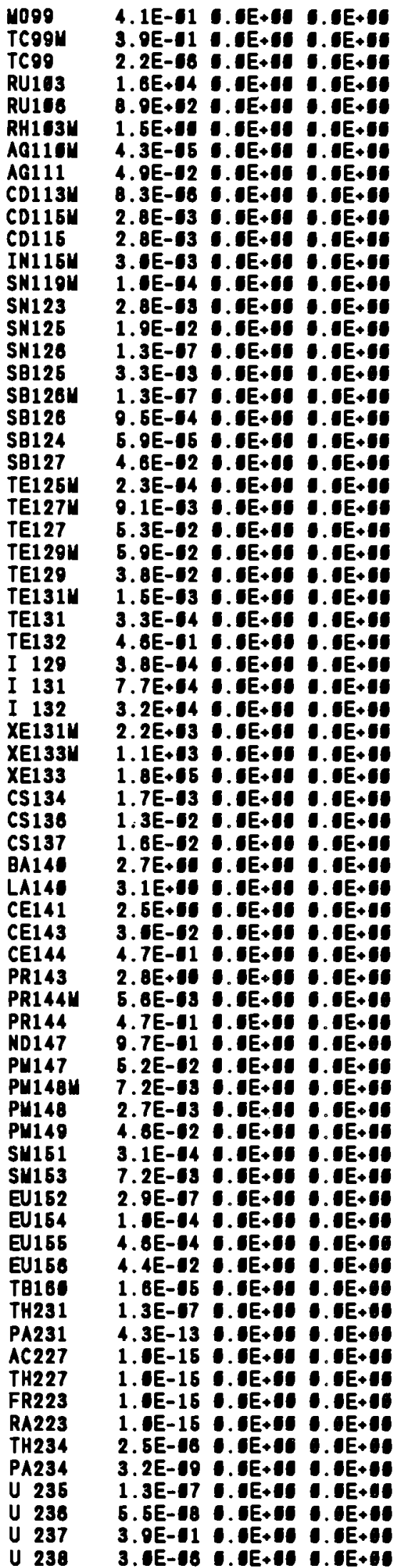




\begin{tabular}{|c|c|}
\hline $\begin{array}{l}\text { NP237 } \\
\text { PA233 } \\
\text { U } 233 \\
\text { TH229 } \\
\text { RA225 } \\
\text { AC225 } \\
\text { NP238 } \\
\text { NP239 } \\
\text { PU238 } \\
\text { PU239 } \\
\text { PU240 } \\
\text { PU241 } \\
\text { AM241 }\end{array}$ & 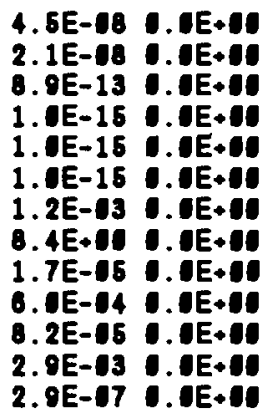 \\
\hline
\end{tabular}

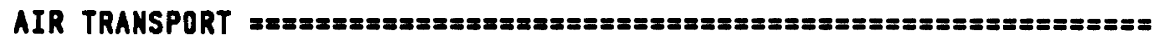
Joint frequency data input.

MI distance, direction, and chi/a rill be selected by the program. Ground level release.

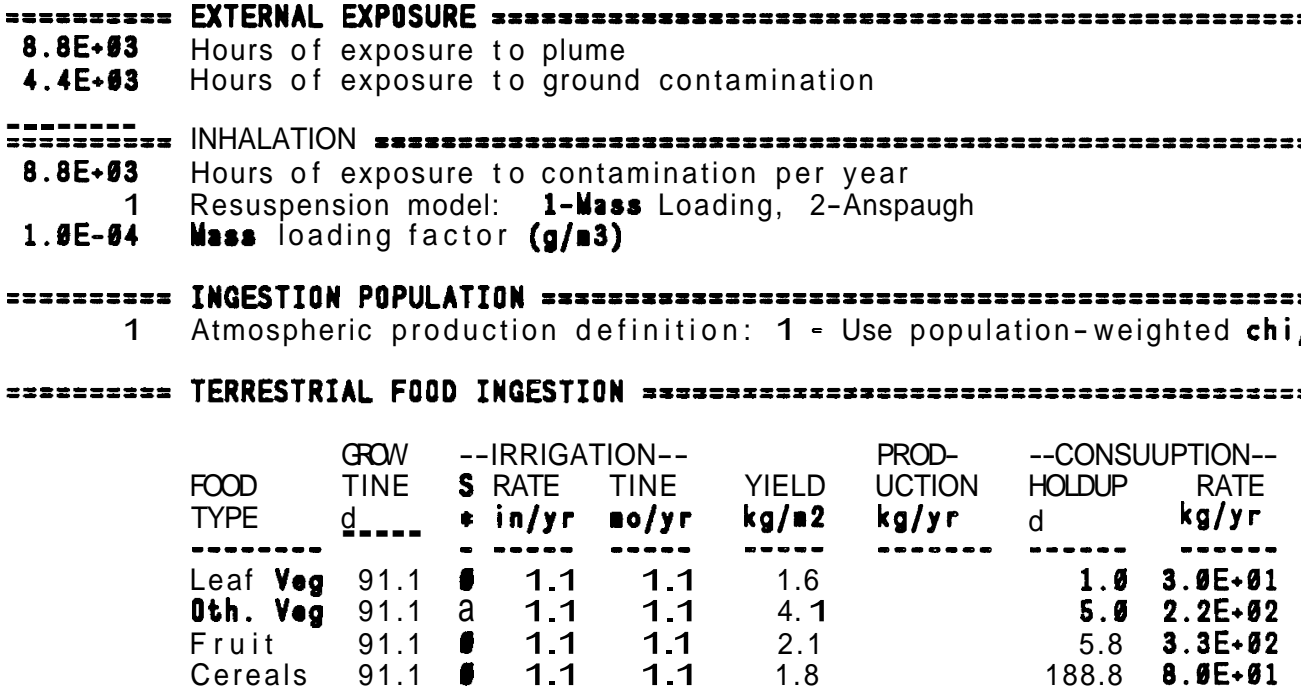

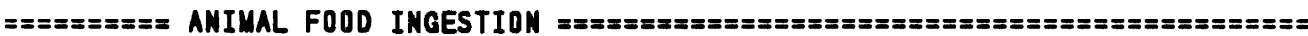

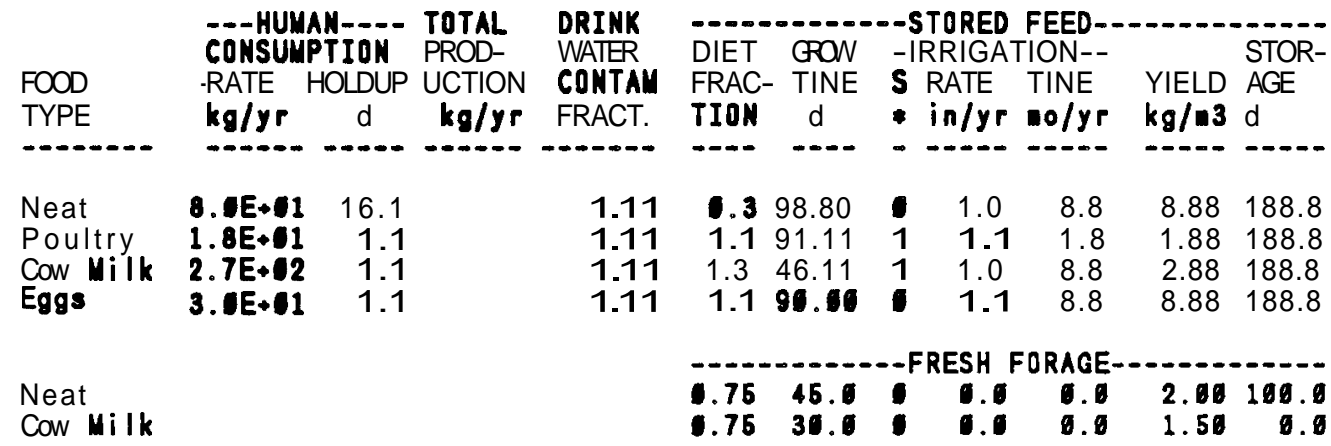


GENII Dose Calculation Program

(Version 1.361 35-Aug-88)

Case title: Top One hundred or so Nuclides, ssemm, 10 DAY COOLED

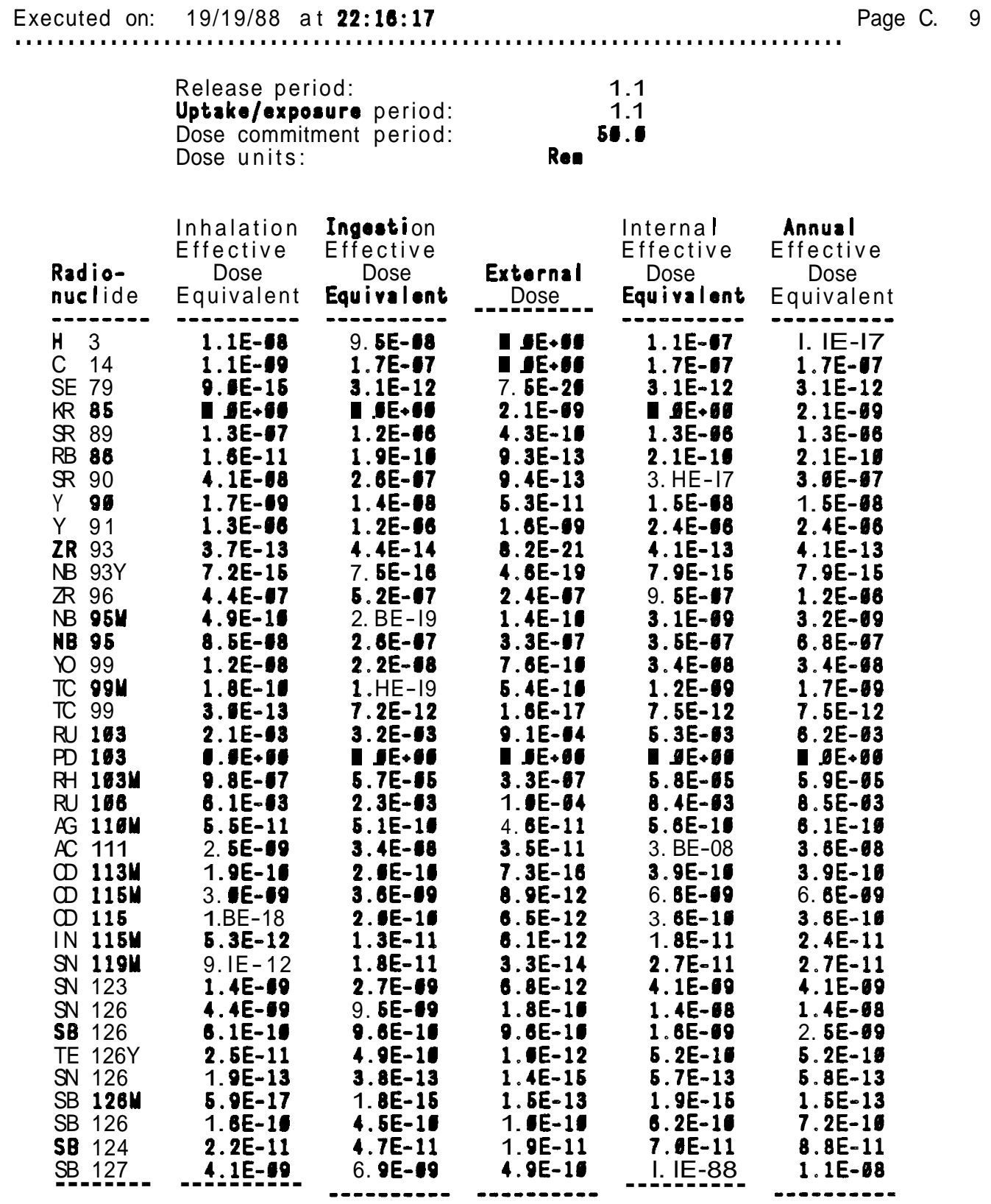




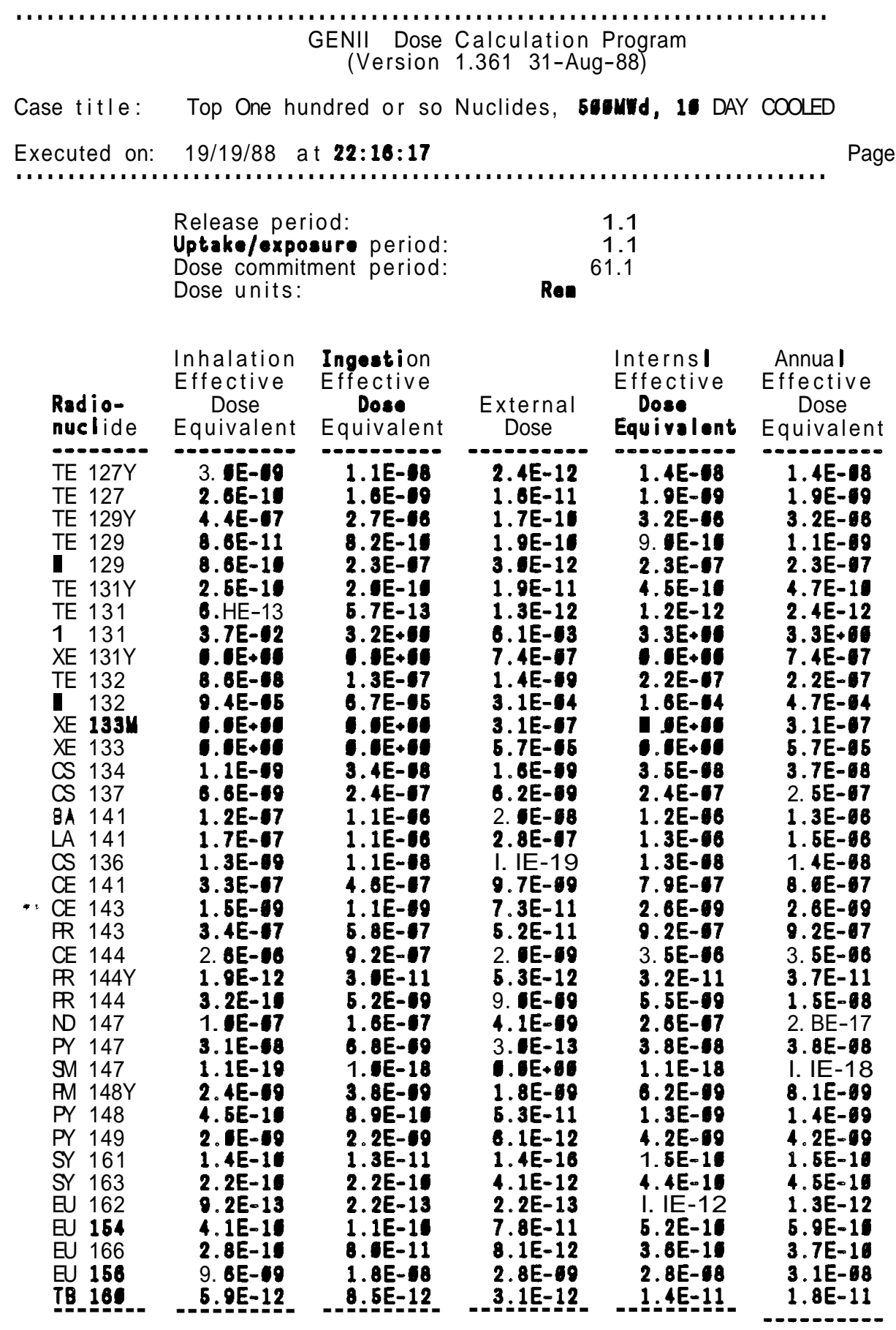




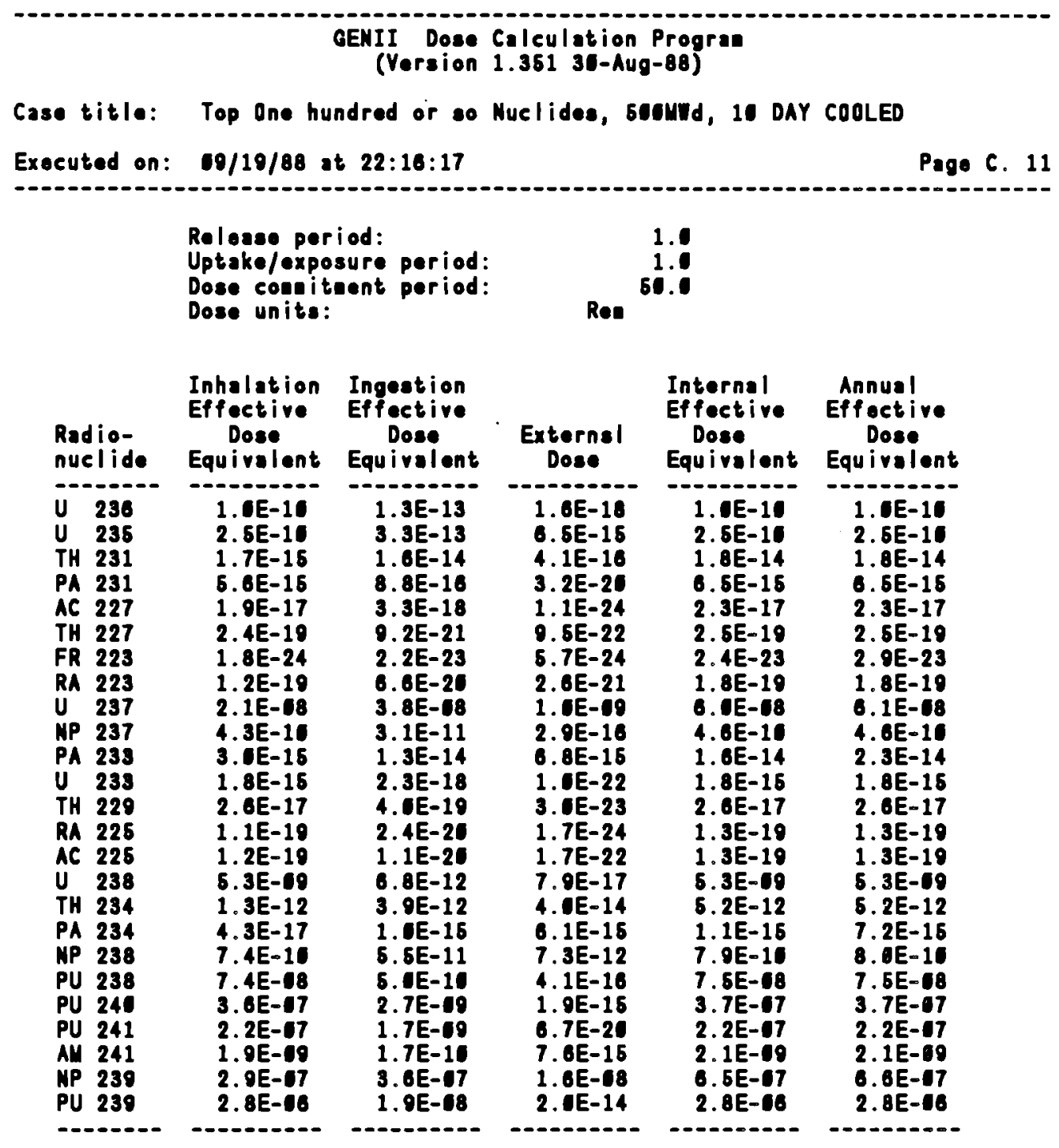




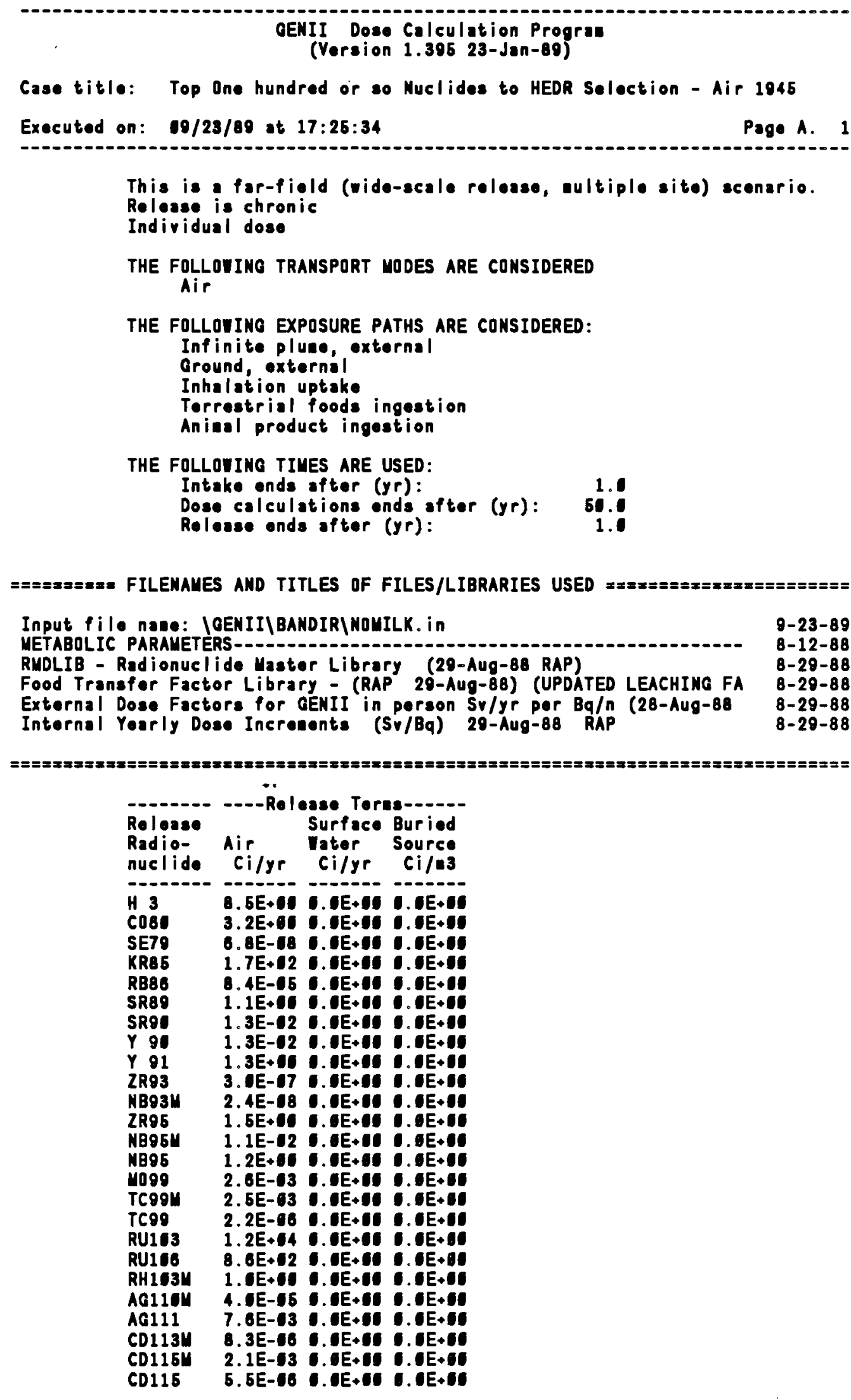

B. 7 


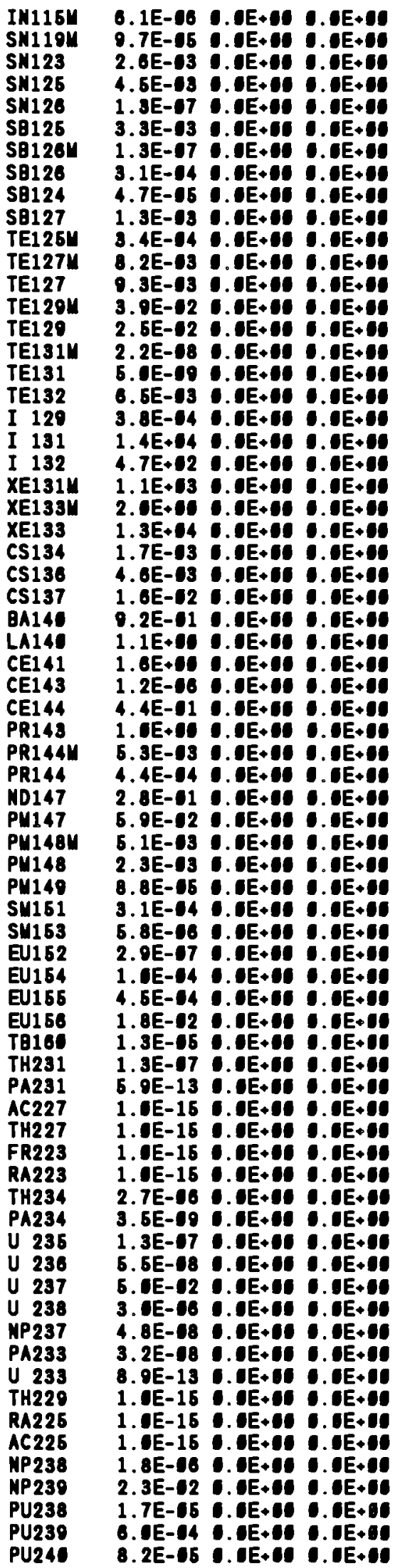




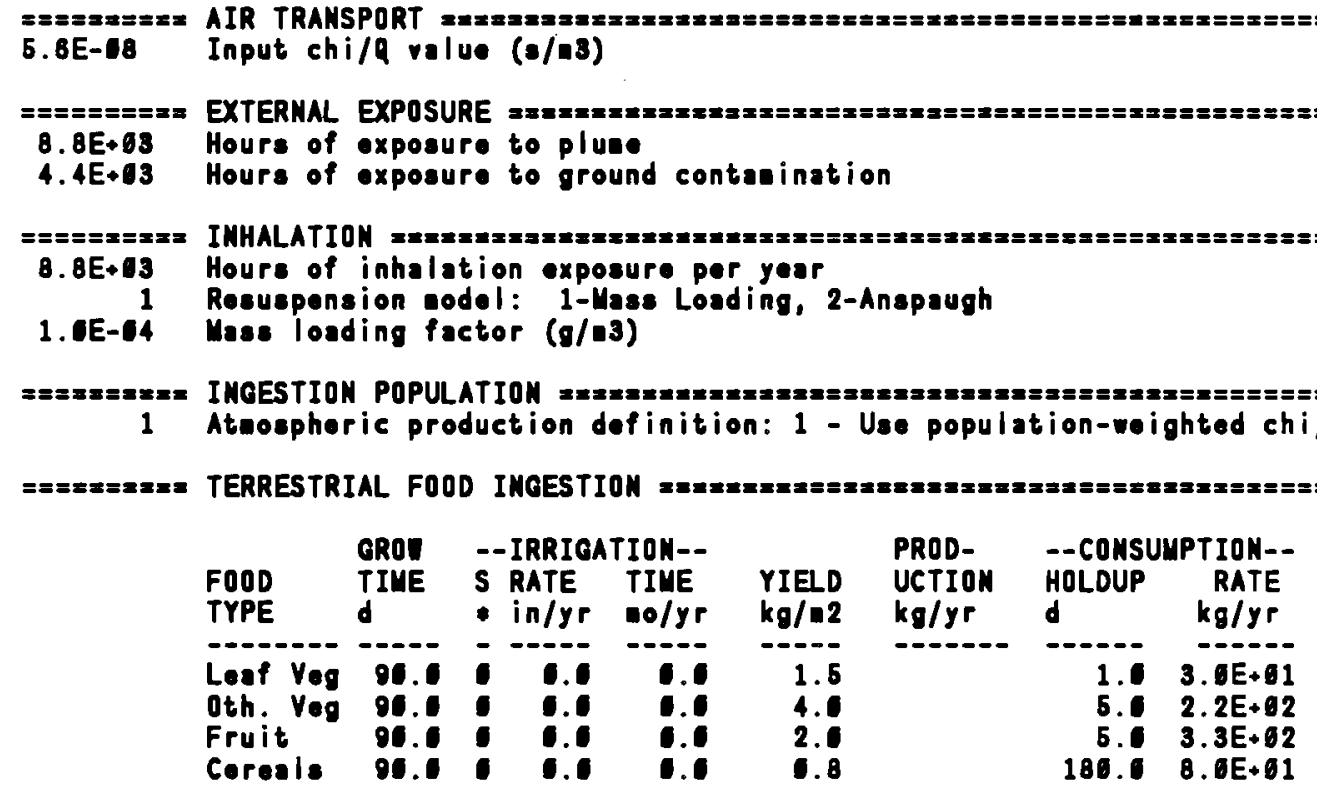

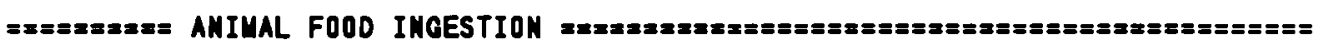

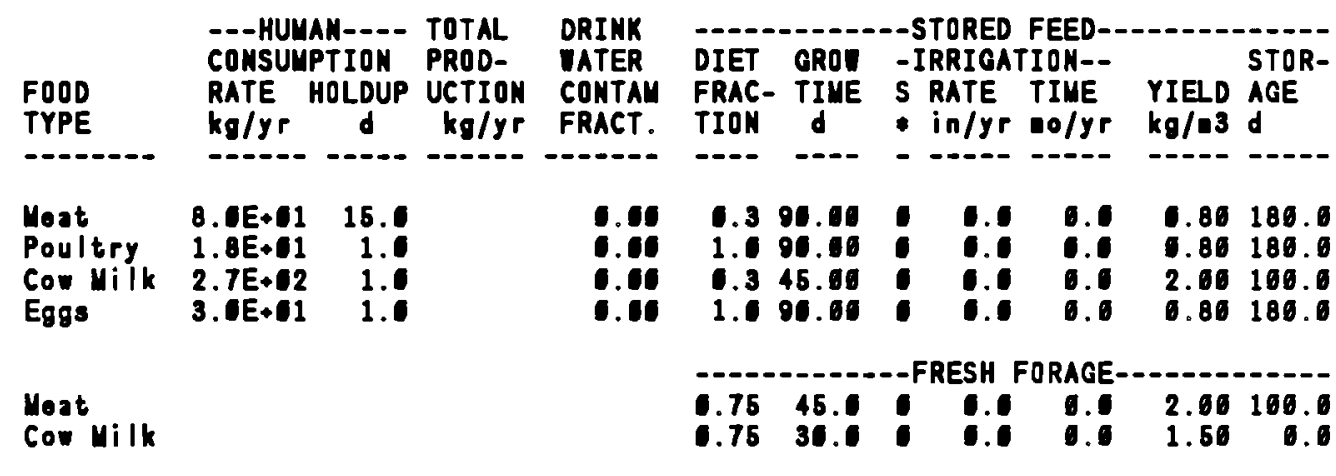

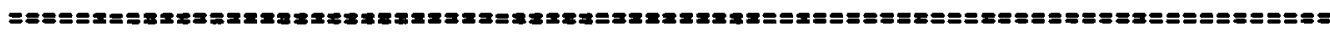

Input prepared by:

Input checked by:
Dote:

Dato: 


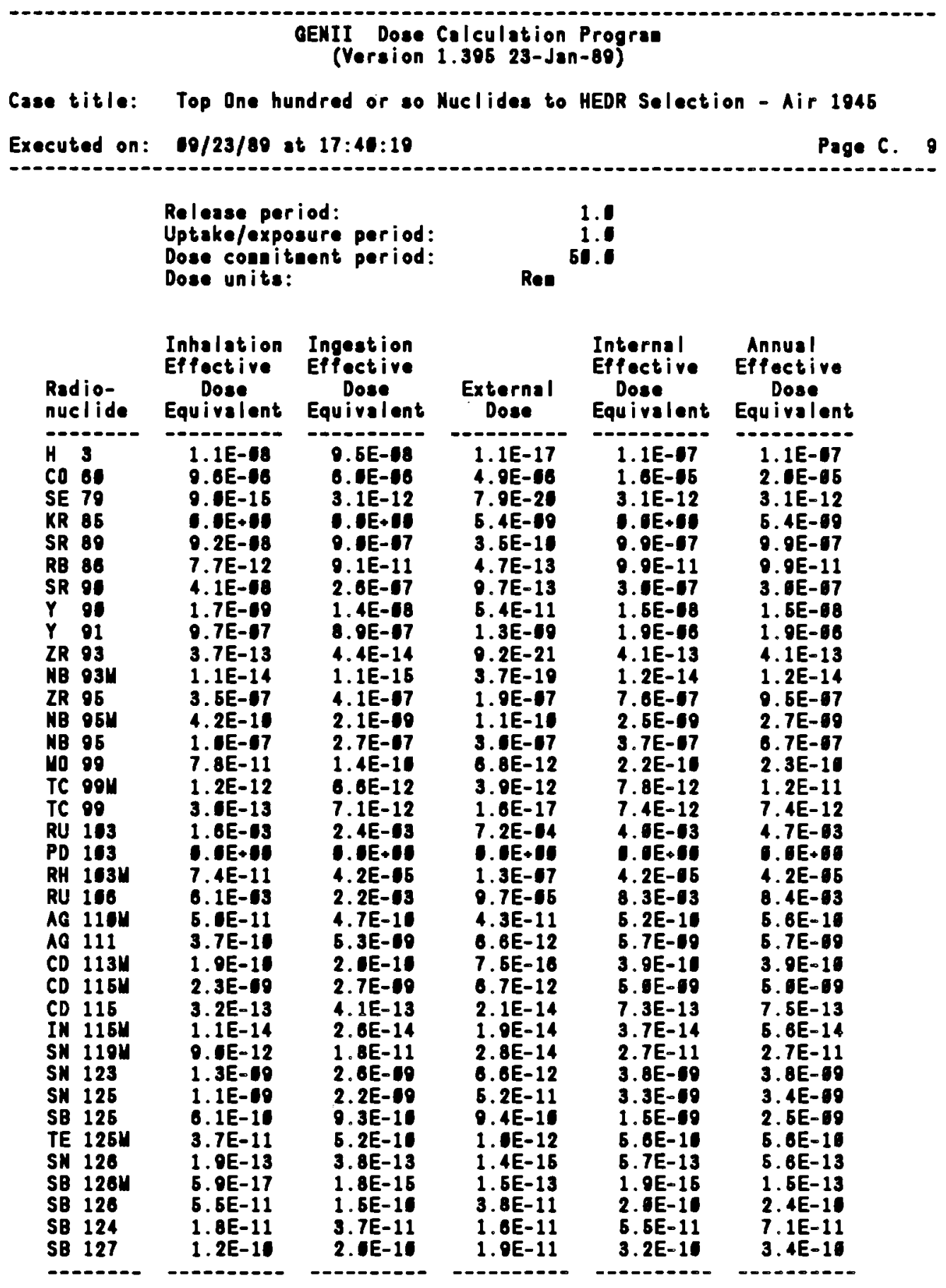


GENII Dose Calculation Progran

(Version 1.395 23-Jan-89)

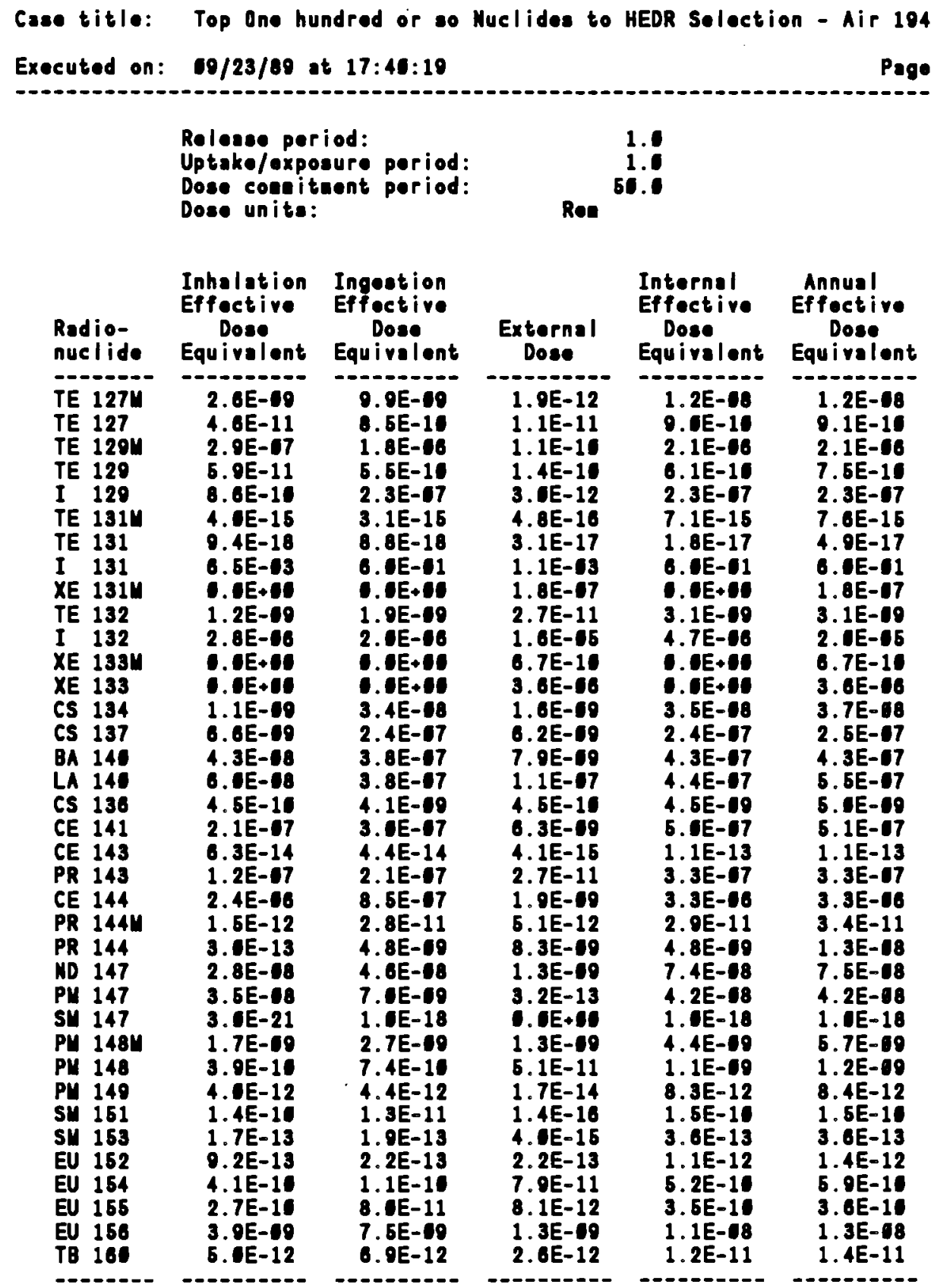




\section{GEMII Dose Calculation Prograo}

(Version 1.395 23-Jan-89)

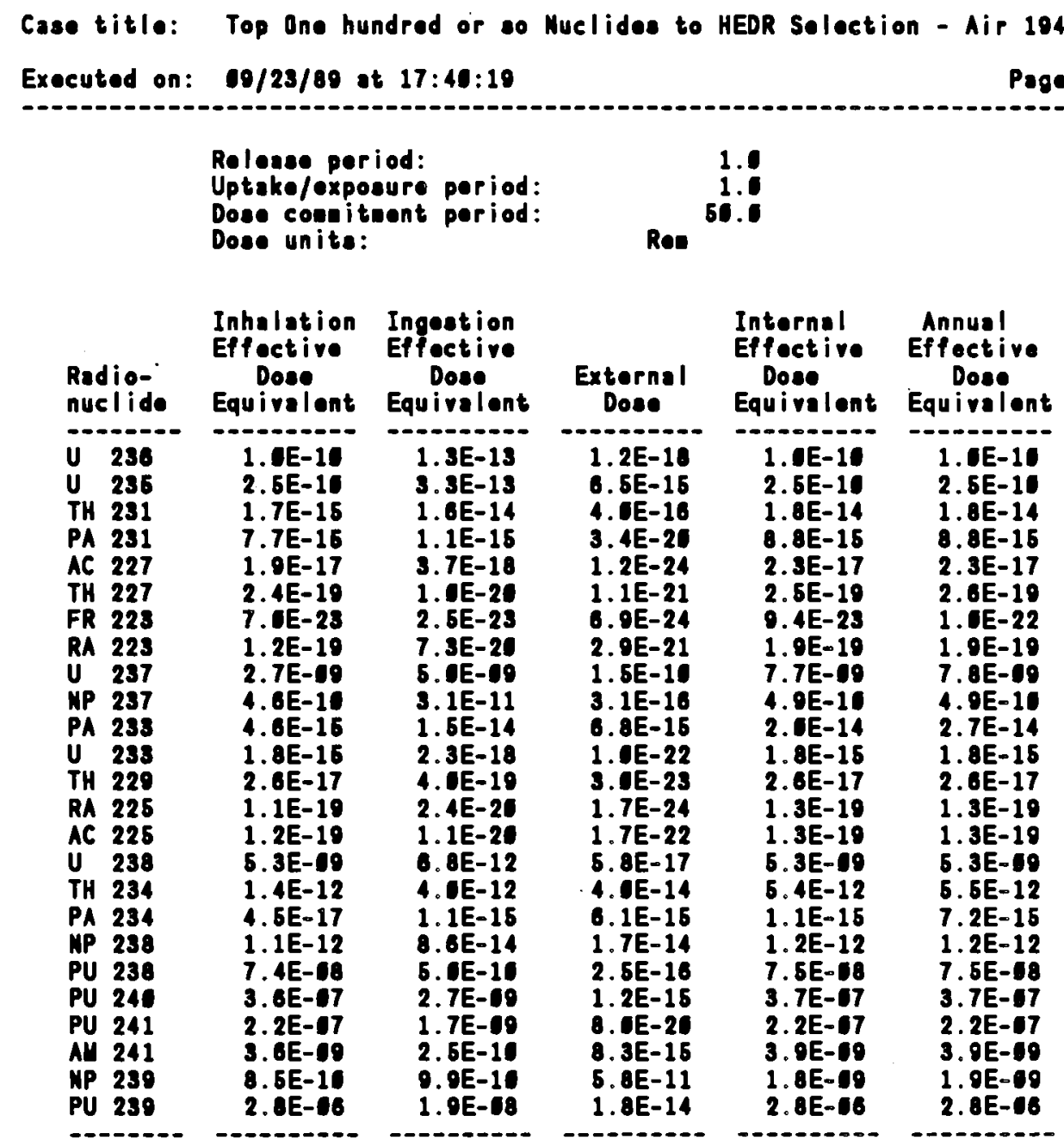


Case title: Top One hundred or so Nuclides, 6eomed, ge DAY COOLED

Executed on: 19/19/88 at 22:16:36

Poge A. 1

This is far-field (ride-scale roleses, aultiple site) scenario.

Release is chronic

Individual dose

THE FOLLOIINO TRANSPORT MODES ARE CONSIDERED Air

THE FOLLOUIMG EXPOSURE PATHS ARE CONSIDERED:

Finite plune, external

Oround, external

Inholation uptake

Torrestrial foods ingestion

Anieal product ingestion

THE FOLLOUING TIMES ARE USED:

Intake enda after $(y r)$ : 1.0

Dose calculations enda ofter $(y r)$ : 50.0

Relesee ende sfter $(y r)$ :

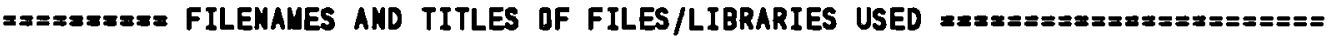

Default Paraneter Values (3-Aug-80 RAP)

RMDLIB - Radionuclide Master Library (28-Aug-88 RAP) 8-28-88

Food Transfer Factor Library - (RAP 20-AUg-88) (UPDATED LEACHIMO FA 8-29-88

External Dose Factora for GENII in person Sv/yr per Bq/n (28-Aug-88 8-29-88

Internsl Yearly Dose Increnents (Sv/Bq) 29-Aug-88 RAP 8

200 Area Population (Somer, Rau, and Robinson, PNL-4010)

-.

2100 AREA - 10 M - Pasquili' $A$ - F (1987)

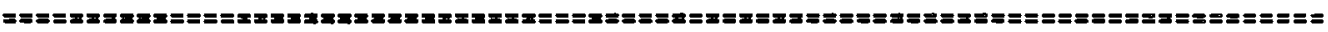

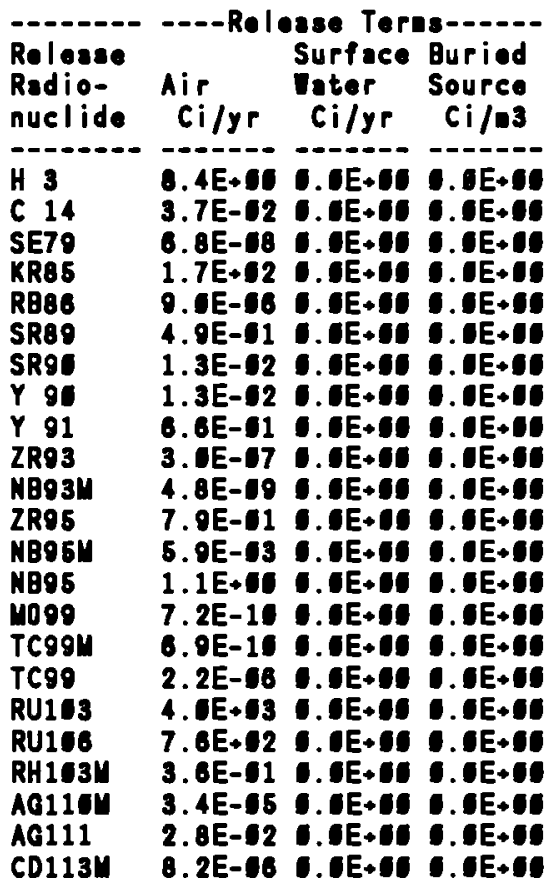

B.13 


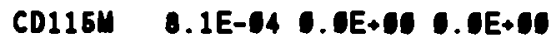

CD115 4.3E-14 O.NE+00 O.OE+CO

IN115M 5.7E-08 O.UE+10 G.UE+OS

SN110M 0.2E-05 O.DE+DO $0.0 E+C O$

SN123 1.0E-93 G.OE+CO G.OE+CE

SN125 6.0E-05 O.OE+CO O.OE+CO

SH126 1.3E-17 O.OE+NO C.OE+QS

$58125 \quad 3.3 E-93-0 E+00$ 0.0E+C

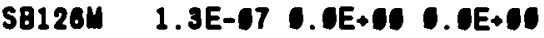

SB126 1.1E-05 -.DE+CO C.NE+CO

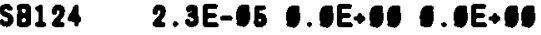

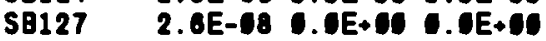

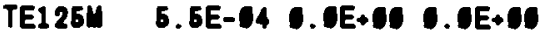

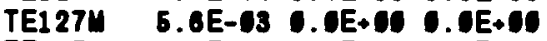

TE127 5.5E-03 $0.0 E+00 \quad 0.0 E+00$

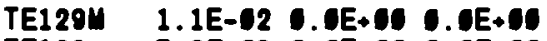

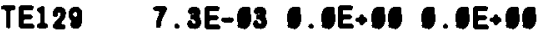

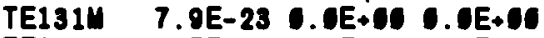

TE131 1.7E-23 G.OE+QS G.OE+QS

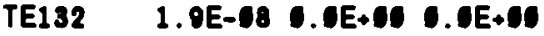

I $120 \quad 3.8 E-040.0 E+00 \quad 0.0 E+00$

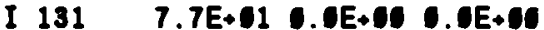

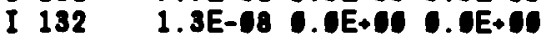

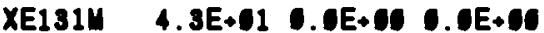

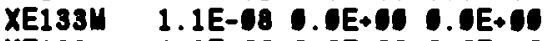

XE133 4.6E+C6 $0.0 E+06-.0 E+95$

CS134 1.0E-63 $. .0 E+015.0 E+96$

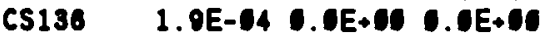

C5137 1.0E-12 $. .0 E+05-.0 E+05$

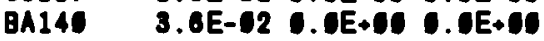

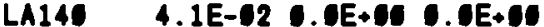

CE141 4.0E-61 ..0E+10 0.0E+10

CE143 $9.2 E-20 \bullet .0 E+05 \cup .0 E+08$

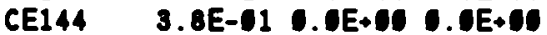

PR143 4.7E-62 $1 . \mathrm{EE}+16 \cup .0 \mathrm{E}+10$

PR144L 4.6E-63 $0.0 E+05 \div .0 E+00$

PR144 3.8E-G1 $5.0 E+95 . J E+90$

ND147 6.4E-63 $. .0 E+60$ C. E + 60

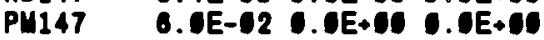

PU148U 1.9E-93 ..JE+95

PV148 1.1E-64 ..JE+65 G.JE+S6

PM149 6.0E-13 -.JE+C5 $0.0 E+60$

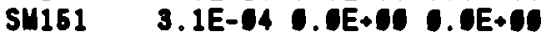

SH153 3.0E-15 0.0E+C6 G.0E+CS

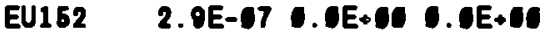

EU154 1.0E-04 $9.0 E+015.0 E+05$

EU155 4.4E-94 $. .0 E+05 \cup .0 E+60$

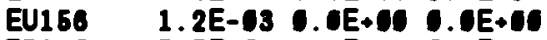

TB165 7.5E-68 @. EE+95 ๑.0E+90

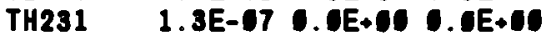

PA231 1.1E-12 0.UE+CS O.JE+G0

AC227 1.0E-15 $\bullet .0 E+06 \bullet .0 E+60$

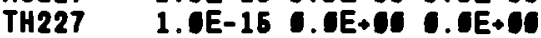

FR223 1.JE-15 $0.0 E+05$ C.JE+GS

RA223 1.JE-15 G.JE+\$S G.JE+CS

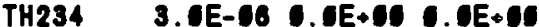

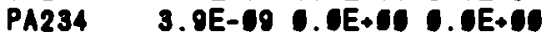

U $235 \quad 1.3 E-67-.6 E+95 \quad .0 E+96$

U $236 \quad 5.5 E-68$ 6.6E+96 $1.0 E+96$

U $237 \quad 1.1 E-64$-.6E+ $10.6 E+90$

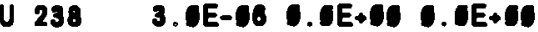

NP237 4.6E-68 G. GE+65 @.GE+60

PA233 4.6E-68 ๑. OE+CS ๑.JE+60

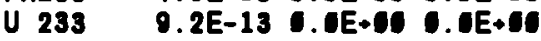

TH229 1.0E-15 O.0E+CS O.JE+00

RA225 1.0E-15 @.0E+00 ०.0E+C0

AC225 1.0E-15 $\bullet .0 E+05 \cup .0 E+60$

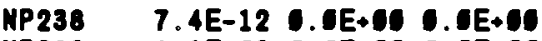

NP239 1.1E-60 G.NE+CO G.JE+CO

PU238 1.7E-65 O.DE+10 $.0 E+10$ 


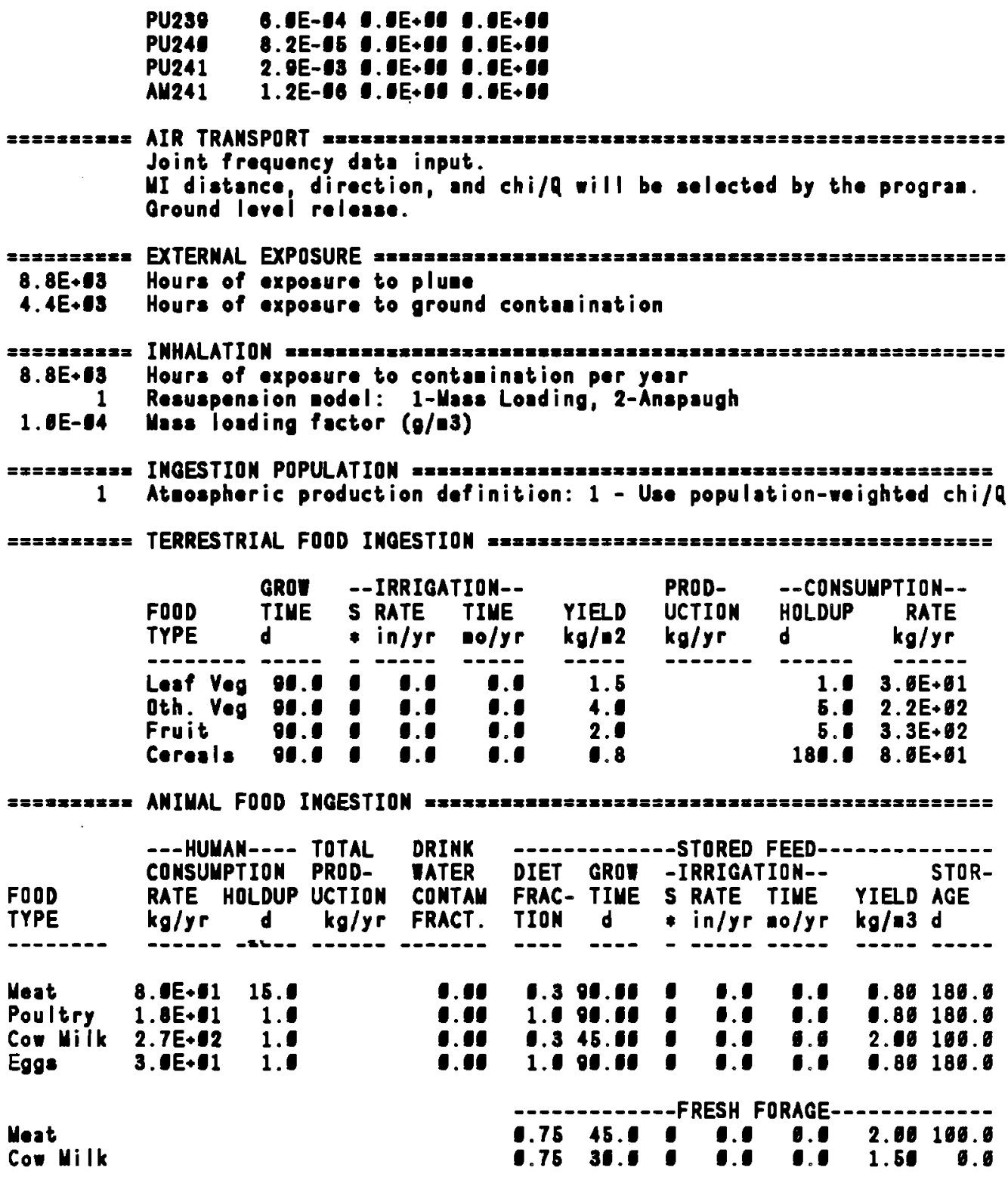

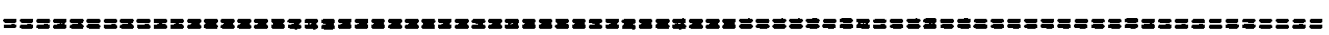

Input prepared by:

Date:

Input checked by:

Date: 


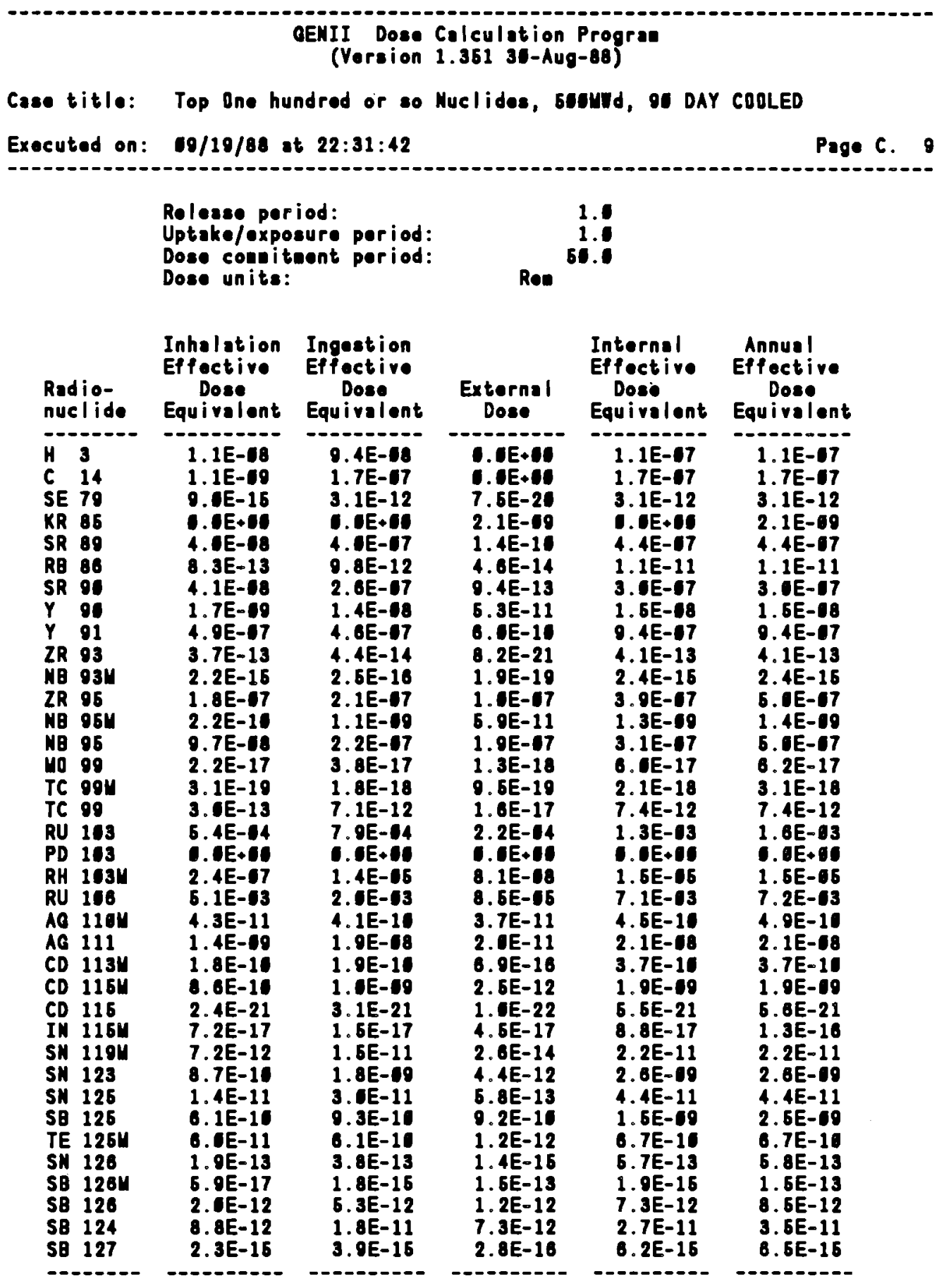




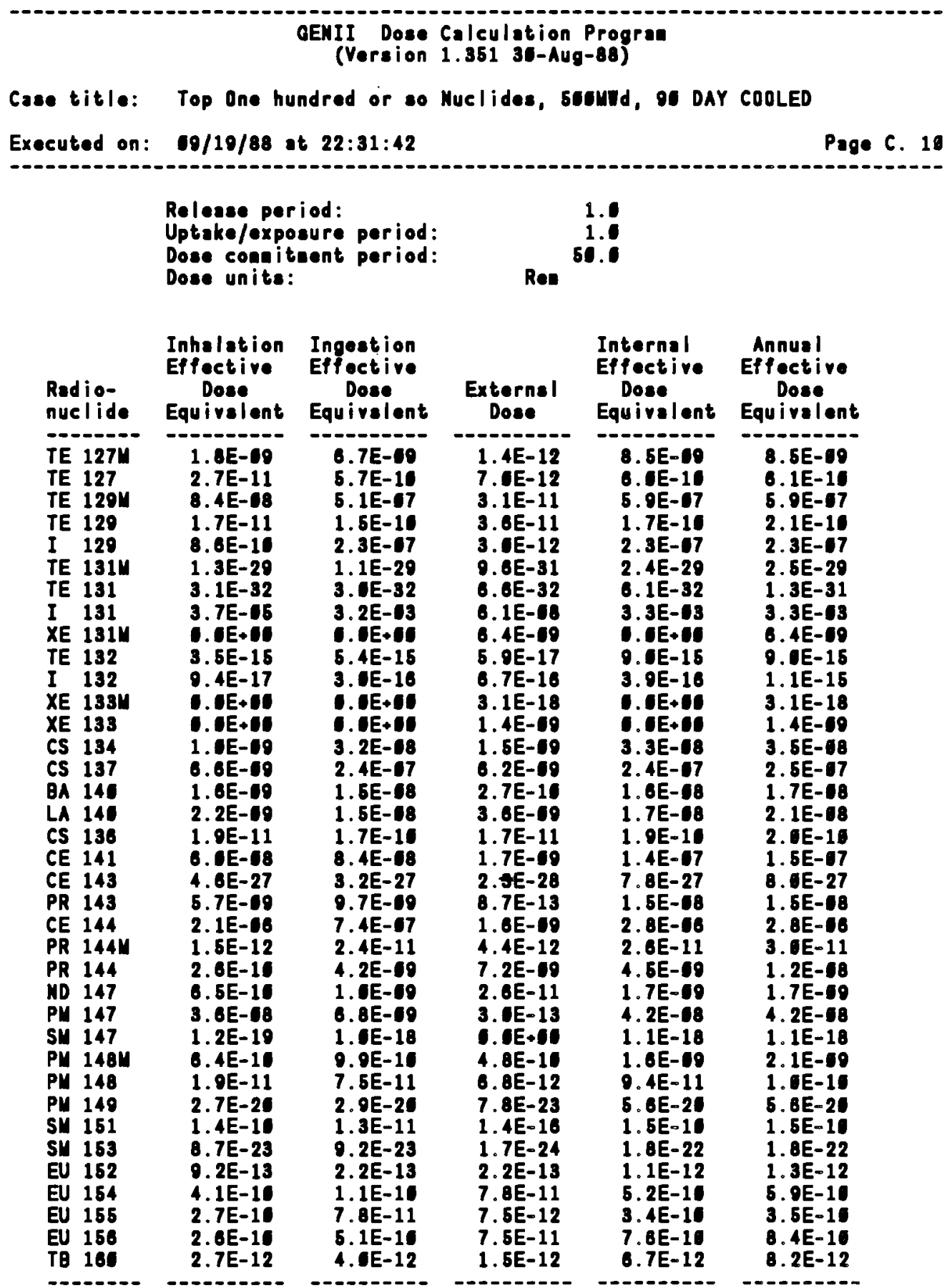




\begin{tabular}{|c|c|c|c|c|c|}
\hline \multirow{2}{*}{$\begin{array}{l}\text { Radio- } \\
\text { nuclide }\end{array}$} & \multicolumn{2}{|c|}{$\begin{array}{l}\text { Release period: } \\
\text { Uptake/exposure period: } \\
\text { Dose contituent period: } \\
\text { Dose units: }\end{array}$} & \multicolumn{2}{|c|}{$\begin{array}{r}1.0 \\
1.0 \\
50.0\end{array}$} & \multirow[b]{2}{*}{$\begin{array}{l}\text { Annus! } \\
\text { Effoct ive } \\
\text { Dose } \\
\text { Equivalent }\end{array}$} \\
\hline & $\begin{array}{l}\text { Inhslation } \\
\text { Effective } \\
\text { Dose }\end{array}$ & $\begin{array}{l}\text { Ingestion } \\
\text { Effective } \\
\text { Dose } \\
\text { Eauivelent }\end{array}$ & Externsl & $\begin{array}{l}\text { Internal } \\
\text { Effective } \\
\text { Dose }\end{array}$ & \\
\hline $\begin{array}{ll}O U & 236 \\
U & 235 \\
T H & 231 \\
P A & 231 \\
A C & 227 \\
T H & 227 \\
F R & 223 \\
R A & 223 \\
U & 237 \\
\text { NP } & 237 \\
P A & 233 \\
U & 233 \\
T H & 229 \\
R A & 225 \\
A C & 225 \\
U & 238 \\
T H & 234 \\
P A & 234 \\
\text { NP } & 238 \\
\text { PU } & 238 \\
\text { PU } & 245 \\
\text { PU } & 241 \\
\text { AU } & 241 \\
\text { NP } & 239 \\
\text { PU } & 239\end{array}$ & $\begin{array}{l}1.0 E-10 \\
2.5 E-16 \\
1.7 E-15 \\
1.6 E-14 \\
1.0 E-17 \\
2.4 E-19 \\
1.8 E-24 \\
1.2 E-19 \\
5.8 E-12 \\
4.3 E-11 \\
8.4 E-15 \\
1.9 E-16 \\
2.8 E-17 \\
1.1 E-19 \\
1.2 E-19 \\
5.3 E-19 \\
1.8 E-12 \\
5.2 E-17 \\
4.6 E-18 \\
7.4 E-18 \\
3.6 E-97 \\
2.2 E-97 \\
7.9 E-19 \\
3.9 E-17 \\
2.8 E-66\end{array}$ & $\begin{array}{l}1.3 E-13 \\
3.3 E-13 \\
1.8 E-14 \\
1.7 E-15 \\
5.2 E-18 \\
1.4 E-20 \\
3.6 E-23 \\
9.9 E-20 \\
1.1 E-11 \\
2.9 E-11 \\
1.7 E-14 \\
2.4 E-18 \\
4.0 E-19 \\
2.4 E-20 \\
1.1 E-29 \\
6.0 E-12 \\
4.3 E-12 \\
1.1 E-15 \\
3.4 E-19 \\
5.0 E-19 \\
2.7 E-69 \\
1.7 E-90 \\
4.6 E-10 \\
4.6 E-17 \\
1.9 E-98\end{array}$ & $\begin{array}{l}1.6 E-18 \\
6.5 E-15 \\
4.1 E-16 \\
4.6 E-20 \\
1.6 E-24 \\
1.5 E-21 \\
8.6 E-24 \\
4.0 E-21 \\
2.9 E-13 \\
2.9 E-16 \\
6.8 E-15 \\
1.1 E-22 \\
3.0 E-23 \\
1.7 E-24 \\
1.7 E-22 \\
7.9 E-17 \\
4.1 E-14 \\
6.1 E-15 \\
4.5 E-20 \\
4.1 E-16 \\
1.9 E-16 \\
6.7 E-20 \\
1.0 E-14 \\
2.1 E-18 \\
2.0 E-14\end{array}$ & $\begin{array}{l}1.6 E-19 \\
2.5 E-10 \\
1.8 E-14 \\
1.6 E-14 \\
2.6 E-17 \\
2.6 E-19 \\
3.6 E-23 \\
2.1 E-19 \\
1.6 E-11 \\
4.6 E-16 \\
2.4 E-14 \\
1.9 E-16 \\
2.6 E-17 \\
1.3 E-19 \\
1.3 E-19 \\
6.3 E-19 \\
6.8 E-12 \\
1.2 E-15 \\
4.8 E-18 \\
7.6 E-18 \\
3.7 E-97 \\
2.2 E-97 \\
8.4 E-09 \\
8.6 E-17 \\
2.8 E-06\end{array}$ & $\begin{array}{l}1.6 E-10 \\
2.6 E-10 \\
1.8 E-14 \\
1.6 E-14 \\
2.6 E-17 \\
2.6 E-19 \\
4.5 E-23 \\
2.2 E-19 \\
1.7 E-11 \\
4.6 E-19 \\
3.1 E-14 \\
1.9 E-15 \\
2.8 E-17 \\
1.3 E-19 \\
1.3 E-19 \\
5.3 E-99 \\
5.9 E-12 \\
7.3 E-15 \\
4.9 E-18 \\
7.6 E-98 \\
3.7 E-97 \\
2.2 E-97 \\
8.4 E-99 \\
8.7 E-17 \\
2.8 E-06\end{array}$ \\
\hline & & & & 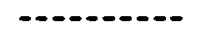 & \\
\hline
\end{tabular}


GENII Dose Calculation Progran

(Vorsion 1.351 30-Aug-8B)

Case title: Top One hundred or so Muclides 0 1el uld, 30 DAYS Coolimg Air 1945

Executed on: 19/20/88 at 18:11:46 Page A. 1

This is a far-field (wide-secole release, aultiple site) scenario.

Release is chronic

Individual dose

THE FOLLOUIMO TRAMSPORT MODES ARE CONSIDERED

Air

THE FOLLOWINO EXPOSURE PATHS ARE CONSIDERED:

Finite pluee, external

Ground, external

Inhalation uptake

Terrestrial foods ingestion

Aniasl product ingestion

THE FOLLOUIMG TIMES ARE USED:

Intake ends after $(y r)$ :

Dose calculations ends after $(y r)$ : $\quad 50.0$

Release ends after $(y r)$ :

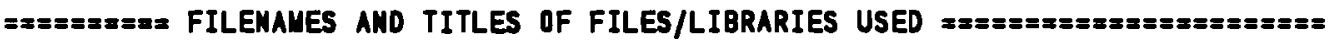

GENII Default Paraneter Values (3-Aug-88 RAP)

IGENII BANDIR Doverfill in $\quad 9-20-88$

RUDLIB - Radionuclide Master Library (29-Aug-88 RAP)

$8-12-88$

Food Transfor Factor Library - (RAP 29-Aug-8B) (UPDATE

$8-29-88$

External Dose Factors for GENII in person Sv/yr per Bg/n (28-Aug-88 8-29-88

Internal Yearly Dose Increnents (SV/Bq) 29-Aug-88 RAP

$8-29-88$

260 Area Population (Sonner, Rau, and Robinson, PNL-4610)

2100 AREA - 10 M - Pasquill A - F (1987)

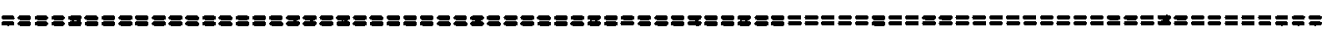

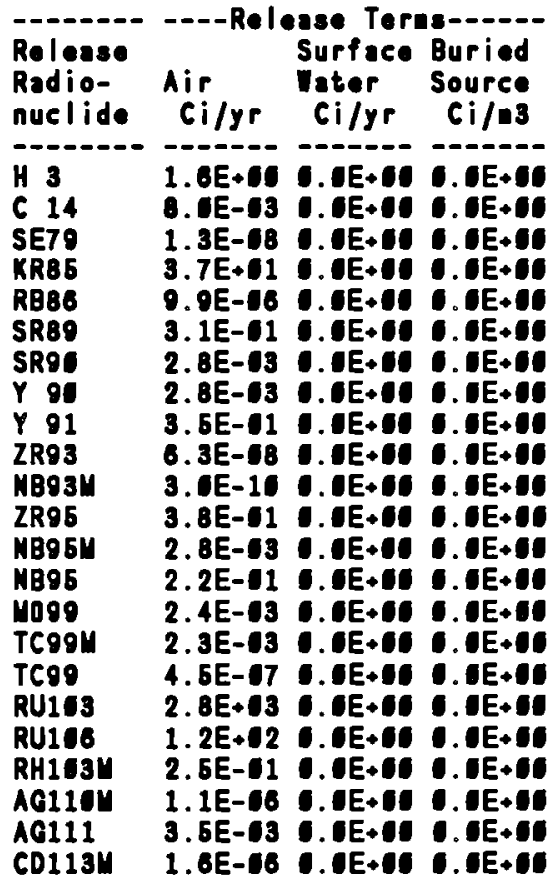

B. 19 
CD115U 5.6E-64 0.6E+CO @.6E+CS

CD115

IN115U

SN119U

SN123

SN125

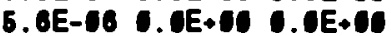

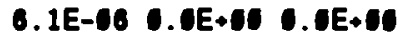

2.2E-65 -. NE+60 C.0E+06

5.TE-64 C.6E+CO O.UE+CS

2.2E-13 0.0E+05 0.0E+01

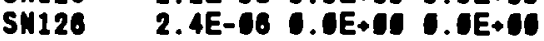

SB125 0.4E-C4 $0.0 E+90$ O.GE+CS

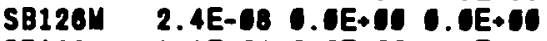

SB126 1.1E-04 $0.0 E+00$ 0.0E+C0

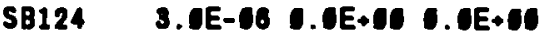

SB127 6.6E-G4 G.UE+06 G.OE+G

TE125U 4.6E-05 $\quad .0 E+00$ C.OE+CS

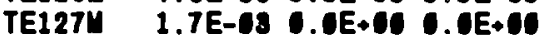

TE127 2.6E-03 $0.0 E+00$ C.UE+CS

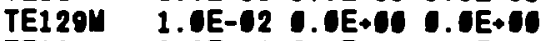

TE129 6.6E-69 ๑.UE+C) ๑. EE+CQ

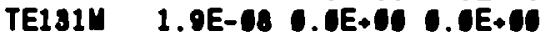

TE131 4.8E-09 ๑.DE+CO ๑.DE+CO

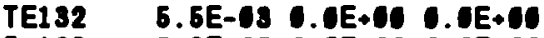

I $120 \quad 5.6 E-65$ G.UE+CO S.OE+CO

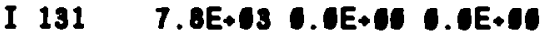

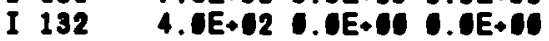

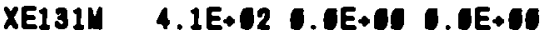

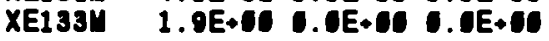

XE133 9.1E+C3 $0.0 E+05 \bullet .0 E+00$

CS134 2.9E-65 O.UE+09 ऽ. UE+00

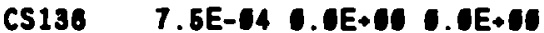

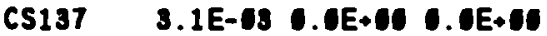

BA146 4.1E-01 G.UE+6) $. .0 E+60$

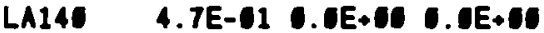

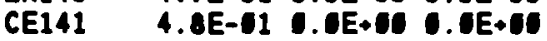

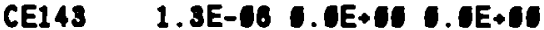

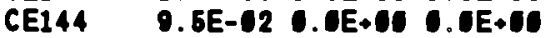

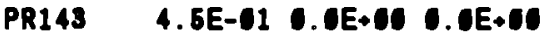

PR144M 1.1E-G3 O.OE+OO O.OE+00

PR144 9.6E-02 O.OE+Q1 O.OE+00

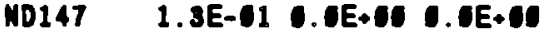

PM147 1.2E-62 J.JE+01 J.0E+00

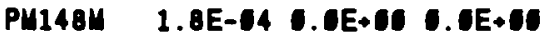

PM148 9.8E-05 G.0E+0S O.OE.AS

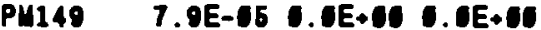

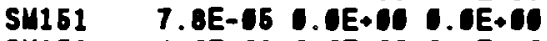

SM153 4.0E-08 $0.0 E+040.0 E+00$

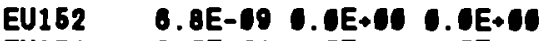

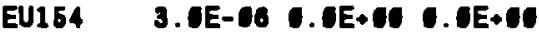

EU155 1.3E- $54.0 E+10$ ๑. $0 E+00$

EU156 3.2E-63 $5.0 E+06 \mathrm{~J} .0 \mathrm{U}+60$

TB185 5.2E-67 $0.0 \mathrm{~J}+01 \mathrm{0.0E+00}$

TH231 1.4E-07 $0.0 E+01 \div .0 E+00$

PA231 3.2E-13 $0.0 E+01$ 0.0E+CO

AC227 1.0E-15 $\quad .0 E+05 \mathrm{O.JE}+00$

TH227 1.UE-15 G.JE+CS G.OE+GS

FR223 1.0E-15 $\quad .0 E+06 \quad 0.0 E+00$

RA223 1.UE-15 $0.0 \mathrm{E}+00 \mathrm{U} .0 \mathrm{E}+0 \mathrm{O}$

TH234 2.1E-18 $1.0 E+08 \cup .0 E+04$

PA234 2.7E-69 $0.0 E+00$ G. $0 E+00$

U $235 \quad 1.4 E-67 \quad 0.0 E+06$ O.0E+40

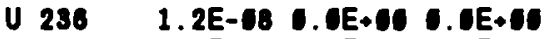

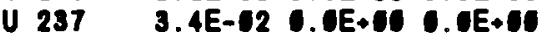

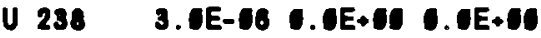

MP237 9.9E-69 G.UE+CS G.UE+60

PA233 4.7E-69 $0.0 E+01$ G.JE+09

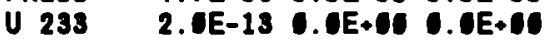

TH229 1.0E-15 $0.0 \mathrm{U}+01 \mathrm{O} .0 \mathrm{E}+00$

RA225 1.UE-15 $0.0 E+C S$ J.UE+US

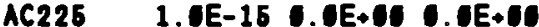

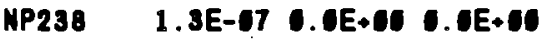

MP239 2.6E-C2 $0.0 \mathrm{E}+61 \mathrm{C.0E+60}$

PU238 3.1E-67 $\triangle .0 E+60 \cup .0 E+60$ 


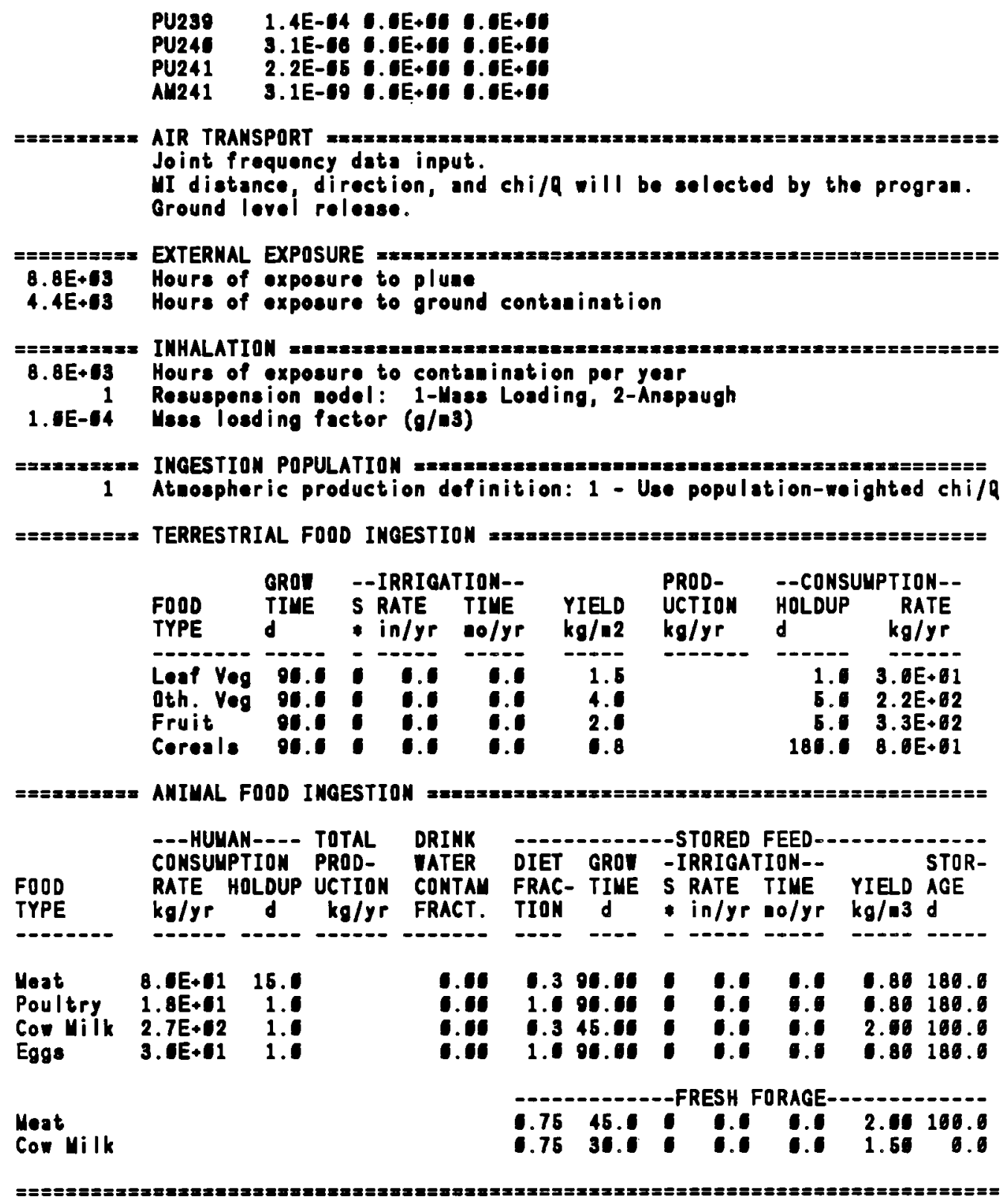

Input prepared by:

Date:

Input checked by :

Date: 


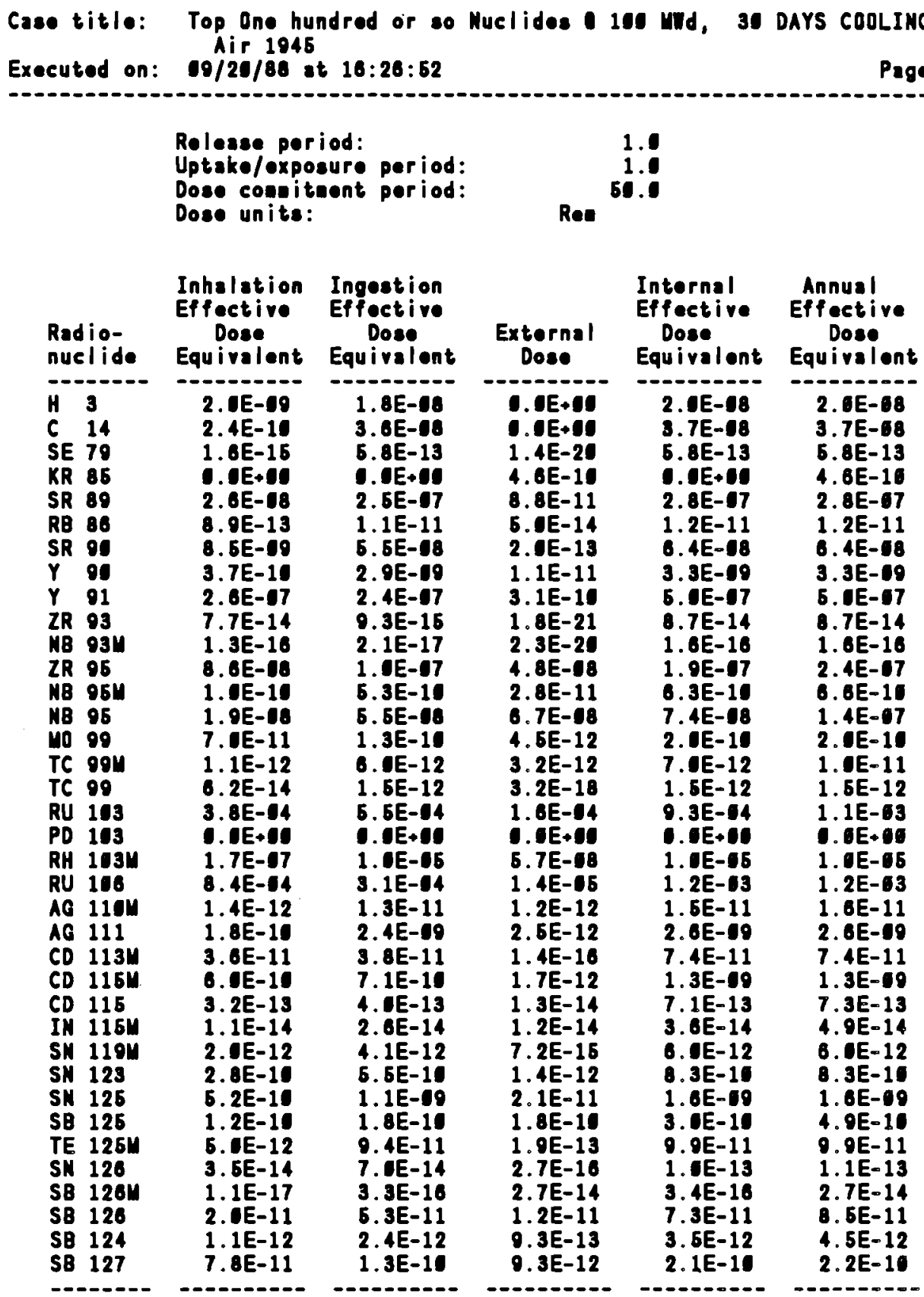


OENII Dose Calculation Progran

(Version 1.351 30-Aug-88)

Case title: Top One hundred or so Nuclides 100 MId, 30 DAYS COOLING -
Executed on: 19/20/88 at 18:28:52

Releses period:

Uptake/exposure period:

Dose conaituent period:

Dose units:

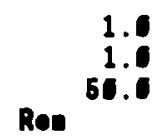

Inhalation Ingestion

Effective Effective

Dose

Radio-

nuclide Equivalent

$$
\text { Dose }
$$

TE 127u

- Equiralont

5. 5E-10

1. $2 E-11$

7. 4 E- 08

1.5E-11

1.3E-10

3. $2 E-15$

7.8E-18

3.7E-13

G. 6 + 01

1.

1.2E-68

U. GE+CE

0.6E+CI

1.8E-11

$1.3 E-69$

1.9E- 68

2. $5 \mathrm{E}-68$

7. 2E-11

6. 2E- 68

$6.7 \mathrm{E}-14$

5. $5 \mathrm{E}-68$

5. $2 \mathrm{E}-97$

3.9E-13

-.3E-11

1. 3E- 68

7.0E-69

2. $4 E-20$

0. EE-11

1.7E-11

3. $6 E-12$

3. 6E-11

1. $1 E-13$

2.1E-14

1. 2E-11

8.1E-11

7. E-10

1. $9 \mathrm{E}-13$
Equiralent

2.1E-09

1. $9 E-10$

4.8E-07

1. 4 E-10

3. 4 E- 68

2. 6 E-15

7. 2E-18

3. 3E- 1

C. EE+C0

1. $6 \mathrm{E}-99$

8. 4E- 07

C. EE+OS

$0.0 \mathrm{E}+00$

5. $9 E-10$

4. $5 \mathrm{E}-\mathrm{68}$

1.7E-07

1.7E- 07

6. 6E-16

8.7E-18

4. 6E-14

๑. 3E- 08

1. 9E-ण7

6. 1E-12

1.0E-60

2.1E- 18

1. $5 \mathrm{E}-69$

2. $2 E-19$

9. $4 E-11$

3.1E-11

3. $8 E-12$

3. $4 E-12$

1. $2 E-13$

5.1E-15

3. 2E-12

2. 3E-11

1. 3E- 60

2. $8 \mathrm{E}-13$
6. $4 E-10$
Internal Annual Effective Effective

External Dose

Dose Equivalent

Dose

$4.4 E-18$

2. 3E-12

2. $8 \mathrm{E}-11$

3. 2E-11

4. . E-13

2.4E-16

1. $6 \mathrm{E}-17$

8. 2E-64

1. JE- 07

1.7E-11

3. 8E-68

2.9E-68

2. $8 E-11$

1.2E-69

3. $1 E-69$

4.2E-18

6. 5E-11

1. $9 \mathrm{E}-69$

3. 2 E-15

B. 3E-12

4. 2 E-10

1. 1E-12

1.9E-69

5. 4E-16

B. 8E-14

C. EE+CO

4. $5 E-11$

1.7E-12

1. EE-14

3.7E-17

2. 3E-15

5. 3E-15

2. 3E-12

2. 3E-12

2. E-10

1. E-13
2.8E- 69

2. EE-10

5. 4E- 67

1. $8 \mathrm{E}-10$

3. 4E-68

5. 8 E- 15

1. $5 E-17$

3. 4E-61

6.6E+00

2. $6 \mathrm{E}-10$

2. $0 E-16$

C. $0 E+08$

0.0E+60

6.1E-10

4.6E-68

1. $9 E-07$

1.9E- 07

7. 3E-10

1. 5E- 67

1.1E-13

1. $6 \mathrm{E}-67$

7. DE-G7

6. 5E-12

1.1E- 09

3. $5 E-08$

8. 5E-19

2. 4E-19

1. 6E-10

4.7E-11

7. 6E-12

3. $9 \mathrm{E}-11$

2. 4E-13

2. BE-14

1. 5E-11

1. DE-10

2. $1 E-69$

4.7E-13
Equivalent

\section{2. $8 E-09$}

2. EE-10

5. $4 E-67$

1. 9E-10

3. 4E- 68

6. E-15

3. 1E-17

3. 4E-61

1. $0 E-67$

2. $8 E-69$

5. 8E- 68

5. $4 E-10$

2. $9 E-10$

6. 4E-10

4.7E-68

1.9E- 07

2. 3E- 67

8. SE-10

1. $5 \mathrm{E}-67$

1. $2 \mathrm{E}-13$

1. $5 \mathrm{E}-67$

7. EE-67

7. 6E-12

3. 0 E-69

3. 5E-68

8. 5E-69

2. $4 E-19$

2. $0 \mathrm{E}-10$

4. $9 \mathrm{E}-11$

7. 5E-12

3. $9 E-11$

2. 4E-13

3.1E-14

1. 8E-11

1. $1 \mathrm{E}-10$

2. 2E-08

5.7E-13 


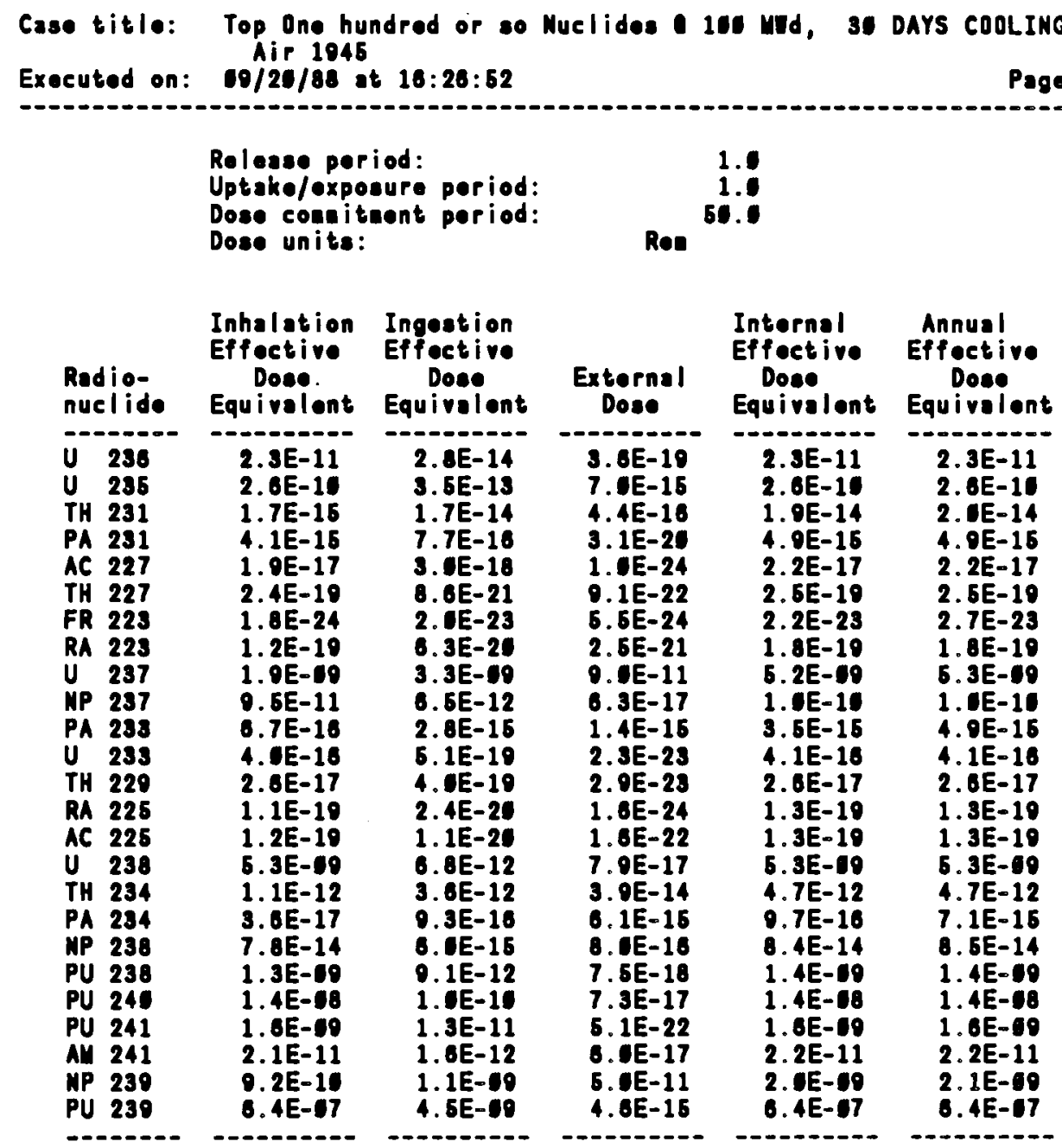




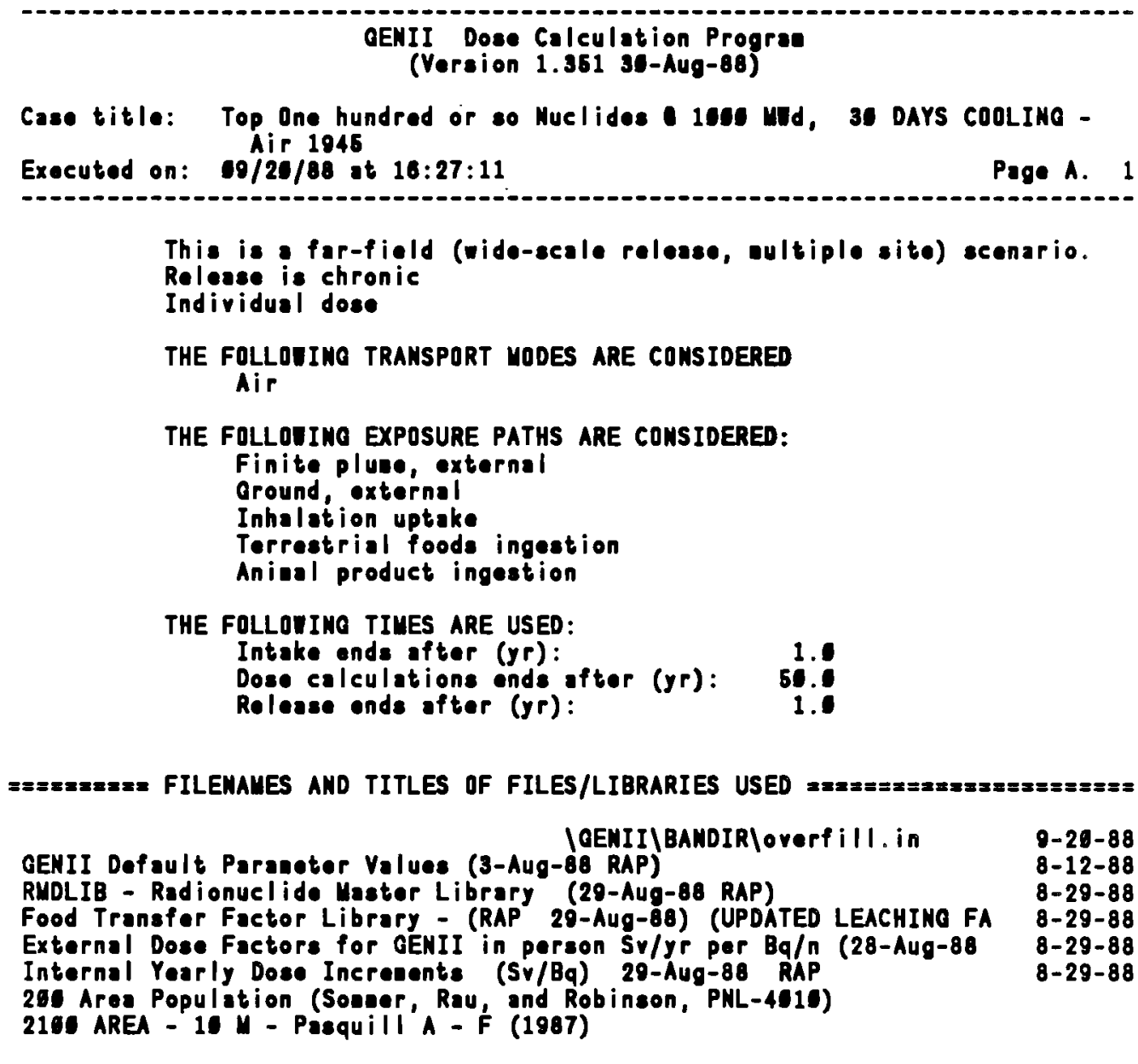

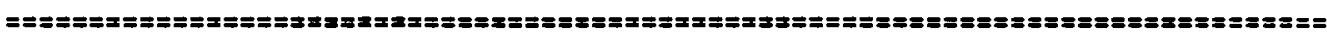

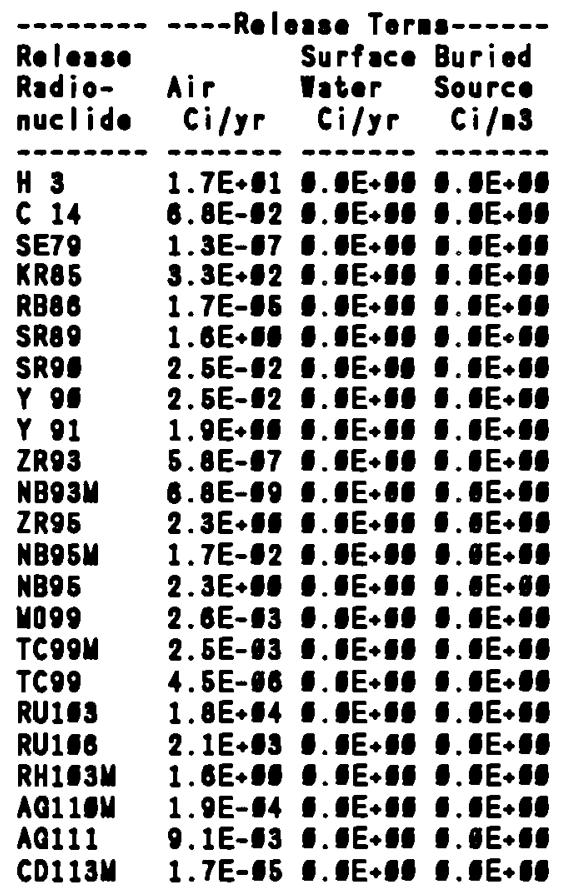


CD115U

CD115

IN1154

SN119U

SN123

SN125

SN128

58125

5B126U

SB126

SB124

5B127

TE125U

TE127U

TE127

TE129U

TE1 29

TE131U

TE131

TE132

I 129

I 131

I 132

XE131V

XE133U

XE133

CS134

CS136

CS137

BA140

LA140

CE141

CE143

CE144

PR143

PR144L

PR144

ND147

PU147

PU148U

PN148

PU149

54151

SN153

EU152

EU154

EU155

EU150

TB168

TH231

PA231

AC227

TH227

FR223

RA223

TH234

PA234

U 235

U 236

U 237

U 238

NP237

PA233

U 233

TH229

RA225

AC226

NP238

NP239

PU238
2.9E-63 $.0 E+90$ 0.0E+ 90

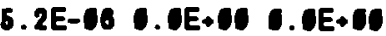
$5.8 \mathrm{E}-66$ ○. $0 \mathrm{E}+10 \mathrm{C.0 \textrm {E } + 0 0}$ $1.8 E-04$ C.OE+00 $.0 E+00$

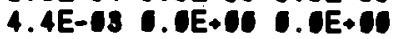
4.8E-63 C.UE+00 $0.0 E+00$ 2.7E-OT S.UE+QS D.UE+OS 6.7E-68 C.UE+CS ..UE+CO

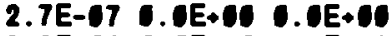
3.9E-64 0.0E+01 O.OE+CO 1.3E-04 -.0E+01 -.0E+90 1.4E-68 6.0E+60 6.0E+60 8.8E-64 0.0E+C6 $0.0 E+00$ 1.5E-62 0.0E+C0 1.UE+C0 1.6E-12 C.OE+65 $.0 \mathrm{OE}+\mathrm{CS}$ 5.8E-02 O. EE+60 G.0E+10 3.8E-62 G. EE+CS G.UE+CS 2. $4 E-18 \bullet .0 E+00 \cup .0 E+08$ 5.8E-69 0. EE+60 O.JE+CO $6.6 E-03$ 0. EE+C0 $0.0 E+10$ 6.7E-64 0.0E+60 C.UE+C6 1.5E+04 0.0E+60 O.JE+06 $4.8 E+62$ O. EE+60 O.UE+60 $1.2 E+03$ O. $\mathrm{EE}+00$ 0.0E+CO

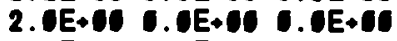
$1.3 E+04$ O. EE+10 O.OE+OO 7. OE-63 O. EE+60 0.0E+60 8.0E-C3 C. EE+CS G.JE+G0

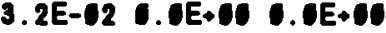
9.8E-61 O. IE+60 G.0E+96 $1.1 E+00$ O.JE+C0 O.UE+60

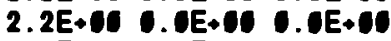
1.2E-66 O. DE+C6 O.JE+CS 8.1E-01 C.0E+01 0.0E+CS 1.1E+19 C.TE+10 O.TE+CO

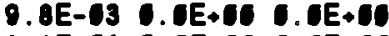
8.1E-61 0.6E+60 0.0E+60 2.8E-61 C. DE+60 6.0E+61 1.1E-61 O.0E+46 C.OE+60 1.3E-62 $0.0 E+00$ O. $0 E+60$

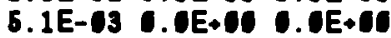
9.DE-65 ๑.DE+C1 $0.0 E+01$ 4.9E-64 0.0E+60 0.0E+60

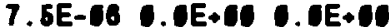
1.3E-68 0.0E+60 0.0E+10

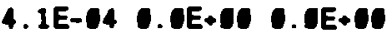

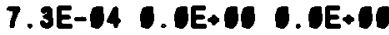

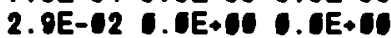

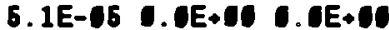
1.2E-02 0.0E+00 0.0E+00 9.0E-13 0.0E+CO 0.UE+CO

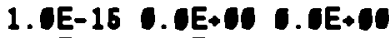
1. EE-15 O. EE+CS 0.OE+CO 1. UE-15 ๑.0E+10 ๑.JE+C0 1.UE-15 O.OE+00 O.OE+00 3. OE-C4 O. EE+DO O.UE+CO 3.8E-09 D. UE+00 0.0E+10 1.2E-67 ๑.DE+01 0.0E+C6

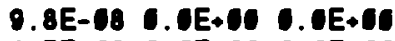
4.7E-12 O. EE+CO O.OE+CO

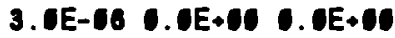
9.2E-08 0.DE+C1 D.DE+CO 7.2E-08 O. EE+C0 C. DE+10 1. OE-15 O. OE+CO O.UE+CO 1. DE-15 O.DE+DO D.DE+DE 1.JE-15 O. DE+00 O.OE+CO

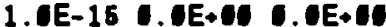

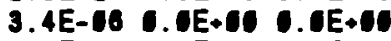
2.0E-12 O. UE+00 O. OE+CO 7.OE-O5 O.OE+DS O.OE+10 


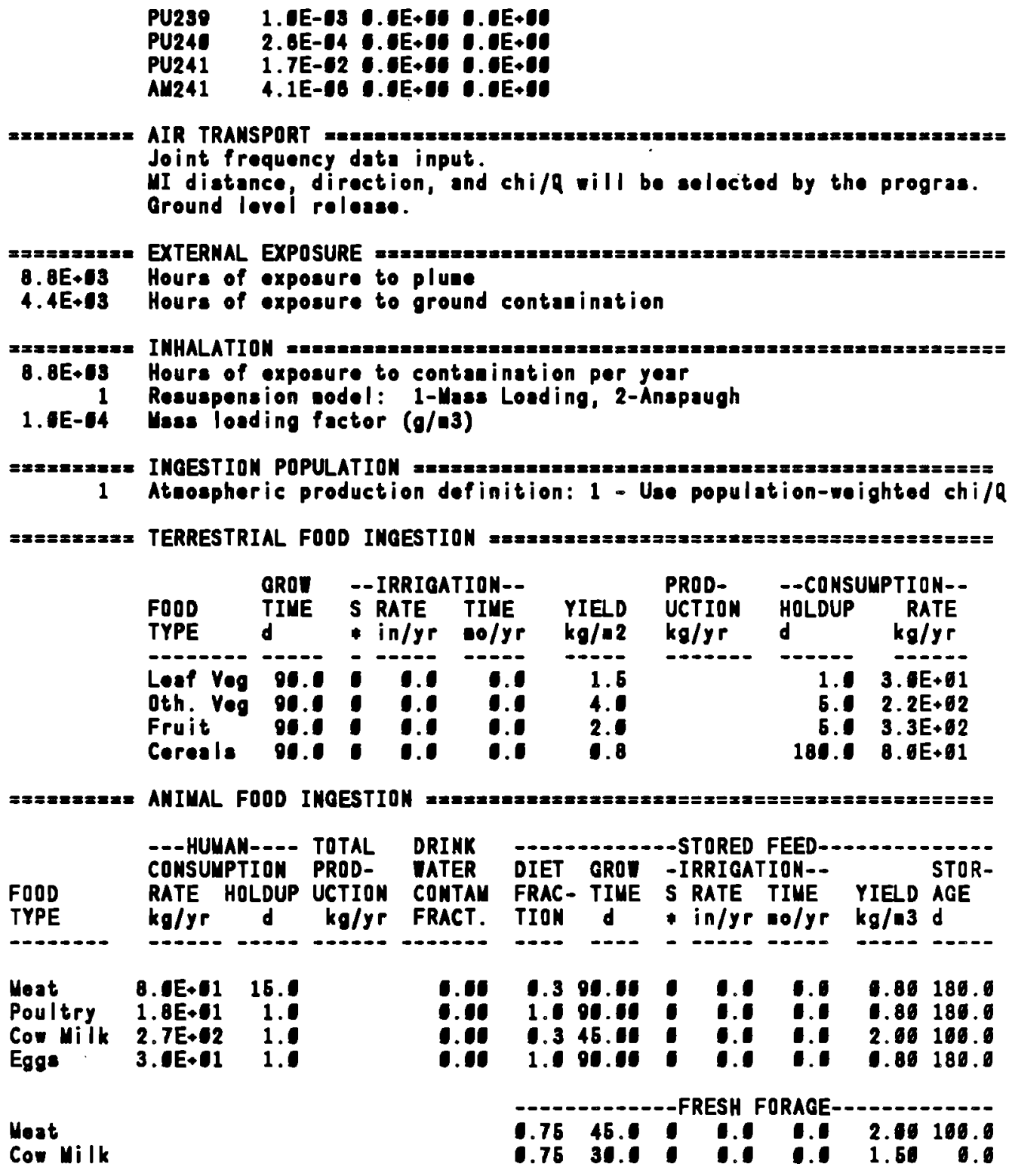

: 


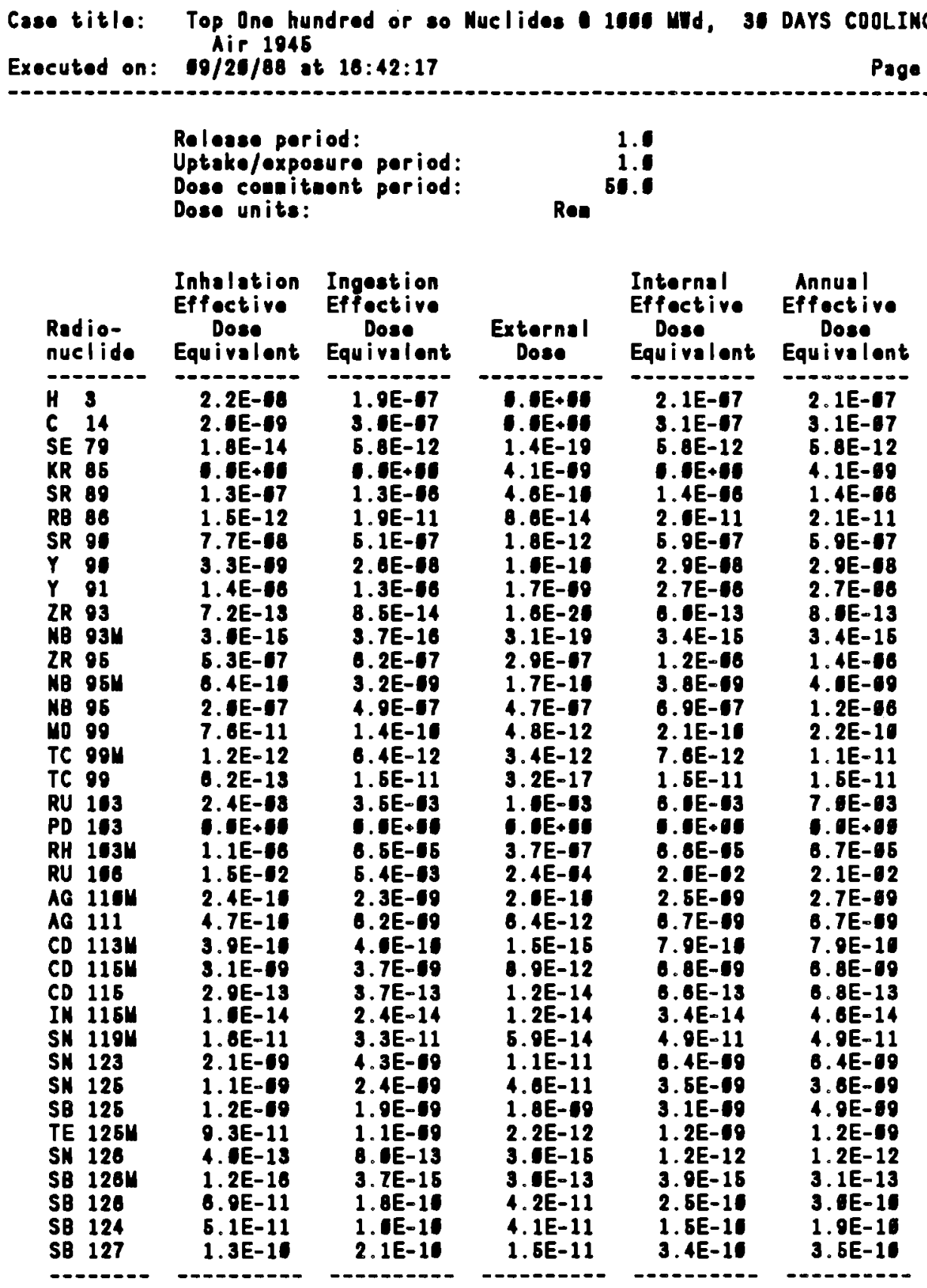




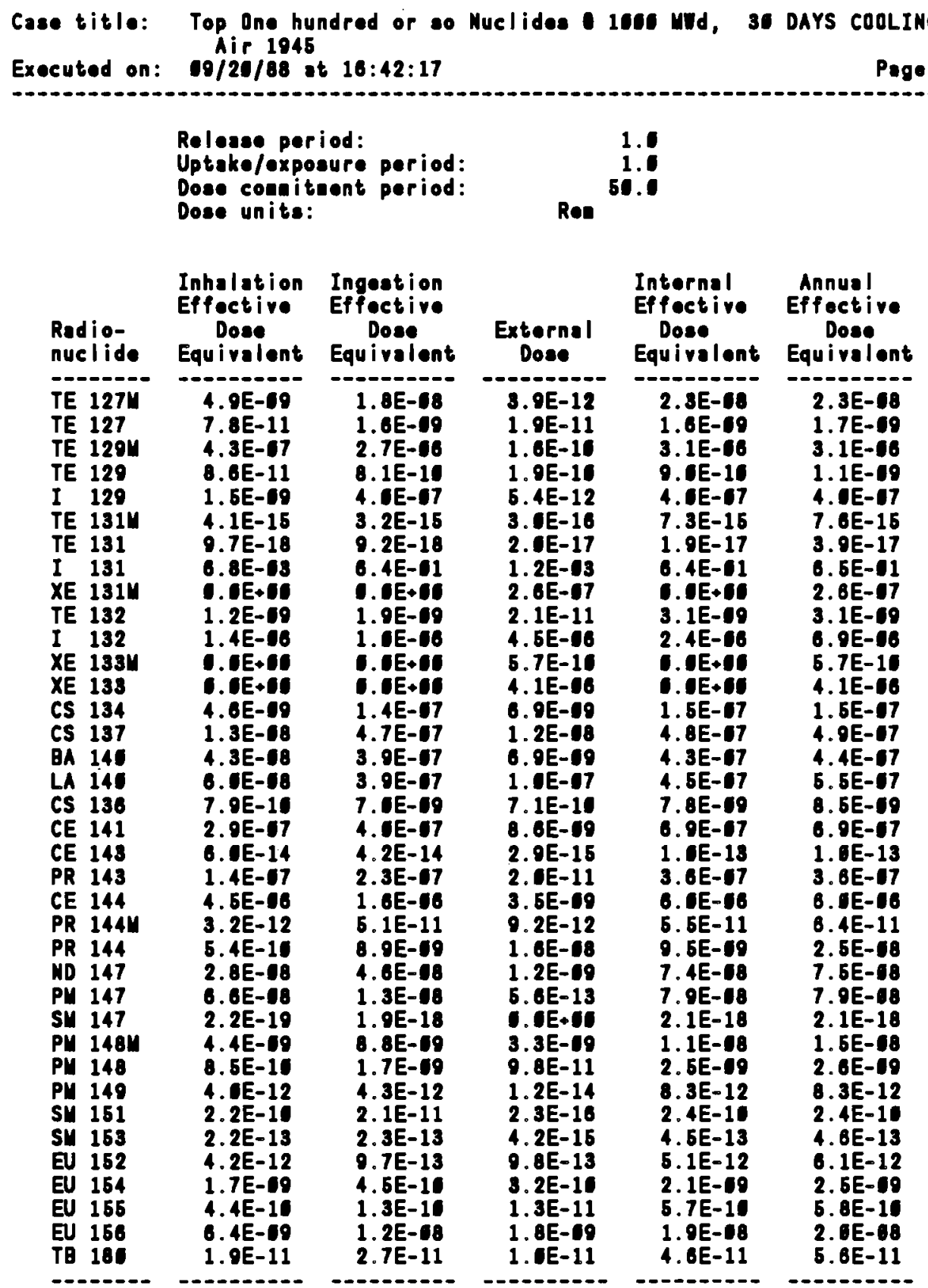




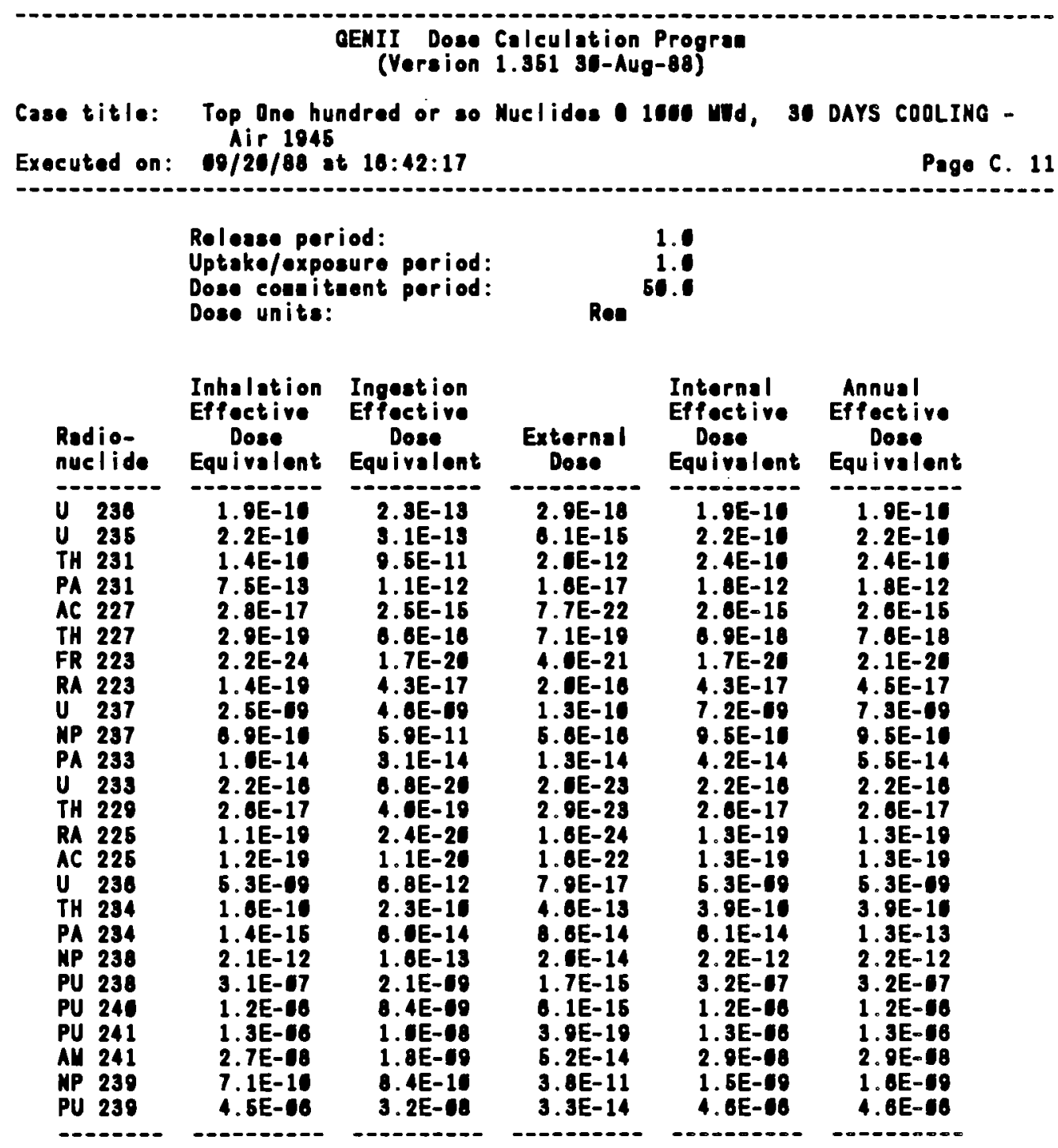


OENII Dose Calculation Progran

(Version 1.395 23-Jan-89)

Case title: Top One hundred or so Nuclides to HEDR Selection - Air 1945
Executed on: $19 / 18 / 89$ at $21: 51: 35$
Page A. 1

This is a far-field (wide-scale relesse, oultiple site) sconario.

Release is chronic

Individual dose

THE FOLLONINO TRANSPORT MODES ARE CONSIDEREO

Air

THE FOLLONINO EXPOSURE PATHS ARE CONSIDERED:

Infinite plune, external

Oround, external

Inhalation uptake

Terrestrial foods ingestion

Aniaal product ingestion

THE FOLLOUINO TIMES ARE USED:

Intake ends after $(y r)$ :

Dose calculations enda after $(y r)$ : 50.0

Release ends ofter ( $y r)$ :

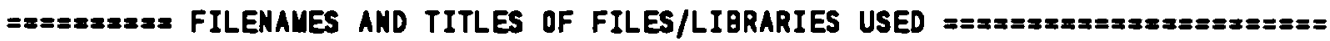

Input fi lo nane: lOENII/GANDIRINOMILK. in

METABOLIC PARAMETERS

RUDLIB - Radionuclide Master Library (29-Aug-88 RAP)

Food Transfor Factor Library - (RAP 29-Aug-88) (UPDATED LEACHING FA

External Dose Factors for GENII in person Sy/yr per Bq/n (28-Aug-8B

Internal Yearly Dose Increments (Sy/Bq) 29-Aug-88 RAP

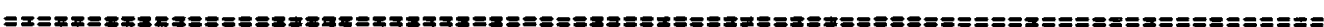

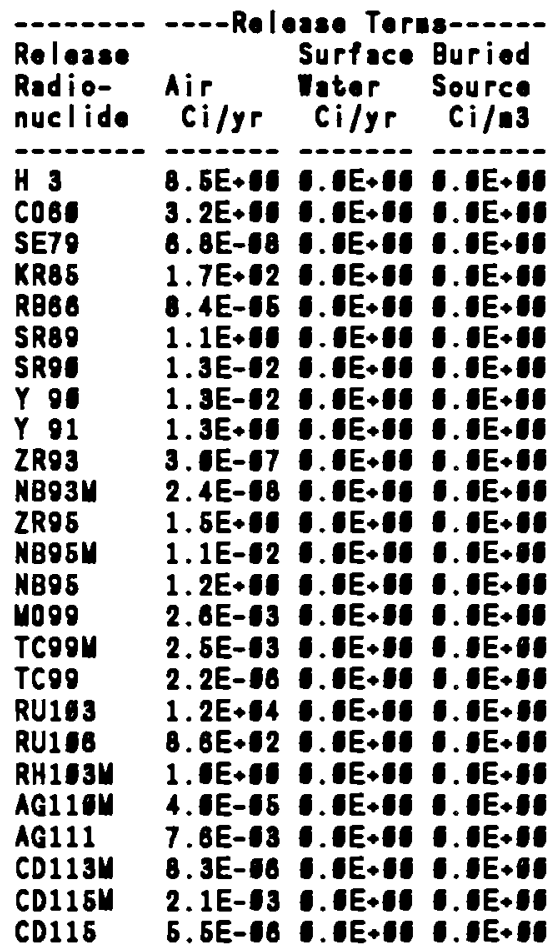




\begin{tabular}{|c|c|c|c|}
\hline 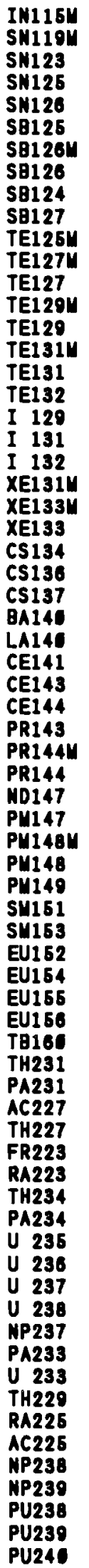 & 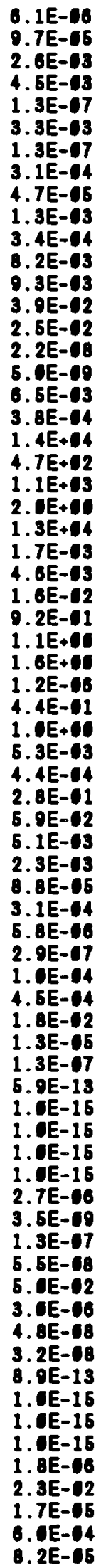 & 6 & 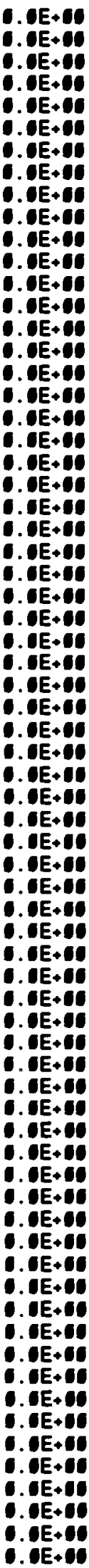 \\
\hline
\end{tabular}


PU241 2.9E-18 0.0E+10 0.0E+10

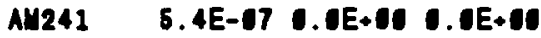

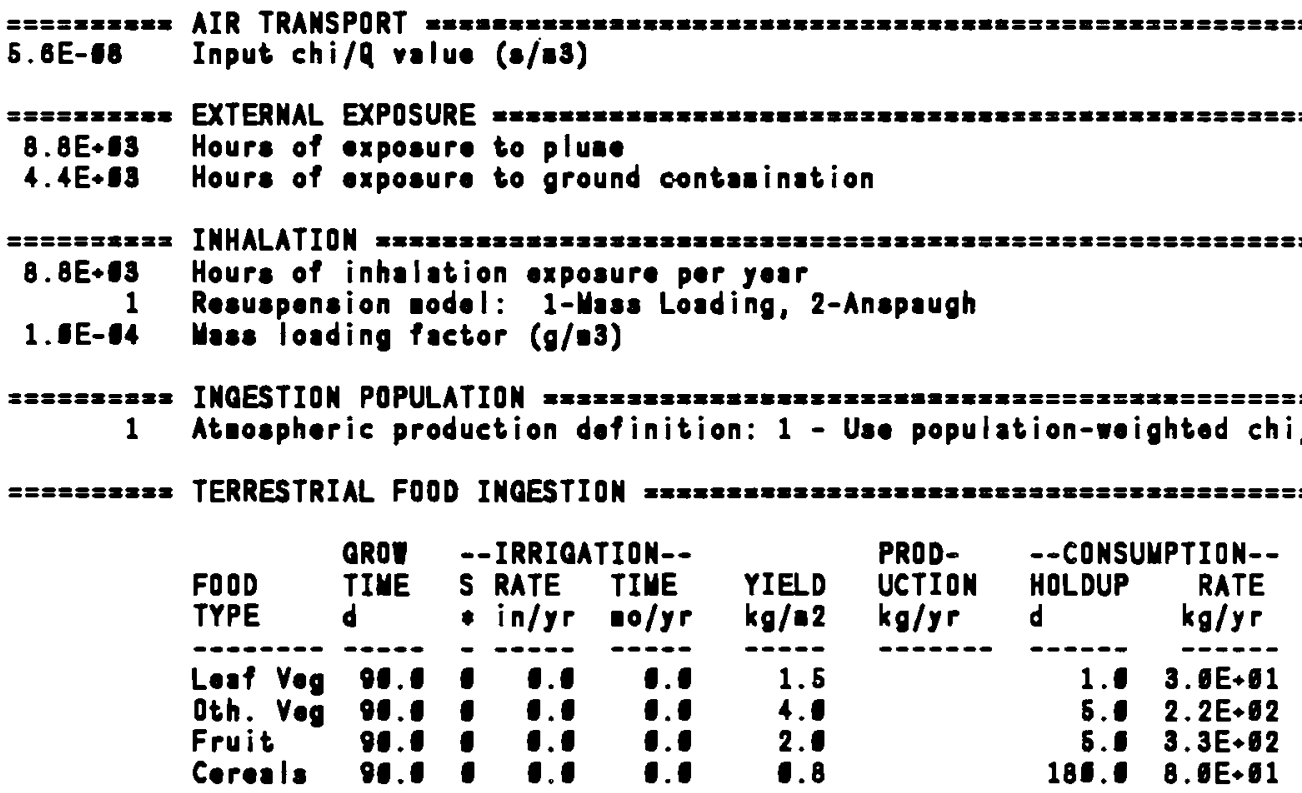

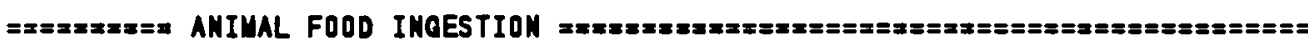

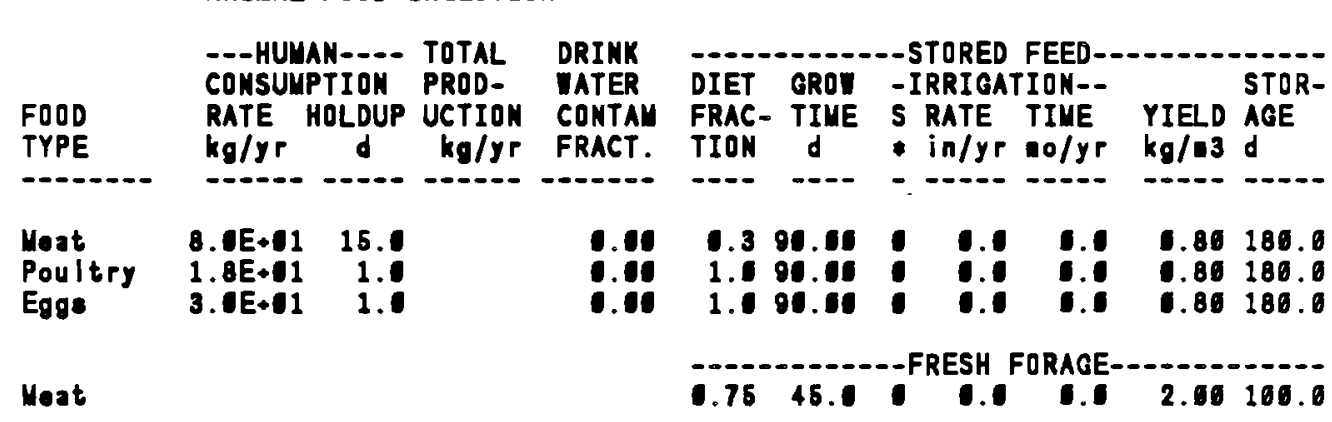

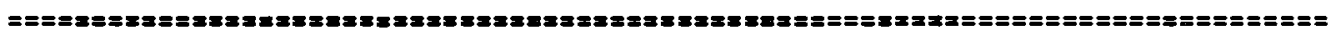

Input prepared by:

Date:

Input checked by:

Date: 


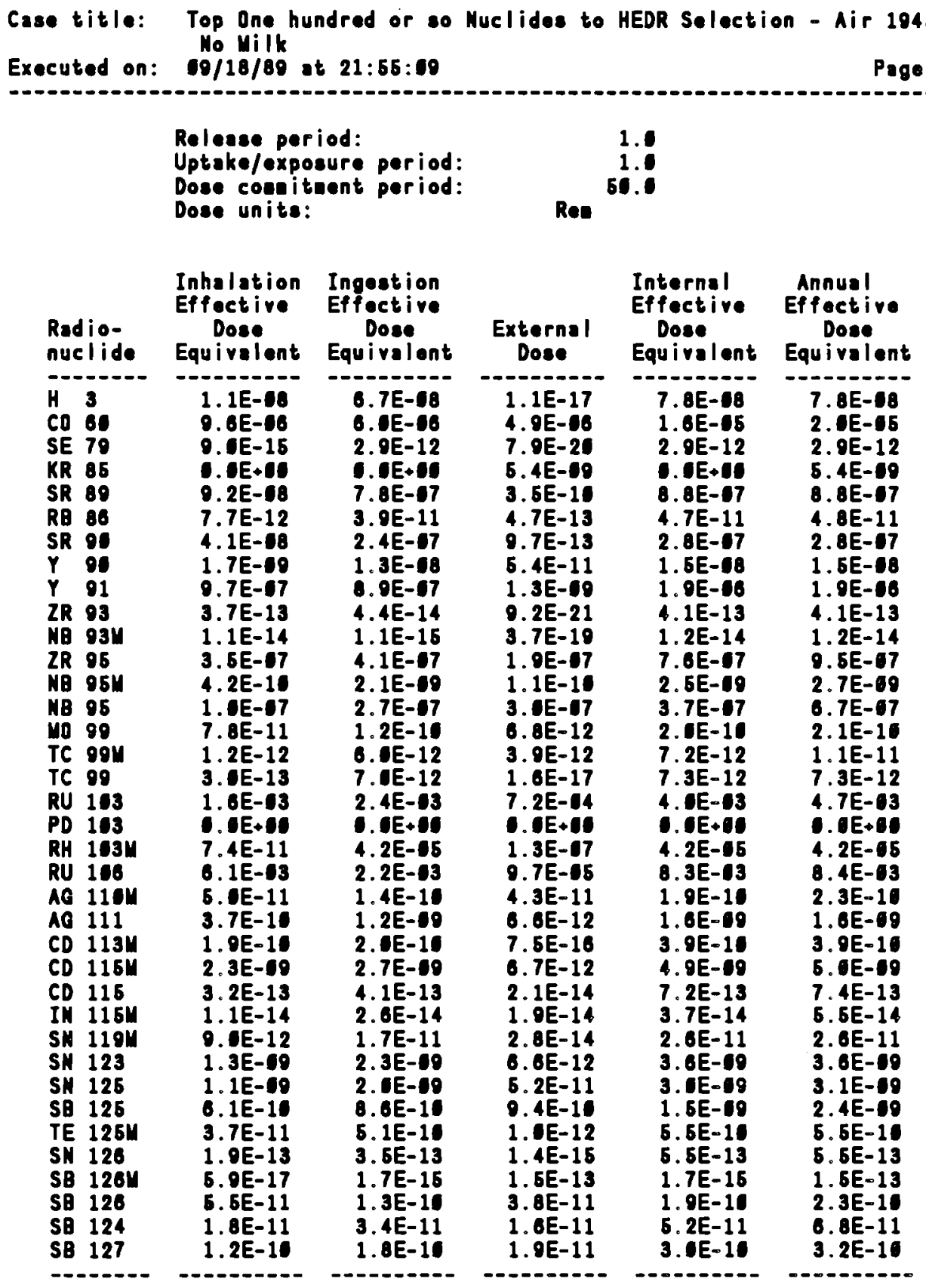


CENII Dose Calculation Progra

(Version 1.395 23-Jan-89)

Case title: Top One hundred or so Nuclides to HEDR Selection - Air 1945
Ex Milk
Executed on: $9 / 18 / 89$ at $21: 65: 09$

Relesee period:

Uptake/exposure period:

Dose counitment period:

Dose units:

Inhalation Ingestion Effective Effective

Radionuc l i de

TE 1274.

Dose

Dose

Equivalent Equivalent

2. 6E-69

4. $6 E-11$

-

2. $9 E-67$

5. $9 E-11$

TE $129 \mathrm{U}$

TE 129

I 120

TE 131U

TE 131

I 131

XE 131M

TE 132

I 132

XE 1334

XE 133

CS 134

C5 137

BA 145

LA 140

CS 138

CE 141

CE 143

PR 143

CE 144

PR 144M

PR 144

ND 147

PU 147

SU 147

PU 148U

PN 148

PN 140

S4 151

SU 153

EU 152

EU 154

EU 155

EU 156

TB 168
8. 6E-10

4. GE-15

9. 4E-18

6. $5 \mathrm{E}-63$

C. $6 E+60$

1. 2E-59

2. 8E-68

6. $0 E+96$

C. $0 \mathrm{E}+10$

1. 1E-10

6. 8E- 69

4. 3E-18

6. GE- 68

4. 5E-10

2.1E-67

6. 3E-14

1. 2E-67

2. 4E- 66

1. $6 \mathrm{E}-12$

3. DE-13

2. BE- 18

3. $6 \mathrm{E}-68$

3. GE-21

1. 7 E-69

3. $9 \mathrm{E}-10$

4. E-12

1. 4E-10

1. $7 \mathrm{E}-13$

0.2E-13

4.1E-10

2. 7 E-10

3. OE-69

5. EE-12
8. 2E-10

1.8E-66

5. 3E-19

1. $6 \mathrm{E}-67$

3. EE-15

8. 6E-18

2. 3E-61

6.6E+65

1. $8 \mathrm{E}-69$

2. JE-66

๑.0E-65

6. $6 E+10$

2. BE-58

1. 8E- 67

3. $6 E-67$

3. 8E-67

2. 1E-09

2.9E- 67

4. 4E-14

2.1E-67

8. 5E- 7

2.8E-11

4. 8E-69

4. 6E- 18

7. UE-69

1. EE-18

2. $7 \mathrm{E}-99$

7. 4E-19

4. 4E-12

1. 3E-11

1. $9 \mathrm{E}-13$

2. 2E-13

1.1E-10

8. EE-11

7. 5E-69

$6.9 E-12$
Externel

$1.9 E-12$

1. 1E-11

1. 1E-10

1. 4E-10

3. $1 E-12$

4.8E-16

3. $1 \mathrm{E}-17$

1.1E-13

1.8E-67

2.7E-11

1. $6 \mathrm{E}-\mathrm{65}$

6. 7E-11

3. 6E-66

1. 6E-69

6. 2E-19

7. 9E- 69

1. 1E-67

4. $5 E-10$

6.3E-69

4. 1E-15

2. $7 E-11$

1. $9 E-09$

5. 1E-12

8. 3E-69

1. 3E-69

3. 2E-13

๑. $6 \mathrm{E}+\mathrm{CO}$

1.3E-69

5. 1E-11

1. TE-14

1. 4E-16

4. EE-15

2. 2E-13

7. OE-11

8. 1E-12

1. 3E- 99

2.6E-12
1.5

1.0

50.5
Internal Annual

Effective Effective

Dose

Equivalent Equivalent

-anivalont

1.2E-68

8.7E-15

2.1E-96

5.9E-10

1.1E-U7

7. DE-15

1. $8 \mathrm{E}-17$

2. 3E-61

U. EE+0S

3.6E-69

4.7E- 06

$0.6 \mathrm{E}+06$

-. EE+95

2.7E-68

1. $9 E-07$

4. SE-G7

4. $4 E-67$

2. 5E-99

5. UE-67

1.1E-13

3. 3E- 7

3. 3E-98

2.9E-11

4.8E-09

7. $4 E-98$

4. $2 E-98$

1. $. E-18$

4. 4E- 99

1. 1E-99

8. 3E-12

1. 5E-15

3. $6 E-13$

1. 1E-12

5. 2E-10

3. $6 \mathrm{E}-10$

1. 1E-98

1. $2 E-11$
$1.2 E-68$

8. 8E-10

2.1E-10

7. 2E-10

1.1E-G7

7. 5E-15

4. 9E-17

2.3E-61

$1.8 E-67$

3. 1E-09

2. DE-05

3. 6E- 06

2.8E- 08

1.9E-97

4. 1E-U7

5. $5 E-07$

3. UE- 99

5. 1E-67

1. 1E-13

3. 3E- 67

3. 3E- 66

3. $4 \mathrm{E}-11$

1. 3E- 98

7. 5E-98

$4.2 E-68$

1. GE-18

5.7E-G9

1. 2E-99

8. 4E-12

1. 5E-10

3. $6 \mathrm{E}-13$

1. $3 E-12$

5. 9E-10

3. 6E-10

1.3E-68

1. $4 \mathrm{E}-11$
6. 7E-10 


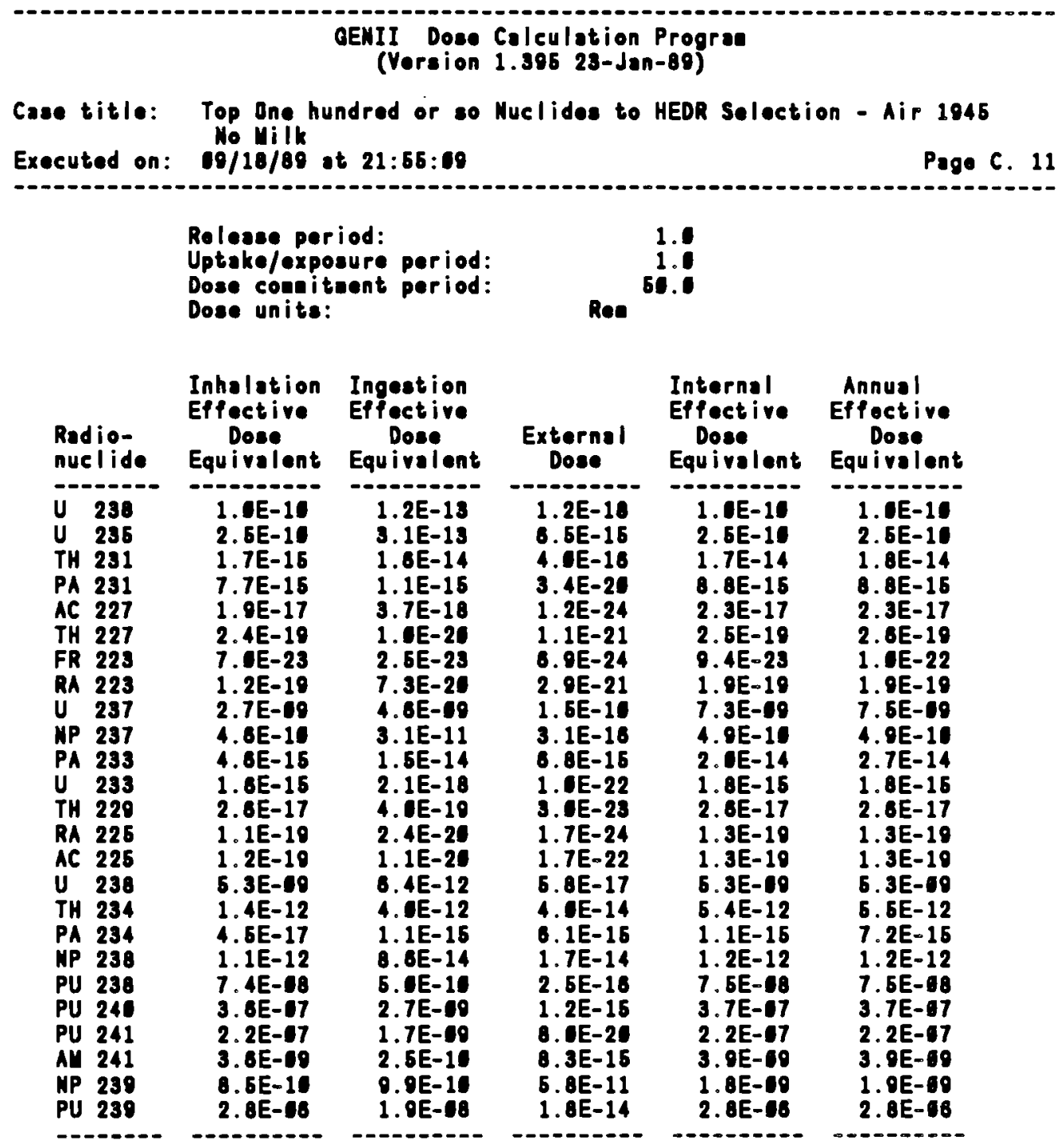




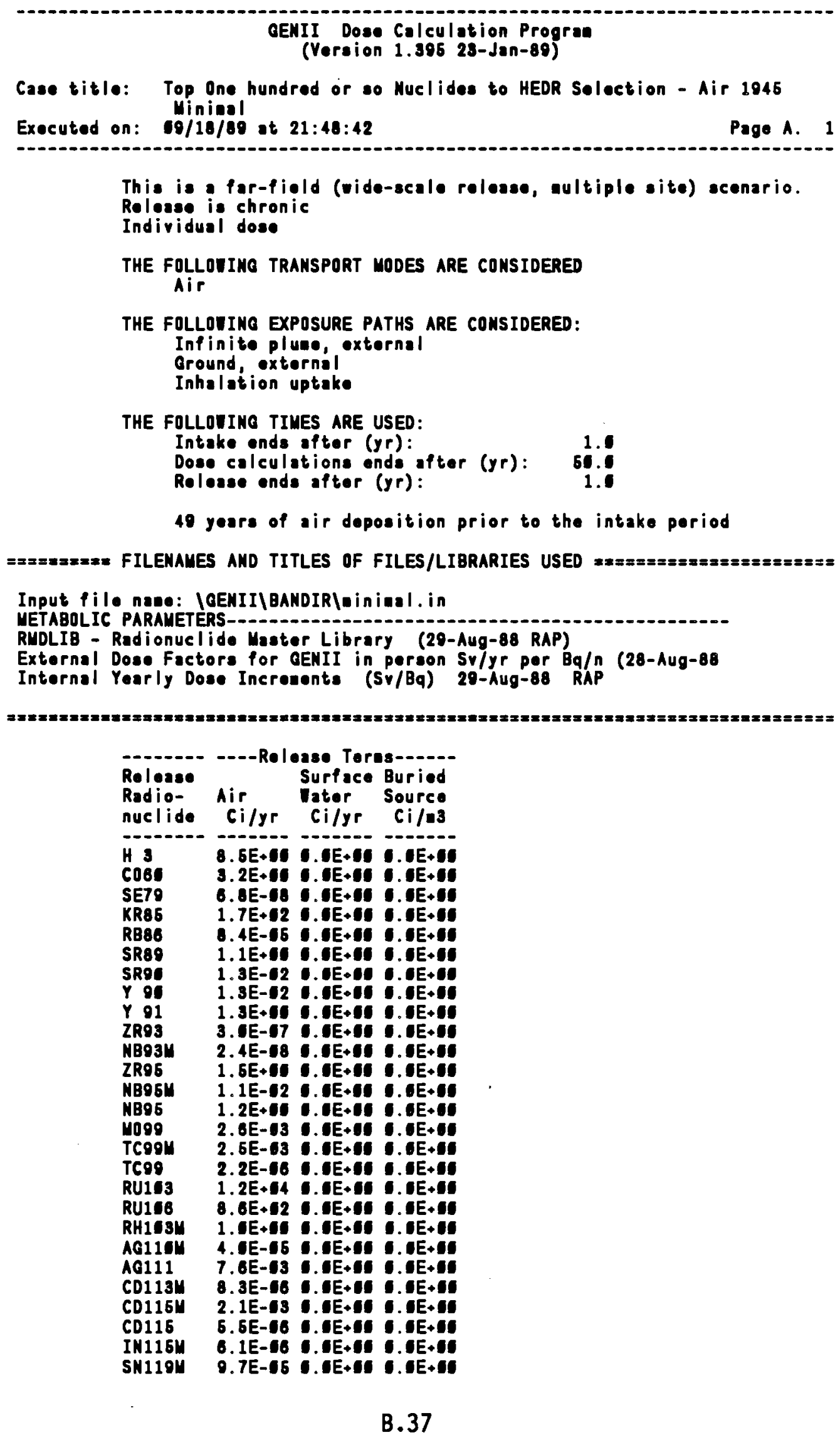




\begin{tabular}{|c|c|c|c|}
\hline 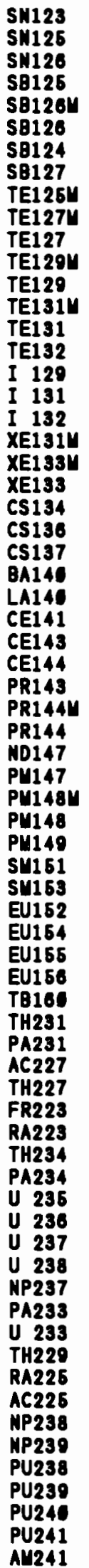 & 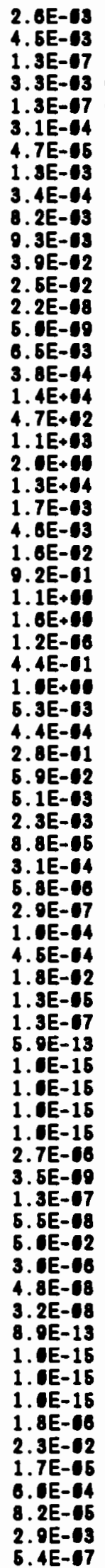 & $\begin{array}{l}\text { E. } \\
\text { EE+ } \\
\text { UE. }\end{array}$ & 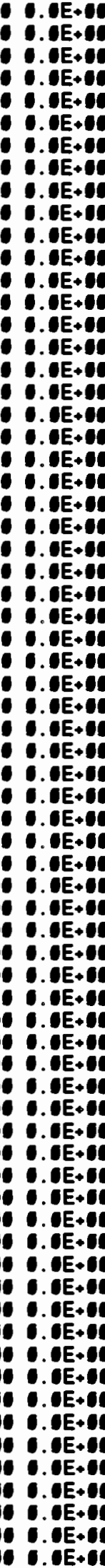 \\
\hline
\end{tabular}




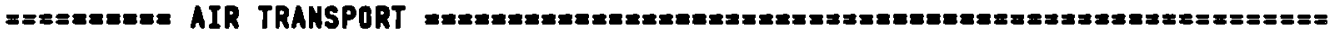
5.6E-08 Input chi/Q value $(e / \mathrm{a})$

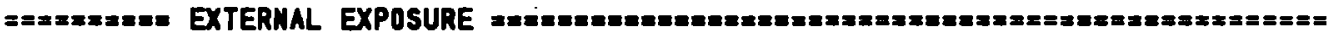

8.8E+53 Hours of exposure to plune

4.4E+Gs Hours of exposure to ground contanination

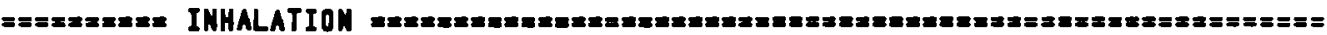

B.8E+C3 Hours of inhalation exposure per year

1 Resuspension nodel: 1-Vase Loading, 2-Anspaugh

1.0E-04 Vess looding factor $(g / 03)$

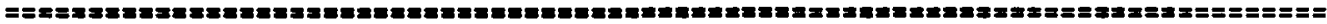

Input prepared by:

Date:

Input checked by:

Date:

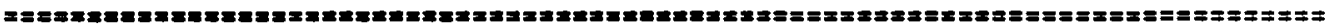


Case title: Top One hundred or so Muclides to HEDR Selection - Air 1945

Executed on: 09/18/89 at 21:51:25 Page C. 9

Release period:

Uptake/exposure period:

Dose counitment period:

Dose units:

Radio-
nuclide Equ

Inhalation

Effective

Dose

Equivalent

H 3

CO 68

SE 79

KR 85

SR 89

RB 86

SR 96

Y 90

$\begin{array}{lll}Y & 91\end{array}$

ZR 93

NB $93 \mathrm{H}$

ZR 95

NB 95U

MB 95

MO 99

TC 994

TC 99

RU 103

PD 103

RH 193U

RU 106

AO $110 \mathrm{Y}$

AQ 111

CD 1134

CD 1164

CD 115

IN $115 \mathrm{H}$

SN 110 U

SN 123

SN 125

SB 125

TE 125U

SN 128

SB 126U

SB 126

SB 124

SB 127
1.1E-18

$9.6 E-16$

9. EE-15

C.GE+CO

$9.2 E-18$

7. 7E-12

4.1E-68

1.7E-09

$9.7 E-67$

3.7E-13

1. 1E-14

3. 6E-67

4. 2E-10

1. EE-67

7. BE-11

1. 2E-12

3. EE-13

1. 6E- 63

-. DE+DS

7.-4E-11

8. 1E-63

5. EE-11

3.7E-1E

1.9E-10

2. 3E-19

3. 2E-13

1. 1E-14

9. E-12

1.3E-99

1.1E-09

6.1E-1E

3. 7E-11

1. $9 \mathrm{E}-13$

5. $9 \mathrm{E}-17$

5. 5E-11

1. $8 E-11$

1. $2 E-10$
Ingestion Effective Dose Equivalent

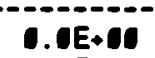

๑. EE+GS

o. $\theta E+0$

G.0E+00

O.DE+0S

-. IE+CS

-. DE+68

C.0E+00

๑. EE+GS

C.OE+OS

G.0E-OS

1. TE+CS

-. $\mathrm{E}+01$

O.OE+OS

C. DE +01

C. $0 \mathrm{E}+\mathrm{AS}$

C. $0 E+01$

C.OE+10

C.OE+105

C.0E+CS

C.UE+CB

-. E +00

I.UE+CS

C. E+CO

C. E+ 10

- IE+CO

C. $\mathrm{E}+10$

-. E +10

C.OE+CO

C. 0 E +6

C. E+ +10

C. E+CO

6. E +06

-. $1 E+60$

-. $6 \mathrm{E}+0 \mathrm{C}$

b. EE+60

C.OE+CO
External

Dose

1.1E-17

3. 2E- 5

2. $5 E-18$

6. 4E-69

3. 5E-10

4. $7 \mathrm{E}-13$

2.6E-11

1. $5 E-69$

1. 3E-99

4. IE-19

1. EE-16

2. 1E-07

1. 2E-10

3.6E- 7

6. 8E-12

3. $9 E-12$

2. OE-17

7. 2E- 14

C. DE +01

1. 3E-67

2. GE- 44

5. 9E-11

6. BE-12

1. 3E-14

B. $7 E-12$

2.1E-14

1. $9 E-14$

4. 6E-14

7. 6E-12

5. $2 E-11$

3. 6E-19

4. 2E-12

6. 5E-14

6. 8E-12

4. E-11

1. 6E-11

1. $9 \mathrm{E}-11$
1.0

1.0

50.6

Internal Annual

Effective Effective Dose

Equivalent

1.1E-08
Dose

Equ ivelent

9. 8E-18

๑. EE-15

C. E. 01

9. 2E-18

7.7E-12

4.1E- 08

1.7E-69

9.7E- 07

3. $7 E-13$

1. $1 E-14$

3. $6 E-67$

4. 2E-10

1. $\mathrm{EE-07}$

7. BE- 11

1. $2 E-12$

3. EE-13

1.6E- 63

๑. $0 \mathrm{E}+10$

7. $4 E-11$

6. 1E- 3

5. TE-11

3.7E-11

1. $9 E-10$

2. $3 E-19$

3. $2 E-13$

1.1E-14

9.UE-12

1. 3E- 09

1.1E-19

6. 1E-10

3. 7E-11

1.9E-13

6. $9 \mathrm{E}-17$

5. $5 E-11$

1. 8E-11

1. 2E-10
1.1E-68

4.2E-65

9.0E-15

5. 4E-09

8. 2E-12

4.1E- 8

3. 2E- 09

$9.7 E-07$

3.7E-13

1.1E-14

5. 6 E- 97

5. 3E-10

4. IE-67

8. 4E-11

5. 1E-12

3. EE-13

2. 3E- 63

-. $0 E+60$

1. 3E- 67

6. 3E-63

1. $1 E-10$

3. 8E-10

1. OE-10

2. 3E-09

3. $4 E-13$

3. $E-14$

๑. EE-12

1. 3E- 69

4.3E-69

4.1E-11

2. BE-13

6. 8E-12

9. BE-11

3. 4E- 11

1. 4E-10
9.3E- 98

1.1E-69 


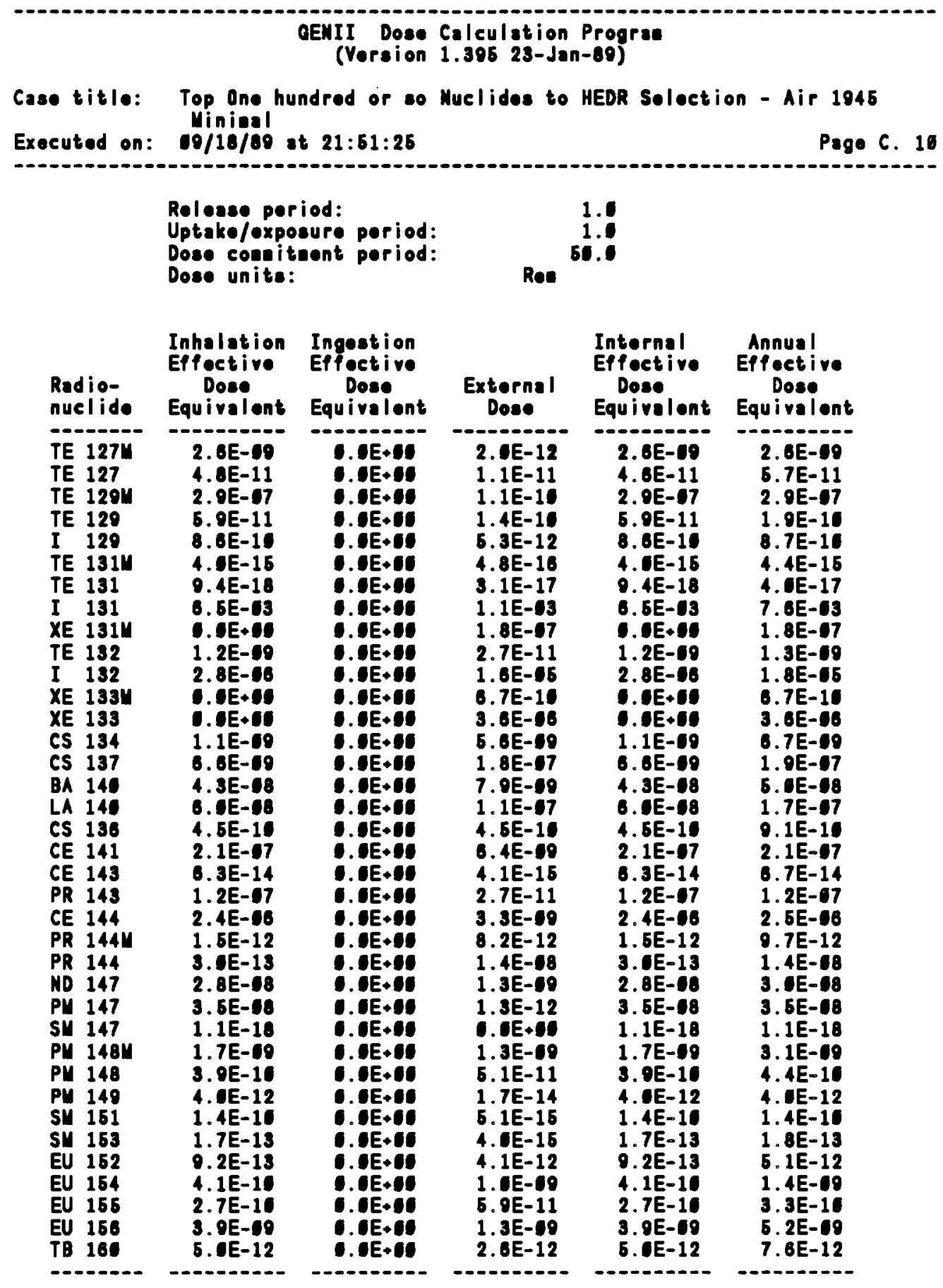




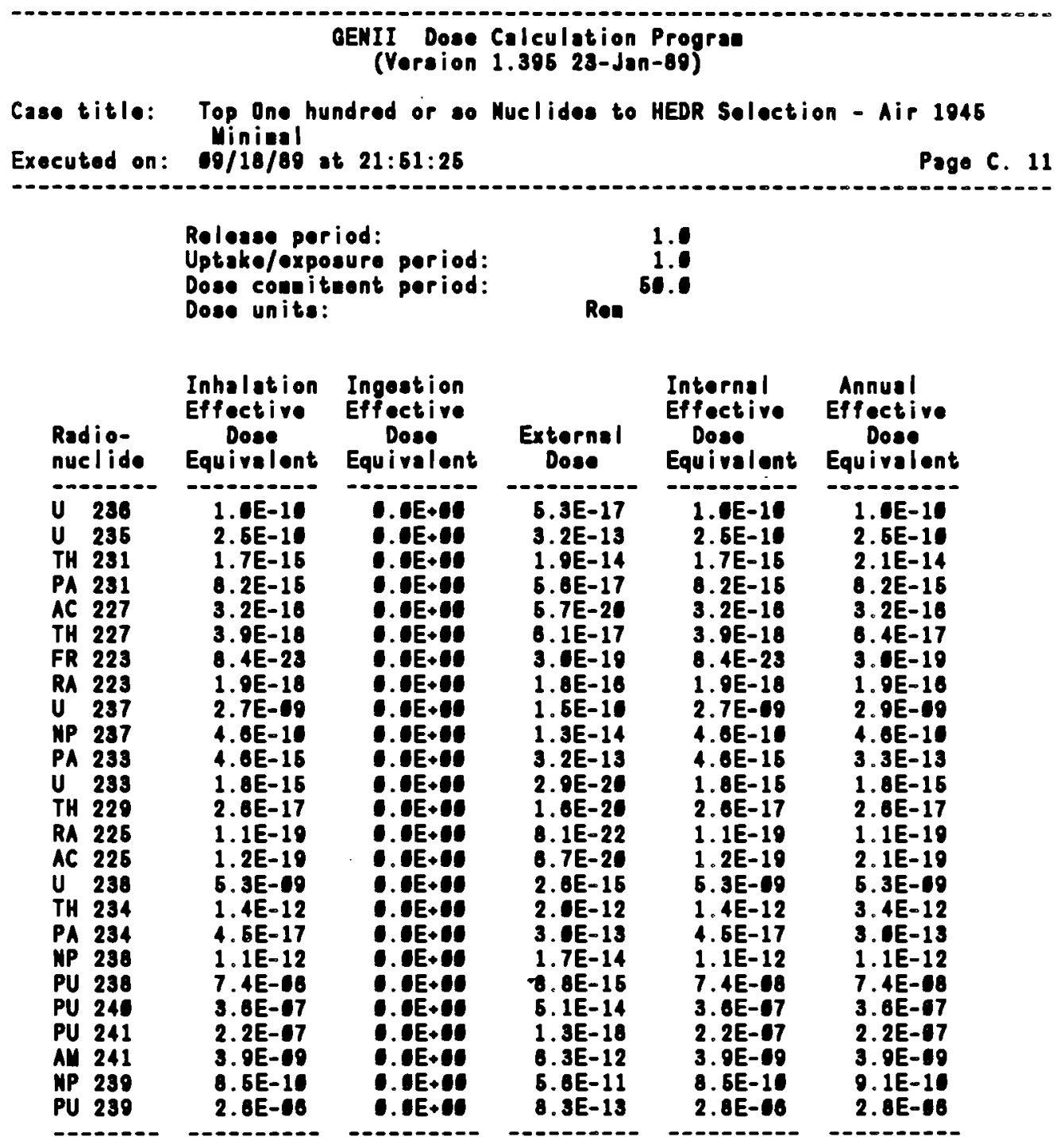




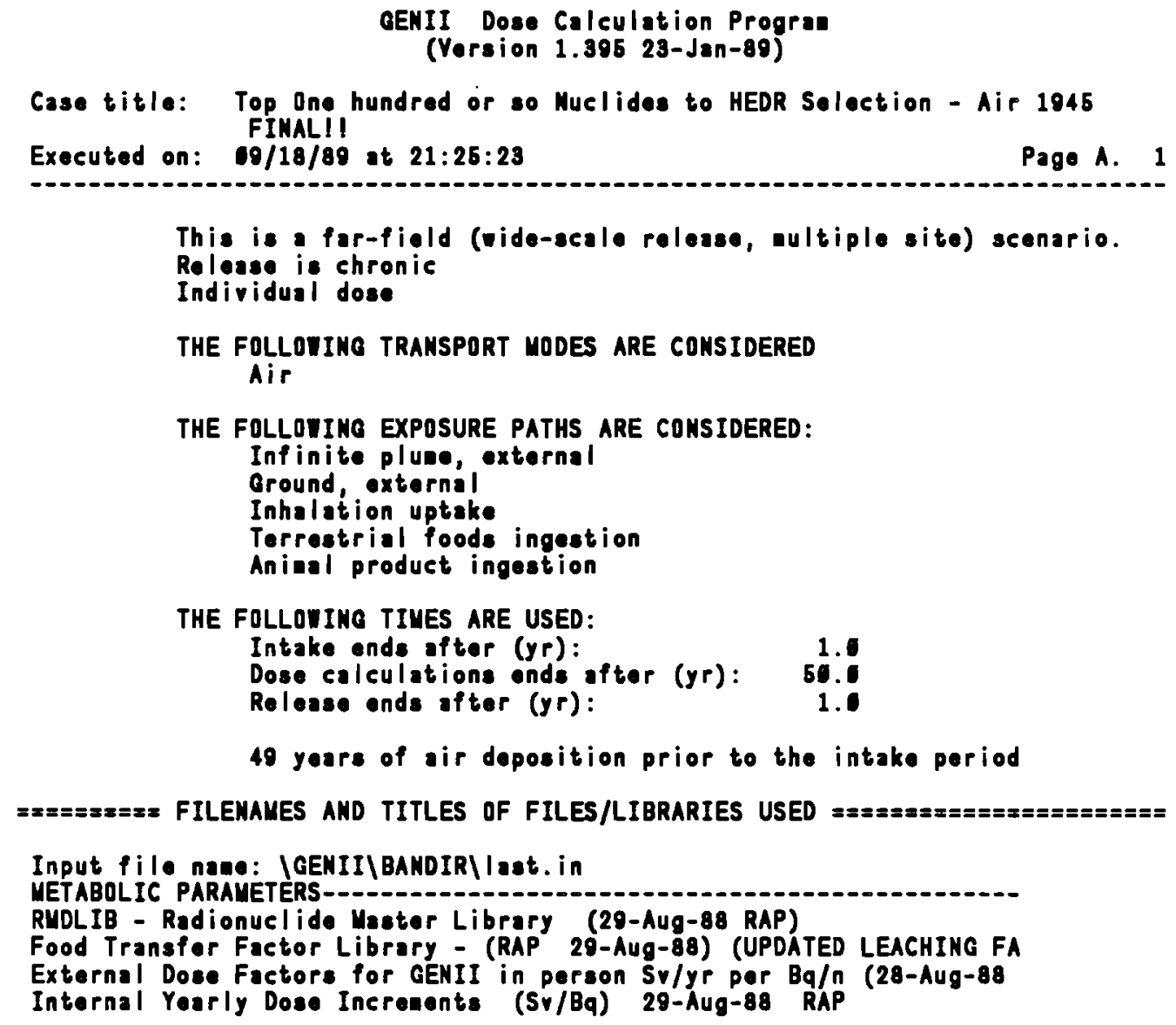




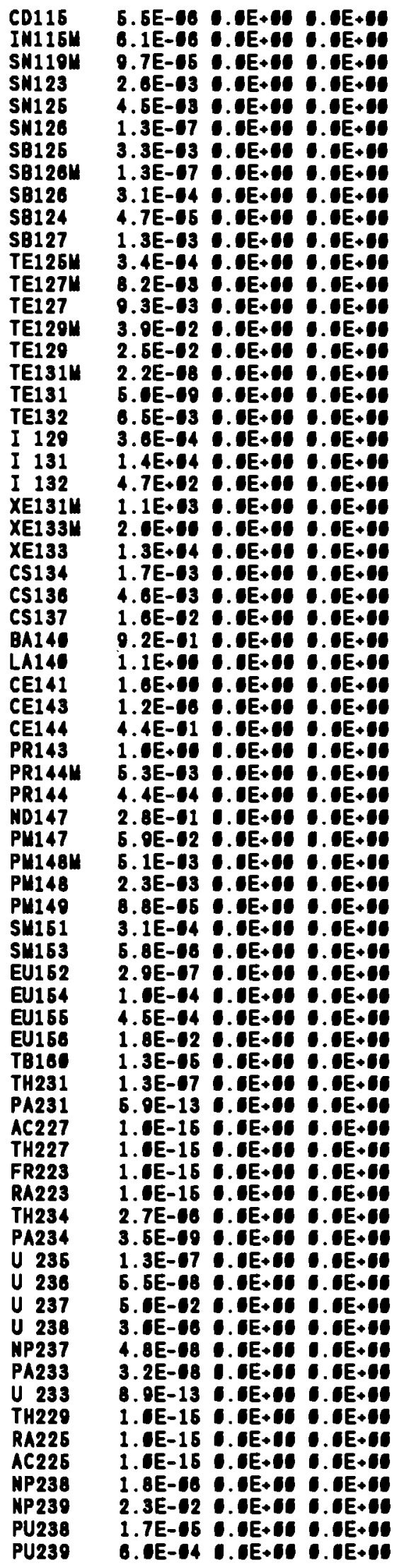




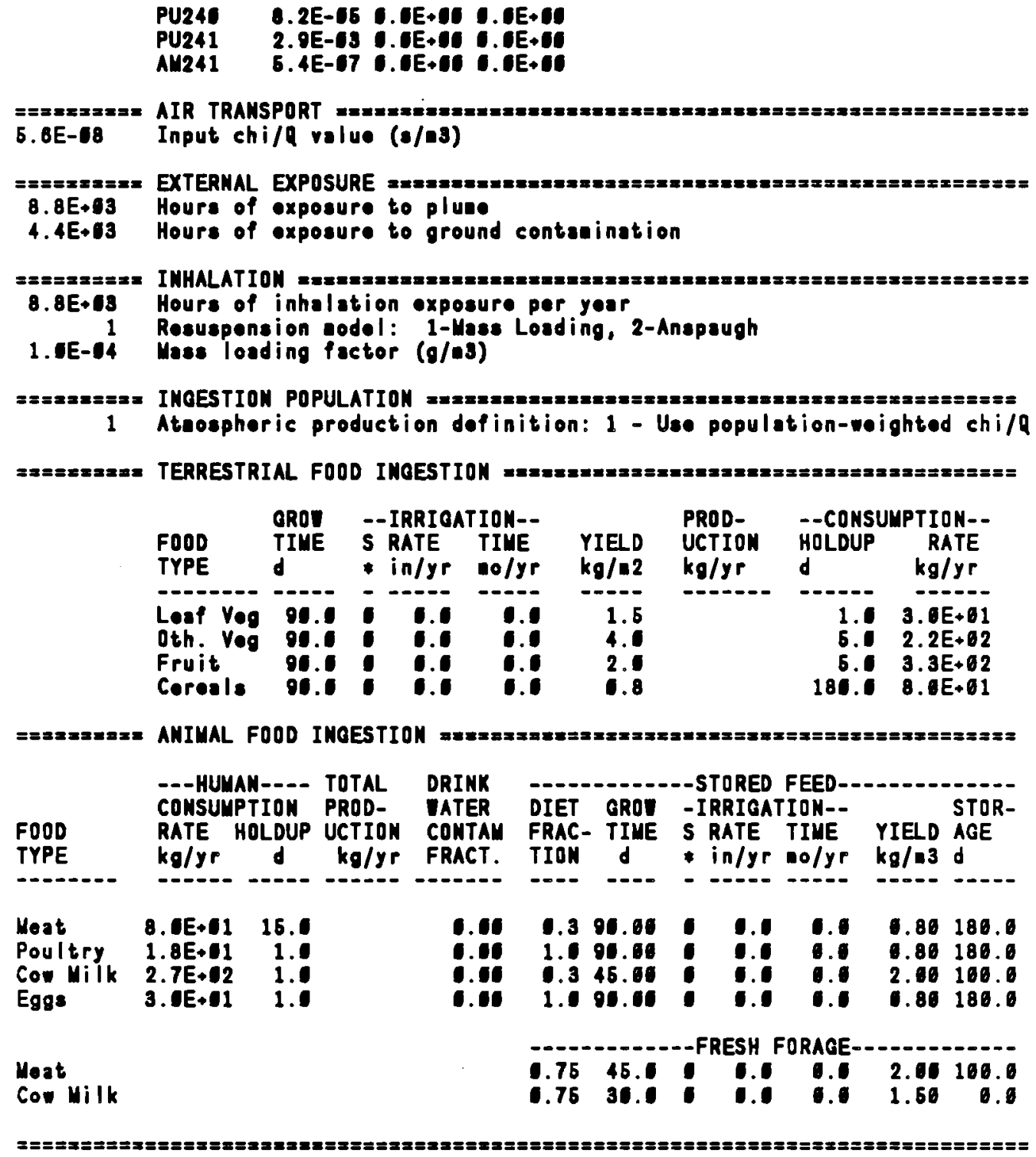

Input propered by:

Date:

Input checked by:

Date: 


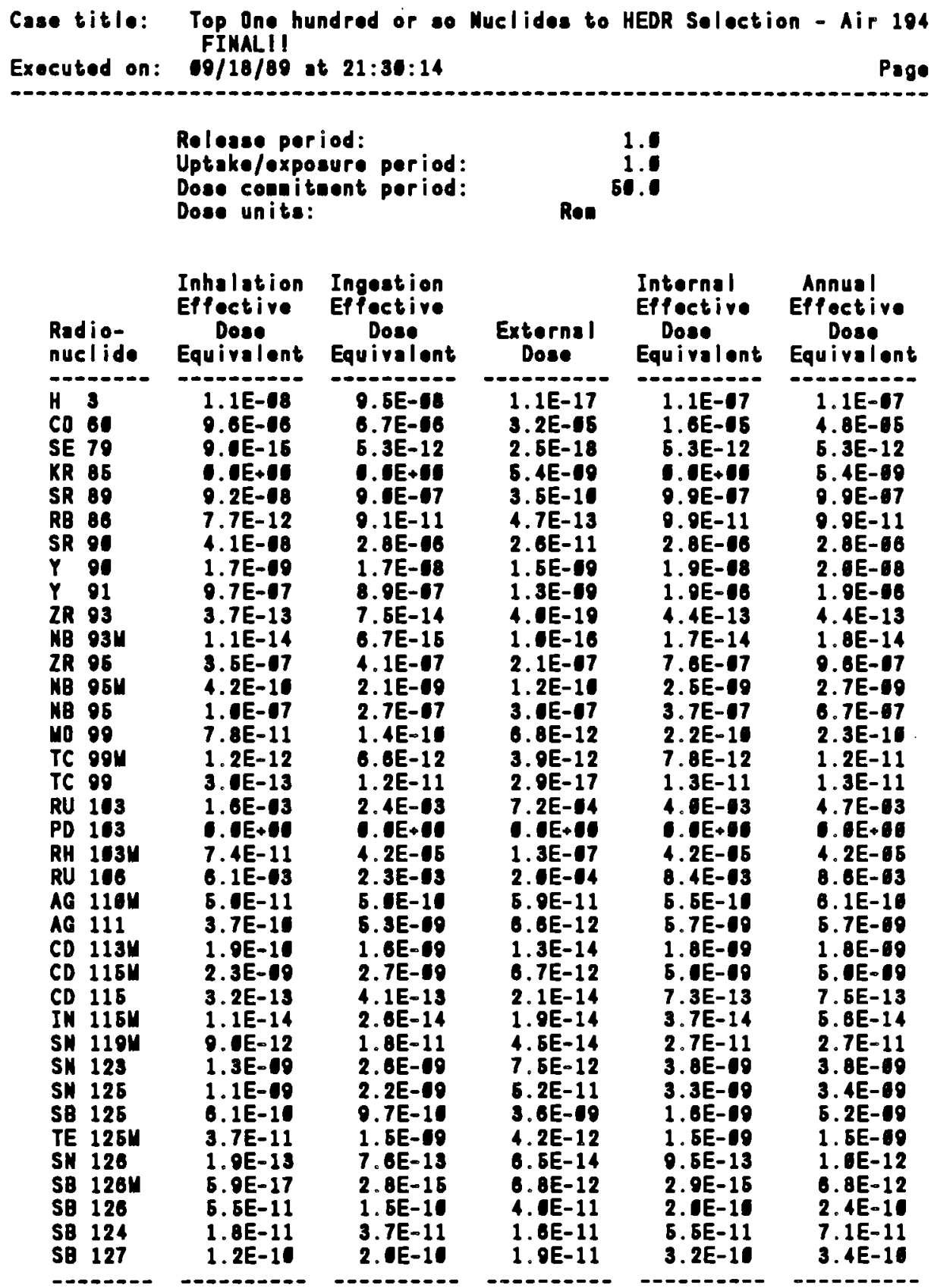




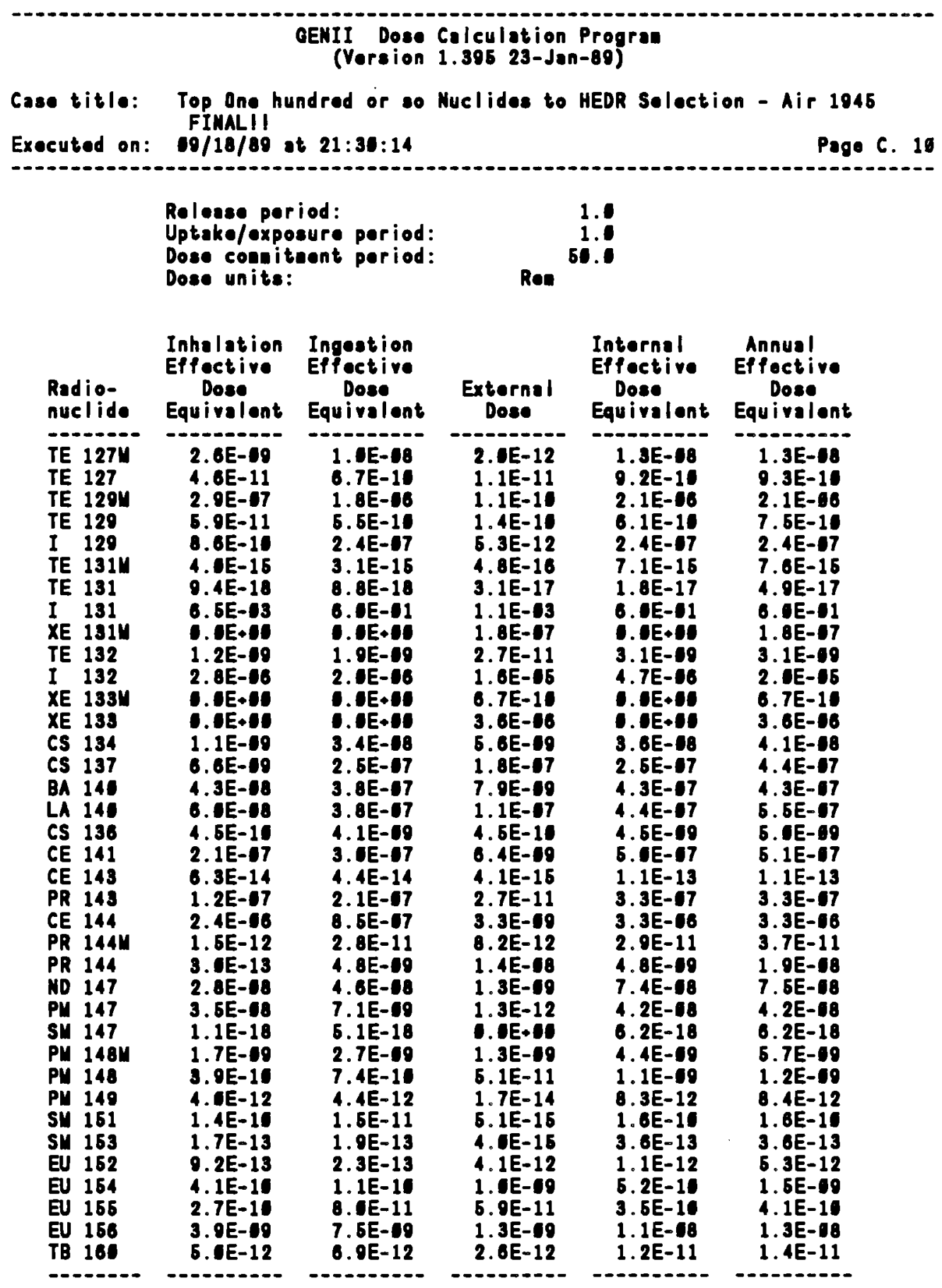




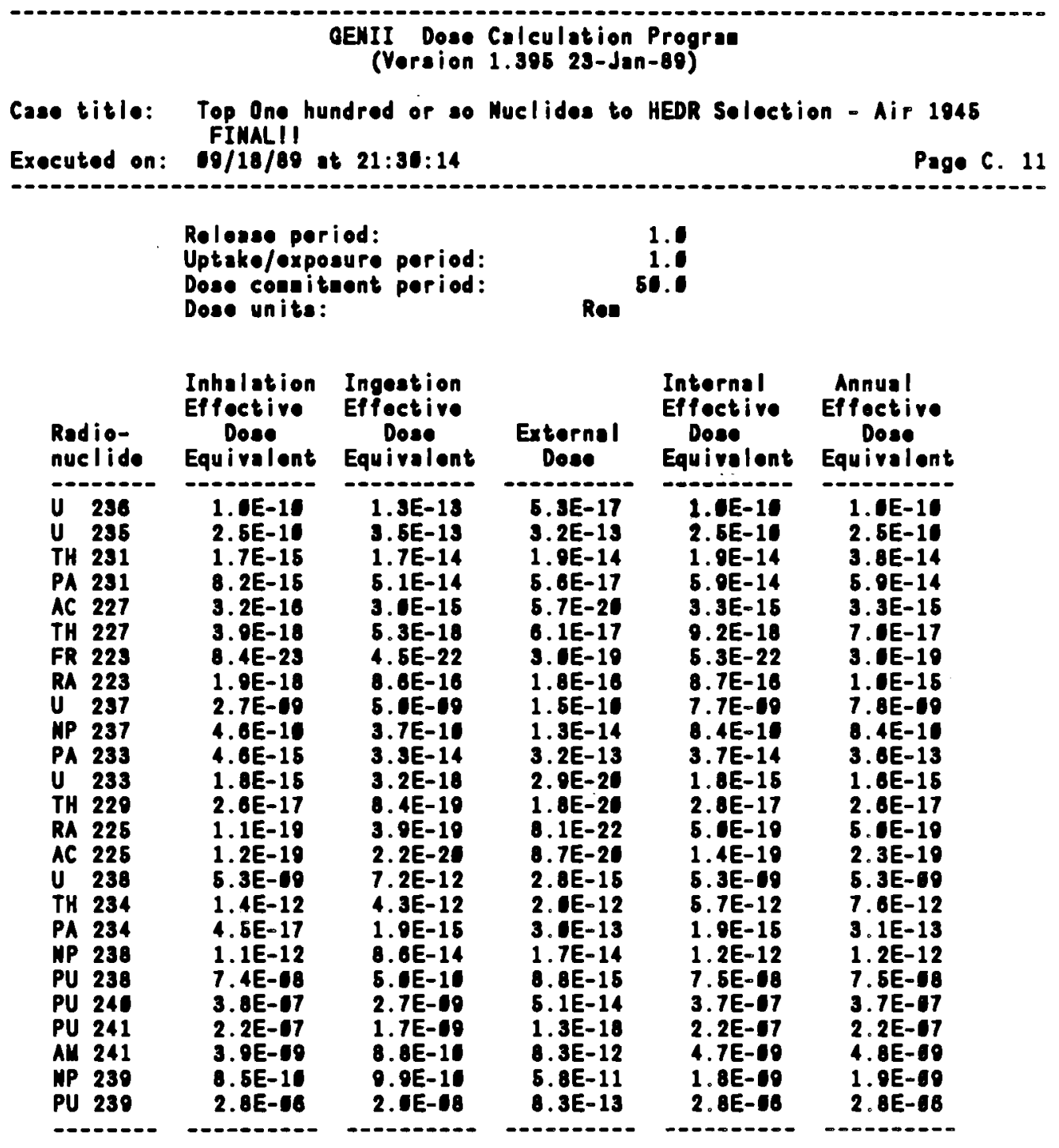


APPENDIX C

GENII RESULTS FOR SURFACE WATER RELEASES 


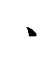

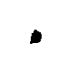

 
APPENDIX C

\section{GENII RESULTS FOR SURFACE WATER RELEASES}

\begin{tabular}{l} 
GENII Dose Calculation Progran \\
(Vorsion 1.395 23-Jan-89) \\
Case title: MI dose fron concentrations neseured in effluent in 1956 \\
Executed on: $19 / 19 / 89$ at $19: 66: 36$ \\
\hline
\end{tabular}

This is a near field (narrowly-focused, ingle site) scenario. Release is chronic

Individual dose

THE FOLLOUIMG TRAMSPORT MODES ARE CONSIDERED Surface Vater

THE FOLLONIMO EXPOSURE PATHS ARE CONSIDERED:

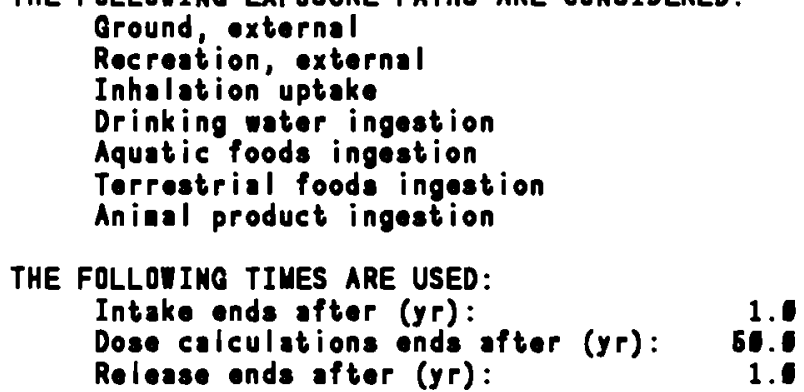

THE FOLLOUIMO TIMES ARE USED:

Intake ends after $(y r)$ :

Dose celculations ond aftor $(y r)$ :

Release ends after $(y r)$ :

\begin{tabular}{|c|c|}
\hline 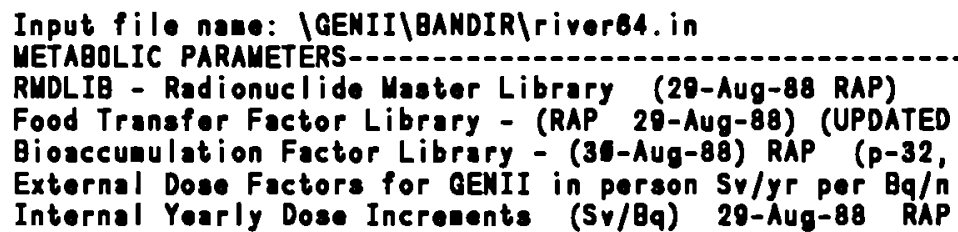 & $\begin{array}{l}\text { LEACHING FA } \\
\text { 2n, np Bodi } \\
(28-A u g-88\end{array}$ \\
\hline
\end{tabular}

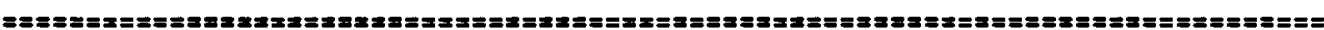

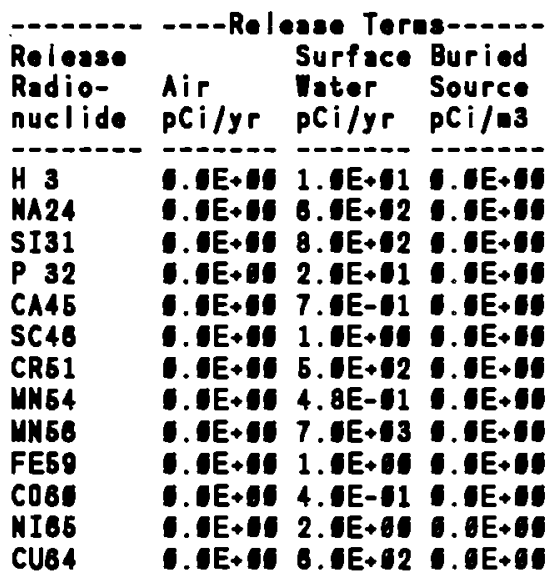

C.1 
ZN65

ZMGOL

OA72

AS76

SRBTU

SRBO

SROS

SRO1

SR92

$Y 90$

$Y 91$

$Y 92$

$Y 93$

ZROS

NB95

M099

TCOS

RU148

RU168

SB122

SB124

I 131

I 132

I 133

I 134

I 135

CS138

CS137

CS138

BA139

BA140

LA140

CE141

CE143

CE144

PR142

PR143

ND147

PM147

PU149

PM151

SM153

EU152U

EU152

EU156

OD153

OD159

TB168

TB181

DY185

HO186

ER169

ER171

TH232

U 238

MP239

PU239

PU24
$0.0 E+001.0 E+01$ ๑. 1.00

C.OE+OS 4.0E+O1 O.OE+CO

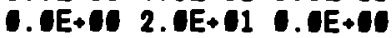

- $E+1$ 1. $1 .+2$ Q

C.UE+CS 5.0E+CS C.UE+CO

C.OE+OS 6.0E-01 O.OE+CO

$0.0 \mathrm{E}+08$ 1.0E-C2 $0.0 \mathrm{E}+\mathrm{CO}$

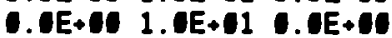

๑.UE+CS 5.UE+C1 U.UE+CO

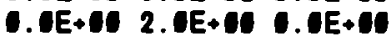

0.UE+CS 3.0E-Q1 O.NE+DO

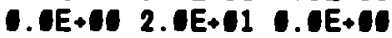

-.UE+CS 5.UE+QS U.UE+CO

$0.0 E+001.0 E+60$ C.0E+C0

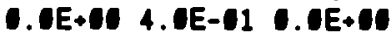

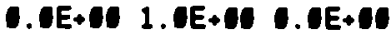

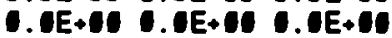

-.OE+OS 4.0E-02 0.0E+CO

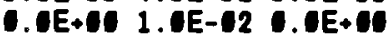

-.UE+C1 1.UE+C1 G.UE+

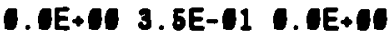

C.UE+CS 5.JE-G1 .UE+OO

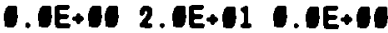

C.UE+CE 7.0E+C6 G.UE+00

-.UE+CO $1.0 E+02$ ט.UE+CO

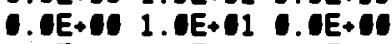

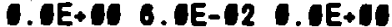

$0.0 \mathrm{E}+101.0 \mathrm{E}-12$ 6.0E+01

$0.0 E+101.0 E+12$ 1.UE+BS

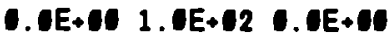

C.JE+CO 1.JE+CO ..UE+QO

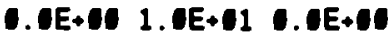

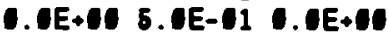

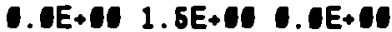

$0.0 E+C 1$ 2.0E-01 C.OE+MO

C.UE+CE 5.0E+CS 6.UE+OS

¿.UE+CE 5.JE-U1 C.UE+UE

- EE+CO 3.JE-11 J.JE+CO

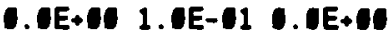

C.UE+CS 7.0E-C1 U.UE+DS

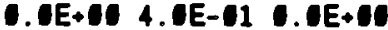

$0.0 E+102.4 E+\$ 1$ Q.UE+QS

C.UE+C6 6.1E+C1 .UE+Q0

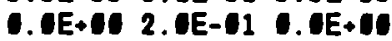

C.UE+CO 3.0E-C1 C.UE+QS

C.UE+CO 2.0E-C2 C.UE+QS

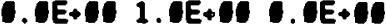

๑. UE+ IO 1.UE- C1 C.UE+CO

-. UE+UE 5.0E-C2 C.UE+CO

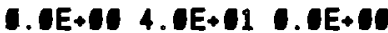

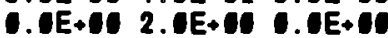

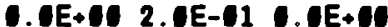

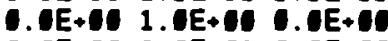

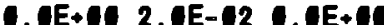

C.UE+C1 7.0E-C3 ๑. EE+CO

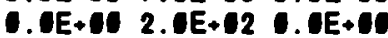

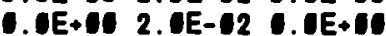

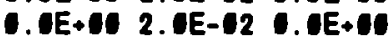

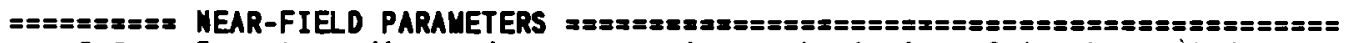
Invontory diaposed $n$ years prior to beginning of intake period LOIC occurred $n$ years prior to beginning of intake period Fraction of roots in upper soil (top $15 \mathrm{ca}$ )

$1.0 E+10$

$0.0+90$

6.OE+90

Fraction of roots in deep soil

Manual redistribution: deep soil/surface soil dilution factor

1250.0

Source area for external dose uodification factor (a2) 


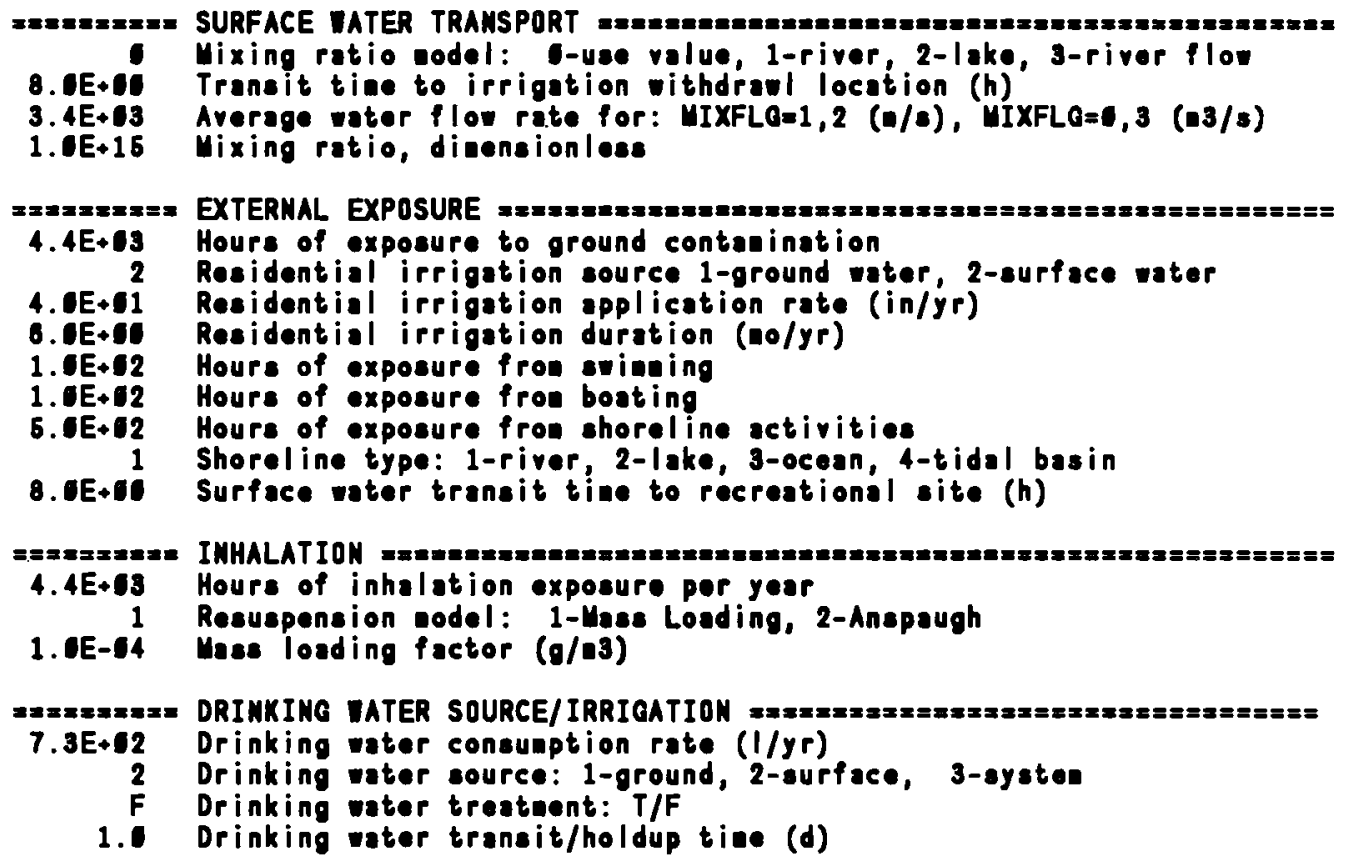

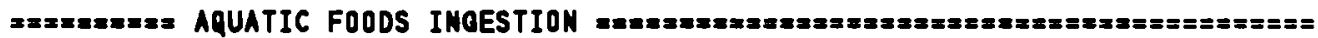

\begin{tabular}{|c|c|c|c|}
\hline $\begin{array}{l}\text { FOOD } \\
\text { TYPE }\end{array}$ & $\begin{array}{l}\text { TRAN- } \\
\text { SIT } \\
h\end{array}$ & $\begin{array}{l}\text { PROD- } \\
\text { UCTION } \\
\mathrm{kg} / \mathrm{yr}\end{array}$ & $\begin{array}{ll}--- \text { CONSUMPTION---- } \\
\text { HOLDUP } & \text { RATE } \\
d & \mathrm{~kg} / \mathrm{yr}\end{array}$ \\
\hline
\end{tabular}

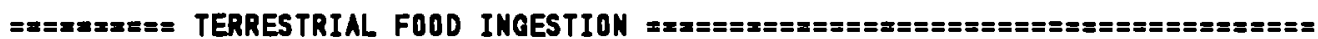

\begin{tabular}{|c|c|c|c|c|c|c|}
\hline $\begin{array}{l}\text { FOOD } \\
\text { TYPE }\end{array}$ & $\begin{array}{l}\text { OROI } \\
\text { TIME } \\
\text { d }\end{array}$ & $\begin{array}{l}\text { - - IRRIGA } \\
\text { s RATE } \\
\text { in/yr }\end{array}$ & $\begin{array}{l}\text { TION-- } \\
\text { TIME } \\
\text { Do/yr }\end{array}$ & $\begin{array}{l}\text { YIELD } \\
\mathrm{kg} / \mathrm{m} 2\end{array}$ & $\begin{array}{l}\text { PROD- } \\
\text { UCTION } \\
\mathrm{kg} / \mathrm{yr}\end{array}$ & $\begin{array}{l}\text {--CONSUUPTION-- } \\
\text { HOLDUP RATE } \\
d\end{array}$ \\
\hline $\begin{array}{l}\text { Leaf Veg } \\
\text { Oth. Yeg } \\
\text { Fruit } \\
\text { Cereale }\end{array}$ & $\begin{array}{l}90.0 \\
90.0 \\
90.0 \\
90.0\end{array}$ & $\begin{array}{rr}2 & 36.0 \\
2 & 45.0 \\
2 & 35.0 \\
2 & 0.0\end{array}$ & $\begin{array}{l}6.0 \\
6.0 \\
6.0\end{array}$ & $\begin{array}{l}1.5 \\
4.5 \\
2.6 \\
0.8\end{array}$ & - & $\begin{array}{rr}1.0 & 3.6 E+61 \\
5.6 & 2.2 E+62 \\
5.6 & 3.3 E+62 \\
180.6 & 8.6 E+61\end{array}$ \\
\hline
\end{tabular}

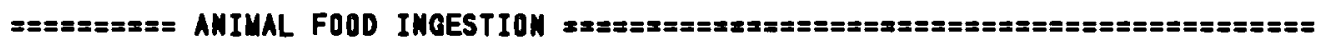

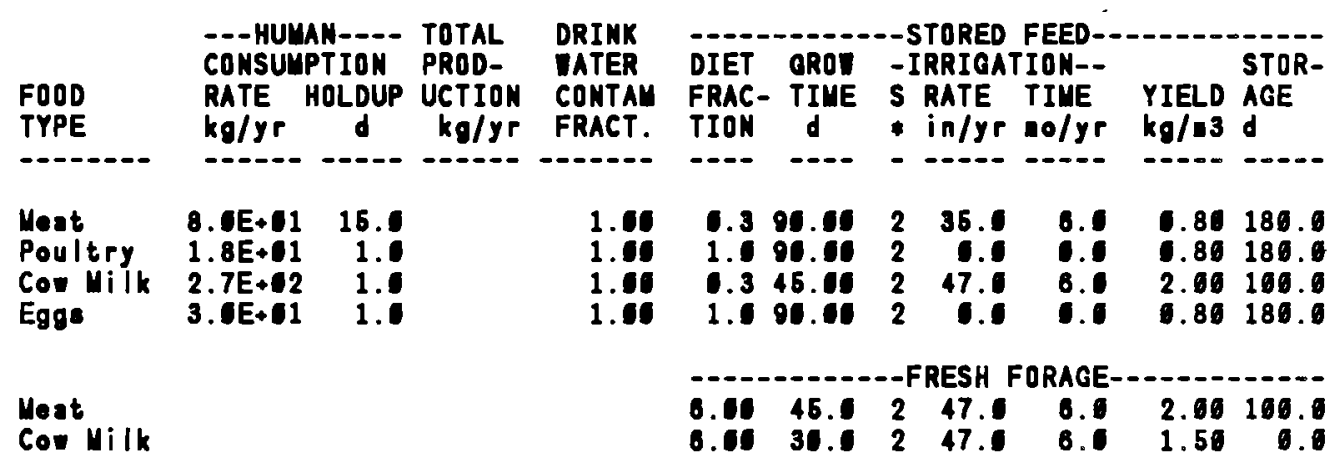




\section{QENII Dose Caleulation Progran}

(Version 1.395 23-Jan-89)

Case title: UI dose fron concentrations aeasured in offluent in 1958

Executed on: $19 / 19 / 89$ st $10: 31: 13$

Page C. 1

Rolesse period:

Uptake/exposure period:

Dose conniteent period:

Dose units:

1.0

1.0

56.0

Rod

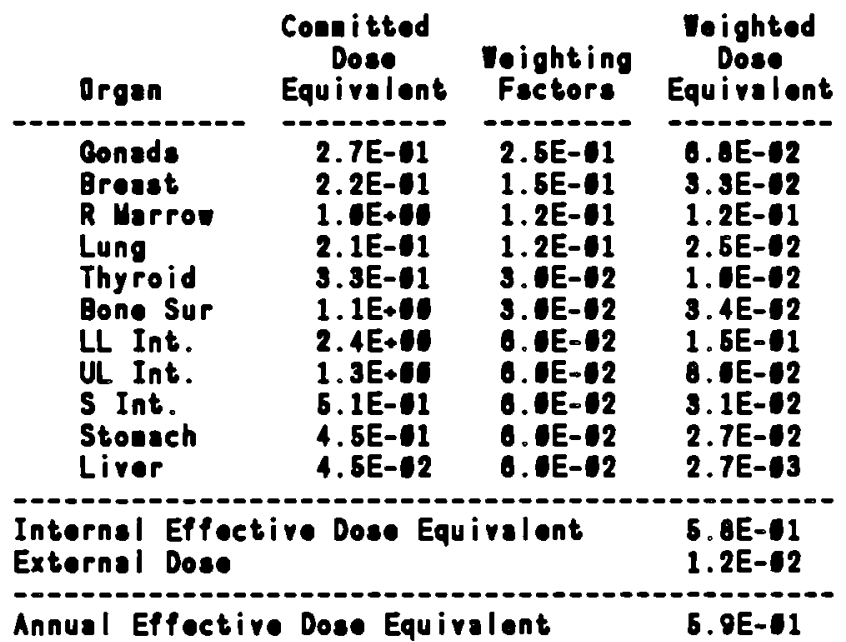

\begin{tabular}{l} 
Controlling Organ: \\
Controlling Pathway: \\
Controlling Radionucl ida: \\
\hline Total Inhalation EDE: \\
Total Ingestion EDE:
\end{tabular}




\section{OENII Dora Calculation Program \\ (Version 1.395 23-Jan-89)}

Case title: MI dose from concentrations weasured in effluent in 1966

Executed on: 19/19/89 at 14:31:13

Page C. 2

Release period:

Uptake/exposure pariod:

Dora connituent pariod:

Dora units:

1.1

1.1

61.1

Ree

Dora Coneitment Year

1

2

$3 \quad \ldots$

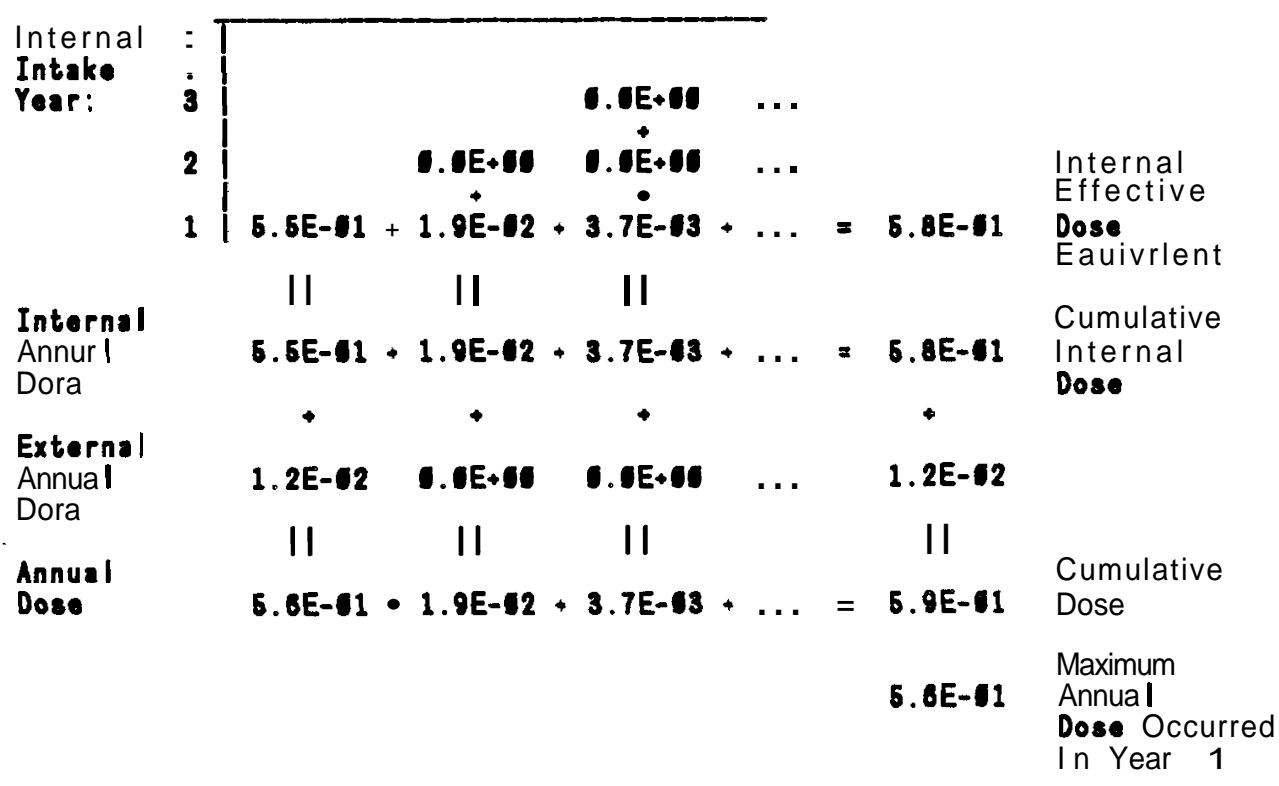


Case title: $\quad$ Ml dose from concentrations aeseured in effluent in 1966

\begin{tabular}{|c|c|}
\hline $\begin{array}{l}\text { Release period: } \\
\text { Uptako/exposure period: } \\
\text { Dose commitment period: } \\
\text { Dose units: }\end{array}$ & $\begin{array}{r}1.1 \\
1.1 \\
61.1\end{array}$ \\
\hline
\end{tabular}

Committed Dose Equivalent by Exposure Pathway

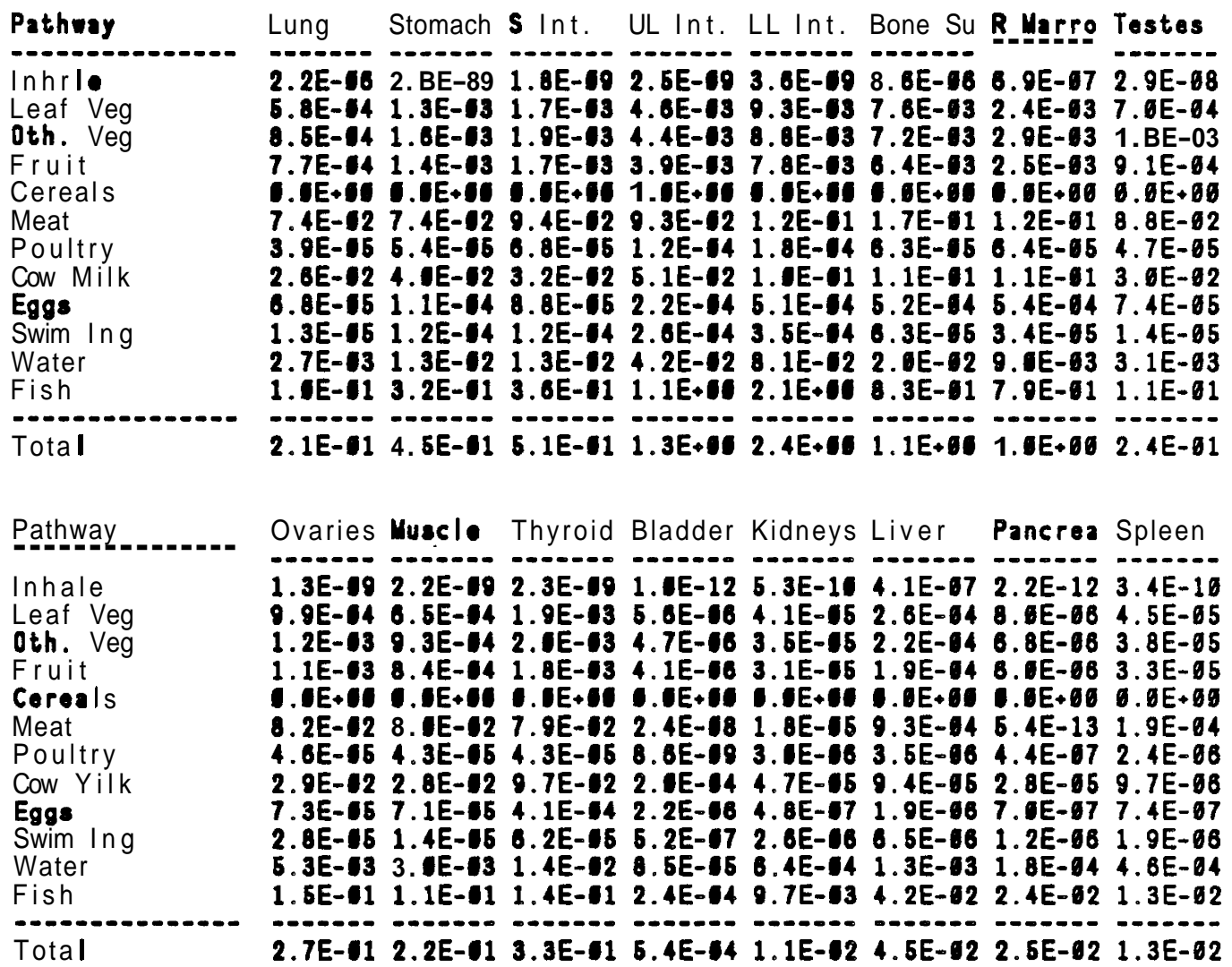




\section{GENII Dose Calculation Program}

(Version 1.396 23-Jan-89)

Case title: MI dose from concentrations measured in effluent in 1966

Executed on: 19/19/89 at 19:31:13

Page C. 4

Release period:

Uptake/exposure period:

Dose commitment period:

Dose unite:

External Dose by Expoeure Pathway

Pathway

Sur Soil

Swim Ext

Boating

Shore

1.7E-c8

6.3E-13

3. 2 E- -13

Total

$9.8 E-64$

Total

1.2E- 12 
CENII Dose Calculation Program

(Version 1.396 23-Jan-89)

Case title: MI dose from concentrations measured in effluent in 1966

Executed on: 19/19/89 at 18:31:13

Page C. 11

Release period:

Uptake/exposure period:

Dose commitment period:

Dose units:

Inhalation

Effective

Radio-

nuclide

H 3

MA 24

SI 31

P 32

CA 46

SC 46

R 61

N 64

YN 66

FE 69

CO 61

NI 66

W 64

$\mathbb{N} 66$

$\mathbb{N}$ 69!

\N 69

GA 72

AS 76

SR 89

SR $87 \mathrm{U}$

R 91

Y 90

SR 91

$Y$ 91U

Y 91

SR 92

Y 92

Y 93

บo 93

$\mathbb{Z R} 93$

NB 93Y

דR 96

N 954

N 96

10 99

TC 994

TC 99
Dose
Equival

Equivalent

\section{- .E+os}

2.0E-11

1. $5 E-13$

1.1E-10

4. 2E-11

1. $6 E-1$

3.1E-1

3.7E-11

2.1E-12

3. $8 E-11$

1.3E-18

4. JE-18

3. EE-12

1. $9 E-99$

8. 9E-13

4.8E-14

1. EE-12

2. 4E-11

1.1E-11

2.3E-16

3. BE-11

4. 5E-12

1. 4E-13

3.6E-15

7.3E-11

3. 2E-14

1. $9 \mathrm{E}-13$

2. IE-13

D. E + II

6. 5E-18

4.8E-20

1. $3 E-19$

1.7E-13

5. 3E-11

3. $0 \mathrm{E}-13$

5. $3 E-16$

3.9E-18
Ingestion
Effective
Dose
Equivalent

8. 5 E-06

1. 3E- 02

3. 1 E-0 4

2. $2 E-01$

4.7E-C4

4.3E-94

1.7E-13

2.3E-64

1.7E-U3

6. EE-U3

1.3E-93

8. 9E- 08

7. 3E-62

1.3E- 1

3.1E-63

2.3E-14

1. 1E-J2

3. 4 E- 62

2.7E-G4

4. $9 E-08$

8.8E-15

3. E-C4

1.1E-14

1.2E-98

9. EE-D5

5. 4 E- 16

6. BE- 05

7. BE-O5

C.0E+00

1. AE-13

5. 1E-17

6. 2E-94

7. 2 E- 7

6.7E-C5

4.1E-S5

1. 1E-66

1 .8E-11.
1.1

1.1

61.1

Rea

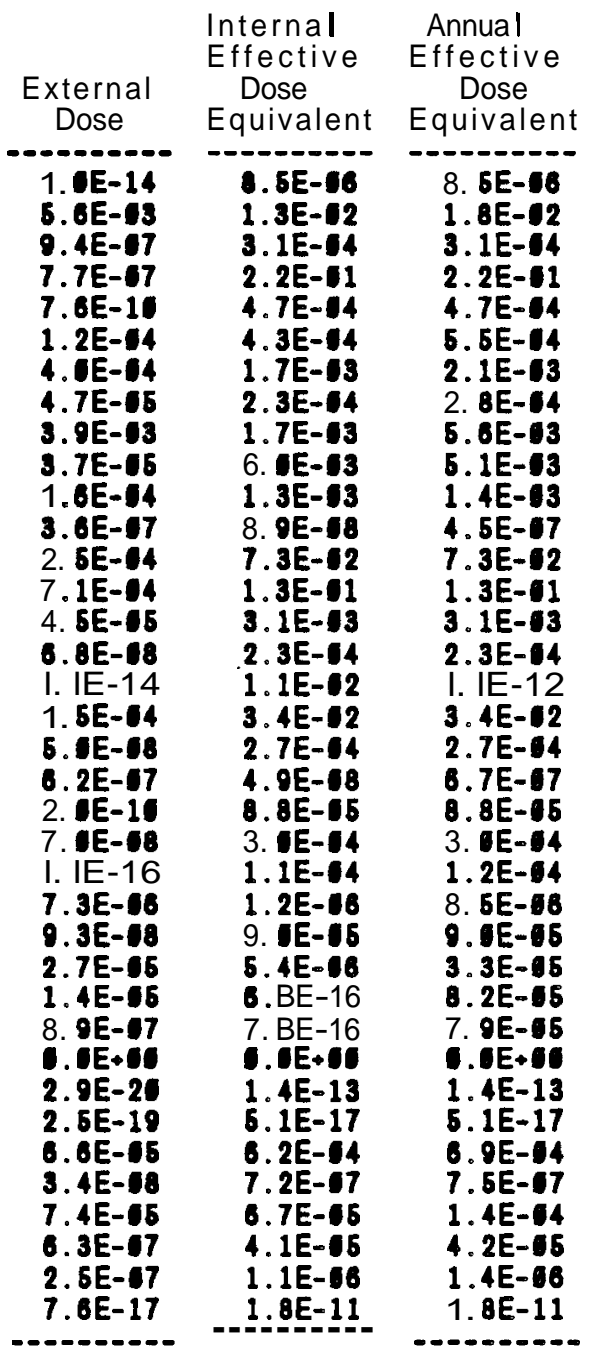




\section{OENII Dose Calculation Program}

(Version 1.396 23-Jan-89)

Case title: MI dose from concentrations measured in effluent in 1966

Executed on: 19/19/89 at 14:31:13

Page C. 12

Release period:

Uptake/oxposure period: Dose commitment period:

Dose units:

Radio-

nuclide

RU 113

PD 1183

$\mathrm{RH}$ 103M

RU 196

SB 122

SB 124

I 131

XE $131 \mathrm{M}$

I 132

I 133

XE 133M

XE 133

I 134

1136

XE 135U

XE 136

CS 136

CS 137

CS 138

BA 139

BA 141

LA 141

CS 136

CE 141

CE 143

PR 143

CE 144

PR 144M

PR 144

PR 142

ND 147

PM 147

SM 147

PM 149

PM 151

SM 161

Su 163
Inhalation

Effective

- Dose

Ingestion

Effective

Equivalent Equivalent

C. EE+ 10

5. 4E-16

6. 1E-11

9.1E-12

3. $8 E-11$

8. 3E-12

I. EE+ge

4.7E-15

1. $8 \mathrm{E}-12$

C. $. E+C 0$

G.OE+CO

6. $7 \mathrm{E}-17$

$9.9 E-14$

ด.DE+00

C.UE+CO

2. 2E-19

4. $9 E-12$

5. $2 E-19$

1. 3E-15

2.7E-12

6.7E-12

3. $6 \mathrm{E}-13$

9. 5E-12

4. $2 \mathrm{E}-13$

5. E -12

8.9E-10

6. 4E-16

1.1E-13

6. EE-13

1. $5 \mathrm{E}-12$

6. 3E-11

4. $2 \mathrm{E}-19$

3. E-13

5. $1 E-14$

7. 8E-15

5.7E-12
-2E-ce

G. E+CS

4. $3 \mathrm{E}-98$

1. 8E-95

4. $9 E-93$

3. 5E- 4

2. $5 E-93$

-.6E+GI

3. $6 E-07$

1. 2E-63

-.E.CO

C. EE+CO

2. DE- 99

6. EE-D5

1.0E+00

C.OE+C6

8. 3E-12

2. $7 E-13$

2. EE-11

6. 2E-68

7.8E-64

1. 2E- 03

2.9E-14

8.1E-04

1. E- 4

9. 6E-G4

3. EE-98

5. 1E-96

1. $9 E-04$

2. 4 E- 5

1. 7E-65

2. EE-15

2. $5 E-04$

8. 2E- 5

5. 8E-10

$6.1 E-13$
9. BE-13

3. 4 E- 13
1.1

1.1

Ren

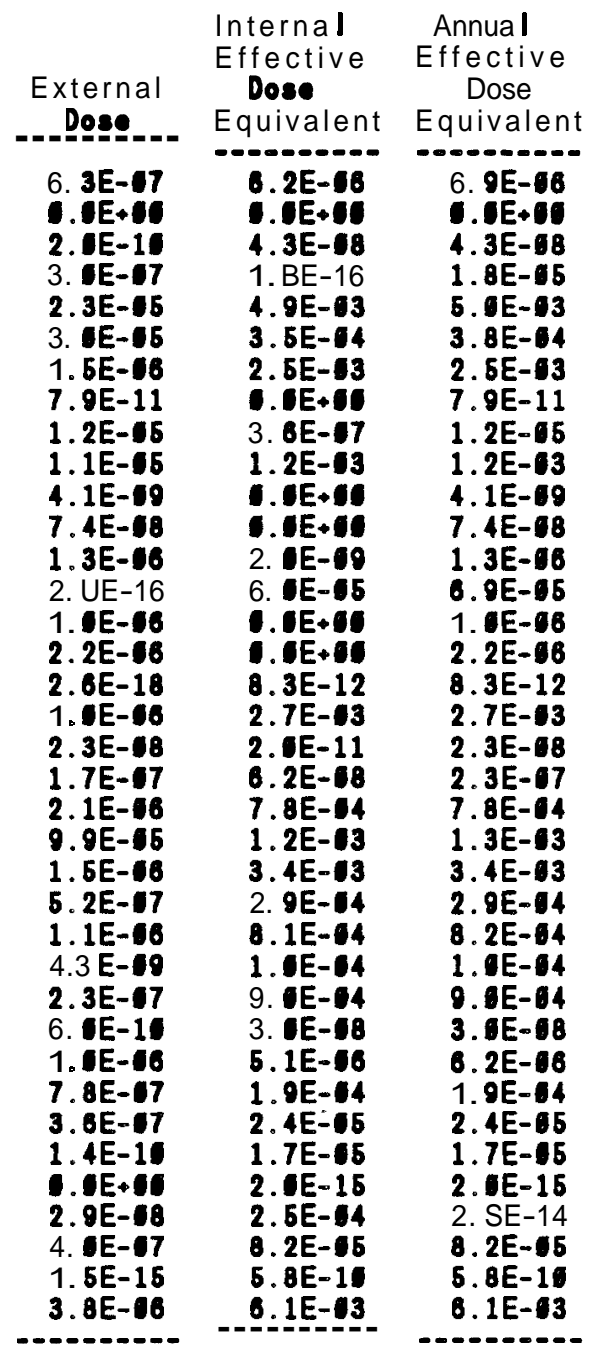


QENII Dora Calculation Progran

(Version 1.396 23-Jan-89)

Case title: MI dose from concentrations ceasured in effluent in 1950

Executed on: 19/19/89 at 16:31:13

Page C. 13

Relesse period:

Uptake/exposure pariod:

Dora commitment period:

Dora unitr:

Inhalation Ingestion

Effactive Effective

Radio-

nuclide

E $162 \mathrm{M}$

EU 162

时 158

(D) 153

(1) 169

TB 160

TB 161

DY 166

HO 186

ER 169

田 171

$\mathrm{TH} 232$

RA 228

AC 228

$\mathrm{TH} 228$

RA 224

PB 212

B I 212

U 238

$\mathrm{TH} 234$

PA 234

PU 241

NP 239

Pบ_239.
Dora

Equivalent Eguivalent

7. 8E-13

7.3E-10

4. $8 \mathrm{E}-12$

5. $1 E-12$

2.8E-14

1.2E-11

7. $9 \mathrm{E}-14$

3. 5E-15

4.1E-13

2. 6E-13

6.1E-15

3. 9E-ด7

8.1E-11

2.5E-12

7. 4E-1S

6. 2E-12

3. $6 E-13$

4.1E-14

1. $5 \mathrm{E}-68$

3. 8E-12

1. $5 E-16$

1.1E-67

7. 3E-11

I. IE-47
2. 4E-93

2.3E-94

3. $2 E-64$

6. EE-00

1. $8 \mathrm{E}-94$

2. 4E-A5

2. 6 E-106

3. 1E-07

7. $4 \mathrm{E}-\mathrm{C} 4$

5.6E-G5

3. EE-05

5. 4 E-O3

1.0E-05

2. SE-08

8. E-T

2. $8 \mathrm{E}-07$

9. 2E-68

2. 4E-69

7. 4 E- 68

3.1E-66

8.3E-10

B. 2E-94

6. EE-02

8. 2 E- 4
1.1

1.1

61.1

Ram

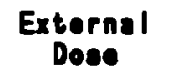

8.0E-05

4. UE-O5

5. 4 E- 06

9.1E-08

$9.4 E-08$

5. AE-98

2.7E-09

2.7E- 07

1. $8 \mathrm{E}-67$

6. 8E-11

5. 5E-17

2. $4 E-10$

6. $9 E-18$

2. E- 17

2. 4E-11

1. $9 \mathrm{E}-10$

2. $4 E-19$

3. 2E-98

3. 6E-11

2. 2E- 68

3. 6E-19

7.7E-11

1. 1 E- 94

1. $8 \mathrm{E}-10$
Internal Annual Effactive Effective Dose Dora Equivalent Equivalent Equivalont Equiralent

2.3E-04 2.7E-64

3.2E-04 3.3E-64

5.0E-06 5.1E-96

$1.6 \mathrm{E}-94$ 1.6E- 4

2.4E-05 2.0E-05

2.6E-60 2.6E-68

3.1E-07 5.7E- 7

7.4E-64 7.4E-94

5.6E-65 5.8E-65

3. IE-65 3.1E-65

5.4E-G3 5.4E-G3

1.0E-05 1.0E-05

2.5E-68 2.3E-67

8. EE- 7 8. 0 E-07

2.6E-67 2.6E- 67

9.2E-68 9. 5E-08

2.4E-69 3.4E-68

7.5E-10 7.5E-96

3.1E-68 3.1E-66

8.3E-10 4.3E- 99

8.2E-64 8.2E-94

5.JE-02 6. EE-02

8.2E-64 8.2E-14 
GENII Dose Calculation Program

(Version 1.396 23-Jan-89)

Case title: MI dose from concentrations measured in effluent in 1964

Executed on: $19 / 19 / 89$ at 16:46:40 $\quad$ Page A. 1

This is a near field (narrowly-focused, single site) scenario.

Release is chronic

Individual dose

THE FOLLOWING TRANSPORT MODES ARE CONSIDERED

Surface Vater

THE FOLLOWING EXPOSURE PATHS, ARE CONSIDERED:

Ground, external

Recreation, external

Inhalation uptake

Drinking water ingestion

Aquatic foods ingestion

Terrestrial foods ingestion

Animal product ingestion

THE FOLLOWING TIMES ARE USED:

Intake ends after (yr):

Dose calculations ends after (yr):

Release ends after (yr):

Input file name: IGENII\BANDIR|river84. in

METABOLIC PARAMETERS-_- $8-12-88$

RYDLIB - Radionuclide Master Library (29-Aug-88 RAP) 8-29-88

Food Transfer Factor Library - (RAP 29-Aug-88) (UPDATED LEACHING FA 8-29-88

Biosccunulation Factor Library - (34-Aug-88) RAP (p-32, zn np modi 9-12-89

External Dose Factors for GENII in person Sv/yr per Bq/n (28-Aug-88 8-29-88

Internal Yearly Dose Increments (Sv/Bq) 29-Aug-88 RAP 8-29-88

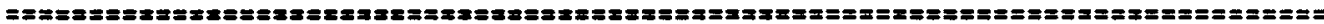

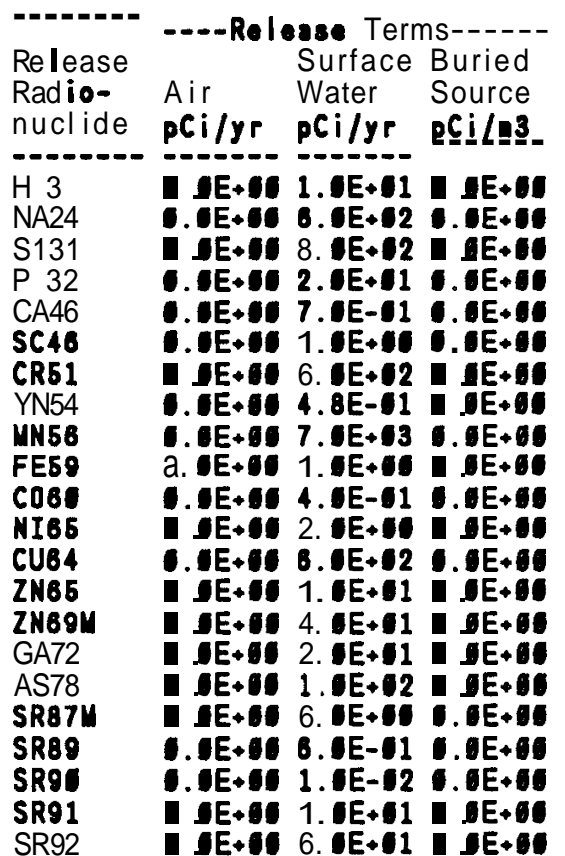




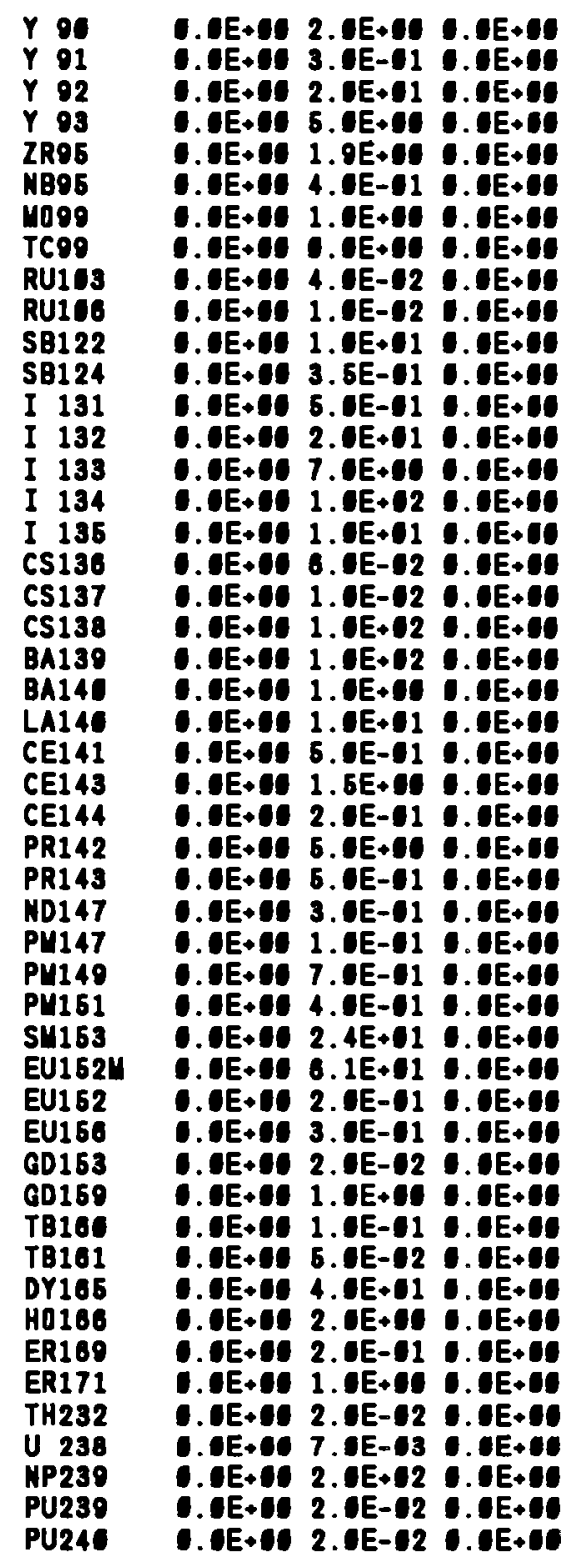

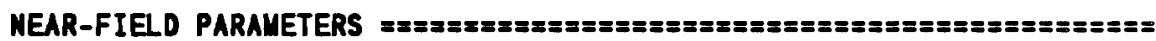

8.8 Inventory disposed $n$ years prior to beginning of intake period LOIC occurred $n$ years prior to beginning of intake period

Fraction of roots in upper soil (top $15 \mathrm{~cm})$ Fraction of roots in deep soil

ME+Gs Manual redistribution: deep soil/surface soil dilution factor
1268.8 Source area for external dose modification factor $(\mathbf{m})$

$\begin{array}{ll}0.60 & \text { Manual redistribution: deep soil/surface soil dilution } \\ 1268.8 & \text { Source area for external dose modification factor }(\mathbf{m})\end{array}$

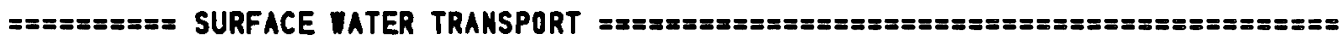
8. Mixing ratio model: 8-use value, 1-river, 2-lake,
Transit time to irrigation vithdraw location (h)

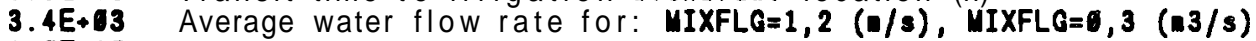

1. $\mathbf{E}+15$ Mixing ratio, dimensionless 


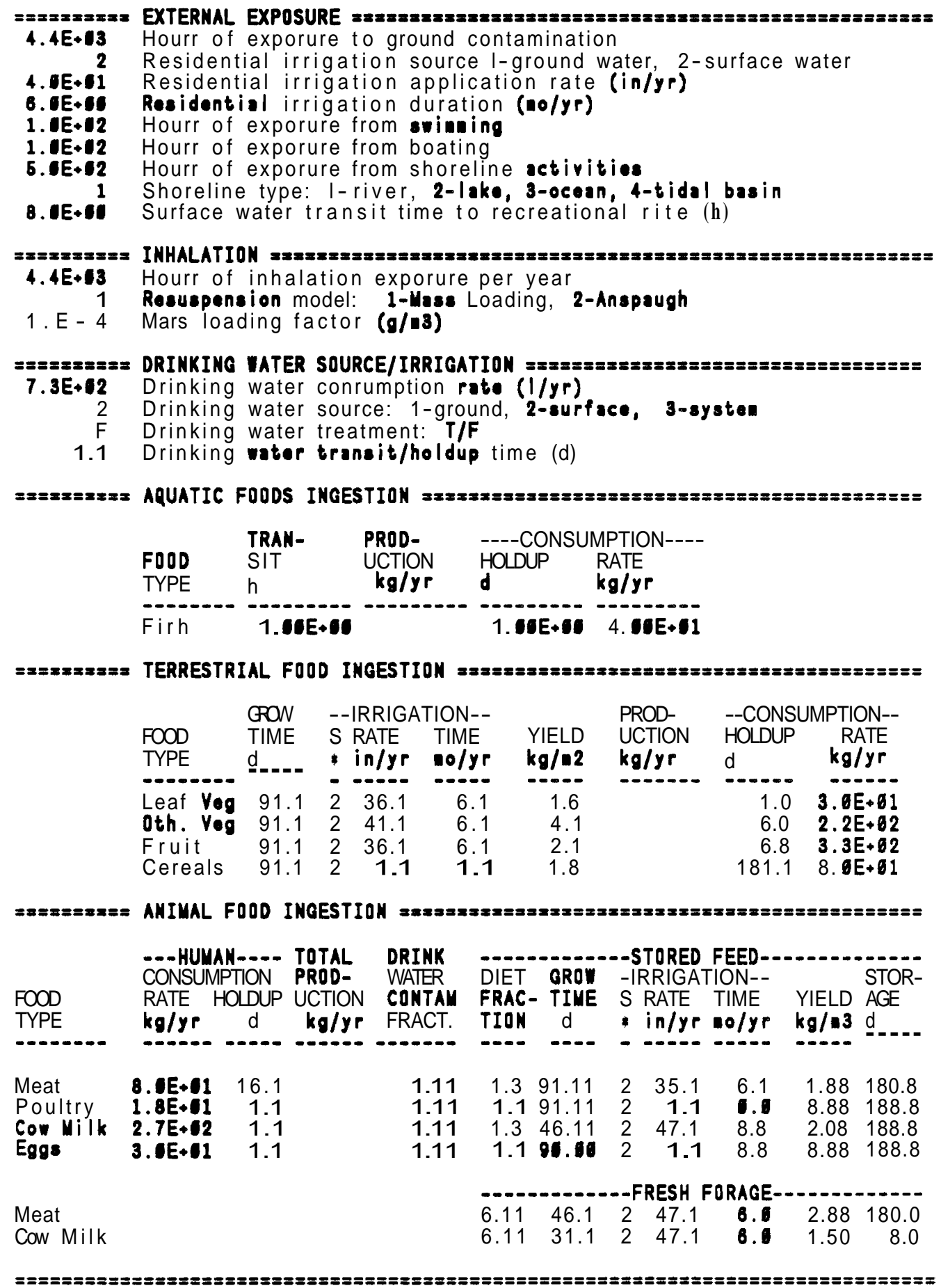


OENII Dose Calculation Program

(Version 1.395 23-Jan-89)

Case title: $\quad Y$ I dose from concentrations measured in effluent in 1964

Executed on: 19/19/89 at 11:07:13

Page E. 11

Release period:

Uptake/exposura period: Dose commitment period:

Dose units:

Inhalation Ingestion

Radio-

nuclide

$\begin{array}{ll}\mathrm{H} & \mathbf{3} \\ \mathrm{NA} & 24\end{array}$

SI 31

P 32

CA 46

$\mathrm{SC} 46$

R 61

YN 64

YN 66

FE 69

CO 60

NI 66

OU 64

$\triangle \mathbb{N} 66$

ZN 69N

\N 69

GA 72

AS 76

SR 89

SR $87 Y$

SR 90

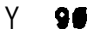

SR 91

$Y$ 91I

Y 91

SR 92

Y 92

Y 93

10 93

ZR 93

NB 93M

ZR 96

$\mathrm{NB} 95 \mathrm{M}$

NB 96

YO 99

TC 99!

TC 99
Effective Effective

Dose Dose

Equivalent Equivalent

- DE+CO

2. IE-11

1. 5E-13

1.1E-10

4. 2E-11

1.6E-10

3.1E-10

3.7E-11

2.1E-12

3. 8E-11

1.3E- 69

4. UE-18

3. E-12

1. 9E-19

8. OE-13

4. $8 \mathrm{E}-14$

1. OE-12

2. 4E-11

1.1E-11

2.3E-18

3. $8 E-11$

4. 5E-12

1. 4E-13

3. .EE-16

7.3E-11

3. 2E-14

1. 9E-13

2. UE-13

C.DE+CO

6. $5 \mathrm{E}-18$

4. $8 E-20$

1. 3E-11

1.7E-13

5.3E-11

3. $6 E-13$

5. 3E-15

- 3. 9E-18.
0.5E-08

1.3E-02

3. 1E-04

2. $2 E-1$

4.7E-94

4.3E-04

1.7E-13

2. $3 E-64$

1.7E-C3

6. GE-93

1.3E-13

8. $9 E-18$

7. 3E- 62

1.3E-91

3. $1 E-93$

2. 3E-94

1. 1E-C 2

3. 4E-62

2.7E- 4

4.9E-08

8. 8E-65

3. E-94

1. 1E- 4

1. 2E-c6

5. 4E- 96

6. $8 E-05$

7. AE-O5

C.EE+CO

1. 4E-13

5. 1E-17

6. 2E-C4

7. 2E- 97

6. 7E-95

4.1E-65

1.1E-CQ

1. $8 \mathrm{E}-11$
9. UE-U5
1.1

1.1

Ren

61.1

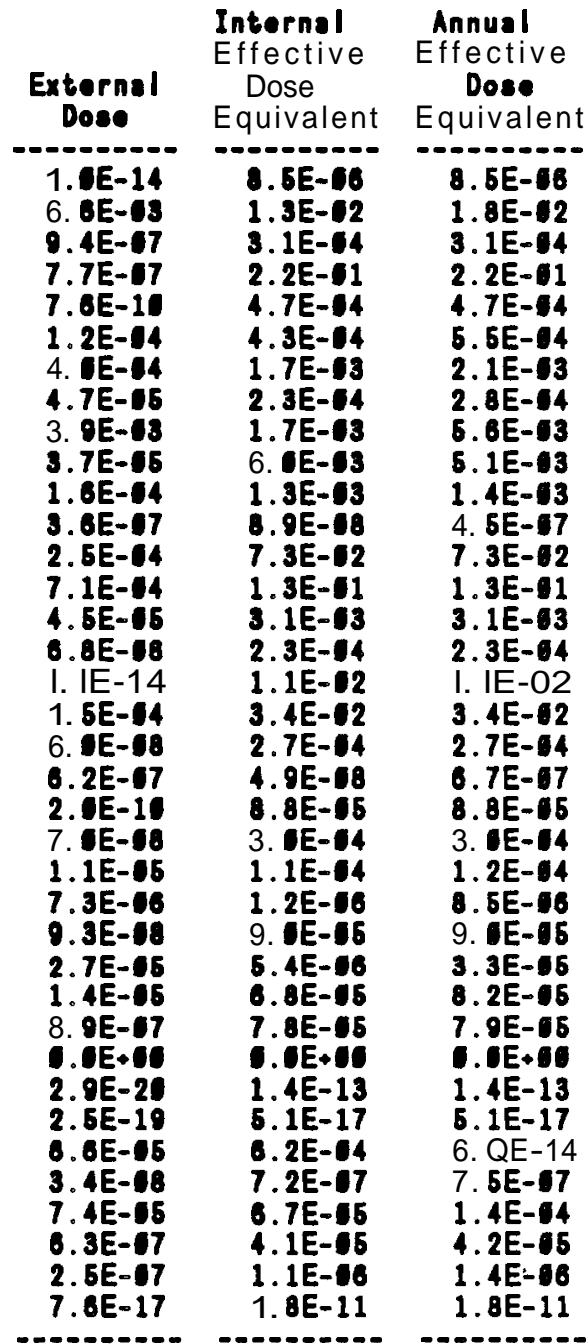




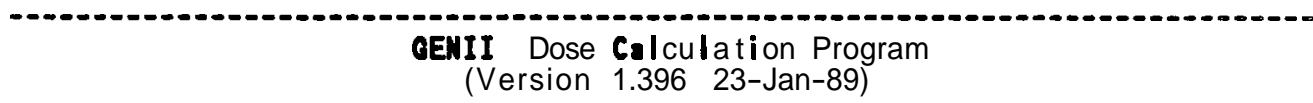

Care title: MI dose from concentrations vessured in offluent in 1964

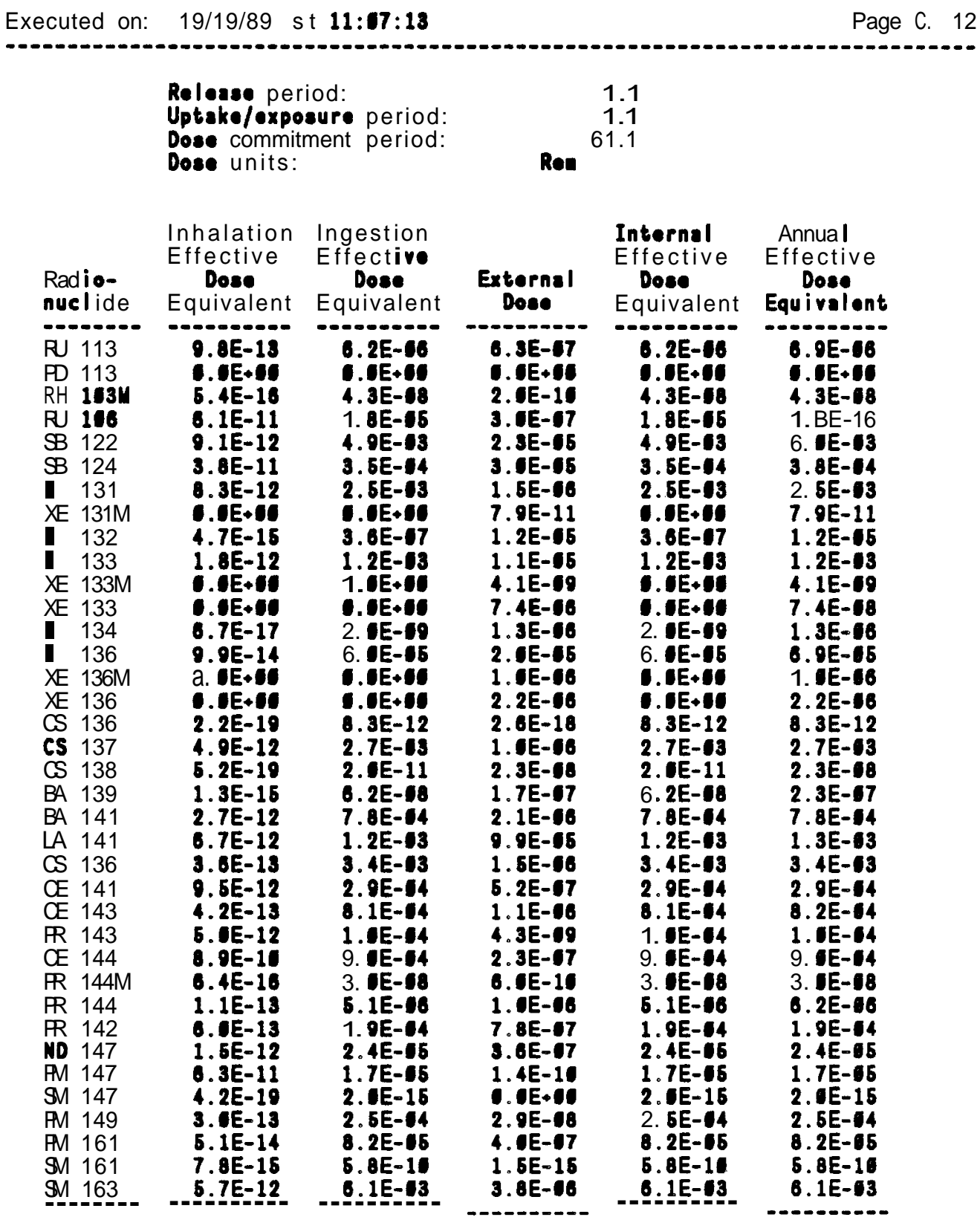


GENII Dora Caleulation Program

(Version 1.396 21-Jan-89)

Case title: MI dose from concentrations eeseured in offluent in 1964

Executad on: 19/19/89 at 11:07:13

Page C. 13

Release period:

Uptake/exposure pariod:

Dora connitment pariod:

Dora units:

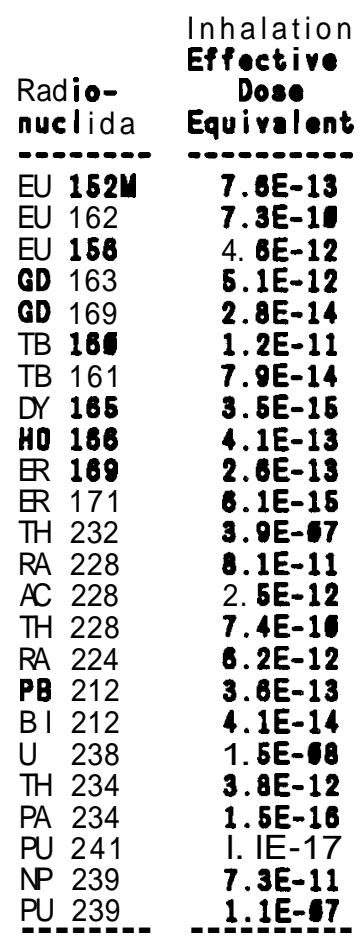

Ingestion

Effactive Dose Equivalent

2. 4E-93

2.3E- 64

3. 2E- 54

6. GE-66

1.6E- 04

2. $4 E-95$

2. $8 \mathrm{E}-68$

3.1E-67

7. 4E-C4

6. $8 E-15$

3. E- 65

5. 4E-C3

1. $E-A 5$

2. 5 E- 68

8. $\mathrm{HE}-17$

2. $6 \mathrm{E}-07$

9. 2E- 98

2. $4 E-69$

7. $4 E-16$

3.1E-68

8. 3E-10

8. 2E-94

6. IE- 2

8. 2 E-c 4
1.1

1.1
61.1

Ram
Internal Annual

Effective Effective Dose Dose

Equivalent Equivalent

Dora

3. UE-65

4. $E-15$
5. $4 E-66$

$9.1 E-68$

9.4E-68

5. 4E-16

2.7E-09

2.7E-97

1.8E-67

6. 8E-11

5. 6E- 7

2. $4 E-10$

6. $9 \mathrm{E}-18$

2. $E-17$

2. 4E-11

1. $9 E-10$

2. $4 E-69$

3. $2 E-68$

3. 6E-11

2. $2 \mathrm{E}-68$

3. 5E- 49

7.7E-11

I. IE-14

1. 6E-10
2. 4E-93

2. 7E- 4

3. $3 E-64$

5. 1E-86

1. $8 \mathrm{E}-94$

2.9E-65

2. $6 E-96$

5.7E- 7

6. 6E-95

3. 1E-95

5. $4 E-93$

1. E-65

2. 3E- 97

8. EE- 7

ด. $5 E-68$

3. $4 \mathrm{E}-68$

7. $5 E-66$

3. 1E-66

4. 3E-69

8. $2 E-94$

5. BE-02

8. 2E-64
7. 4 E- 94

2. 6E- 97 
OENII Dora Calculation Program

(Version 1.396 23-Jan-89)

Case title: $Y$ I dose from concentrations measured at Richland in 1968

Executed on: 19/19/89 at 19:32:32 Page A. 1

This is a near field (nerrowly-focused, single site) scenario.

Release ir chronic

Individual dose

THE FOLLOWING EXPOSURE PATHS ARE CONSIDERED:

Ground, external

Recreation, external

Inhalation uptake

Drinking woter ingartion

Aquatic foodr ingeation

Terrestrial foods ingartion

Animal product ingartion

THE FOLLONIMO TINES ARE USED:

Intake ends aft $a r$ ( $y r)$ :

Dora calculations ends after $(y r)$ :

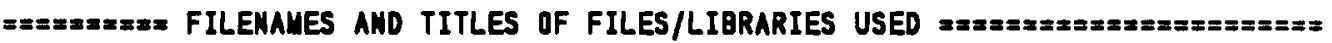

Input file nane: lGENII/BANDIR/river68. in

METABOLIC PARAMETERS-
RYDLIB - Radionuclida Master Library (29-Aug-88 RAP)
Food Transfer Factor Library - (RAP 29-Aug-88) (UPDA

$9-18-89$
$8-12-88$

8-29-88

Food Transfer Factor Library - (RAP 29-Aug-88) (UPDATED LEACHING FA 8-29-88

Biosccuaulation Factor Library - (34-Aug-88) RAP (p-32, zn, np modi 9-12-89

External Dora Factors for GENII in person Sy/yr par Bq/n (28-Aug-88 8-29-88

Internal Yearly Dose Increnents (Sv/Bq) 29-Aug-88 RAP 8-29-88

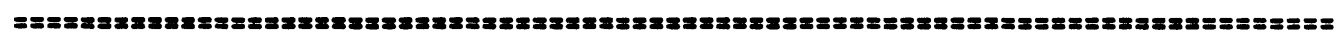

1 Surface soil input unit: (1-n2, 2-13, 3-kg)

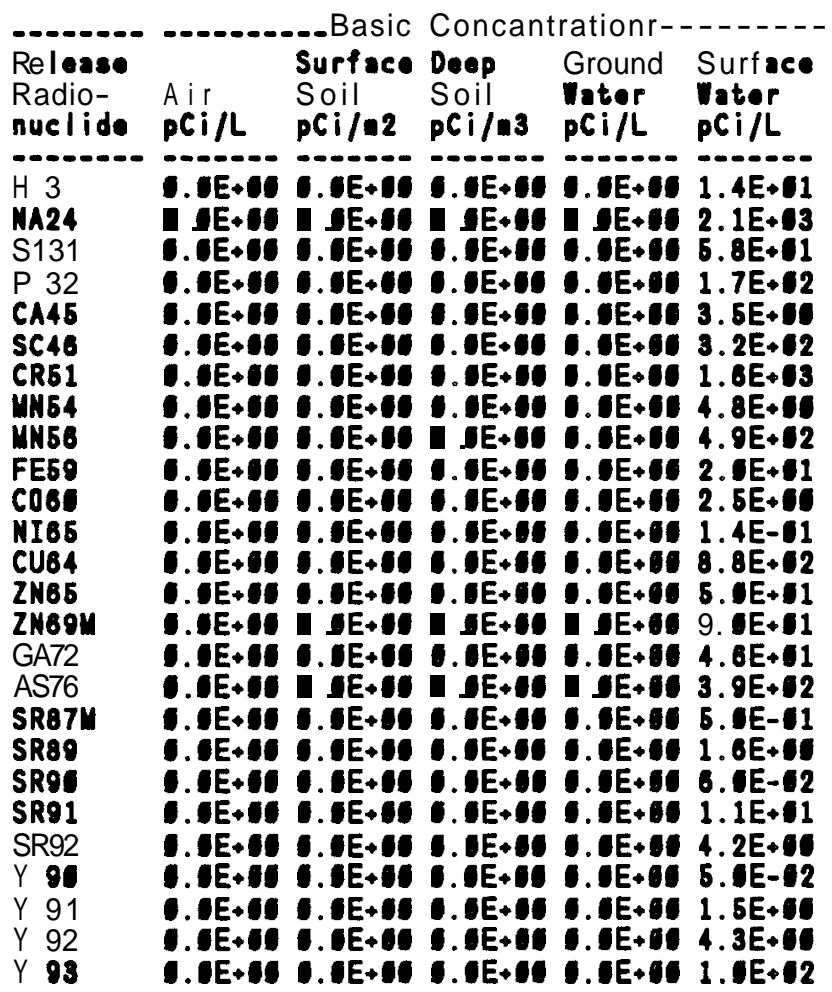




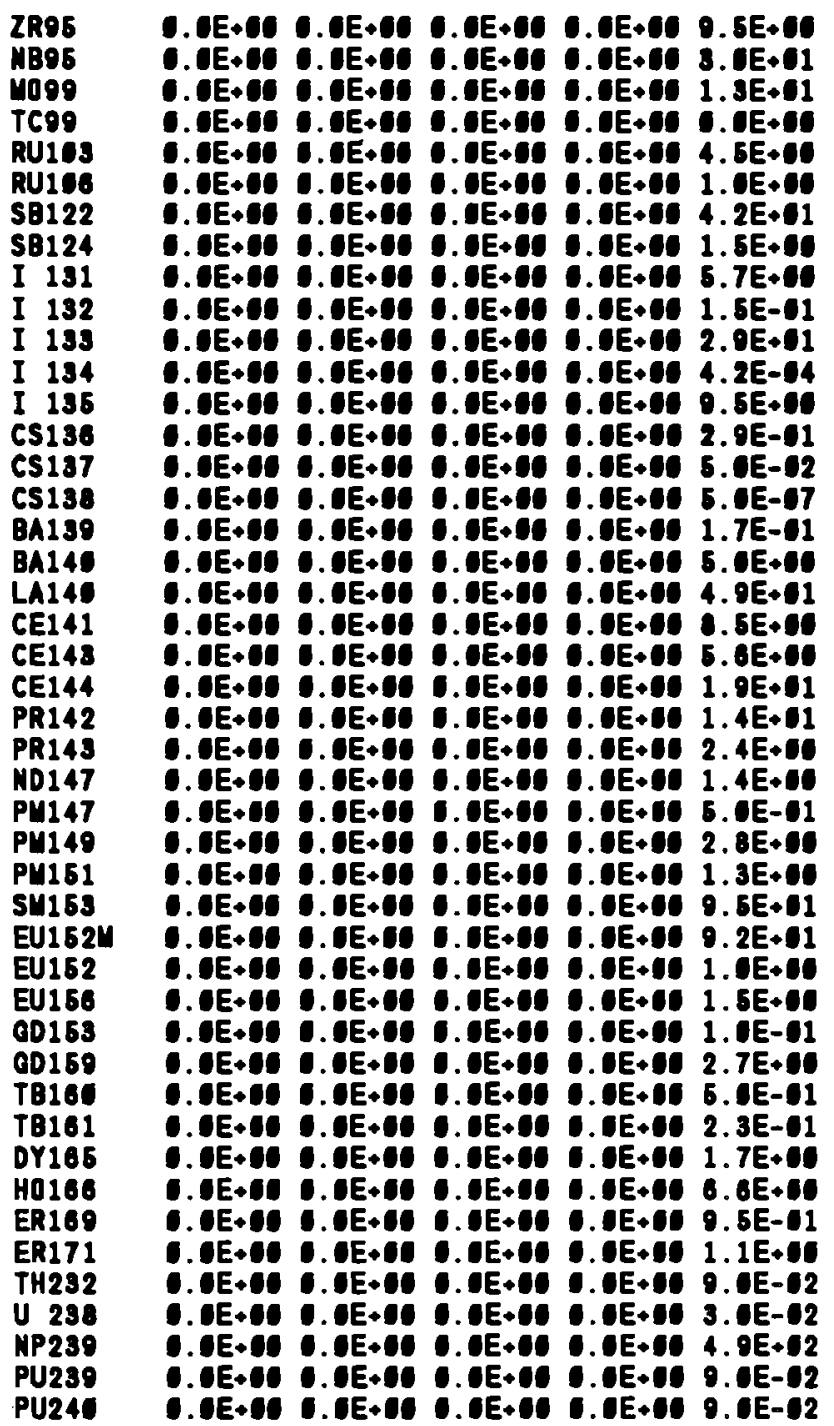

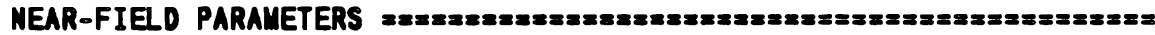
Inventory disposed $n$ years prior to beginning of intake period

- LOIC occurred $n$ years prior to beginning of intake period

1. EE+S Fraction of roots in upper soil (top $16 \mathrm{~cm}$ )

-. EE+U1 Fraction of roots in deep roil

-.UE +0 Manual redistribution: deep soil/surface roil dilution factor

1250.5 Source area for external dose modification factor ( $(\mathbf{2})$

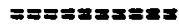

$4.4 E+03$

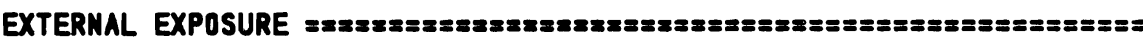
Hours of exporure to ground contamination

2 Residential irrigation soures 1-ground water, 2-surface water

4.0E+01 Residential irrigation application rate (in/yr)

6.DE+OI Residential irrigation duration (no/yr)

1.0E+02 Hours of exporure from swimming

1. EE+12 Hourr of exporurr from boating

5. EE+ 02 Hours of exporurr from rhoreline activities

1 Shoreline type: 1-river, 2-lake, 3-ocean, 4-tidal basin 


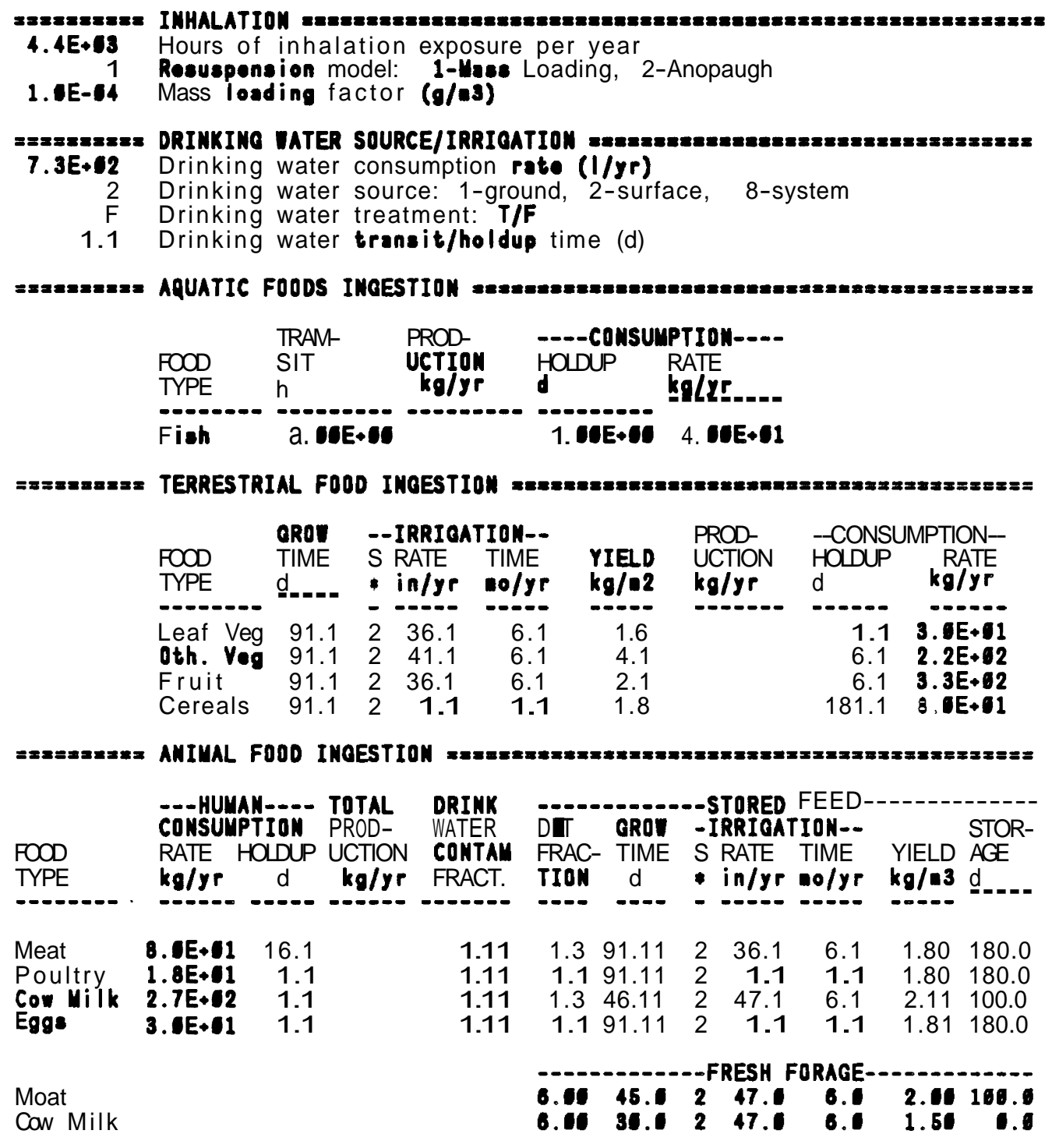

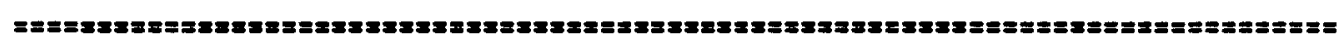


GENII Dose Calculation Program

(Version 1.396 23-Jan-89)

Case title: Ml dose from concentrations measured at Richland in 1968

Executed on: 19/19/89 at $99: 49: 44$

Page C. 11

Uptake/exposure period:

Dose commitment period:

Dose units:

Inhalation Ingestion

Radio-

nuclide

H 3

NA 24

SI 31

P 32

CA 46

SC 46

CR 61

UN 64

MN 58

FE 69

CO 61

NI 66

CU 64

ZN 66

2N B9I

ZN 69

GA 72

AS 76

SR 89

SR $87 \mathrm{M}$

SR 91

Y 91

SR 91

$Y$ 91U

Y 91

SR 92

Y 92

Y 93

yo 93

דR 93

NB 93M

ZR 96

NB 95M

MB 96

MO 99

TC $99 \mathrm{M}$

TC 99
Effective

Dose

\section{Effective}

Equivalent

a. $\mathbf{E}+\boldsymbol{I I}$

1. $1 E-11$

9.7E-15

1. EE-10

2. 2E-11

5.4E-69

1. 1E-10

4. E E-11

1. $4 E-13$

8. 1E-11

8. 8E-10

2. 8 E-17

6. 0 E-13

I. IE-89

3. 2E-13

1. 5E-14

3. $8 \mathrm{E}-13$

1.3E-11

3. $3 E-12$

1. $8 \mathrm{E}-17$

2. 2E-11

$9.6 E-13$

2. $9 E-14$

7. EE-16

3. 4E-11

2. $2 E-15$

7. E-15

7.4E-13

C.OE+CO

1. 1E-17

1. EE-10

6. 8E-11

6.8E-14

6. 8E-11

5. $4 E-13$

7. 5E-15

5. 5E-18.
Dose

Equivalent

1.3E-16

5. 3E-93

2. $6 E-06$

2. EE- 1

2. $8 \mathrm{E}-64$

1. $6 \mathrm{E}-12$

5. 8 E- 44

2. 5E- 64

1.7E-65

1. 1E-02

8. 4 E- 4

1. 4E-69

1. $2 E-02$

6. 9E- 12

7.7E-94

5. 6 E- 05

2.7E-03

1. 4E-C2

7. 8E-05

1. 6 E- 99

5. 7E-OS

2.6E-66

1. 4E-D5

1. 4E- 07

4. EE-95

I. IE-17

8. 2E-67

1.7E-64

c. 0 + +0

2. 9E-13

1.1E-16

3. $3 E-14$

3. $9 E-07$

4. 2E-94

6. E-S5

1. $6 \mathrm{E}-08$

2.5E-11
1.1

Ren

61.1

Internsl Annual

Dose

Equivalent Dose

1.6E-15

3.1E-13

6. 2E- 18

7.2E-67

4. EE-10

4. EE-13

1. 4E- 14

5. 2E-15

2. $5 E-94$

8.1E-65

1.1E-04

2. 4E-68

6.1E-65

3. $9 E-04$

1. $8 E-65$

2. $E-69$

4. 2E-65

7. 5E- 65

1. 4E-68

4. 8E-68

1. 3E-10

7. EE-O9

2. 4E- 98

1. 2E-67

4. 2E-98

1. $9 E-68$

3. BE-17

3. 2E-68

๑.UE +61

5. OE-20

5. 4E-19

3. $6 E-65$

1. BE-18

I. IE-14

9.6E-47

1.7E-G7

1. 1E-16

\section{3E-66}

5. 3E- 63

2.6E- 06

2. IE-J1

2.6E-64

1. 5E-62

5. $6 \mathrm{E}-\mathrm{G} 4$

2. 5E-64

1.7E-65

1.1E- 2

8. 4E- 4

1. 4E- 69

1. 2E- 2

6. $9 E-02$

7. 7E-64

5. 6E- 55

2.7E-63

1. 4E- 2

7.8E-C5

1. TE-19

5. 7E- 5

2.6E-66

1. 4E- 65

1. $4 E-67$

4. EE- 5

I. IE-17

8. 2E-67

1. $7 E-4$

1. $0 E+60$

2.9E-13

1.1E-16

3. 3E- 64

3. 9E- 17

4. 2E-64

6. AE-U5

1. 6E-D6

2.5E-11
Equivalent

1.3E- 16

8. $4 \mathrm{E}-93$

2.7E- 68

2. E- 1

2. $8 E-64$

1. QE-92

7. EE-04

3. E- 4

2.7E-64

I. IE-12

9. 5E-g4

2. 5E-98

1. 2E-g2

7. EE-62

7.9E-64

5.6E- 5

2.7E-63

1. $4 E-62$

7. BE-16

4. $9 \mathrm{E}-68$

5.7E-65

2. $8 \mathrm{E}-96$

1.6E- 5

2.7E- 67

4. EE- 65

2.

1. 2E-66

1. 8E- 4

6. $6 \mathrm{E}+90$

2. 9 E-13

1. $1 \mathrm{E}-16$

3.7E-64

4.1E-67

5. 2E-94

6. EE- 5

$1.8 \mathrm{E}-66$

2. 5E-11 


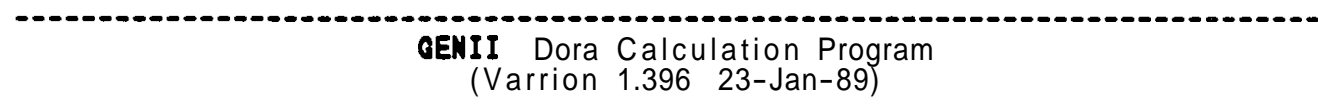

Case title: MI dose from concentrations measured at Richland in 1968

Exacutrd on: $19 / 19 / 89$ at $\mathbf{9 9 : 4 9 : 4 4}$
$\begin{aligned} & \text { Uptake/exposure pariod: } \\ & \begin{array}{l}\text { Dora conpitaent pariod: } \\ \text { Dora unitr: }\end{array}\end{aligned}$

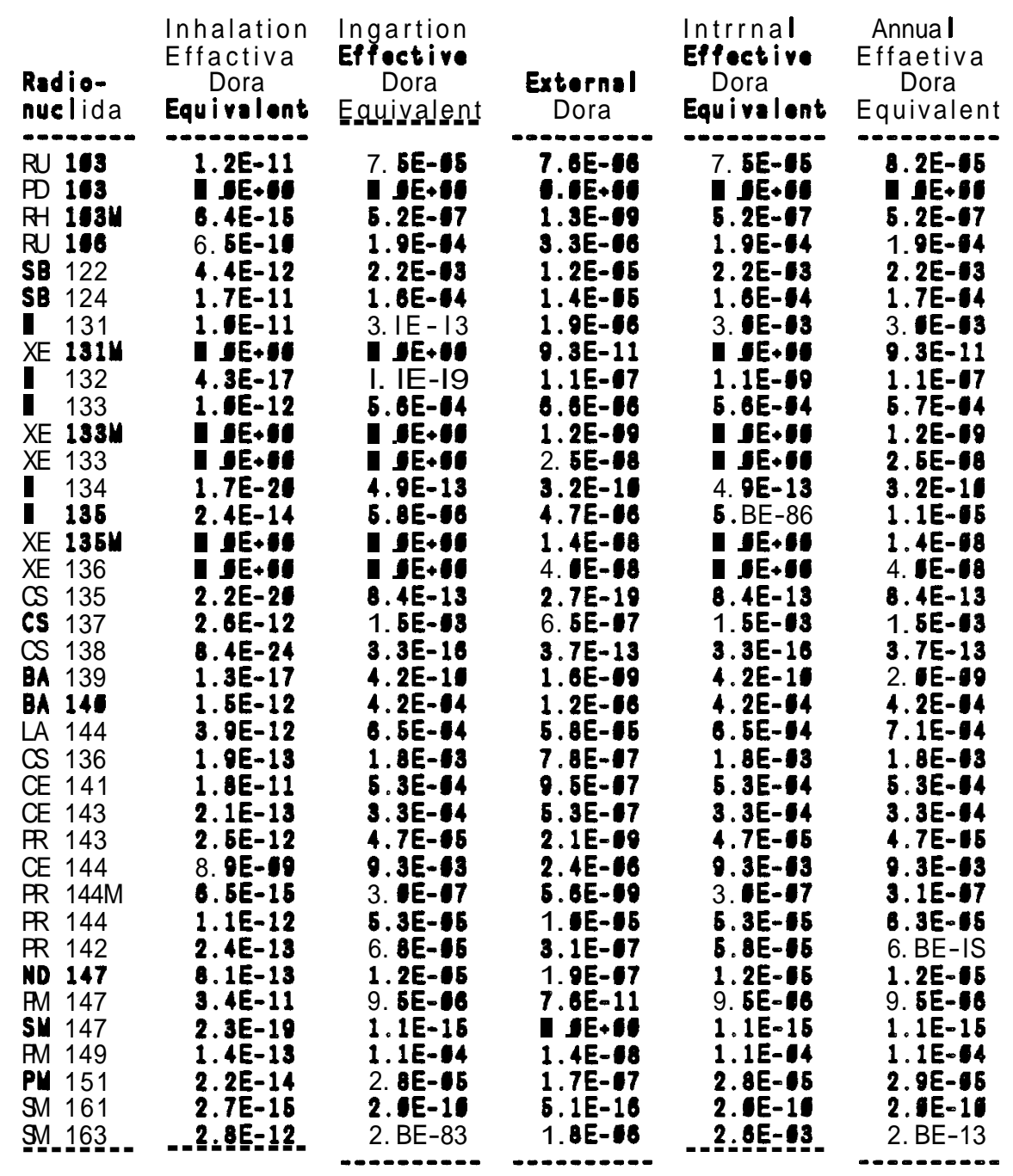




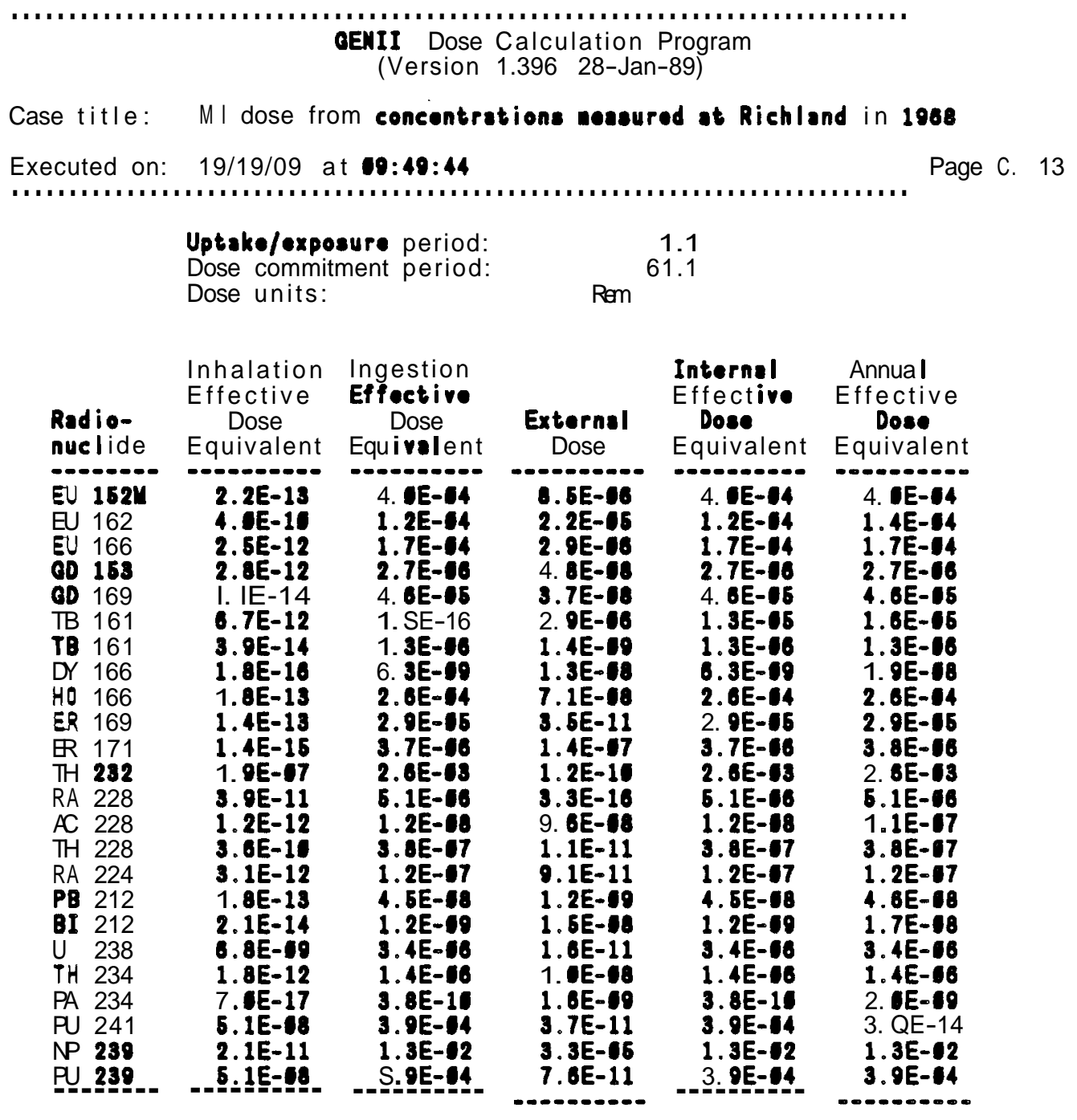




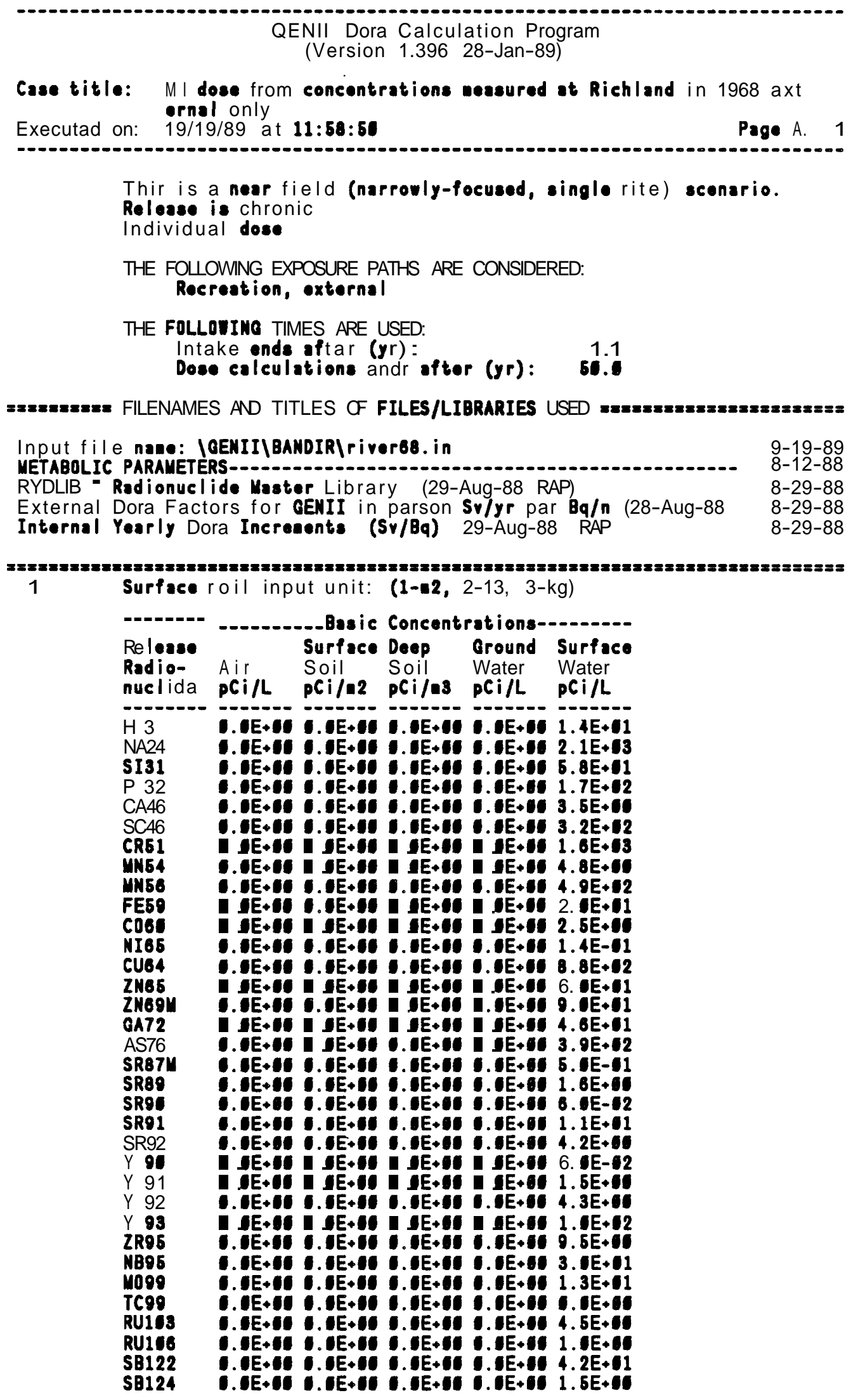




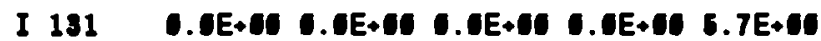

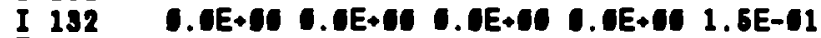

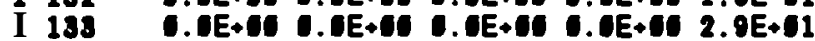

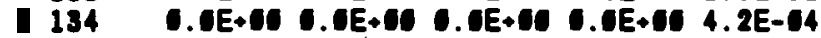

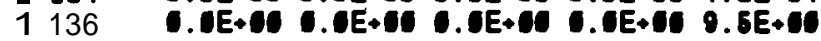

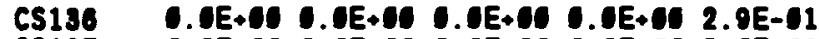

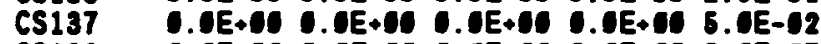

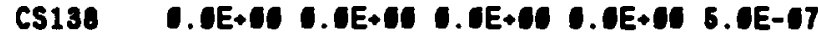

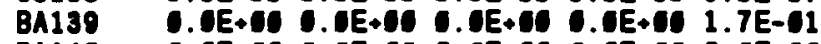

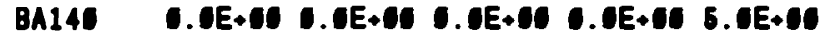

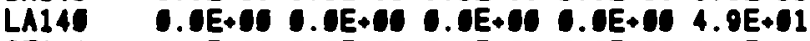

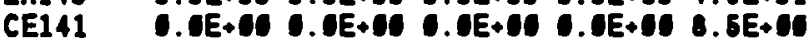

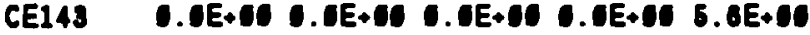

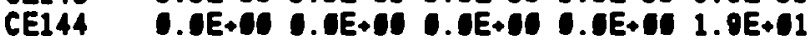

PR142 0.0E+01 0.0E+01 0.0E+00 0.0E+00 1.4E+01

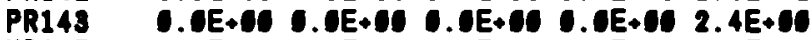

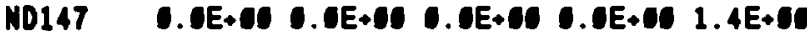

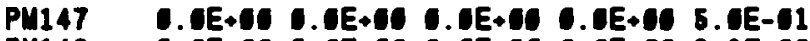

PU149 0.0E+06 5.0E+00 J.0E+60 J.0E+00 2.8E+10

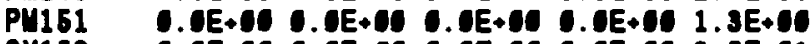

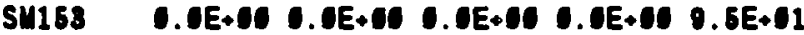

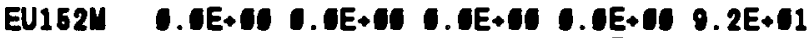

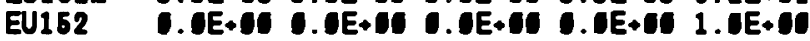

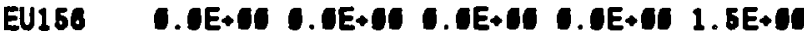

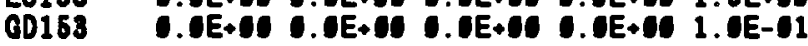

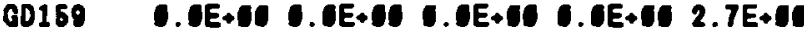

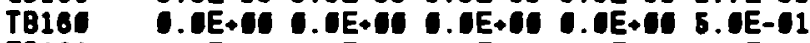

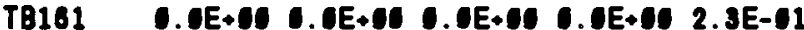

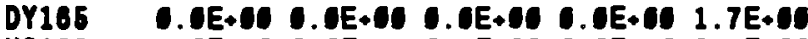

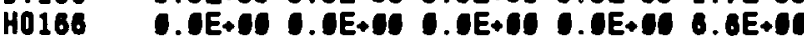

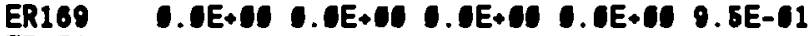

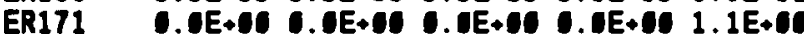

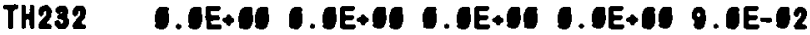

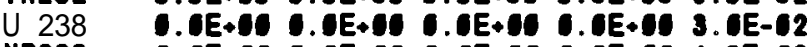

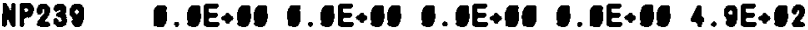

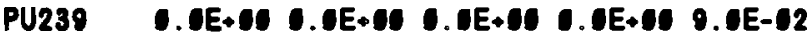

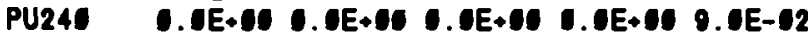

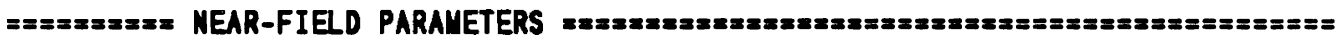
- Inventory disposed $\boldsymbol{n}$ years prior to beginning of intake period
LOIC occurred $n$ years prior to beginning of intake period Fraction of roots in upper soil (top $16 \mathrm{~cm}$ ) Fraction of roots in deep roil

$1.0 E+9$

6.SE+G0

Manual redistribution: deep soil/surface soil dilution factor

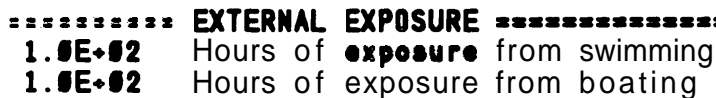

5.JE+12 Hours of exposure from shoreline activities

1 Shoreline type: 1-pivor, 2-lake, I-ocean, 4-tidal basin
} 


\section{OENII Dose Calculation Program}

(Version 1.396 23-Jan-89)

Care title: MI dose from concentrations measured at Richland in 1968 ext ernal only

Executed on: 89/19/89 at 12:09:34

Page C. 9

Uptake/exposure period:

Dose commitment period:

Dose units:

Inhalation Ingestion

Effective Effective

Radio-

nuelide

H 3

NA 24

SI 31

P 32

CA 46

SC 46

CR 61

YN 64

YN 66

FE 69

CO 60

NI 66

CU 64

$\mathbb{Z N} 66$

$\mathbb{N}$ 69N

¿N 69

GA 72

AS 76

R 89

S 87M

界 9

$Y$ os

S 91

$Y$ 911

Y 91

R 92

Y 92

Y 93

YO 93

XR 93

NB 93Y

$\mathbb{Z R} 96$

N 95M

N 96

YO 99

TC 994

TC 99
Dose

Equivalent

IE+CO

IE+OO

IE+CO

I. SE+CO

- SE+DO

C.SE+CO

I SE+CS

I SE+WO

G.SE+CO

IE+CO

I $\mathrm{E}+\boldsymbol{D S}$

CE+CO

IE-CS

IE+II

- IE+CS

IE+GO

I $S E+C 0$

IE+CO

IE+CI

I $E+00$

IE+US

IE+UI

IE+CO

IE+OS

IE+OS

I SE+CS

IE+UI

- DE+CO

- $S E+Q 0$

IE+CY

IE+CS

I E + US

IE+DO

IE+UI

I $S E+0$

I GE+

IE+ 19
Dose

Equivalent

1. BE-19

5. 7E- 06

6. 8E-C8

2. $9 E-10$

2. 2E-18

3. $9 E-16$

4.6E- 17

2. $7 E-18$

9.8E-67

2.6E- 67

6. GE-18

1.8E-10

8. $8 \mathrm{E}-67$

1. $3 E-68$

2. 3E- 07

I. $\mathrm{E}+00$

4.3E- 7

4.1E-66

2. 8E-18

1. 2E-10

1. 5E-18

I. IE-19

5. 4E- 18

I $5 E+01$

2. 8E- 48

1. $4 E-68$

1. 7E- 88

9. 6E- 07

- $\mathrm{EE}+00$

IE+

IE+UI

7. 1 E- 98

IE+US

1. 5E-97

8. UE-08

a. $\mathbf{E}+00$

IEE+I!
1.9

Pem

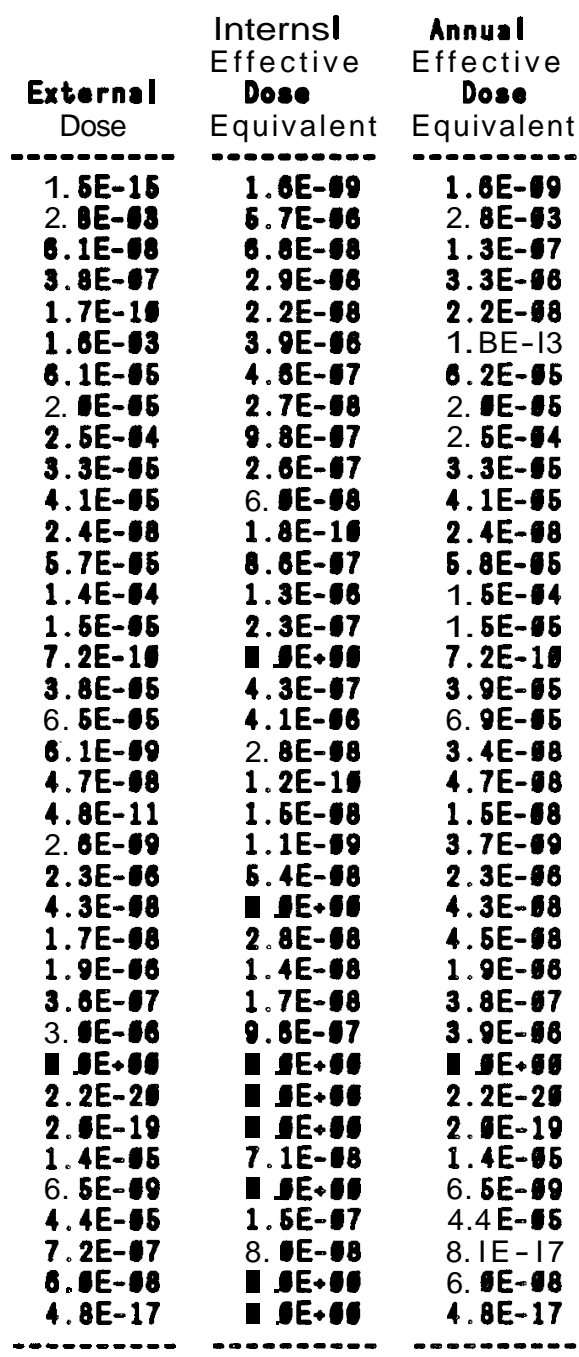


GENII Dose Calculation Program

(Version 1.396 23-Jan-89)

Case title: MI dose from concentrations measurod at Richland in 1968 ext

Executed on: 19/19/89 at 12:19:34

Page C. 10

Uptake/exposure period:

Dose commitment period:

Dose units:

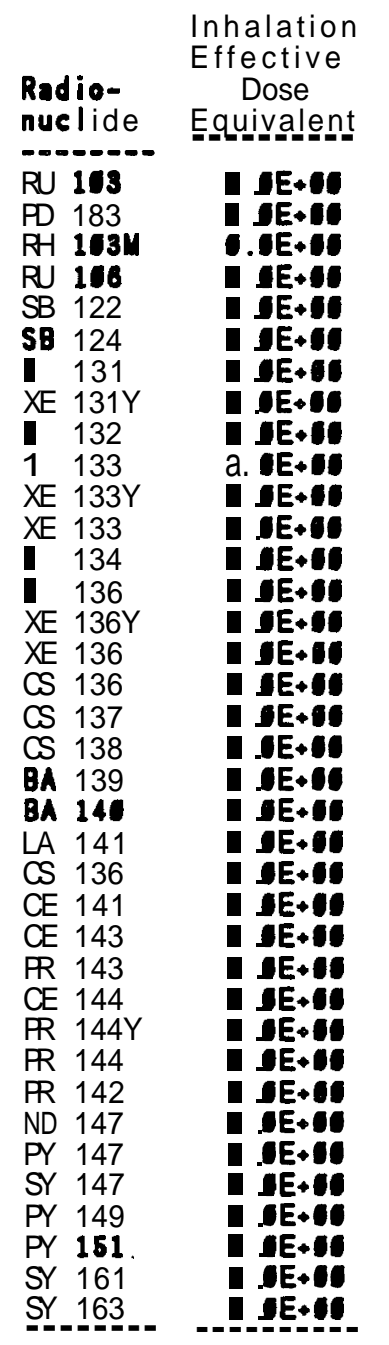

Ingestion

Effective

Dose

Equivalent

$2.75-68$

2.7E-98

IE+ $T$

6. 3E- 68

6. 2 E- 7

3. $5-98$

5. 5E- 07

I $E+00$

2.1E-19

5. $6 \mathrm{E}-67$

IE+CE

- $A E+C$

2. $2 E-13$

4. E-18

I. $\mathrm{E}+00$

IE+DS

$1 E E+C 0$

4. BE-A9

1. $9 E-16$

1. 4 E-10

9. 1E-c8

8.1E-67

6. EE-69

4. $9 E-08$

5. 1E-88

2.3E- 68

8. $-\mathrm{E}-67$

- EE+CI

IE+CI

1. 5E- 7

1. $2 E-08$

1. $0-19$

IE+CE

2. $3 \mathrm{E}-08$

7. $9 E-09$

IE+

6.7E-67
1.1

Ren

61.0

Internal Annual

Dose Dose

External

Dose

Equivalent

Equivalent

3.2E-18 2.7E-98 3.3E-68

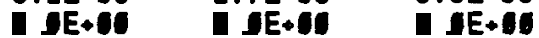

4.9E-10 $\square E+10$ 4. $9 E-10$

$1.2 E-18$ 5.3E-98 $1.3 \mathrm{E}-96$

8.7E-66 6.2E- $17 \quad 9.3 E-66$

5.6E- 36 3. 5 E- $98 \quad 5.7 \mathrm{E}-66$

1.1E-16 6. 5E-07 1.7E-96

3.5E-11 IE+90 3.5E-11

I. IE-I7 2.1E-10 I. IE-17

6. $8 E-98$

4.3E-10

9. 4E-99

3. $2 E-10$

4. $5 E-10$

5.1E- 19

1. $5 E-98$

9. $5 \mathrm{E}-25$

2. SE-D7

3. $7 E-13$

1. BE- 09

6. 3E-67

4. $2 \mathrm{E}-65$

3.9E-G7

4. $3 E-67$

4. $5 E-07$

1.1E-U9

9. $\mathrm{TE}-97$

2. $E-10$

3. 7E-68

2. $8 E-67$

1.GE-T

2. $9 E-11$

IE+O0

1. 1E-18

1. 5E- 1

1. $9 E-16$

1. $6 E-60$
$5.65-17$

IE+CO

(.) $\mathrm{E}+00$

2. 2E-13

4. GE-08

I. $. \mathrm{E}+00$

(1) $E+00$

IE+CL

4. BE-69

1. $9 E-16$

1. 4E-10

9.1E-18

8. 1E-J7

6. E-19

4. 9E-18

$5.1 E-98$

2. 3E-18

8. E-T7

IE+DE

IE $\mathrm{SES}$

1. 5E- 67

1. 2E-98

1. $\mathrm{UE}-99$

I. $\mathrm{E}+10$

2. 3E-48

7. OE-O9

I $\mathrm{EE}+00$

5.7E-97.
6. $4 \mathrm{E}-96$

4.3E-10

9. 4E- 69

3. 2E-10

4. $5 E-66$

5. 1E-69

1. 5E-08

9.5E-20

2.1E-97

3. $7 E-13$

1.7E-69

7. 2E-67

4. 3E-95

4. EE-07

4. $8 E-67$

5. E- 07

2. 4 E- 98

1.7E- 66

2. बE-69

3. $7 E-66$

4. 3E-07

I. IE-I7

1. 1E-09

a. $E+80$

3. 4 E- 08

1. BE-I7

1. $9 E-16$

2. 1E- 86 


\section{GENII Dose Calculation Program}

(Version 1.396 23-Jan-89)

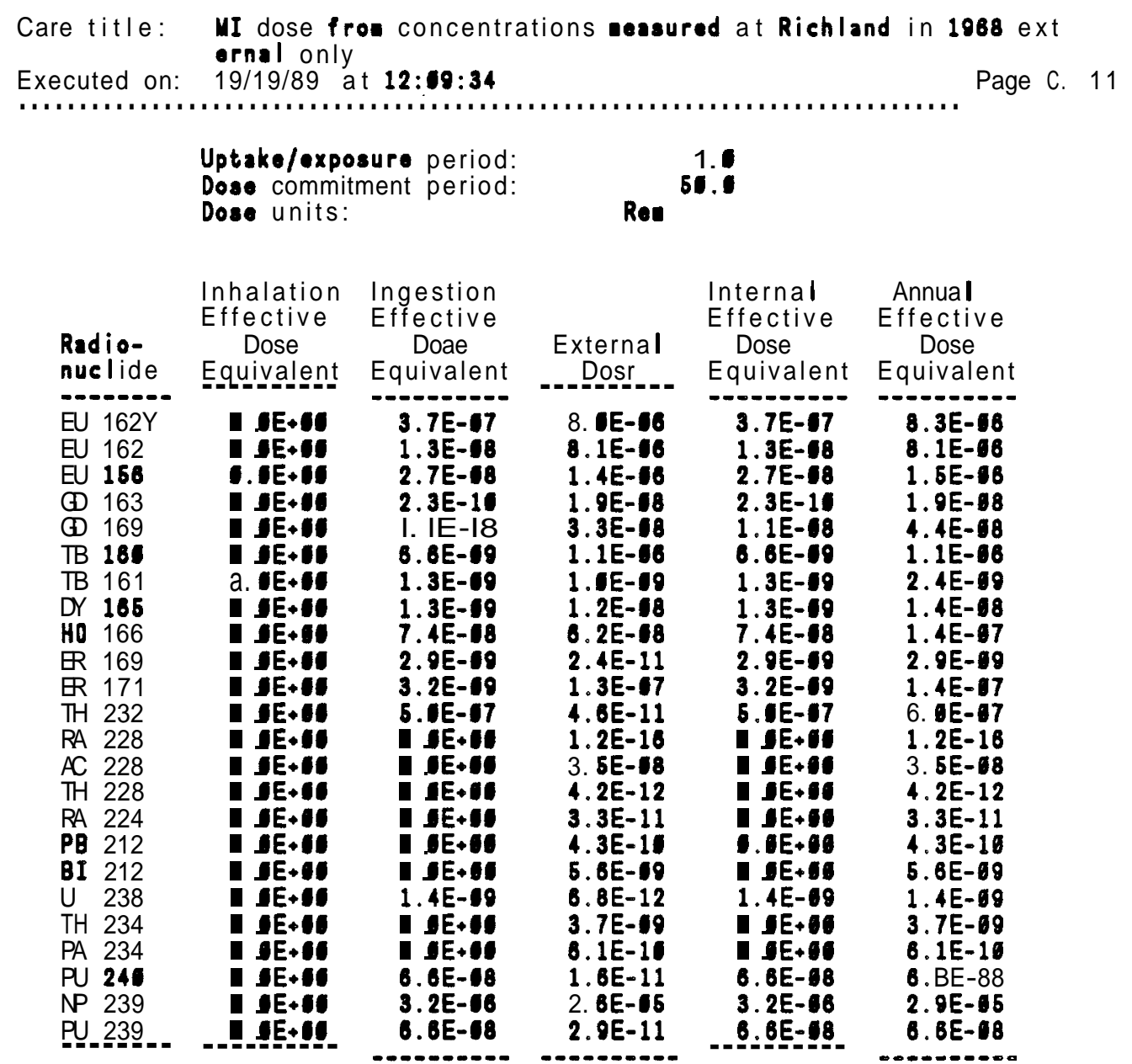




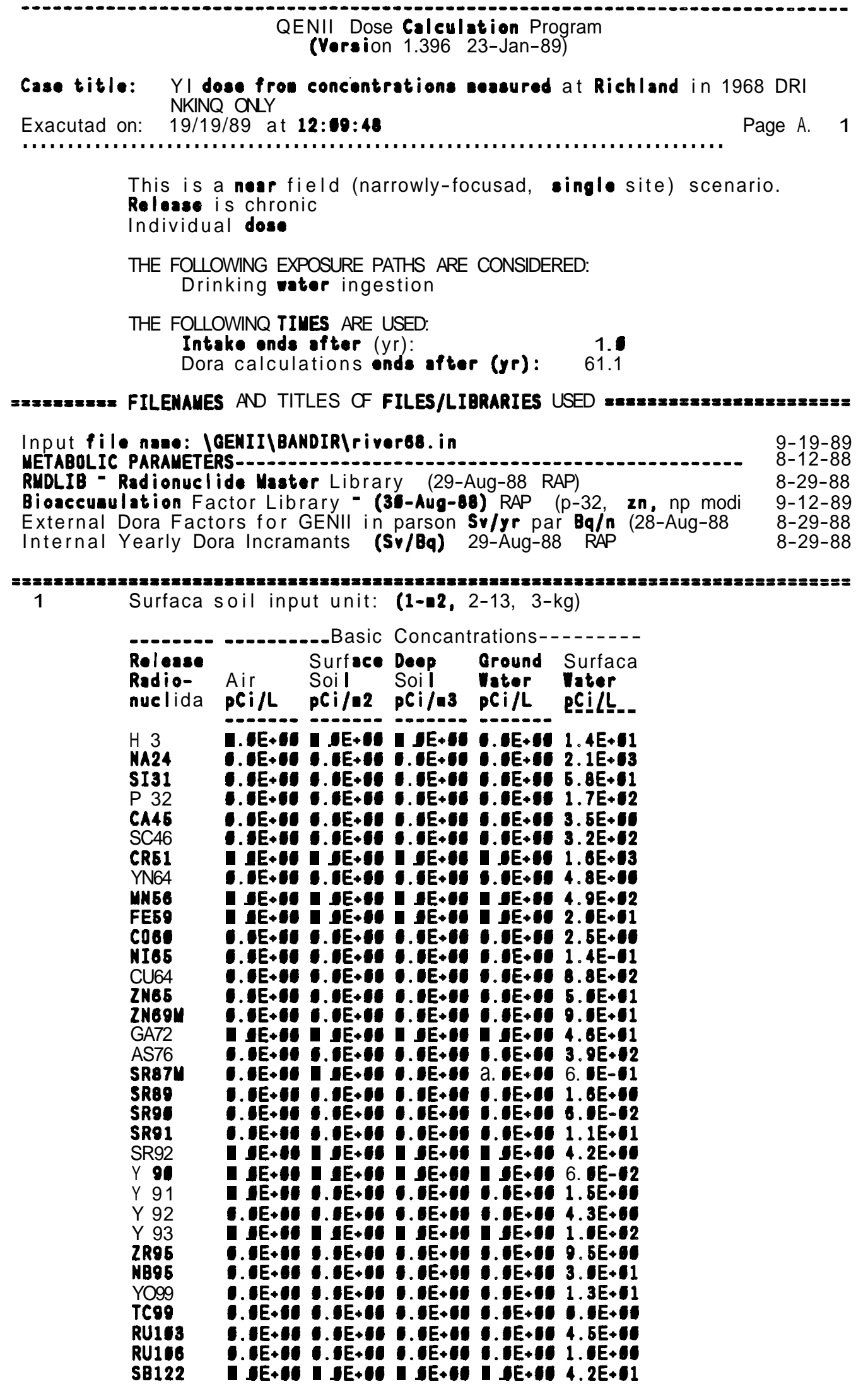




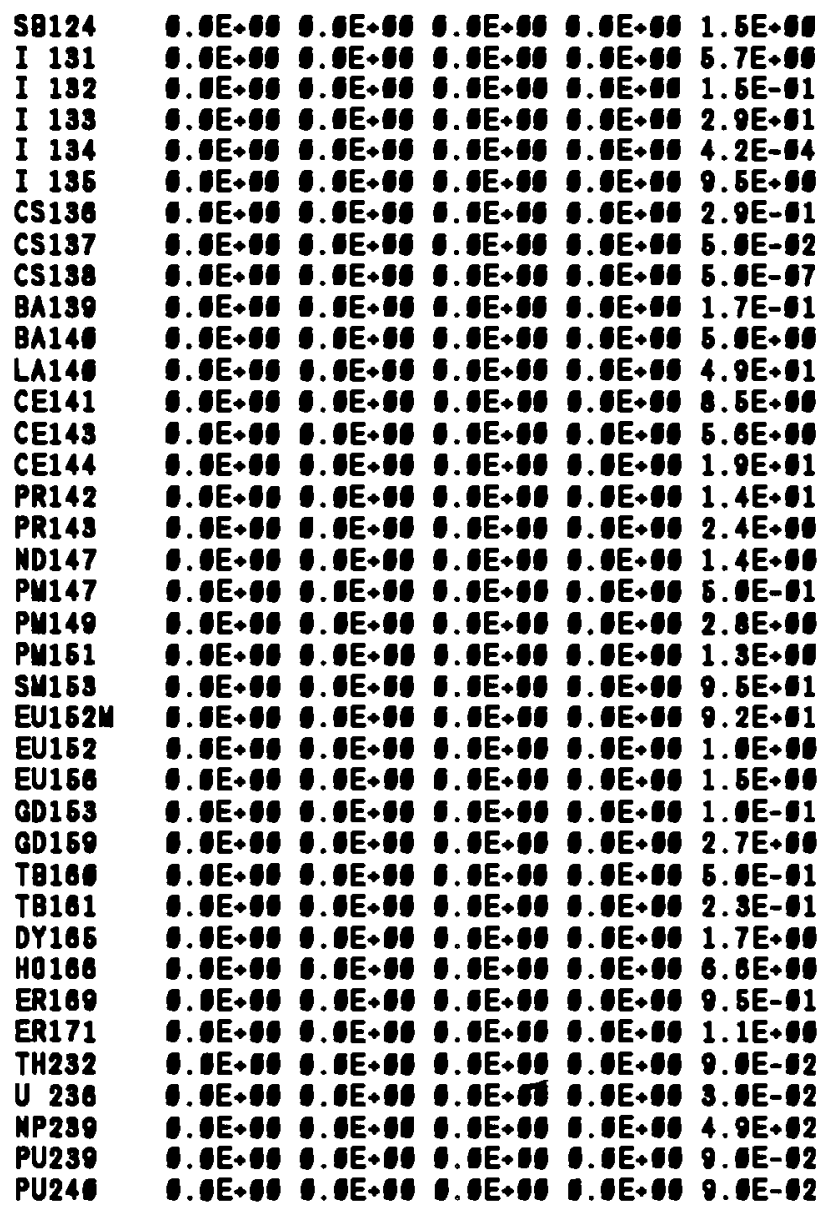

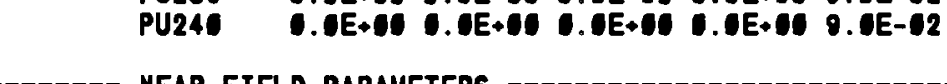

\begin{tabular}{|c|c|}
\hline I. \# & $\begin{array}{l}\text { Inventory disposed } n \text { years prior to beginning of intake period } \\
\text { LOIC occurred } n \text { years prior to beginning of intake period } \\
\text { Fraction of roots in upper soil (top } \mathbf{1 6} \mathbf{c a} \text { ) } \\
\text { Fraction of roots in deep soil } \\
\text { Manusl redistribution: deep soil/surface soil dilution factor }\end{array}$ \\
\hline 7.3E & $\begin{array}{l}\text { Drinking rater consueption rate (1/yr) } \\
\text { Drinking water source: 1-ground, 2-surface, 3-system } \\
\text { Drinking water treatment: T/F } \\
\text { Drinking water transit/holdup time (d) }\end{array}$ \\
\hline
\end{tabular}


GENII Dorm Calculation Program

(Version 1.396 23-Jan-89)

Case title: MI dose from concentrations measured at Richland in 1988 DRI MKIMe ONY

Executed on: 89/19/89 at 12:24:28 Page C. 9

Uptake/exposure period:

Dose commitaent period:

Dose units:

Inhalation Ingestion

Effective Effective

Radio-

Dose

nuclide

H 3

NA 24

SI 31

P 32

CA 46

sc 46

CR 61

IN 64

MN 66

FE 69

CO 85

NI 66

a 64

ZN 66

\N B9Y

ZN 89

GA 72

AS 76

SR 89

S 87M

SR 91

$Y 9$

SR 91

$Y$ 91I

Y 91

SR 92

Y 92

Y 93

Mo 93

דR 93

NB 93M

דR 96

NB 95U

NB 96

MO 99

TC 994

TC 99
Equivalent

I.

IE+OO

C. E+ +00

IE+15

IE+09

IE+DS

-. E+CI

IE+CS

IE+CS

CE+CS

$\mathrm{DE}+00$

I $E+0 S$

IE+GI

IE+CI

IIE+OS

IE+CO

I. $E+10$

- $D E+00$

I SE+CO

IE+CS

ISE+CI

8. $\mathrm{E}+01$

IE+II

I. $. E+B S$

IE+CS

IE+OS

I SE+MS

I $\mathrm{JE}+\mathrm{CO}$

I SE+GS

- IE+CO

IE+OS

- EE+CS

IE.UE

IE+OS

- IE+CO

I $E+00$

IE+II Dose

Equivalent

5.7E- 7

6. $9 E-04$

4.2E-98

1. EE- 3

7. 8E-68

1. 4 E-C3

$1.6 E-04$

9. 7E-06

6. 6E-07

9. 2E-A5

1.8E-C5

9.1E-11

8.1E-65

4. $6 E-4$

2.6E-15

1.9E- 10

4.7E-15

8. EE-G4

1. JE-O5

1. 2E-10

5. 3E- 60

4.1E- 7

3. 4E-98

3. $8 \mathrm{E}-68$

1. UE-G5

1.1E-08

2. IE-17

6.7E-05

I EE+OS

7. 2E-14

1.8E-18

2. 8E-06

2. 2E-18

5. 6 E- -5

2. 3E- 5

5. $5 E-97$

1. TE-13
1.1

$\mathrm{Rem}$

50.0

Internal Annual

Dose Dose

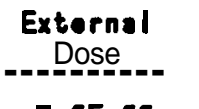

Equivalent

Equivalent

- . $. E+00$

I.OE+00

- SE+CO

IE+DI

I. $. E+C 0$

IE+CS

- EE+U1

I $. E+00$

I SE+OS

- DE+DS

IE+CO

IE+OU

IE+DI

- SE+DI

I E.OSO

G.OE+CO

I GE+GS

I EE+g0

IEE+OS

IE.0S

C.DE+U1

I EE+0S

- IE+

I. $\mathrm{E}+00$

IE. IO

I. EE+00

IE+US

- IE+OS

- $1 E+00$

- $J E+00$

IE $E+0$

(1) $\mathrm{E}+00$

IEE+OS

- E +00

I. $. E+00$

IE+US

I $\mathrm{E}+00$
.7E- 7

6. 9E-94

4. 2E- 98

1. EE-G3

7.8E-C8

1. 4E- 13

1. BE-C4

9.7E- 16

5. 6E- 57

$9.2 E-65$

1.8E-45

-.1E-11

8.1E-65

4.6E-64

2.6E-65

1.9E-66

4.7E-65

8. IE- I4

1. E-A5

1. 2E-1E

5. 3E-98

4.1E- 17

3. 4 E- 08

3. $6 \mathrm{E}-18$

1. EE-C5

1. 1E-68

2. E- 67

6. 7E- 55

IE +1

7. 2E-14

1.8E-18

2.6E-65

2. 2E- 98

5. 6E-G5

2. 3E- 5

5. 5E-47

1.7E-13
5. 7E-G7

$6.9 \mathrm{E}-64$

4. 2E-98

1.OE-G3

7. 8E- 66

1. 4E- 3

1.6E-64

9.7E-๑6

6. BE- 17

9. 2E-65

1.8E-95

9.1E-11

8.1E-65

4. BE-14

2. $6 E-65$

1.9E-66

4.7E-65

8. OE- 94

1.0E-65

1. 2E-10

5. $3 E-96$

4.1E-97

3. 4 E-66

3. BE-I8

1. $E-55$

1.1E- 88

2. $6 E-07$

B. $7 E-65$

- $6 \mathrm{E}+60$

T. 2E-14

1. $8 \mathrm{E}-18$

2. BE- 16

2. 2E-68

6. 6E-45

2. $3 E-95$

6. SE-I7

1.7E-13

\begin{abstract}
--.--.-.--
\end{abstract}
$-$ 


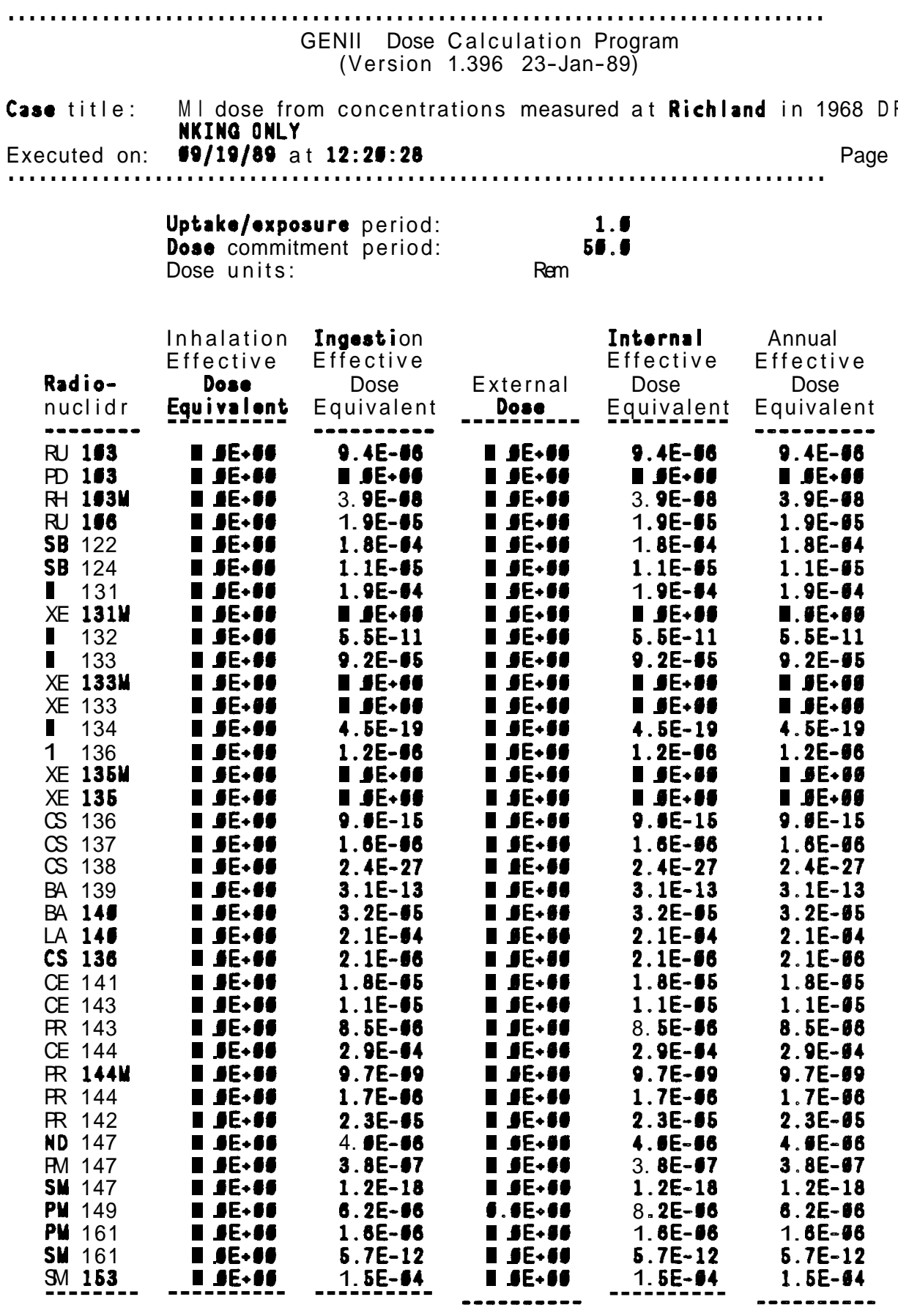


CENII Dorm Calculation Program

(Version 1.896 28-Jan-89)

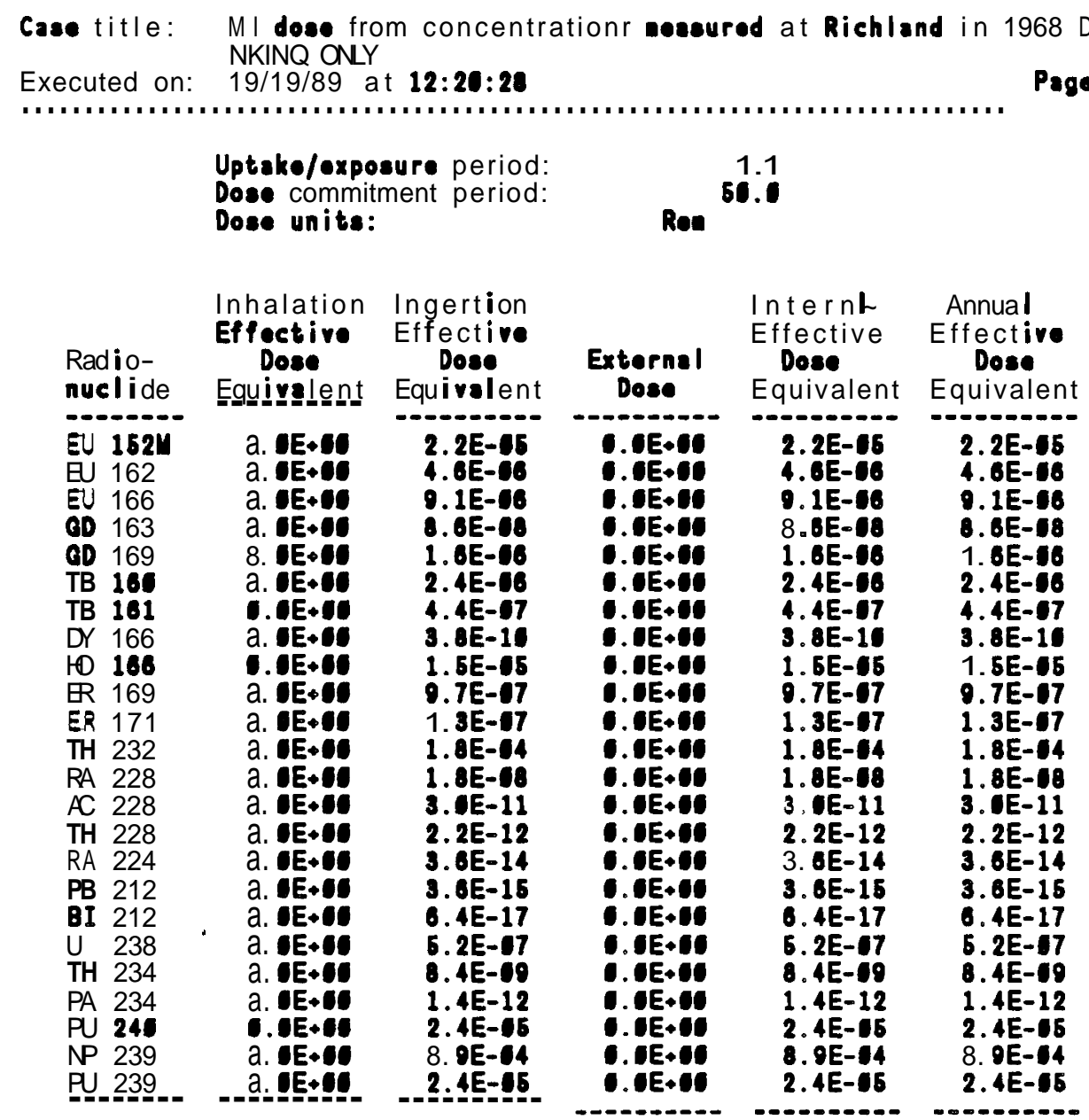




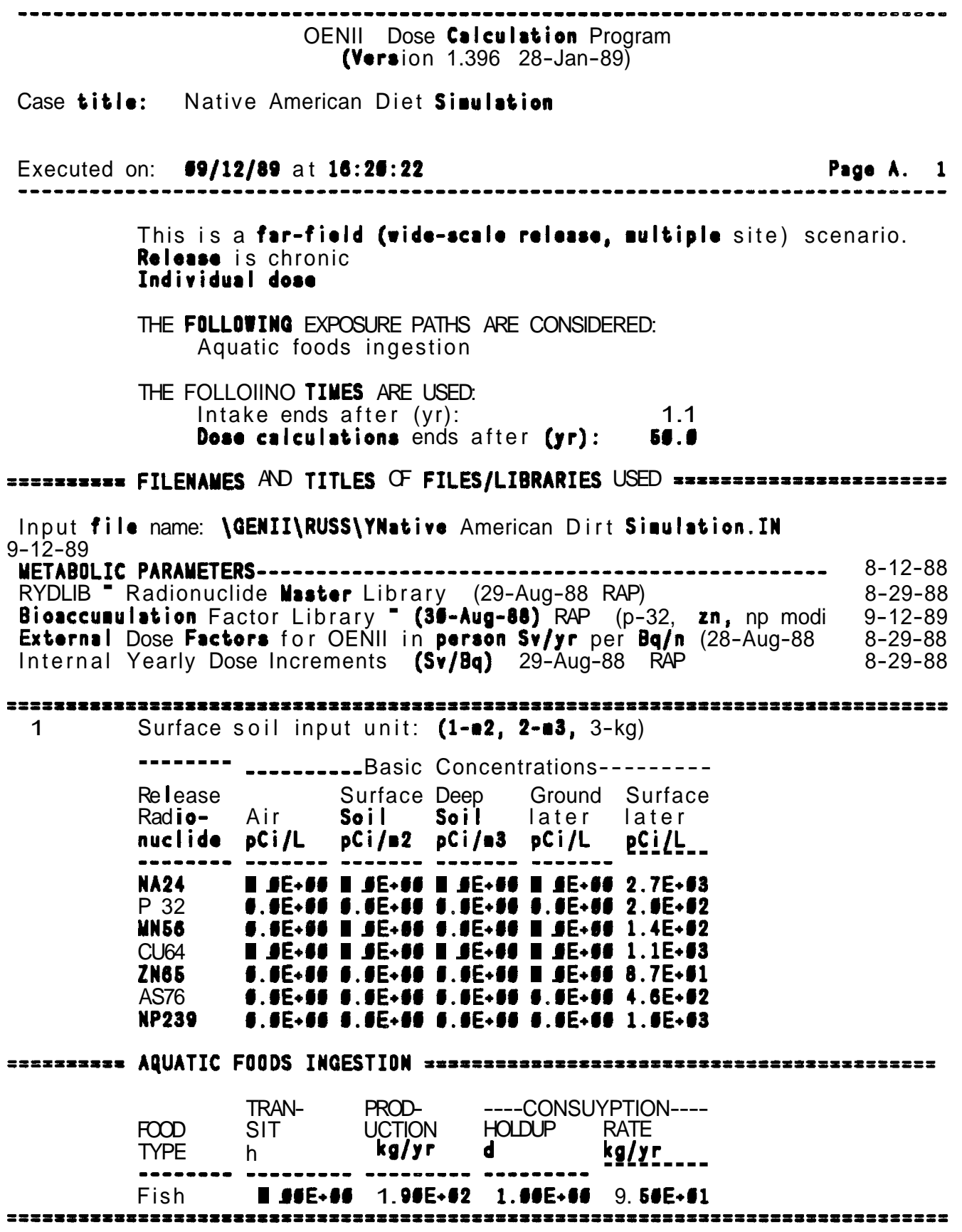


OENII Dose Calculation Program

(Version 1.395 28-Jan-89)

Care title: Native American diet Simulation

Executed on: $\mathbf{1 9 / 1 2 / 8 9}$ at $\mathbf{1 6 : 2 1 : 4 1}$

Uptake/exposure period: Dose commitment period: Dose unitr:

\section{0 \\ Ron}

Page C. 5

\begin{tabular}{|c|c|c|c|c|c|}
\hline $\begin{array}{l}\text { Radio- } \\
\text { nuelide }\end{array}$ & $\begin{array}{c}\text { Inhalation } \\
\text { Effective } \\
\text { Dose } \\
\text { Equivalent }\end{array}$ & $\begin{array}{l}\text { Ingertion } \\
\text { Effective } \\
\text { Dose } \\
\text { Equivalent }\end{array}$ & $\begin{array}{l}\text { External } \\
\text { Doget }\end{array}$ & $\begin{array}{l}\text { Internal } \\
\text { Effoctive } \\
\text { Dose } \\
\text { Equivalent }\end{array}$ & $\begin{array}{c}\text { Annual } \\
\text { Effective } \\
\text { Dose } \\
\text { Equivalent }\end{array}$ \\
\hline $\begin{array}{ll}\text { NA } & 24 \\
\text { P } & 32 \\
\text { UN } & 58 \\
\text { CU } & 64 \\
\text { ZN } & 66 \\
\text { AS } & 76 \\
\text { NP } & 239 \\
\text { PU } & 239 \\
\end{array}$ & 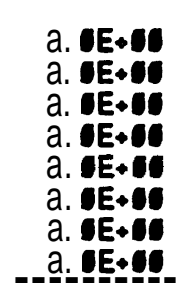 & $\begin{array}{l}\text { a. } 0 E-08 \\
4.9 E-01 \\
8.3 E-08 \\
3.4 E-02 \\
5.3 E-02 \\
3.7 E-02 \\
5.9 E-02 \\
6.9 E-07\end{array}$ & 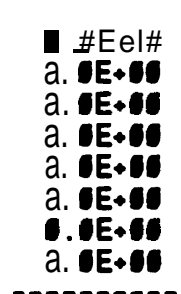 & 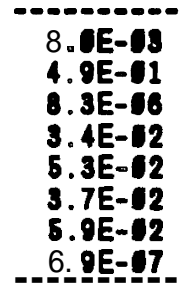 & $\begin{array}{l}\text { 8. } 0 E-93 \\
4.9 E-91 \\
8.3 E-96 \\
3.4 E-62 \\
5.3 E-62 \\
3.7 E-62 \\
5.9 E-12 \\
\text { 5. } 9 E-97\end{array}$ \\
\hline
\end{tabular}




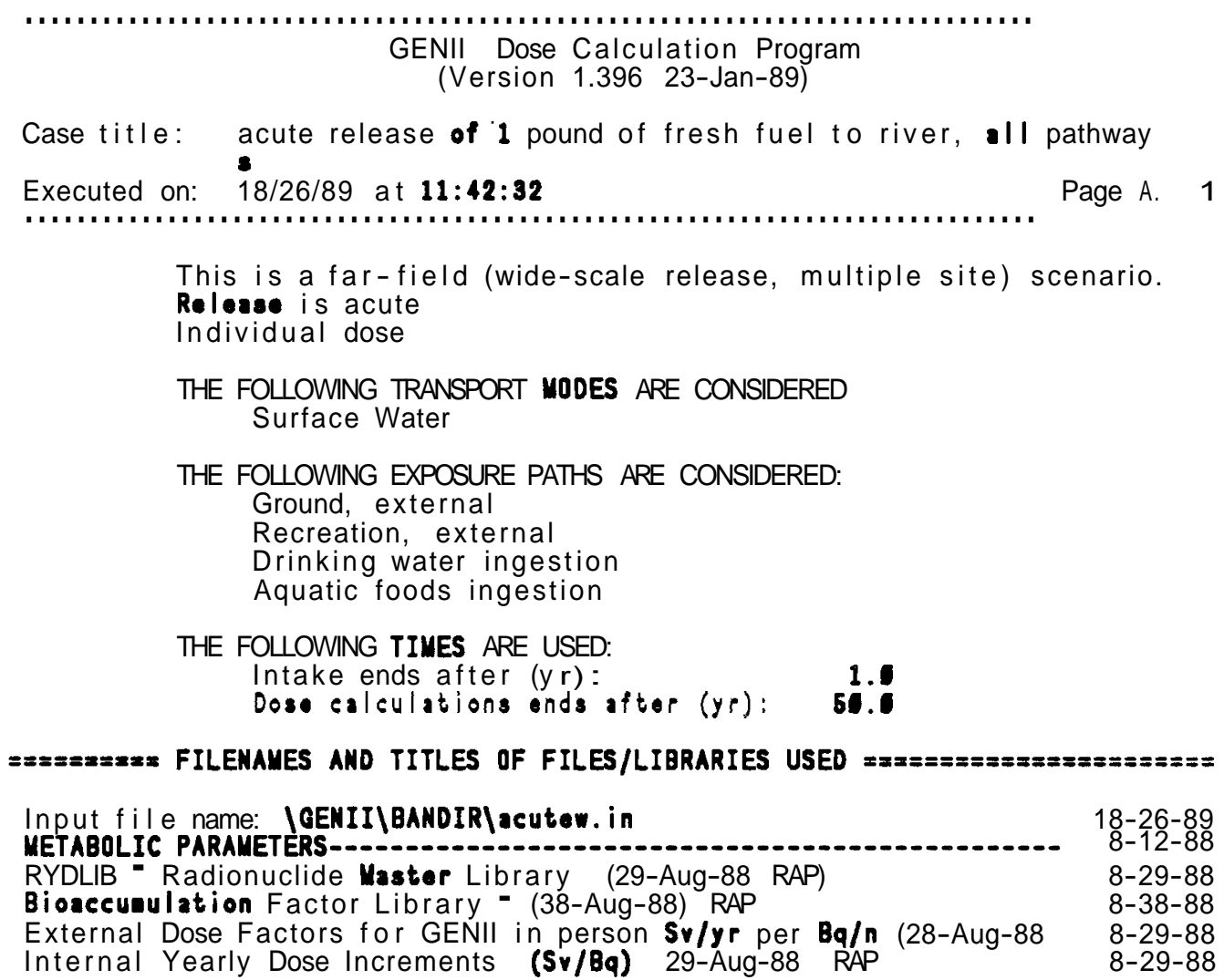

THE FOLLOWING TIMES ARE USED: 


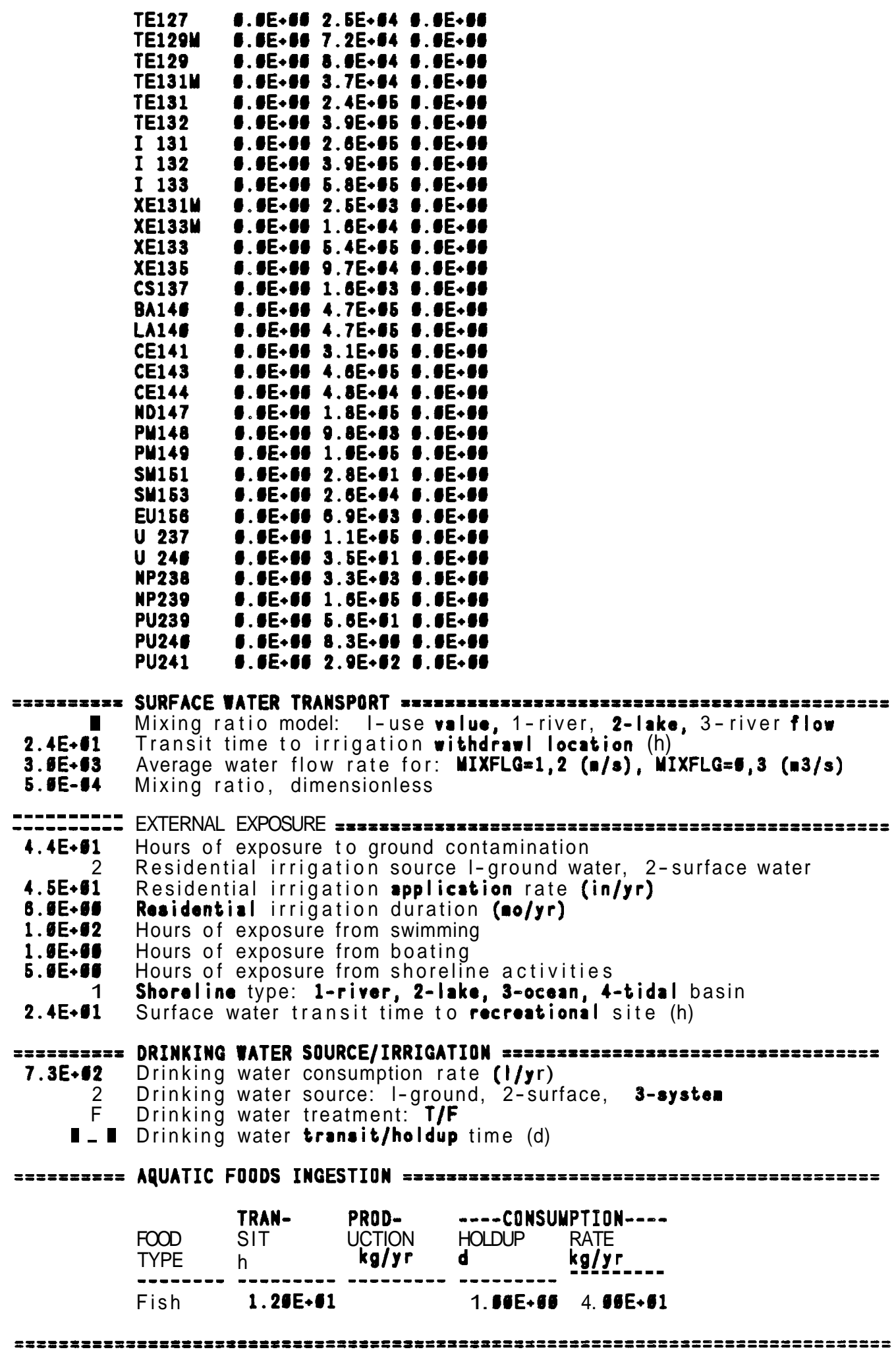


OENII Dose Calculation Program

(Version 1.905 23-Jan-89)

Cass title: acute release of 1 pound of fresh fuel to river, all pathway

Executed on: ${ }^{8} 11 / 26 / 89$ at 11:50:56

Page C. 1

Acute release

Uptake/exposure period:

Dose commitment period:

Dose unit::

1.1

Ren

61.1

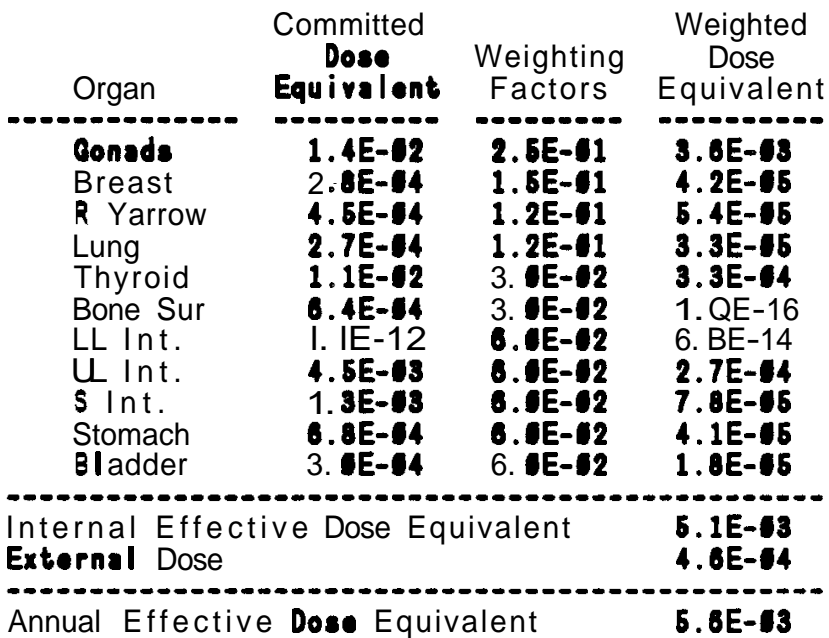

Control ling Organ:
Controlling Pathway:
Controlling Radionuclide:
Total Inhrlation EDE:
Total Ingestion EDE:




CENI Dora Calculation Program
(Yeroion 1.896 28-Jan-89)

Case title: scute release of 1 pound of fresh fuel to river, all pathway

Exacutod on: $11 / 26 / 89$ at 11:54:55

Page C. 3

Acute relrare

Uptake/exposure pariod:

Dora connitment period:

Dora unitr:

\author{
Ram $\begin{array}{r}1.1 \\ 61.1\end{array}$
}

Cormittad Dora Equivalent by Radionuclide

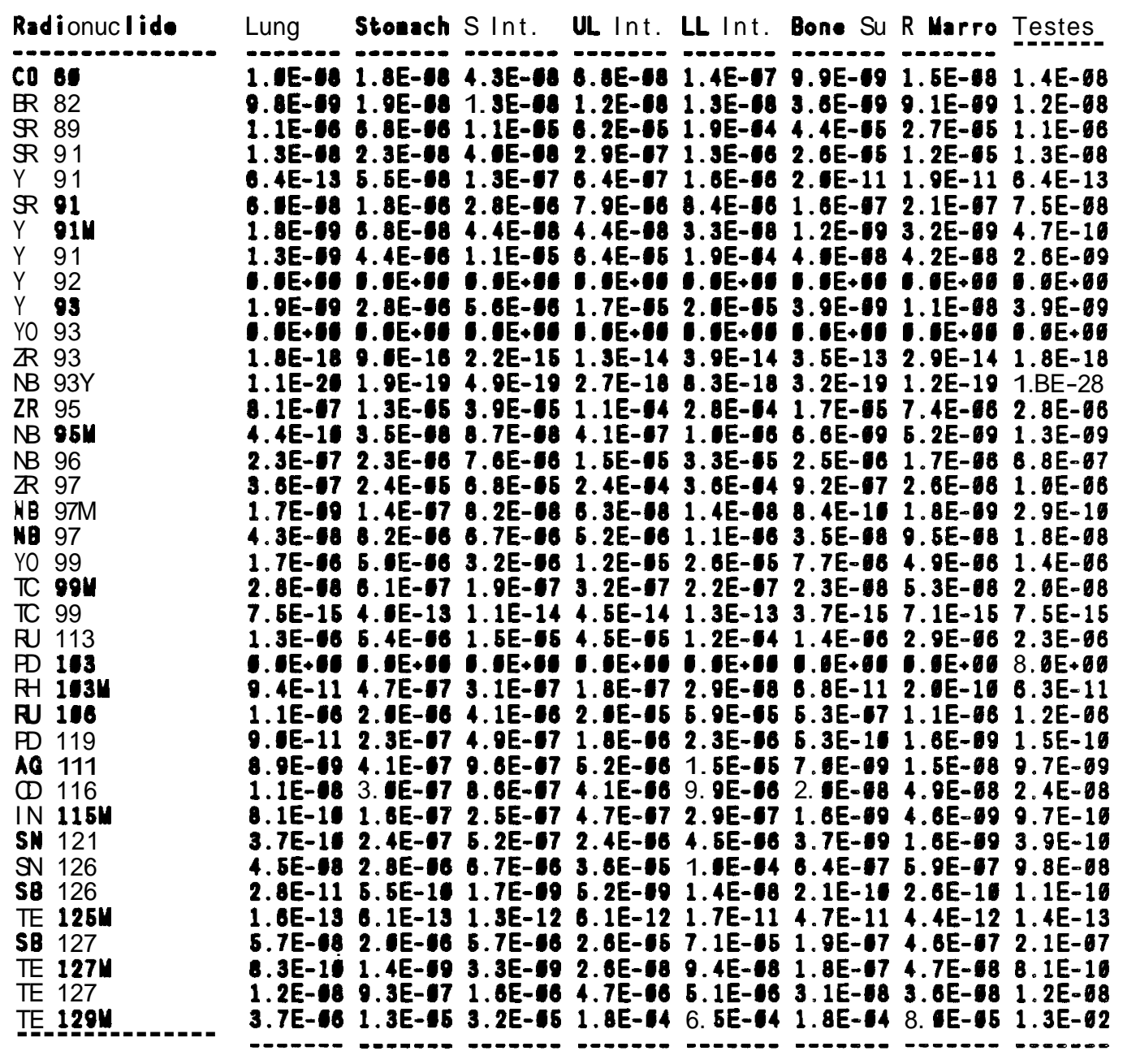




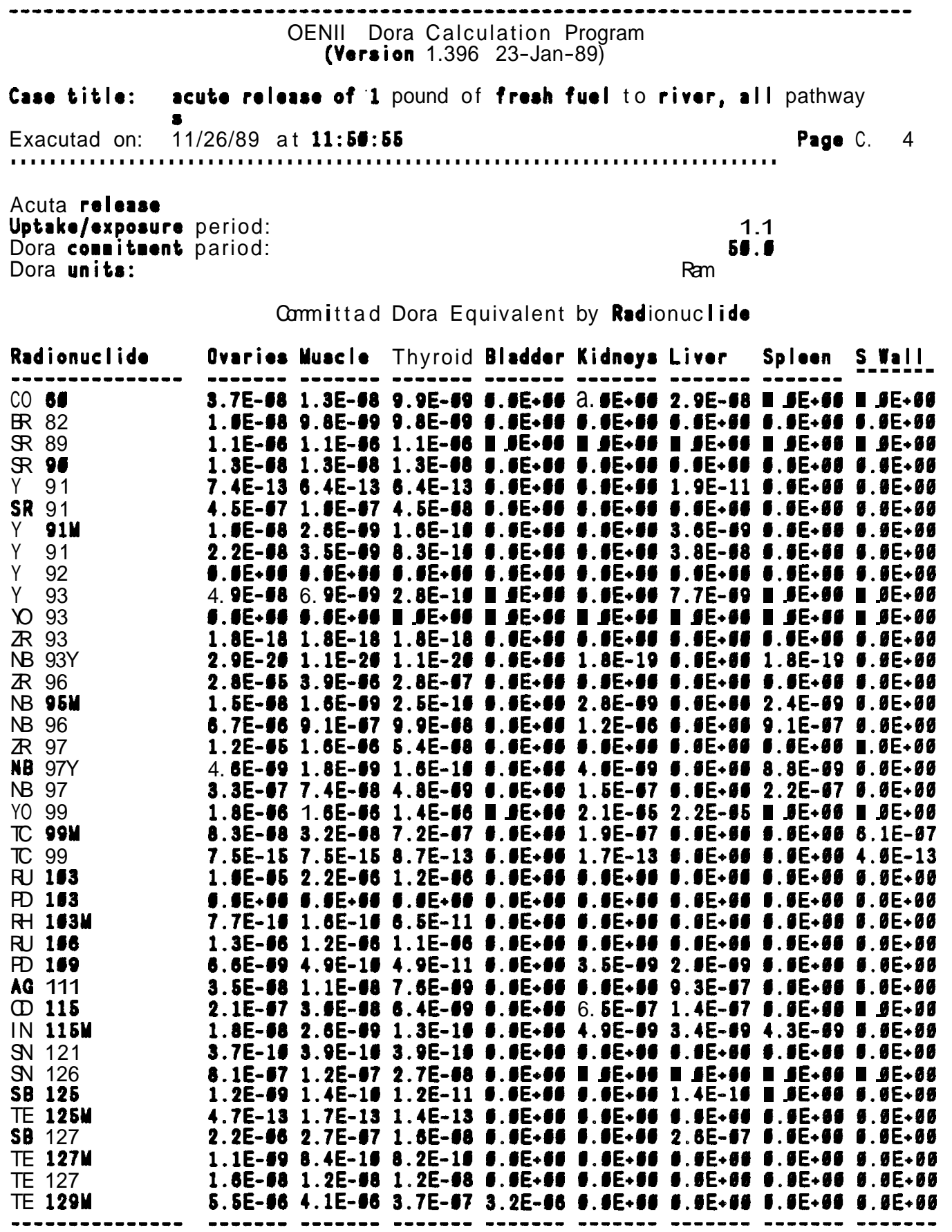




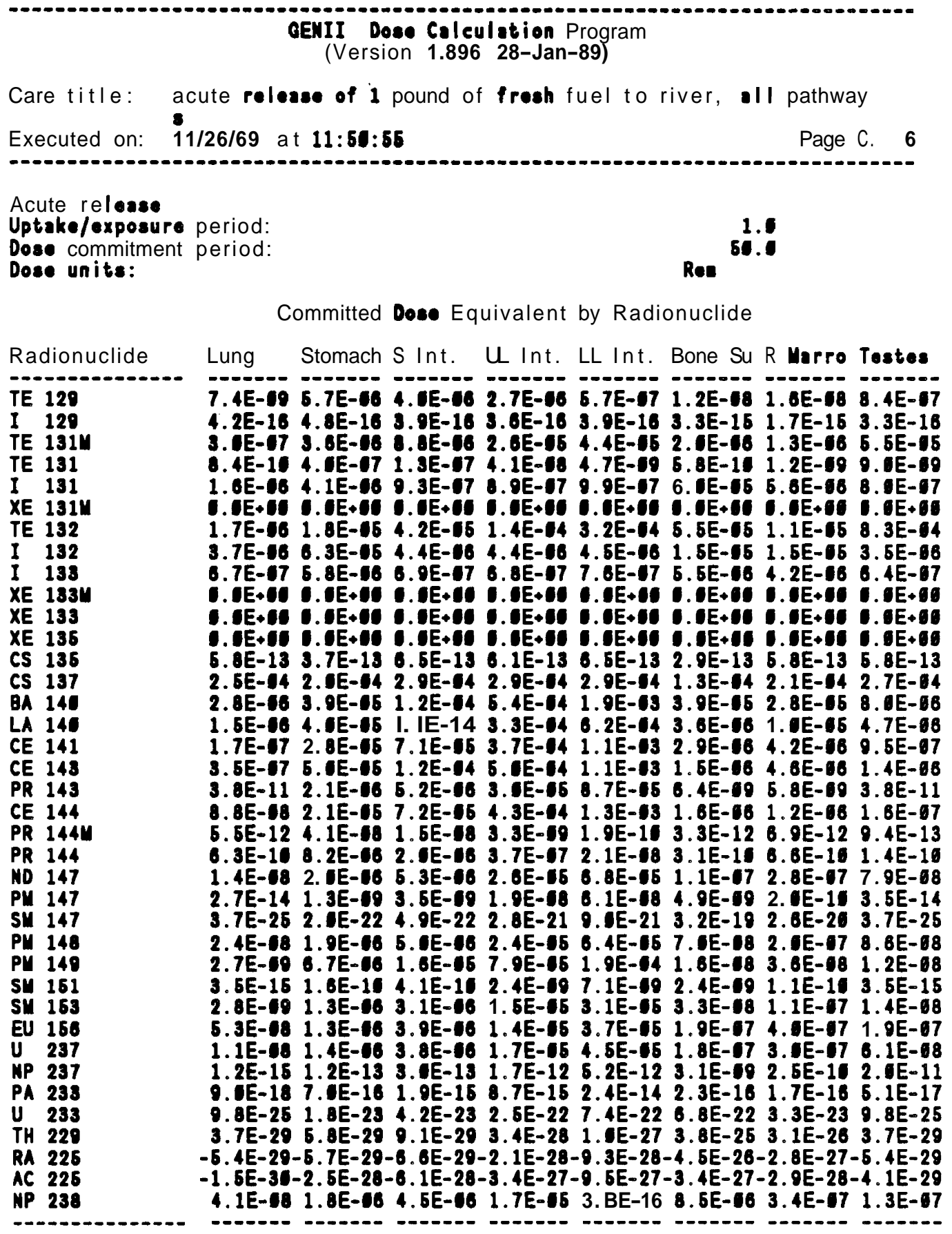


CENII Dose Calculation Program

(Version 1.396 23-Jan-89)

Case title: acute release of $\mathbf{1}$ pound of fresh fuel to river, all pathway

Executed on: 10/26/89 at 11:54:56 Page C. 6

Acute release

Uptake/exposure period:

Dose commitment period:

Dose units:

$\operatorname{Rer}^{50.0}$

Committed Dose Equivalent by Radionuclide

Radionuclide

TE 129

129

TE 131M

TE 131

I 131

XE 131U

TE 132

1132

I 133

XE 133M

XE 133

XE 136

CS 136

CS 137

BA 140

LA 141

CE 141

CE 143

R 143

CE 144

PR 144M

PR 144

ND 147

PM 147

SU 147

PM 148

PM 149

SU 161

SU 163

时 168

U 237

N 237

PA 233

U 233

$\mathrm{TH} 229$

RA 226

AC 226

N 238
Oraries Wuscle Thyroid Bladder Kidnoys Liver Spleen S_Ial!

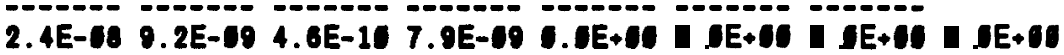

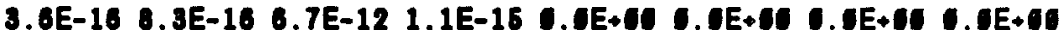

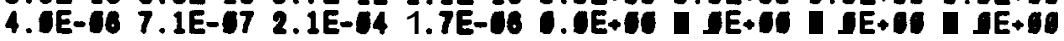

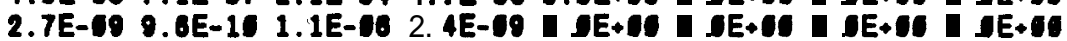

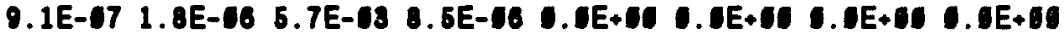

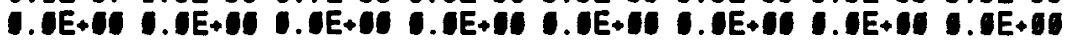

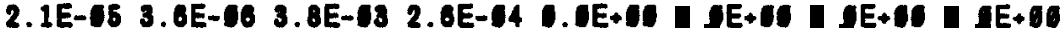

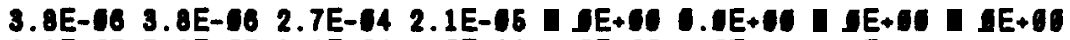

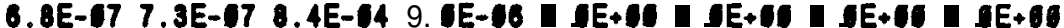

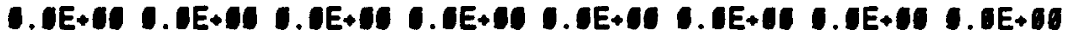

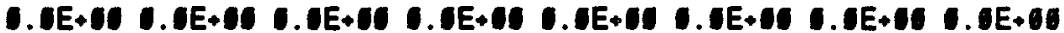

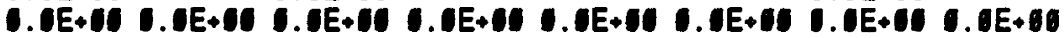

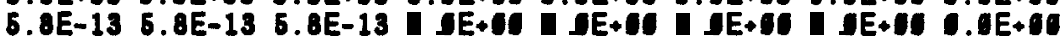

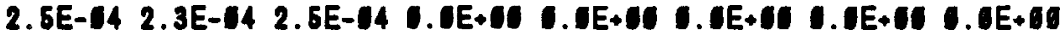

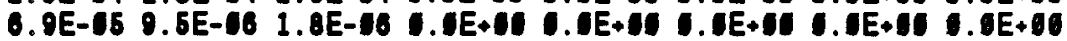

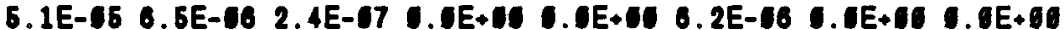

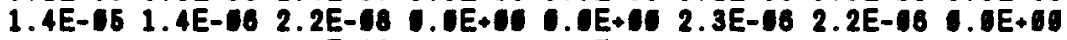

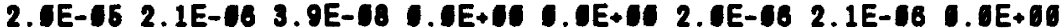

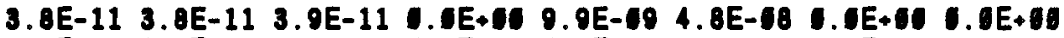

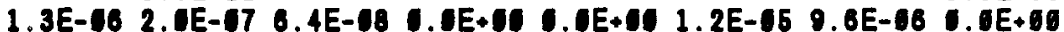
1.4E-11 6.8E-12 4.1E-13 ..UE+10 1.2E-11 7.6E-12 .0E+01 $.0 \mathrm{E}+00$

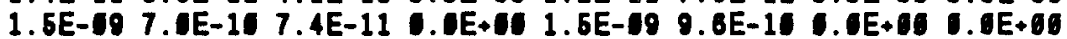

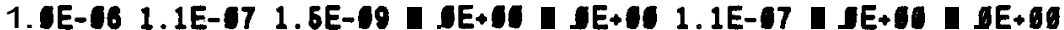
$1.6 E-133.8 E-142.6 E-140.0 E+005.0 E+091.2 E-690.0 E+001.0 E+00$

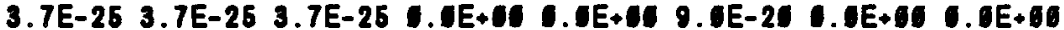

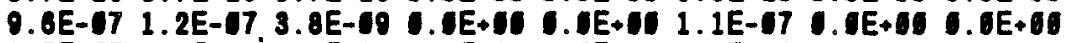

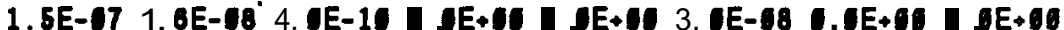

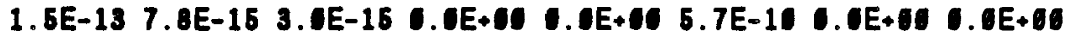

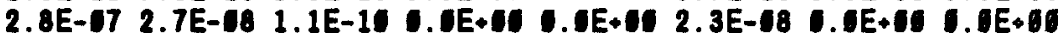

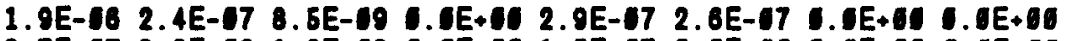

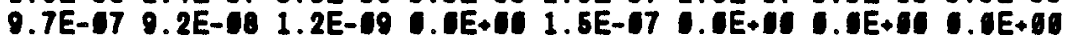

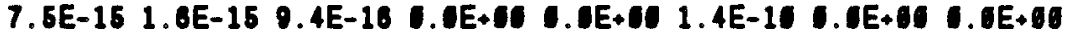
6.1E-16 6.5E-17 1.2E-18 0.0E+09 8.2E-17 5.8E-17 0.0E+01 $6.0 E+90$

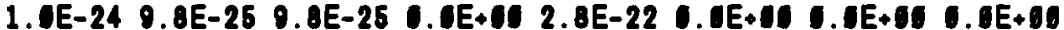

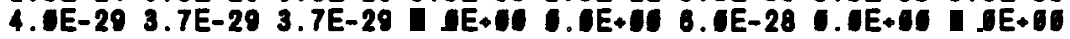

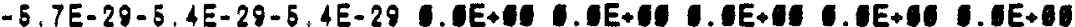

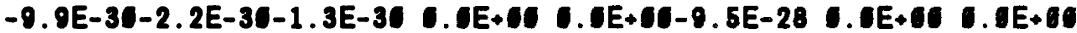

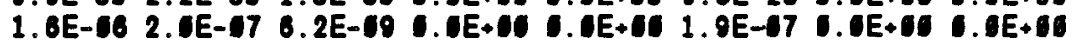




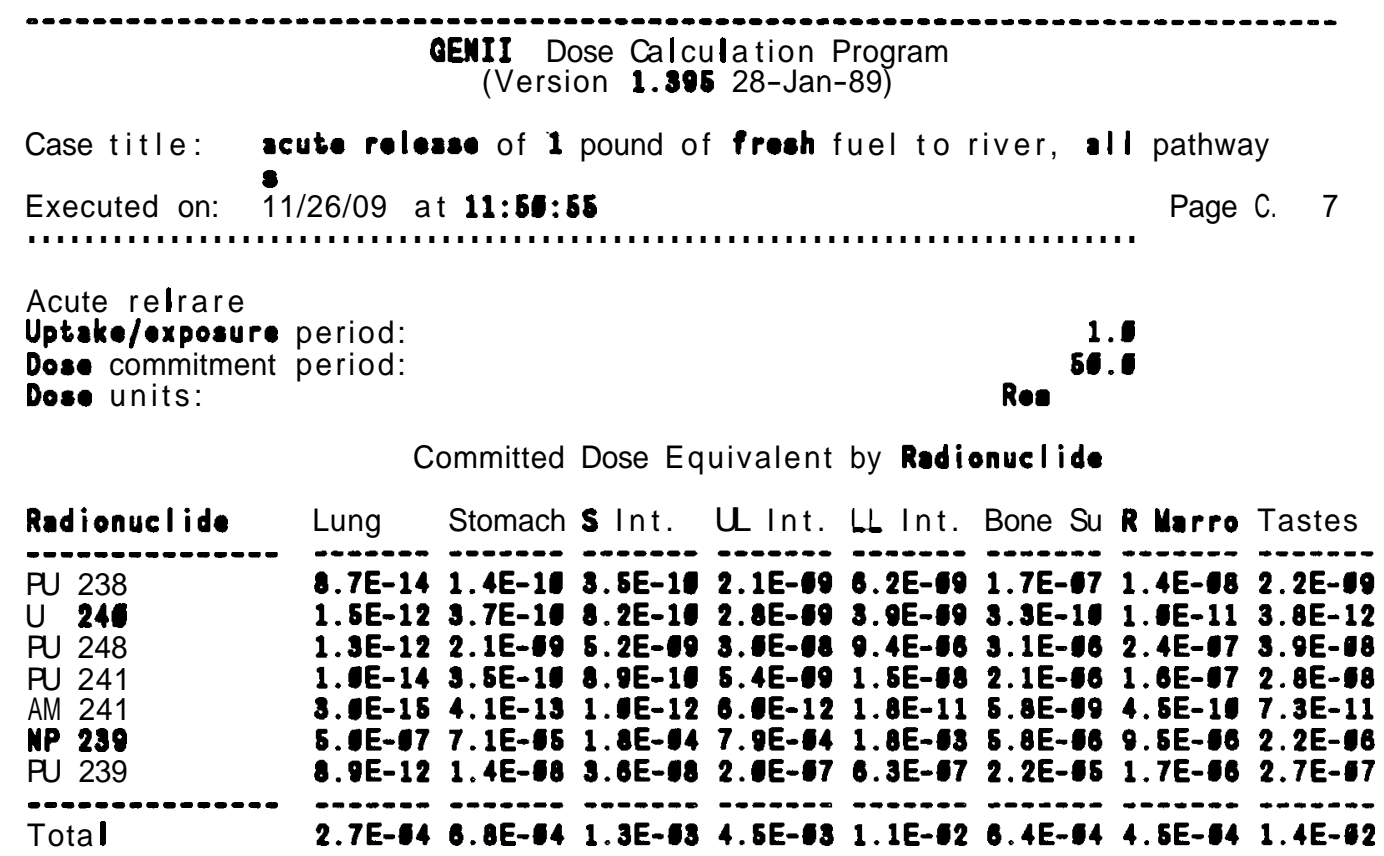




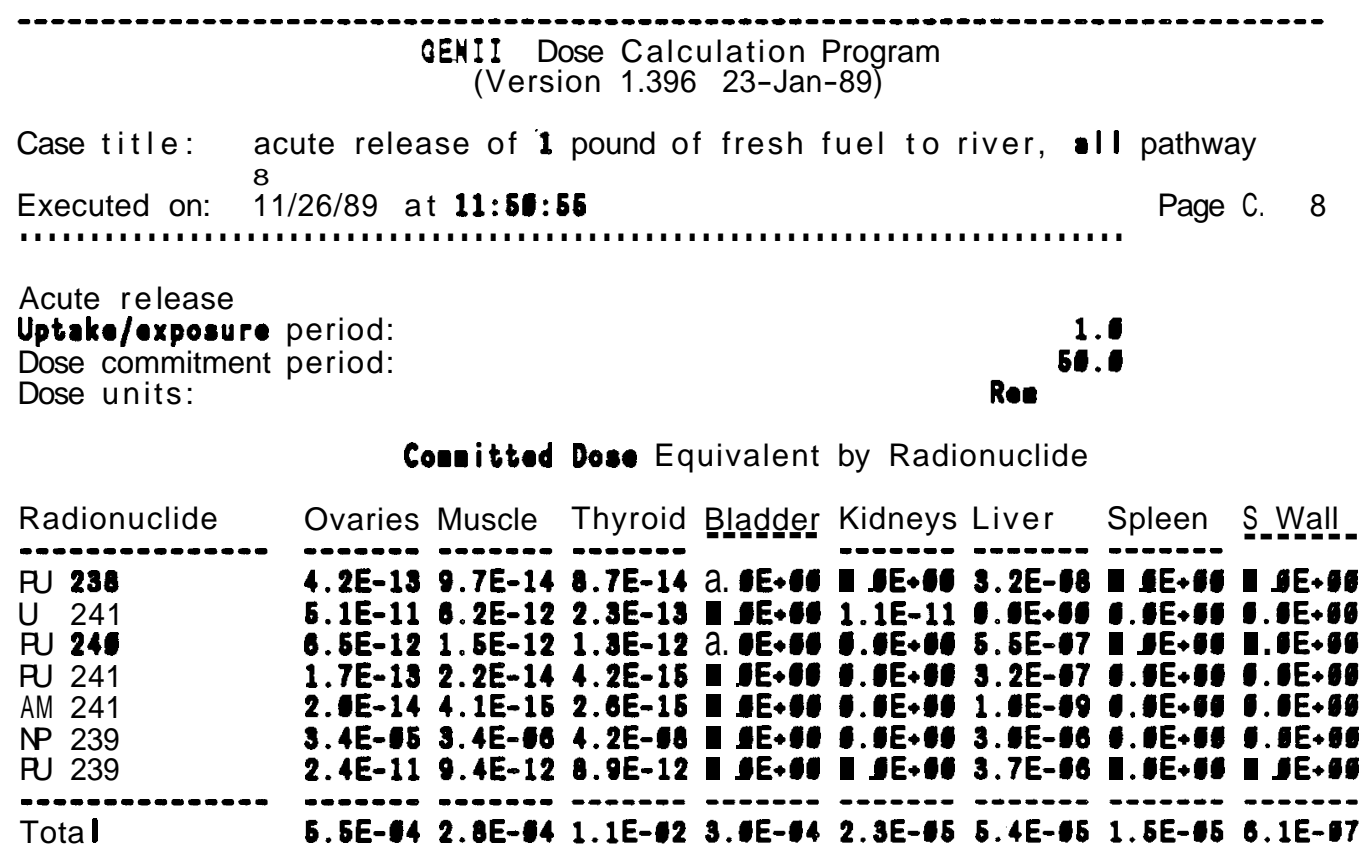




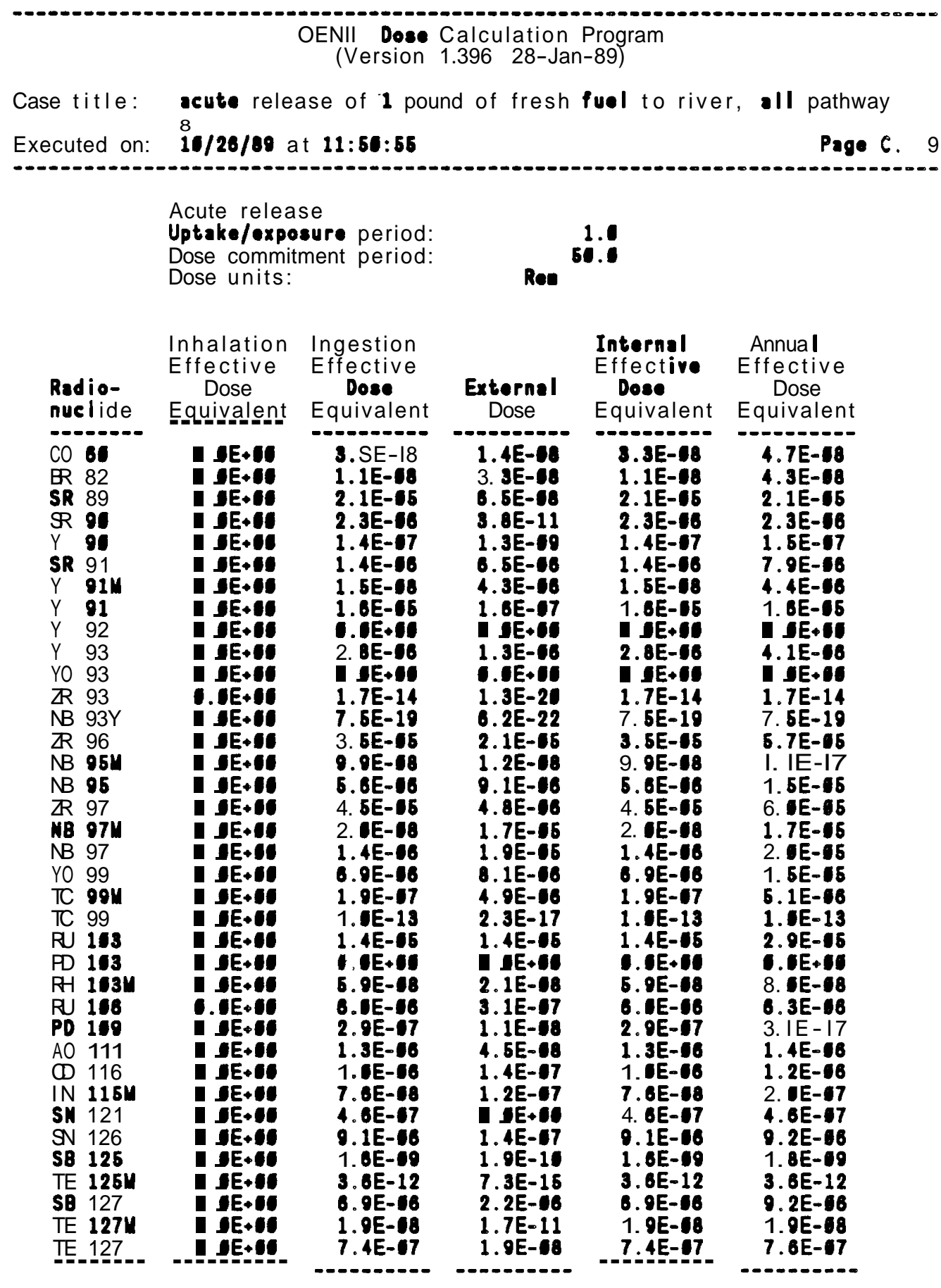


OENII Dora Calculation Program

(Version 1.396 23-Jan-89)

Case title: scute relesse of 1 pound of fresh fuel to river, all pathway
Executed on: 8 18/26/89 at 11:50:55

Acute release

Uptake/exposure pariod:

Dora coneitment pariod:

Dora units:

Radio-
nuclida E

Inhalation Ingestion

Effactiva Effactiva

Dose Dose

TE 129u

Equivalent Eguiyalent

$\square$ DE+OO

- EE+CE

129

TE 131Y

TE 131

- 131

XE 131Y

TE 132

I 132

I 133

XE 133Y

XE 133

XE 136

CS 136

CS 137

BA 140

LA 141

CE 141

CE 143

PR 143

CE 144

PR $144 \mathrm{Y}$

PR 144

N 147

PY 147

SY 147

PY 148

PY 149

SY 161

SY 163

EU 156

U 237

NP 237

PA 233

U 233

TH 229

BA 226
- $\mathrm{E}+\boldsymbol{O C O}$

IE+CI

IE+GI

IE+BS

IE+CI

IE+CO

IE+CI

IE+CO

IE+GS

I. $\mathrm{E}+\boldsymbol{D O}$

I $E+65$

I GE+GS

IE+OS

IE+OS

- DE+OO

$1 \mathrm{GE}+\mathrm{OS}$

IE+GS

IE+IS

IE+DI

IE+DO

IE+US

IE+CS

I $J E+00$

- SE+00

IE+OS

IE+DS

- $J E+01$

IE+O0

IE+UI

IE+G0

IE+CS

IE+CO

GE+CI

IE+g.
3. 3E-93

1. EE-68

2. E-13

2. 5E-G5

7. E-108

1. 8E-G4

IE+OS

3.7E-94

1.8E-05

2.7E-O5

I $\mathrm{E}+00$

- SE+G0

IE+OS

5. 3E-13

2. 3E-J4

1.8E-J4

8.1E-65

$9.7 E-45$

1.1E-94

7. 4 E- 66

1.1E- 94

3. $6 E-99$

6. 3E- 07

6. 4 E- 96

5. $4 \mathrm{E}-19$

1. 9 E-25

6. DE-06

1.8E-G5

7.2E-10

3.1E- 16

3. 9 E- 98

4. 4E-66

1. 4E-10

2. $3 E-15$

1. 1E-22

1. 5E-26
$-1.8 E-27$
1.1

Ram

56.)

Dora

3. 6E-97

3. 9E- 67

1. 2E-17

4. EE-G6

2.7E- 1

1.3E-D5

2. $9 E-99$

$9.7 E-68$

1.1E-14

2.3E-15

6. 2E-98

1. $9 \mathrm{E}-08$

5. 1E-67

7.1E-2

1. 4E-97

1.3E-95

1. $5 \mathrm{E}-64$

2. 3E-96

9. $5 E-66$

2. E-

9. 2E-c8

5. $8 \mathrm{E}-10$

2. $9 E-97$

3. E-66

3. $9 E-13$

I. $\mathrm{E}+60$

7. $8 \mathrm{E}-67$

1.1E-67

2. JE-14

1. 2E-14

1.2E-06

1.6E-66

1. $9 \mathrm{E}-16$

3. $8 E-17$

1. $7 \mathrm{E}-28$

1. $7 \mathrm{E}-31$

-2 . IE- 31

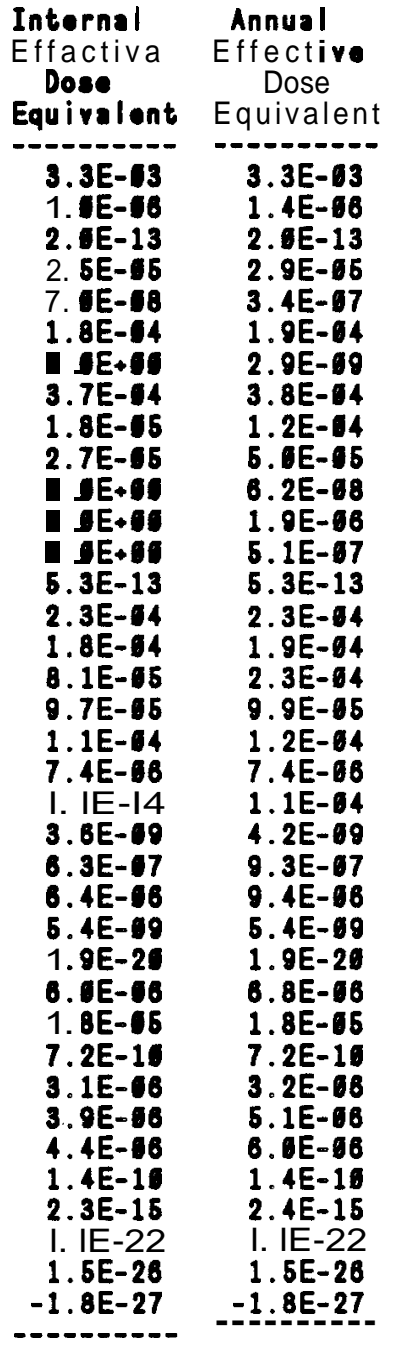




CENI Dose Calculation Program
(Version 1.396 23-Jan-89)


QENII Dora Calculation Program

(Version 1.396 23-Jan-89)

Case title: acute releses of'l pound of fresh fuel to river, drinking only

$\begin{array}{ll}\text { Executed on: 10/26/89 at 10:25:28 Page A. } 1 & \end{array}$

This is a far-field (wide-seale rolease, nultiple rite) scenario.

Release is acute

Individual dose

THE FOLLOWINQ TRANSPORT MODES ARE CONSIDERED

Surface Iater

THE FOLLOWINQ EXPOSURE PATHS ARE CONSIDERED:

Drinking water ingartion

THE FOLLOWINQ TIMES ARE USED:

Intake ends after $(y r)$ : 1.1

Dora calculations ends after $(\mathbf{y} r): \quad 61.1$

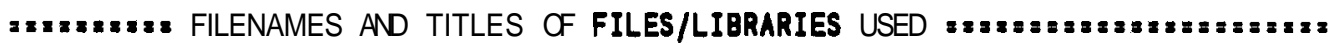

Input $\uparrow$ ile nane: JOENIIJBANDIR lacutow. in

METABOLIC PARAMETERS-_.

RMDLIB - Radionuelide Master Library (29-Aug-88 RAP) 8-29-88

Biosccunulation Factor Library - (30-Aug-88) RAP 8-38-88

External Dora Factors for QENII in person Sy/yr par Bq/n (28-Aug-88 8-29-88

Internal Yearly Dora Inereents (Sv/Bq) 29-Aug-88 RAP 8-29-88

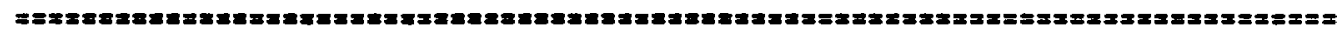

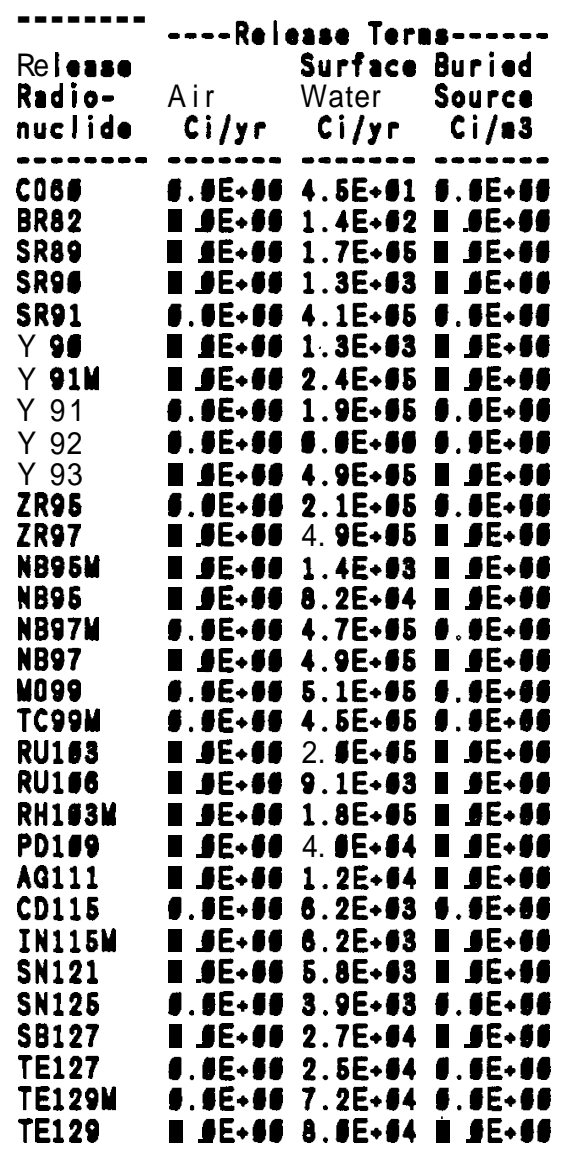




\begin{tabular}{|c|c|}
\hline 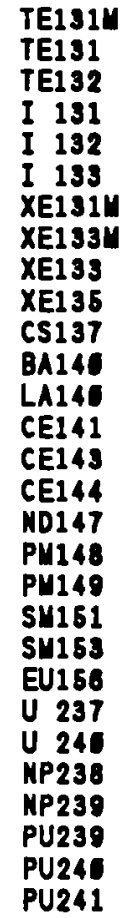 & 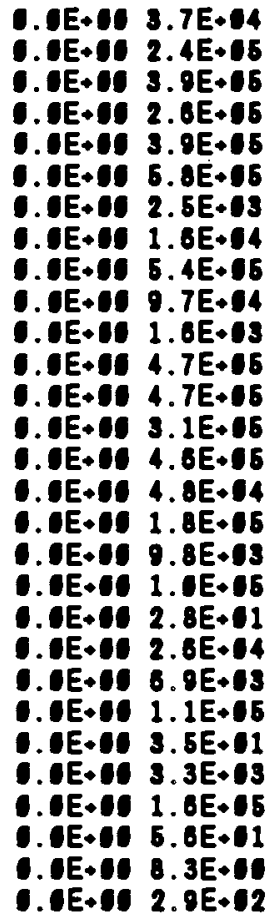 \\
\hline
\end{tabular}

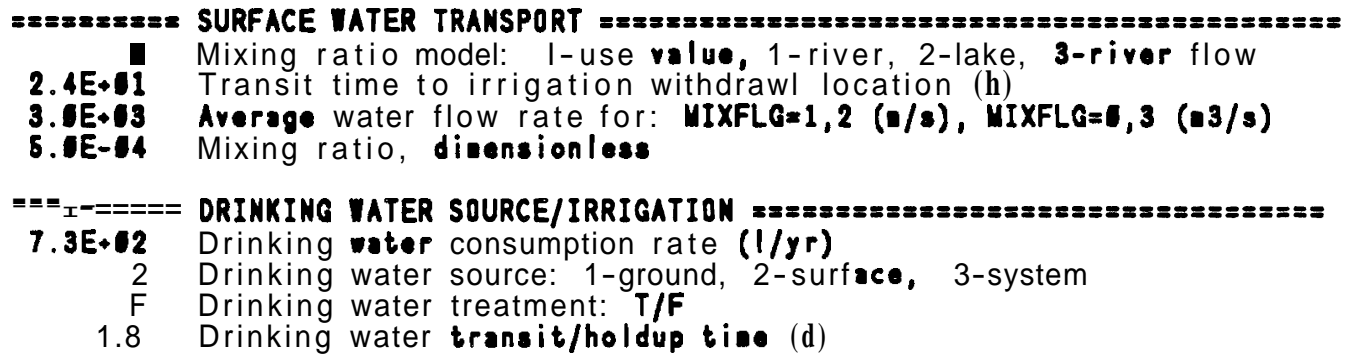

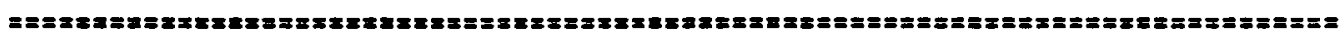




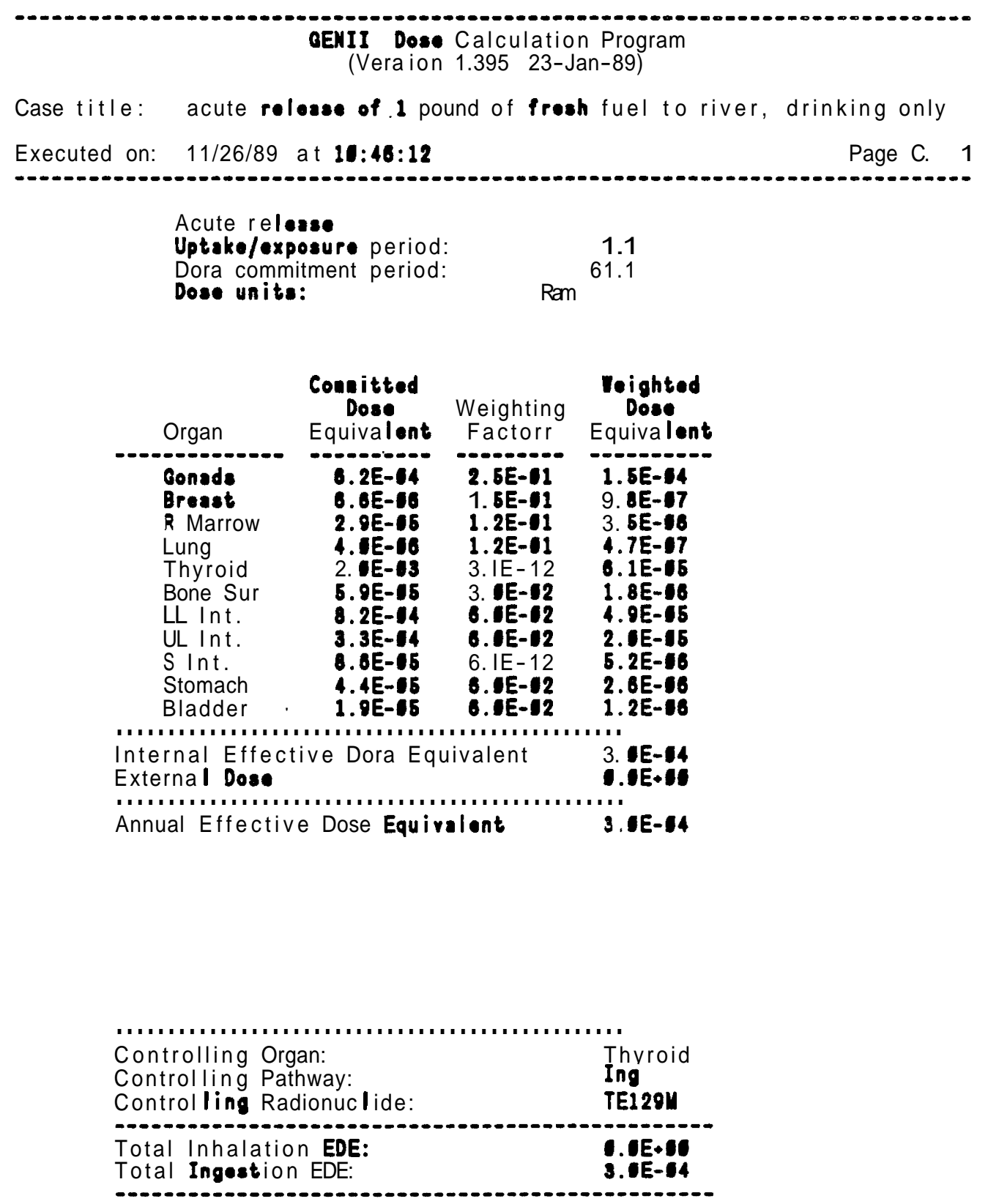

Controlling Organ:
Controlling Pathway:
Controlling Radionuclide:


GENII Dorm Caleulation Program

(Version 1.396 28-Jan-89)

Case title: seute relesse of 1 pound of fresh fuel to river, drinking only

Executed on: $11 / 26 / 89$ at 11:48:12 Page C. 3

Acuta release

Uptake/exposure period:

Dora coneituent period:

Dora units:

Ram $\begin{array}{r}1.1 \\ 61.1\end{array}$

Committad Dose Equivalent by Radionuclide

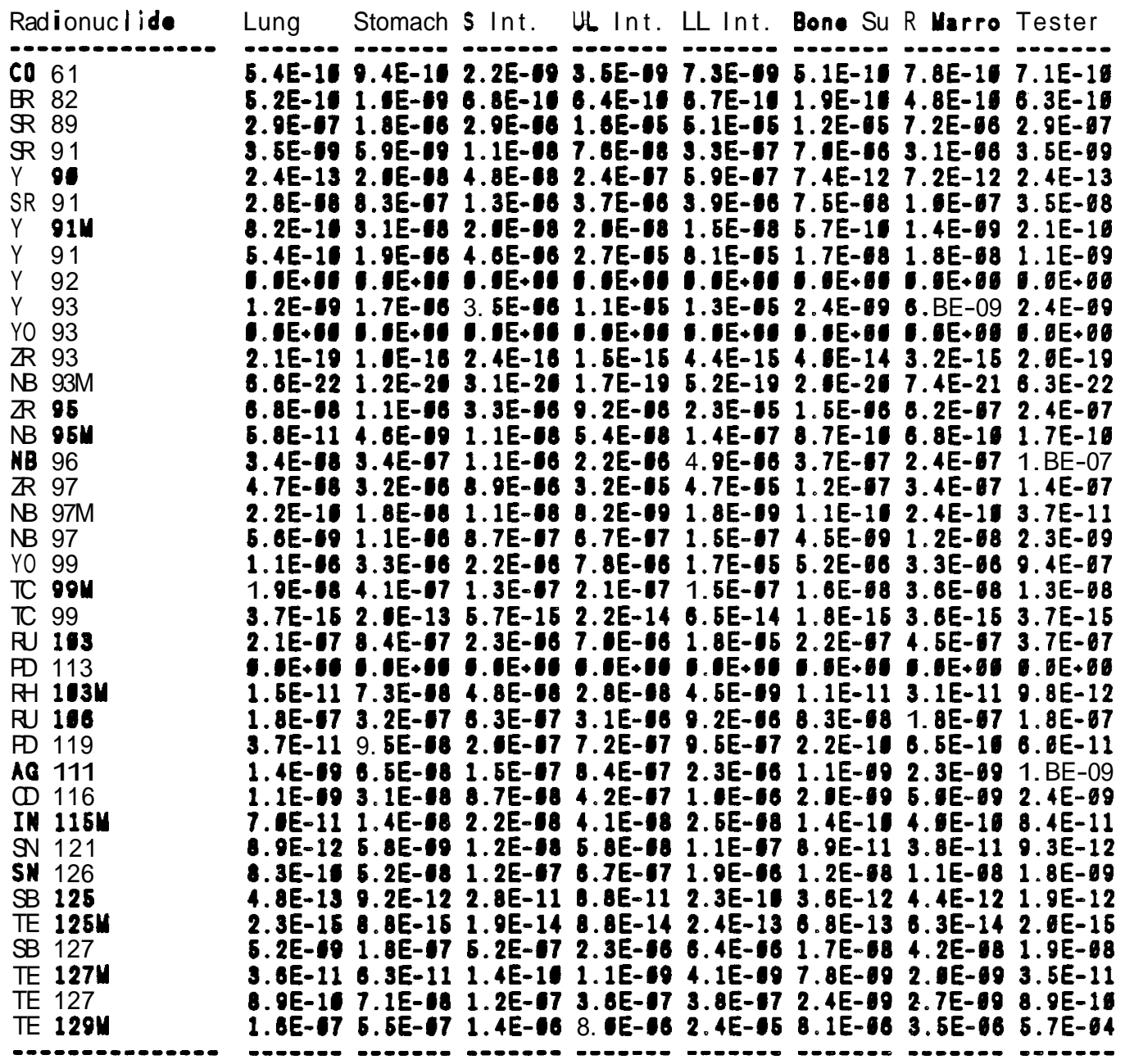




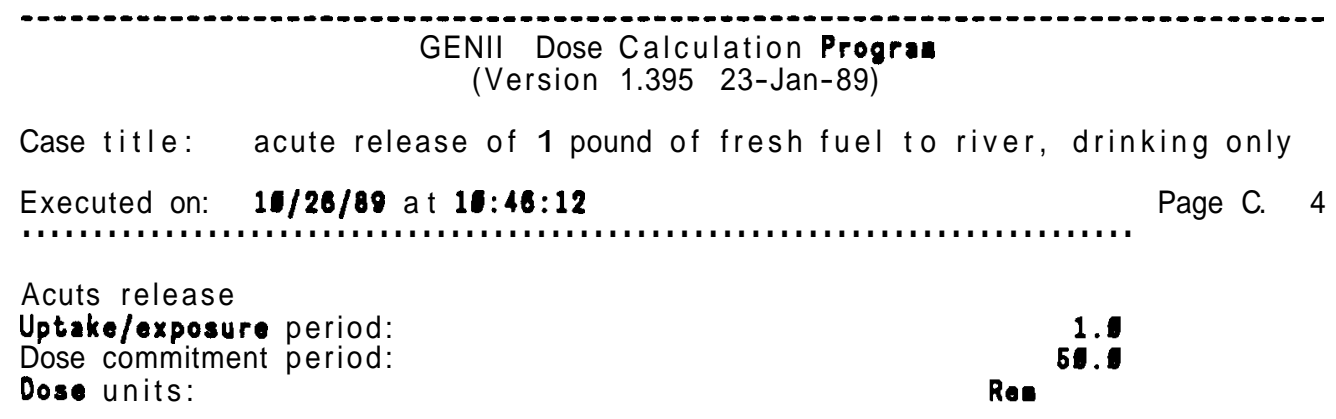

Committed Dose Equivalent by Radionuclide

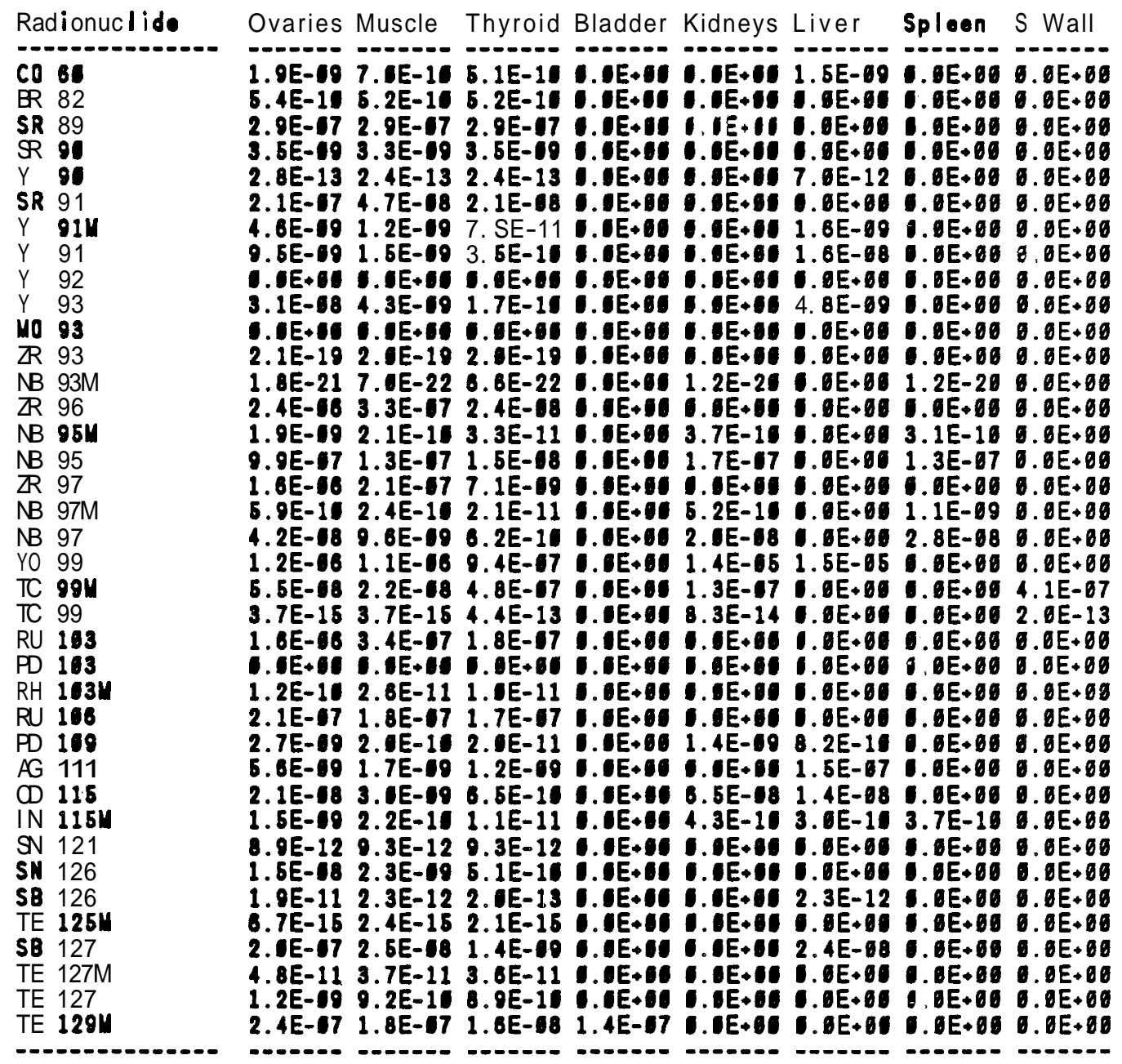


GEMII Door Calculation Program

(Version 1.396 23-Jan-89)

Case title: acute release of 1 pound of fresh furl to river, drinking only

Executed on: 11/26/89 at 18:46:12 Page C. 6

Acutr pelese

Uptake/exposure period:

Dose cormiteent period:

Dose unitr:

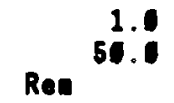

1.9

Conaitted Dose Equivalrnt by Radionuclide 


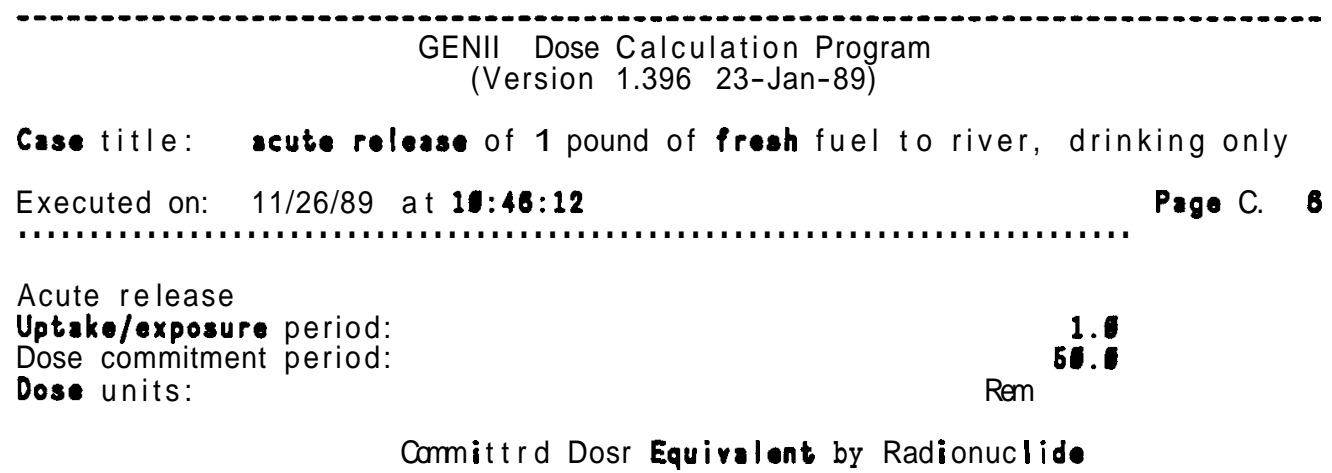

Radionuclide

TE 129

I 129

TE $131 \mathrm{Y}$

TE 131

I 131

XE 131Y

TE 132

I 132

1133

XE 133Y

XE 133

XE 136

CS 136

CS 137

BA 140

LA 140

CE 141

CE 143

PR 143

CE 144

PR 144Y

PR 144

N 147

PY 147

SY 147

PM 148

PY 149

SY 161

Sע 163

EU 166

U 237

NP 237

PA 233

U 233

TH 229

PA 225

AC 225

NP 238
Ovaries Muscle Thyroid Bladder Kidneys Liver Spleen S_la!l

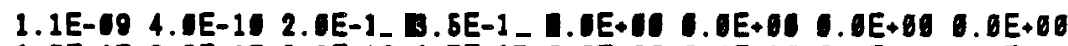

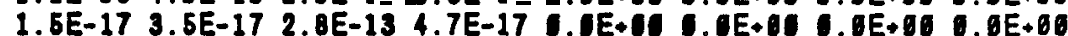

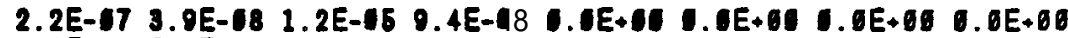

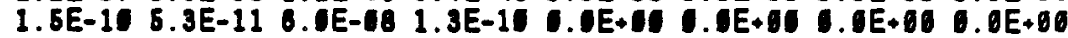

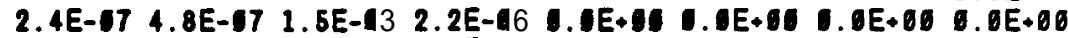

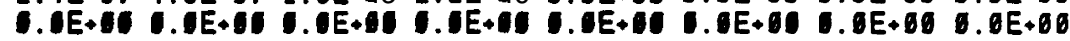

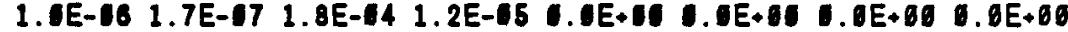

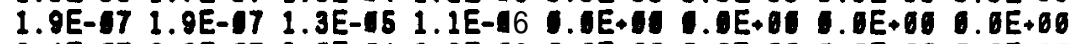

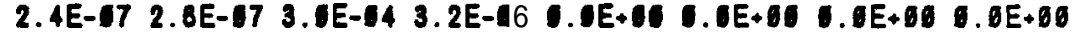

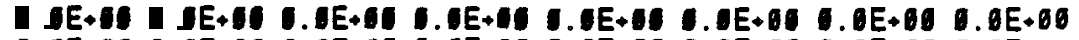

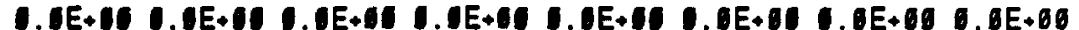

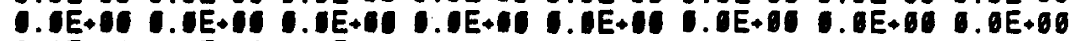

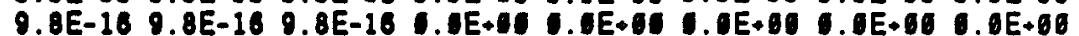

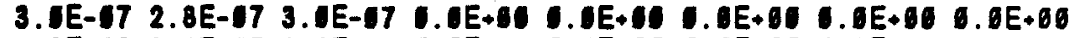

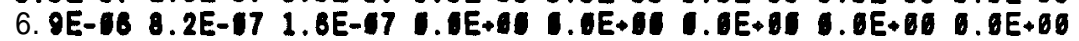

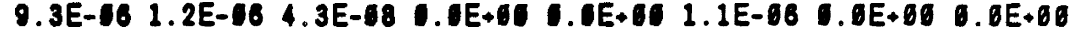

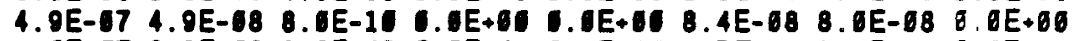

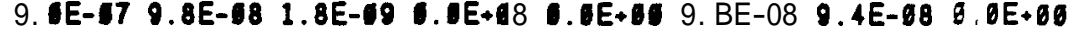

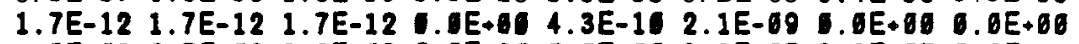

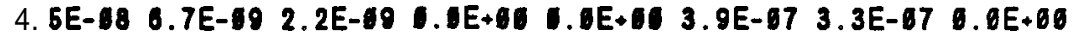
5. EE-13 2.3E-13 1.4E-14 $1.0 \mathrm{E}+00$ 4.2E-13 2.6E-13 $0.0 \mathrm{E}+00 \quad 0.0 \mathrm{E}+60$ $5.2 E-112.4 E-112.5 E-112$. $0 E+60$ 5.0E-11 3.3E-11 $0.0 E+\theta 00.0 E+\theta 0$

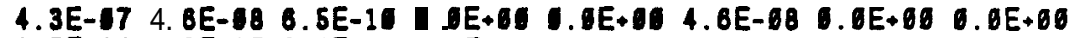
1.5E-14 3.6E-15 2.4E-15 6.0E+09 0.0E+001.1E-10 0.0E+60 0.0E+60

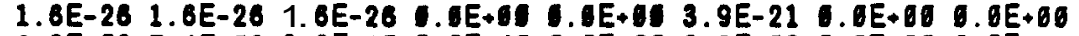

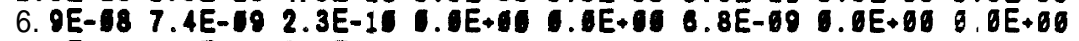

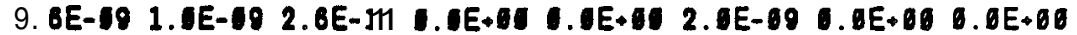
8.5E-15 4.5E-16 1.8E-J16 0.0E+09 ๑.0E+00 3.3E-11 .0E+00 8.8E+00

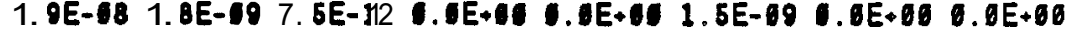

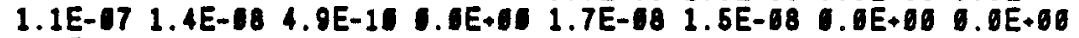

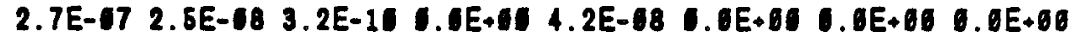
$1.0 E-162.1 E-17$ 1.3E-17 5.0E+61 0.0E+61 $1.8 E-120.0 E+00 \quad 0.0 E+60$

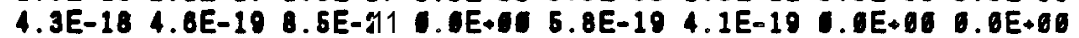

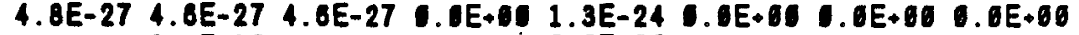

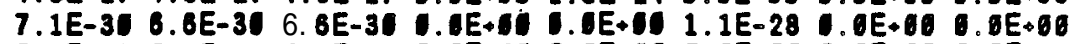

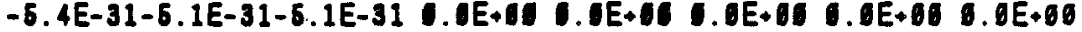

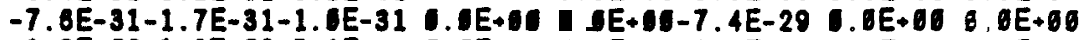

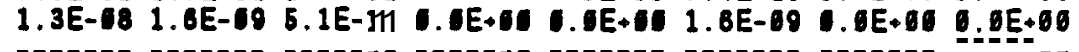




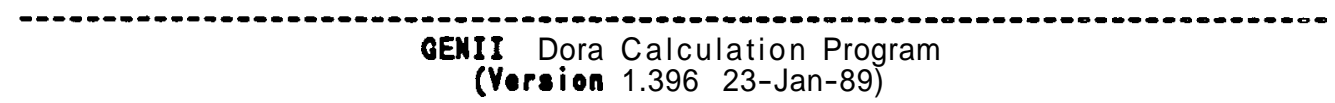

Case title: acute relesse of 1 pound of fresh furl to river, drinking only

Exrcutad on: $\mathbf{1 0 / 2 6 / 8 9}$ at 16:46:12
Acute release
Uptake/exposure period:
Dora commitment prriod:
Dora unitr:

Committad Dora Equivalent by Radionuclide

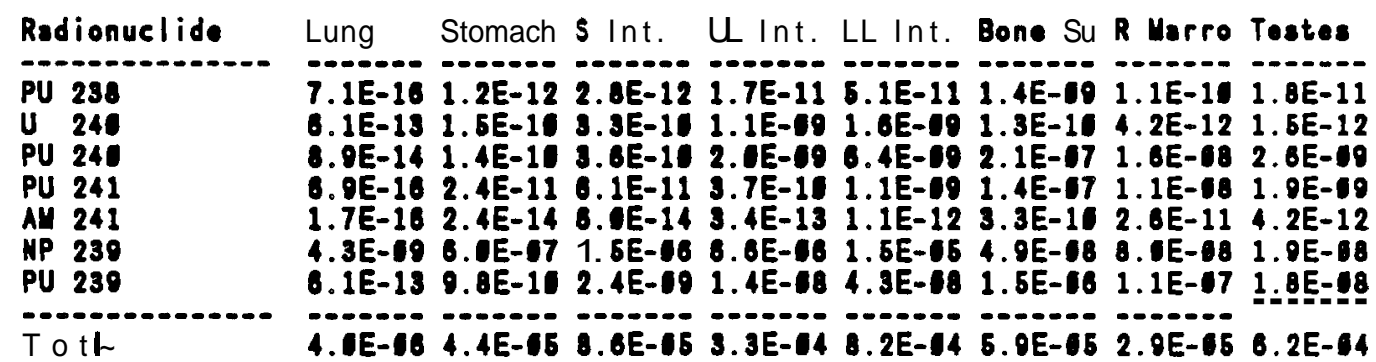


(Version 1.396 23-Jan-89)

Case title: scute rolesse of 1 pound of fresh fuel to river, drinking only

Executed on: 10/28/89 at 10:46:12

Page C. 8

Acute release

Uptake/exposure period:

Coseittad Dora Equivalent by Radionuclida

Radionuclida

PU 238

U 240

PU 241

PU 241

A4 241

NP 239

PU_239

Total
Ovaries Huscle Thyroid Bladder Kidneys Liver Spleen $\mathbf{s}$ Wall

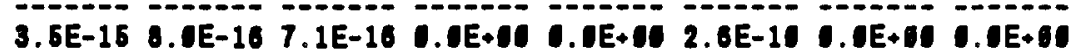

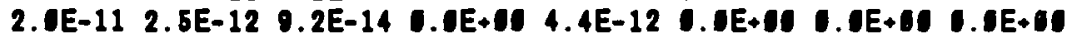
4.4E-13 9.0E-14 0.0E-14 0.0E+00 0.0E+06 3.7E-08 0.UE+00 $0.0 E+06$

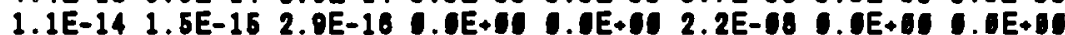

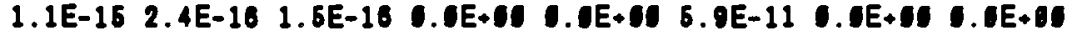

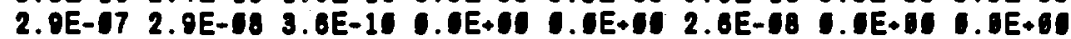

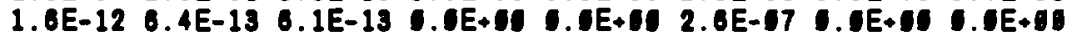
2. 
Case title: acute release of 1 pound of fresh fuel to river, drinking only

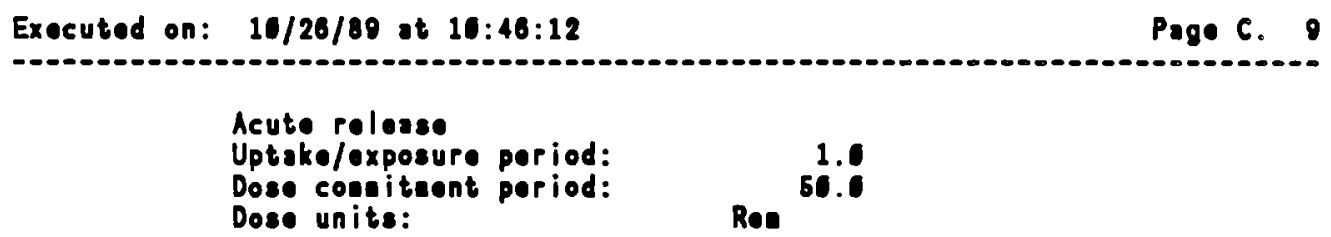

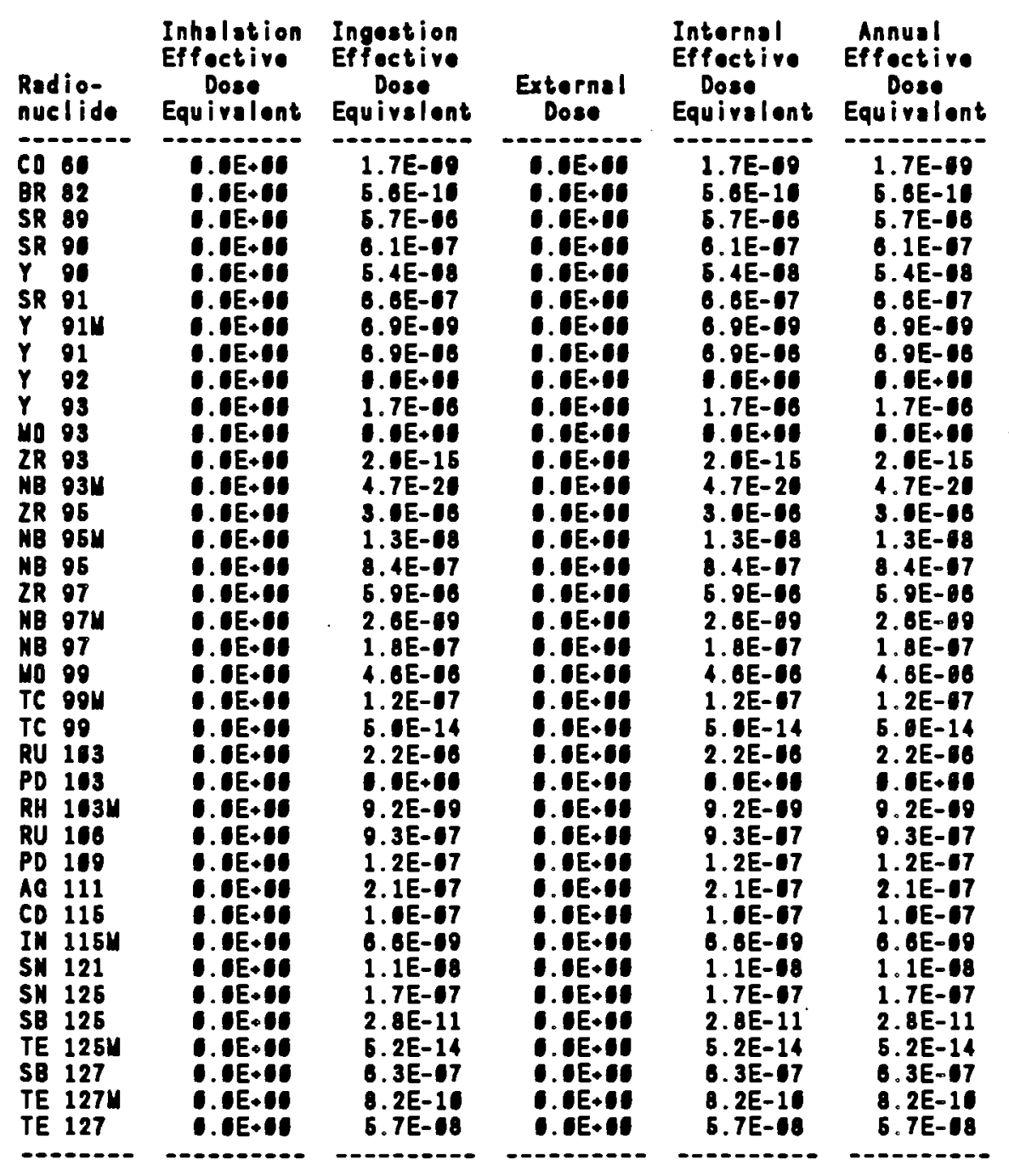




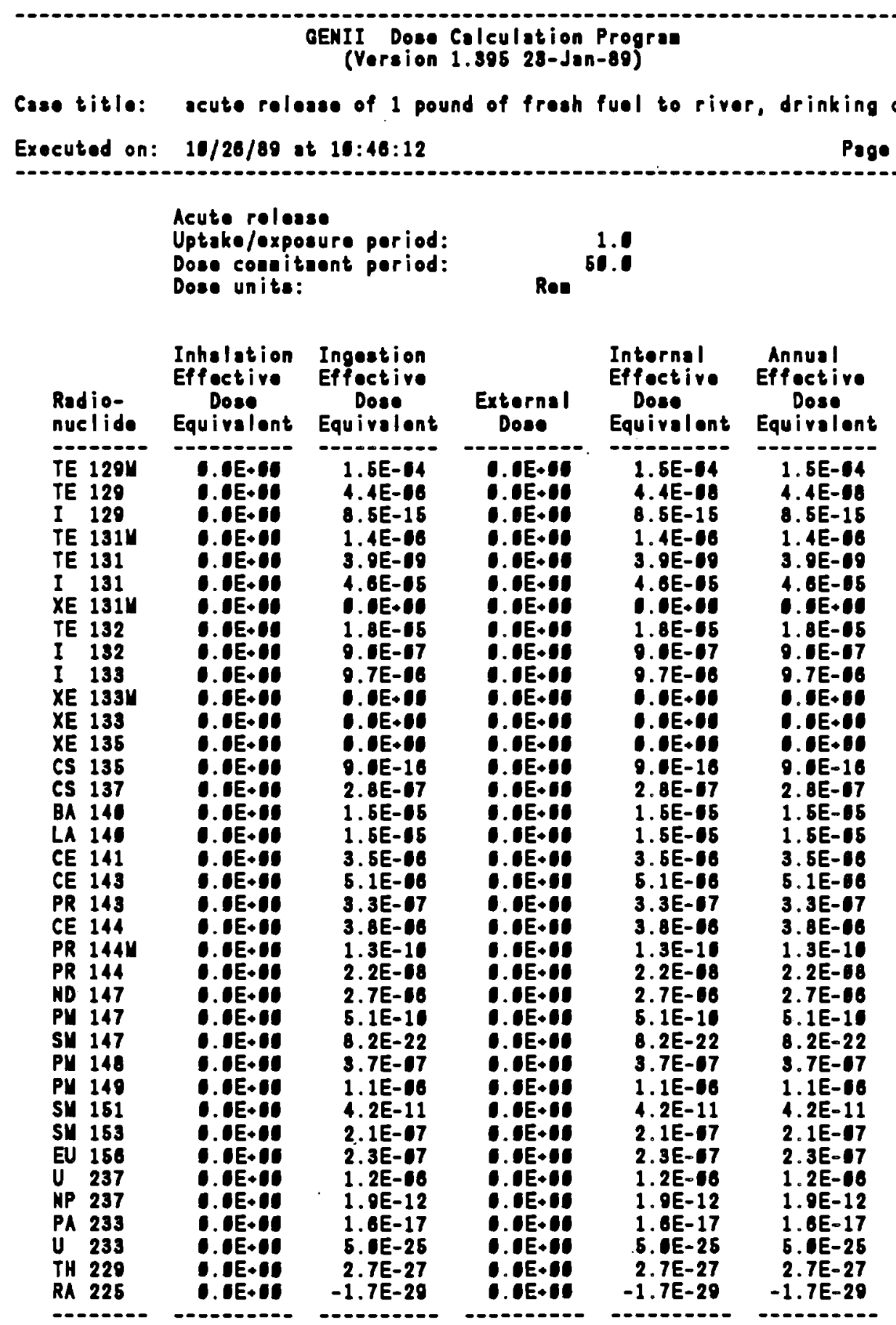




OENII Dose Calculation Progras
(Version 1.395 23-Jen-89)




\section{APPENDIX D}

NCRP SCREENING FACTOR RESULTS

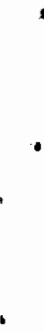




\begin{tabular}{|c|c|c|c|c|c|c|c|c|c|c|c|c|}
\hline \multirow{2}{*}{$\begin{array}{l}\text { Effective } \\
\text { MUCLIDE }\end{array}$} & \multicolumn{3}{|c|}{ - Dose Air Scroening Factors } & \multicolumn{2}{|c|}{ (aren per $\mathrm{Ci} / \mathrm{m} 3$ ) } & \multicolumn{2}{|c|}{ Buildup tine 34} & \multirow{2}{*}{$\begin{array}{l}y \\
\text { TOTAL. }\end{array}$} & \multicolumn{2}{|l|}{$\begin{array}{l}\text { ORIGEN }(\mathrm{C} i) \\
\text { Inventory }\end{array}$} & \multirow{2}{*}{$\begin{array}{l}\text { Weighted } \\
\text { Relative } \\
\text { Dose factor } \\
\text { (TDF x INY } \\
\text { oren-n3 }\end{array}$} & \multirow{2}{*}{$\times R F)$} \\
\hline & Half-life & INHW & PLUME & GROUND & VEG & MILK & MEAT & & $\begin{array}{l}\text { FP/AP/Ac+D } \\
\text { bvg mhu/I } \\
\text { Эed Buildup }\end{array}$ & & & \\
\hline $\begin{array}{l}-13 i \\
y-103 \\
y-106 \\
0-61 \\
-95 \\
-132 \\
-144 \\
-137 \\
-96 \\
-91 \\
r-89 \\
-146\end{array}$ & $\begin{array}{r}8.04 \\
39.28 \\
368.2 \\
5.271 \\
63.98 \\
2.31 \\
284.3 \\
30.6 \\
29.12 \\
68.61 \\
54.6 \\
12.74 \\
35.16 \\
33.6\end{array}$ & $\begin{array}{l}2 \\
12 \\
11 \\
10 \\
12 \\
11 \\
13 \\
1 \\
11 \\
10 \\
10\end{array}$ & 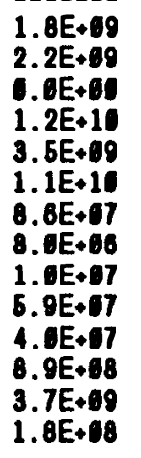 & $\begin{array}{l}4.4 \mathrm{E}+11 \\
2.6 \mathrm{E}+12 \\
1.8 \mathrm{E}+13 \\
5.3 \mathrm{E}+14 \\
1.3 \mathrm{E}+13 \\
3.6 \mathrm{E}+11 \\
6.6 \mathrm{E}+12 \\
3.8 \mathrm{E}+14 \\
6.7 \mathrm{E}+13 \\
5.0 \mathrm{E}+11 \\
3.9 \mathrm{E}+11 \\
4.1 \mathrm{E}+12 \\
3.6 \mathrm{E}+12 \\
5.7 \mathrm{E}+11\end{array}$ & $\begin{array}{l}9.0 \mathrm{E}+12 \\
1.1 \mathrm{E}+12 \\
1.3 \mathrm{E}+13 \\
1.6 \mathrm{E}+13 \\
1.6 \mathrm{E}+12 \\
1.7 \mathrm{E}+66 \\
9.7 \mathrm{E}+12 \\
3.4 \mathrm{E}+13 \\
3.6 \mathrm{E}+14 \\
3.7 \mathrm{E}+12 \\
3.7 \mathrm{E}+12 \\
2.2 \mathrm{E}+12 \\
9.0 \mathrm{E}+11 \\
3.7 \mathrm{E}+12\end{array}$ & 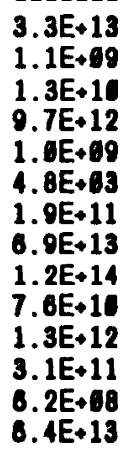 & $\begin{array}{l}5.3 E+12 \\
1.7 E+11 \\
2.1 E+12 \\
3.6 E+13 \\
6.1 E+68 \\
2.4 E-13 \\
1.6 E+12 \\
6.6 E+13 \\
2.9 E+13 \\
6.0 E+11 \\
3.0 E+11 \\
3.0 E+10 \\
7.1 E+67 \\
2.9 E+12\end{array}$ & $\begin{array}{l}4.7 E+13 \\
4.0 E+12 \\
3.7 E+13 \\
6.0 E+114 \\
1.6 E+13 \\
4.4 E+16 \\
2.1 E+13 \\
6.5 E+14 \\
5.9 E+14 \\
6.3 E+12 \\
6.6 E+12 \\
6.6 E+12 \\
4.6 E+12 \\
7.2 E+13\end{array}$ & $\begin{array}{l}2.0 E+64 \\
1.2 E+65 \\
8.6 E+63 \\
3.2 E+65 \\
1.6 E+65 \\
6.7 E+62 \\
4.4 E+64 \\
1.6 E+03 \\
1.3 E+63 \\
1.3 E+65 \\
1.1 E+65 \\
9.2 E+64 \\
1.2 E+\theta 5 \\
3.9 E+03\end{array}$ & $\begin{array}{l}7 . \\
1 . \\
1 . \\
1 . \\
1 . \\
7 . \\
1 . \\
1 . \\
1 . \\
1 . \\
1 . \\
1 . \\
1 . \\
1 .\end{array}$ & & 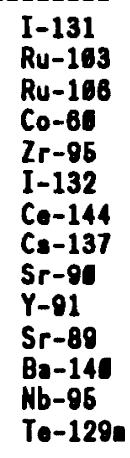 \\
\hline $\begin{array}{l}11 \\
39 \\
13 \\
13 \\
16 \\
34 \\
17 \\
16 \\
11 \\
25 \\
32 \\
18\end{array}$ & $\begin{array}{r}240 \\
5.2 \\
13 . \\
45.2 \\
2.0 \\
18 . \\
65 \\
1.57 \\
14 \\
2 . \\
78 \\
13 \\
41\end{array}$ & $\begin{array}{l}10 \\
15 \\
01 \\
16 \\
10\end{array}$ & $\begin{array}{l}3.8 \\
1.7 \\
1.8 \\
1.1 \\
7.4 \\
6.3 \\
4.2 \\
3.9 \\
7.8 \\
2.0 \\
1.0 \\
1.0 \\
9.4\end{array}$ & $\begin{array}{l}3.9 \mathrm{E}+11 \\
3.5 \mathrm{E}+11 \\
1.0 \mathrm{E}+60 \\
4.1 \mathrm{E}+10 \\
4.9 \mathrm{E}+11 \\
1.5 \mathrm{E}+14 \\
2.4 \mathrm{E}+11 \\
7.7 \mathrm{E}+11 \\
2.0 \mathrm{E}+13 \\
3.9 \mathrm{E}+11 \\
5.8 \mathrm{E}+13 \\
1.1 \mathrm{E}+12 \\
3.8 \mathrm{E}+12 \\
1.1 \mathrm{E}+13\end{array}$ & $\begin{array}{l}1.1 \\
1.1 \\
3.1 \\
3 . \\
9.1 \\
2.1 \\
1.1 \\
4.1 \\
1.1 \\
7 . \\
2 .\end{array}$ & $\begin{array}{l}2.1 \mathrm{E}+18 \\
6.9 \mathrm{E}+69 \\
1.0 \mathrm{E}+68 \\
2.4 \mathrm{E}+16 \\
4.8 \mathrm{E}+69 \\
9.2 \mathrm{E}+13 \\
2.0 \mathrm{E}+16 \\
6.9 \mathrm{E}+69 \\
6.8 \mathrm{E}+14 \\
1.6 \mathrm{E}+68 \\
7.3 \mathrm{E}+11 \\
1.2 \mathrm{E}+13 \\
7.6 \mathrm{E}+12 \\
6.8 \mathrm{E}+16\end{array}$ & $\begin{array}{l}1 \\
1 \\
1 \\
1 \\
19 \\
3 \\
1 \\
1 \\
4 \\
19 \\
1 \\
1 \\
2 \\
1\end{array}$ & $\begin{array}{l}4 . \\
1 . \\
1 . \\
8 . \\
3 . \\
1 . \\
4 . \\
1 . \\
8 . \\
6 . \\
1 . \\
1 .\end{array}$ & $\begin{array}{l}65 \\
61 \\
64 \\
05 \\
65 \\
02 \\
64 \\
65 \\
04 \\
62 \\
02 \\
62 \\
02 \\
02\end{array}$ & $\begin{array}{l}1 . \\
1 . \\
1 . \\
1 . \\
1 . \\
1 . \\
1 . \\
7 . \\
1 . \\
1 . \\
1 . \\
1 .\end{array}$ & & $\begin{array}{l}-141 \\
1-239 \\
-133 \\
-143 \\
-146 \\
-134 \\
-147 \\
1-246 \\
129 \\
-241 \\
-125 \\
-132 \\
-136 \\
-148 .\end{array}$ \\
\hline 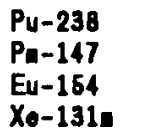 & $\begin{array}{r}87.74 \\
2.6234 \\
8.6 \\
11.9\end{array}$ & 1 & $8.9 E$ & $\begin{array}{l}7.2 E+11 \\
5.6 E+68 \\
4.3 E+14 \\
6.0 E+96\end{array}$ & $\begin{array}{l}1.9 E+14 \\
5.6 E+11 \\
4.6 E+12 \\
1.0 E+90\end{array}$ & $\begin{array}{l}0.2 \\
9.8 \\
9.2\end{array}$ & $\begin{array}{l}1 \\
1 \\
1 \\
1\end{array}$ & $\begin{array}{l}15 \\
11 \\
14 \\
97\end{array}$ & $\begin{array}{l}60 \\
63 \\
63\end{array}$ & 1.0 & $\begin{array}{l}10 \\
15 \\
15 \\
10\end{array}$ & $\begin{array}{l}-238 \\
-147 \\
-164 \\
-131 .\end{array}$ \\
\hline
\end{tabular}




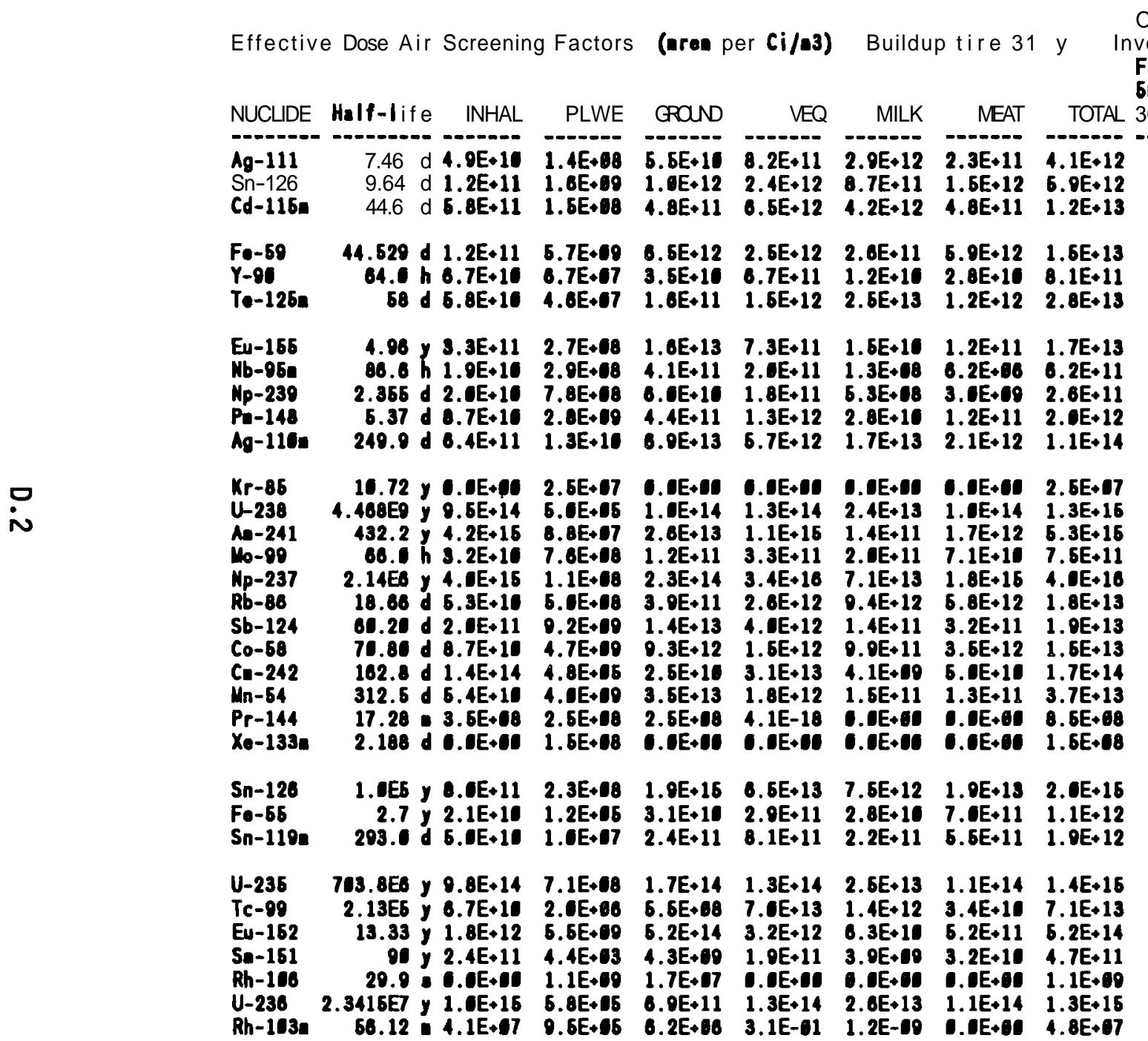

ORIGEN (Ci)

FP/AP/Ac+D

FP/AP/Ac+D

Weighted

Relative

Dose factor

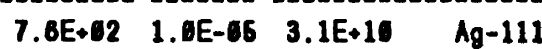

4.6E-12 1.0E-05 2.7E+10 Sn-126

2.1E+02 1.0E-ब5 2.6E+10 Cd-115

$8.8 E+91 \quad 1.0 E-15 \quad 1.3 E+10$

Fe-69

$1.3 \mathrm{E}+13$ 1.GE-95 $1.1 \mathrm{E}+10$

$Y-90$

$3.4 E+91$ 1. EE-95 $9.6 E+09$ To-125

$4.6 E+01 \quad 1.0 E-05 \quad 7.7 E+09$

$1.1 \mathrm{E}+63 \quad 1.0 \mathrm{E}-65 \quad 7.0 \mathrm{E}+08$

$2.3 \mathrm{E}+13$ 1.0E-06 $\quad 6.1 \mathrm{E}+09$

2.3E+12 1.0E-06 $4.7 E+09$

$4.1 \mathrm{E}+01 \quad 1.0 \mathrm{E}-06 \quad 4.6 \mathrm{E}+00$

Eu-156

$\mathrm{Nb}-05 \mathrm{n}$

Mp-239

$P \square-148$

$1.7 E+12 \quad 1.0 E+00 \quad 4.4 E+09$

Ap-110

3E- 1 EE-55 $3.0 E+90$

$5.4 E-9$ 1. E- 25 .

2.7E+12 1. EE-65 2.0E+

4. BE- $1.0 \mathrm{E}-05$ 2.0E+09

4.8E-13 1.6E-66 1.9E+68

$8.4 E+65$ 1.0E-05 1.6E+68

4.7E+11 1.0E-05 8.0E+68

$6.3 \mathrm{E}+100 \quad 1.0 \mathrm{E}-05 \quad 7.0 \mathrm{E}+68$

3.3E-61 1.0E-65 6.8E+08

1.6E+89 1.0E-96 6.6E+88

$4.4 E+84$ 1. IE-95 3.8E+80

$2.0 E+601.0 E+00 \quad 3.0 E+68$

$\mathrm{Kr}-8 \mathrm{85}$

U-238

An-241

Mo-90

Np-237

$\mathrm{Rb}-68$

Sb-124

Co-58

Co-68

Can-242

Pr-144

Pr-144
Xe-133

1.3E- $02 \quad 1.0 E-05 \quad 2.0 E+68$

$1.0 E+01$ 1.0E-06 2.1E+80

$5 n-126$

$0.7 E+15$ 1. DE-95 1.8E+68

Sn-110

1.3E- 12 1.0E- $16 \quad 1.8 \mathrm{E}+68$

2.3E-11 1.0E-95 1.6E+68

$\begin{array}{lll}2.3 \mathrm{E}-11 & 1.6 \mathrm{E}-06 & 1.6 \mathrm{E}+68 \\ 2.0 \mathrm{E}-12 & 1.0 \mathrm{E}-05 & 1.6 \mathrm{E}+08\end{array}$

$3.1 E+61$ 1.0E-15 1.4E+69

8.6E+13 1.0E-65 $9.4 E+97$

6.6E-03 1.0E-05 7.2E+07

1. $0 E+65 \quad 1.0 E-15 \quad 6.0 E+07$

U-235

Tc-99

Eu-162

Sn-161

Rh-106

U-238

Rh-103a 


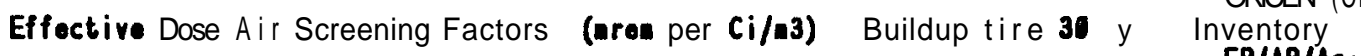

Relative

FP/AP/AC+D $\quad$ Dose factor

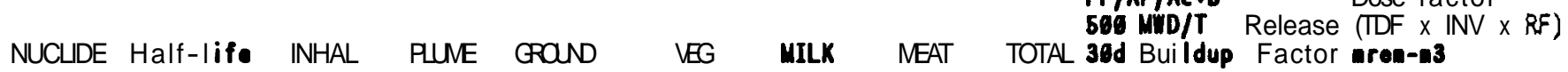

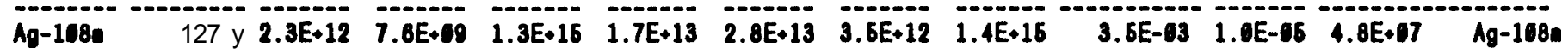

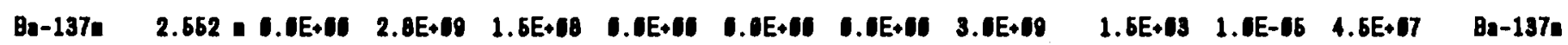

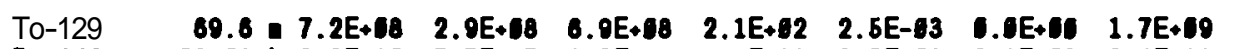

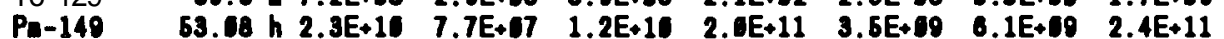

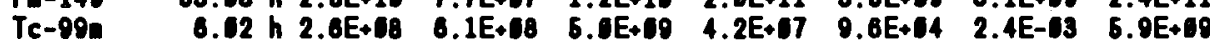

Th-234 24.10 d 2.8E+11 $3.0 \mathrm{E}+17 \quad 3.7 \mathrm{E}+11 \quad 4.2 \mathrm{E}+12 \quad 7.4 \mathrm{E}+69 \quad 3.2 \mathrm{E}+10 \quad 4.9 \mathrm{E}+12$

Pu-236 2.851 y $1.3 \mathrm{E}+15 \quad 5.8 \mathrm{E}+15 \quad 3.6 \mathrm{E}+13 \quad 7.5 \mathrm{E}+13 \quad 2.4 \mathrm{E}+11 \quad 3.1 \mathrm{E}+11 \quad 1.4 \mathrm{E}+16$

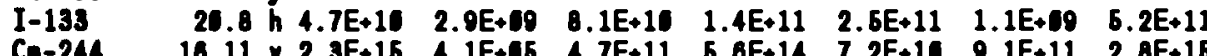

Cu-242 3.763E5 $3.9 E+15$ 4.1E+16 4.7E+11 $5.6 E+14$ 7.2E+16 $9.1 E+11$ 2.8E+16

Cd-116 53.46 h $3.4 E+10 \quad 9.0 E+18$ 1.2E+11 $3.0 E+11$ 1.7E+11 $4.6 E+10 \quad 6.3 \mathrm{E}+11$

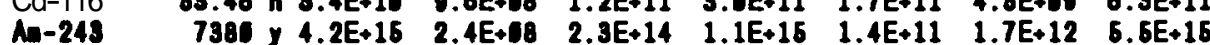

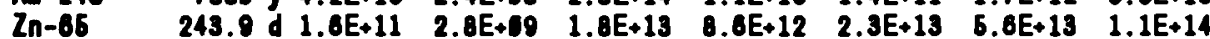

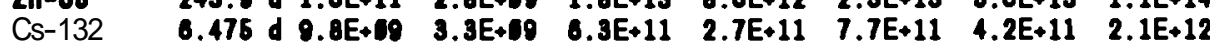

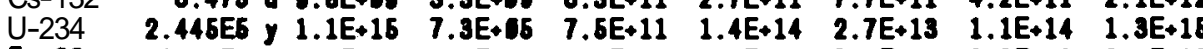

$\begin{array}{lllllllll}2 r-93 & 1.63 E 0 & 2.8 E+12 & 0.0 E+16 & 4.6 E+11 & 8.3 E+11 & 6.3 E+08 & 3.3 E+16 & 3.8 E+12\end{array}$

St-163 46.7 h $1.0 E+10 \quad 2.6 E+10 \quad 2.1 E+10 \quad 1.3 E+11 \quad 2.2 E+10 \quad 3.0 E+10 \quad 1.7 E+11$

2.3E0 y 3.6E+16 6.4E+15 $5.6 E+10 \quad 5.4 E+12 \quad 1.0 E+13 \quad 9.6 E+12 \quad 2.6 E+13$

96 y $5.0 E+10 \quad 0.0 E+10 \quad 0.0 E+10 \quad 5.2 E+11 \quad 1.1 E+11 \quad 5.7 E+10 \quad 7.4 E+11$

$\mathrm{Ni}-83$

$\mathrm{Cr}-61$

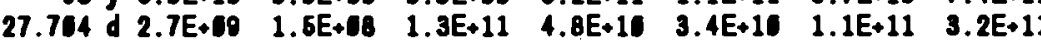

Sn-117. $13.61 \mathrm{~d} 3.6 \mathrm{E}+10 \quad 7.0 \mathrm{E}+08 \quad 3.1 \mathrm{E}+11 \quad 7.1 \mathrm{E}+11 \quad 2.6 \mathrm{E}+11 \quad 4.8 \mathrm{E}+11 \quad 1.8 \mathrm{E}+12$

$\begin{array}{llllllllll}\text { Cd-163 } 242 & d & 1.9 E+11 & 4.1 E+18 & 4.1 E+12 & 6.3 E+11 & 1.1 E+10 & 8.7 E+10 & 4.9 E+12\end{array}$ $H-3 \quad 12.35$ y $5.0 E+18 \quad 0.0 E+00 \quad 0.0 E+00 \quad 1.0 E+09 \quad 1.6 E+09 \quad 4.7 E+08 \quad 4.4 E+09$

C-14

C. -143

$\mathrm{C}-243$ 6736 y $1.7 E+10 \quad 2.7 E+16 \quad 0.6 E+10 \quad 4.6 E+11 \quad 2.3 E+11 \quad 2.7 E+11 \quad 9.8 E+11$

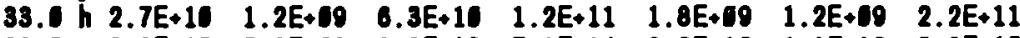

28.6 y $2.8 E+16 \quad 6.9 E+08 \quad 9.2 E+13 \quad 7.1 E+14 \quad 9.2 E+16 \quad 1.1 E+12 \quad 3.6 E+16$

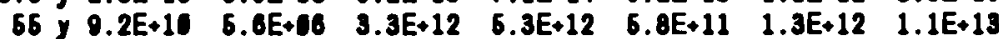

2. $6 E+63 \quad 1.0 \mathrm{E}-65 \quad 4.3 \mathrm{E}+67$

$6.8 \mathrm{E}+61$ 1.0E- 65 2.1E+67 Pa-149

$2.6 \mathrm{E}+62$ 1.0E-65 1.6E+17 Te-908

2.7E-01 1.0E-06 1.3E+67 Th-234

6.1E-64 1.0E-05 8.5E+06 Pu-236

2.3E-65. 7.0E-11 8.4E+66 I-133

2.7E-64 1.0E-65 $7.6 \mathrm{E}+66 \quad \mathrm{Cm}-244$

1.4E-14 1.0E-15 5.8E+16 Pu-242

6.6E-11 1.0E-16 $3.6 \mathrm{E}+16 \quad \mathrm{Cd}-115$

6. 2E-15 1.0E-15 $3.4 E+16 \quad A_{0}-243$

2.6E-63 1.0E- 25 2.0E+16 Zn-65

$1.1 E-61$ 1.0E-65 2.4E+16 C -132

$1.4 E-14$ 1.0E-15 $1.8 \mathrm{E}+16 \quad \mathrm{U}-234$

3.0E-62 1.0E-05 1.2E+66 Zr-93

5.8E-61 1.0E-65 $9.9 E+06$ SII-163

3.7E-63 1.0E- $05 \quad 9.3 E+15 \quad C_{3}-135$

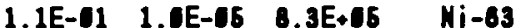

2.4E-G1 $1.0 E-05$ 7.7E.05 $\mathrm{Cr}-61$

2.8E-62 1.0E-65 6.0E+16 Sn-117.

$9.7 \mathrm{E}-103 \quad 1.0 \mathrm{E}-106 \quad 4.8 \mathrm{E}+165$

$8.6 \mathrm{E}+00$ 1.0E- 06 3.7E+06

3.7E-12 1.0E-05 $3.6 E+05$

1.3E-11 1.0E-15 2.8E+15

6.3E-16 1.0E-105 2.3E+105

2.1E-13 1.0E-16 $2.3 E+16$

Gd-153

$\mathrm{H}-3$

C-14

Ce-143

Cd-109

$484 d \quad 9.1 E+11 \quad 1.0 E+07 \quad 6.0 E+11 \quad 1.0 E+13 \quad 4.4 E+12 \quad 6.6 E+11 \quad 1.7 E+13$

Sb-122

2.70 d $4.1 E+10 \quad 2.1 E+09 \quad 1.8 E+11 \quad 4.6 E+11 \quad 1.4 E+10 \quad 9.9 E+09 \quad 7.1 E+11$

7.9E-14 $1.0 \mathrm{E}-105 \quad 1.3 \mathrm{E}+105$

$\mathrm{Sn}-121$.

In-115.

$\begin{array}{lllllll} & \end{array}$

$1.6 \mathrm{E}-62$ 1.0E-65 $1.1 \mathrm{E}+16 \mathrm{6}$

Cd-109

6.1E-11 1.0E-05 $3.8 \mathrm{E}+104$

$\mathrm{Sb}-122$

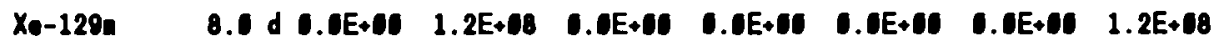

2.0E-104 1.0E+100 2.4E+104

$X_{0}-129=$

Nb-94 2.03E4 y $3.3 E+12 \quad 7.6 E+19 \quad 1.4 E+16 \quad 4.1 E+12 \quad 2.4 E+19 \quad 3.0 E+18 \quad 1.4 E+16$

$\begin{array}{lll}1.1 \mathrm{E}-166 & 1.0 \mathrm{E}-65 & 1.6 \mathrm{E}+104 \\ 2.6 \mathrm{E}-63 & 1.0 \mathrm{E}-65 & 1.4 \mathrm{E}+104\end{array}$

Nb-94

Pu-237 46.3 d $1.6 E+10 \quad 2.2 E+48 \quad 3.6 E+11 \quad 1.6 E+11 \quad 6.6 E+66 \quad 1.3 E+68 \quad 6.4 E+11$

$\mathrm{Pu}-237$ 
RADIONUCLIDE RANKINGS BASED ON ORIGEN INVENTORY, NCRP SCREENING FACTORS, AND RELEASE FACTORS

PACE 4

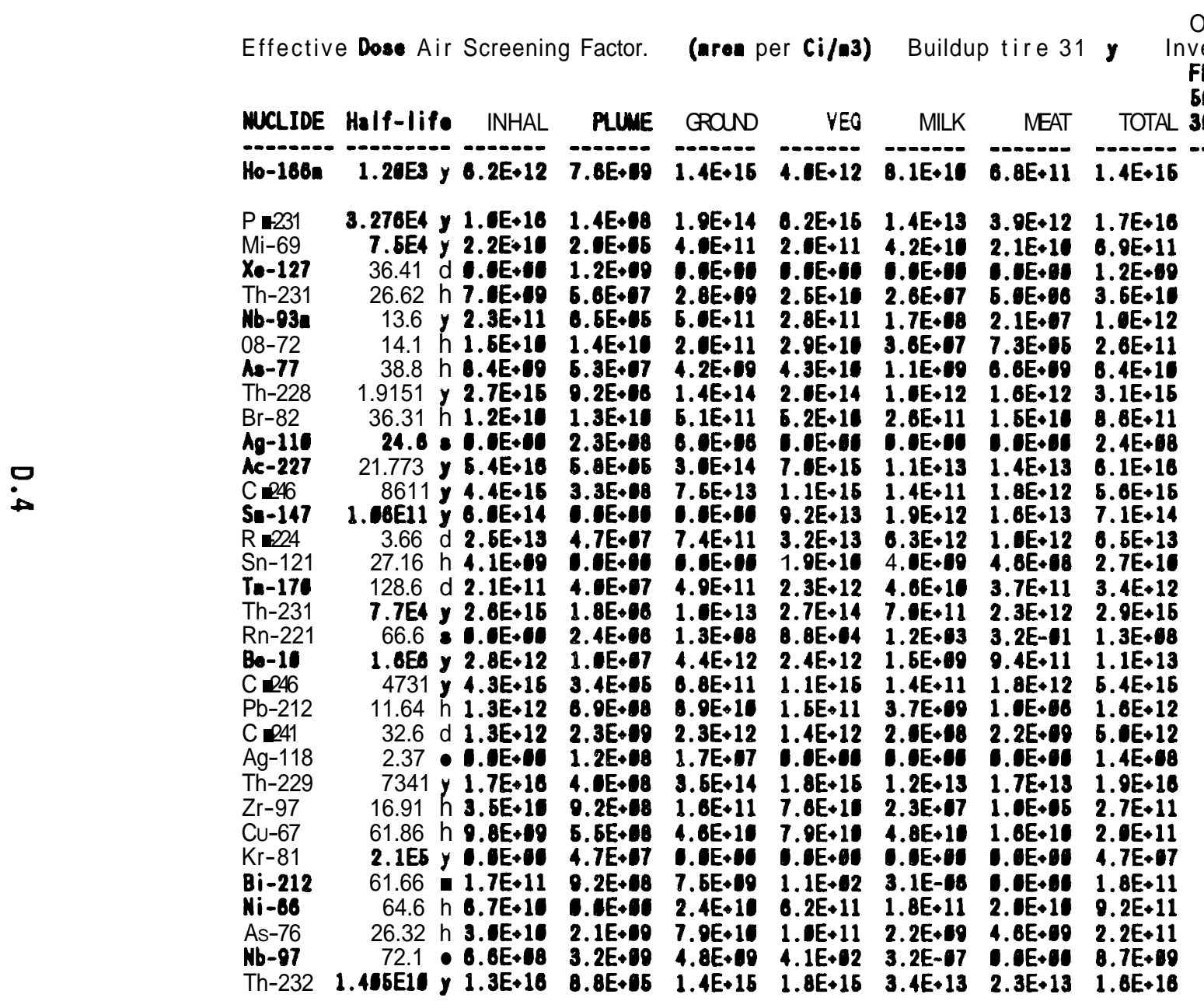

ORIGEN (Ci)

FP/AP/AC+D

EP/AP/AC+D

Relative

Relative

Dose factor

Geg UTD/T Release (TDF $\times$ INV $\times$ RF)

TOTAL 38d Buildup Factor mren-n

0.4E-\$7 1.0E-45 $\quad 1.3 E+04 \quad \mathrm{Ho}-1661$

5. 9E-18 1.9E-05 1.0E+04

$.6 E-04 \quad 1.0 E-06 \quad 5.9 E+63 \quad \mathrm{Ni}-59$

4.1E-66 1.0E+06 6.0E+03 $X_{0}-127$

1.3E-02 1.0E-66 4.6E+83 Th-231

2.4E-04 1.0E-05 2.4E+03 Nb-93R

7. $5 E-94$ 1.0E-05 2.0E+13 G2-72

2.7E-03 1.0E-06 $1.7 \mathrm{E}+13 \mathrm{A3} \quad \mathrm{As}-77$

4.6E-08 1.0E-05 1.4E+63 Th-228

$1.041 .0 E-05$ 1. $0 E \cdot 02$

$5.4 E-12$ 1. $0 E-05$ 1.3E+ 12

2.0E-10 1.0E-05 1.2E+ 12

1.4E-00 1.0E-05 O.0E-01

4.4E-00 1.0E-06 3.1E+61

4.0E-08 1.0E-05 2.6E+61

4.7E-95 1.0E-95 1.3E+81

2. 5E-07 1.0E-16 8.7E+10

1.0E-10 1.0E-06 6.6E+66

4.0E-108 1. $1 \mathrm{E}+90 \quad 5.1 \mathrm{E}+010$

$4.4 E-18$ 1.0E-05 4.0E+BO

1.4E-11 1.0E-G5 $6.0 \mathrm{E}-\mathrm{B}$

1.2E-11 1.0E-06 $0.0 \mathrm{E}-1$

$8.8 E-09$ 1.0E-05 4.4E-

3.1E-04 1.0E-06 4.3E-01

$1.3 E-12$ 1. OE-O5 2. EE-01

7.3E-08 1.0E-06 2.0E-01

$8.9 E-188$ 1.0E- 15 1.8E- 11

$1.7 \mathrm{E}-69$ 1.0E+60 $8.1 \mathrm{E}-62$

4.0E-08 1.0E- 05 7.1E-02

$1.9 \mathrm{E}-90$ 1.0E- 15 1.8E-62

3.4E-00 1.0E-06 7.4E-63

Br-82

Ag-110

Ac-227
Ca-245

Ca -245
Sa -147

Ra-224

Sn-121

To-170

Th-23:

Rn-220

Bo-10

$\mathrm{C} P-246$
$\mathrm{~Pb}-212$

$\mathrm{Pb}-212$
$\mathrm{C}-241$

$A g-100$

Th-229

Zr-97

Cu-67

Kr-81

Bi-212

$\mathrm{Mi}-66$

$7.9 E-68$ 1.0E-65 8.9E-63 $\mathrm{As}-70$

Th-232 $1.466 E 10$ y $1.3 E+16 \quad 8.8 E+95 \quad 1.4 E+15 \quad 1.8 E+15 \quad 3.4 E+13 \quad 2.3 E+13 \quad 1.8 E+16$

4.1E-14 1.0E-15 $\quad 0.8 E-103$

Th-232 


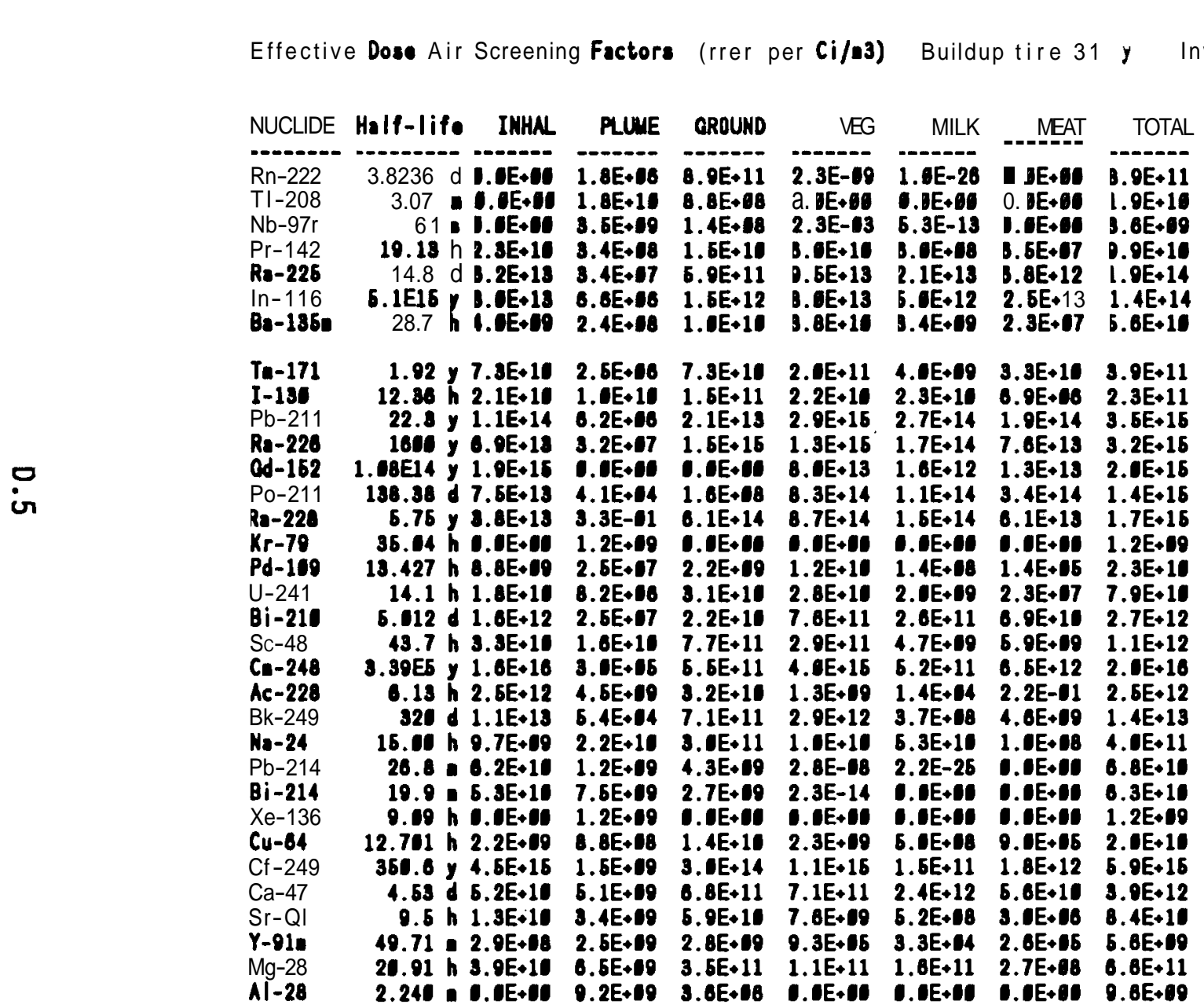

ORIGEN $(\mathrm{Ci})$

FP/AP/Ac+D

Weighted FP/AP/Ac+D
Go6 MIID/T Rolese (TDF $\times$ INV $\times$ RF)

NUCLIDE Half-life INHAL PLUNE aRoUND VEG MILK MEAT TOTAL 30d Buildup Factor eren-as

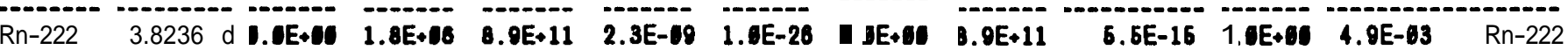

$\begin{array}{llllllll} & \end{array}$

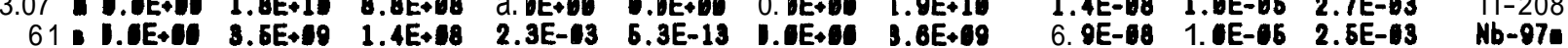

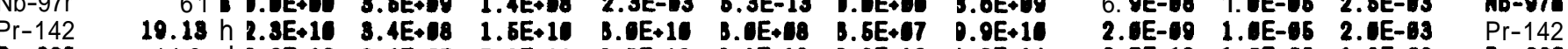

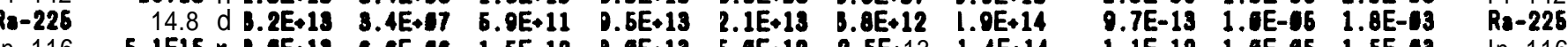

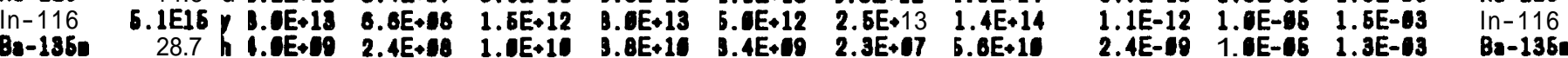

TI-171 1.92 y $7.3 E+102.6 E+16 \quad 7.3 E+10 \quad 2.0 E+11 \quad 4.0 E+19 \quad 3.3 E+10 \quad 3.0 E+11$

$T=17$

$T \mathbf{m}-17$

1.6E-15 7.0E-11 2.7E- $14 \quad \mathrm{I}-135$

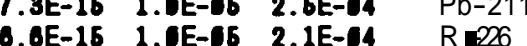

$\begin{array}{llll}0.0 \mathrm{E}-16 & 1.0 \mathrm{E}-165 & 2.1 \mathrm{E}-14 & \mathrm{R} 226 \\ 1.1 \mathrm{E}-15 & 1.0 \mathrm{E}-15 & 2.2 \mathrm{E}-16 \mathrm{G} & \mathrm{Gd}-162\end{array}$

1.1E-16 1.0E-15 2.2E-16 Gd-162

\begin{tabular}{llll}
$0.7 E-16$ & $1.0 E-15$ & $9.4 E-18$ & $\mathrm{PO}-211$ \\
\hline
\end{tabular}

3.6E-16 1.0E-16 5.9E-66 $\mathrm{Ra}-228$

3.2E-12 1. EE-05 7.4E-07 Pd-109

-

.5E-15 $1.0 E-15$ 1.7E-17

O.

$\begin{array}{llll}4.4 E-15 & 1.0 E-05 & 4.8 E-18 & \mathrm{SC}-48 \\ 1.5 \mathrm{E}-10 & 1.0 \mathrm{E}-15 & 3.1 \mathrm{E}-18 & \mathrm{C}-248\end{array}$

1.6E-19 1.0E-65 3.1E-18 CA-248

$\begin{array}{llll}3.6 E-16 & 1.0 E-05 & 8.0 E-19 & A C-228 \\ 4.0 E-17 & 1.0 E-15 & 5.0 E-19 & B k-249\end{array}$

$1.0 \mathrm{E}-\mathrm{O}$.

5.5E-15 1.0E-15 3.7E-19 $\quad \mathrm{Pb}-214$

5.5E-15 1.0E-65 3.5E-19 Bi-214

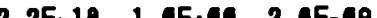

2.2E-18 $1.0 \mathrm{E}$.

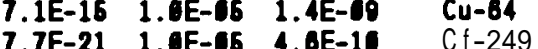

$\begin{array}{llll}7.7 \mathrm{E}-21 & 1.0 \mathrm{E}-16 & 4.0 \mathrm{E}-10 & \mathrm{C}-249 \\ 2.0 \mathrm{C}-18 & 1.0 \mathrm{E}-15 & 7.0 \mathrm{E}-11 & \mathrm{Ca}-47\end{array}$

$\begin{array}{llll}\text { 2.0E-18 } & 1.0 \mathrm{E}-05 & 7.9 \mathrm{E}-11 & \mathrm{Ca}-47 \\ \mathbf{3} .3 \mathrm{E}-18 & 1.0 \mathrm{E}-05 & \mathbf{5} .3 \mathrm{E}-12 & \mathrm{Sr}-\mathrm{Q}\end{array}$

4.0E-18 1.0E-16 2.2E-13 Y-910

1.3E-20 1.0E- 05 6. $0 \mathrm{E}-14 \quad \mathrm{Mg}-28$

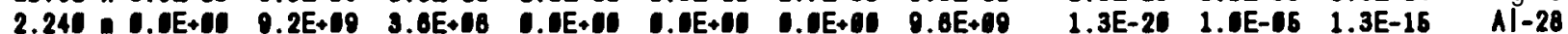


RADIONUCLDE RANKINGS BASED ON ORIGEN INVENTORY, NCPP SCREENING FACTORS, AND REEEASE FACTORS

PACE 6

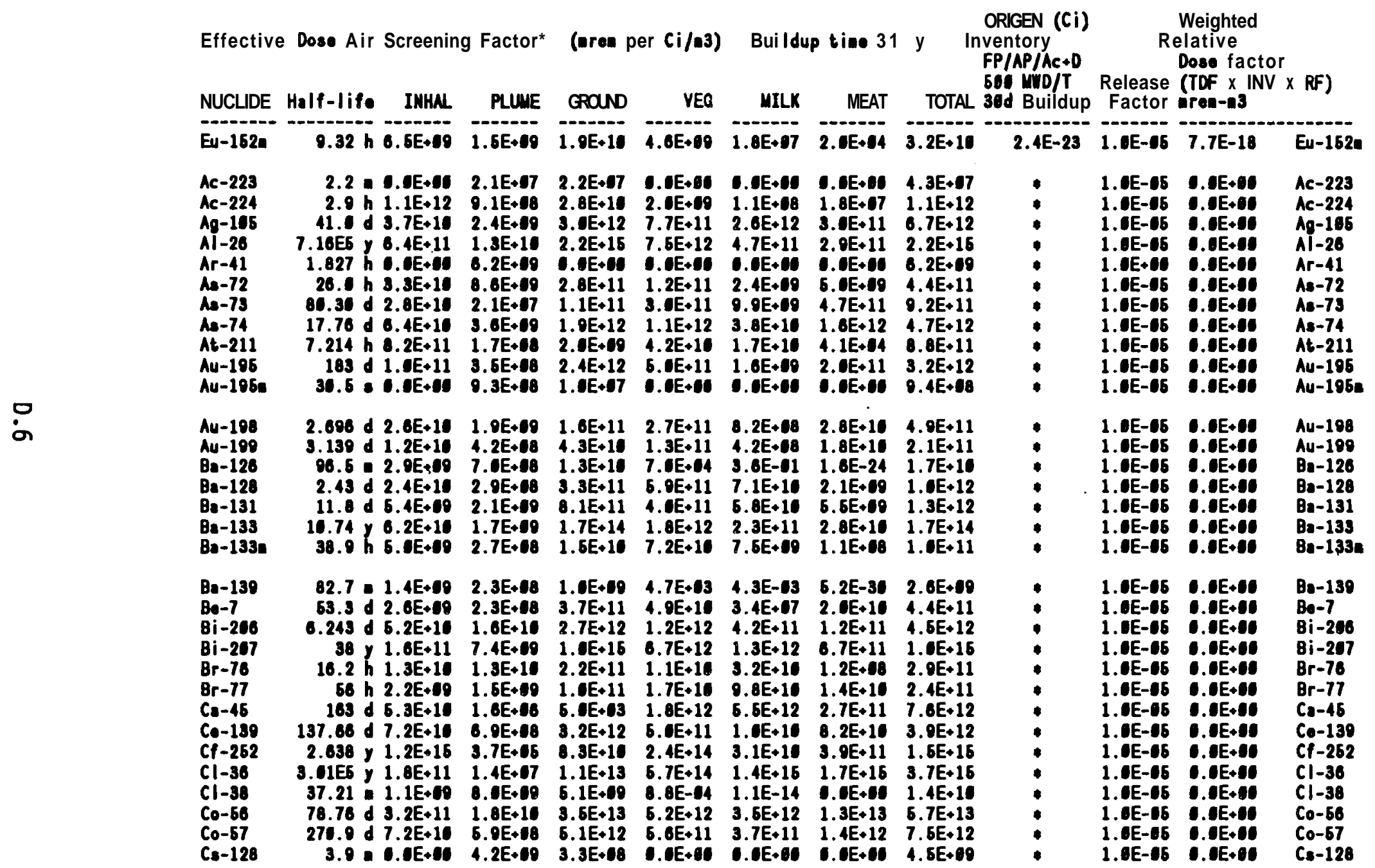




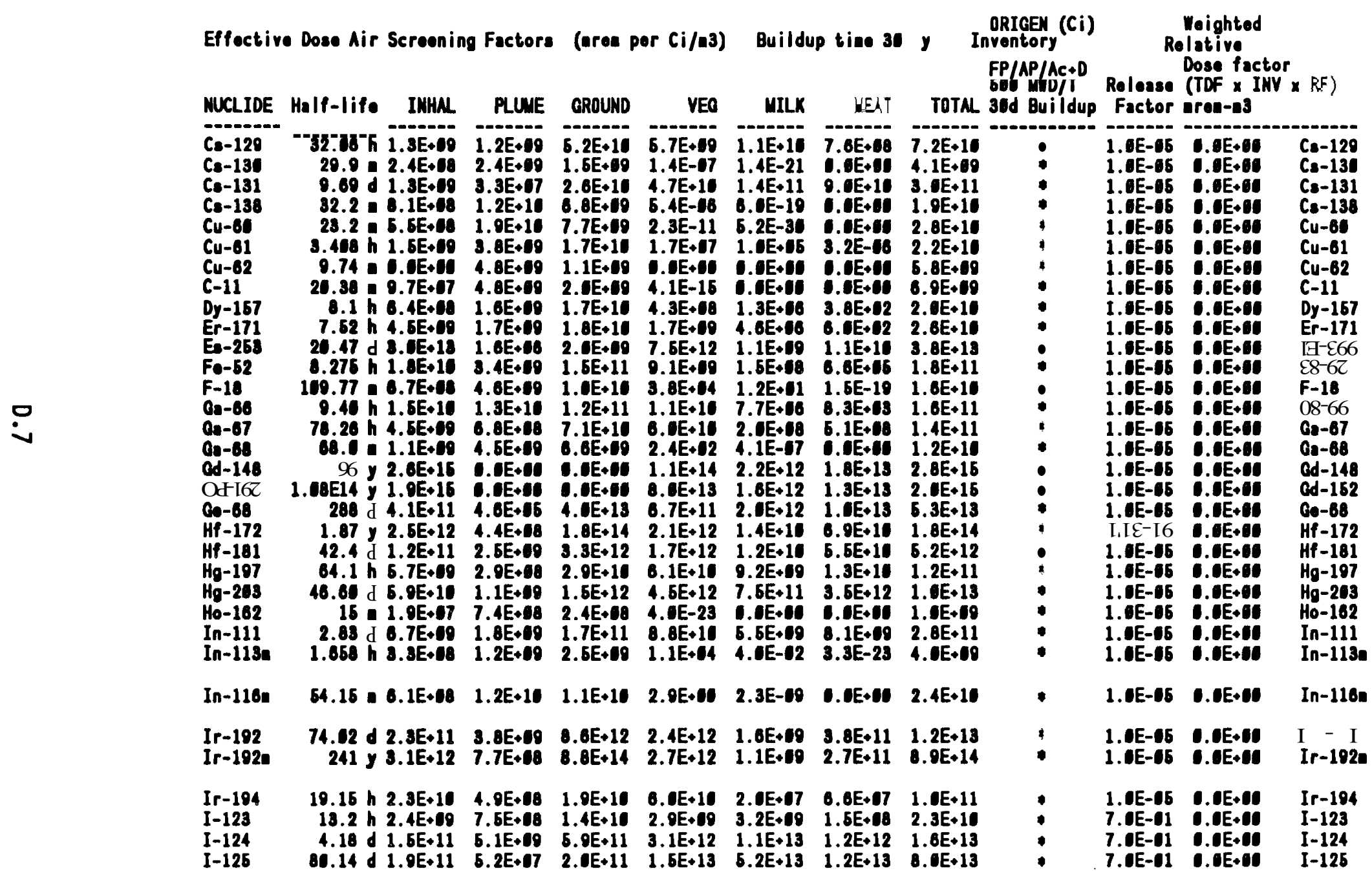




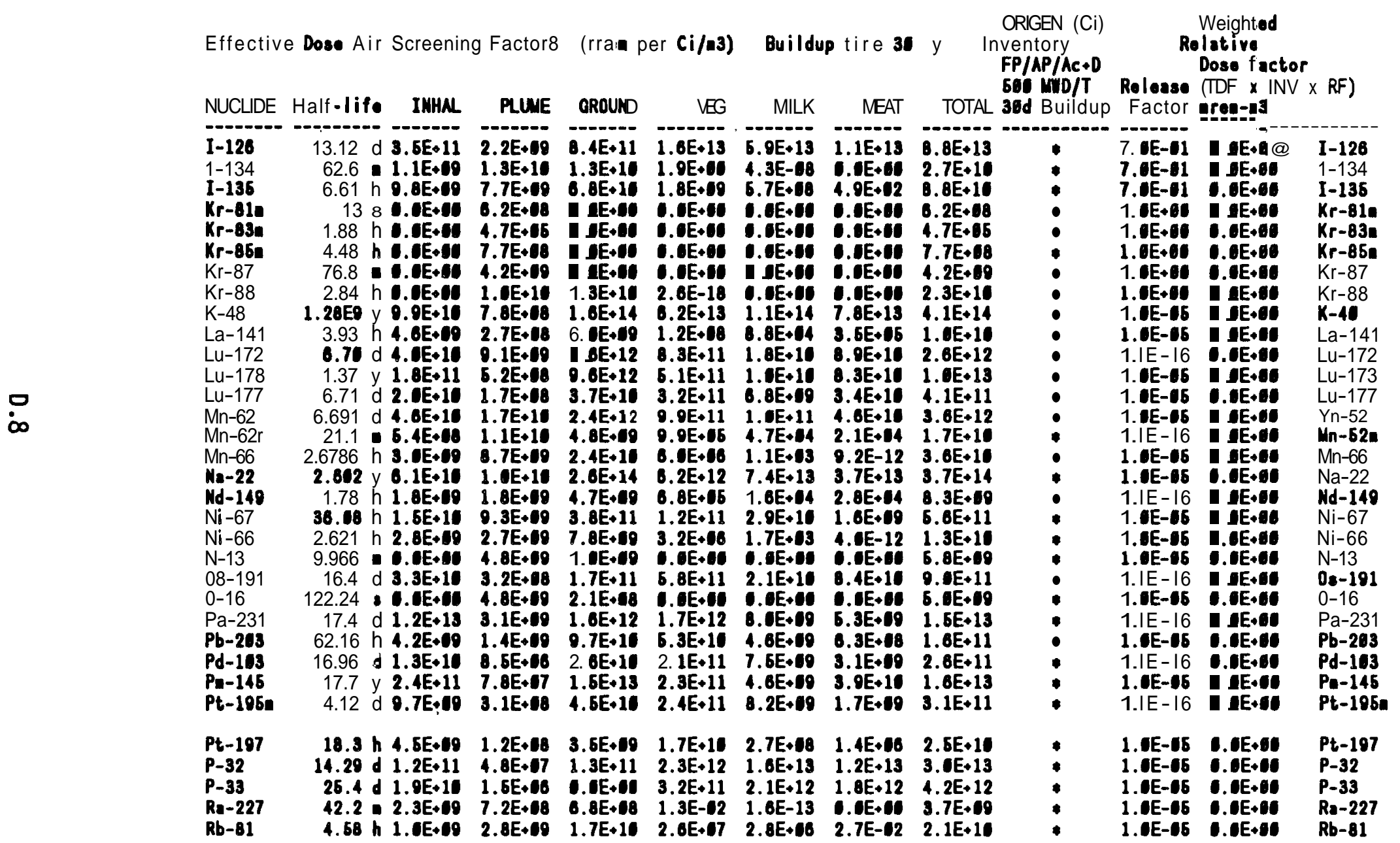


RADIONUCLDE RAHKIMOS BASED ON ORIGEN INVEMTORY, NOPP SCREENINQ FACTORS, AD REEASE FACTORS

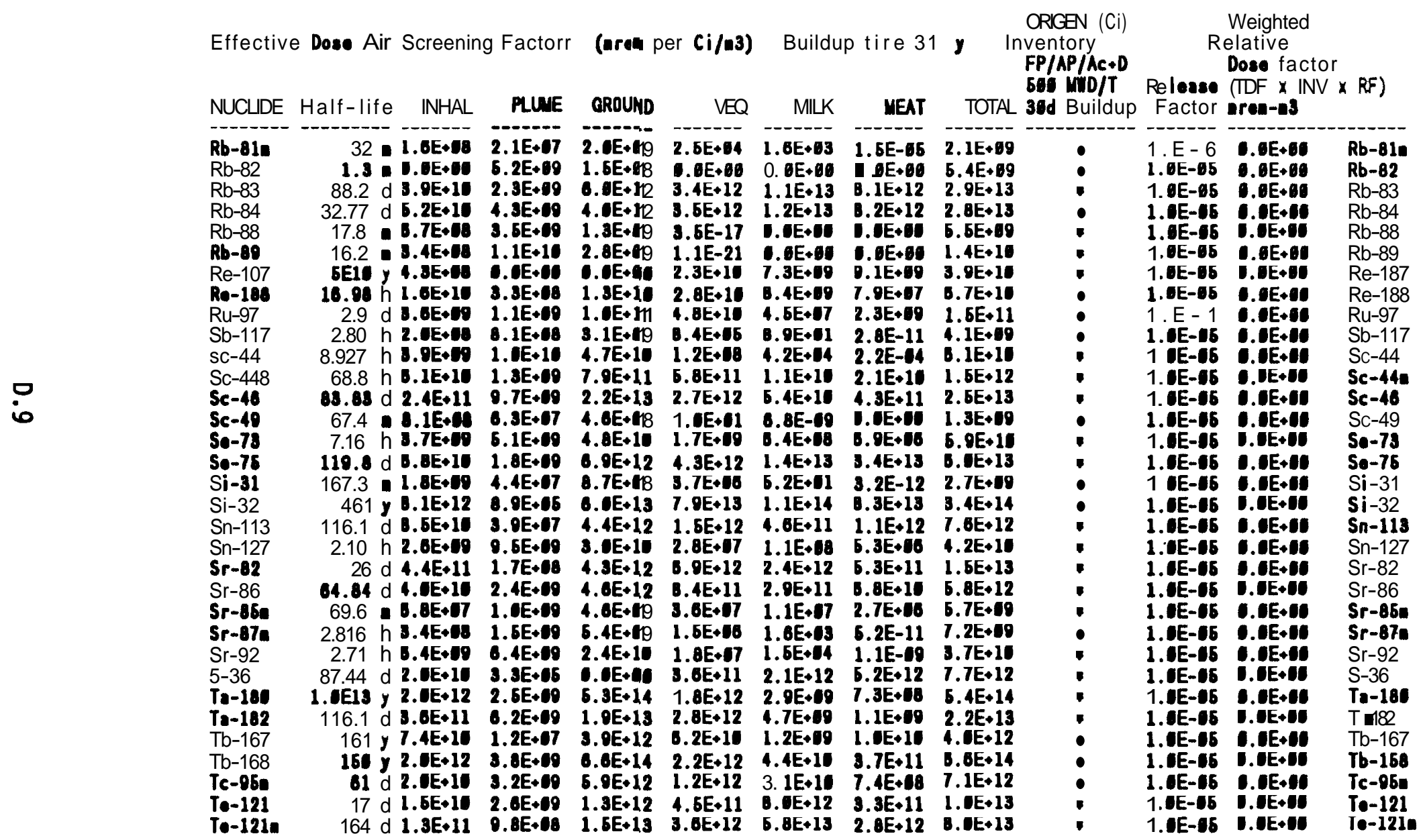


RADIONUCLDE RAMKIMGS BASED ON ORIGEM INENTORY, NCPP SCREEMIMO FACTORS, AN REEASE FACTORS

PAGE 10

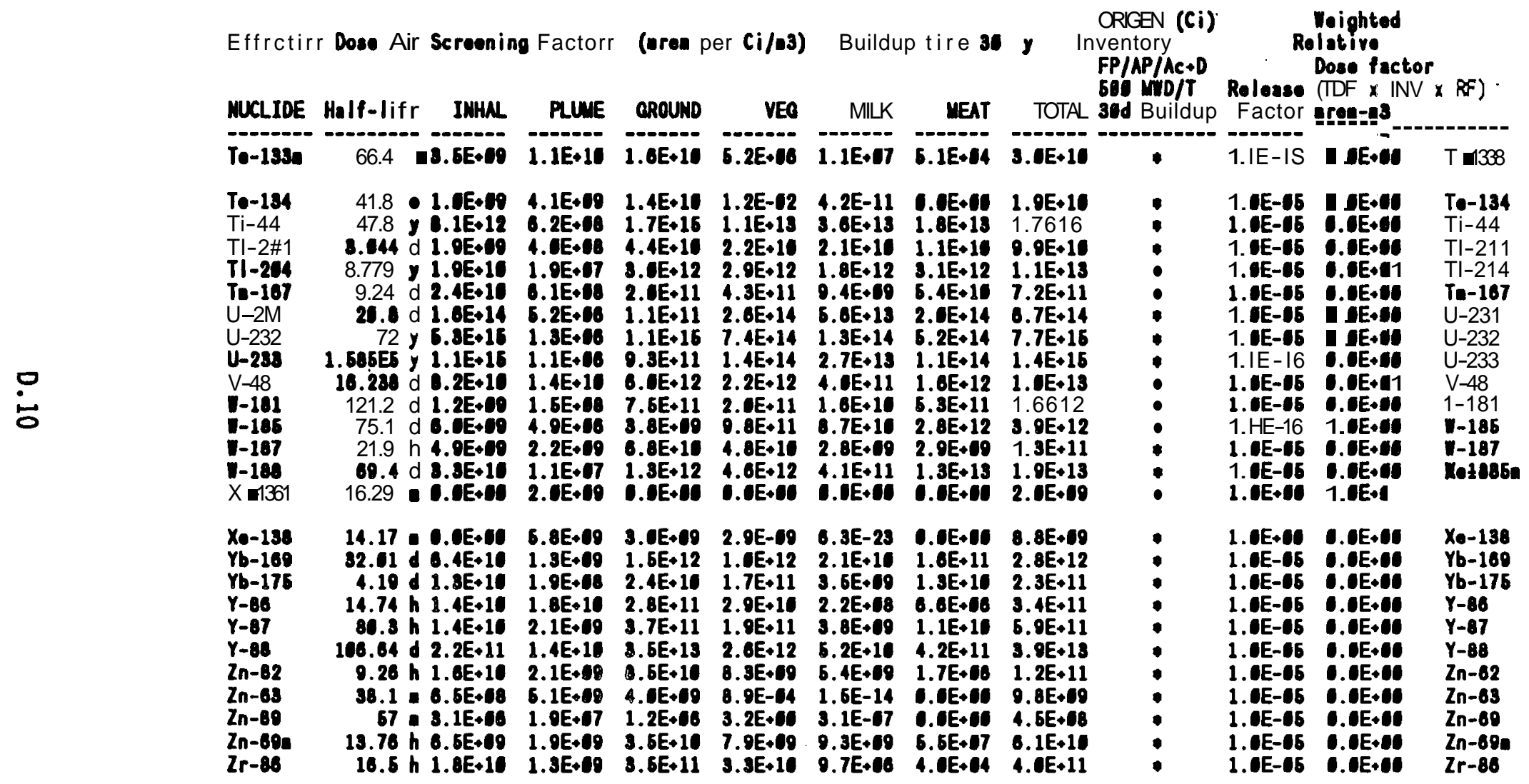

- Indicates isotopes for which ORICEN inventory is oithrr I or aissing 
RADIOMUCLIDE RAMKIMGS BASED ON ORIGEN IMVENTORY AMD MCRP SCREENINO FACTORS

Effective Dose Air Scrooning Factors (aree per Ci/as) Buildup ties $30 y$
MUCLIDE Half-life IMHAL PLUME GROUND VEO MILK MEAT TOTA

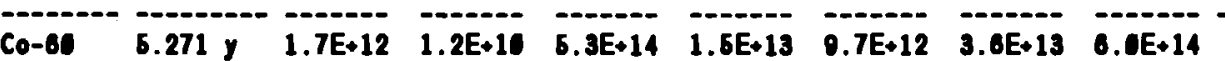

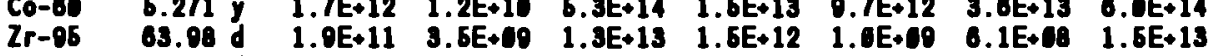

$\begin{array}{lllllllll}I-131 & 0.04 d & 2.0 E+11 & 1.0 E+10 & 4.4 E+11 & 0.0 E+12 & 3.3 E+13 & 5.3 E+12 & 4.7 E+13\end{array}$

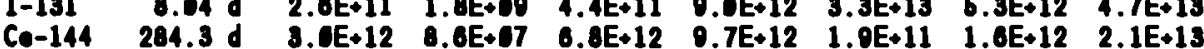

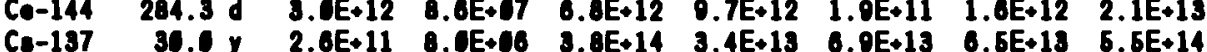

$\begin{array}{llllllll}\text { Sr-90 } 29.12 \text { y } & 1.0 E+13 & 1.0 E+17 & 0.7 E+13 & 3.6 E+14 & 1.2 E+14 & 2.0 E+13 & 5.0 E+14\end{array}$

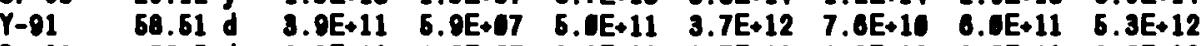

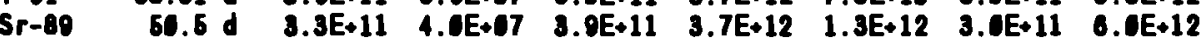

Ba-140 $12.74 d \quad 3.0 E+10 \quad 0.9 E+18 \quad 4.1 E+12 \quad 2.2 E+12 \quad 3.1 E+11 \quad 3.0 E+10 \quad 6.0 E+12$

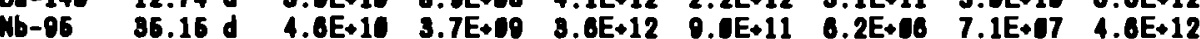

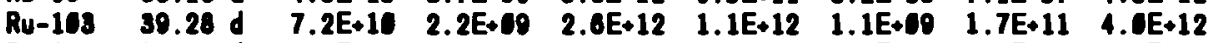

Bu-106 368.2 d $3.8 E+12 \quad 0 E+10 \quad 1.8 E+13 \quad 1.3 E+13 \quad 1.3 E+10 \quad 2.1 E+12 \quad 3.7 E+13$

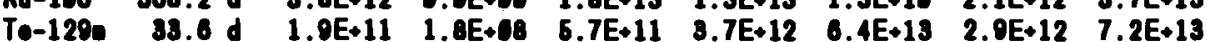

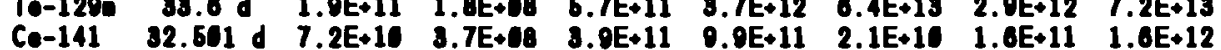

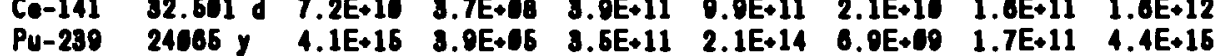

Pr-143 13.56 d $6.6 E+10 \quad 1.0 E+07 \quad 4.1 E+10 \quad 1.1 E+12 \quad 2.4 E+10 \quad 1.0 E+11 \quad 1.4 E+12$

L.-140 40.272 h $3.9 E+10$ l.1E+10 4.9E+11 3.0E+11 4.8E+19 $5.1 E+09 \quad 8.5 E+11$

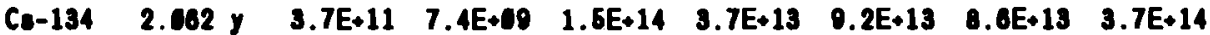

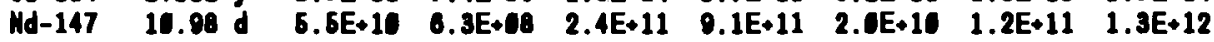

Pu-249 6537 y $4.1 E+15$ 4.2E+15 7.7E+11 2.1E+14 $6.9 E+19$ 1.7E+11 $4.4 E+15$

Pu-241 14.4 $8.3 E+13 \quad 7.6 E+13 \quad 3.9 E+11 \quad 4.5 E+12 \quad 1.6 E+18 \quad 3.6 E+00 \quad 8.8 E+13$

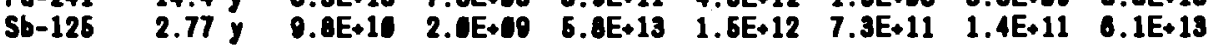

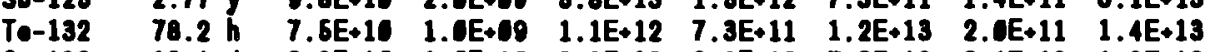

$\begin{array}{llllllll}C & -136 \quad 13.1 \mathrm{~d} \quad 6.0 E+10 & 1.0 E+10 & 3.8 E+12 & 2.0 E+12 & 7.6 E+12 & 5.4 E+12 & 1.9 E+13\end{array}$

$P=-148041.3 d \quad 1.8 E+11 \quad 0.4 E+10 \quad 1.1 E+13 \quad 2.8 E+12 \quad 5.8 E+10 \quad 4.4 E+11 \quad 1.5 E+13$

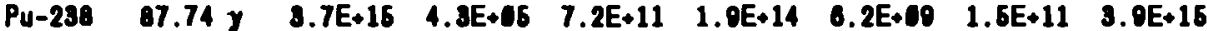

$\begin{array}{llllllllll}P a-147 & 2.0234 & \text { y } & 3.1 E+11 & 8.0 E+05 & 5.0 E+08 & 6.0 E+11 & 0.8 E+00 & 0.1 E+10 & 0.0 E+11\end{array}$

EU-154 8.8 y $2.3 E+12$ 6.0E+10 $4.3 E+14$ 4.6E+12 $9.2 E+107.6 E+11$ 4.4E+14

$\begin{array}{lllllllll}A g-111 & 7.45 \mathrm{~d} & 4.9 E+10 & 1.4 E+08 & 5.6 E+10 & 8.2 E+11 & 2.0 E+12 & 2.3 E+11 & 4.1 E+12\end{array}$

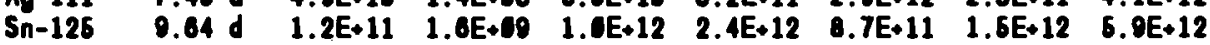

$\begin{array}{lllllllll}C d-115 & 44.6 d \quad 6.8 E+11 & 1.6 E+(8) & 4.8 E+11 & 6.6 E+12 & 4.2 E+12 & 4.8 E+11 & 1.2 E+13\end{array}$

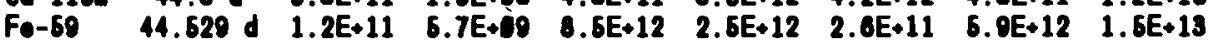

Fo-69 44.629 d $1.2 \mathrm{EE}+11$ 6.7.7E+09
PAGE 1

$\begin{array}{ll}\text { ORIGEY (Ci) } & \text { woighted } \\ \text { Inventory } & \text { Rolative } \\ \text { FP/AP/AC+D } & \text { Dose factor }\end{array}$

Dose factor
SOS IITD/T Release (TDF $\times$ INV $\times$ RF)

3ed Buildup Factor iren-as

$3.2 \mathrm{E}+105$

$1.0 \mathrm{E}+20$

2.3E+18

$0.6 \mathrm{E}+17$

$0.3 \mathrm{E}+17$

3.7E+17

7. $8 E+17$

7.1E+17

6. $7 \mathrm{E}+17$

6.1E+17

5. $7 E+17$

4.6E+17

$3 \mathrm{~F}+17$

$2.2 E+17$

2. $8 E+17$

$2.6 E+17$

2.6E+17

$1.4 E+17$

$0.0 E+16$

6. $3 E+16$

3. $6 E+16$

3. $6 \mathrm{E}+16$

$2.6 E+16$

2. $E$ + 16

$0.2 E+15$

8. $8 E+16$

7.7E+15

$6.6 \mathrm{E}+15$

5. $3 E+16$

$4.6 E+15$

3.1E+15

$2.7 E+15$

2.5E+15

1.3E+16

$8.8 E+01$

$1.3 \mathrm{E}+16$ 
RAOIOMUCLIDE RAMKIMOS OASED ON ORICEN IMVENTORY AMO MCRP SCREENIMO FACTORS

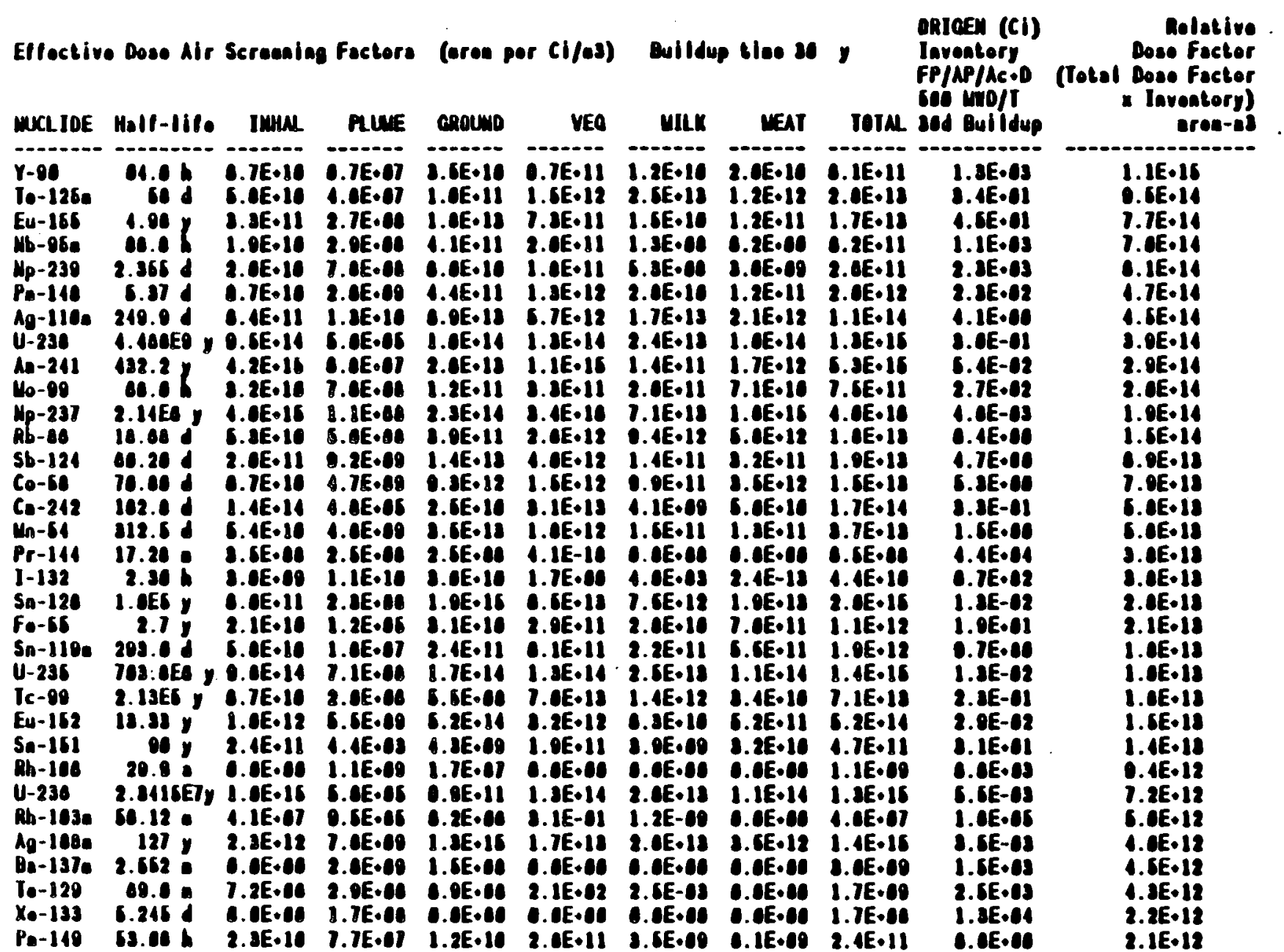


Ellective Dose Air Screening Factore (oren per Ci/ja) Bulldup tiace 30 y

onjegl (Cl)

Relative

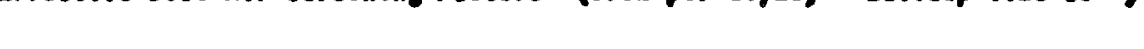

Dose Faclor

(Tolal Dose Factor

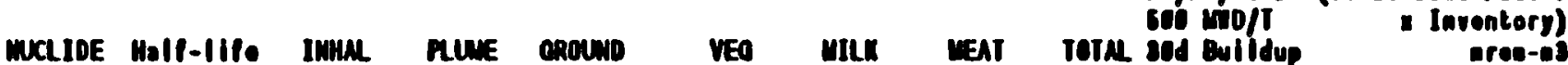

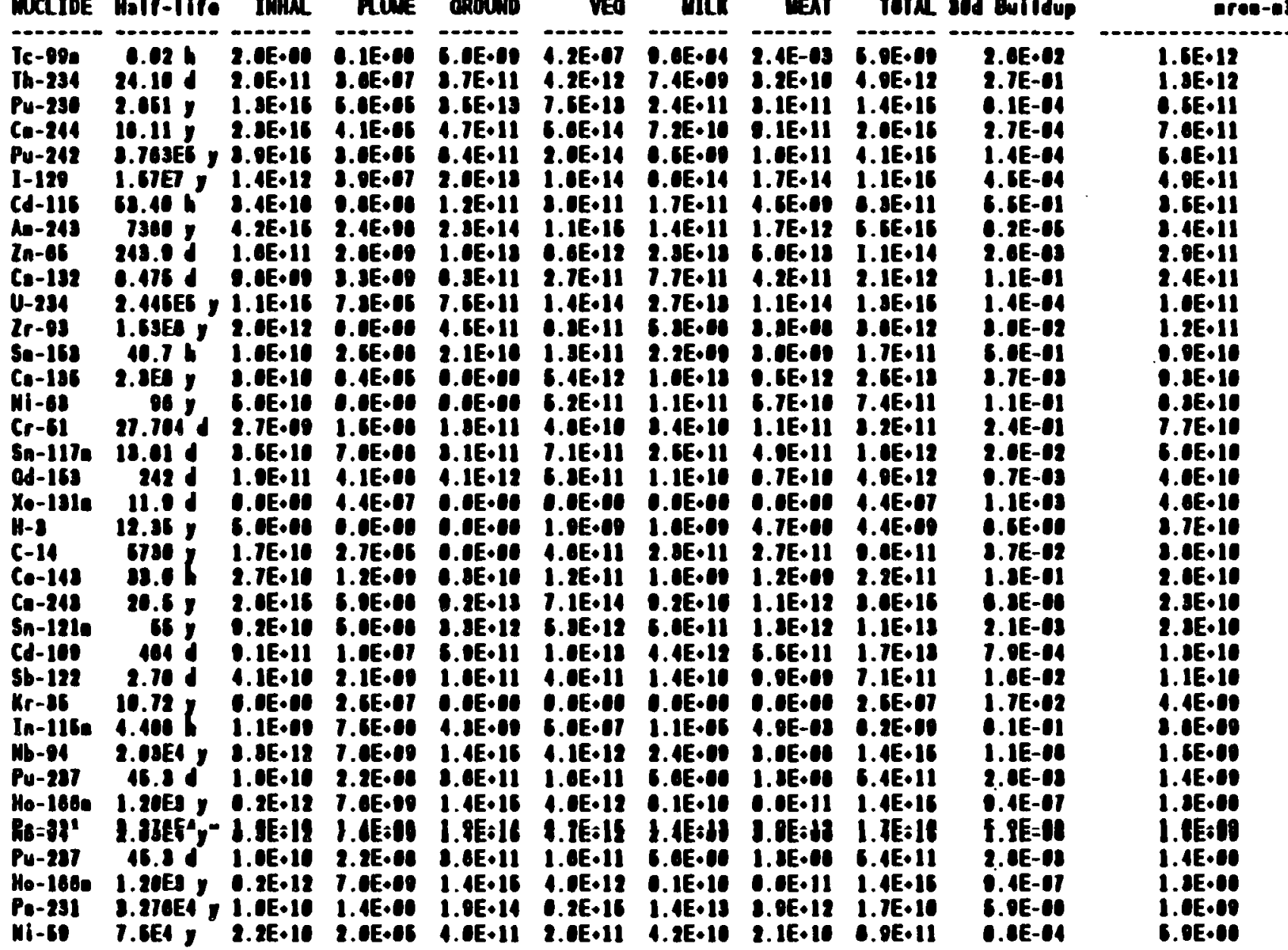




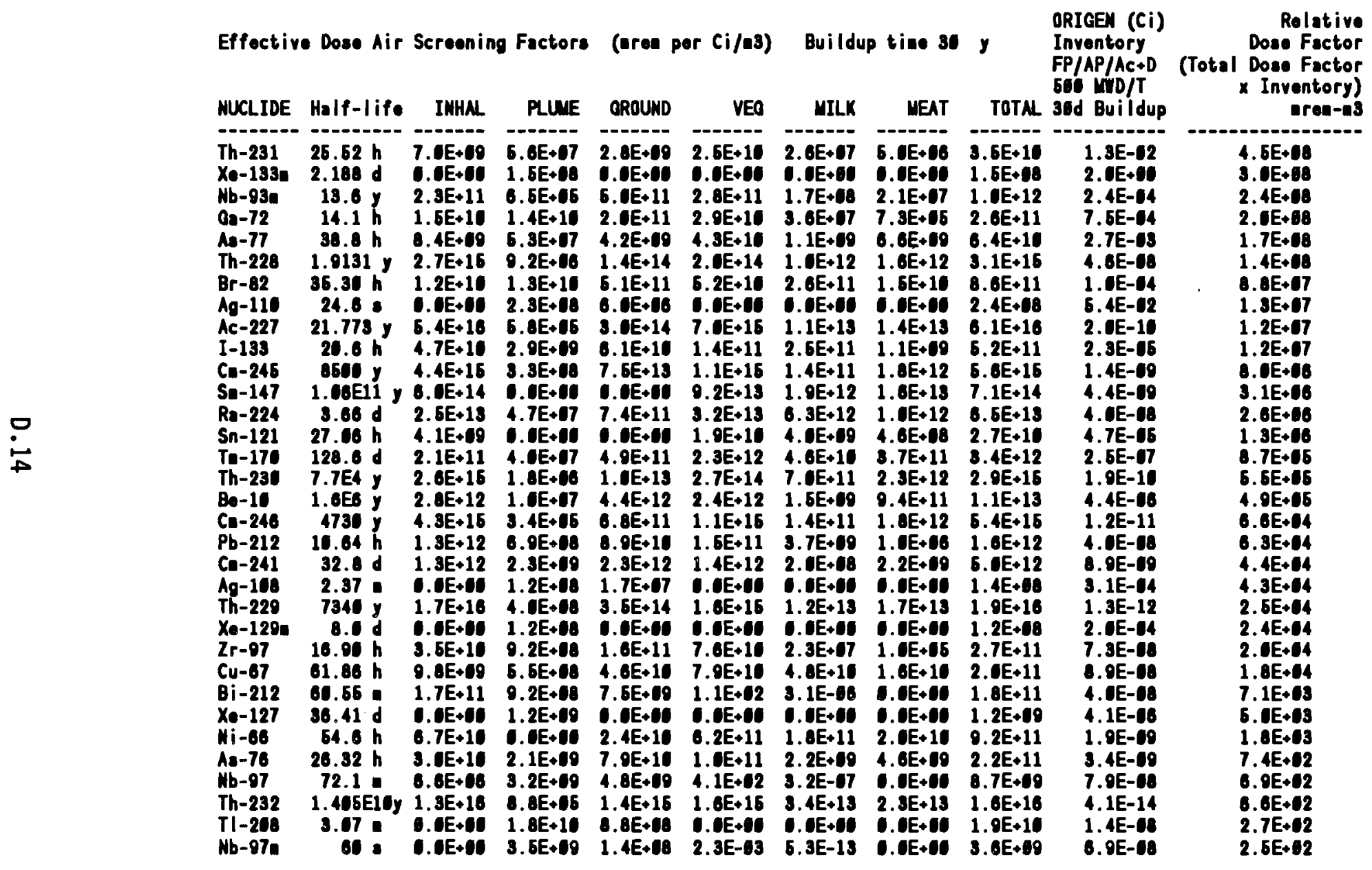


RADIONUCLIDE RAMKINGS BASED ON ORIGEN INVENTORY AND NCRP SCREENINO FACTORS

Effective Dose Air Screening Factors (aren por Ci/m3) Buildup tine 30 y

MUCLIDE Half-life INHAL PLUME GROUND YEQ MILK MEAT TOTAL Pr-142 10.13 h $2.3 E_{10}$ 3.4E+

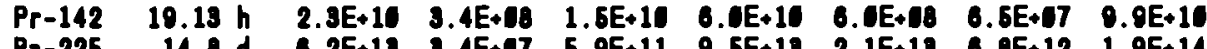
$\begin{array}{lllllllll}R 2-225 & 14.8 d & 6.2 E+13 & 3.4 E+17 & 5.9 E+11 & 0.6 E+13 & 2.1 E+13 & 0.8 E+12 & 1.9 E+14\end{array}$ In-116 5.1E15 y $3.0 E+13 \quad 6.6 E+16 \quad 1.6 E+12 \quad 8.0 E+13 \quad 6.0 E+12 \quad 2.6 E+13 \quad 1.4 E+14$ $B a-135028.7 \mathrm{~h} \quad 4.0 \mathrm{E}+10 \quad 2.4 \mathrm{E}+18 \quad 1.0 \mathrm{E}+10 \quad 3.8 \mathrm{E}+10 \quad 3.4 \mathrm{E}+00 \quad 2.3 \mathrm{E}+07 \quad 5.0 \mathrm{E}+10$

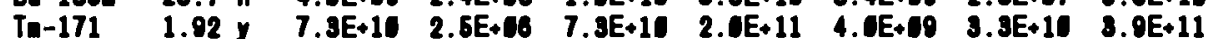

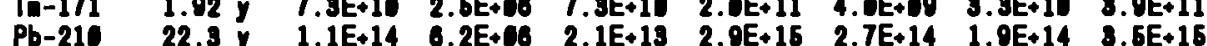

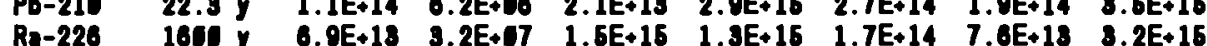

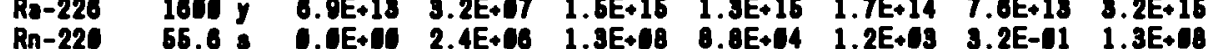

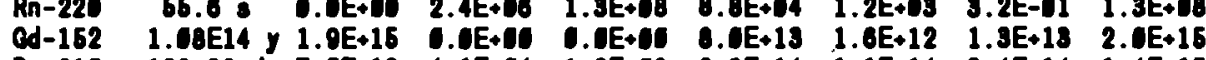
Po-210 138.38 d $7.6 E+18 \quad 4.1 E+14 \quad 1.6 E+18 \quad 8.3 E+14 \quad 1.1 E+14 \quad 3.4 E+14 \quad 1.4 E+16$ Re-228 5.75 y $8.8 E+13$ 3.3E-11 B.1E+14 B.7E+14 1.6E+14 8.1E+18 1.7E+15

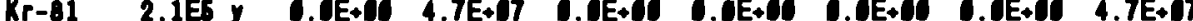

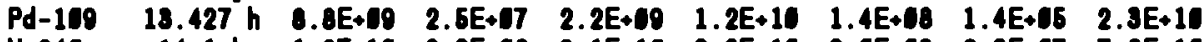
$\begin{array}{lllllllll}\mathrm{U}-24 \mathrm{~S} & 14.1 \mathrm{~h} & 1.8 \mathrm{E}+10 & 8.2 \mathrm{E}+16 & 3.1 \mathrm{E}+10 & 2.8 \mathrm{E}+10 & 2.0 \mathrm{E}+10 & 2.3 \mathrm{E}+17 & 7.9 \mathrm{E}+10\end{array}$ $B i-2105.112$ d $1.6 E+12$ 2.5E+17 2.2E+10 $7.8 E+112.0 E+11 \quad 0.9 E+11 \quad 2.7 E+12$

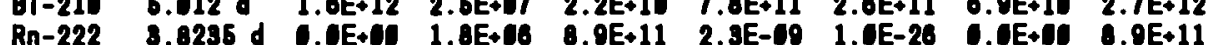

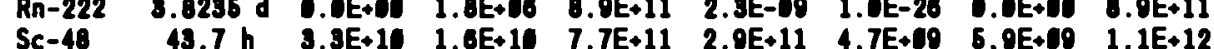

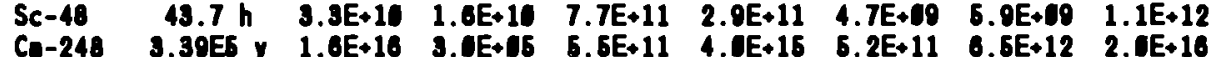

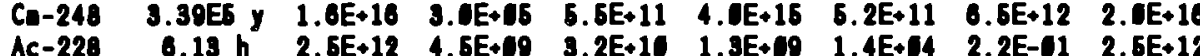

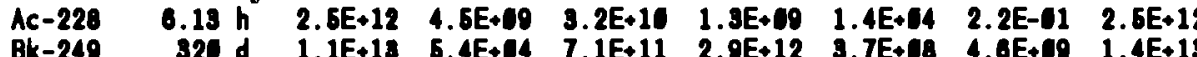

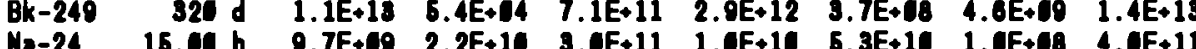

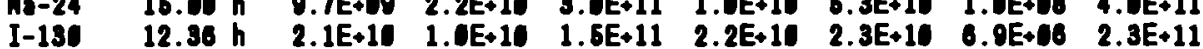

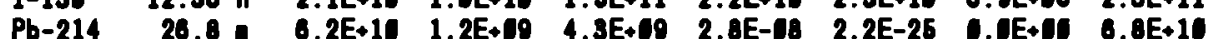

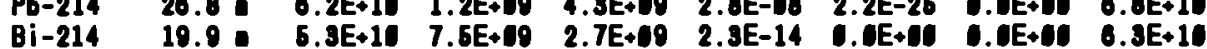

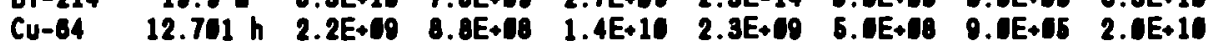

Cf-249 350.0 y $4.6 E+15 \quad 1.6 E+19 \quad 3.0 E+14 \quad 1.1 E+15 \quad 1.5 E+11 \quad 1.8 E+12 \quad 5.0 E+15$

$\begin{array}{llllllllll}C 0-47 & 4.63 & d & 5.2 E+10 & 6.1 E+10 & 6.8 E+11 & 7.1 E+11 & 2.4 E+12 & 6.6 E+10 & 3.9 E+12\end{array}$

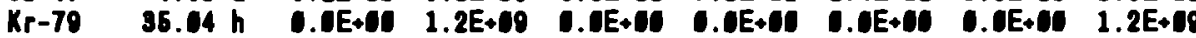

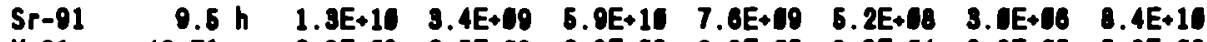

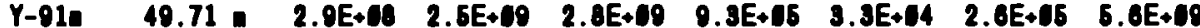

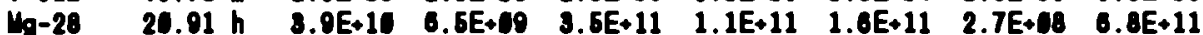

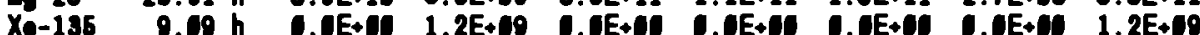

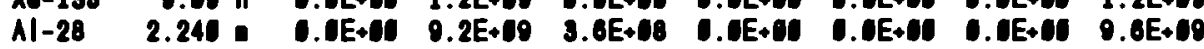

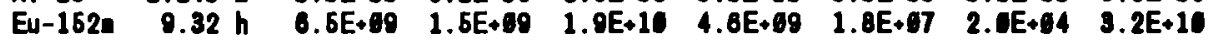

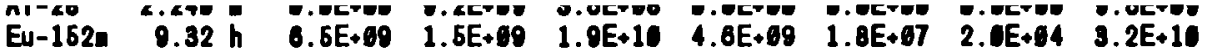

PAGE 5

ORIGEY (Ci)

Inventory Dose Factor

FP/AP/Ac+D (Total Dose Factor

Ges

600 NWD/T

2.0E-10

1.1E-12

2. 1 E- 19

2.3E-10

7.3E-15

8. $8 \mathrm{E}-15$

4.0E-18

1.1E-15

6.7E-10

3. 5E-16

1.7E-19

3. 2E-12

6.0E-13

6.5E-15

5.5E-15

4. $4 E-15$

1. $5 \mathrm{E}-19$

3. $5 \mathrm{E}-10$

4. $\mathrm{E}-17$

9.0 E-16

1.6E-15

5. $5 \mathrm{E}-15$

5. $5 E-15$

7.1E-15

7.7E-21

2. UE-18

4.1E-16

6. 3E-10

4.0E-10

1.3E-20

2. $2 E-18$

1. $3 E-20$
$2.4 E-23$

2.05-<E-23 ares-az
2. $0 \mathrm{E}+02$

1. $8 \mathrm{E}+\mathrm{Q} 2$

1. $5 E+112$

1.

$9.1 E+11$

2.5E+11

2.1E+U1

$5.1 E+01$

$2.2 E+00$

9.4E-11

5. 9E-U1

Q.1E-12

7.4E-12

$4.7 \mathrm{E}-12$

1.7E-12

4. 9 E-03

4.8E-13

3.1E-13

8.6E-04

5. 6E-C4

3. QE-14

8. BE- 14

7 .

3.7E-04

3. 5E-04

1. 4 E-C4

4.6E-05

7. OE-18

5. JE-10

5. 3E- 7

2. 2E- 19

2.2E- 10

Q. OE-DO

2.8E-68

1.3E-10

7.TE-13

7. 7 E-13 
APPENDIX E

MEASURED CONCENTRATIONS OF RADIONUCLIDES

IN THE COLUMBIA RIVER 



\section{APPENDIX E}

\section{MEASURED CONCENTRATIONS OF RADIONUCLIDES IN THE COLUMBIA RIVER}

J. K. Soldat (Draft 1969)

INTRODUCTION

The concentrations of about 70 radionuclides have been measured occasionally in samples of the cooling water leaving the Hanford production reactors and entering the Columbia River. These analyses, together with appropriate factors for dilution and radioactive decay, have been used to estimate the concentrations of the radionuclides in the river at Richland, Washington. This evaluation was done principally to generate an order of magnitude estimate of the concentrations of several radionuclides that might be present in the Columbia River at levels below their detection 1imit.

\section{RADIOCHEMICAL ANALYSES}

The three detailed radiochemical analyses of reactor effluent water available for the present study were performed in 1956, 1964, and February 1968. The results are summarized in Table I. (a) Not all 70 nuclides were reported in each sample. The 1968 data were used whenever possible. If no analysis was reported in 1968 or if the result was quoted as a less than value, the 1964 data were applied. The 1956 results were not used in the present analysis but were included for comparison with the later data. Note that these older data were reported as concentrations 4 hours after sample time, while the 1964 and 1968 results were corrected for radioactive decay back to time of sampling.

The different nuclides reported in each analysis depend, among other things, on the expertise of the laboratory personnel in interpreting gamma spectrometric analyses of the samples and upon the degree of chemical separation applied prior to this gamma analysis. Certain gamma energies present in

(a) The analytical data was furnished in a private communication from J. P. Corley to J. K. Soldat, March 20, 1969. 
trace amounts could easily be interpreted as different nuclides by different laboratories or by the same laboratories at different points in time.

Radiochemical analyses of certain radionucl ides are performed routinely on samples of Columbia River water collected at Richland, Washington. During 1968, the long lived nucl ides were measured in weekly and monthly cumulative samples, while the shorter-1ived nucl ides were measured in monthly grab samples. The results of these analyses for the sampling periods closest to the data of the effluent water analysis $(2 / 20 / 68)$ are presented in Table II.

\section{DILIION FACTOR}

The apparent dilution of the reactor effluent in the Columbia River was estimated by comparison of the concentrations measured at Richland and the corresponding concentrations measured in the effluent water. For the nuclides with half-lives of 2 weeks or less, account was taken of the amount of radioactive decay which had occurred between the discharge point and the measurement point. The ratio of concentrations in the river $(\mathrm{pCi} / \mathrm{L})$ to that in the effluent $(\mathrm{pC} \mathrm{i} / \mathrm{mL})$ was then calculated. Table III summarizes the results of this comparison.

The apparent dilution ratios in Table III range from 1 to 10 . Even though the ratio should depend upon the number of reactors operating, there is no large difference between the range of values for those nuclides for which 1968 data were available and for those for which 1964 data were appl ied. The average of the ratios listed in Table III is 6 . If those which seem to have been significant7y reduced by sedimentation and/or biological removal are deleted (e.g., ${ }^{46} \mathrm{Sc},{ }^{54} \mathrm{Mn}$, and ${ }^{56} \mathrm{Mn}$ ), the average becomes 8 . The one nuclide which is least affected by radioactive decay or biological uptake in the river is ${ }^{51} \mathrm{Cr}$ and $\mathrm{it}$ has the highest apparent dilution ratio $(-10)$. Three other nuclides have similar ratios. Those with smaller ratios are probably significantly removed from the river water by biological activity and/or sedimentation. The latter is true for ${ }^{46} \mathrm{Sc}$ which is one of the major contributors to river shoreline contamination. It seems appropriate therefore to use a ratio of 10 to calculate the concentrations in the river at the point of discharge. Concentrations further downstream can then be calculated assuming radioactive 
decay is the only removal process. This is obviously an over-simplification, which can result in values which are up to an order of magnitude too high, for those radionuclides that are accumulated in biota and/or sediment.

\section{$\underline{\text { RADIOACIIVE DECAY }}$}

By 1968, the majority of the effluent activity was being discharged to the river form the $100-\mathrm{K}$ reactor area. The river flow rate in late February 1968 was 75,000 cfs. ${ }^{(1)}$ The travel time in the river from the $100-\mathrm{K}$ reactor discharge point to the Richland sample point at the stated flow is 16 hours. (2) Prior to discharge into the river, the reactor effluent passes through effluent retention basins where a short delay time is experienced. This delay time is small compared to the travel time in the river and is within the range of errors present the travel-time estimate. Therefore, the total decay time between effluent sample analysis and Columbia River at Richland was taken to be 16 hours.

\section{$\underline{\text { RESULTS }}$}

The results of applying the appropriate dilution ratio and decay factors to the effluent concentrations, are shown in Table IV. The concentrations in the river at the point of discharge, assuming no radioactive decay or deposition in the effluent basins are shown in Column 2. Decay during travel through the basin is significant only for ${ }^{134} \mathrm{I}$ and ${ }^{138} \mathrm{Cs}$. The estimated concentrations in the river at Richland, Washington, are shown in Column 5, and the concentrations actually measured there are shown in Column 6 for comparison. Excluding those nuclides for which fallout of weapon test debris is a significant source $\left({ }^{3} \mathrm{H},{ }^{90} \mathrm{Sr}_{-}{ }^{90} \mathrm{Y}\right.$, and ${ }^{131_{\mathrm{I}}} \mathrm{I}$, there is reasonable agreement (within an order of magnitude) between the predicted and measured values. This is true even for those nuclides where the prediction is based on the 1964 effl uent analyses.

The values in Table $\mathbf{N}$ represent only an approximation to the concentrations at Richland at one point in time. The quantities of radionucl ides discharged to the river with the reactor effluent water vary with time. The river flow rate also varies widely throughout the year, affecting 1) the 
amount of water available for dilution, 2) the travel time from the point of discharge to Richland, and 3) the rate of deposition of certain nuclides to the river bottom and the shoreline mud. Thus, not only do the numerical values of the concentrations change, but also the relative abundance of the radionuclides can change with river flow rate.

\section{REFERENCES}

1. Woolridge, C. B., ed. "Environmental Surveillance in the Vicinity of Hanford for February 1968," BNWL-778-2, (Uncl assified), Apri1 30, 1968.

2. J. K. Soldat, "Columbia River Flow Time Calculations," HW-58312 Rev., (Uncl assified), July 1, 1962. 
TABLE 1- Concentrations of Several Radi onuclides in Reactor Effluent Water, $\mathrm{pCi} / \mathrm{mL}$

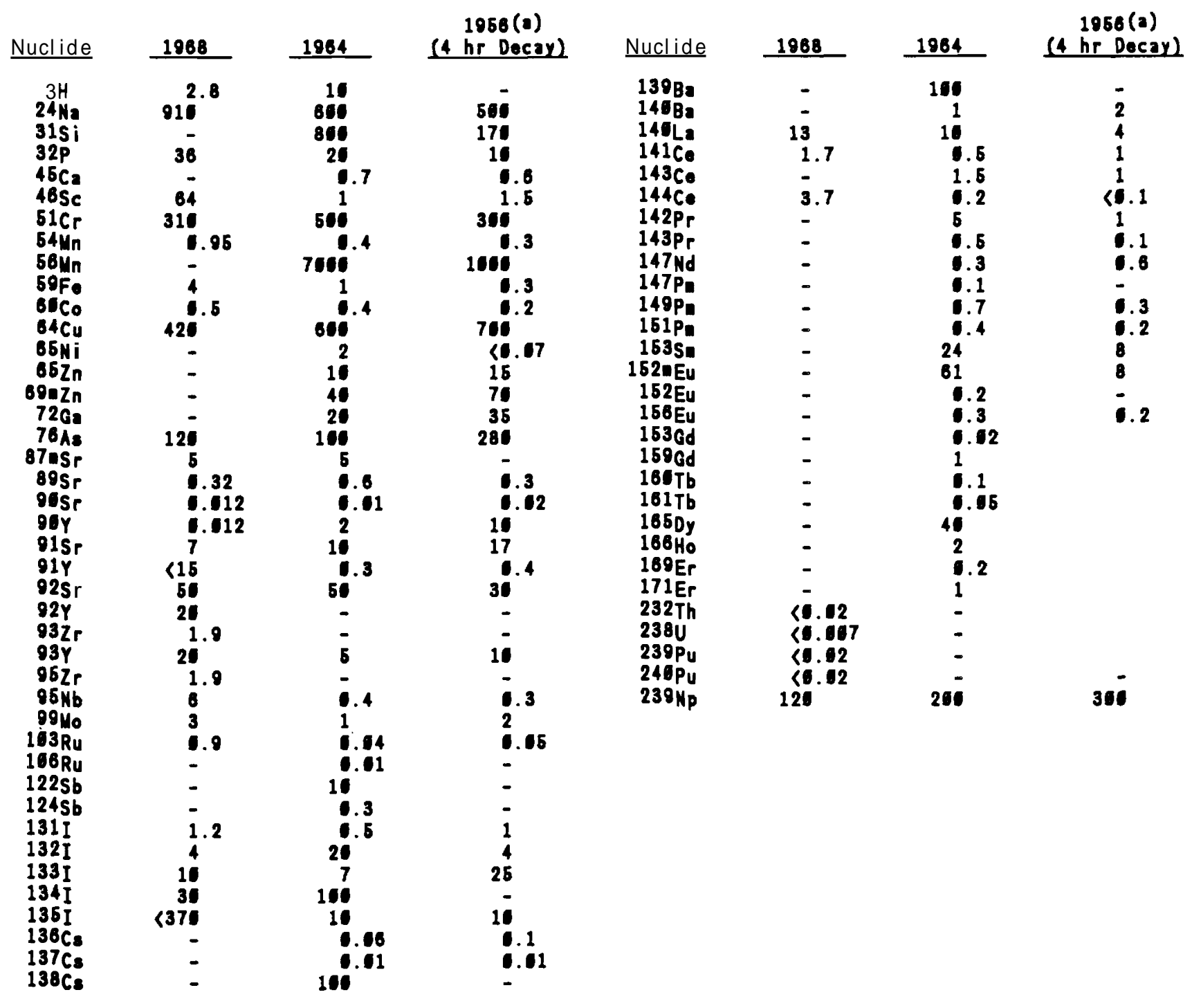
(a) 1958 data are reported as of 4 hours after sample time. The 1964 and $\mathbf{1 9 6 8}$ data were
corrected for radioactive decay back to the time of sample collection. 
TABLE II. Concentrations of Selected Radionuclides Measured in Columbia River at Richland, Washington (February 1968), pCi/L

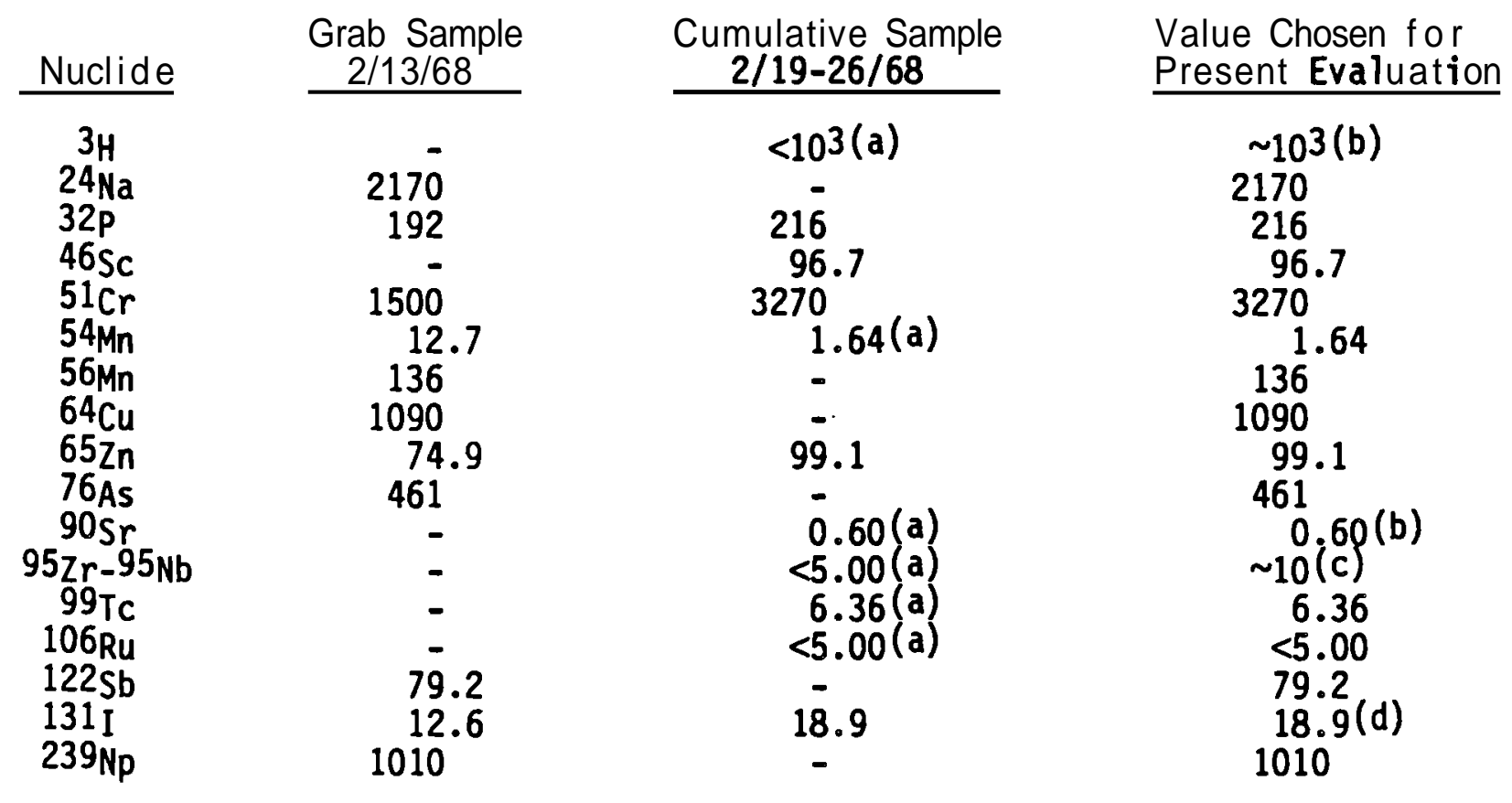

(a) Analysis performed on a monthly composite of the weekly cumulative samples.

(b) The source of $3 \mathrm{H}$ and $90 \mathrm{Sr}$ in the Columbia River is fallout of debris from weapons testing. The value for $3 \mathrm{H}$ for January was 1900 and for March was 1300.

(c) The results for the February monthly composite seem inordinately low.

The values for January and March were 7.4 and 12.8, respectively.

(d) Occasionally increased $131_{\text {I }}$ is also present from fallout. 
TABLE III. Estimated "Dilution Factors" for Reactor Effluent Water in the Columbia River

\begin{tabular}{|c|c|c|c|c|c|}
\hline \multirow[b]{2}{*}{ Nuclide } & \multicolumn{3}{|c|}{ Concentration in River, $\mathrm{PC} / \mathrm{L}$} & \multirow[b]{2}{*}{$\begin{array}{c}\text { Concentration } \\
\text { in Effluent, } \\
\mathrm{pCi} / \mathrm{mL} \\
\end{array}$} & \multirow[b]{2}{*}{$\begin{array}{c}\text { Ratio } \\
\text { River, } \mathrm{pCi} / \mathrm{L} \\
\text { Effluent, } \mathrm{pCi} / \mathrm{mL}\end{array}$} \\
\hline & $\begin{array}{c}\text { Measured } \\
\text { at Richland } \\
\end{array}$ & $\begin{array}{r}\text { Decay (a) } \\
\text { Correction } \\
\end{array}$ & $\begin{array}{c}\text { Calculated } \\
\text { at Discharge } \\
\text { Point } \\
\end{array}$ & & \\
\hline $24 \mathrm{Na}$ & 2170 & 0.477 & 4550 & 910 & 5.0 \\
\hline $32 p$ & 216 & 0.968 & 223 & 36 & 6.2 \\
\hline $46 \mathrm{Sc}$ & 96.7 & - & 96.7 & 64 & 1.5 \\
\hline${ }^{51} \mathrm{Cr}$ & 3270 & - & 3270 & 310 & 10.5 \\
\hline $54 \mathrm{Mn}$ & 1.64 & - & 1.64 & 0.95 & 1.7 \\
\hline $56 \mathrm{Mn}$ & 136 & 0.0140 & 9710 & $(7000)$ & $(1.4)$ (b) \\
\hline $64 \mathrm{Cu}$ & 1090 & 0.420 & 2600 & 420 & 6.2 \\
\hline $65 \mathrm{Zn}$ & 99.1 & - & 99.1 & (10) & (9.9) \\
\hline 76 As & 461 & 0.657 & 702 & 120 & 5.8 \\
\hline $122 \mathrm{Sb}$ & 79.2 & 0.848 & 93.4 & (10) & $(9.3)$ \\
\hline $239_{N p}$ & 1010 & 0.821 & 1230 & 120 & 10.0 \\
\hline
\end{tabular}

(a) For a travel time of 16 hours.

(b) Numbers () are based on 1964 data 
TABLE IV. Comparison of Estimated and Measured Concentrations of Radionual ides in Columbia River Water ( $\mathrm{pCi} / \mathrm{L}$ )

Estimated River Concentration

at Point

Nucl ide Discharge $\underline{\mathrm{t} 1 / 2(\mathrm{hr})} \quad \underline{\mathrm{e}^{-\lambda t(\mathrm{a})} \text { Richland }}$ Concentration

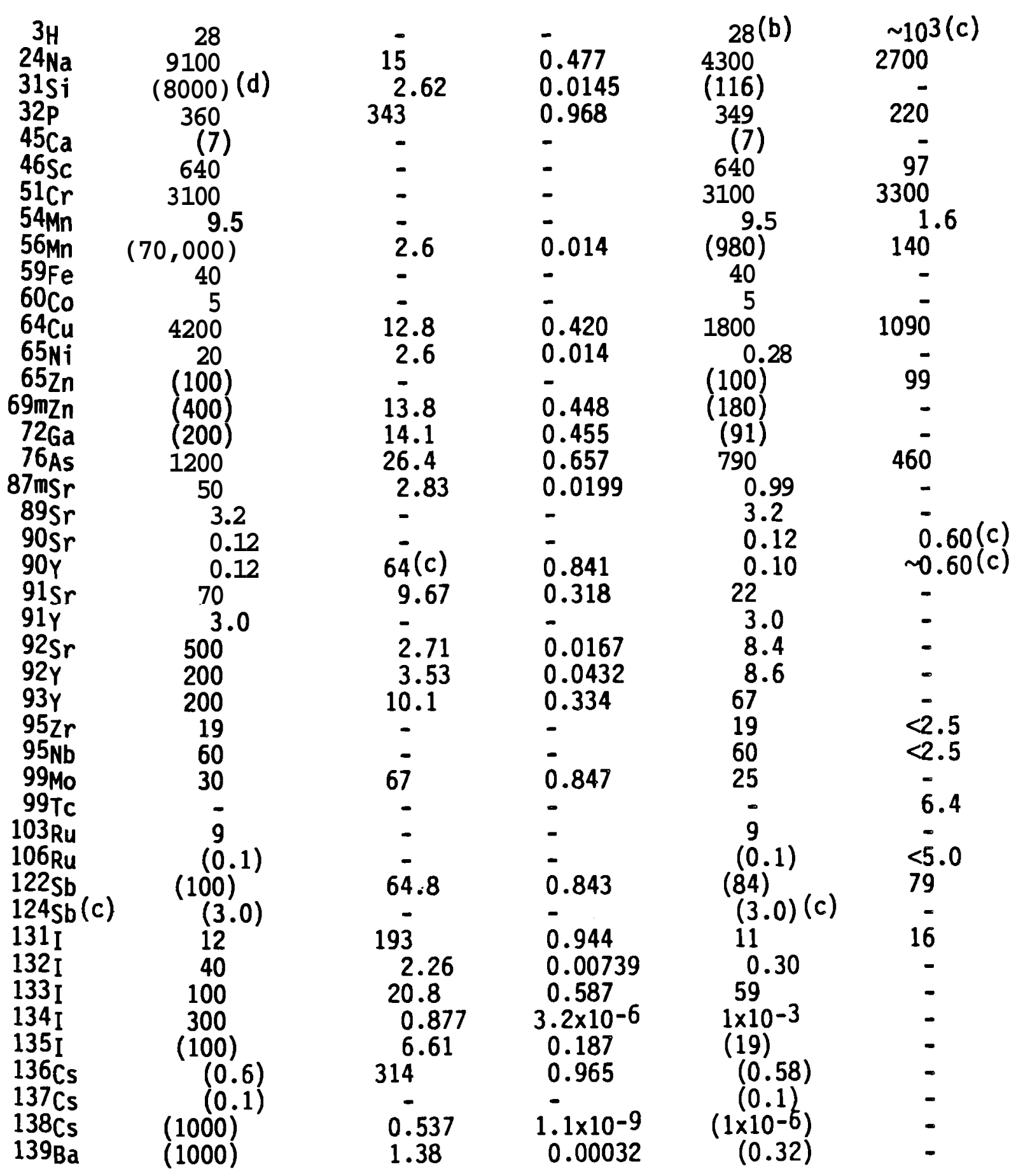


TABLE IV. (contd)

Estimated River Concentration

\begin{tabular}{|c|c|c|c|c|c|}
\hline Nucl ide & $\begin{array}{c}\text { of } \\
\text { Discharge }\end{array}$ & $\mathrm{tl} / 2(\mathrm{hr})$ & $e^{-\lambda t(a)}$ & $\begin{array}{c}\text { at } \\
\text { Richland }\end{array}$ & $\begin{array}{c}\text { Measured } \\
\text { Concentration }\end{array}$ \\
\hline $140 \mathrm{Ba}$ & (10) & - & - & (10) & - \\
\hline $140 \mathrm{La}$ & (30) & 40.2 & 0.759 & 99 & - \\
\hline $141 \mathrm{Ce}$ & 17 & - & & 1.7 & - \\
\hline $143 \mathrm{Ce}(\mathrm{c})$ & (15) & 33 & 0.715 & (11) & - \\
\hline $\begin{array}{l}144 \mathrm{Ce}(\mathrm{c}) \\
142 \mathrm{Pr}\end{array}$ & $\begin{array}{l}37 \\
50\end{array}$ & $\overline{19}_{2}$ & $\overline{0} .561$ & $\begin{array}{r}3.7 \\
(28)\end{array}$ & - \\
\hline $143 \mathrm{pr}$ & $(5.5)$ & 326 & 0.967 & $(4.8)$ & - \\
\hline $147 \mathrm{Pr}$ & (3) & 266 & 0.9592 & $(2.9)$ & - \\
\hline $147 \mathrm{Pm}$ & (1) & - & & (1) & - \\
\hline $149 \mathrm{Pm}$ & (7) & 53.1 & 0.812 & $(5.7)$ & - \\
\hline $151 \mathrm{Pm}$ & (4) & 27.8 & 0.671 & $(2.7)$ & - \\
\hline $153 \mathrm{sm}$ & (240) & 46.8 & 0.789 & (190) & - \\
\hline 152mEu & (610) & 9.3 & 0.303 & (180) & - \\
\hline $152 \mathrm{Eu}$ & (2) & & & (2) & - \\
\hline $156 \mathrm{Eu}$ & (3) & 365 & 0.970 & $(2.9)$ & - \\
\hline $153 \mathrm{Gd}$ & $(0.2)$ & - & & $(0.2)$ & - \\
\hline $159 \mathrm{Gd}$ & (10) & 18.0 & 0.540 & $(5.4)$ & - \\
\hline $160 \mathrm{~Tb}$ & $(1.0)$ & - & & (1) & - \\
\hline $161 \mathrm{~Tb}$ & $(0.5)$ & 166 & 0.935 & $(0.47)$ & - \\
\hline $165 \mathrm{Dy}$ & $(400)$ & 2.33 & 0.00857 & $(3.4)$ & - \\
\hline $166 \mathrm{HO}^{\circ}$ (c) & (20) & 26.9 & 0.662 & (13) & - \\
\hline $\begin{array}{l}169 \mathrm{Er} \\
171 \mathrm{Er}\end{array}$ & (10) & 223 & 0.951 & $(1.9)$ & - \\
\hline $232 \mathrm{Th}$ & $<0.2$ & $1.3<$ & 0.29 & $\begin{array}{l}(3.0) \\
<0.2\end{array}$ & $=$ \\
\hline $238 \mathrm{U}$ & $<0.07$ & - & - & $<0.07$ & $\sim 0.5(\mathrm{e})$ \\
\hline $239 \mathrm{pu}$ & $<0.2$ & 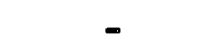 & - & $<0.2$ & - \\
\hline $240 \mathrm{Pu}$ & $<0.2$ & - & - & $<0.2$ & - \\
\hline $239 \mathrm{~Np}$ & 1200 & 56.5 & 0.822 & 990 & 1010 \\
\hline
\end{tabular}
(a) At a river flow of 75,000 cfs, the travel time is 16 hours.
(b) The February monthly average at Priest River Dam was $2500 \mathrm{pCi} / \mathrm{L}$; the January and March averages at Richland were 1900 and 1300, respectively.
(c) Essentially all from fallout.
(d) Values in () were obtained from 1964 data.
(e) Naturally occurring at $\sim 1 \mu \mathrm{g} / \mathrm{L}$. 
APPENDIX F

SUMMARY OF TSP COMMENTS AND BATTELLE RESPONSES 

SUMARY OF TSP COMMENTS AND BATTELLE RESPONSES

Document Number PNL-7'231 HER

Document Title Selection of Dominant Radionuclides for 


\section{SUMARY OF TSP COMMENTS AND BATTELLE RESPONSES}

Document Title Selection of Dominant Radionuclides for Phase I of the HEDR Project

\begin{tabular}{|c|c|c|c|c|}
\hline $\begin{array}{l}\text { Comment } \\
\text { Number }\end{array}$ & Commenter & $\begin{array}{c}\text { Page, } \\
\text { Paragraph }\end{array}$ & Comment Summary & Resolution \\
\hline 4. & $\mathrm{KLG}$ & & $\begin{array}{l}\text { The criteria for selecting the isotopes is not } \\
\text { clear. In the water pathway isotope tables, some } \\
\text { of the percentages add up to more than } 100 \% \text {, and } \\
\text { some to only } 80 \% \text { or so. }\end{array}$ & $\begin{array}{l}\text { term by applying monitoring data } \\
\text { for a number of years (thus } \\
\text { implicitly covering a range of } \\
\text { reactor power levels and fuel } \\
\text { conditions). A paragraph describ- } \\
\text { ing this matrix of investigations } \\
\text { has been added to Section } 3 \text {. } \\
\text { Tables } 4.1 \text { through } 4.8 \text { are pre- } \\
\text { pared from the detailed informa- } \\
\text { tion in Appendixes B and C. } \\
\text { Rounding of the detailed values } \\
\text { sometimes yields results that } \\
\text { slightly exceed } 100 \% \text {, but for } \\
\text { simplicity (and as an implicit } \\
\text { acknowledgment of the uncer- } \\
\text { tainty), only } 2 \text { significant fig- } \\
\text { ures are used in the tables. The } \\
\text { radionuclides were selected for } \\
\text { final recommendation based on both } \\
\text { the frequency in which they } \\
\text { appeared in the general tables in } \\
\text { Section } 4 \text { and the relative mag- } \\
\text { nitude of the doses presented in } \\
\text { the appendixes. A paragraph has } \\
\text { been added to Section } 4.2 \text { to } \\
\text { elucidate this concept. }\end{array}$ \\
\hline$N A=N_{0}$ & action. & & & \\
\hline
\end{tabular}


SUMMARY OF TSP COMMENTS AND BATTELLE RESPONSES

Document Number PNL-7231 HER

Document Title Selection of Dominant Radionuclides for

\begin{tabular}{|c|c|c|c|c|}
\hline $\begin{array}{l}\text { Comment } \\
\text { Number }\end{array}$ & Commenter & $\begin{array}{l}\text { Page, } \\
\text { Paragraph }\end{array}$ & Comment Summary & Resolution \\
\hline 5. & $\mathrm{KLG}$ & & $\begin{array}{l}\text { I am not convinced that it is wise to label many } \\
\text { of the isotopes "dominant" at this stage. There } \\
\text { is no doubt about the iodine- } 131 \text { being dominant, } \\
\text { but for the water-pathway isotopes, the term } \\
\text { seems rather arbitrary. }\end{array}$ & $\begin{array}{l}\text { NA - Of the potentially hundreds } \\
\text { of radionuclides released to the } \\
\text { Columbia River during historical } \\
\text { Hanford operations, at most a few } \\
\text { dozen contribute essentially all } \\
\text { of the dose. Those listed in the } \\
\text { tables in Section } 4 \text { or the recom- } \\
\text { mendation of Section } 5 \text { are gen- } \\
\text { erally those that actually do } \\
\text { "dominate" the dose to most peo- } \\
\text { ple, although some may contribute } \\
\text { only a few percent to the total } \\
\text { for any one individual. }\end{array}$ \\
\hline 6. & $\begin{array}{l}\text { G.S. } \\
\text { Roessler } \\
\text { (GSR) }\end{array}$ & & Tables in Appendix are hard to read. & $\begin{array}{l}\text { Appendix tables are direct com- } \\
\text { puter printouts. The only way to } \\
\text { make them clearer would be to } \\
\text { retype each page. }\end{array}$ \\
\hline 7. & GSR & & $\begin{array}{l}\text { A list of important elements and their abbrevia- } \\
\text { tions would be helpful for persons not intimately } \\
\text { familiar with all } 100+\text { elements. }\end{array}$ & $\begin{array}{l}\text { A I is t of abbreviations was added } \\
\text { on page xi. }\end{array}$ \\
\hline 8. & GSR & $\begin{array}{l}\text { Page ii i }, \\
\text { para. } 1\end{array}$ & Add TSP, Direction, etc. in paragraph. & Added. \\
\hline 9. & GSR & $\begin{array}{l}\text { Page } 1.1 \\
\text { para. } 1 \\
\text { line } 14\end{array}$ & Project, not Pproject & Corrected. \\
\hline$N A=N o$ & ction. & & & \\
\hline
\end{tabular}




\section{SUMMARY OF TSP COMMENTS AND BATTELLE RESPONSES}

Document Number PNL-7231 $\quad<D R$

Document Fitle Selection of Nominznt Rad ionuclidss for Phase I of the H\&Dळ Project

\begin{tabular}{|c|c|c|c|c|}
\hline $\begin{array}{l}\text { Comment } \\
\text { Number }\end{array}$ & Commsnter & $\begin{array}{l}\text { لage, } \\
\text { Paragraph }\end{array}$ & Oomment Summay & Resolwtion \\
\hline 10. & $\begin{array}{l}\text { B. Shl } \operatorname{Si} \text { on } \\
(\mathrm{BS})\end{array}$ & & $\begin{array}{l}\text { I am concern } d \text { by the lack of smphasis on ncer } \\
\text { tainty analysis and estimates of accwracy nd } \\
\text { precision which appear to be a sociated wi h the } \\
\text { Phase I results. }\end{array}$ & ND - Fwtvre work \\
\hline 11. & BS & & $\begin{array}{l}\text { Th following pathways need attention: Air- } \\
\text { Ra oout-Runoff-River Water-Irrigation-Backyard } \\
\text { Co -Milk-Humans, and Air-Rainout-Runoff- } \\
\mathrm{Ci}_{\text {O ? erns-Irrigation-Background Cow-Milk-Humans }}\end{array}$ & 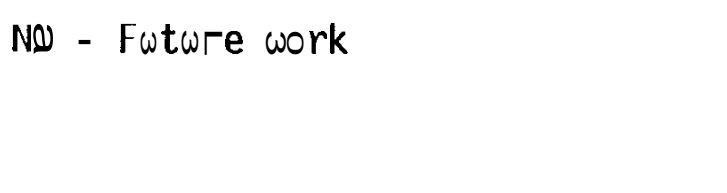 \\
\hline 12. & $3 S$ & & $\begin{array}{l}\text { If the dose cut-off limit is set low, say } 10 \text { mrem } \\
\text { whole body and } 30 \text { mrem thyroid, would we not have } \\
\text { to consider many more radionucl des? When would } \\
\text { this consideration occur? }\end{array}$ & $\begin{array}{l}\text { NA - Rationales for scoping } \\
\text { decisions wili be proposed for } \\
\text { fwtwre work. }\end{array}$ \\
\hline 13 & D Price & Nage 33 & $\begin{array}{l}\text { Te diet items are not we } 1 \text { defi, ned and many com- } \\
\text { mon fems ar missing. }\end{array}$ & 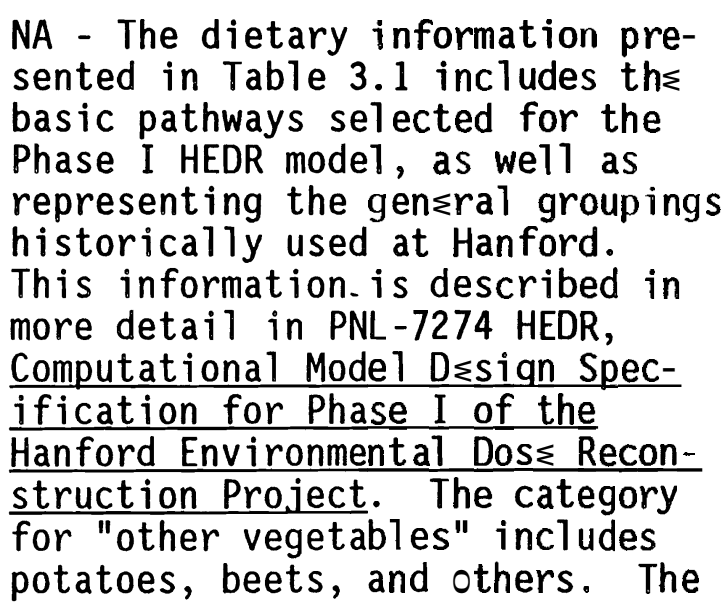 \\
\hline$N A=N_{0}$ & stion & & & \\
\hline
\end{tabular}

I am concern $d$ by the lack of emphasis on ncer tainty analysis and estimates of accuracy nd precision which appear to be associated wi $h$ the I results. Co -Milk-Humans, and Air-Rainout-Runoff-

If the dose cut-off limit is set low, say 10 mrem whole body and 30 mrem thyroid, would we not have to consider many more radionucl des? When would

Tre diet items are not well defu, ned and many comn tems ar missing. 


\section{SUMMARY OF TSP COMMENTS AND BATTELLE RESPONSES}

Document Number PNL-7231 HEDR

Document Title Selection of Dominant Radionuclides for Phase I of the HEDR Project

14.

G.G.

Caldwe 11

$(G G C)$

15

GGC

GGC

\begin{tabular}{l|l|l} 
& & Table 3.1 \\
& & \\
\hline
\end{tabular}

\section{6 ; page}

2.3, para.

2 , lines

2-5

Page vii, para. 1, 1 ines 14 22
These statements are true only for the transport by ground water to the river. How will well water be handled? nuclides, if only to explicitly indicate their small quantity and relative unimportance.

Does "drinking water" include that from both the river and wells?

\section{Resolution}

"milk" category includes butter, cheese, and other milk products. The holdup times assigned to each pathway deal with the fractionation between fresh and stored. The diet is derived from Hanfordrelated dietary surveys performed in the 1950s and 1960s.

NA - Future work in FY 1991.

NA - Selection of "biologically important" radionuclides depends on the pathways being considered and the interests of the reader. They are all included in Appendixes $B$ and $C$. Inclusion here is not feasible without greatly increasing the complexity of the summary.

$N A$ - In the instance referred to, only river water is included. Groundwater is not explicitly 
SUMARY OF TSP COMMENTS AND BATTELLE RESPONSES

Document Number PNL-7231 HEDR

Document Title Selection of Dominant Radionuclides for Phase I of the HEDR Project

M.A. Robk in (MAR)

18. MAR
Page 2.1

17.

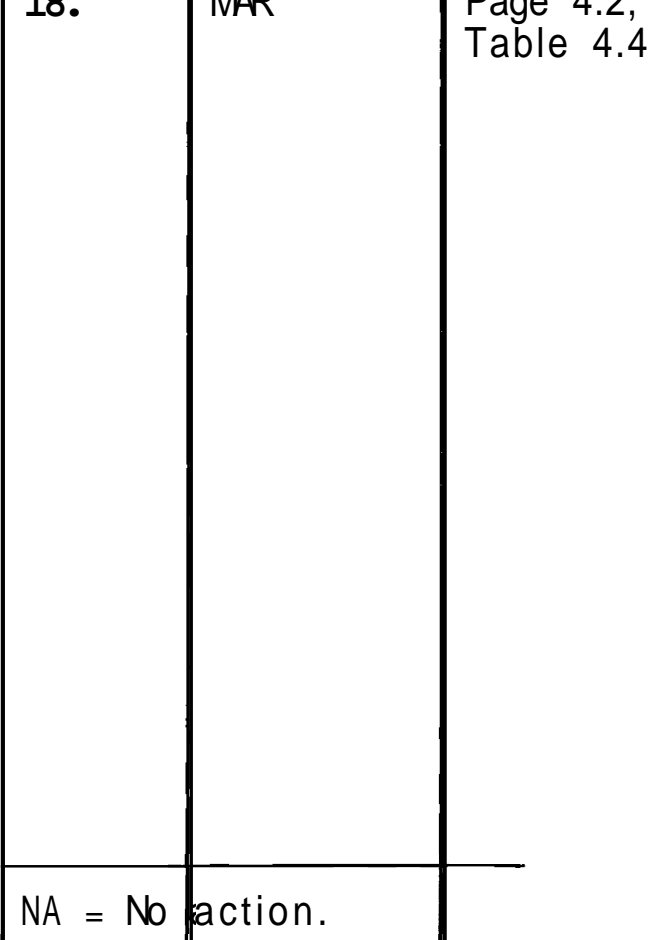

The statement of how radionuclide quantities are determined is not complete. The quantities of short-1ived radionuclides are determined by the fuel power level.

The importance of the milk pathway is not clear from the table, which shows its contribution to the dose from iodine-131 to be only about $6 \%$.

\section{Resolution}

treated in this report, and drinking from riparian wells should be bounded by doses from drinking from the river itself.

A sentence has been added to complete the discussion.

NA - The tables show only relative magnitude for each exposure scenario, not absolute magnitude. The percentages can be added downwards only, not across. What is indicated in Table 4.4 is that, even without including milk, iodine dominates the dose through inhalation and crop ingestion.

The absolute results presented in Appendix $B$ indicate that the milk pathway for these scenarios could provide over $60 \%$ of the dose to an individual. 
SUMMARY OF TSP COMMENTS AND BATTELLE RESPONSES

Document Number PNL-7231 HEDR

Document Title Selection of Dominant Radionuclides for Phase I of the HEDR Project

\begin{tabular}{|c|c|c|c|c|}
\hline $\begin{array}{l}\text { Comment } \\
\text { Number }\end{array}$ & Comm $<n t \varepsilon r$ & $\begin{array}{l}\text { Page, } \\
\text { Daragraph }\end{array}$ & Coinm nt Summary & Resolution \\
\hline 19. & $\begin{array}{l}\text { M. L. } \\
\text { Bi azek }\end{array}$ & Pag $\leq 31$ & $\begin{array}{l}\text { The "highly desirable additions" should be added } \\
\text { as well as iodine-131 in Phase I. }\end{array}$ & $\begin{array}{l}\text { NA - Thw nine river pathway radio } \\
\text { nuclides stated in the text were } \\
\text { studigd in Phase I. Iodine-131 } \\
\text { will } \$ \text { studied for the river } \\
\text { pathway in future work. }\end{array}$ \\
\hline 20. & A. Sic & & Is this a final determination? & $\begin{array}{l}\text { No; future work will determine } \\
\text { contribution of other radio- } \\
\text { nuclides to dose. }\end{array}$ \\
\hline 21. & 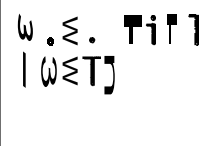 & & $\begin{array}{l}\text { Insert tables and figures in the text after } \\
\text { callout, rather than at end of report. }\end{array}$ & $\begin{array}{l}\text { Tables and figures now follow } \\
\text { their callouts as closely as } \\
\text { possible. }\end{array}$ \\
\hline 22. & $\begin{array}{r}\omega E \\
1\end{array}$ & $\begin{array}{l}\text { Psge vii, } \\
\text { line } 5\end{array}$ & "to the environment from Hanford..." & Wording was changed. \\
\hline 23. & WET & $\begin{array}{l}\text { Psge vii, } \\
\text { line } 10\end{array}$ & "1964-1966 for releases to surface water..." & Wording was changed. \\
\hline 24. & WET & $\begin{array}{l}\text { Psge vii, } \\
\text { line } 14\end{array}$ & $\begin{array}{l}\text { Make it clear in the summary that we are refer- } \\
\text { ring to effective dose equivalent. This is } \\
\text { important to understand for the } 1944-47 \text { releases. }\end{array}$ & $\begin{array}{l}\text { A sentence has been added to the } \\
\text { Summary in response to this } \\
\text { comment. }\end{array}$ \\
\hline 25. & $\omega<T$ & Pag $\Sigma$ vi & $\begin{array}{l}\text { Sixth lino from bottom: Again, say "releases to } \\
\text { surface wstor..." }\end{array}$ & Wording was changed. \\
\hline 26. & WET & $\begin{array}{l}\emptyset \exists g \leq 2.1 \\
\infty \text { sra } 2\end{array}$ & $\begin{array}{l}\text { Begin first sentence with "The results of the } \\
\text { study to date indicate the largest releases ..." }\end{array}$ & Change was made. \\
\hline$N A=N_{0}$ & action & & & \\
\hline
\end{tabular}


SUMMARY OF TSP COMMENTS AND BATTELLE RESPONSES

Document Number PNL-7231 HEDR

Document Title Selection of Dominant Radionuclides for Phase I of the HEDR Project

\begin{tabular}{|c|c|c|c|c|}
\hline $\begin{array}{l}\text { Comment } \\
\text { Number }\end{array}$ & Commenter & $\begin{array}{c}\text { Page, } \\
\text { Paragraph }\end{array}$ & Comment Summary & Resolution \\
\hline 27. & JET & $\begin{array}{l}\text { Page 2.3, } \\
\text { para. } 3\end{array}$ & $\begin{array}{l}\text { Second sentence: Define "processed," and specify } \\
\text { "brief." }\end{array}$ & $\begin{array}{l}\text { The sentence has been revised to } \\
\text { address this comment. }\end{array}$ \\
\hline 28. & JET & Page 2.4 & $\begin{array}{l}\text { First sentence: } \quad 70 \text { additional radionuclides } \\
\text {..." }\end{array}$ & Change was made. \\
\hline 29. & JET & $\begin{array}{l}\text { Page } 3.1, \\
\text { para. } 3, \\
\text { line } 2\end{array}$ & $\begin{array}{l}\text { We usually refer to "immersion" in air rather } \\
\text { than submersion. }\end{array}$ & $\begin{array}{l}\text { NA - At Hanford, the terminology } \\
\text { has historically been "submersion" } \\
\text { when describing air, and "immer- } \\
\text { sion" when describing water. This } \\
\text { distinction has been retained for } \\
\text { continuity with the historical } \\
\text { documents. }\end{array}$ \\
\hline 30. & JET & $\begin{array}{l}\text { Page } 4.1 \\
\text { para. } 4\end{array}$ & $\begin{array}{l}\text { Figures of the data in Tables } 4.1 \text { and } 4.2 \text { would } \\
\text { be useful. }\end{array}$ & $\begin{array}{l}\text { Figures } 4.1 \text { and } 4.2 \text { have been } \\
\text { added in response to this comment. }\end{array}$ \\
\hline 31. & JET & $\begin{array}{l}\text { Page 4.2, } \\
\text { para. 2, } \\
\text { line } 3\end{array}$ & "radionuclides" & Corrected. \\
\hline 32. & JET & $\begin{array}{l}\text { Page 4.3, } \\
\text { Table } 4.3\end{array}$ & What is meant by NCRP Screening Fractions? & $\begin{array}{l}\text { NA - The headings read "NCRP } \\
\text { Screening, Release Fractions" and } \\
\text { "NCRP Screening, No Release Frac- } \\
\text { tions", as described in Section } \\
4.1 .\end{array}$ \\
\hline 33. & JET & $\begin{array}{l}\text { Page 4.8, } \\
\text { Table } 4.8\end{array}$ & $\begin{array}{l}\text { Did you carry out a similar evaluation for a } \\
\text { pulse release to air? }\end{array}$ & $\begin{array}{l}\text { NA - No acute release calculations } \\
\text { were performed for the air. }\end{array}$ \\
\hline$N A=N_{0}$ & action. & & & \\
\hline
\end{tabular}


SUMMARY OF TSP COMMENIS AND BATTELLE RESPONSES

Document Number PNL-7'231 HEDR

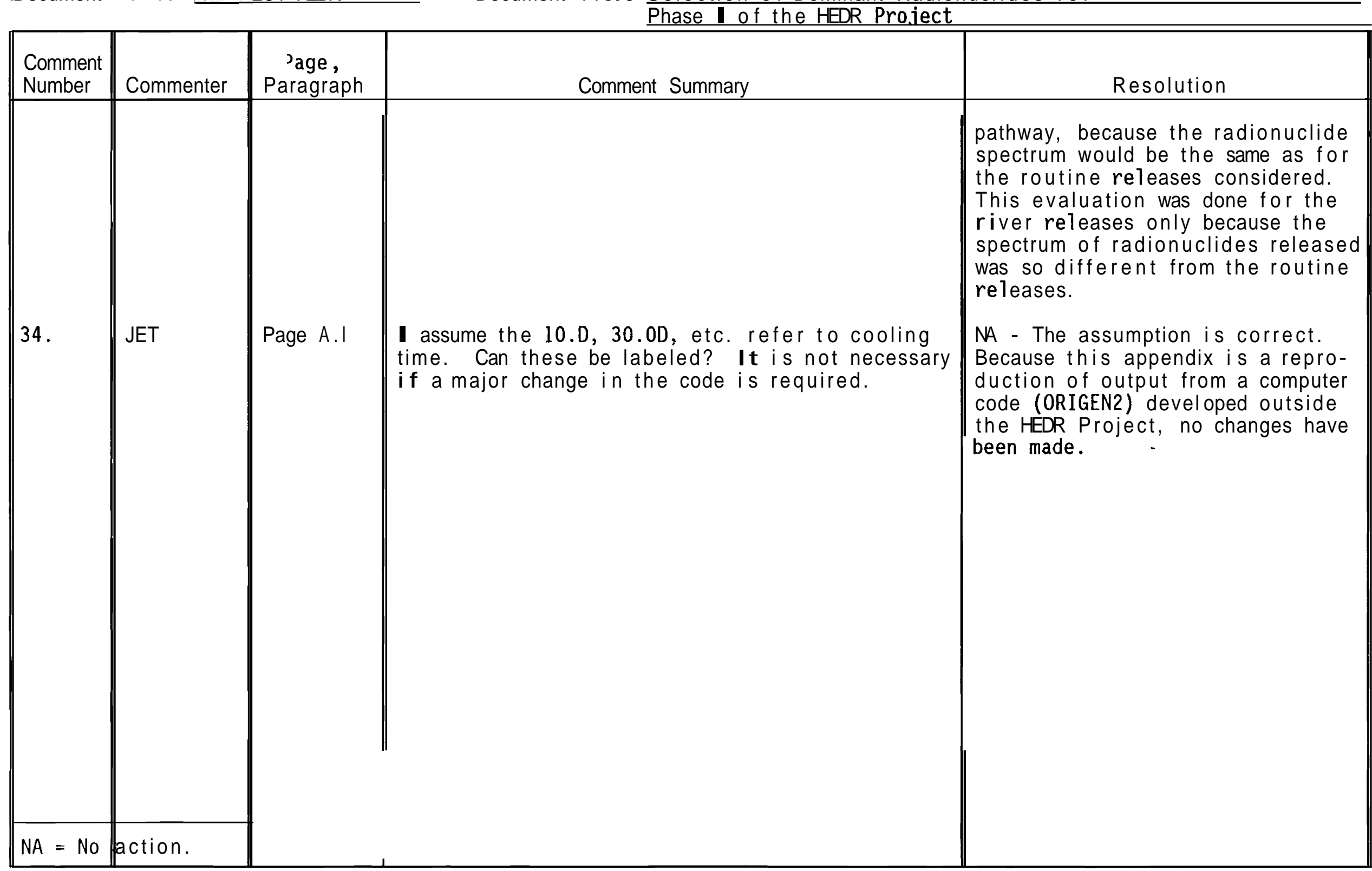



DISTRIBUTION

No. of

$\underline{\text { Copies }}$

\section{OFFSITE}

18 Technical Steering Panel

D. S. Barth

University of Nevada

4505 Maryland Parkway

Las Vegas, N 89154

W. A. Bishop

2503 Wedgewood Court SE

Olympia, WA 98501

M. L. Blazek

Oregon Department of Energy

625 Marion Street N.E.

Salem, OR 97310

G. G. Caldwell

Director

Tulsa C ity-County Health Dept

4616 East 15th Street

Tulsa, OK 74112

S. N. Davis

Dept. of Hydrology \& Water Resources

Bldg. 11

University of Arizona

Tucson, AZ 85721

N. J. Germond

224 Iron Mountain Blvd.

Lake Ozwego, OR 97034

P. C. Klingeman

Civil Engineering Dept.

Oregon State University

Corvallis, OR 97331-2302
No. of

Copies

K. J. Kopecky

Fred Hutchinson Cancer

Research Center

1124 Columbia Street

Seattle, WA 98104

R. L. Morrill

Dept. of Geography

DP-10

University of Washington

Seattle, WA 98195

A. H. Murphy

c/o Climate Analyses Center

National Weather Service, NOAA

W/NMC51, WWB Room. 604

Washington, DC 20233

V. Nguyen

EWA Inc.

133 1st Ave. N.

Minneapolis, MN 55401

D. W. Price

Agricultural Economics

Hulbert Hall Room 211

Washington State University

Pullman, WA 99164-6210

M. A. Robkin

Radiological Sciences SB-75

University of Washington

Seattle, 98195

G. S. Roessler

Rt. 1, Box 139H

Elysian, MN 56028 
No. of

Copies

B. Shleien

2421 Homestead Drive

Silver Springs, MD 20902

A. P. Slickpoo, Sr.

P. 0. Box 331

809 Nez Perce Lane

Kamiah, ID 83536

J. E. Till

Rt. 2 Box 122

Neeses, SC 29107

D. E. Walker, Jr.

P.O. Box 4147

Boulder, CO 80306

2 DOE Office of Scientific and Technical Information

Technical Information Center

P.O. Box 62

Oak Ridge, TN 37830

18 K. CharLee

Office of Nuclear Waste Mgmt.

Department of Ecology

99 South Sound Center

Mail Stop PV-11

Olympia, WA 98504
No. of

Copies

3 M. Sage

Centers for Disease Control

$1600 \mathrm{Clifton}$ Road

At1anta, GA 30333

J. Thomas, HEAL

1720 N. Ash

Spokane, 99205

2 DOE Richland Operations

R. F. Brich, SED

A5-55

M. W. Tiernan, SED

A5-55

$34 \quad$ Pacific Northwest Laboratorv

J. M. Daer (10)

R. 0. Gilbert

W. A. Glass (6)

S. P. Gydesen

A. H. McMakin

B. A. Napier (5)

W. L. Templeton

Public Reading Room (5)

Publishing Coordination

Records Center

Technical tibrary

(2)

K6-89

K7 -34

K4-13

P8-55

K6-86

K3-54

$\mathrm{K} 1-30$

Al- 65

$\mathrm{K} 1-11$

K3-70

P8-55 